Drug Metabolism Reviews

ISSN: 0360-2532 (Print) 1097-9883 (Online) Journal homepage: https://informahealthcare.com/loi/idmr20

\title{
Poster Abstracts
}

To cite this article: (2011) Poster Abstracts, Drug Metabolism Reviews, 43:sup2, 37-211, DOI: 10.3109/03602532.2011.629832

To link to this article: https://doi.org/10.3109/03602532.2011.629832

曲 Published online: 07 Nov 2011.

Submit your article to this journal $₫$

Џll Article views: 7813 


\title{
Poster Abstracts
}

\section{P1. A method for monitoring drug exposure in hematopoietic stem cell transplant recipients}

\author{
$\underline{\text { Isabelle Laverdiere }}^{1}$, Patrick Caron ${ }^{2}$, Félix Couture $^{3}$, Éric Lévesque ${ }^{4}$ and Chantal Guillemette ${ }^{5}$ \\ ${ }^{1}$ Laval University, CHUL Research Center, Quebec, QC, Canada, ${ }^{2}$ CHUL Research Center, Quebec, QC, Canada, \\ ${ }^{3}$ Hematology-oncology, Hôtel-Dieu de Québec Hospital, Québec, QC, Canada, ${ }^{4}$ Pharmacogenomics Laboratory, \\ CHUQ Research Center, Québec, QC, Canada, ${ }^{5}$ Pharmacogenomics Laboratory, CHUQ Research Center and Faculty of \\ Pharmacy, Laval University, Québec, QC, Canada
}

The use of combinations of immunosuppressant drugs is considered the therapeutic gold standard for post-allogeneic hematopoietic stem cell transplantation (HSCT) to prevent serious complications such as graft versus host disease and rejection. However, these drugs have a narrow therapeutic index and wide inter-individual pharmacokinetic fluctuations, resulting in unpredictable levels of drugs in the blood. Systemic concentrations of several immunosuppressive drugs have been correlated with their efficacy and potential life-threatening complications, thus supporting the need for a pharmacokinetic monitoring. Some chromatographic techniques have been proposed for the simultaneous monitoring of multiple immunosuppressive drugs, but none offers the possibility of measuring cyclosporine, tacrolimus, methotrexate, prednisone, prednisolone, and methylprednisone in addition to total and free mycophenolic acid (MPA) and its phenolic (MPAG) and acyl (AcMPAG) glucuronides. To address this critical deficiency, a liquid chromatography-coupled tandem mass spectrometry method was developed for the quantification of circulating levels of these multiple immunosuppressant drugs. Linearity, precision and accuracy were validated within the typical therapeutic range of concentrations for each compound. The method could measure individual drugs with high sensitivity, accuracy (bias $<14 \%$ ), and reproducibility $(\mathrm{CV}<12.8 \%)$. Its clinical application was validated by measuring levels of these drugs in samples obtained from HSCT recipients treated with combined immunosuppressive drug therapy. Our results indicate that this approach is suitable for simultaneous determination of in vivo levels of immunosuppressive drugs commonly used in combined therapies. This method requires a small-volume peripheral blood sample, an attribute that may be beneficial to HSCT patients in the clinic. Indeed, owing to the frequent administration of myeloablative conditioning regimens in the pre-transplantation period, the recovery of hematologic function is slow and gradual after engraftment. It is therefore favorable to limit blood sample collection in the immediate post-transplantation period. This method may be convenient for large-scale clinical studies aimed at improving and optimizing patient outcomes. It could also be useful in therapeutic drug monitoring to promote the efficient use of immunosuppressive drugs and thus to limit life-threatening complications related to non-optimal exposure to immunosuppressive medications.

\section{P2. Abstract withdrawn}

\section{P3. Absolute UGT protein quantification by LC-MS3}

\author{
$\underline{\text { Richard King }^{1} \text {, Carmen Fernandez-Metzler }}{ }^{1}$ and J. Larry Campbell ${ }^{2}$ \\ ${ }^{1}$ PharmaCadence Analytical Services, LLC, Hatfield, PA, ${ }^{2} A B$ SCIEX, Concord, ON, Canada
}

The absolute quantification of proteins involved in drug metabolism is a new area that is gaining in popularity and utility. By far the most often used strategy is the surrogate peptide approach. These methods rely on detection of peptides created from the protein of interest by enzymatic or chemical digestion. Typically, the protein of interest and a stable isotopically labeled internal standard peptide are digested in a relevant sample such as a microsomal fraction or plasma containing often large amounts of other proteins. The final digested sample may contain thousands of peptides derived from any combination of the proteins present in the sample matrix. The complexity of the digested sample makes selective detection a challenge, even 
by LC-MS/MS. Selected reaction monitoring (SRM) of HPLC separated samples is most often considered the gold standard for selectivity; however, SRM often does not have the selectivity needed to allow absolute quantification of surrogate peptides from biological matrices. Vastly improved selectivity can be realized using MS3. MS3 is a term used to describe multiple steps of mass dependent isolation and fragmentation followed by mass analysis and detection. The extra step of isolation and fragmentation associated with MS3 provides the enhanced selectivity of the technique. In this presentation, we discuss the implementation of MS3 with 40ms cycle time on the 5500QTRAP and we compare the quantitative results for six UGT isoforms measured from human liver microsomes using selected reaction monitoring and MS3. The work was done using the ABSciex QTRAP 5500 mass spectrometer and an Eksigent HT Express HPLC with $1.0 \mathrm{~mm}$ ID $\times 30 \mathrm{~mm}$ C18 reverse phase column and gradient elution. The enhanced selectivity of MS3 improves assay precision as measured by \%CV and the assay LLOQ as compared to SRM. The improvement in signal to noise also makes automated data processing of the MS3 data for quantitative analysis of protein practical. The time required to integrate the MS3 mass chromatograms and provide quantitative results is approximately $30 \%$ of the time required to process the SRM data for the same samples.

\title{
P4. Application of the narrow window mass extraction technique for in vitro adme screening in drug discovery
}

\author{
Barry Press, Rima Al-Awar and Ahmed Aman
}

\section{Medicinal Chemistry, Ontario Institute for Cancer Research, Toronto, ON, Canada}

Incorporating higher throughput analytical methods and enhancing the ability for data mining of in vitro ADME samples can greatly benefit drug discovery teams during lead optimization. Standard bioanalytical techniques generally rely on triple quadrupole mass spectrometers using the technique of multiple reaction monitoring (MRM) for quantification (QUAN). Although this conventional MRM approach can provide high specificity and sensitivity, this practice is also time-consuming since it is a two-step process and requires the optimization of multiple parameters (cone voltage, collision energy, and Q1/Q3 m/z values) for each and every compound being analyzed. Furthermore, MRM techniques only detect the initial compound of interest and data mining for other, qualitative (QUAL) information (such as metabolite formation) is not possible. The use of a sensitive hybrid Q-Tof instrument in a full scan acquisition mode and subsequent narrow window mass eliminates the need for compound optimization while also providing both qualitative and quantitative (QUAL/QUAN) data to assist chemistry efforts in structure-property-relationships (SPR). The Xevo-Q-TOF mass spectrometer is a high resolution accurate mass instrument which possesses the speed, sensitivity, linearity, and wide mass range needed to screen in vitro ADME samples. The high resolution capabilities of Q-TOF-MS, combined with mass defect filtering and monitoring of isotopic peak patterns, allows for the discrimination against background noise when performing narrow window mass extraction from a full scan analysis. Samples were generated from multiple ADME assays including cellular (Caco-2) permeability, microsomal and hepatocyte stability, plasma stability, protein binding, and CYP450 inhibition of (known) metabolite formation. Chromatographic separations were obtained utilizing an Acquity UPLC and generic gradient conditions. Quantitative results obtained by narrow $\mathrm{m} / \mathrm{z}$ windows were compared with traditional MRM analysis of the same samples analyzed using the high-sensitivity QTRAP 5500 system.. Data mining was then performed on the full scan analysis to identify known metabolites such as glucuronide, de-methylated, and hydroxylated metabolites from metabolic assays, as well as to determine possible non-hepatic compound instability and isomerization from in vitro plasma stability and permeability samples. Results indicate that the use of the Xevo-QTOF, combined with narrow window mass extraction and full scan analysis, gives comparable quantitative data (e.g. percent loss of parent compound) to conventional MRM techniques without the need for time-consuming compound optimization of new chemical entities (NCEs) or known CYP-dependent metabolites. In addition, valuable qualitative information could be obtained only from Xevo-Q-TOF full scans showing the susceptibility of reference compounds and NCEs for Phase I oxidative metabolism, Phase II conjugation, and non-hepatic, compound instability.

\section{P5. Comparison of high resolution accurate mass spectrometry and triple quadrupole MRM in quantitative bioanalysis}

\author{
Yuan-qing Xia ${ }^{1}$, Ragu Ramanathan ${ }^{2}$, Suma Ramagiri ${ }^{3}$, Jeffrey D. Miller ${ }^{1}$ and Mohammed Jemal ${ }^{4}$ \\ ${ }^{1}$ AB SCIEX, Framingham, MA, ${ }^{2}$ Biotransformation, Bristol-Myers Squibb, Princeton, $N J,{ }^{3} M D S$ Analytical Technologies, \\ Concord, ON, Canada, ${ }^{4}$ Bioanalytical Discovery Analytical Sciences, Bristol-Myers Squibb, Princeton, NJ
}

Novel Aspect: Comparison of results acquired using full-scan high resolution MS and triple quadrupole MRM in quantitative bioanalysis to support DMPK in early drug discovery. Introduction: A new paradigm shift using full scan 
high-resolution mass spectrometry (HRMS) for simultaneously qualitative and quantitative analysis of drugs and their metabolites has become a very promising approach in assessing DMPK properties for drug discovery analyses. Unlike the targeted acquisition approach using triple quadrupole MRM, the HRMS using time-of-flight MS provides large volume of data that can be used in post-acquisition data mining to obtain both qualitative and quantitative results related to the drugs, metabolites and biomarkers. Herein, we present our evaluation of the performance of full scan HRMS and triple quadrupole MRM for quantification of drugs in plasma, comparing sensitivity, selectivity, accuracy/precision, and ruggedness. Methods: 25 model drug compounds marketed and non-marketed including buspirone, nafazadone, prednisolone, prednisone and reserpine were used. Acetonitrile-precipitated plasma samples were injected onto a LC-MS system. A QqTOF (TripleTOFTM 5600 system) was operated in full scan MS mode with scan range of $\mathrm{m} / \mathrm{z} 100-\mathrm{m} / \mathrm{z}$ 1000, and full scan MS followed by information dependent acquisition (IDA) triggered scan MS/MS. All the experiments maintained resolution of $\sim 30,000$ or greater. A triple- quadrupole (Thermo TSQ Quantum) mass spectrometer was operated in ESI mode with specific MRM transitions for the model compounds. Preliminary Data: The lower limits of quantitation (LLOQ) level for each analyte, using HRMS or MRM, were determined based on accuracy and precision, analyte-signal-to- background ratio, and response/concentration proportionality. Similar or even better LLOQ and linear dynamic ranges were obtained using QqTOF in full scan HRMS. Of the 25 compounds evaluated, QqTOF full scan HRMS achieved LLOQ of $0.2 \mathrm{ng} / \mathrm{mL}$ for 18 compounds, and $1-2 \mathrm{ng} / \mathrm{mL}$ for 4 compounds and $5 \mathrm{ng} / \mathrm{mL}$ for 2 compounds. The mass accuracy was better than $\pm 5 \mathrm{ppm}$. Sufficient data points across chromatographic peaks with peak width of 3 seconds were observed. The accuracy/precision and ruggedness achieved were comparable for the two methods. A similar result was obtained using TOF-MS IDA $2 \mathrm{MS} / \mathrm{MS}$ scans, and it demonstrated the feasibility of performing such Qual/Quant assays. Therefore, one instrument and one sample can be used for obtaining qualitative and quantitative information of ECNs in support of drug discovery.

\title{
P6. Development of collagenase as an effective tool in tissue homogenization for bioanalytical drug quantitation
}

\author{
Ann Ran-Ran Qin, Stephanie Dale, Brian Dean and Sheerin Shahidi-Latham
}

\section{Drug Metabolism and Pharmacokinetics, Genentech Inc., South San Francisco, CA}

A bioanalytical group's function is to extract and quantitate the amount of drug present in a set of samples. While compounds are usually extracted from plasma, other matrices may be analyzed as well, depending on the specific target of the project. Some tissue matrices are easy to homogenize when using beat beater or polytron devices, only taking a few seconds to a few minutes. While these devices are effective for soft tissues, such as brain or tumor, they are not as effective for tough fibrous tissues, such as the more challenging skin, muscle and heart. To combat these tissues, a novel approach for tissue homogenization was developed using the enzyme collagenase, purified from the fermentation of Clostridium histolyticum bacteria (Collagenase Sigma Product Information), to break down the collagen fibers present in epithelial and connective tissue. Prior experiments have demonstrated that enzymatic digestion alone on brain tissue is a good alternative of sample preparation for LC/MS/MS analysis (Chongwoo Yu, L.D.P., John Hollembaek, Wenlin Li, Lucinda H. Cohen (2004). "Enzymatic Tissue Digestion as an Alternative Sample Preparation Approach for Quantitative Analysis Using Liquid Chromatography-Tandem Mass Spectrometry." Analytical Chemistry 76(6): 17611767). In this experiment, the benefits of tissue homogenization using bead beater with the help of collagenase as opposed to using bead beating alone was explored, as well as studying the matrix and stability effects on compounds with different physical chemical properties. Collagenase solution was incubated with a variety of difficult matrices, including heart, skeletal muscle and skin. The tissue and collagenase mixture were incubated overnight at room temperature, while another set of samples were heated and agitated in a $37^{\circ} \mathrm{C}$ water bath ranging from 15 minutes to a few hours. This time course incubation was stopped after a uniform homogenate was detected with the naked eye as well as microscope magnification. Cloazapine, Tolbutamide and Atenolol compounds were spiked into tissues with and without the addition of collagenase at $247,2220,6670 \mathrm{ng} / \mathrm{ml}$ concentrations, then analyzed at time zero and after two week storage in $-80^{\circ} \mathrm{C}$ freezer. The addition of collagenase to the tough tissues resulted in significant tissue degradation compared to bead-beater and polytron devices alone. Heating and agitating the mixture in a $37^{\circ} \mathrm{C}$ water bath did not accelerate collagenase activity or tissue degradation. Matrix effects were observed in all tissues, but collagenase does not appear to cause any major ion suppression or stability effects. Collagenase was found to be a simple, cost effective and efficient practice for challenging tissue homogenization without the need for additional expensive devices. The addition of collagenase allows for the analysis of difficult, fibrous tissues and can expedite the bioanalytical quantitation of new chemical entities. 


\title{
P7. Abstract withdrawn
}

\section{P8. Dried matrix spot analysis of loxapine phase II metabolites in rat urine}

\author{
$\underline{\text { Keith Huie }}^{1}$, Lori H. Takahashi ${ }^{1}$, Andrea Reed ${ }^{1}$ and James Cassella ${ }^{2}$ \\ ${ }^{1}$ PK/ADME, Alexza Pharmaceuticals Inc, Mountain View, CA, ${ }^{2}$ Alexza Pharmaceuticals Inc, Mountain View, CA
}

Loxapine is a dibenzoxazepine antipsychotic. Gender differences in the plasma pharmacokinetic and metabolic profiles in male and female Sprague Dawley rats were observed following oral or intravenous administration of loxapine succinate. Plasma was collected and analyzed, and in order to fully investigate the differences, urine samples were collected from 0-24 hours post-dose, and analyzed for loxapine and its metabolites. Urine volumes were measured, and glacial acetic acid $(0.1 \%)$ added. The samples were stored at $-70^{\circ} \mathrm{C}$ until analysis. One set of samples were spotted $(15 \mu \mathrm{L}$ each spot) onto Whatman FTA-DMPK cards (Type A, B, and C), dried, and then stored for 96 hours at ambient temperature. Another set of samples was treated by centrifugation after thawing. Hydrolysis of Phase II metabolites was accomplished by the addition of glucuronidase/sulfatase, followed by incubation for 2 hours at $37^{\circ} \mathrm{C}$. The hydrolyzed samples were also spotted onto DMPK cards or treated by centrifugation. Samples were punched $(6 \mathrm{~mm})$ from each card, and extracted using methanol containing internal standards. The extracts and centrifuged samples were analyzed by LC-MS-MS for loxapine, amoxapine, loxapine $\mathrm{N}$-oxide, 7-OH loxapine, and 8-OH loxapine using authentic standards and their respective deuterated internal standards. Following both intravenous and oral administration of loxapine to rats, the major metabolite observed in plasma was 7-OH-loxapine, with amoxapine and loxapine N-oxide observed to a lesser extent. No 8-OH loxapine (major human metabolite) was observed in rat plasma samples. Very little $(<3 \%)$ loxapine or its metabolites were excreted into the urine over 24 hours post dose following either route of administration. The main metabolites recovered from the urine were 7-OH loxapine and loxapine $\mathrm{N}$-oxide, with the amount of 7-OH-loxapine recovered from female rats higher than from the male rats. Treatment with a glusulase enzyme indicated that loxapine, 7-OH loxapine and 8-OH loxapine were excreted as glucuronides, and males appeared to exhibit more glucuronidation than females. DMPK Card B had the highest \% recovery of all aglycones, and showed that $47 \%$ of $7-\mathrm{OH}$ loxapine was conjugated into phase II metabolites. The results from Card B were comparable to the analysis with centrifuged processed samples, and therefore, dried matrix spot analysis is suitable for analysis of loxapine Phase II metabolites in rat urine.

\section{P9. Evaluation of accelerator mass spectrometry (AMS) analysis at two AMS laboratories with samples from a microtracer study of $\left[{ }^{14} \mathrm{C}\right]$ saxagliptin in dogs: Preparation for a clinical absolute bioavailability study}

\author{
Lisa J. Christopher ${ }^{1}$, Xiaohui (Sophia) Xu' ${ }^{1}$, Vikram Roongta ${ }^{1}$, Kai Cao ${ }^{1}$, Brad D. Maxwell ${ }^{1}$, Roger \\ Demers $^{2}$, Bruce Stouffer ${ }^{1}$, Ali Arjomand ${ }^{3}$, Qi Song 3 , Stephen Dueker ${ }^{4}$, Peter Lohstroh ${ }^{4}$, Ramaswamy \\ A. Iyer ${ }^{1}$, W. Griffith Humphreys ${ }^{1}$ and Mark E. Arnold ${ }^{1}$ \\ ${ }^{1}$ Bristol-Myers Squibb, Princeton, NJ, ${ }^{2}$ Tandem Labs, West Trenton, NJ, ${ }^{3}$ Accium Biosciences, Seattle, WA, ${ }^{4}$ Vitalea \\ Sciences, Davis, CA
}

Accelerator mass spectrometry (AMS) is an ultra-sensitive analytical technique that has been used in the pharmaceutical setting for determining concentrations of drug-derived C-14 in biological samples after administration of nanocurie or low microcurie levels of C-14-labeled compounds. In recent years, AMS has been utilized to support absolute bioavailability (BA) studies where an intravenous (IV) $\left[{ }^{14} \mathrm{C}\right]$ microdose of drug is administered concurrently with an oral therapeutic dose. In this approach, plasma concentrations of unlabeled drug are quantitated with a validated LC-MS/MS method and $\left[{ }^{14} \mathrm{C}\right]$ drug concentrations are measured by AMS after chromatographic isolation. There are currently no universally accepted guidelines for AMS validation. Although accuracy and reproduciblity of AMS instrument measurements are considered to be good, quantitation is ultimately dependent upon sample processing. In particular, issues related to extraction recovery and verification of chromatographic separation of the parent compound from other drug-related components need to be addressed in order to obtain accurate drug measurement. A clinical absolute BA study of Onglyza" (saxagliptin) was required for drug approval in some countries. This was the first time, to our knowledge, that an absolute BA study with the IV $\left[{ }^{14} \mathrm{C}\right]$ microdose was used in support of a regulatory approval. We therefore, elected to take a conservative approach for evaluating the AMS methodology, which included a validation and analysis of preclinical study samples prior to analysis of samples from the clinical study. The current investigation describes the evaluation of the methodology for measurement of $\left[{ }^{14} \mathrm{C}\right]$ saxaglitpin by AMS using a dog microtracer study. For the dog study, plasma samples were collected from 3 
animals receiving an oral mictotracer dose of $\left[{ }^{14} \mathrm{C}\right]$ saxagliptin $(0.071 \mathrm{mg} / \mathrm{kg}, 3.4 \mathrm{nCi} / \mathrm{kg})$. At each timepoint, the samples from individual dogs were pooled, mixed well, and were then divided into 3 aliquots. The first aliquot was analyzed for saxagliptin using a validated LC-MS/MS assay. The remaining two aliquots were analyzed at two separate contract laboratories by AMS after chromatographic separation of saxagliptin from its metabolites. Standards and QC samples were also included in the AMS evaluation. Results indicated acceptable assay performance at both laboratories. Furthermore, plasma concentrations of saxagliptin in dog study samples determined by AMS were similar to those from the validated LC-MS/MS method and the values generated by the two AMS labs were comparable to each other.

\title{
P10. Simultaneous quantification method of human CYPS3A4, 3A7, 2B6, 2C9 and 2C19 in humanaized mouse liver microsomes
}

\author{
Yasuko Tsukazaki $^{1}$, Naoto Senda ${ }^{1}$, Shin-ichiro $\mathrm{Nitta}^{1}$, Yasutoshi Ito ${ }^{2}$, Shigeru Yamada ${ }^{2}$, Yasuhiro \\ Kazuki $^{3}$, Kinya Kubo ${ }^{3}$ and Mitsuo Oshimura ${ }^{3}$ \\ ${ }^{1}$ Analytical Technology Research Department, Mitsubishi Chemical Medience Corporation, Tsukuba, Japan, ${ }^{2}$ K. K. AB \\ SCIEX, Shinagawa, Tokyo, Japan, ${ }^{3}$ Chromosome Engineering Research Center, Tottori University, Tottori, Japan
}

We evaluated the metabolic profiles of several CYP3A4 substrates which were metabolized by the humanized CYP3A transchromosomic mouse (CYP3A-HAC/Cyp3a KO mouse) liver microsome. It is confirmed that this mouse has humanCYP3A gene cluster (CYP3A4, CYP3A5, CYP3A7, CYP3A43) (Kazuki, Y. et al., a poster presentation at the 16th North American ISSX Meeting, 2009). As we reported, those were observed that the metabolic functions of human CYP3A4/CYP3A5 introduced were kept in CYP3A-HAC/Cyp3a KO mice (Tsukazaki et al, a poster presentation at the 9th ISSX International Meeting, 2010). In the present study, we estimated a simultaneous quantification method for human CYPs3A4, 3A7, 2B6, 2C9 and 2C19 in adult and fetal CYP3A-HAC/Cyp3a KO mouse liver microsomes. Each CYP specific digested fragment was assigned by a high resolution LC-MS/MS system (AB SCIEX Triple TOF 5600 equipped with ekisigent nano flow LC-system), and specific digested fragments for each human CYPs were determined by Peak View ${ }^{\mathrm{mat}}$. Human CYPs in mouse liver microsome were quantified by MRM method using a high sensitive LC-MS/MS system (AB SCIEX QTRAP® 5500 equipped with Shimadzu LC-20A system). The specificity and calibration curves were estimated by wild type mouse liver microsome samples which were spiked each human CYP independently. Microsome was digested by trypsin, and after digested samples were extracted using the OASIS HLB $\mu$ Elution plate. Human CYP3A4 was found of $8.47 \pm 0.706 \mathrm{pmol} / \mathrm{mg}$ protein from adult CYP3A-HAC/Cyp3a KO mouse liver microsome, but CYPs3A7, 2B6, 2C9 and 2C19 were not detected by the AB SCIEX Triple TOF 5600. The other hand, both of CYPs3A7 and 3A4 were detected from fetal CYP3A-HAC/Cyp3a KO mouse liver microsome $(5.37 \pm 0.660,0.153 \pm 0.0338 \mathrm{pmol} / \mathrm{mg}$ protein, respectably). All isoformes of human CYPs were not detected from wild type mouse liver microsomes. Furthermore, we measured DHEA hydroxylation activity using Triple TOF 5600 equipped with conventional HPLC system. Fetal CYP3A-HAC/cyp3a KO mouse liver microsome was catalyzed 16-alpha hydoroxylation of DHEA, mainly caused by CYP3A7. The other hand, only 7-beta hydroxy DHEA, mainly caused by CYP3A4 was detected from liver microsome of adult CYP3A-HAC/cyp3a KO mouse. In conclusion, we established the quatification method on human CYPs 3A4, 3A7, 2B6, 2C9 and 2C19 spiked on mouse liver microsome Furthermore, we could detected not only CYPs 3A4 and 3A7 in CYP3A-HAC/Cyp3a KO mice but also some metabolites formed by those enzymes. It shows that human CYP3As introduced in CYP3A-HAC/Cyp3a KO mouse is functional.

\section{P11. Strategy to increase detection of low concentration of radioactive metabolites in biological fluids by HPLC fractionation with solid scintillant counting using TopCount}

\author{
Jie Chen, Wing Lam, Fran Xu, Jose Silva and Heng Keang Lim
}

Dmpk, Johnson \& Johnson Pharmaceutical Research and Development, Raritan, NJ

Purpose: Evaluate and compare various strategies to increase sensitivity of detection and quantification of low concentrations of radioactive metabolites in biological fluids using HPLC fractionation combined with TopCount microplate solid scintillant counting. Methods: Two different methods were evaluated for increasing the amount of materials fractionated into 384-well micoplate. The first method involved increase loading using larger bore column and with maximum amount diverted to fraction collector. The other method involved collection of multiple HPLC runs into same 384-well microplate (LumaPlate, Perkin-Elmer, Shelton, CT) at the rate of 5 or $6 \mathrm{~s}$ /well with a fraction collector (PAL, Leap Technologies, Carrboro, NC). HPLC solvents in microplate were evaporated using a speed vacuum system (SpeedVac SPD-2010, Thermo Savant, Holbrook, NY). Radioactivity (CPM value) of the fractions in 384-well plates was determined with TopCount 
(Perkin-Elmer, Shelton, CT). Up to 12 well were counted simultaneously with a 5 min counting time. Results: Biological samples such as plasma, urine, and feces with low concentrations of radioactivity were analyzed using HPLC with on-line radioactivity flow detection and off-line TopCount detection with multiple injections. Comparing the on-line and off-line approaches, the radioactivity profile from TopCount showed much more detail and the sensitivity was improved since the multiple injections allowed the double or triple radioactivity accumulated in 384-well microplate. Conclusion: The data from this study strongly suggest that HPLC with multiple injections in combination with TopCount is a viable alternative analytical tool for detection and quantification of low levels of radioactive metabolites in biological fluids.

\title{
P12. Bovine serum albumin in Caco-2 permeability testing and effect on Papp values determined with highly protein bound compounds
}

\author{
Elke S. Perloff, Sudarshan Kapadnis, Andrew K Mason and David M. Stresser
}

BD Gentest ${ }^{S M}$ Contract Research Services, Woburn, MA

Objective: Determination of in vitro apparent permeability $\left(\mathrm{P}_{\text {app }}\right)$ in Caco-2 cell monolayers is a routine aspect of many drug discovery programs. For many compounds, however, non-specific binding to the cells and/or filter plate assembly, possibly combined with limited solubility, results in substantially reduced compound recovery from the test system $(<<$ $50 \%$ ), and/or the lack of quantifiable concentrations in the receiver sample. To mitigate non-specific binding and improve solubility, the assay buffer is frequently supplemented with protein (e.g. bovine or human serum albumin, fetal bovine serum, calf serum, etc), however, limited systematic data is available on the impact this may have on $\mathrm{P}_{\text {app }}$. The present study determined the impact of adding $2 \%$ BSA to the assay buffer on $\mathrm{P}_{\text {app }}$ values for a set of compounds covering a wide range of plasma protein binding. Methods: Bidirectional permeability in Caco-2 cell monolayers was determined for a set of compounds covering a wide range of reported plasma protein binding. The assays were performed using proteinfree assay buffer (HBSS with $10 \mathrm{mM}$ HEPES, pH 7.4) as well as buffer supplemented with $2 \%$ bovine serum albumin. Compounds were assayed at $10 \mu \mathrm{M}$ and incubated for $90 \mathrm{~min}$. Donor ( 0 and $90 \mathrm{~min}$ ) and receiver samples $(90 \mathrm{~min})$ were quantified by LC/MS/MS and $\mathrm{P}_{\text {app }}$ and mass balance values were calculated and compared between conditions. Results: The impact of $2 \%$ BSA in the Caco-2 assay buffer varied widely for different compounds tested. $P_{\text {app }}$ values for certain highly protein bound compounds (e.g. naproxen, warfarin) decreased substantially (e.g. 10-fold or more) in presence of BSA, while effects on $\mathrm{P}_{\text {app }}$ values for compounds with moderate (verapamil, propranolol) or low (metoprolol, labetalol) protein binding were limited. Not surprisingly, impact on efflux ratios was less pronounced as the $\mathrm{P}_{\text {app }}$ values are reduced for both directions. Conclusions: Addition of protein to the assay buffer is useful and frequently used to mitigate non-specific binding and improve solubility in permeability screening assays, however, caution should be exercised when interpreting the resulting data. $P_{\text {app }}$ values may be substantially decreased by the presence of protein in the assay buffer, particularly for compounds exhibiting high plasma protein binding, which may result in erroneous permeability classification.

\section{P13. Effect of compatibility on isofraxidin pharmacokinetic after oral administrated with Yinxieling or Sarcandra glabra (Thumb.) Nakai}

\author{
Chuanjian $\mathrm{Lu}^{1}$, Jinghong $\mathrm{Yu}^{2}$, Buqing $\mathrm{Lu}^{2}$ and ${\underline{\mathrm{Rui}} \mathrm{Zhi} \mathrm{Zhao}^{3}}^{3}$
}

${ }^{1}$ Guangdong provincial academy of Chinese Medicine, Guangzhou, China, ${ }^{2}$ Second Affiliated Clinical College, guangzhou university of Chinese medicine, Guangzhou, China, ${ }^{3}$ Second Affiliated Clinical College, guangzhou university of Chinese Medicine, Guang zhou, China

In traditional Chinese medicine, compatibility of different crude drugs is usually used to enhance the cure effect and reduce the side effects, more and more studies showed that compatibility could change the pharmacokinetics characteristics such as metabolism, excretion and tissue distribution of the main components in the drug, as a sequel affect the pharmacodynamic. Therefore, it is essential to study the effect of compatibility on the pharmacokinetics of the main components for understanding the mechanism of compatibility.Isofraxidin is the active components of Sarcandra glabra(Thumb.) Nakai, and the latter is the components of Yinxieling, a complex prescription of Chinese medicine usued to treat psoriasis, and in order to study the effect of compatibility on the pharmacokinetics of isofraxidin, the analysis method by HPLC was established. The linearity of the calibration curve was good in the range of $0.25 \mathrm{I} \mathrm{Ig} / \mathrm{mL} \sim 12 \mathrm{I} \mathrm{Ig} / \mathrm{mL}$ for isofraxidin and the detection limit for isofraxidin were $0.25 \mathrm{IIg} / \mathrm{mL}$. The extraction recoveries of isofraxidin were $70.9 \% \sim 71.9 \%$ with RSD was $1.1 \%$; The intra-day precision and inter-day precision of isofraxidin were lower than $8.9 \%$. Pharmacokinetics was studied by administrated Yinxieling thumb or sarcandra glabra(thumb.) nakai respectively to Sprague-dawley rats.The results 
were analyzed by pharmacokinetic software DAS(2.0).Results The difference of pharmacokinetic parameters between the group of Yinxieling(thumb.) and Sacandra glabra(thumb.)nakai were unremarkable. The results indicated that compound prescription of Chinese medicine did not affect the pharmacokinetics of isofraxidin. Keywords $£^{\circ} Y$ Yinxieling; sarcandra glabra(thumb.)nakai; Isofraxidin; pharmacokinetics Acknowledgements: This research was financially supported by the found of Guangdong Natural Science Foundation (T004847, 10251040701000001), the technology found of Guangdong province (2010A03010008), and the technology found of Guang zhou city (2010J-E031)

\title{
P14. Evaluation of oral bioavailability and intestinal availability from pharmacokinetic changes associated with drug-drug interaction
}

\author{
$\underline{\text { Akihiro Hisaka }}{ }^{1}$, Akiko Mori², Saori $\mathrm{Koh}^{3}$, Ayako Tsukihashi², Hirotaka Ando ${ }^{2}$ and Hiroshi Suzuki
}

${ }^{1}$ Pharmacology and Pharmacokinetics, The University of Tokyo Hospital, Tokyo, Japan, ${ }^{2}$ Department of Pharmacy, The University of Tokyo Hospital, Tokyo, Japan, ${ }^{3}$ Laboratory for Safety Assessment and ADME, Pharmaceuticals Research Center, Asahi Kasei Pharma Corporation, Izunokuni, Shizuoka Pref., Japan

Scheme: Basic Equations of DDI Method

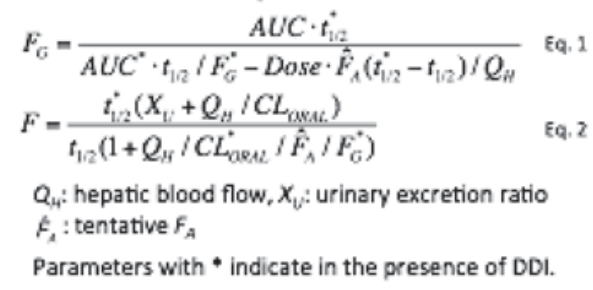

Figure: Comparison of $\mathbf{F}$ Evaluated by DDI Method and Conventional Method

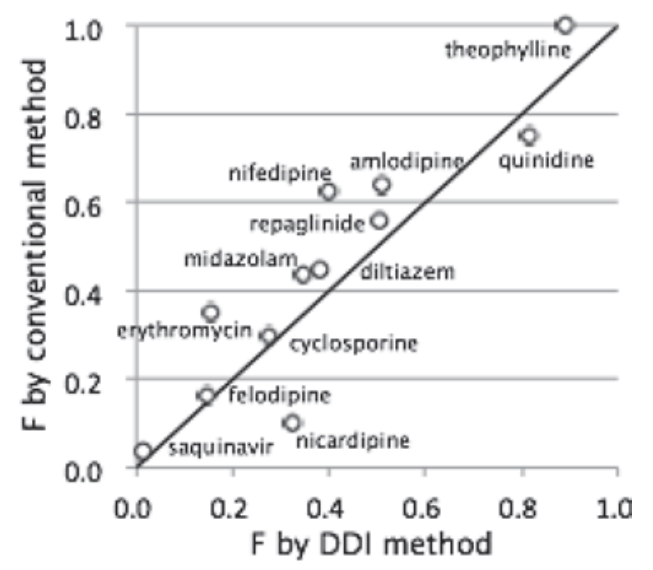

[Background] In general, oral bioavailability $(\mathrm{F})$ and intestinal availability $\left(\mathrm{F}_{\mathrm{G}^{\prime}}\right.$, a ratio of drug escaping from intestinal metabolism during absorption) are calculated by comparing the clearances evaluated after intravenous and oral doses. Thus, an intravenous-dose study is necessary for their evaluations, and moreover, inter-individual and inter-study differences in the clearance may produce serious errors. In the previous study, we demonstrated that $\mathrm{F}_{\mathrm{G}}$ is evaluable from changes in the AUC and plasma half-life $\left(\mathrm{t}_{1 / 2}\right)$ associated with drug-drug interaction (DDI) for relatively well-absorbed substrate drugs of CYP3A4 when its inhibitor is co-administered ${ }^{1}$ ). In this study, the application of this method (DDI method) is extended to less absorbed drugs, and furthermore, to the evaluation of F. [Method] Basic equations of the DDI method (Scheme) were derived from the clearance theory. To evaluate $\mathrm{F}_{\mathrm{G}}$ and $\mathrm{F}$ in the DDI method, the absorption ratio $\left(F_{A}\right)$ needs to be approximated, but unnecessary to be very precise, hence it is named tentative $F_{A}$ in the scheme. Tentative $\mathrm{F}_{\mathrm{A}}$ was fixed previously $\left.{ }^{1}\right)$, but in this study, approximated statistically or by the membrane permeability determined in vitro experiments. It was assumed that $\mathrm{F}_{\mathrm{G}}$ becomes 1.0 when strong inhibitors are co-administered $\left(\mathrm{F}_{\mathrm{G}}{ }^{*}\right)$. Extensive Monte-Carlo simulations were conducted to check preciseness of the DDI method. Furthermore, $\mathrm{F}_{\mathrm{G}}$ and $\mathrm{F}$ of approximately 60 drugs (mainly substrates of CYP3A4) were evaluated based on literature information, and compared with the observed values if available. [Results and Discussion] The simulation study indicated that the modified DDI method provides reasonable estimation of $F_{G}$ using tentative $F_{A}$ approximated statistically even for poorly absorbed drugs (i.e. $F_{A}<0.3$ ). For evaluation of $\mathrm{F}$, tentative $\mathrm{F}_{\mathrm{A}}$ needs to be more precise; accordingly approximated by in vitro information such as the permeability determined in the Caco-2 system. Overall, $\mathrm{F}$ and $\mathrm{F}_{\mathrm{G}}$ obtained by the DDI method were in excellent accord with those 
evaluated by other methods using the systematic clearance after intravenous dose (Figure). Since evaluation of a DDI is frequently carried out within a single study using the same subjects, it seemed that the DDI method provides more stable evaluation compared with the conventional methods in many situations. [Conclusion] The DDI method was proposed as a robust technique for evaluation of $\mathrm{F}_{\mathrm{G}^{\prime}}$, and in addition, it may become an alternative method for evaluation of $\mathrm{F}$ when an intravenous-dose study is unavailable. Reference: ${ }^{1}$ ) Tsukihashi et al., A new calculation method of intestinal availability from pharmacokinetic changes by drug-drug interaction, 25th JSSX Annual Meeting, 2010, Tokyo, Japan.

\title{
P15. Methodology optimisation in intestinal microsome preparation: Implications for scaling approaches?
}

\author{
Oliver Hatley ${ }^{1}$, Christopher Jones ${ }^{2}$, Aleksandra Galetin ${ }^{1}$ and Amin Rostami-Hodjegan ${ }^{1}$ \\ ${ }^{1}$ School of Pharmacy and Pharmaceutical Sciences, University of Manchester, Manchester, United Kingdom, ${ }^{2}$ Discovery \\ DMPK, AstraZeneca, Alderley Park, United Kingdom
}

The lack of validated subcellular intestinal scaling factors currently limits prediction of first-pass metabolism and bioavailability of orally administered xenobiotics through in vitro-in vivo extrapolation (IVIVE). Therefore, the objective of this study was to ascertain and validate microsomal scaling factors for quantifying intestinal metabolism in vivo.Following initial difficulties in using a recently published method ${ }^{[1]}$, previously reported superiority in activity of microsomes prepared via elution verses scraping ${ }^{[2]}$, and limitations through lack of standardized methods in the literature, the process of enterocyte preparation via the elution method was evaluated and optimized using the rat. Alterations in conditions for enterocyte isolation ("incubation time" and "EDTA concentration"), homogenization (20, 60 and 100\% amplitude sonication) and sub-cellular fraction preparation (addition of heparin and/or glycerol) were systematically evaluated. In order to compare variant effects, the protein yields were normalized and microsome quality was analysed by measurement of cytochrome P450 (CYP450) ${ }^{[3]}$. The results indicated thatcomparable CYP450 normalized protein yields were obtained irrespective of alterations to the preparation of intestinal microsomes post enterocyte isolation. However, variability was positively associated with the severity of homogenization $(\mathrm{CV}=7.3,7.2$ and $22 \%$, reported for 20,60 and $100 \%$ amplitude sonication respectively), an effect increased by use of glycerol ( $\mathrm{CV}=77 \%)$. The addition of heparin decreased this variability, but subsequently reduced overall specific CYP450 contents (reduced $<40 \%$ ). Enterocyte isolation was implicated as the most critical determinant of the intestinal scaling factor value, since mean normalised protein yield varied over 2 fold when experimental steps applied to the enterocytes were altered. In conclusion, a reproducible method based on elution of intestinal enterocytes was developed. The optimal balance between protein yield and functional CYP450, based on the degree of homogenization and microsomal purification conditions, should be carefully considered for provision of functional microsomes and a reproducible scaling factor. The technique applied for enterocyte isolation was shown to be a critical aspect in determining an intestinal scalar. Current investigation is focused on IVIVE using the derived intestinal scaling factor applied to a selected set of model reference compounds to evaluate its accuracy in predicting intestinal clearance within the Qgut model ${ }^{[4]}$.

\section{References}

1. Bruyere, A., et al., Development of an optimized procedure for the preparation of rat intestinal microsomes: comparison of hepatic and intestinal microsomal cytochrome P450 enzyme activities in two rat strains.Xenobiotica, 2009.39(1): p. 22-32.

2. Galetin, A. and J.B. Houston, Intestinal and hepatic metabolic activity of five cytochrome P450 enzymes: impact on prediction of first-pass metabolism. J Pharmacol Exp Ther, 2006. 318(3): p. 1220-9.

3. Wilson, Z.E., et al., Inter-individual variability in levels of human microsomal protein and hepatocellularity per gram of liver. Br J Clin Pharmacol, 2003. 56(4): p. 433-40.

4. Yang, J., et al., Prediction of intestinal first-pass drug metabolism.Curr Drug Metab, 2007.8(7): p. 676-84.

\section{P16. Pharmacokenetics comparison of astilbin after orally administered with Yin Xie Ling or Smilacis Glabrae in rats}

\author{
Yin Jie Wang ${ }^{1}$, $\underline{\text { Rui Zhi Zhao }}^{2}$ and Chuan jian $\mathrm{Lu}^{1}$ \\ ${ }^{1}$ Guangzhou university of Chinese medicine, Guangzhou, China, ${ }^{2}$ Second Affiliated Clinical College, guangzhou \\ university of Chinese Medicine, Guang zhou, China
}

Pharmacokinetic reflect drug absorption, distribution, metabolism and elimination, it is a key factor related with its action. Therefore it is an indirect method to evaluate drug action with less harmful, and usually used to predict pharmaceutical 
preparations and also used in study the synergy and attenuation mechanism in traditional Chinese medicine. Astilbin is a compound comes from Smilacis, zhongjiefeng et al. It has anti-inflammatory effect, inhibited T lymphocytes and contacting hypersensitivity, and inhibited the proliferation of horny cell and promoted its apoptosis, and is the main active components of Yin Xie Ling which used in clinical to treat psoriasis. In order to study the mechanism of Yin Xie Ling compatibility, pharmacokinetics of astilbin after administration of Rhizome Smilacis Glabrae or Yin Xie Ling were studied. Drug was administered to rats orally, and at 5, 15, 30min, 1, 1.5, 2, 4, 6, 8h blood were collected, extracted by SPE, and analyzed by HPLC. The separation was performed on a reverse- phase column with gradient elution composed of methanol, acetonitrile and $0.05 \%$ formic acid water. The detection wavelength is $330 \mathrm{~nm}$, the linear range was $265.5 \mathrm{ng} /$ $\mathrm{ml} \ll \times 3.1 ; \mathrm{Ig} / \mathrm{ml}$. The recovery was $102 \%$. The intra-day and inter-day precision were $7.87 \%$ and $10.1 \%$ respectively. The concentration of astilbin after orally administered with Yin Xie Ling were much higher than the concentration of astilbin after orally administered with Smilacis Glabrae, the Cmax increased almost 3 times. There were two peaks in the profile of Yin Xie Ling group, but only one peak in the profile of Smilacis Glabrae group. The results indicated that other drugs in Yin Xie Ling may be enhanced the absorption of astilbin and affected the pharmacokinetics of astilbin, and this effect may be helpful for its clinical. Acknowledgements: This research was financially supported by the found of Guangdong Natural Science Foundation (T004847, 10251040701000001), the technology found of Guangdong province (2010A03010008), and the technology found of Guang zhou city (2010J-E031)

\title{
P17. Pharmacokinetics and oral bioavailability of a novel PPAR-sparing insulin sensitizer and its pharmacologically active hydroxy metabolite in monkeys and humans
}

\author{
Wade J. Adams ${ }^{1}$, Jonathan G. Bissland ${ }^{2}$, Carter D. Brooks ${ }^{3}$, Lori L. Cochrane ${ }^{2}$, Jerry R. Colca ${ }^{1}$, M. \\ Melissa Peet ${ }^{4}$, Natalie D. Schmock ${ }^{2}$ and James T. VanderLugt ${ }^{1}$ \\ ${ }^{1}$ Metabolic Solutions Development Company, Kalamazoo, MI, ${ }^{2}$ Analytical Sciences, MPI Research, Inc., Mattawan, MI,
${ }^{3}$ Jasper Clinic, Inc., Kalamazoo, MI, ${ }^{4}$ Drug Discovery, MPI Research, Inc., Mattawan, MI
}

MSDC0602 (5-\{4-[2-(3-methoxyphenyl)-2-oxoethoxy]benzyl\}-1,3-thiazolidine-2,4-dione]) is being developed for the treatment of Type 2 diabetes. It is an insulin sensitizer that is related to the thiazolidinedione pioglitazone (ACTOS ${ }^{\mathrm{m}}$ ) except MSDC0602 has significantly reduced ability to activate the nuclear transcription factor PPAR. These compounds exert their pharmacology by selectively modulating mitochondrial metabolism resulting, among other things, in improved insulin sensitivity and an increase in brown adipose tissue. The PPAR-sparing nature of this compound and its pharmacologically active hydroxy metabolite (MSDC-0597) may provide increased therapeutic ratio in clinical practice by avoiding PPAR?-related side effects including fluid retention and weight gain. The intravenous and oral dose pharmacokinetics of MSDC-0602 and MSDC-0597 and the bioavailability of orally administered MSDC-0602 were evaluated in the cynomolgus monkey in preclinical studies and the oral dose pharmacokinetics of both compounds were evaluated in healthy humans as part of a Phase I single and multiple dose tolerance and safety study. Validated non-chiral reversed-phase LC-MS/MS analytical methodology that utilized negative electrospray ionization was used for analysis of monkey and human sodium heparin plasma samples. The analytical ranges for MSDC-0602 and MSDC-0597 were 0.300 to $300 \mathrm{ng} / \mathrm{mL}$ and 10.0 to $10,000 \mathrm{ng} / \mathrm{mL}$, respectively, in both monkey and human plasma. Intravenous doses of MSDC-0602 and MSDC-0597 had systemic clearances of 140 and $0.531 \mathrm{~mL} / \mathrm{min} / \mathrm{kg}$, respectively, in the monkey and confirmed that MSDC-0602 was acting as a prodrug for the delivery of MSDC-0597. Low but significant concentrations of MSDC-0602 were observed in plasma after intravenous administration of MSDC-0597, confirming that the alcohol was converted to the ketone parent in vivo. Assuming quantitative conversion of MSDC-0602 to MSDC-0597, the MSDC0597 clearance was slightly lower $(0.444 \pm 0.047 \mathrm{~mL} / \mathrm{min} / \mathrm{kg})$ after intravenous administration of MSDC-0602 than after intravenous administration of MSDC-0597. The steady state volumes of distribution of MSDC-0602 and MSDC-0597 in monkeys were 27.2 and $0.480 \mathrm{~L} / \mathrm{kg}$, respectively. In monkeys, the absolute oral bioavailabilities of MSDC-0597, when administered as unformulated MSDC-0602, were $12 \%$ for the non-micronized free acid, $61.6 \%$ for the micronized free acid, $67.7 \%$ for the non-micronized sodium salt and $83.3 \%$ for the non-micronized potassium salt. Single and multiple oral doses of unformulated non-micronized and micronized MSDC-0602 were administered to healthy human subjects at doses ranging from 30 to $1620 \mathrm{mg}$ in the tolerance and safety study. As in the monkey, MSDC-0602 was rapidly metabolized to MSDC-0597 in humans, with the ratio of MSDC-0602 and MSDC-0597 concentrations remaining essentially constant over the entire concentration-time profile. The occurrence of secondary maxima in concentration-time profiles, some of which occurred as long as $24-48 \mathrm{hr}$ post-dose in humans, suggested that enterohepatic recycling occurred. Administration of unformulated micronized vs non-micronized MSDC-0602 resulted in 5.1- and 5.5-fold increases in oral bioavailability in monkey and man, respectively. These data confirmed that the monkey was a suitable animal model for assessing oral bioavailability of MSDC-0602 in humans. Solid dosage forms were selected for further evaluation in humans based on preliminary monkey bioavailability studies. 


\title{
P18. Prediction of time-course of drug concentrations in enterocytes and blood during oral absorption by translocation model, a new physiological pharmacokinetic model for gastrointestinal tracts
}

\author{
$\underline{\text { Hirotaka Ando }}^{1}$, Akihiro Hisaka ${ }^{2}$ and Hiroshi Suzuki ${ }^{1}$
}

\begin{abstract}
${ }^{1}$ Department of Pharmacy, The University of Tokyo Hospital, Faculty of Medicine, The University of Tokyo, Tokyo, Japan, ${ }^{2}$ Pharmacology and Pharmacokinetics, The University of Tokyo Hospital, Faculty of Medicine, The University of Tokyo, Tokyo, Japan
\end{abstract}

[Background] In the previous study, in order to consider various physiological issues occurred during oral absorption of drugs, such as dissolution, uptake/efflux transport, metabolism, and transfer to the blood stream, we proposed translocation model (TM), a new local pharmacokinetic model for gastrointestinal (GI) tracts ${ }^{1}$ ). TM is composed of four compartments (formulation, lumen, enterocyte, and lamina propria) which are trans-located along the length of the GI tracts, and the kinetic parameters associated with those compartments are time- and location-dependent. The objective of the present study is to predict the time-course of drug concentrations in the enterocytes and in the blood stream from in vitro parameters by using TM. [Methods] To explain movements of the drug formulation or solution in the GI tracts, location and dispersion terms were involved in TM, and the parameters were obtained by a fitting analysis to the observed movements in the human GI tracts. The luminal and tissue volumes, luminal surface area, and blood flows were determined based on literature information and the luminal and villous structure. Data from Caco-2 assays were used to estimate kinetic parameters of the active transport by P-gp at apical membrane and of the passive transport at both apical and basolateral membranes of the enterocyte. The time-courses of drug concentrations in the portal and systemic blood and in the enterocytes were calculated, and the bioavailability $(F)$, intestinal availability $\left(F_{G}\right)$ and dose- $F_{A} \cdot F_{G}$ relationships were predicted for 16 drugs (alfentanil, alprazolam, atorvastatin, cyclosporine, felodipine, methadone, midazolam, nifedipine, nisoldipine, quinidine, repaglinide, saquinavir, sildenafil, tacrolimus, triazolam, and zolpidem) by using TM. To confirm accuracy of the predictions, $F$ and $F_{G}$ were compared with the observed values in vivo. The cause of non-linearity was considered by comparing $K_{m}$ values of the intestinal metabolism and transport with the estimated drug concentrations in the enterocytes. [Results] $F$ and $F_{G}$ values predicted by TM agreed with the observed values within errors of less than 1.5-fold for most drugs analyzed in this study. Moreover, TM explained the time courses of drug concentrations in the systemic blood in a reasonable manner. For some drugs, the time courses of concentration in the enterocytes exceeded beyond $K_{m}$ values of metabolism and/or transport, suggesting a mechanism responsible for the non-linear pharmacokinetics. Incorporation of an adequate description on permeability across the basolateral membrane improved accuracy of the predictions. TM also explained the blood-flow limited absorption in some situations. [Conclusion] This study revealed the usefulness of TM for prediction of local pharmacokinetics of GI tracts. In order to consider issues occurred in the enterocytes, adequate description of the basolateral membrane was important for appropriate predictions. Reference: ${ }^{1}$ ) Ando et al., Simulation of drug concentrations in enterocyte during absorption with a new pharmacokinetic model of gastrointestinal tracts; translocation model, International Symposium on BA/BE of Oral Drug Products, 2011, Kobe, Japan.

\section{P19. Preparation of controlled release tablets of Rheum palmatum and evaluation in vivo}

\section{Ruizhi Zhao}

\section{Second Affiliated Clinical college, Guangzhou university of triditional Chinese medicine, Guangzhou, China}

Release rate of pharmaceuticals is very important to preparations, it directly influence on the pharmacodynamics and side effect of drugs. People realized it for many years and developed many methods for controlling the release rate of preparations, the study of the release rates has been the hotspot of pharmaceutical research, such as sustained release preparations, controlled release preparations, immediate release preparation especially for poor dissolution drugs and so on, in modern times, We could use different form of preparation or different matrix to control the release rate almost as we like. But this is not for the Traditional Chinese Medicine (TCM). In ancient times of China, people usually use decoction and powder to realize immediate release of drugs. But it is very inconvenience, and the taste is terrible. So in modern times, people try to make it to injection and oral liquid pharmaceutical, and Dropping Pill, some of it is very successful, such as injection of Qingkailing and Bingduling oral liquid, and composite Salviae Dropping Pill, etc, but most drugs are not suited to prepare to liquid pharmaceutical, because liquid pharmaceuticals are easily changed, the drugs property limited its pharmaceuticals. And Droppling Pill can carry very little drug, to most of the TCM drugs it is 
not suit. So how to achieve a rapid and prolonged release is an important problem in the pharmaceutical study of TCM. However, little research has been found in the literature relating to in vivo performance of preparation of TCM especially for immediate release. The aim of this study was to prepare controlled release tablets of Chinese Traditional Medicine. The Rheum palmatum was chosen as the model drug, and the Anthraquinone free derivatives being the index of Rheum palmatum, concentrations of Anthraquinone free derivatives in serum were determined by HPLC. The tablets were prepared by directly compressed method, and the release behavior in vivo was evaluated by the serum concentration in dogs. Drug release from tablets containing HPMC and lactose is rapidly and prolonged for a long time than capsules, and the AUC of tablets was 4.6 times compared to capsules. It indicates that the mixture of HPMC and lactose could be used as the matrix of the rapid and prolonged release of Traditional Chinese Medicine. Key wards: Rheum palmatum, controlled release tablets, in vivo evaluation

\title{
P20. Substantial impact of P-glycoprotein on bioavailability in rats (1)
}

\author{
Megumi Irie, Fumihiro Nakamori, Ai Tamura, Katsuhiro Yamano, Shigeyuki Terashita and Toshio \\ Teramura
}

\begin{abstract}
Analysis \& Pharmacokinetics Research Labs., Institute for Drug Discovery Research, Astellas Pharma Inc., Tsukuba-shi, Japan
\end{abstract}

Here we investigated the pharmacokinetic (PK) profiles and the role of P-glycoprotein (P-gp) on the intestinal absorption of compound A in rats. In spite of the high solubility, good permeability in a parallel artificial membrane permeability assay (PAMPA) (apparent permeability $\left(P_{a p p}\right)=14.1 \times 10^{-6} \mathrm{~cm} / \mathrm{sec}$ at $\left.\mathrm{pH} 6.5\right)$ and the moderate metabolic rate in liver microsomes, in PK (i.v. and p.o.) studies compound A exhibited high total clearance (CLt) and poor bioavailability $(<$ $10 \%)$. However, its metabolites were not detected in plasma after oral administration. Furthermore, relatively high excretion into the urine (27\%) and bile (9\%) was detected after i.v. administration. FaFg of Compound A was extremely low as well (0.03). This was determined using the portal-systemic concentration difference method, which calculates the difference in AUC between the portal vein and the systemic circulation. We observed a nonlinear increase in compound A's plasma concentration when administered orally at $3 \mathrm{mg} / \mathrm{kg}$ or $10 \mathrm{mg} / \mathrm{kg}$. Furthermore, transport studies in MDCK cells expressing MDR1 revealed that compound A was a P-gp substrate. Compound A's $P_{a p p}$ in Caco-2 cells was only $0.6 \times 10^{-6}$ $\mathrm{cm} / \mathrm{sec}$ in the absorptive direction at pH 6.5. These findings suggested that P-gp accounts for compound A's poor FaFg. To clarify whether P-gp limits compound A's intestinal absorption, a PK (i.v. and p.o.) study was performed using $M d r 1 a$ deficient mice. Compound A's bioavailability in Mdrla-deficient mice (20\%) was 7 -fold higher than wild type (3\%). We also performed a drug-drug interaction study in rats using elacridar to inhibit P-gp. Concurrently administering elacridar increased compound A's bioavailability approximately 6-fold, while no change in CLt was observed. These studies clearly demonstrate that P-gp caused, in part, poor bioavailability of compound A in rats. Limited intestinal absorption due to P-gp is likely to affect the pharmacokinetics of other P-gp substrates in rats and humans alike.

\section{P21. Substantial impact of P-glycoprotein on bioavailability in rats (2)}

\author{
Fumihiro Nakamori, Megumi Irie, Ai Tamura, Katsuhiro Yamano, Shigeyuki Terashita and Toshio \\ Teramura

\begin{abstract}
Analysis \& Pharmacokinetics Research Labs., Institute for Drug Discovery Research, Astellas Pharma Inc., Tsukuba-shi, Japan
\end{abstract}

[Introduction] Oral bioavailability (F) is a product of absorption (Fa), intestinal availability (Fg), and hepatic availability $(\mathrm{Fh})(\mathrm{F}=\mathrm{FaFg} \times \mathrm{Fh})$. While Fh has been investigated extensively, recent studies have focused more on FaFg instead. In the present study, an in-house compound $\mathrm{B}^{*}$ was used to investigate factors affecting FaFg in rats, since intestinal absorption/metabolism is known to influence pharmacokinetics in rats, as revealed by the low FaFg value (0.15) using the portal-systemic difference method. [Methods and Results] Metabolic stability in rat intestinal microsomes showed that compound B is stable against rat intestinal cytochrome P450 enzymes. Parallel artificial membrane permeable assay (PAMPA) experiments indicated that compound B is highly permeable with a permeability value of $14 \times 10^{-6} \mathrm{~cm} / \mathrm{s}$. However, experiments using cells stably expressing human multidrug resistance (MDR1) showed the apparent permeability to be $0.45 \times 10^{-6} \mathrm{~cm} / \mathrm{s}$ for apical to basal flux, and $29.7 \times 10^{-6} \mathrm{~cm} / \mathrm{s}$ for basal to apical flux, indicating that compound $\mathrm{B}$ is a typical substrate of P-glycoprotein (P-gp). Pharmacokinetic studies using normal mice and mdrla-deficient (KO) mice showed that the respective FaFg values of compound B were 0.25 and almost 1.0, indicating that intestinal P-gp 
limited the intestinal absorption of compound B. [Conclusion] Our present findings suggest that the low FaFg of compound B in rats is caused by intestinal P-gp, implying that intestinal P-gp substantially affects bioavailability by limiting intestinal absorption ( $\mathrm{Fa}$ ) in rats. *The structure of compound B is totally different from compound A used in the study of "Substantial impact of P-glycoprotein on bioavailability in rats (1)".

\title{
P22. Accurate prediction of hepatic clearance for low-clearance compounds using micropatterned hepatocyte-stromal cell co-cultures (HepatoPac ${ }^{\circledR}$ )
}

\author{
Tom S. Chan ${ }^{1}$, Salman Khetani ${ }^{2}$, Amanda Moore ${ }^{3}$ and Hongbin $\mathrm{Yu}^{1}$ \\ ${ }^{1}$ Drug Metabolism and Pharmacokinetics, Boehringer Ingelheim Pharmaceuticals Inc., Ridgefield, CT, ${ }^{2}$ Hepregen \\ Corporation, Medford, MA, ${ }^{3}$ Research and Development, Hepregen Corporation, Medford, MA
}

Generating accurate in vitro intrinsic metabolic stability and clearance data is an important aspect of predicting in vivo human clearance and establishing a safe dosing range in early clinical studies. Primary hepatocytes in suspension are routinely used for determination of metabolic clearance. However, hepatocytes in suspension are only viable for several hours, which is not long enough to appropriately evaluate the metabolic stability of low clearance compounds. Using HepatoPac, a recently developed micropatterned hepatocyte-stromal cell co-culture system which can be used for continuous incubations for up to 7 days, we have successfully determined the metabolic stability of 15 commercially available low clearance drugs $(\mathrm{CL}<5 \mathrm{~mL} / \mathrm{min} / \mathrm{kg}$ ). Intrinsic clearance was calculated using the in vitro half-life determined for each compound and hepatic clearance was subsequently calculated using the well-stirred model with and without a correction for protein binding. Without correction for plasma protein binding, hepatic clearance was accurately predicted for only 1 compound, as determined by prediction of clearance within 2-fold of published clinical values. However, with correction for plasma protein binding, hepatic clearance was accurately predicted for 11 compounds. These results clearly demonstrate the utility of HepatoPac for the prediction of in vivo hepatic clearance of low-clearance compounds.

\section{P23. An in vivo-in vitro correlation of hepatic clearance for metabolism by non-CYP with custom pooled hepatocytes}

\author{
Takafumi Akabane $^{1}$, Nicolas Gerst² ${ }^{2}$ Jeffrey N. Masters ${ }^{2}$ and Kouichi Tamura ${ }^{2}$ \\ ${ }^{1}$ Analysis \& Pharmacokinetics Research Labs., Astellas Pharma Inc., Ibaraki, Japan, ${ }^{2}$ Astellas Rsch Inst of America, Skokie, IL
}

The use of human pooled hepatocytes as a preclinical predictor of human pharmacokinetics for non-cytochrome P450 (CYP) metabolism has attracted attention at drug discovery due to their broad spectrum of metabolic enzyme activity, large lots production, and tight variation. In order to verify usability of human pooled hepatocytes for non-CYP metabolism evaluation, first, we evaluated 18 compounds primarily metabolized by non-CYP enzymes including UDPglucuronosyltransferase, reductase, aldehyde oxidase, flavin-containing monooxygenase, and monoamineoxidase in individual hepatocytes and pooled hepatocytes from the same donors. The intrinsic clearance in pooled hepatocytes showed more than $70 \%$ of average clearance value in individuals in 16/18 compounds which suggest pooled hepatocytes maintained an average activity of the individual hepatocytes. Subsequently, in vivo-in vitro correlation (IVIVC) analysis using pooled hepatocytes were performed in 24 compounds. Although further studies are needed for IVIVC using hepatocytes as under-prediction trend or large variation were observed in this analysis, quantitative hepatic clearance prediction was successfully observed in aldehyde oxidase substrates using empirical scaling factor. The possible reasons for underprediction or variation, and the possibility to improve the predictability for other non-CYP enzyme were also discussed.

\section{P24. An integrated approach for validating and delivering in silico human liver microsome clearance models to discovery scientists}

\author{
Ignacio Aliagas ${ }^{1}$, Alberto Gobbi ${ }^{1}$, Cyrus Khojasteh ${ }^{2}$, Jane Kenny ${ }^{2}$, Man Ling Lee ${ }^{1}$ and Daniel Ortwine ${ }^{1}$ \\ ${ }^{1}$ Discovery Chemistry, Genentech Inc., South San Francisco, CA, ${ }^{2}$ Dmpk, Genentech Inc., South San Francisco, CA
}

Metabolism is one of the major clearance pathways of drugs from the body. High-throughput liver microsome and hepatocyte stability assays are now in routine use in the pharmaceutical industry. Such assays produce a substantial amount 
of consistent data, permitting predictive computational models to be developed. Use of such models to forecast the stability of compounds ahead of synthesis could be a valuable tool for prioritizing compound synthesis and compound design. We have developed a model to predict clearance from human liver microsome (HLM) cells using a dataset of $>20,000$ compounds. The model output is expressed as categories and probabilities. The cross-validated Q-square was 0.50 for the human liver microsome model with a RMS error of 3.4. The R-square for a prospective set of 5,500 newly synthesized compounds was 0.41 with a RMS error of 3.8. This model, along with others that predict various DMPK assay end points, have been integrated into a number of desktop tools available to discovery scientists. Calculations are also performed on all registered compounds and results uploaded to our corporate database. All models are updated on a regular basis. In addition to desktop availability of all models, we have developed easy to use tools to evaluate model performance on individual compounds, chemical series, or project-wide sets of compounds. These tools provide easy-to-interpret charts and plots, giving discovery scientists an instant assessment of how well the models are working for their compounds. These capabilities, along with strong support from management for their use, has resulted in an across the board decrease in the percentage of synthesized compounds that are unstable in HLM cells. We will present statistics from the HLM model using the validation tools we developed to generate charts and plots to show prediction accuracy on compounds not in the training set. We will also show a three year history of measured HLM stability data, demonstrating that if the model is consistently used as a filter in synthesis decisions, an overall decrease of HLM in vitro clearance can be achieved.

\title{
P25. Be careful what you ask for: Challenges of predicting human clearance for a low metabolic turnover compound, ELND006
}

\author{
$\underline{\text { Kevin Quinn }}^{1}$, David Nakamura ${ }^{1}$, Heather Zhang ${ }^{1}$, Shawn Gauby ${ }^{1}$, Colin Lorentzen ${ }^{1}$, Erich \\ Goldbach $^{1}$, Amanda Moore ${ }^{2}$, Salman Khetani ${ }^{3}$, Earvin Liang ${ }^{4}$, John-Michael Sauer ${ }^{1}$ and George \\ Tonn $^{1}$
}

${ }^{1}$ Drug Metabolism and Pharmacokinetics, Elan Pharmaceuticals, South San Francisco, CA, ${ }^{2}$ Research and Development, Hepregen Corporation, Medford, MA, ${ }^{3}$ Hepregen Corporation, Medford, MA, ${ }^{4}$ Elan Pharmaceuticals, South San

Francisco, $C A$

The discovery of a new chemical entity (NCE) suitable for CNS drug development is a challenging endeavor, as many different properties require simultaneous optimization. One of these properties, metabolic stability, is a surrogate for in vivo clearance (CL). A goal for many discovery project teams is to select an NCE with the greatest metabolic stability; however, very high metabolic stability can also pose challenges for development. The prediction of human CL, with the objective of selecting compounds with half-lives suitable for once daily dosing, has become an important selection criterion for many discovery organizations. ELND006, a potent and APP selective gamma secretase inhibitor demonstrating CNS reduction of Beta Amyloid in rodents and non-human primates ${ }^{1}$ was selected from a series of NCEs based, in part, on its metabolic stability in both NADPH and UDPGA supplemented liver microsomes. Further experiments aimed at calculating the predicted human intrinsic CL (i.e., incubations in human liver microsomes and hepatocytes) revealed that this compound exhibited very low turnover $(<5 \%)$. In an attempt to improve turnover, incubations with increased microsomal protein and cell numbers were attempted; however, consistent with the lipophilicity $(\log \mathrm{D} 3.9)$ of ELND006, high nonspecific binding leading to low free concentrations (Fu,microsomes $=0.06$ ) likely obscured any benefit of increased enzyme levels. While it was evident from these data that ELND006 would exhibit low CL, the actual magnitude of the CL could not be accurately determined. Phase I clinical data revealed that the average apparent terminal half-life in healthy elderly subjects following oral dosing ranged from 220 to 306 hours ${ }^{2}$, yielding a total body clearance $(\mathrm{CL} / \mathrm{F})$ of $1 \mathrm{~mL} / \mathrm{min} / \mathrm{kg}$. After estimations for bioavailability, this value is suggestive of a human CL of 0.2 to $0.3 \mathrm{~mL} / \mathrm{min} / \mathrm{kg}$. The availability of micropatterned co-cultures of hepatocytes and fibroblasts in a 96-well plate format ${ }^{3}$ prompted a retrospective analysis to determine whether the human CL of ELND006 could be predicted. This system mimics elements of in vivo liver physiology and increases cell viability and function of drug metabolizing enzymes on a scale of days rather than hours. ELND006 was metabolized under these conditions resulting in a prediction of human clearance $(0.9 \mathrm{~mL} / \mathrm{min} / \mathrm{kg})$ that was within three to four-fold of that observed in vivo. In addition, the CL values for the positive controls, warfarin and quinidine, agreed well with literature reports. Our data suggests that while further investigation is warranted, micropatterned co-cultures of hepatocytes may offer an alternative to empirical methods for predicting the human CL low turnover compounds in drug discovery.

1) Brigham, et al. 2010. Pharmacokinetic and pharmacodynamic investigation of ELND006, a novel APP-selective gamma-secretase inhibitor, on amyloid- $\beta$ concentrations in the brain, CSF and plasma of multiple nonclinical species following oral administration. ICAD. Honolulu, Hawaii. S546

(c) 2011 Informa Healthcare USA, Inc. 
2) Liang et al. 2011. A Phase I dose escalation study to evaluate the safety, tolerability, PK/PD of multiple oral dosing of D6 in healthy elderly subjects. ICAD. Paris, France. P2-476

3) Khetani and Bhatia. 2008. Microscale culture of human liver cells for drug development. Nature Biotechnology 26 , $120-126$

\title{
P26. Prediction of PK parameters of human using human chimeric mice
}

\author{
$\underline{\text { Seigo Sanoh }}{ }^{1}$, Aya Horiguchi ${ }^{1}$, Kazumi Sugihara ${ }^{2}$, Chise Tateno ${ }^{3}$, Toru Horie ${ }^{4}$, Shigeyuki Kitamura ${ }^{5}$ \\ and Shigeru Ohta ${ }^{1}$
}

${ }^{1}$ Graduate School of Biomedical Sciences, Hiroshima University, Hiroshima, Japan, ${ }^{2}$ Faculty of Pharmaceutical Sciences, Hiroshima International University, Kure, Japan, ${ }^{3}$ PhoenixBio Co., Ltd., Higashihiroshima, Japan, ${ }^{4}$ De Three Reseach Laboratories, Ibaraki, Japan, ${ }^{5}$ Nihon Pharmaceutical University, Saitama, Japan

The prediction of drug metabolism and phramacokinetics (PK) in human is important during pre-clinical stage in pharmaceuticals. Several prediction methods have been developed using cryopreserved human liver microsomes or hepatocytes. However, accurate human clearances prediction is difficult since a lot of new drug candidates with diversities of chemical structures have increased to be metabolized by non-CYP enzymes. In the present study, we used chimeric mice with humanized liver (PXB mice, PhoenixBio, Co., Ltd, Hirohisma, Japan), whose livers are replaced with approximately $80 \%$ of human hepatocytes. The expression levels and metabolic activities of CYP and non-CYP in liver of PXB mice are similar to the patterns of human. In this study, we attempted to verify the predictability of PK parameters in PXB mice using several model compounds which have been known to be mainly metabolized by CYP and/or non-CYP in liver. (1) In vitro metabolic study; Fresh hepatocytes were isolated from PXB mice using in situ collagenase perfusion method and purified by removal of mice hepatocytes. Suspended hepatocytes were incubated for $2 \mathrm{hrs}$ after treatment of each drug. In vitro intrinsic clearances were calculated from time course of disappearance of parent compounds after incubation in human chimeric hepatocytes. (2) In vivo PK study; PXB mice substituted with human hepatocytes were administrated bolus intravenously (i.v.) of drugs at $0.3-5 \mathrm{mg} / \mathrm{kg}$ body weight. (3) Human PK data for verification; total clearance (CLt) and plasma unbound fraction (fu) in actual human are applied from literature values. In vivo intrinsic clearance (CLint,in vivo) were calculated from the CLt, fu, and average hepatic blood flow (Qh) using the analysis based on well-stirred model. Directly comparison between in vitro intrinsic clearance (CLint,in vitro) of PXB mice hepatocytes and CLint,in vivo calculated by well-stirred model in observed in human, which showed the moderate correlation. On other hand, we compared CLt between human and PXB mice, which was showed the good correlation. Additionally, there was also good correlation of half life (t1/2) after i.v. administration between human and PXB mice. In this study, the predictability of hepatocytes from PXB mice was similar to that of reported using cryopreserved human hepatocytes. However, abundant fresh hepatocytes are available again by transplantation of donor hepatocytes. Consequently, this in vitro approach may be utility for screening the metabolic stability of chemical library. Furthermore, in vivo experiments should be also useful for optimization of new chemical entities with good bioavailability and stable plasma level in human.

\section{P27. Utilization of chimeric mice with humanized liver as a useful model for predicting human pharmacokinetics (PK) of a drug candidate with major aldehyde oxidase (AO) metabolism}

\author{
Maria D. Bacolod, Kimloan Nguyen, Zack Zou, Guiying Li, Hao Zhou, Dario Doller and Gamini \\ Chandrasena
}

Chemical and Pharmacokinetic Sciences, Lundbeck Research USA, Inc., Paramus, NJ

It is common practice to extrapolate human pharmacokinetics (PK) of drug candidates using in vitro and in vivo data from preclinical animal species, provided the metabolite profiles are qualitatively similar. In vitro metabolism studies of the drug candidate, Lu AF20253 in a number of species, including human, showed significant differences in the metabolite profile. In addition, the predicted hepatic clearance (CLh) of Lu AF20253 in the well-stirred model using corresponding intrinsic clearance (CLint) values found to be low in microsome, but moderate to high in hepatocyte incubations. The subsequent rat and dog intravenous (IV) PK showed that this compound has low plasma clearance (CLp) in rat $(10 \mathrm{~mL} / \mathrm{min} / \mathrm{kg})$ and $\operatorname{dog}(2.8 \mathrm{~mL} / \mathrm{min} / \mathrm{kg})$, with excellent oral bioavailability $(\sim 100 \%$ in both species $)$ consistent 
with predicted CLh in the well-stirred model utilizing microsomal CLint. The in vitro metabolic profiling from human cytosolic and S9 incubations with appropriate chemical inhibitors suggested that Lu AF20253 is primarily metabolized by aldehyde oxidase (AO), a cytosolic enzyme reported to be predominantly expressed in both human and cynomolgus monkey liver with observed low or no activity in either rats or dogs. The moderate IV CLp (20 mL/min/ kg) and low bioavailability (18\%) in cynomolgus monkey was consistent with predicted CLh fromin vitro hepatocyte incubations. Since there are significant differences in the expression levels and substrate specificity of AO between preclinical species and human, an accurate human PK prediction for Lu AF20253 using preclinical species data alone was found to be very challenging. Thus, we explored the use of a chimeric mouse model with humanized liver (uPA ${ }^{+/+} / \mathrm{SCID}_{\text {transgenic mouse }}$ liver is replaced with $\sim \mathbf{8 0 \%}$ of human hepatocytes) since this animal model has been reported to be a very useful tool in predicting human ADME properties (Strom SC, et al., 2010). The presence of high levels of circulating AO-dependent major oxidative metabolite following both IV and PO dosing, as well as the observed higher CLp (58 mL/min/ $\mathrm{kg}$ ) and lower oral bioavailability (30\%) compared to control mice without humanized liver (CLp of $24 \mathrm{~mL} / \mathrm{min} / \mathrm{kg}$ and oral bioavailability $\sim 100 \%$ ) confirmed the AO-dependent first-pass metabolism of Lu AF20253. Collectively, these data support the use of the chimeric humanized mouse as a potential in vivo model to address AO-mediated liver drug metabolism and to provide accurate human PK predictions.

\title{
P28. Biotransformation of abused synthetic cannabinoids, JWH-018 and JWH-073, by human cytochromes P450 and UDP-glucuronosyltransferases
}

\author{
Anna Radominska-Pandya ${ }^{1}$, Krishna C. Chimalakonda ${ }^{2}$, Stacie M. Bratton ${ }^{1}$, Barbara Fedejko-Kap ${ }^{1}$, \\ Vi-Huyen Le ${ }^{3}$, Kan Hui “Nicole” Yiew ${ }^{1}$, Cindy Moran, Laura P. James ${ }^{5}$ and Jeffery H. Moran ${ }^{3}$ \\ ${ }^{1}$ Biochemistry and Molecular Biology, University of Arkansas for Medical Science, Little Rock, AR, ${ }^{2}$ Pharmacology \\ \& Toxicology, University of Arkansas for Medical Science, Little Rock, AR, ${ }^{3}$ Department of Health, AR Public Health \\ Laboratory, Little Rock, AR, ${ }^{4}$ Arkansas State Crime Lab, Little Rock, AR, ${ }^{5}$ Univ of Arkansas for Med Sci and Arkansas \\ Children's Hospital, Department of Pediatrics, Little Rock, AR
}

K2/Spice is a very popular emerging drug of abuse that is touted as "legal marijuana". Most K2 products consist of plant materials laced with several synthetic cannabinoids possessing psychoactive properties similar to those produced by the $\Delta^{9}$-tetrahydrocannabinol $\left(\Delta^{9}\right.$-THC) found in marijuana. While up to 10 different K2-aminoalkyl indoles (AAIs) are reported to be present in various K2 preparations, the two most commonly observed are JWH-018 [naphthalen-1-yl-(1-pentylindol-3-yl)methanone] and JWH-073 [naphthalen-1-yl-(1-butylindol-3-yl)methanone]. Our preliminary data using human clinical specimens from individuals known to have ingested either JWH-018 or JWH-073 have identified several products of Phase I and II metabolism. Many of the hydroxylated metabolites have been shown to be monohydroxy derivatives of the naphthalene, indole, or side-chain moieties that are excreted in urine primarily as glucuronic acid conjugates, thus implicating the involvement of both Cytochrome P450s (CYPs) and UDP-glucuronosyltransferases (UGTs) in this process. We hypothesize that metabolism of K2-AAIs by specific CYP and UGT isoforms is an obligatory step in the biotransformation and elimination of these compounds. This study is the first to evaluate specific human recombinant CYPs and UGTs as well as and human liver and intestinal microsomes for their in vitro activity toward JWH-018 or JWH-073 and six hydroxylated metabolites of each. Metabolites from these assays were identified and characterized using LC-MS/MS and HPLC-UV/Vis methods, and steady state kinetic analyses were carried out. CYP2C9 and 2C19 were shown to be major enzymes involved in the hydroxylation of JWH-018 and JWH-073, with the M5 product of JWH-018, (1-(5-hydroxypentyl)-1H-indol-3-yl) (naphthalen-1-yl)methanone, and the M3 product of JWH-073, (1-butyl-6-hydroxy-1H-indol-3-yl)(naphthalen-1 -yl)methanone, being the predominant products of these reactions. UGT1A1, 1A3, 1A9, 1A10 and $2 B 7$ were shown to be major enzymes involved in glucuronidation, with relatively high affinity and capacity for these hydroxylated compounds, indicating that CYP-produced metabolites of these compounds may be rapidly glucuronidated and eliminated from the body. Inhibition studies were also done with selected CYP isoforms to analyze the affect of increasing concentrations of JWH-018 and JWH-073 and two of their most abundant metabolites found in human urine, M5 [(1-(5-hydroxypentyl)] and M6 [(1-(5-carboxypentyl)]. These data show significant inhibition of CYP activity by these compounds and reinforces the importance of UGT conjugation in the clearance of these compounds, preventing potential drug-drug interactions. Identification of the mechanism of K2-AAI biotransformation and the enzymes involved in this process is essential for future pharmacokinetic and pharmacogenetic studies. Without this information, understanding of adverse drug reactions related to polymorphisms and drug-drug interactions cannot be achieved. (NIH-GM075893 to AR-P; APHL Innovation Award to JHM; UAMS Arkansas Center for Clinical and Translational Research to LPJ,AR-P,JHM,CM) 


\title{
P29. Characterization of in vitro glucuronidation of five anthraquinones from Rhubarb
}

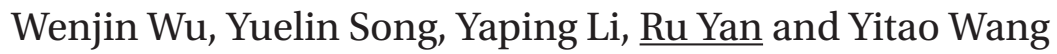

State Key Laboratory of Quality Research of Chinese Medicine, Institute of Chinese Medical Sciences, Universtiy of Macau, Macau, China

Background Anthraquinones (aloe-emodin, rhein, emodin, chrysophanol, physcion) and their glucosides are major polyphenol constituents in Rhubarb. An in vivo study revealed anthraquinone glucuronides as the predominant forms in rat plasma when a Rhubarb extract was orally administered, implying impact of glucuronidation on in vivo fates and consequent actions of anthraquinones. Rhubarb anthraquinones share the core structure of danthron (1,8-dihydroxyanthracene9,10-dione) with different substituents at beta-position (C-3 and/or C-6). The present study investigated, for the first time, the in vitro glucuronidation of rhubarb anthraquinones to determine the structure-metabolism relationship. Methods Aloeemodin, emodin, chrysophanol, physcion and danthron $(0.5$ and $5 \mu \mathrm{M})$ were individually incubated with pooled hepatic microsomal proteins $(0.05 \mathrm{mg} / \mathrm{ml})$ from humans (HLMs) or rats (RLMs) for $10 \mathrm{~min}$ in the presence of UDPGA. The incubation of rhein was $30 \mathrm{~min}$ and $0.5 \mathrm{mg} / \mathrm{ml}$ of proteins. In incubations with recombinant human UGT $(0.05 \mathrm{mg} / \mathrm{ml}, 12$ isozymes $)$, four anthraquinones except for rhein were incubated for $10 \mathrm{~min}$. Main metabolites of aloe-emodin and emodin were prepared from scale-up reactions with RLMs, isolated using pre-HPLC, and structures identified using LC-MS/MS and NMR analysis. Chrysophanol-8-O-glucoside and physcion-8-O-glucoside were used to aid structural identification of chrysophanol and physcion glucuronides by glucuronidation and hydrolysis. Quantitative determination was performed using HPLC-MS/ MS. Results In microsomal proteins of both species, danthron only produced one glucuronide due to its structural symmetry. All other anthraquinones formed 1-O-glucuronide and 8- $\mathrm{O}$-glucuronide. One additional glucuronide resulting from conjugation of the 3-OH or 3-COOH was identified with emodin and rhein, respectively. RLMs showed higher activities towards glucuronidation of the same anthraquinone than human with exception of emodin, the elimination rate of which was comparable between species. The average elimination rates of test anthraquinones in HLMs and RLMs were in the same descending order (rat vs human, $\mathrm{nmol} / \mathrm{min} / \mathrm{mg}$ protein): emodin (6.80 vs 6.83), aloe-emodin (1.39 vs 0.56), danthron (1.24 vs 0.46), chrysophanol (0.69 vs 0.14), physcion (0.36 vs 0.054) and rhein (0.035 vs 0.016). Substituent(s) at beta-position(s) of anthraquinones also affected positional preference of glucuronidation. In HLMs when substituted with- $\mathrm{CH}_{2} \mathrm{OH}$ (aloeemodin) or- $\mathrm{CH}_{3}$ (physcion, $-\mathrm{OCH}_{3}$ at ring $\mathrm{B}$ ) at ring A, both compounds showed a preference to- $\mathrm{OH}$ at ring $\mathrm{B}$. The hydroxyl group at the same benzene ring with- $\mathrm{CH}_{3}$ of chrysophanol tended to be the preferential site of conjugation. With a beta-OH, emodin showed an absolute positional preference with the 3 - $O$-glucuronide accounting for $>95 \%$ of emodin glucuronides. Aloe-emodin and rhein showed different preference of glucuronidation in RLMs. Human UGT1A1, 1A3, 1A7, 1A8 (except for aloe-emodin), 1A9 and 1A10 catalyzed glucuronidation of all anthraquinones at varied activities and positional preference. UGT 1A9 exhibited the highest activities in most cases. While UGT 2B subfamily showed low or no activities towards antranquinones glucuronidation, except that 2B7 and 2B15 exhibited similar activities to $1 \mathrm{~A} 3$ towards emodin glucuronidation. All UGTs showed highest capability and predominant preference towards beta-OH of emodin. Conclusions Rhubarb anthranquinones, except for rhein, undergo extensive glucuronidation in liver microsomes of humans and rats. The elimination rate and positional preference of glucuronidation varied with substituted position and moiety.

\section{P30. Comparison of UDP-glucuronosyltransferase 2B7 absolute protein quantification and metabolic activity using zidovudine as selective probe substrate}

\author{
$\underline{\text { Staffan Thorén }}^{1}$, Marie Wallin $^{1}$, Johan Lengqvist ${ }^{2}$ and Pawel Baranczewski ${ }^{1}$
}

\begin{abstract}
${ }^{1}$ CNSP iMed DMPK, AstraZeneca R\&D Södertälje, Södertälje, Sweden, ${ }^{2}$ Molecular Toxicology Global Safety Assessment, AstraZeneca R\&D Södertälje, Södertälje, Sweden
\end{abstract}

The existing in vitro methods often underestimate in vivo clearance and magnitude of drug-drug interactions (DDIs) related to metabolic reactions catalyzed by the UDP-glucuronosyltransferases (UGTs). More accurate scaling factors for extrapolation of data obtained under in vitro conditions to the in vivo environment would improve predictions of clearance and DDIs. In order to improve in vitro predictions of the contribution of UGT2B7 to metabolic clearance and DDIs, metabolic activity was compared with absolute protein quantification in a set of individual human liver microsomes (HLMs) from 20 subjects. The catalytic rates of UGT2B7 were measured under optimal incubation and analytical (UPLC-MS/MS) conditions for a selective probe substrate, zidovudine (AZT). An absolute protein quantification method was developed to measure UGT2B7 protein. After trypsin digestion of $24 \mu \mathrm{g}$ HLM, unique signature peptides for UGT2B7 were measured by isotope dilution mass spectrometry relative to heavy labelled peptide standards. The limits of quantification $(<20 \mathrm{fmol})$ were determined by UPLC-ESI-MS/MS performed with a Waters TQD operated in the MRM mode. A Km value of $831 \mu \mathrm{M}$ 
was obtained for AZT in incubations with pooled HLMs using the Michaelis-Menten plot. The following final concentrations of AZT were chosen for estimation of UGT2B7 activity: 100, 1000 and $5000 \mu \mathrm{M}$. The zidovudine glucuronidation rate obtained at the substrate concentration of $5000 \mu \mathrm{M}$ varied between $134 \mathrm{and} 703 \mathrm{pmol} / \mathrm{min} / \mathrm{mg}$ protein. The absolute UGT2B7 protein quantification assay revealed values between 17.5 and $66.9 \mathrm{pmol} / \mathrm{mg}$ of microsomal proteins. The Pearson correlation, $\mathrm{r}=0.70$, between UGT2B7 activity and protein abundance was statistically significant at $\mathrm{p}<0.0001$. Additionally, the relative ratio between the maximal and minimal values was similar: 5.2-fold for UGT2B7 activity and 3.8fold for protein abundance, respectively. The measurement of UGT2B7 enzyme activity combined with accurate UGT2B7 abundance data provides scaling factors for extrapolation of in vitro data generated in recombinant human UGTs or HLMs to the whole human liver environment. This will significantly improve IVIVE for UGT2B7 enzymes.

\title{
P31. Determination of 7-hydroxycoumarin specificity using recombinant human enzymes of uridine 5'-diphospho-glucuronosyltransferase (UGT)
}

\author{
Timothy A. Moeller, Terrel Rose and Scott Heyward
}

Celsis In Vitro Technologies, Baltimore, MD

7-Hydroxycoumarin (7-HC) has been used as a probe substrate for phase II enzymes uridine 5'-diphospho-glucuronosyltransferase (UGT) and sulfotransferase (ST) in both microsomal and in cellular systems. It may be used directly as a substrate to measure phase II activities or may be the intermediate metabolite from the phase I metabolism of the parent substrates 7-ethoxycoumarin or couarmin. In humans, 7-HC glucuronide is the predominant phase II metabolite. However, the specificity of 7-HC as a phase II substrate for the UGT isozymes has not been reported. To investigate 7-HC specificity as a substrate for UGT isozymes, we used a panel of recombinant human enzymes (UGT1A1, 1A3, 1A4, 1A6, 1A9, 1A10, 2B4, 2B7, 2B15 and 2B17). Rank order of the activity showed a stratification amongst the UGTs tested: (1A6, 1A9) > (1A1, 1A10, $2 \mathrm{~B} 7,2 \mathrm{~B} 15)>(1 \mathrm{~A} 3,1 \mathrm{~A} 4,2 \mathrm{~B} 4,2 \mathrm{~B} 17)$. These groups spanned a wide range of activities from UGT1A6 at $3808 \mathrm{pmol} / \mathrm{min} / \mathrm{mg}$, to UGT1Al at $289 \mathrm{pmol} / \mathrm{min} / \mathrm{mg}$ to UGT1A3 at $31 \mathrm{pmol} / \mathrm{min} / \mathrm{mg}$. Km and Vmax determination was performed to confirm the rank order. The results show that 7-HC is not metabolized equally between the UGT isozymes. This data may aid in interpreting metabolism differences between tissues or donors due to differential distribution of UGT isozymes.

\section{P32. Effects of various incubation mediums on UDP-glucuronosyltransferase 2B7 (UGT2B7) activity}

\author{
Dustin M. Smith, Arenas Raquel, David D. Boyer and Michael B Fisher
}

Drug Discovery Support, Boehringer-Ingelheim Pharmaceuticals Inc, Ridgefield, CT

UGTs are a class of drug metabolizing enzymes located in the endoplasmic reticulum of various tissues including the liver, kidney and intestine. Like cytochrome P450s, the role of UGTs in the clearance of various chemical entities is commonly determined by in vitro methodology. Unlike P450s, the effects of various incubation buffers, concentration of organics and other varables in incubation procedures has not been systematically investigated. The goal of this study is to survey the effect of various incubation conditions on the activity of UGT2B7. UGT2B7 retained normal activity when exposed up to $10 \%$ DMSO. However, the enzyme showed the greatest sensitivity to acetonitrile, losing $95 \%$ of its activity at $10 \%$ acetonitrile. When exposed to methanol, normal activity was observed up to $6 \%$ and only a $50 \%$ decrease in activty was observed in the presence to $10 \%$ methanol. UGT2B7 had slightly elevated activity when incubated in $50 \mathrm{mM}$ HEPES instead of $100 \mathrm{mM}$ potassium phosphate. However, a 2.5 fold increase in activity was observed in $50 \mathrm{mM}$ Tris. The potential use of the pore forming protein $\alpha$-hemolysin was explored. When compared to typical concentrations of alamethicin, a maximum of 35\% of UGT2B7 activity was discerned. These data indicate optimum incubation conditions as well as general tolerabilities when exploring UGT2B7 metabolism in vitro.

\section{P33. Evaluation of toxic potential of acylglucuronides by combination of orthogonal factors}

\author{
Yoshiaki Shimada, Toshihiro Matsuda, Kohichiro Tanaka, Kazuyoshi Nozaki, Shigeyuki Terashita \\ and Toshio Teramura
}

Analysis \& Pharmacokinetics Research Labs., Astellas Pharma Inc., Tsukuba, Japan

Although acylglucuronides (AGs) are known to be toxic metabolites, no method of evaluating idiosyncratic drug toxicity (IDT) of AGs has been well established. We focused here on using orthogonal factors, which vary substantially between 
compounds, such as a daily dose (D), excretion ratio (E), and degradation rate constant (k) to evaluate toxic potential of AGs. Eleven model compounds with highly metabolic contribution ratios of AG were selected and placed into one of three categories based on clinical results (safe, warning and withdrawn). k values for each compound were obtained via biosynthesized AG assay using human liver microsomes and UDP-glucuronic acid followed by spiked human plasma or HSA solution. a Values of two combined parameters $(\mathrm{D} \times \mathrm{k})$ were then divided into two groups (safe vs. warning or withdrawn) as the values could not be divided into the above-mentioned three categories. Combination of three parameters $(\mathrm{D} \times \mathrm{E} \times \mathrm{k})$, however, did enable division into the above-mentioned three categories, indicating that $\mathrm{D} \times \mathrm{E} \times \mathrm{k}$ is a powerful index for evaluating IDT in AGs. Reference a) Chen Z, Holt T, Pivnichny J and Leung K (2007) A simple in vitro model to study the stability of acylglucuronides. Journal of Pharmacological and Toxicological Methods 55:91-95.

\title{
P34. Evidence for homodimerization of UDP-glucuronosyltransferase $2 B 7$ and identification of the dimerization domain by protein homolgy modelling
}

\author{
John O. Miners, Benjamin C. Lewis and Peter I. Mackenzie \\ Clinical Pharmacology, Flinders University School of Medicine, Adelaide, Australia
}

There is accumulating evidence that UDP-glucuronosyltransferase (UGT) enzymes, particularly members of the UGT1A subfamily, exist as oligomers in homodimeric and heterodimeric states. Although the importance of UGT2B7 in the metabolism of drugs, environmental chemicals and endogenous compounds is well established, direct evidence for protein dimerization is currently lacking. This study aimed to characterize the homodimerization of UGT2B7 by coimmunoprecipitation (Co-IP) and generate a homology model that identified the dimerization domain. UGT2B7 was tagged separately with hemagglutinin (UGT2B7-HA) and cMYC (UGT2B7-cMYC) and Co-IP of solubilized protein was performed with an anti-HA affinity matrix. Co-IP was demonstrated for co-expressed UGT2B7-HA and UGT2B7-cMYC, but not for a mixture of the two proteins expressed separately. Derived $S_{50}$ values and Hill coefficients for 4-methylumbelliferone glucuronidation were essentially identical for wild-type UGT2B7, UGT2B7-HA, UGT2B7-cMYC, and the co-expressed tagged proteins. A UGT2B7 homology model, based on plant and human templates, was constructed using SYBYLX1.2 with all residues energy minimized using the Tripos Force Field. A cluster of highly hydrophobic amino acids on the surface of the UGT2B7 model, within the B'-C loop, was identified as the putative protein dimerization domain. The domain spans residues 183 to 200 of the $\mathrm{N}$-terminus and includes the signature motif FPPSYVPVVMS. Several key interactions spanning the dimerization domain potentially stabilize the dimer interface. These include the presence of proline 'brackets', aromatic $\pi-\pi$ stacking interactions, two salt bridges, and two S-aromatic (face) interactions. The dimerization signature motif identified by the UGT2B7 homology model is highly conserved in all human UGT2B enzymes suggesting that all proteins in this subfamily potentially form homo- and hetero- dimers. The homology model provides important insights into UGT2B7 structure-activity relationships and the mechanism responsible for the atypical glucuronidation of many substrates of this enzyme (including 4-methylumbelliferone).

\section{P35. Liquid-chromatographic mass spectrometric quantitation of uridine 5'-diphospho- Glucuronosyltransferase (UGT) enzymes: Analysis of 50 individual human liver microsomes and preliminary statistical results}

\author{
Christopher Ray Gibson ${ }^{1}$, Richard King², Carmen Fernandez-Metzler², Jorge Quiroz ${ }^{3}$, Thomas \\ Rushmore $^{4}$ and Kevin Bateman ${ }^{5}$
}

\begin{abstract}
${ }^{1}$ Dept of Drug Metab/WP75B-200, Merck \& Co Inc, West Point, PA, ${ }^{2}$ PharmaCadence Analytical Services, LLC, Hatfield, $P A,{ }^{3}$ Nonclinical and Pharmaceutical Sciences Statistics, Merck Research Laboratories, Kenilworth, NJ, ${ }^{4}$ Life Technologies, Durham, NC, ${ }^{5}$ Dmpk, Merck Research Laboratories, West Point, PA
\end{abstract}

In order to gain a better understanding of the quantitative levels and variability of uridine 5'-diphospho-glucuronosyltransferase (UGT) in the human liver, liquid-chromatography mass-spectrometric methods were developed to quantify the concentrations of the proteins in human liver microsomes. The methods were based upon a previously published isotope-dilution technique with some modifications (Fallon et. al, 2008). Since pure recombinant UGTs of known concentration are not available, the analytical approach is divided into 3 parts: 1) qualification of the recombinant protein standard using isotope dilution, 2) use of this qualified standard to build calibrators in a surrogate matrix, and 3) quantification of the endogenous protein in the relevant matrix. Assays were developed to determine the concentrations of UGT1A1, 1A4, 1A6, 1A9, 2B7 and 2B17 in 50 individual human liver microsome samples. The pooled mean (\%CV) 
concentration values (pmol UGT / mg liver microsomal protein) of UGT1A1, 1A4, 1A6, 1A9, 2B17, and 2B7 were 18.1 (48), 11.0 (50), 4.0 (45), 5.7 (45), 3.4 (162) and 19.9 (66), respectively. Statistical analysis of log-transformed concentration data suggests no correlation between the measured UGT concentrations and age or body-mass-index (BMI) of the donor. Additionally, there are the suggestions that 1.) the concentration of UGT1A6 correlate with the concentrations of UGT1A1, 1A4 and 1A9 and 2.) the concentrations of UGT1A4 correlate to the concentration of UGT2B7. The availability of quantitative information on the concentration of the UGT enzymes in the human liver may enable the generation of Inter-System Extrapolation Factors (ISEF) for UGT enzymes making currently available recombinant human UGT enzymes an alternative in vitro system to predict UGT-mediated metabolic intrinsic clearance. Furthermore, knowledge of the co-variation (or lack thereof) between the concentration of UGT enzymes and other physiological parameters may help improve predictive models of UGT-mediated metabolic clearance in the human population. Additional studies, such as correlation to probe substrate enzymatic activity, are required the fully understand the accuracy and precision of the LC-MS based concentration data presented above. Fallon JK, et al., Absolute Quantification of Human UridineDiphosphate Glucuronosyl Transferase Enzyme Isoforms 1A1 and 1A6 By Tandem LC-MS. Drug Metabolism Letters. 2; 210-222, 2008.

\title{
P36. Optimized assays for human UDP-glucuronosyltransferase (UGT) activities: Altered alamethicin concentration and utility to identify UGT inhibitors
}

\author{
Robert L. Walsky ${ }^{1}$, Jonathan N. Bauman ${ }^{1}$, Karine Bourcier ${ }^{2}$, Georgina Giddens ${ }^{2}$, Kimberly Lapham¹, \\ Tim F. Ryder ${ }^{1}$, R. Scott Obach ${ }^{1}$, Ruth Hyland ${ }^{2}$ and Theunis C. Goosen ${ }^{1}$ \\ ${ }^{1}$ Pharmacokinetics, Dynamics and Metabolism, Pfizer Inc., Groton, CT, ${ }^{2}$ Department of Pharmacokinetics, Dynamics, \\ and Metabolism, Pfizer Inc., Sandwich, United Kingdom
}

The measurement of the effect of new chemical entities on human UDP-glucuronosyltransferase (UGT) marker activities using in vitro experimentation represents an important experimental approach in drug development to guide clinical drug interaction study design or support claims that no in vivo interaction will occur. Selective high-performance liquid chromatography-tandem mass spectrometry functional assays of authentic glucuronides for five major hepatic UGT probe substrates were developed: $\beta$-estradiol-3-glucuronide (UGT1A1), trifluoperazine- $N$-glucuronide (UGT1A4), 5-hydroxytryptophol-O-glucuronide (UGT1A6), propofol-O-glucuronide (UGT1A9), and zidovudine-5'-glucuronide (UGT2B7). High analytical sensitivity permitted characterization of enzyme kinetic parameters at low human liver microsomal and recombinant UGT protein concentration $(0.025 \mathrm{mg} / \mathrm{ml})$, which led to a new recommended optimal universal alamethicin activation concentration of $10 \mu \mathrm{g} / \mathrm{ml}$ for microsomes. Alamethicin was not required for recombinant UGT incubations. Apparent enzyme kinetic parameters, particularly UGT1A1 and UGT1A4, were impacted by nonspecific binding and unbound intrinsic clearance for UGTs 1A9 and 2B7 increased significantly following addition of $2 \%$ bovine serum albumin, with minimal changes for UGT1A1, UGT1A4, and UGT1A6. Eleven potential UGT and characterized cytochrome P450 inhibitors were evaluated as UGT inhibitors in 96-well plates, resulting in nonselective UGT inhibition by chrysin, mefenamic acid, silybinin, tangeretin, ketoconazole, itraconazole, ritanovir, and verapamil. The pan-cytochrome P450 inhibitor, 1-aminobenzotriazole, minimally inhibited UGT activities and may be useful in reaction phenotyping of mixed UGT and cytochrome P450 substrates. These methods should prove useful in the routine assessments of the potential for new drug candidates to elicit pharmacokinetic drug interactions via inhibition of human UGT activities and the identification of UGT-enzyme selective chemical inhibitors.

\section{P37. Prediction of the metabolic clearance of a spiropiperidine BACE1 inhibitor by glucuronidation and cytochrome P450-mediated oxidations}

\author{
Jianhua Liu' ${ }^{1}$ Zhuang Miao² and Louis Leung ${ }^{3}$ \\ ${ }^{1}$ Dept of PDM/CNS, Pfizer Inc., Groton, CT, ${ }^{2}$ Pfizer Global Research and Development, Groton, CT, ${ }^{3}$ Pharmacokinetics \\ Dynamics and Metabolism, Pfizer Inc., Groton, CT
}

Species differences in the quantitative metabolite profile of a spiropiperidine BACE1 (Beta-site Amyloid precursor protein Cleavage Enzyme 1) inhibitor were noted in incubations from liver microsomes and cryopreserved hepatocytes of mouse, rat, dog, monkey or human, and in plasma of rats and dogs after oral drug administration. Biotransformation pathways were preliminarily characterized as cytochrome P450 (CYP)-mediated oxidations and UDP-glucuronosyltransferase (UGT)-catalyzed direct phenol glucuronidation. Direct phenol-glucuronidation was detected as a 
predominant metabolite pathway in dogs but a minor pathway in rats. In order to predict the overall metabolic clearance in human and to assess the contribution of glucuronidation versus the oxidation pathways, an in vitro system was used consisting of combined CYP and UGT cofactors in alamethicin-activated human liver microsomes in the absence or presence of $2 \%$ BSA. The unbound intrinsic clearance $\left(\mathrm{CL}_{\text {int,u }}\right)$ was determined to be approximately $70 \mu \mathrm{L} / \mathrm{min} / \mathrm{mg}$ in the presence of both CYP and UGT cofactors and $2 \%$ BSA. The fraction metabolized $\left(\mathrm{f}_{\mathrm{m}}\right)$ was estimated to be approximately $35 \%$ by UGT and $65 \%$ by CYP when $\mathrm{CL}_{\text {int,u }}$ was determined in systems consisting of the combined or separated UGT and CYP cofactors. Together with knowledge gained from in vitro studies pertaining to the specific CYP and UGT enzymes responsible for the respective clearance pathways in human, results from the current study greatly facilitates the prediction of overall drug clearance, inter-subject variability due to potential involvement of polymorphic enzymes, and the potential for clinical drug-drug interactions due to UGT or CYP-mediated process.

\title{
P38. Abstract withdrawn
}

\section{P39. Telmisartan non-selectively inhibits UDP-glucuronosyltransferase (UGT) 1A and UGT2B proteins 3}

\author{
Kathleen M. Knights, Shane M. Spencer and John O. Miners \\ Department of Clinical Pharmacology, Flinders University, Adelaide, Australia
}

Telmisartan, 4-((2- $n$-propyl-4-methyl-6-(1-methylbenzimidazol-2-yl)-benzimidazol-1-yl)methyl)-biphenyl-2-carboxylic acid is a highly selective lipophilic non-peptide angiotensin type 1 (AT1) receptor antagonist. In humans the major metabolite of telmisartan is the 1-O-acylglucuronide, which is transported by MRP2, P-gp and BCRP. In vitro studies using human liver microsomes and recombinant UDP-glucuronosyltransferases (UGT) 1A1, 1A4 and 1A6 identified a role for UGT1A1, whereas UGT1A4 and UGT1A6 were inactive (Ebner and Roth 1999). The aim of the present study was to investigate telmisartan as an inhibitor of glucuronidation using a panel of recombinant UGT proteins. Telmisartan was screened for inhibition of UGT 1A1, 1A3, 1A6, 1A7, 1A8, 1A9, 1A10, 2B15 and $2 \mathrm{~B} 17$ activities using the non-selective UGT substrate 4-methylumbelliferone, whereas lamotrigine and aldosterone were used as the substrate probes for UGT1A4 and UGT2B7, respectively. Incubations were performed at substrate concentrations corresponding to the $\mathrm{K}_{\mathrm{m}}$ or $\mathrm{S}_{50}$ of each enzyme in the absence or presence of telmisartan $(1,10,100$ and $1000 \mu \mathrm{M})$. Subsequently mechanisms of telmisartan inhibition and inhibitor constants were determined using UGT 1A1, 1A3, 1A4, 2B7 and 2B17. Telmisartan inhibited glucuronidation of all probe substrates with $\mathrm{IC}_{50}$ values ranging from $1.2 \mu \mathrm{M}-35.9 \mu \mathrm{M}$. Telmisartan inhibited UGT 1A1, $1 \mathrm{~A} 3,1 \mathrm{~A} 4,2 \mathrm{~B} 7$ and $2 \mathrm{~B} 17$ according to a competitive mechanism with $\mathrm{K}_{\mathrm{i}}$ values of $3.2,0.4,0.8,3.5$, and $3.7 \mu \mathrm{M}$, respectively. These data indicate that telmisartan is a non-selective inhibitor of both UGT1A and UGT2B proteins. Further studies are required to elucidate potential drug-drug interactions involving glucuronidated drugs in addition to the documented interactions involving telmisartan and ABC-transporters.

Ebner, T. \& Roth, W.In vitro glucuronidation of telmisartan, a novel angiotensin II receptor antagonist. J. Hum. Hypertension 1999; 113: Suppl. 3, S11.

\section{P40. The role of intestine and liver in glucuronidation of the imidazo- and triazoloacridinone antitumor agents, C-1311 and C-1305: High affinity substrates for UGT1A10}

\author{
$\underline{\text { Barbara Fedejko-Kap }}^{1}$, Stacie M. Bratton², Moshe Finel ${ }^{3}$, Zofia Mazerska ${ }^{1}$ and Anna Radominska- \\ Pandya $^{2}$ \\ ${ }^{1}$ Department of Pharmaceutical Technology and Biochemistry, Gdansk University of Technology, Gdansk, Poland, \\ ${ }^{2}$ Biochemistry and Molecular Biology, University of Arkansas for Medical Science, Little Rock, AR, ${ }^{3}$ Centre for Drug \\ Research, University of Helsinki, Finland
}

C-1311 (5-Diethylaminoethylamino-8-hydroxyimidazoacridinone) and C-1305 (5-dimethylaminopropylamino-8hydroxytriazoloacridinone) are antitumor agents that have also been shown to be active against autoimmune diseases. Although these compounds are close structural analogs, C-1305 is expected to have more beneficial pharmacological properties than C-1311. Both compounds have been shown to bind covalently to DNA only after activation by metabolism. It was previously demonstrated that flavin-containing monooxygenases play a significant role in the microsomal biotransformation of both compounds, while hepatic cytochromes P450 do not metabolize these compounds even though they 
are selectively inhibited by the studied agents. Moreover, in vivo studies have shown the formation of C-1311 glucuronide in mice, with high levels being found in both plasma and liver. It is hypothesized that similar glucuronidation of C-1311 and C-1305 will take place in humans. The aim of this work was to identify human UDP-glucuronosyltransferases (UGTs) capable of glucuronidation of these compounds. HPLC analysis with UV-Vis and MS/MS detection was used to analyze the activities of 12 human recombinant UGTs from the 1A and 2B subfamilies, 7 human liver microsomes (HLM) from individual donors, 12 pooled human intestinal microsomes (HIM) from individual donors, and microsomes from 7 sequential segments of gastrointestinal (GI) tract from 4 individual donors. We have found that both compounds were conjugated by several UGT1A family isoforms, with the glucuronidation activity of each active isoform being about 4 fold higher toward C-1305 than toward C-1311. Extrahepatic UGT1A10 was found to be the most active toward both compounds, and LC-MS/ MS analysis suggested that this isoform targets the 8-hydroxyl group of each. Previous structure-function relationship studies of UGT1A10 identified F90, V92 and F93 as being involved in the binding of phenol substrates, and D393 as being essential in the binding of the glucuronic acid moiety of the co-substrate, UDP-glucuronic acid. Using 7 single point mutants of UGT1A10, we have demonstrated that F90 is critical and F93 is significant for the glucuronidation of C-1305 and C-1311, as well as confirmed the importance of D393 in the glucuronidation of these compounds. We have also observed significant interindividual differences in glucuronidation activity among the HIM and HLM donors toward C-1305 and C-1311. Activity in the GI tract was generally low in the stomach and colon, and higher in the duodenum and small intestine segments. Overall, the activity in the HIM was significantly higher than in HLM. This is in agreement with the high activity seen with recombinant UGT1A10, which is expressed in the intestine and absent in the liver. These in vitro metabolic investigations can provide the a basis for predicting the pharmacological outcomes of these drugs in humans and shed light on the differences in first pass metabolism after oral versus intravenous administration, which can have implications for therapy of colon and other intestinally derived tumors. (NIH-GM075893 to AR-P)

\title{
P41. Abstract withdrawn
}

\section{P42. Transcriptional diversity at the UGT2B7 locus is dictated by extensive pre-mRNA splicing}

\author{
Vincent Ménard, Olivier Eap, Joannie Roberge, Mario Harvey, Éric Lévesque and Chantal \\ Guillemette
}

Pharmacogenomics Laboratory, CHUQ Research Center and Faculty of Pharmacy, Laval University, Québec, QC, Canada

UGT2B7 is a key member of the UDP-glucuronosyltransferase (UGT) family that participates in glucuronidation of a vast array of endogenous compounds as well as pharmaceuticals. Much evidence suggests a large inter-individual variability of UGT2B7-mediated glucuronidation, which is still unexplained by polymorphisms. We hypothesized that alternative splicing may be at least in part responsible for the variability in UGT2B7 function. In-depth analysis of the UGT2B7 gene structure led to the discovery of six novel exons, four in the 5' of the gene and two in its 3' region. Kidney and liver samples presented the greatest enrichment of tissue-specific splicing, with a total of 21 newUGT2B7 transcripts isolated. Furthermore, transcription from the proximal promoter (exon 1), associated with the active UGT2B7 mRNA isoform 1 (UGT2B7_v1), is most commonly observed in the gastrointestinal tract, whereas a distal promoter (exon la) induces the exclusion of the canonical exon 1 and is active in hormone-related tissues. We also showed that novel transcripts with alternative 3' ends could be translated into proteins lacking transferase activity in human cells but acting as negative regulators upon glucuronosyltransferase activity when co-expressed with the active UGT2B7_il protein. Overall, our findings point toward a significant variability in structure, abundance and tissue-specific UGT2B7 transcriptome, in addition to novel functions for UGT2B7-derived proteins, all of which may ensure the production of tissue-specific proteomes and functions.

\section{P43. 6-Methoxybenzo[d]thiazole-2-carbonitrile: A highly selective luminogenic substrate for CYP1A2 induction and inhibition assays}

\author{
Łames J. Cali ${ }^{1}$, Dongping Ma ${ }^{1}$, Timothy A. Moeller ${ }^{2}$, James Unch ${ }^{1}$ and Poncho Meisenheimer ${ }^{1}$ \\ ${ }^{1}$ Research Department, Promega Corporation, Madison, WI, ${ }^{2}$ Celsis In Vitro Technologies, Baltimore, MD
}

CYP1A2 plays a prominent role in drug metabolism. Its inhibition or induction by a first drug can alter metabolism of a second drug and cause an adverse drug-drug interaction. To predict in vivo CYP inhibition new pharmaceutical entities 
are tested against liver microsomes or recombinant CYP enzymes with a probe CYP substrate, which may also be used for induction testing with cultured hepatocytes. Because liver microsomes and hepatocytes contain multiple potentially cross-reacting CYP enzymes, a highly selective probe substrate is required when effects on a specific CYP are studied. Here we describe 6-methoxybenzo[d]thiazole-2-carbonitrile as a highly selective luminogenic CYP1A2 substrate suitable for in vitro assays with liver microsomes, recombinant CYP1A2 and cultured hepatocytes. This compound is a pro-substrate for firefly luciferase that is converted by CYP1A2 to a luminogenic form that produces light in a second reaction with luciferase. As light output is proportional to CYP activity, CYP1A2 inhibitors and inducers are scored as they respectively decrease light from a microsome assay or increase light from a hepatocyte assay. Compared to a fluorescent assay the luminogenic assay showed better CYP1A2 selectivity while eliminating the problem of interference by fluorescent test articles. While the assay results are well correlated to conventional HPLC or LCMS based assays, the rapid plate-based formats are easier to use, inexpensive and enabling for high throughput applications.

\title{
P44. Changes in hepatic cytochrome P450 expression after citrobacter rodentium infection in Cyp4a10- and Cyp4a14-deficient mice
}

\author{
Beatrice A. Nyagode and Edward T. Morgan
}

Emory University School of Medicine, Atlanta, GA

Previously we reported that following infection with Citrobacter rodentium, the equivalent of enteropathogenic Escherichia coli in humans, murine hepatic cytochrome P450 mRNAs are selectively regulated and that Cyp4a mRNAs were among the most sensitive to down-regulation. Here we investigate the role of Cyp4a10 or Cyp4a14 in mediating the regulation of P450s of the mouse liver during C. rodentium infection by comparing responses in WT and Cyp4adeficient mice. In this study either female or male wildtype (WT), Cyp4a10-deficient (Cyp4a10--) or Cyp4a14-deficient $\left(\right.$ Cyp $\left.4 a 14^{-/}\right)$mice were infected orally with $C$. rodentium and the hepatic expression of several P450s mRNAs were examined seven days later. In general, the mRNA expression level of untreated female and male Cyp4a-deficient animals were between $20-80 \%$ of those found in WT animals, the exception being an almost 3-fold higher expression of Cyp2a5 in male animals of either null genotype when compared with WT. In all genotypes, when significant sex differences were recorded, generally male animals had lower basal expression levels of mRNAs than female except with Cyp2c29 where WT female and male had similar expression levels but the Cyp4a-deficient males had between 2- and 20-fold higher expression. During infection, all WT and Cyp4a10-deficient groups of animals behaved similarly in their regulation of Cypla2 and 2c29. In female animals, when compared with WT, the absence of Cyp4a10 attenuated the up-regulation of Cyp2c9. The up-regulation of Cyp4f18 and down-regulation of Cyp2a5, 2d22, 3a11 and 3a25 seen in WT mice were all abrogated in Cyp4a10 $0^{--}$mice. In male animals, when compared with WT, the absence of Cyp4a10 resulted in the downregulation of both Cyp2b9 and 2e1 and the up-regulation of Cyp3a13 and $4 \mathrm{f} 18$ while the down-regulation of Cypla2, 2a5, 2c29 and 2d9 were abrogated. All WT and Cyp4a14-deficient groups of mice had similar trends in their regulation of Cypla2, 2b9, 2d9, 2el and 4a10 during infection. For both female and male animals, when compared to WT, the absence of Cyp4a14 resulted in the up-regulation of Cyp2c29 while the up-regulation of Cyp4f18 and the down-regulation of both Cyp3a11 and 3a25 were abrogated. While in WT male mice Cyp3a13 was elevated and Cyp2d22 depressed, the absence of Cyp4a14 abrogated these effects. These results indicate that the presence or absence of Cyp4a10 or Cyp4a14 affects the hepatic response to enteral C. rodentium infection reflected in the differential but overlapping impacts of Cyp4a10 and Cyp $4 a 14$ genotypes in the regulation of $\mathrm{P} 450 \mathrm{mRNAs}$. We speculate that these results may indicate a broader role of Cyp4as in the response to infection.

\section{P45. Characterization of Cyp2b-Knockdown mouse reveals changes in lipid metabolism}

\author{
Basma Damiri and William Baldwin
}

Biology/ Environmental Toxicology, Clemson Univeristy, Clemson, SC

The Cyp2b subfamily contains five members (Cyp2b9, Cyp2b10, Cyp2b13, Cyp2b19, and Cyp2b23) of which three (Cyp2b9, Cyp2b10, Cyp2b13) are hepatic enzymes involved in xenobiotic detoxification. We made a Cyp2b-knockdown mouse using lentiviral-promoted shRNA homologous to all five Cyp2b subfamily members to characterize Cyp2b's role in xenobiotic detoxification. Western blots from 8-week old mice demonstrate that hepatic Cyp2b's are repressed 5-10X by the shRNA. Unexpectedly, Cyp2b-KD mice showed significant changes in some organ weights, especially an increase in abdominal, inguinal, and renal adipose. Both male and female Cyp2b-KD mice showed significant increase in fat to body weight ratio 
(112.1\% and $73.5 \%$ for males and females, respectively). Interestingly, associated with changes in fat to body ratios was changes in non-fasting triglycerides levels. Non-fasting serum triglyceride was increased $18-39 \%$ in 8-10 weeks old female and male mice, respectively with a statistically significant increase in males. Therefore, wild-type and Cyp2b-knockdown mice were housed for 35 weeks and necropsies performed to test whether older Cyp2b-knockdown mice show perturbed lipid utilization. Thirty-five week old Cyp2b-KD mice exhibited a 17 to $22 \%$ increase in their body weight caused by a significant increase in fat deposition compared to wild-type (FVB) mice. This was accompanied by increased triglyceride and low density lipoprotein levels. Subsequent studies were performed with $100 \mathrm{ml}$ of corn oil or the CAR activator and CYP-inducer ТСРОВOP at $3 \mathrm{mg} / \mathrm{kg} /$ in corn oil as a carrier. Surprisingly, 8-12 weeks Cyp2b-knockdown mice showed some difficulty in dealing with the corn oil. Significant increases in total serum cholesterol (25-39\%) was observed in the corn oil control Cyp2b-knockdown male and female mice, respectively, compared to the WT mice, probably because the Cyp2b-KD mice were unable to respond to the unsaturated fatty acids in the corn oil. The increases in non-fasting serum total cholesterol and triglyceride was reversed by ТСРОВОP treatment in Cyp2b-knockdown male mice but not in Cyp2bknockdown female mice as ТСРОВОР treatment caused a significant increase in triglyceride in Cyp2b-KD females compared to WT mice. Cyp2b-KD mice appear to have perturbations in clearing corn oil from the liver as corn oil treatment increased Oil Red O staining indicating accumulation of lipids in the livers of Cyp2b-KD mice compared to WT mice. FoxA2 and Cptla, involved in triglyceride homeostasis, were increased 2-6 fold, albeit not significantly, in Cyp2p-KD mice treated with corn oil. In conclusion, changes in Cyp2b expression led to perturbation in lipid metabolism in Cyp2b-KD mice. This suggests that Cyp2b is more than a detoxification enzyme, but is also involved in the metabolism of unsaturated fatty acids, as Cyp2b-KD mice have increased fat deposition and show increased serum and liver lipid levels.

\title{
P46. Comparative analyses of in vitro biotransformation in human liver microsomes and MetaSite based in silico predictions for 70 new chemical entities
}

\author{
Mithat Gunduz, Jennifer L. Bushee, Upendra A. Argikar, Giuliano Berellini, Kevin Colizza, Hong Jiang, \\ Michelle Dennehy, Amanda Cirello, Nigel Waters, Amin Kamel, Franco Lombardo and Shawn Harriman
}

\author{
Metabolism and Pharmacokinetics, Novartis Institutes for Biomedical Research Inc., Cambridge, MA
}

MetaSite, a software program developed by Molecular Discovery Ltd., that predicts CYP-mediated sites of metabolism based on the CYP enzyme's active site, and orientation of a substrate via GRID molecular interaction field descriptors. It further accounts for accessibility and reactivity of a given CYP and a given substrate respectively, to determine each atom's probability of metabolisml. MetaSite is routinely employed in drug discovery to understand metabolic liabilities of new chemical entities2. The objective of the present investigation was (i) to compare in vitro human liver microsomal metabolite identification results with in silico soft-spot predictions by Metasite and (ii) to understand the molecular and ADME properties leading to high predictive accuracy as well as those drivers implicated in low predictive accuracy. To this effect, human liver microsomal biotransformation studies were carried out for 70 new chemical entities. Top three metabolic soft spots were computed by Metasite, for liver as a metabolic model, with reactivity correction enabled. Comparison between in silico and in vitro results revealed approximately $70 \%$ accuracy of prediction by Metasite for new chemical entities. A weighted average of MetaSite's top three sites of metabolism was calculated from the Metasite score and was compared with human microsomal Clint. Preliminary observations indicate a tendency that the prediction of MetaSite is better for new chemical entities with high microsomal extraction ratios i.e. higher Clint ( $>300 \mathrm{uL} / \mathrm{min} / \mathrm{mg}$ of microsomal protein). Similarly a general tendency toward increase in confidence of prediction by Metasite for the top three metabolic soft spots was seen with increase in the number of observed metabolites in human liver microsomal incubations. 1. Cruciani, G.; Carosati, E.; De Boeck, B. et al. (2005) J. Med. Chem. 48, 6970-6979. 2. Trunzer, M.; Faller, B.; and Zimmerlin, A. (2009) J. Med. Chem. 52:329-335.

\section{P47. Comparison of P450 2D and 3A-dependent drug oxidation activities in cynomolgus monkeys, microminipigs, and humans}

\author{
Hiroshi Yamazaki $^{1}$, Chie Emoto ${ }^{1}$, Ryo Koizumi ${ }^{1}$, Makiko Shimizu $^{1}$, Norie Murayama ${ }^{1}$, Kazuhide \\ Iwasaki $^{2}$, Masahiro Utoh ${ }^{2}$ and Yasuhiro Uno ${ }^{2}$
}

\section{${ }^{1}$ Showa Pharmaceutical Univ, Machida, Japan, ²Shin Nippon Biomedical Laboratories, Kainan, Japan}

Cynomolgus monkeys (Macaca fascicularis, $\mathrm{mf}$ ) are widely used to determine pharmacokinetics and toxicological potential of many drug candidates as human models in the drug discovery and development. Small minipigs (Microminipig; registered as a novel variety of pig in Japan) were found and developed ( $<10 \mathrm{~kg}$ body weight at 7 months-old). Cytochrome 
P450s (P450, CYP), one of the most important enzymes in drug metabolism, in monkey livers are generally similar to corresponding human P450s exhibiting high degrees of homologies in cDNAs and amino acid sequences. Therefore, species differences regarding important liver P450 3A and 2D function were examined among cynomolgus monkeys, microminipigs, and humans using typical human P450 probe reactions. CYP3A-mediated midazolam 1'-hydroxylation activities in liver microsomes from individual monkey were highly correlated with midazolam 4'-hydroxylation activities but not correlated with dextromethorphan $N$-demethylation activities. Apparent dextromethorphan $O$-demethylation and bufuralol 1'-hydroxylation activities of monkey or microminipig liver microsomes were higher than those of human liver microsomes. Recombinant monkey CYP2D17 and CYP2D44 catalyzed dextromethorphan $\mathrm{O}$ - and $\mathrm{N}$-demethylation as well as monkey mfCYP3A4 and mfCYP3A5 did, although contributions of corresponding CYP2D6 and CYP3A4/3A5 to dextromethorphan $\mathrm{N}$ - and $\mathrm{O}$-demethylation, in human liver microsomes were negligible. These results demonstrate that monkey P450 2D and 3A enzymes showed similar substrate selectivity to those of human P450 enzymes, but exhibited wider substrate selectivity toward P450 2D substrate oxidation than those in human livers. Special attention should be paid when enzymatic and pharmacokinetic data are extrapolated from non-rodents to humans.

\title{
P48. Drug interactions of thalidomide with midazolam in human hepatoma cell line system with differentiated HepaRG cryopreserved
}

\author{
Norie Murayama, Kanako Yajima, Makiko Shimizu, Masahiro Utoh and Hiroshi Yamazaki
}

Showa Pharmaceutical Univ, Machida, Japan

We previously reported the drug interactions of thalidomide with midazolam and cyclosporine A using recombinant human cytochrome P450 3A enzymes and liver microsomes. Heterotropic cooperativity of human P450 3A5 mediated by thalidomide was shown in these in vitro systems. The purpose of this study was to investigate these drug interactions in cultured human liver cell line system more closely resembled livers. A new hepatic cell line system with differentiated HepaRG cryopreserved (Biopredic International, Rennes, France) was adopted. After thawing and culturing of differentiated HepaRG cells cryopreserved, typical oxidation activities were analyzed with midazolam (CYP3A4), omeprazole (CYP2C19), dextromethorphane (CYP2D6), and diclofenac (CYP2C9) by LC with tandem MS spectrometry system. These marker drug oxidation activities of HepaRG cells tended to be increased up to 1 week gradually and maintained for 2 weeks. Under these conditions, midazolam 1'-hydroxylation activity was suppressed by $50 \%$ in the presence of $100 \mu \mathrm{M}$ thalidomide while no effects were seen on midazolam 4-hydroxylation. On the contrary, both midazolam 1'- and 4-hydroxylation activities were suppressed by $100 \mu \mathrm{M}$ of 5-hydroxythalidomide, a primary human metabolite of thalidomide. These results suggest that cultured human cell line system with differentiated HepaRG cryopreserved could be applicable to thalidomide drug interaction study. Further oxidative metabolism of 5-hydoroxythalidomide to 5-hydroxythalidomide GSH-conjugate formation is under investigation using the new HepaRG cell system mimicking in vivo human livers.

\section{P49. Effect of 6 Schisandrins from Wuzhi tablet (Schisandra sphenanthera extract) on the activity of cytochrome P450 3A and P-glycoprotein in rats}

\author{
Bi Huichang ${ }^{1}$, Qin Xiaoling ${ }^{1}$, Xue Xinping ${ }^{1}$, Wang Ying ${ }^{1}$, Chen Xiao ${ }^{2}$ and Huang Min ${ }^{1}$ \\ ${ }^{1}$ School of Pharmaceutical Sciences, Sun Yat-sen University, Guangzhou, China, ${ }^{2}$ The First Affiliated Hospital, Sun \\ Yat-sen University, Guangzhou, China
}

Objective We recently reported that the blood concentrations of Tacrolimus (FK506) in rats were markedly increased following the intake of a Chinese herbal preparation, Wuzhi Tablet (WZ, Schisandra sphenanthera extract), which might be due to the inhibition of CYP3A and/or P-gp via substances in Wuzhi tablet. Thus, in this study the effect of 6 Schisandrins from Wuzhi tablet on the rat hepatic and intestinal CYP3A and P-gp activity after short-term pretreatment was studied. Method Male Sprague-Dawley rats were randomized to several groups: for oral dosing, Midazolam (MDZ, $2 \mathrm{mg} / \mathrm{kg}$ ) was administered orally to rats with or without an oral dose of water or 6 kinds of schisandins $(0.024 \mathrm{mM} / \mathrm{kg}$, including Schisandin A, B, C or Schisandol A, B or schisantherin A), 30 min after MDZ dosing, rats were given another substrate Digoxin (DG, $0.25 \mathrm{mg} / \mathrm{kg}$ ); for intravenous dosing, DG $(0.1 \mathrm{mg} / \mathrm{kg})$ as well as MDZ $(1 \mathrm{mg} / \mathrm{kg})$ was administered intravenous injection to rats with or without an oral dose of water or 6 kinds of schisandrins. Blood samples were collected from the right cannulated jugular vein at proper time. Target analytes were determined by a fully-validated LC-MS/MS method. Pharmacokinetic parameters were calculated by non-compartment model, and statistical analysis was performed using SAS software. Results When MDZ and DG was orally administered to rats in the presence of most of the schisandrins, 
plasma concentrations of MDZ and DG were increased in different extent. Schisandrol B showed the strongest effect and can increase 8.7 folds and 1,0 fold of the $\mathrm{AUC}_{0-4 \mathrm{~h}}$ of MDZ and DG, respectively. The next was Schisantherin A which increased $189.1 \%$ and $73.5 \%$ of the AUC of MDZ and DG, respectively. When MDZ and DG were administered to rats intravenously, plasma concentrations of MDZ were also increased and elimination was delayed when coadministered Schisandrol B, but the extent of effect was much slighter than that of oral groups. When coadministered with Schisandrol B, the AUC o-4h of MDZ was only increased by $67.8 \%$ for intravenous dosing, indicating Schisandrol B could dramatically inhibit the intestinal and liver CYP 3A activity and Schisandrol B possessed much stronger inhibiting effect on intestinal CYP3A than liver CYP 3A. However, Schisandrol B showed similar effect on oral and intravenous DG, indicating Schisandrol B possessed much stronger inhibiting effect on liver P-gp activity.Conclusion Plasma concentrations of MDZ and DG were increased when co-administration with the schisandrins by inhibiting intestinal and hepatic CYP3A and P-gp activity. Schisandrol B showed the strongest effect. Schisandrol B had direct inhibitory effect on intestinal CYP3A and hepatic P-gp activity. Schisandrol B possessed much stronger inhibitory effect on intestinal CYP3A than that of hepatic P-gp activity.

\title{
P50. Effects of CYP2C9*3, *28 and *30 on CYP2C9 inhibition profiles and glyburide metabolism in vitro
}

\author{
Keiko Maekawa, Noriko Harakawa, Su-Ryang Kim, Jun-ichi Sawada and Yoshiro Saito \\ National Institute of Health Sciences, Tokyo, Japan
}

Background and purpose:Cytochrome P450 2C9 (CYP2C9) is a polymorphic enzyme responsible for the oxidative metabolism of up to $15 \%$ of the drugs that undergo phase I metabolism. We have already reported that $C Y P 2 C 9 * 3, * 28$ and $* 30$ exhibit reduced CYP2C9 activities in a substrate-dependent manner. This study was aimed to evaluate the effects of these alleles on CYP2C9 inhibition and glyburide metabolism in vitro. Methods: The wild-type and three variant proteins were expressed using a baculovirus-insect cell system, and microsomal fractions were prepared. IC50 values of 15 drugs to the wild-type and variant enzymes were measured using the P450-Glo assay which employed a luminogenic CYP2C9 probe substrate, Luciferin-H. In addition, kinetic parameters of the wild-type and variant enzymes for glyburide hydroxylation were assessed. Results:All variants exhibited significantly lower Luciferin-H hydroxylation activities than CYP2C9.1 due to increased $K_{\mathrm{m}}$ values and decreased $V_{\max }$ values. When Luciferin-H was used at concentrations equal to the apparent $K_{\mathrm{m}}$ value of each variant, IC50 values of ketoconazole to all three variants were significantly lower than that of CYP2C9.1. The inhibitions of CYP2C9.28 by 9 drugs including sulfaphenazole were significantly weakened compared with those of CYP2C9.1. The IC50 values of two and five drugs were significantly lower and higher, respectively, to CYP2C9.30 than those to CYP2C9.1. Especially, glyburide exhibited 64-fold higher IC50 value to CYP2C9.30 than that to CYP2C9.1, suggesting that CYP2C9.30 had decreased binding affinity to glyburide in the substrate recognition site. As expected from the inhibition profile of glyburide to CYP2C9.30, the catalytic activity of CYP2C9.30 to glyburide was decreased by $>70 \%$ due to the increased $K_{\mathrm{m}}$ value. Conclusion: $C Y P 2 C 9 * 3, * 28$ and $* 30$ alleles would exhibit distinct enzyme activities and drug-drug interactions from $C Y P 2 C 9 * 1$.

\section{References}

Maekawa, K., Harakawa N. et al. (2009) Substrate-dependent functional alterations of seven CYP2C9 variants found in Japanese subjects. Drug Metab Dispos. 37:1895-1903.

\section{P51. Evaluation of broad-spectrum, irreversible CYP450 inhibitors and an inhibition cocktail for metabolite identification studies}

\author{
Jennifer L. Bushee, Mithat Gunduz, Kevin Colizza, Amin Kamel, Shawn Harriman and Upendra A. \\ $\underline{\text { Argikar }}$
}

\section{Metabolism and Pharmacokinetics, Novartis Institutes for Biomedical Research Inc., Cambridge, MA}

Characterization of metabolites and understanding metabolic soft-spots in a chemical scaffold is routinely carried out in drug discovery in liver microsomal models across multiple species. However, oxidative metabolites identified in microsomal incubations in the presence of NADPH may arise from either CYP or FMO enzymes. The objective of our investigation was to evaluate the efficiency of commercial, irreversible, non-selective CYP inhibitors, either individually or in combination with one another, for application in biotransformation studies. Buspirone, is largely metabolized by CYPs and the metabolites have been well characterized. Therefore it was chosen as a probe substrate. Known time dependent CYP inhibitors 
such as 1-aminobenzotriazole, (1-ABT), proadifen or 2-(diethylamino)ethyl 2,2-diphenylpentanoate (SKF525A) and a cocktail (I-cocktail) of known specific irreversible CYP inhibitors were evaluated for their CYP knockout efficiencies. The I-cocktail consisted of furafylline (CYP1A2), 8-methoxypsoralen (CYP2A6), ticlopidine (CYP2B6), amiodarone (CYP2C8), tienilic acid (CYP2C9), ticlopidine (CYP2C19), paroxetine (CYP2D6), diethylthiocarbamate (CYP2E1) and erythromycin (CYP3A4). The experiments were carried out using rat and human liver microsomes fortified with NADPH with and without mechanism based inhibitor pre-treatment. Metabolite(s) identification of buspirone was carried out on a ThermoFinnigan LTQ-Orbitrap-XL ${ }^{\circledR}$ with accurate mass measurement and MSn capabilities. Control experiments revealed that less than $5 \%$ buspirone remained at the end of the one hour incubation period, in contrast to $89 \%, 69 \%$ and $77 \%$ remaining for 1-ABT, SKF525A and I-cocktail pre-treatments respectively in human liver microsomes. Formation of an N-dealkylated carboxylic acid metabolite was inhibited in all three pre-treatments. Another metabolite formed subsequent to piperazine ring opening was absent in human liver microsomal incubations pre-treated with I-cocktail, although present in reduced peak areas in the incubations pre-treated with 1-ABT and SKF525A. Effects of the above time dependent inhibitors on each of the fourteen or more identified buspirone metabolites in rat and human liver will be presented.

\title{
P52. Human CYP3A4 mediates the biotransformation of sorafenib to its major oxidized metabolites
}

\author{
Michael Murray, Sussan Ghassabian, Fanfan Zhou, Bruce N. Tattam, Munikumar R. Doddareddy, \\ David E. Hibbs and Tristan Rawling
}

Pharmacy, The University of Sydney, Sydney, Australia

Sorafenib (4-[4-([4-chloro-3-(trifluoromethyl)phenyl]carbamoylamino)phenoxy]- $N$-methyl-pyridine-2-carboxamide) is an orally active tyrosine kinase inhibitor that is used in the treatment of liver and renal cancers. Despite its clinical value adverse effects frequently necessitate dose interruption or termination of therapy, and under-dosage due to rapid clearance may lead to therapeutic failure. Information on the human cytochromes P450 (CYPs) that mediate the oxidation of sorafenib to its principal metabolites could be used in the development of dose optimization strategies. In this study the major phase I metabolites of sorafenib were synthesized and the roles of individual human CYPs in biotransformation pathways were characterized using a novel liquid chromatography/tandem mass spectrometry assay. cDNA-expressed CYP3A4 was active in the formation of the major $N$-oxide and $N$-hydroxymethyl metabolites of sorafenib ( 68 and $30 \mathrm{pmol} / \mathrm{pmol} \mathrm{CYP} / \mathrm{h}$, respectively). The closely related CYP3A5 exhibited only $~ 5 \%$ of the activity of CYP3A4 in the formation of both metabolites; eleven other CYPs and 3 flavin-containing monooxygenase (FMO) enzymes were inactive. CYP3A4 also selectively catalyzed sorafenib $N$-demethylation, but metabolite formation was extremely low. In human hepatic microsomes $(\mathrm{n}=14)$ sorafenib $N$-oxide formation varied 6.9 -fold $(56-385 \mathrm{pmol} / \mathrm{mg}$ protein $/ \mathrm{min}$ ) and $N$-hydroxymethylsorafenib formation varied over a 5.7 -fold range (25-143 pmol/mg protein/min). Both reactions were correlated with CYP3A4-mediated midazolam 1'-hydroxylation, but not with CYP1A2, CYP2A6, CYP2C8, CYP2C9 or CYP2D6-dependent reactions. In accord with these findings the CYP3A4 inhibitor ketoconazole inhibited sorafenib oxidation in human liver but other CYP and FMO inhibitors were inactive. $\mathrm{K}_{\mathrm{m}}$ values for microsomal sorafenib oxidation varied over the range 8-28 $\mu \mathrm{M}$, while $\mathrm{V}_{\max }$ values were in the range 120-236 and $64-120 \mathrm{pmol} / \mathrm{mg}$ protein $/ \mathrm{min}$ for the $N$-oxide and $N$-hydroxymethyl metabolites, respectively. Modeling of sorafenib in the CYP3A4 active center was undertaken using Glide software after structural manipulation by Maestro in MacroModel (Schrödinger Inc). In the optimal pose the atoms in the structure of sorafenib that undergo biotransformation were within $\sim 5.4$ Å of the CYP3A4 heme iron. Hydrogen bonding interactions between the terminal amide group and pyridine nitrogen in sorafenib and the amino acids Ser119, Arg212 and Glu374 of CYP3A4 were identified. The present findings indicate that sorafenib is oxidized selectively to one minor and two major metabolites by human CYP3A4. Inter-individual variation in hepatic CYP3A4 activity is a likely contributor to sorafenib efficacy and toxicity in cancer patients.

\section{P53. Human cytochrome P450 2E1 inhibits cytochrome P450 2B4's activity by competing for cytochrome $\mathbf{P 4 5 0}$ reductase through a synergistic interaction}

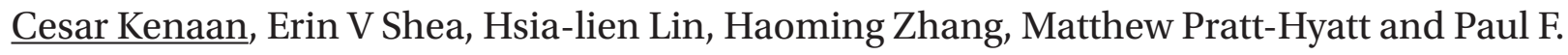 \\ Hollenberg
}

Department of Pharmacology, University of Michigan, Ann Arbor, MI

For efficient catalysis to occur, cytochromes P450 (CYPs or P450s) require an interaction with their physiological redox partner, cytochrome P450 reductase (CPR). However, CPR is in limited supply as it exists in a 1:10 to 1:25 ratio to P450 
in vivo. Furthermore, studies in both microsomal and reconstituted systems have shown that the presence of one P450 isoform can significantly influence the catalytic activity of another isoform. In this study, we assessed whether CYP2E1 could influence CYP2B4's catalytic properties under steady-state turnover conditions. The results show that CYP2E1 can inhibit the CYP2B4-mediated metabolism of benzphetamine (BNZ) with a $\mathrm{K}_{\mathrm{i}}$ of $0.05 \mu \mathrm{M}$. However, CYP2B4 was incapable of inhibiting CYP2E1-mediated p-nitrophenol hydroxylation. When these inhibition studies were performed with an artificial electron donor, tert-butyl hydroperoxide, CYP2B4 was resistant to inhibition by CYP2E1. CYP2B4 was also significantly less susceptible to inhibition by a CYP2E1 Y422D mutant, which exhibits impaired binding to CPR. Determining the apparent $\mathrm{K}_{\mathrm{M}}$ of CYP2B4 for CPR in the presence of increasing concentrations of CYP2E1 revealed the dual competitive and non-competitive nature of CYP2E1 inhibition since at low concentrations of CYP2E1 (0.125- 0.75 $\mu \mathrm{M}$ ), CYP2E1 increased CYP2B4's $\mathrm{K}_{\mathrm{M}}$ by 13-fold but had no effect on $\mathrm{k}_{\mathrm{cat}}$. Yet at higher concentrations of CYP2E1 (1.25$1.50 \mu \mathrm{M}) \mathrm{K}_{\mathrm{M}}$ decreased to similar levels that were observed with CYP2B4 in the absence of CYP2E1; however, $\mathrm{k}_{\text {cat }}$ also decreased by 10-fold. Additionally, CYP2E1 increased the $\mathrm{K}_{\mathrm{M}}$ of CYP2B4 for BNZ by 8-fold, although the $\mathrm{K}_{\mathrm{M}}$ was recovered when saturating concentrations of CPR were used. When CYP2B4 and CYP2E1 were incubated together in the presence of a chemical cross-linker (bis(sulfosuccinimidyl)suberate, BS ${ }^{3}$ ), no CYP2E1-CYP2B4 cross-links were observed indicating that CYP2B4 and CYP2E1 do not interact with each other and suggesting that CYP2E1's inhibitory properties are not likely due to direct P450-P450 interactions. Although the individual $\mathrm{K}_{\mathrm{M}}$ values of CYP2E1 and CYP2B4 for CPR are virtually identical, the $\mathrm{K}_{\mathrm{M}}$ of CYP2E1 for CPR when measured in the presence of CYP2B4 decreased to $0.07 \mu \mathrm{M}$ suggesting that a synergistic interaction is a likely mechanism for CYP2E1's competitive properties. (Supported in part by NIH grant CA16954).

\title{
P54. Abstract withdrawn
}

\section{P55. Identification of cytochrome P450 enzymes involved in microsomal metabolism of 20(S)- protopanaxadiol in human liver and intestine}

\author{
Nga Ting Colette Chiu ${ }^{1}$, Subrata Deb $^{2}$, Hans Adomat ${ }^{2}$, William Jia ${ }^{1}$ and Emma Guns ${ }^{2}$ \\ ${ }^{1}$ Department of Surgery, University of British Columbia, Vancouver, BC, Canada, ${ }^{2}$ Vancouver Prostate Centre, \\ Vancouver, BC, Canada
}

Cytochrome P450 (CYP) enzymes are mainly involved in oxidative metabolism of xenobiotics in humans. 20(S)protopanaxadiol (aPPD), a gastrointestinal bacterial metabolite of ginseng saponin, may be responsible for the antidepressant activity of ginseng. Previously, we have identified three monohydroxylated and five minor dihydroxylated products of aPPD following in vitro incubations with human intestinal microsomes (HIM) and human liver microsomes (HLM). However, the enzymes responsible for oxidative metabolism of aPPD are unknown. The present study aims to determine the contribution of individual CYP enzymes in the formation of monohydroxylated aPPD metabolites in HLM and HIM. Reaction phenotyping was carried out by using specific CYP chemical inhibitors and a panel of human recombinant CYP enzymes. CYP chemical inhibitors used in the study were as follows: $\alpha$-naphthoflavone (10 $\mu \mathrm{M}$; CYP1A1), furafylline (20 $\mu \mathrm{M}$; CYP1A2), 8-methoxypsoralen (1 $\mu \mathrm{M}$; CYP2A6), orphenadrine (200 $\mu \mathrm{M}$; CYP2B6), sulfaphenazole $(20 \mu \mathrm{M}$; CYP2C9), quinidine (10 $\mu \mathrm{M}$; CYP2D6), diethyldithiocarbamate $(50 \mu \mathrm{M}$; CYP2E1), ketoconazole (1 $\mu \mathrm{M}$; CYP3A4/5), troleandomycin ( $20 \mu \mathrm{M}$; CYP3A4), and SKF 525A (500 $\mu \mathrm{M}$; a broad spectrum CYP inhibitor). CYP inhibitors were co-incubated with $4 \mu \mathrm{M}$ and $11 \mu \mathrm{M}$ aPPD in HLM $(0.15 \mathrm{mg} / \mathrm{ml})$ and HIM $(0.25 \mathrm{mg} / \mathrm{ml})$, respectively, in $67 \mathrm{mM}$ phosphate buffer $(\mathrm{pH} 7.4)$ and NADPH-regenerating system for $10 \mathrm{~min}$. A panel of baculovirus-expressed human recombinant enzymes (30 pmol/ml; CYP1A1, CYP1A2, CYP2A6, CYP2B6, CYP2C8, CYP2C9, CYP2C19, CYP2D6, CYP2E1, CYP3A4, CYP3A5, and CYP4A11) was incubated with $11 \mu \mathrm{M}$ aPPD as described above. The hydroxylated metabolites of aPPD were resolved using a liquid chromatography/mass spectrometry (LC/ MS) on a BEH C ${ }_{18}$ column $(1.7 \mu \mathrm{m}, 2.1 \times 100 \mathrm{~mm})$ in positive ionization mode. Monohydroxylated metabolites of aPPD were identified by multiple reaction monitoring of the $m / z 477>459$ and $459>441$ transitions in the LC/MS assay. Ketoconazole potently inhibits metabolite formation (85-95\% inhibition) in both HLM and HIM, while diethyldithiocarbamate only shows weak inhibition (20-40\%). Interestingly, troleandomycin moderately inhibits (65-70\%) metabolite formation in HIM but shows weak inhibition (30-35\%) in HLM. Among the human recombinant P450 enzymes examined, recombinant CYP3A4 showed the highest activity in metabolizing aPPD, followed by CYP3A5. In summary, our results show that CYP3A isoforms are the predominant enzymes responsible for aPPD monohydroxylation in HLM and HIM. 


\title{
P56. In vitro assessment of methylene blue hydrate as a multiple CYP450 inhibitor and a mechanism-based inhibitor
}

\author{
HyunJi Jeong, Yeonjung Choi, Myoungki Baek and Hongsik Moon
}

\author{
Preclinical Research Lab, SK Biopharmaceuticals, Daejeon, South Korea
}

Methylene blue hydrate (MBH) is used to treat methemoglobinemia and urinary tract infections. $\mathrm{MBH}$ is also used as a dye or staining agent to make certain body fluids and tissues. Recently, MBH is developed as an investigational drug for Alzheimer's disease ${ }^{1), 2), 3}$. Despite many uses in a range of different fields, there are no known drug interactions of MBH. Drug-drug interactions (DDIs) remain an important issue in the drug discovery and development. Especially, cytochrome P450 (CYP450) inhibition and mechanism-based inhibition (MBI) might cause clinically significant DDIs ${ }^{4)}$ ${ }^{5)}$. Consequently, to assess the risk of DDIs of MBH, we studied the potential for CYP450 inhibition and MBI of MBH on multiple CYP450 enzymes using human liver microsomes. For CYP450 $\mathrm{IC}_{50}$ determinations, multiple concentrations of $\mathrm{MBH}$ were prepared and pre-incubated with human liver microsomes and post-incubated with substrate and NADPH at $37.5^{\circ} \mathrm{C}$ to initiate the reaction. The reactions were terminated by combining with ice-cold acetonitrile and the supernatant from each sample after centrifugation was analyzed by LC/MS/MS. MBH at a concentration of $10 \mu \mathrm{M}$ showed more than $87 \%$ inhibition for six CYP450 enzymes, CYP1A2, 2C8, 2C9, 2C19, 2D6, and 3A4 (using two substrates). $\mathrm{IC}_{50}$ value for $1 \mathrm{~A} 2$ was $0.63 \mu \mathrm{M}, 2 \mathrm{C} 8$ was $2.66 \mu \mathrm{M}, 2 \mathrm{C} 9$ was $1.46 \mu \mathrm{M}, 2 \mathrm{C} 19$ was $0.33 \mu \mathrm{M}, 2 \mathrm{D} 6$ was $1.67 \mu \mathrm{M}, 3 \mathrm{~A} 4$ using nifedipine as a substrate was $2.71 \mu \mathrm{M}$, and $3 \mathrm{~A} 4$ using testosterone as a substrate was $1.01 \mu \mathrm{M}$, respectively. As a result, $\mathrm{MBH}$ showed moderate to potent inhibition ( $\mathrm{IC}_{50}<10 \mu \mathrm{M}$ ) in tested six CYP450 isoforms. To evaluate whether $\mathrm{MBH}$ is also possible to show MBI, we further performed MBI test using human liver microsomes and major CYP450 enzymes with industry recommended probe substrates ${ }^{4), 5), 6)}$. $\mathrm{MBH}$ at a concentration of $1 \mu \mathrm{M}$ showed similar MBI on CYP1A2, 2C8, 2C9, 2C19, 2D6, and 3A4 (using two substrates) by comparison with reference compounds known as selective mechanism-based inhibitors. These data indicate that MBH has the potential as a multiple CYP450 inhibitor and mechanism-based inhibitor of CYP1A2, 2C8, 2C9, 2C19, 2D6 and 3A4.

1) Sabbagh MN, Drug development for Alzheimer's disease: Where are we now and where are we headed?, Am. J. Geriatr. Pharmacother., 7(3), 167-185, 2009

2) Wiklund L, Basu S, et al., Neuro- and cardioprotective effects of blockade of nitric oxide action by administration of methylene blue, Ann. N. Y. Acad. Sci., 1122, 231-244, 2007

3) Oz M, Lorke DE, et al., Methylene blue and Alzheimer's disease, Biochem. Pharmacol., 78(8), 927-932, 2009

4) Watanabe A, Nakamura K, et al., Risk assessment for drug-drug interaction caused by metabolism-based inhibition of CYP3A using automated in vitro assay systems and its application in the early drug discovery process, Drug Metab. Dispos., 35(7), 1232-1238, 2007

5) FDA Draft Guidance, Guidance for industry, Drug interaction studies - study design, data analysis, and implications for dosing and labeling, 1-52, 2006

6) Obach RS, Walsky RL, Mechanism-based inactivation of human cytochrome P450 enzymes and the prediction of drug-drug interactions, Drug Metab. Dispos., 35(2), 246-255, 2007

\section{P57. In vitro metabolism of corydaline and characterization of cytochrome P450 enzymes responsible for its metabolism in human liver microsomes}

\author{
$\underline{\text { Hye Young } \mathrm{ii}^{1}}$, Sae Rom Lim${ }^{1}$, Hye Ri Lee ${ }^{2}$ and Hye Suk Lee ${ }^{1}$
}

\begin{abstract}
${ }^{1}$ College of Pharmacy, The Catholic University of Korea, Wonmi-gu, Bucheon, Gyeonggi-do, South Korea, ${ }^{2}$ Agricultural Biotechnology, The Catholic University of Korea, Wonmi-gu, Bucheon, Gyeonggi-do, South Korea
\end{abstract}

Corydaline is the bioactive alkaloid isolated from Corydalis Tubers with various antiacetylcholinesterase, antiallergic, and antinociceptive activities. The purposes of this study were to identify the in vitro metabolism of corydaline in human liver microsomes and to characterize the cytochrome P450 (CYP) enzymes responsible for the metabolism of corydaline. Incubation of corydaline with human liver microsomes in the presence of an NADPH generating system resulted in the formation of eight metabolites including O-desmethylcorydaline (M1, M2, M3, and M4), hydroxydesmethylcorydaline (M5), didesmethylcorydaline (M6, M7), and hydroxycorydaline (M8). The formation of desmethylcorydaline, M1 from corydaline was major metabolic pathway, and the formation of desmethylcorydaline, M2, M3, and M4 and hydroxycorydaline (M8) were minor metabolic pathways. The human CYP enzymes responsible for corydaline metabolism were characterized by evaluating on the basis of a combination of correlation analysis and experiments 
including immunoinhibition of corydaline in human liver microsomes and metabolism of corydaline by human cDNAexpressed CYP enzymes. CYP2C9 and CYP3A4 were major enzymes involved in the formation of a major metabolite, O-desmethylcorydaline, M1 from corydaline. CYP3A4 was the major enzyme for the formation of O-desmethylcorydaline, M2 and M3, and hydroxycorydaline, M8 from corydaline with a little involvement of CYP2D6, CYP1A2, or CYP2C8, respectively. These results suggest that the pharmacokinetics of corydaline may be affected by CYP2C9 and CYP3A4 responsible for the metabolism of corydaline or by the co-administration of appropriate CYP2C9 and CYP3A4 inhibitors or inducers.

\title{
P58. Inhibitory properties of trapping agents, reducing agents, radical scavengers or chemical inhibitors against major human cytochrome P450 isoforms
}

\author{
Jasleen Sodhi ${ }^{1}$, Erlie Delarosa ${ }^{2}$, Patrick M. Dansette ${ }^{3}$ and Cyrus Khojasteh ${ }^{1}$
}

${ }^{1}$ Drug Metabolism and Pharmacokinetics, Genentech Inc., South San Francisco, CA, ${ }^{2}$ Dmpk, Genentech Inc., South San Francisco, CA, ${ }^{3}$ Cnrs Umr 8601, Uninersité Paris Descartes, Paris CX 06, France

Characterization of reactive metabolites can be assessed by incubating the drug of interest, liver microsomes and NADPH with one of the following types of agents: a trapping agent (nucleophiles or electrophiles), reducing agent, radical scavenger or chemical inhibitor. In this study, we assessed the capacity of these agents to inhibit the major cytochrome P450 isoforms. Each agent was tested at a different concentration in order to mimic concentrations commonly used in vitro (these concentrations are noted next to each compound below). Of all eight trapping agents tested, mercaptoethanol $(14.3 \mathrm{mM})$ showed inhibition with IC50 values of $0.603 \mathrm{mM}$ for CYP1A2, $0.535 \mathrm{mM}$ for CYP2C9, $0.283 \mathrm{mM}$ for CYP2C19, $17.6 \mathrm{mM}$ and $10.1 \mathrm{mM}$ for CYP3A4. Thiocyanate $(4 \mathrm{mM})$ showed inhibition of 2C19 with an IC50 value of $1.3 \mathrm{mM}$. Morpholine (11.4 mM), N-methyl-piperidine (8.2 $\mathrm{mM})$, dimedone $(0.050 \mathrm{mM})$, $\mathrm{N}$-acetyl-lysine $(2.5 \mathrm{mM}), \mathrm{N}$-acetyl-cysteine $(1 \mathrm{mM})$ and 5-methyl-1,3-cyclohexanedione $(0.050 \mathrm{mM})$ did not inhibit the enzymes at concentrations noted. Of the three reducing agents tested, arsenite $(5 \mathrm{mM})$ showed inhibition with IC50 values of $2.5 \mathrm{mM}$ for CYP1A2 and $1.8 \mathrm{mM}$ for CYP2D6. Both tris(2-carboxyethyl)phosphine (2 mM) and dithiothreitol $(4 \mathrm{mM})$ did not show inhibition. Ascorbic acid $(3 \mathrm{mM})$, a radical scavenger, did not inhibit the enzymes. Trichloropropeneoxide $(1 \mathrm{mM})$, a chemical inhibitor of epoxide hydrolase, did not show inhibition. Two electrophiles were tested and both showed inhibition. N-ethylmaleimide $(0.400 \mathrm{mM})$ showed inhibition with IC50 values of $0.0042 \mathrm{mM}$ for CYP1A2, $0.0252 \mathrm{mM}$ for CYP2D6, $0.0312 \mathrm{mM}$ and $0.0063 \mathrm{mM}$ for CYP3A4. N-methylmaleimide $(0.4 \mathrm{mM})$ showed inhibition with IC50 values of $0.008 \mathrm{mM}$ for CYP1A2, $0.126 \mathrm{mM}$ for CYP2D6 and $0.0178 \mathrm{mM}$ for CYP3A4. Our results suggest that many of these agents may be used at these concentrations for trapping studies. However, mercaptoethanol, thiocyanate, aresenite, $\mathrm{N}$-ethylmaleimide and $\mathrm{N}$-methylmaleimide cause inhibition at millimolar concentrations and addition of these compounds may decrease the amount of reactive metabolites being formed by inhibiting cytochrome P450 activity.

\section{P59. Mechanism-based inactivation of cytochrome P450 2B6 by methadone}

\section{Hemali Amunugama}

\section{Pharmacology, University of Michigan, Ann Arbor, MI}

MECHANISM-BASED INACTIVATION OF CYTOCHROME P450 2B6 BY METHADONE Hemali Amunugama, Haoming Zhang and Paul F. Hollenberg Department of Pharmacology, University of Michigan, Ann Arbor, MI, USA 48109. Methadone is a $\mu$-opioid receptor agonist widely used in the treatment of narcotic addiction and in chronic pain conditions. Methadone is metabolized predominantly in the liver by cytochrome P450s (CYPS) to its pharmacologically inactive primary metabolite 2-ethylidene-1,5-dimethyl-3,3-diphenylpyrrolidine. Initial in vitro data suggested that CYP3A4 is the major isoform responsible for the in vivo clearance of methadone in humans. However, recent clinical data have identified that CYP2B6 is actually the major isoform responsible for in vivo methadone metabolism and clearance.In this study, methadone was analyzed for its ability to act as a mechanism-based inactivator of CYP2B6. Methadone inactivates CYP2B6 in a time, concentration and NADPH dependent manner with a $\mathrm{KI}=10.0 \mu \mathrm{M}$ and kinact $=0.02 \mathrm{~min}-1$. The activity loss of 2B6, in the presence of methadone and NADPH occurred with a concurrent loss of the reduced CO spectrum of the P450. Moreover, a good correlation between the loss of 2B6 activity and the loss of CO-binding was observed. HPLC analysis of P450-associated heme demonstrated that approximately $80 \%$ loss of native heme is accompanied by a comparable inactivation of 2B6. A labeled protein or stable heme adducts were not observed by LC/MS/MS analysis. 
These results suggest that covalent modification or destruction of heme is the major mechanism leading to the inactivation of 2B6 by methadone. (Supported in part by the grant CA 16954 from the NCI)

\title{
P60. Metabolism and metabolic activation of food-composition by human P450 variants representing single nucleotide polymorphisms
}

\author{
Hiromasa Imaishi, Tatsushi Goto, Taiki Mori, Mai Hatta, Maki Matui and Takashi Hikawa
}

Research Center for Environmental Genomics, Kobe University, Kobe, Japan

[Purpose] Natural food-composition such as furanocoumarin 5-methoxypsoralen (5-MOP) and methyleugenol, contained in plants, are suggested to have cytotoxic or mutagenic properties. Human Cytochrome P450 subfamily is a candidate to convert natural compounds in foods into reactive intermediates. This study aimed to identify metabolites of 5-MOP and methyleugenol by CYP2A13 and CYP1A1. Furthermore, we want to clarify functional differences among CYP2A13 and CYP1A1 variants representing single nucleotide polymorphisms (SNPs). [Methods] Membrane fractions were prepared from E. coli expressing CYP2A13 variants (CYP2A13-1 (wild type), 4 (R101Q), 5 (F453Y), 6 (R494C), 8 (D158E) and 9 (V323L)) and CYP1A1 variants. These membrane fractions were incubated with 5-MOP and methyleugenol, and the metabolites of 5-MOP and methyleugenol were analyzed by HPLC. [Results and Discussion] All CYP2A13 and CYP1A1 variants were successfully expressed in E. coli system, and the contents of CYP2A13 polypeptides were about 5\% of total protein. CYP2A13-1 converted 5-MOP into 5-MOP dihydrodiol, Km and Vmax values of the reaction were $1.44 \mathrm{micro} \mathrm{M}$ and $4.23 \mathrm{nmol} / \mathrm{min} / \mathrm{nmol} \mathrm{P} 450$, respectively. The generation of dihydrodiol implies the production of reactive intermediates in the converting process. Though most variants of CYP2A13 had similar catalytic activity of 5-MOP, the activity of CYP2A13-4 was not detectable. [Conclusion] This study suggests that the CYP2A13 enzyme is involved in the toxicity of 5-MOP and that the SNPs within CYP2A13, except for allele CYP2A13-4, have little effect on the severity of toxicity.

\section{P61. Milk thistle extracts and components differentially modulate hepatic CYP1A2, CYP2C19, and CYP2D6 activity}

Vanessa González-Pérez ${ }^{1}$, Scott J. Brantley ${ }^{1}$, Tyler N. Graf ${ }^{2}$, David J. Kroll ${ }^{3}$, Nicholas H. Oberlies ${ }^{2}$ and Mary F. Paine ${ }^{1}$

${ }^{1}$ Division of Pharmacotherapy and Experimental Therapeutics, UNC Eshelman School of Pharmacy, The University of North Carolina at Chapel Hill, Chapel Hill, NC, ${ }^{2}$ Department of Chemistry and Biochemistry, The University of North Carolina at Greensboro, Greensboro, NC, ${ }^{3}$ Brite, North Carolina Central University, Durham, NC

Purpose. A large portion of adults in Western countries ( 20\%) admits to taking herbal supplements regularly. Milk thistle (Silybum marianum) is one such supplement and is used to self-treat liver disorders and for chemoprevention. Widespread use of herbal supplements, combined with their lack of strict regulation, raises concerns for interaction with conventional medications. Inhibition of cytochrome P450 (CYP) enzyme activity is a major mechanism underlying drug-xenobiotic interactions. The inhibitory effects of milk thistle extracts and single components (flavonolignans) on CYP2C9 and CYP3A activity have been evaluated previously. This study aimed to evaluate the inhibitory effects of seven flavonolignans (silybin A, silybin B, isosilybin A, isosilybin B, silychristin, isosilychristin, silydianin), as well as two commercially available extracts, silibinin (1:1 mixture of silybin A and silybin B) and silymarin (mixture of seven flavonolignans), on CYP1A2, CYP2C19, and CYP2D6 activity using human liver microsomes (HLM). Methods. Reaction mixtures consisted of HLM $(0.03,0.2$, or $0.04 \mathrm{mg} / \mathrm{mL}$ for CYP1A2, CYP2C19, or CYP2D6, respectively), probe substrate [phenacetin $(50 \mu \mathrm{M}), S$-mephenytoin $(50 \mu \mathrm{M})$, or dextromethorphan $(2.5 \mu \mathrm{M})]$, flavonolignan/extract $(1,10$, or 100 $\mu \mathrm{M})$, and phosphate buffer (0.1 M, pH 7.4). Reactions were initiated with NADPH (final concentration, $1 \mathrm{mM}$ ); the final organic solvent (DMSO) concentration was $0.1 \%(\mathrm{v} / \mathrm{v})$. Fluvoxamine $(3 \mu \mathrm{M})$, omeprazole $(50 \mu \mathrm{M})$, and quinidine $(2 \mu \mathrm{M})$ were used as positive controls for inhibition of CYP1A2, CYP2C19, and CYP2D6 activity, respectively. Reactions were quenched after 40 (CYP1A2), 25 (CYP2C19), or 15 (CYP2D6) minutes with cold acetonitrile. After centrifugation, supernatants were analyzed for the metabolites acetaminophen, 4'-hydroxymephenytoin, or dextrorphan by LC/MS/MS. IC ${ }_{50}$ values for selected flavonolignans/extracts (concentration range, 1-200 $\mu \mathrm{M}$ ) towards dextromethorphan $O$-demethylation were determined by nonlinear least squares regression using WinNonlin. Results. Of the three CYP reactions tested, only dextromethorphan $O$-demethylation showed sensitivity to inhibition $(\geq 50 \%)$ by more than one flavonolignan/extract; silymarin was the most potent, followed by silibinin and silybin $\mathrm{B}\left(\mathrm{IC}_{50}\right.$ 
values of $40 \pm 4.3,98 \pm 7.4$, and $96 \pm 9.6 \mu \mathrm{M}$, respectively). $S$-Mephenytoin 4 '-hydroxylation showed sensitivity only to silymarin, which inhibited activity by $\sim 80 \%$ at $100 \mu \mathrm{M}$. Phenacetin $O$-deethylation showed modest sensitivity to silibinin, which inhibited activity by $\sim 30 \%$ at $100 \mu \mathrm{M}$; all flavonolignans and silymarin activated this reaction at each concentration tested, by 120-170\%. Conclusions. One of the mixtures (silymarin), rather than any single flavonolignan, inhibited both CYP2D6-mediated dextromethorphan O-demethylation and CYP2C19-mediated S-mephenytoin 4'-hydroxylation, suggesting that a non-flavonolignan contributes to the inhibition potency of silymarin. In contrast, silymarin and all flavonolignans activated CYP1A2-mediated phenacetin $O$-deethylation. These results highlight that herbal supplements differ in modulatory effects on CYP enzyme activity and that single components do not necessarily reflect effects of the mixture.

\title{
P62. Modification of cytochrome P450 3A4 by soft electrophiles leads to differential residual activity
}

\author{
Dan A. Rock ${ }^{1}$, John A. Davis ${ }^{2}$ and Brooke M. VandenBrink ${ }^{1}$
}

${ }^{1}$ Pharmacokinetics and Drug Metabolism, Amgen Inc, Seattle, WA, ${ }^{2}$ Pharmacokinetics and Drug Metabolism, Amgen Inc., Seattle, WA

Modification of Cytochrome P450 3A4 by Soft Electrophiles Leads to Differential Residual Activity Dan A. Rock, John A. Davis and Brooke M. VandenBrink Mechanism-based inactivators of cytochrome P450 (CYP) 3A4 lead to reduced enzyme activity by apo-adduct formation or perturbation of the heme via direct alkylation, metabolic-intermediate complex formation or heme destruction. The extent of inactivation caused by an apo-adduct to CYP3A4 can varying from no reduction in activity to complete loss of activity over a short time course, depending on the size of the alkylating moiety as well as the site of apo-adduct formation. For example, raloxifene and pyrene iodoacetamide have been characterized to selectively modify cysteine 239 in recombinant CYP3A4 (Baer et al., 2007). Despite labeling the same amino acid, raloxifene leads to significant inactivation (rate $=0.18 \mathrm{~min}-1$ ) of CYP3A4; whereas, pyrene iodoacetamide does not lead to diminished activity. In contrast, pyrene maleimide (a close structural analog to pyrene iodoacetamide) leads to a significant decrease in CYP3A4-mediated metabolism of midazolam and testosterone with maximal inactivation rates of $0.29 \mathrm{~min}-1$ and $0.35 \mathrm{~min}-1$, respectively. The aim of this work was to characterize multiple soft electrophiles towards CYP3A4 by kinetic profiling with multiple CYP3A4 probe substrates. In addition, the degree and sites of alkylation on CYP3A4 is characterized by mass spectroscopy to help in further understanding of the disparity in CYP3A4 inactivation between electrophiles with distinct physiochemical properties. Ultimately, knowledge of the mechanistic features of the inactivation of CYP3A4 will provide additional insights into CYP3A4 biochemistry and its vulnerability to the effects of reactive intermediates. Baer BR, Wienkers LC and Rock DA (2007) Time-dependent inactivation of P450 3A4 by raloxifene: identification of Cys239 as the site of apoprotein alkylation. Chem Res Toxicol 20:954-964.

\section{P63. NNK-induced malignant transformation in human breast epithelial cells expressing CYP2A13}

\author{
Jun-Yan Hong ${ }^{1}$, Ding Ding ${ }^{2}$, Yu Guo ${ }^{3}$ and Shuqing Chen ${ }^{2}$ \\ ${ }^{1}$ Environmental and Occupational Health, School of Public Health, University of Medicine and Dentistry of New Jersey, \\ Piscataway, NJ, ${ }^{2}$ College of Pharmaceutical Sciences, Zhejiang University, Hangzhou, China, ${ }^{3}$ College of Basi Medical \\ Science, Wuhan University, Wuhan, China
}

Cigarette smoking is a well-established cause of human lung cancer and many other types of cancers. However, its etiological role in the development of human breast cancer is not clear. Previously, we demonstrated that among all the human cytochrome (CYP) enzymes, CYP2A13 is the most efficient in the metabolic activation of 4-(methylnitrosamino)-1-(3pyridyl)-1-butanone (NNK), a major tobacco-specific carcinogen. It can also metabolically activate another carcinogen 4-aminobiphenyl (ABP) in tobacco smoke. Based on the report that tobacco smoke constituents, including carcinogens, can reach human breast and our recent finding that CYP2A13 protein is selectively expressed in human breast ductal cells, we hypothesize that CYP2A13-mediated metabolic activation of NNK in the breast ductal cells plays an important role in the development of breast cancer. In this study, we transfected MCF-10A cells, a non-malignant human breast epithelial cell line, with human CYP2A13 cDNA and confirmed the expression of CYPA13, at both protein and activity levels, in the stable transfectant cells (MCF-10A/2A13). In comparison with the vector transfected cells (without 
CYP2A13 expression), MCF-10A/2A13 cells showed a significantly increased susceptibility to NNK-induce malignant transformation (as determined by soft agar assay). This result provides a strong support to our working hypothesis and suggests that CYP2A13 genetic variation might affect human susceptibility to breast cancer. It is also of interest to observe that human bronchial epithelial cells expressing CYP2A13 (BEAS-2B/2A13) are much more susceptible to NNK-induced malignant transformation than MCF-10A/2A13 cells, which may explain why the establishment of etiological role of cigarette smoking in human breast cancer is more difficult than in lung cancer (Supported by FAMRI Research Award CIA-082500)

\title{
P64. Novel catalytic properties and physiologic functions of cytochrome P450 from streptomyces coelicolor
}

\author{
Qian Cheng and Fred \& Peter Guengerich
}

Biochemistry, Vanderbilt University, Nashville, TN

With ongoing genome-sequencing efforts, more and more bacterial cytochrome P450s (P450) without defined functions are emerging in the database, representing a rich source for novel biocatalysts and new chemical entities. To harness the potential of these enzymes, we established a novel metabolomic approach to characterize the function of these enzymes. A recent successful example of this approach is the discovery that Streptomyces coelicolor P450 $154 \mathrm{Al}$ catalyzes an unexpected intramolecular [2+2] cycloaddition of a cellular dipentaenone to yield an oxetanecontaining product without oxidation-reduction. Here we report our recent study on Streptomyces coelicolor P450 107U1. Knockout of P450 107U1 blocked the formation of spores in the aerial hyphae, thus the first P450 identified to participate in the sporulation process in Streptomycetes. Metabolome analysis has identified one endogenous substrate of P450 107U1, and P450 107U1 was found to catalyze the dehydrogenation of this substrate in the presence of redox partners and NADPH. The structure elucidation of the identified substrate is currently in progress. (Supported in part by USPHS R01 GM066970)

\section{P65. Predicting oxidation sites with order of occurrence among multiple sites for CYP4A- mediated reactions}

\author{
Yoshiyuki Yamaura $^{1}$, Kouichi Yoshinari² and Yasushi Yamazoe ${ }^{2}$ \\ ${ }^{1}$ DMPK Research Group, Discovery Technology Laboratories, Minase Research Institute, Ono Pharmaceutical Co., Ltd., \\ Osaka, Japan, ${ }^{2}$ Graduate School of Pharmaceutical Sciences, Tohoku University, Sendai, Japan
}

Cytochrome P450 4A (CYP4A) enzymes selectively catalyze $\omega$-hydroxylation of physiologically important lipid components such as eicosanoids, prostaglandins and arachidonic acid. This enzyme are also involved in the metabolism of drugs such as febuxostat. Therefore, prediction of CYP4A-mediated metabolism is important in the discovery stage of new drug development. For the prediction of the CYP madiated metabolism, the classical ligand-property-based empirical approaches or X-ray crystallography-based docking model approaches have been reported. However, the prediction of multiple sites of oxidation of a substrate is not well handled in most cases, and classification of molecules as substrates and non-substrates is also difficult. In the present study, we developed a two-dimensional templatescoring system based on published data from assays using CYP4A recombinant enzymes. The system predicts the order of occurrence among multiple oxidation sites, as well as the regioselectivity. The template has a linearly arranged honeycomb shape and an adjacent area. Molecules are overlaid on the template with the locations of the atoms restricted to the corners of hexagonal blocks. The overlaid conformers are then checked to determine whether they reside within the template area, and their position occupancy and position function scores are calculated. The sum of these scores is compared among possible conformers, and the conformer with the highest total score is predicted to be preferentially metabolized. In the present study, prediction of sites of CYP4A-mediated oxidation and classification into substrates and non-substrates were performed for collected compounds, and agreement between predicted and experimental data exceeded $95 \%$ for substrates and non-substrates. The template-scoring system can be easily linked to databases of two-dimensional chemical structures. Thus, this system may be useful for studies of drug metabolism and new drug development. 


\title{
P66. Predicting regioselectivity and lability of cytochrome P450 metabolism using quantum mechanical simulations
}

\author{
Matthew D. Segall
}

Optibrium Ltd., Cambridge, United Kingdom

Many computational methods have been developed that predict the regioselectivity of metabolism by drug metabolising isoforms of the Cytochrome P450 superfamily of enzymes (P450). These include methods based on: the accessibility of potential sites of metabolism in enzyme-bound conformations [1]; statistically trained models that recognise likely sites of metabolism [2], quantum mechanical calculations to identify metabolic 'hot spots' [3]; and sub-structural matches to known reactive sites [4]. We will describe a method for predicting P450 metabolism that combines quantum mechanical simulations to estimate the reactivity of potential sites of metabolism on a compound [5] with a ligand-based approach to accounting for the effects of orientation and steric constraints due to the binding pockets of different P450 isoforms. We will present validation results for this method for prediction of regioselectivity by the major drug metabolising isforms of P450. However, while valuable, predicting the relative proportion of metabolite formation at different sites on a compound is only a partial solution to designing more stable compounds in drug discovery. The advantage of a quantum mechanical approach is that it provides a quantitative estimate of the reactivity of each site, from which additional information can be derived regarding the vulnerability of each site to metabolism in absolute terms. One such measurement is the site lability, as calculated by StarDrop ${ }^{\mathrm{mw}}$, which is a measure of the efficiency of the product formation step by comparison of the rate of product formation with that of decoupling to form water and inactivation of the oxidative enzyme species. This is an important factor in determining the overall rate of metabolism and we will illustrate how this provides valuable guidance regarding the potential to redesign compounds to overcome issues due to rapid metabolism by P450s.

[1] Gabriele Cruciani G. et al. (2005) J. Med. Chem. 48(22) pp. 6970-6979

[2] Hasegawa K et al. (2010) Mol. Inf. 29(3) pp. 243-249

[3] M. Hennemann e. al. (2009) ChemMedChem 4(3) pp. 657-69

[4] P. Rydberg et al. (2010) ACS Med. Chem. Lett.1(3) pp. 96-100

[5] Jones JP et al. (2002) Drug Metab. Dispos.30(1) pp. 7-12

\section{P67. Protein binding, transmembrane transport and enzyme interactions on the processing of schisandrin in rat liver}

\author{
Łing-jing Wu, Yan-yan Zhang, Chun-zhi Ai and Ling Yang
}

\section{Laboratory of Pharmaceutical Resource Discovery, Dalian Institute of Chemical Physics, Chinese Academy of Sciences, Dalian, China}

The schisandrin (SZ) is a major absorbed effective constituent of Fructus schisandrae, which is extensively applied in Chinese medicinal formula. Envidence has indicated that SZ is a selective cytochrome P450 3A (CYP3A) substrate. Aim to examine whether the plasma substrate (SZ) depletion clearance can characterize the hepatic CYP3A activity in vivo, we investigated the role of protein binding, transmembrane and enzyme interactions in the hepatic clearance of SZ in the recirculating perfused rat liver preparation. An initial dose of 7!İmol SZ was introduced into the perfusate reservoir (200ml) as a bolus to study the hepatic disposion of SZ. The permeability surface area product for influx (PSinf), characterizing the hepatic distribution of SZ was estimated using the indicator dilution method, with fluorescein isothiocyanate dextran 4000 (FD-4) as an extracellular reference. The unbound fraction of SZ in 2\% bovine serum albumin (BSA) Krebs-Henseleit bicarbonate (KHB) buffer (fu,p) and that in rat liver cytosols (fu,t) were investigated using an ultrafiltration method. Additionally, the effect of BSA on the recovery of SZ in reservoir and total metabolite 8(R)-hydroxyl-schizandrin formation was simulated with the simple liver physically based pharmacokinetics (PBPK) model. The results indicated that the distribution phase of SZ in rat liver was negligible for the influx clearance was comparable with the metabolic clearance (PSinf 24.6j $\mathrm{A} 5.2 \mathrm{ml} /$ $\mathrm{min} / \mathrm{g}$ v.s. CLint,met $23.8 ¡ \mathrm{~A} 3.7 \mathrm{ml} / \mathrm{min} / \mathrm{g})$. And the detected values of fu,p and fu,t (0.08 and 0.15 for SZ, respectively) were then used for liver PBPK modeling. The fitting data showed that the elimination rate constant (ke) of SZ was decreased by $60 \%$ (from $0.483 \mathrm{~h}-1$ to $0.193 \mathrm{~h}-1$ ) when $2 \%$ BSA was added. Simultaneously, the $8(\mathrm{R})$-hydroxyl-schizandrin formation rate constant (kmf) was decreased from $0.472 \mathrm{~h}-1$ to $0.189 \mathrm{~h}-1$. However, the kmf value was constantly close to ke. In conclusions, the metabolism of SZ to 8(R)-hydroxyl-schizandrin via CYP3A predominantly contributed to its hepatic clearance in 
spite of a significantly effect of plasma protein binding and the hepatic clearance of SZ was enzyme-capacity limited, which suggested that SZ plasma depletion clearance might characterize the hepatic CYP3A activity in vivo.

\title{
P68. Single nucleotide polymorphisms in prostaglandin $I_{2}$ synthase gene (CYP8A1) in a Korean population and their comparative analysis with other ethnic groups
}

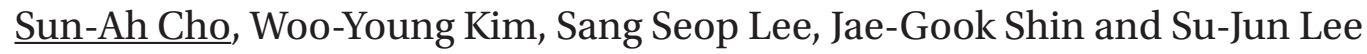 \\ Pharmacogenomics Research Center, Inje University College of Medicine, BuSan, South Korea
}

Prostaglandin $\mathrm{I}_{2}$ (prostacyclin) synthase gene, the unique member of family 8 of the cytochrome P450 superfamily (CYP8Al), is a key enzyme in the biosynthesis of prostacyclin $\left(\mathrm{PGI}_{2}\right)$. $\mathrm{PGI}_{2}$ is a strong vasodilator and the most potent endogenous inhibitor of platelet aggregation. Therefore, it is considered to play an important role in cardiovascular disease development. In an effort to screen for CYP8A1 single nucleotide polymorphisms (SNPs) in Koreans, the CYP8A1 gene was directly sequenced in 50 normal subjects. A total of 25 variants were identified: 7 in exons, 8 in introns, and 10 in 5'-untranslated region (UTR). The distribution of VNTR polymorphisms, was such that the most frequent allele was R6T (69\%), followed by R4 (23\%), R5T (4\%), and R6 (4\%). Twenty-five variants were used to characterize linkage disequilibrium (LD) structures at the CYP8A1 locus, which resulted in one LD block. Ethic different distribution of CYP8A1 variants and haplotypes were found. Identification of CYP8A1 SNPs with LD block and the tagging SNPs creates an important resource for genotype/phenotype association studies for future clinical studies

\section{P69. Stable transfection of CYP2D6 gene into hepaRG cells}

\author{
Lai Peng, Steven N. Hart and Xiao-bo Zhong
}

Pharmacology, Toxicology, and Therapeutics, University of Kansas Medical Center, Kansas City, KS

HepaRG cells become a promising alternative to primary human hepatocytes for xenobiotic metabolism and toxicity studies as they express various liver-specific functions. However, HepaRG cells lack the activity of an important cytochrome P450, CYP2D6, which limits their usage in studies involving CYP2D6. We discovered the CYP2D6*2 and CYP2D6*9 alleles in HepaRG cells by genotyping, which may partly contribute to low activity of CYP2D6 in HepaRG cells. In order to recover CYP2D6 activity in this cell line, we stably transfected a plasmid containing CYP2D6*1 cDNA into HepaRG cells. Expression of functional CYP2D6 up to a level comparable to primary human hepatocytes was achieved in one of the selected clones. With stable transfection of CYP2D6 into undifferentiated HepaRG cells, we would be able to generate large amount of differentiated, hepatocyte-like HepaRG cells with CYP2D6 activity, which will provide a new tool for studies assessing CYP2D6-dependent drug metabolism and toxicity.

\section{P70. Structural features of midazolam allostery by cytochrome P450 3A4}

\section{Arthur G. Roberts}

Department of Pharmaceutical and Biomedical Sciences, University of Georgia, Athens, GA

Human cytochrome P450 3A4 (CYP3A4) metabolizes a majority of known drugs and endogenous compounds. The enzyme exhibits atypical kinetics, involving homotropic and heterotropic cooperativity between bound ligands. The hydroxylation of the sedative midazolam (MDZ) is known to exhibit homotropic cooperativity by a decrease in the ratio of 1'-OH-MDZ to 4-OH-MDZ at high MDZ concentrations. We show that MDZ exhibited heterotropic cooperativity with the anti-epileptic drug carbamazepine (CBZ) with decreases in the 1'-OH-MDZ to 4-OH-MDZ ratios. To build a drug-bound CYP3A4 model, MDZ and CBZ bound to CYP3A4 were probed using paramagnetic relaxation enhancement (PRE) NMR and molecular docking with AutoDock 4.2. The distances calculated from the PRE were used to constrain the molecules to the substratefree X-ray crystal structure of CYP3A4 during simulated annealing. These simulations revealed that either two MDZ molecules or an MDZ molecule and a CBZ molecule assume a stacked configuration within the CYP3A4 active site. In either case, the proton at position- 4 of the MDZ molecule was closer to the heme than the protons of the $1^{\prime}-\mathrm{CH}_{3}$ group. On the other hand, molecular docking of a single molecule of MDZ revealed that the molecule was preferentially oriented with the $1^{\prime}-\mathrm{CH}_{3}$ position closer to the heme than the 4-position. A stacked configuration of drug molecules within the cytochrome P450 active site may represent a common structural mechanism for homotropic and heterotropic cooperativity. 


\title{
P71. Abstract withdrawn
}

\section{P72 . The biomonitoring of naphthols in urine and some oxidative stress parameters in workers exposed to jet fuel (JP-8)}

\author{
$\underline{\text { Ahmet Sayal }^{1}, \text { Onur Erdem², Ay }{ }^{\circ} \text { Eken }}{ }^{2}$, Cemal Akay ${ }^{2}$ and Ahmet Aydin ${ }^{3}$ \\ ${ }^{1}$ Department of Toxicology, Gulhane Military Medical Academy, Ankara, Turkey, ${ }^{2}$ Toxicology, Gulhane Military Medical \\ Academy, Ankara, Turkey, ${ }^{3}$ Department of Toxicology, Yeditepe University, Faculty of Pharmacy, Ýstanbul, Turkey
}

Jet propulsion fuel 8 (JP-8) is the largest source of chemical exposures among Air Force personnel. Over 2 million military and civilian personnel per year are occupationally exposed JP-8. There is limited data on possible health risk from exposure to JP-8 in workers. Urinary naphthols have been suggested as useful biomarkers of exposure to JP-8. Urinary 1- and 2-naphthols as a biomarker of occupational exposure to jet fuel and some antioxidant enzyme parameters; erythrocyte copperzinc superoxide dismutase (CuZn-SOD), erthrocyte and plasma selenium dependent glutatione peroxidase (Se-GSHPx), erythrocyte catalase (CAT) enzyme activity and thiobarbituric acid reactive substances (TBARS) levels have been compared among workers $(n=43)$ and subjects not occupationally exposed to JP-8 $(n=38)$. The antioxidant enzyme parameters were not statically different between study and control group. For the study group we determined higher erythrocyte TBARS data in smokers than nonsmokers $(\mathrm{p}<0.05)$. Our data reveal that jet fuel during aircraft fuel system maintenance operations are absorbed by personnel. In the lightning of the oxidative stress results obtained, it is difficult to claim that the occupational exposure to jet fuel can have possible oxidative response in human. However, there were interaction between cigarette smoking and JP-8 exposure for the increased lipid peroxidation and urinary naphthols levels.

\section{P73. The effect of aluminium on testese and antioxidant system in rats}

\author{
Cemal Akay ${ }^{1}$, Onur Erdem ${ }^{1}$, Ahmet Aydin $^{2}$, Ayse Eken $^{1}$ and Ahmet Sayal ${ }^{1}$ \\ ${ }^{1}$ Toxicology, Gulhane Military Medical Academy, Ankara, Turkey, ${ }^{2}$ Pharmaceutical Toxicology, Yeditepe University, \\ Istanbul, Turkey
}

Aluminum (Al) is the third most abundant element and the most common metal in the earth's crust. Al enters into the body from the environment and from diet and medication. The most common route of exposure to Al is oral intake. Food, drugs, drinking water and kitchen tools can lead to Al toxicity. In recent times, Al toxicity is a major area of interest and many studies are carried out to make its effects clear on biological systems. In recent years, it became clear that aluminum is a potential toxic agent in humans and has been implicated in the pathogenesis of several clinical disorders, such as dementia, respiratory tract disorders and allergic reactions. Chronic exposure to aluminum fumes, inhalation of aluminum and aluminumoxide powder increase the risk to develop serious central nervous system pathology, in particular Alzheimer's disease and amyotrophic lateral sclerosis. The aim of this study was to evaluate the effect of aluminium on rats' testese, erythrocyte and antioxidant system. For this purpose forty rats(F-344) were divided into two equal groups as control and experimental ones. The solution of $\mathrm{AlCI}_{3} \cdot 6 \mathrm{H}_{2} \mathrm{O}$ was given orally $(75 \mathrm{mg} / \mathrm{kg} /$ day $)$ to the experimental group daily with a special canule throughout one month. One month later the rats were sacrificed and the testese and erythrocyte were taken out. Al, Malondialdehyde (MDA), superoxide dismutase (SOD) and gluthathione peroxidase (GSH Px) levels were measured. The results showed that the levels of Aluminium on testese and erythrocyte were found higher compared to control ones $(\mathrm{p}<0.05)$. While MDA levels were higher compared to control group, GSH Px and SOD levels were found lower compared to conrol one ( $<<0.05)$. In conclusion these results show that Al may have toxic effect on testes and antioxidant system in rats

\section{P74. In vitro identification of CYP450 and FMO mediated metabolic pathways to support drug discovery}

\author{
Yiding Hu, Sirimas Sudsakorn, Nanqun Zhu, Hanlan Liu and Thomas O'Shea
}

\section{DMPK \& Pharmaceutics, Genzyme Corp, Waltham, MA}

Cytochrome P450 enzymes (CYP450) and flavin-containing monooxygenses (FMO) are membrane-bound metabolizing enzymes catalyzing the metabolism of a variety of xenobiotics. Oxidative metabolism of xenobiotics by CYP450 or 
FMO often generate the same metabolites. Consequently, elucidation of the enzymes responsible for biotransformation is required to appropriately direct the medicinal chemistry campaign and to understand the potential drug-drug interaction (DDI) risks. In this work, a reaction phenotyping assay was developed to differentiate metabolism by CYP450 or FMO enzymes in liver microsomes. Benzydamine was employed as a marker substrate for both CYP450 mediated pathway (N-demethylation) and FMO catalyzed metabolism (N-oxidation). In an example of this approach, the assay was implemented to support a discovery project in which the major metabolite of the lead chemical scaffold was the N-oxidation of a tertiary amine in vitro across species (i.e., human, rat, dog, and monkey) and in vivo in rat and dog. Species differences were observed between human and preclinical species in the formation of N-oxide metabolite. In human and monkey liver microsomes, the N-oxidation was almost completely inhibited by 1-aminobenzotriazole (1-ABT), indicating the dominant presence of the CYP450 mediated metabolic pathway. In contrast, in dog and rat liver microsomes, the N-oxidation was only partially blocked by either 1-ABT or FMO inhibition (via heat inactivation or by methimazole), indicating dual CYP450 and FMO pathways were involved in the formation of N-oxide metabolite. This finding provided the appropriate direction for in vitro CYP450 reaction phenotyping studies of the lead candidate to assess potential DDI.

\title{
P75. Characterization of the impact of life stage on xenobiotic metabolizing enzyme expression and gene - chemical interactions in the liver
}

\author{
Janice S. Lee ${ }^{1}$, William O. Ward ${ }^{1}$, Hongzu Ren ${ }^{1}$, Beena Vallanat ${ }^{1}$, Michael J. DeVito ${ }^{2}$ and Jon C. Corton ${ }^{1}$ \\ ${ }^{1}$ EPA, Research Triangle Park, NC, ${ }^{2}$ Toxicology Branch, NIEHS/NTP, Research Triangle Park, NC
}

Differences in responses to environmental chemicals and drugs between life stages are likely due in part to differences in the expression of xenobiotic metabolizing enzymes and transporters (XMETs). We have carried out a comprehensive analysis of the mRNA expression of XMETs through life stages in mice and humans with the goal of using the information to predict differences in chemical responsiveness between life stages. Using full-genome arrays, the mRNA expression of all XMETs and their regulatory proteins was examined during fetal (gestation day (GD) 19), neonatal (postnatal day (PND) 7), prepubescent (PND32), middle age (12 months), and old age (18 and 24 months) in the male C57BL/6J (C57) mouse liver and compared to adults (2-6 months). Fetal and neonatal life stages exhibited dramatic differences in XMET mRNA expression compared to the relatively minor effects of old age. At all life stages except PND32, under-expressed genes outnumbered over-expressed genes and included those in Phases I, II and III. In the fetus and neonate, parallel increases in expression were noted in the dioxin receptor, Nrf2 components and their regulated genes while nuclear receptors and regulated genes were generally down-regulated. Suppression of male-specific XMETs was observed at early (GD19, PND7) and to a lesser extent, later life stages (18 and 24 months). To determine if aged humans exhibit changes in XMET expression, we examined gene expression profiles from young (21-45 years) and old (69+ years) men and women. Compared to mice, there were relatively few consistent changes in gene expression in the livers from aged humans compared to younger humans. We identified 370 genes that were altered between young and old men and 1163 genes that were altered between young and old women. Age caused minimal numbers of changes in the gene expression of XMETs ( 8 in males and 33 in females between young and old). Most of these changes were in the expression of Phase III genes. The expression of solute carriers increased with age in men while the majority decreased with age in women. These studies indicate that the livers from aging humans exhibit a number of changes which may lead to differences in the transport of xenobiotics. We have initiated a project to identify chemicals that may be differentially metabolized or transported at different life stages due to changes in XMETs. Using the Comparative Toxicogenomics Database (CTD), chemicals were identified which interacted with proteins with functions that affect the metabolism or transport of the chemical. Preliminary analysis of the differences between the fetus and adult showed that chemicals of similar structure and toxicity mode of action cluster together. In summary, our analysis revealed dramatic differences in the expression of the XMETs through different life stages in the mouse with more subtle differences in older humans. XMET expression patterns and known gene-chemical interactions could be used to predict life stage-specific responses to environmental chemicals and drugs. These predictions could be validated in relevant in vitro models. (This abstract does not reflect US EPA policy.)

\section{P76. Gene expression of hepatic metabolizing enzymes and transporters in a traumatic brain injury (TBI) rodent model}

\author{
Gail D. Anderson ${ }^{1}$, Fred M. Farin², Theo Bammler ${ }^{3}$, Richard Beyer ${ }^{3}$ and Michael R. Hoane ${ }^{4}$
}

${ }^{1}$ Phamacy, University of Washington, Seattle, WA, ${ }^{2}$ Environmental Health, Univeristy of Washington, Seattle, WA, ${ }^{3}$ Environmental and Occupational Health Sciences, University of Washington, Seattle, WA, ${ }^{4}$ Psychology, Southern Illinois University, Carbondale, IL 
Objective: Clinically, TBI results in an increased clearance of drugs metabolized by cytchrome P450s (Cyps) and glucuronyltransferase (UGT) within a few days of injury. In contrast, rat TBI models have suggested a decrease in metabolism. Methods: In a study designed to determine the effects of nicotinamide (NAM) on gene expression, rats received either saline or NAM (bolus + infusion for $72 \mathrm{hrs)} \mathrm{after} \mathrm{a} \mathrm{moderate} \mathrm{cortical} \mathrm{contusion} \mathrm{injury} \mathrm{(CCI).} \mathrm{For} \mathrm{this} \mathrm{analysis,} \mathrm{fresh} \mathrm{liver}$ samples were harvested in 5 animals in each treatment arm at $24 \mathrm{hr}, 72 \mathrm{hr}$ and $168 \mathrm{hrs}$ post-CCI and 5 intact sham controls. Samples were removed with a tissue punch, were snap frozen and stored at $-80^{\circ} \mathrm{C}$. RNA was isolated from the tissue punches. RNA samples passed stringent quality control, were processed and submitted to microarray analysis using Affymetrix GeneChip Rat Gene 1.0 ST arrays. Microarray data analysis was performed with the Bioconductor package limma. The effect of TBI+saline compared to sham on hepatic gene expression of the cytochrome P450s (CYP), non-CYP Phase I enzymes (esterase, dehydrogenases, FMOs, etc), Phase II enzymes, ABC transporters and SLC transporters was determined.Results: The number of differentially expressed genes (>1.5-fold up or down, $\mathrm{p}<0.05$ ) at 24,72 and $168 \mathrm{hrs}$ post-TBI for TBI+saline compared to sham is given in table.

\begin{tabular}{|c|c|c|c|c|c|c|}
\hline & \multicolumn{2}{|c|}{$24 \mathrm{hrs}$} & \multicolumn{2}{|c|}{$72 \mathrm{hrs}$} & \multicolumn{2}{|c|}{$168 \mathrm{hr}$} \\
\hline & Up & Down & Up & Down & Up & Down \\
\hline Cytochrome P450s $(\mathrm{n}=75)$ & 4 & 3 & 6 & 7 & 3 & 3 \\
\hline Other Phase I enzymes $(n=90)$ & 6 & 2 & 2 & 7 & 1 & 8 \\
\hline Phase II enzymes $(\mathrm{n}=78)$ & 1 & 8 & 3 & 9 & 1 & 7 \\
\hline $\mathrm{ABC}$ transporters $(\mathrm{n}=47)$ & 0 & 1 & 1 & 5 & 0 & 5 \\
\hline SLC transporters $(n=345)$ & 3 & 10 & 4 & 20 & 1 & 39 \\
\hline
\end{tabular}

21 Cyp genes (28\%) were differentially expressed in TBI compared to sham animals with an equal number of genes increased and decreased. Only 7 Cyp genes were up (Cyp2c12, 3a72, 1lb1) or down (Cyp2bl, 2f4, 4f39, 2r1) regulated at more than one time point. There was no effect of TBI on CYP1a1, 2al, 2a2, 2b1, 2C11, 2C13, 3a23/3, 2d, 2d2, $2 \mathrm{~d} 3,2 \mathrm{~d} 4,2 \mathrm{~d} 5$ or $2 \mathrm{e} 1$. TBI primarily decreased the expression of enzymes involved in non-CYP phase 1 and phase II metabolism. Ephxl were down-regulated at 24 and $72 \mathrm{hr}$ and Ephx2 was down-regulated at all three time points. TBI decreased expression of Nat8, Nat15, Sulf2, Gsta2, Gsta5, Gst1, Gstm1, Gstm2, Gstm3, Gstm4, Gstm5, Gstm7, Gstt1, Gstt2 and Gsst3. There was no effect on the Ugts. Of the 47 ABC transporters, 6 were differentially expressed in TBI compared to sham animals. 61 of the 345 SLC transporters were differentially expressed. There was no effect of TBI on Abcc1 (Mrp1), Abcb1a (Mdr1), Abcg2 (Bcrp), Abce1 (Oabp), Slc22a2 (Oct2), Slc22a3 (Oct3), Slc22a6 (Oat1), Slc22a8 (Oat3), Slc22a9 (Oat7) and Slcolb3 (Oatp-8).Conclusion: The effect of TBI on hepatic enzymes and transporters was highly variable with no effect on the majority of enzymes and transporters involved in drug disposition. As differences in mRNA do not always translate into differences in protein expression, further studies are needed to confirm the significance of these findings.

\title{
P77. Species differences in metabolic clearance due to UDP-glucuronosyltransferase substrate selectivity: Impact during early drug discovery
}

\author{
Mark Niosi ${ }^{1}$, Theunis C. Goosen ${ }^{2}$, Brendon Kapinos ${ }^{1}$, Matthew D. Troutman ${ }^{3}$, Kentaro Futatsugi ${ }^{4}$ \\ and Alfin D. N. Vaz
}

${ }^{1}$ Pharmacokinetics, Dynamics \& Metabolism, Pfizer, Groton, CT, ${ }^{2}$ Pharmacokinetics, Dynamics and Metabolism, Pfizer Inc., Groton, CT, ${ }^{3}$ Dept of PDM/MS 8118W-150, Pfizer Inc., Groton, CT, ${ }^{4}$ Pfizer, Groton, CT, ${ }^{5}$ Pharmacokinetics, Dynamics \& Metabolism, Pfizer Global R \& D, Groton, CT

During drug discovery and development, experimental animals are frequently used for pharmacokinetic (PK) studies to facilitate in vitro-in vivo extrapolation (IV-IVE) of human hepatic drug clearance $\left(\mathrm{CL}_{\mathrm{H}}\right)$. Observed species differences could impact early drug discovery screening, compound selection for progression to humans, and selection of preclinical species for toxicity assessment. An in vivo disconnect in clearance was observed between rat, monkey, and mouse for a series of new chemical entities, which required further assessment of potential differences in clearance mechanism. Early metabolite profiling indicated a significant contribution of $N$-glucuronidation in human and monkey hepatocytes, while the UDP-glucuronosyltransferase (UGT)-mediated clearance mechanism was not observed in rat and dog hepatocytes. Limited information is available on species differences in glucuronidation and in order to access the impact of UGT-mediated clearance on overall predicted $\mathrm{CL}_{\mathrm{H}}$ within this series, we assessed (1) in vitro intrinsic clearance $\left(\mathrm{CL}_{\text {int }}\right)$ in microsomes with the substrate-depletion method, supplemented with NADPH or UDPGA in the presence of bovine serum albumin (BSA); (2) scaled $\mathrm{CL}_{\mathrm{H}}$ from in vitro $\mathrm{CL}_{\text {int }}$ employing the well-stirred model; (3) human PK projections from in vitro and single-species allometric scaling; and (4) in vitro metabolic profiles 
across species. A high degree of concordance was observed between in vitro $\mathrm{CL}_{\text {int }}$ scaled to $\mathrm{CL}_{\mathrm{H}}$ when microsomes were supplemented with UDPGA and high intravenous CL in monkey. In contrast, intravenous clearance in rat and dog was generally low to moderate and addition of UDPGA to liver microsomes did not result in significant increases in $\mathrm{CL}_{\text {int' }}$ compared to microsomes supplemented with NADPH. Accordingly, IV-IVE was readily scalable within species, resulting in deselection of compounds predicted to have high human $\mathrm{CL}_{\mathrm{H}}$ based on observed microsomal $\mathrm{CL}_{\text {int }}$. Reaction phenotyping with human recombinant UGT enzymes identified UGT1A9 as the major hepatic UGT contributing to conjugation. UGT othologs across species are hard to elucidate, but lower observed rat $\mathrm{CL}_{\mathrm{H}}$ may be related to rat UGT1A9 being a pseudogene. These findings indicate single-species allometic scaling of $\mathrm{CL}_{\mathrm{H}}$ is possible from monkey PK for UGT1A9 substrates while rat and dog PK would underpredict human $\mathrm{CL}_{\mathrm{H}}$. Scaled $\mathrm{CL}_{\mathrm{H}}$ from in vitro UGT microsomal $\mathrm{CL}_{\text {int }}$ in the presence of BSA is considered most reliable when attempting human PK predictions for UGT substrates.

\title{
P78. Sulfotransferase expression and activity is altered by human metabolic disease
}

\author{
Emine Bihter Yalçin, Karissa Neira, Renae Gupta, Angela L. Slitt and Roberta S. King
}

Biomedical and Pharmaceutical Sciences, University of Rhode Island, Kingston, RI

Obesity-related disease is a rapidly growing problem in the United States and the developed world. Obesity has shown significant association with diabetes and other metabolic disease clusters, such as insulin resistance, fatty liver disease, high blood pressure, and abnormal cholesterol and triglyceride levels. A consequence of obesity or diabetes is development of fatty liver (also called steatosis), which can progress to steatohepatitis and cirrhosis of the liver. In humans, diabetes has been observed to alter hepatic excretion function and affect the pharmacokinetics of commonly used drugs such as acetaminophen. As the liver is progressively altered, protein expression and activity of the drug metabolizing enzymes (DMEs) and transporters change. Our purpose is to determine how the expression and activity of the major cytosolic sulfotransferases are altered by various metabolic disease states. While these sulfotransferases have been well-characterized in tissues from healthy humans, it is not known how they are affected by metabolic disease. It is our hypothesis that hepatic steatosis and cirrhosis alter sulfotransferase expression and activity in human liver. The rationale for this hypothesis is that liver disease has been shown in human and animal models to alter nuclear and non-nuclear transcription factor expression and activity; and several of these transcription factors subsequently regulate sulfotransferase expression. Since SULTS play important roles in drug metabolism and detoxication and in metabolism of endogenous molecules, it is necessary to characterize SULT expression and activity in populations which exhibit steatosis or cirrhosis due to obesity or excessive alcohol intake. As the number of children and adults who are overweight and obese continue to rise, there is a significant portion of patients for whom it is necessary to know whether they are at risk for decreased drug efficacy or increased drug toxicity. In addition, sulfotransferases act on many endogenous compounds within the metabolic pathways such as cholesterol, bile acids, estrogens and androgens. In this role, alteration of sulfotransferase activity may contribute to the disease process itself. This poster presents results obtained by mRNA, protein (Western) and activity assays for human SULT1A1, SULT1A3, SULT1E1, SULT2A1 and SULT2B1b in livers from male and female humans with non-fatty liver, steatosis, alcoholic cirrhosis, or diabetic cirrhosis.

\section{P79. The effect of sterculic oil intervention on drug metabolism enzyme activity and transporter expression in LETO/OLETF model of obesity}

\author{
Manoj S. Chiney ${ }^{1}$, Harrison Tam ${ }^{1}$, Laura C. Ortinau², James W. Perfield II ${ }^{2}$ and L'Aurelle Anna Lee \\ Johnson $^{1}$ \\ ${ }^{1}$ Experimental and Clinical Pharmacology, University of Minnesota, Minneapolis, $M N,{ }^{2}$ Department of Nutrition and \\ Exercise Physiology, University of Missouri, Columbia, MO
}

Previous in vitro studies have shown that metabolic syndrome associated ectopic lipid accumulation regulates the expression and activity of drug metabolizing enzymes (DME) and transporters $(1,2)$. The lipogenic enzyme stearoylCoA desaturase-1 (SCD1) is associated with ectopic lipid deposition and has emerged as a target for the treatment of the metabolic syndrome. Sterculic oil (SO) is a known natural inhibitor of SCD1 and we hypothesized that SO supplementation would alter obesity-associated changes in DME and transporter expression and activity. Therefore, the current study examines the role of SO treatment on expression and activity of DME and transporters in lean LETO and 
obese OLETF rats. Liver tissue samples were isolated from 14 week old male LETO and OLETF rats fed with a standard AIN-93G diet or OLETF animals that were fed an AIN-93G diet supplemented with $0.5 \%$ SO (OLETF-SO). Microsomes were isolated from the livers using a previously described method, while RNA was isolated using a Qiagene ${ }^{\circledR}$ RNA isolation kit. CYP1A, NAT and XO activity was determined using Ethoxyresorufin, p-aminobenzoic acid as probe substrates and Cayman ${ }^{\circledR}$ XO assay kit respectively. The mRNA expression was determined using qRT-PCR. Our results indicate that the hepatic XO activity was 2.5 times higher in the OLETF obese rats when compared to the LETO lean rats (891.2 units/mg vs. 374.8 units/mg protein). The addition of SO to the diets of OLETF rats resulted in significant reversal of the elevated XO activity (450.4 units/mg protein) similar to that of the LETO lean rats. The hepatic mRNA expression of SLCO1A4 and ABCC2 transporters was 5 and 2.5 fold lower in the OLETF rats when compared to lean LETO rats, $(\mathrm{p}<0.001,0.01)$ respectively; however, this effect was not reversed in the OLETF-SO rats. Interestingly OLETF rats fed an AIN-93G diet supplemented with SO showed an increase in hepatic mRNA expression of $A B C B 1$ ( 2.5 fold, $\mathrm{p}<0.01$ ) and SLC22A6 (1.63 fold; $\mathrm{p}<0.05$ ) as compared to the OLETF and LETO rats. No differences were observed between LETO, OLETF, and OLETF-SO rats with respect to hepatic CYP1A and NAT enzyme activity and hepatic ABCC1 and SLC22A6 mRNA levels. In conclusion, this study demonstrates that inhibition of SCD1 by SO reverses the upregulation of XO activity. Also SO was found to differentially alter transporter mRNA expression levels. Additional studies are required to determine the exact mechanism of the SO mediated alterations and its role in affecting the disposition of drugs.

\title{
References
}

1. Hirunpanich V, Sethabouppha B, Sato H. Inhibitory effects of saturated and polyunsaturated fatty acids on the cytochrome P450 $3 \mathrm{~A}$ activity in rat liver microsomes. Biol Pharm Bull. 2007 Aug;30(8):1586-8.

2. Zangar RC, Novak RF. Effects of fatty acids and ketone bodies on cytochromes $\mathrm{P} 4502 \mathrm{~B}, 4 \mathrm{~A}$, and $2 \mathrm{El}$ expression in primary cultured rat hepatocytes. Arch Biochem Biophys. 1997 Jan 15;337(2):217-24.

\section{P80. Accurate mass imaging MALDI MS of whole animal tissues to assess the label-free disposition of drug}

\author{
$\underline{\text { Sheerin K. Shahidi-Latham }}{ }^{1}$, Cristine Quiason ${ }^{1}$, D. Shannon Cornett ${ }^{2}$, Katherine A. Kellersberger ${ }^{2}$ \\ and Brian J. Dean ${ }^{1}$
}

${ }^{1}$ Drug Metabolism \& Pharmacokinetics, Genentech Inc., South San Francisco, CA, ${ }^{2}$ Bruker Daltonics, Billerica, MA

In the drug discovery and development process, it is necessary to obtain information about the absorption, distribution, and metabolism of an administered compound. Currently, traditional methods such as liquid chromatography tandem mass spectrometry (LC-MS/MS) or whole-body autoradiography (WBA) are employed to help elucidate drug disposition. Unfortunately, these techniques have their limitations. For example, LC-MS/MS can provide qualitative and quantitative information but requires the excision and homogenization of organs or tissues of interest, thus losing information regarding drug and metabolite localization. Although WBA studies can provide distribution information, it cannot resolve localization of parent drug from biotransformation products that have retained the radiolabel. Furthermore, WBA studies are usually reserved for compounds advancing to the clinic due to high-cost and often laborious synthesis of a radiolabeled-analog. Coupling matrix-assisted laser desorption/ionization (MALDI) with high-resolution mass spectrometry allows for the label-free detection of individual drug and metabolite distributions from a single imaging experiment. The objective of this study was to demonstrate the capabilities and advantages of this technique to assess drug disposition in the drug discovery phase by obtaining label-free drug and metabolite distributions across whole-rat tissue sections without any a priori knowledge. Male Sprague-Dawley rats were administered a single $30 \mathrm{mg} / \mathrm{kg}$ PO dose of GNE-A, euthanized at 1 and $8 \mathrm{hrs}$ post dose, and $20 \mu \mathrm{m}$ thick whole-body sections analyzed on a 7.0T SolariX FTMS system equipped with a dual ESI-MALDI source. The large dynamic range of the 7 Tesla FTMS made it an ideal platform for imaging complex samples such as tissue. A systematic raster of the tissue section was performed at $400 \mathrm{um}$ steps and at each position a spectrum was obtained over the range of 100 to $1000 \mathrm{Da}$ with high mass resolution (resolving power of $\sim 200,000 @ m / z 400$ ). Data analysis software employing a multiple mass defect filtering script (<2 ppm mass tolerance) aided the identification of Phase I and Phase II metabolites from the dataset. Several biotransformations were identified including oxidations and dealkylations. Since the data were acquired at known x,y coordinates, a two-dimensional grid could be produced, whereby each spectrum represents a pixel in the ion image. By selecting the corresponding drug and metabolites peaks, individual ion images were then extracted, revealing unique distributions of each molecular species across the whole-rat section. This study clearly demonstrated the advantages of a mass spectrometric approach for the early assessment of drug disposition by providing a label-free means to detect and display the individual distributions of drug and its subsequent metabolites. 


\title{
P81. Disposition of 14C-RRx-001 in rats after a single intravenous administration and in blood
} from rats, dogs, monkeys, and humans

\author{
Jan Scicinski ${ }^{1}$, Bryan Oronsky ${ }^{1}$, William Fitch ${ }^{2}$, Michael Taylor $^{3}$, Gang Luo $^{4}$, Timothy Musick ${ }^{4}$, \\ Joseph Marini ${ }^{4}$, Chris Adams², Michael Schicker ${ }^{4}$, Mark Gohdes ${ }^{4}$, Don Mckenzie ${ }^{4}$ and Richard \\ Ridgewell $^{4}$
}

${ }^{1}$ RadioRx Inc., Mountain View, CA, ${ }^{2}$ Stanford University, Stanford, CA, ${ }^{3}$ NonClinical Safety Assessment, Mountain View, CA, ${ }^{4}$ Covance Laboratories Inc., Madison, WI

RRx-001, a derivative of a geminal dinitroazetidine (structure shown), has demonstrated promise as a novel cancer therapeutic agent in a number of cell lines and tumor models and is proposed to enter a Phase I clinical trial in cancer patients. RRx-001 is believed to elicit an anticancer effect through nitrogen oxides released from decomposition of conjugates formed in vivo. In this study, the in vivo and in vitro disposition of ${ }^{14} \mathrm{C}-\mathrm{RRx}-001$ was characterized. After a single intravenous administration of ${ }^{14} \mathrm{C}-\mathrm{RRx}-001(10 \mathrm{mg} / \mathrm{kg})$ to rats, the volume of distribution of total ${ }^{14} \mathrm{C}-\mathrm{RRx}-001$ derived total radioactivity, based on plasma and blood concentrations, was 0.926 and $0.084 \mathrm{~L} / \mathrm{kg}$, respectively. These results indicated that most of the ${ }^{14} \mathrm{CRRx} 001$ derived radioactivity partitioned into rat erythrocytes. Similarly, the ratios of blood to plasma concentrations of radioactivity ratio were 12.4 and 222, respectively, at 0.083 and 168 hours postdose. The radioactivity was eliminated from plasma with a terminal half-life of 66.1 hours but no elimination phase was observed in blood through 168 hours. The remaining radioactivity in blood at 168 hours was as high as $61.8 \%$ of the total dose. ${ }^{14} \mathrm{CRRx}$-001 derived radioactivity was recovered at 36.3 and $2.22 \%$ of the dose, respectively, in 168-hour urine and feces samples. The blood-to-plasma partitioning of ${ }^{14} \mathrm{C}-\mathrm{RRx}-001(1$ and $20 \mu \mathrm{M})$ was further examined in vitro in blood from rats, dogs, monkeys and humans. Four major soluble metabolites were identified from rat and human blood incubation samples. The structures of these metabolites support the initial hypothesis.

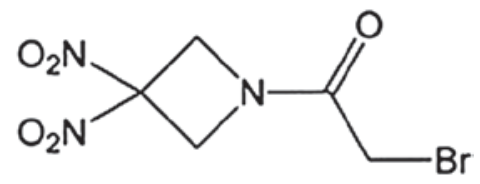

\section{P82. Metabolism and excretion of $\left[{ }^{14} \mathrm{C}\right]$ crizotinib in rats and dogs}

Evan B. Smith $^{1}$, Lance Goulet ${ }^{1}$, Gregory S. Walker ${ }^{2}$, Shinji Yamazaki ${ }^{1}$, Theodore R. Johnson ${ }^{1}$, Deepak Dalvie $^{1}$ and Bill J. Smith ${ }^{1}$

${ }^{1}$ Pharmacokinetics, Dynamics \& Metabolism, Pfizer Inc., San Diego, CA, ${ }^{2}$ Pharmacokinetics, Dynamics \& Metabolism, Pfizer Inc., Groton, CT

Crizotinib [PF-02341066: (R)-3-[1-(2,6-dichloro-3-fluoro-phenyl)-ethoxy]-5-(1-piperidin-4-yl-1H-pyrazol-4-yl)pyridin-2-ylamine] is a small-molecule inhibitor of the anaplastic lymphoma kinase (ALK) receptor tyrosine kinase and its oncogenic variants and is currently in development for the treatment of ALK-positive advanced non-small cell lung cancer. The objective of this study was to characterize the mass balance and metabolic disposition of $\left[{ }^{14} \mathrm{C}\right]$ crizotinib in rats and dogs, the nonclinical species used in toxicology evaluation. Rats and dogs were administered a single oral dose of $\left[{ }^{14} \mathrm{C}\right]$ crizotinib at $10 \mathrm{mg} / \mathrm{kg}(200 \mu \mathrm{Ci} / \mathrm{kg}$ and $20 \mu \mathrm{Ci} / \mathrm{kg}$, respectively). Blood and excreta were collected at various time intervals for 24 and 168 hours postdose, respectively, in rats and for 120 and 168 hours postdose, respectively, in dogs. In a separate study in bile duct-cannulated rats, bile samples were collected at various time intervals for 48 hours after dosing. Radioactivity in plasma and urine was determined directly by liquid scintillation counting (LSC). Whole blood and fecal samples were combusted prior to analysis by LSC. Metabolite profiles in pooled urine, fecal, bile and plasma samples were generated by radio-HPLC. Metabolites in these samples were quantified and then characterized by LC/ MS. In rats and dogs, mean total recoveries of the administered radioactive dose were $102 \%$ and $89 \%$, respectively. There were no marked gender-related differences in urinary or fecal excretion. The primary route of elimination after oral administration of $\left[{ }^{14} \mathrm{C}\right]$ crizotinib was the feces, accounting for $99 \%$ and $83 \%$ of the administered dose in rats and dogs, respectively. Excretion of drug-related material via the feces was essentially complete by 24 and 72 hours postdose in rats and dogs, respectively. Less than $3 \%$ of dose-related radioactivity was recovered in the urine of either species. In the rat, biliary excretion accounted for $38-62 \%$ of the dose. Crizotinib was the predominant radiolabeled component in plasma of rats and dogs, accounting for $44-53 \%$ and $59-71 \%$ of circulating radioactivity, respectively. In both species, the 
primary circulating metabolites resulted from oxidation of the piperidine ring and included crizotinib lactam (trace in dog), crizotinib nitrone and other minor oxidative metabolites. In addition, direct sulfate conjugation of crizotinib was a major pathway in female rats. Crizotinib was the major drug-related component in feces, accounting for $60-79 \%$ and 47-70\% of the dose in rats and dogs, respectively. Excretion of unchanged crizotinib in urine was minimal. Drug-related components in rat bile included $O$-desalkyl crizotinib lactam, conjugates of $O$-dealkylated metabolites, and crizotinib sulfate. Biliary excretion of unchanged crizotinib was minimal. Overall, fecal/biliary excretion represented the major route of elimination of $\left[{ }^{14} \mathrm{C}\right]$ crizotinib-derived radioactivity in rats and dogs, and elimination was essentially complete. Qualitatively, the metabolism of crizotinib in rats was representative of that observed in humans.

\title{
P83. Metabolism, excretion and pharmacokinetics of $\left[{ }^{14} \mathrm{C}\right]$ crizotinib following oral administration to healthy subjects
}

\author{
Theodore R. Johnson $^{1}$, Lance Goulet ${ }^{1}$, Evan B. Smith ${ }^{1}$, Shinji Yamazaki ${ }^{1}$, Gregory S. Walker ${ }^{2}$, Weiwei \\ $\mathrm{Tan}^{3}$, Chunze $\mathrm{Li}^{3}$, Yao Ni ${ }^{4}$, Gabriella Bedarida ${ }^{5}$, Nicoletta Brega ${ }^{6}$, Deepak Dalvie ${ }^{1}$ and Bill J. Smith ${ }^{1}$ \\ ${ }^{1}$ Pharmacokinetics, Dynamics \& Metabolism, Pfizer Inc., San Diego, CA, ${ }^{2}$ Pharmacokinetics, Dynamics \& Metabolism, \\ Pfizer Inc., Groton, CT, ${ }^{3}$ Clinical Pharmacology, Pfizer Inc., San Diego, CA, ${ }^{4}$ Clinical Operations, Pfizer Inc., Groton, CT, \\ ${ }^{5}$ Clinical Research Unit, Pfizer Inc., New Haven, CT, ${ }^{6}$ Clinical Oncology, Pfizer Inc., Milan, Italy
}

Crizotinib [PF-02341066: (R)-3-[1-(2,6-dichloro-3-fluoro-phenyl)-ethoxy]-5-(1-piperidin-4-yl-1H-pyrazol-4-yl)-pyridin2-ylamine] is a small-molecule inhibitor of the anaplastic lymphoma kinase (ALK) receptor tyrosine kinase and its oncogenic variants and is currently in development for the treatment of ALK-positive advanced non-small cell lung cancer. The objective of this study was to characterize the mass balance, metabolic disposition and pharmacokinetics of $\left[{ }^{14} \mathrm{C}\right]$ crizotinib in healthy subjects. In this open-label study, 6 healthy men, aged $41-45$ years, received a single oral dose of $\left[{ }^{14} \mathrm{C}\right]$ crizotinib $(250 \mathrm{mg} / 100 \mu \mathrm{Ci})$. Blood, urine and feces were collected predose and at various intervals up to 480 hours postdose. Radioactivity in plasma and urine was determined directly by liquid scintillation counting (LSC). Whole blood and fecal samples were combusted prior to analysis by LSC. Crizotinib concentration in plasma and urine samples was determined by validated liquid chromatography/mass spectrometry (LC/MS) methods. Plasma pharmacokinetic parameters for total radioactivity and crizotinib were calculated using standard noncompartmental methods. Metabolite profiles in pooled urine, fecal and plasma samples were generated by radio-HPLC or HPLC with fraction collection followed by LSC. Metabolites in these samples were quantified and then characterized by LC/MS. The overall mean recovery of administered radioactive dose was $85 \%$ over the 480 -hour study, with mean recoveries of $63 \%$ in feces and $22 \%$ in urine. A majority of administered radioactivity (78\%) was recovered in the first 120 hours postdose. Total radioactivity measured in plasma peaked between 3 and 6 hours after dosing, similar to the $\mathrm{T}_{\max }$ for crizotinib. Mean $\mathrm{C}_{\max }$ values for total radioactivity and crizotinib were $436 \mathrm{ng} \mathrm{eq} / \mathrm{mL}$ and $109 \mathrm{ng} / \mathrm{mL}$, respectively, and crizotinib represented approximately $12 \%$ (AUC (0-last) $_{\text {) of }}$ drug-related material in plasma. The $t_{1 / 2}$ of total radioactivity was prolonged compared with that of crizotinib, with a mean crizotinib $t_{1 / 2}$ value of 94 hours. A mean of $1.3 \%$ of parent drug was excreted unchanged in urine. Metabolic profiling demonstrated that crizotinib was the major circulating component in plasma, accounting for $33 \%$ of circulating radioactivity in the 0-96 hour pooled sample. A lactam metabolite of crizotinib (PF-06260182), formed via oxidation of the piperidine ring, represented $10 \%$ of circulating radioactivity. Other metabolites, representing $<10 \%$ of circulating radioactivity individually, included glucuronide and sulfate conjugates of $O$-desalkyl crizotinib, $O$-desalkyl crizotinib lactam, and a sulfate conjugate of $O$-desalkyl crizotinib lactam. Unchanged crizotinib was the major excreted component in feces, accounting for $53 \%$ of total administered dose. The major excreted component in urine ( $4.5 \%$ of dose) was a sulfate conjugate of $O$-desalkyl crizotinib lactam. No other metabolites accounted for $>1 \%$ of the total administered dose in excreta. In conclusion, elimination of $\left[{ }^{14} \mathrm{C}\right]$ crizotinib-derived radioactivity was essentially complete, with fecal/biliary excretion representing the major route of elimination. Oxidative metabolism plays a significant role in the clearance of crizotinib.

\section{P84. Metabolic fate and disposition of GSK2251052, a novel boron-based antimicrobial against Gram-negative bacteria, in sprague dawley rats and cynomolgus monkeys}

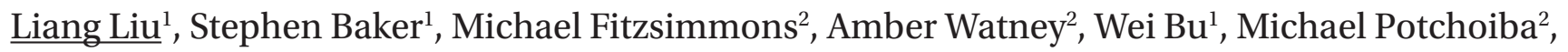 \\ Ming $\mathrm{Qi}^{2}$, Michael R. K. Alley ${ }^{1}$, Lisa Feng ${ }^{1}$, Vincent Hernandez ${ }^{1}$, Irwin Heyman ${ }^{1}$ and Sanjay Chanda ${ }^{1}$ \\ ${ }^{1}$ Anacor Pharmaceuticals Inc., Palo Alto, CA, ${ }^{2}$ Covance Inc., Madison, WI
}

GSK2251052 [(S)-3-(aminomethyl)-7-(3-hydroxypropoxy)1-hydroxy-1,3-dihydro-2,1-benzoxaborole hydrochloride], formerly known as AN3365, a novel boron-containing leucyl-tRNA synthetase inhibitor, is currently being developed for 
the treatment of serious Gram-negative bacterial infections. Distribution, metabolism, and excretion of GSK2251052 were investigated following a single intravenous administration of ${ }^{14} \mathrm{C}$-GSK2251052 in male and female Sprague Dawley rats, male Long Evans rats, at $50 \mathrm{mg} / \mathrm{kg}(150 \mu \mathrm{Ci} / \mathrm{kg})$, and male and female cynomolgus monkeys at $25 \mathrm{mg} / \mathrm{kg}(150 \mu \mathrm{Ci} / \mathrm{kg})$. QWBA in rats showed radioactivity was widely and rapidly distributed to most tissues with insignificant retention in pigmented ocular tissues by 35 days, and the majority of radioactivity was eliminated from most tissues by $168 \mathrm{~h}$. Potentially preferential distribution of radioactivity into the cellular component of blood with blood to plasma ratio of 1.3 for $24 \mathrm{~h}$ was observed. The overall mean recoveries of radioactivity in male and female rats and monkeys were $95 \%$ and $82 \%$, respectively. Most of the total ${ }^{14}$ CGSK2251052derived radioactivity was excreted within 48 hours, with urinary excretion as the primary route of elimination, representing $67.5 \%$ and $51.7 \%$ of the total dose in rats and monkeys by $168 \mathrm{~h}$, respectively. Metabolism of GSK2251052 was moderate in rats and extensive in monkeys. Significant quantities of an oxidative metabolite (M3), whereby the hydroxypropyl moiety of GSK2251052 was oxidized to a propanoic acid moiety, were present in monkey plasma, while moderate amounts of N-acetylated M3 (M7) were evident in rat plasma. Differences in urinary excretion profiles were demonstrated, with GSK2251052 as the major component excreted in rat urine accounting for $46 \%$ of the radioactivity dose, in comparison to less than $10 \%$ of the dose present in urine from monkeys. Metabolite M3 was the major component excreted in urine from monkeys. No gender difference on metabolic profile and excretion of GSK2251052 was observed in rats and monkeys. The species differences in the metabolic profiles suggest renal clearance of GSK2251052 predominates in rats, while the metabolic clearance is higher in monkeys than rats.

\title{
P85. Molecular mechanism of altered ezetimibe disposition in nonalcoholic steatohepatitis
}

\author{
Rhiannon N. Hardwick, Craig D. Fisher, Stephanie M. Street, Mark J. Canet and Nathan J. \\ Cherrington
}

Pharmacology \& Toxicology, University of Arizona, Tucson, AZ

Ezetimibe (EZE) lowers serum lipid levels by blocking Neimann-Pick C1-Like 1-mediated cholesterol uptake in the intestine. Disposition of EZE and its pharmacologically active glucuronide metabolite (EZE-GLUC) to the intestine is dependent on biliary efflux from the liver. Previous studies in our laboratory suggest that hepatobiliary transporter expression and function may be altered during non-alcoholic steatohepatitis (NASH). Therefore, the purpose of the current study was to determine the molecular mechanism by which disposition of clinically relevant drugs may be altered in NASH. Rats fed a methionine-choline deficient (MCD) diet for 8 weeks were administered $10 \mathrm{mg} / \mathrm{kg}$ EZE either by intravenous bolus or oral gavage. Plasma and bile samples were collected over 2 hours followed by terminal urine, liver and intestinal tissue collection. EZE and EZE-GLUC concentrations were determined by LC-MS/MS. Hepatic expression of the sinusoidal Abcc3 transporter was induced in MCD animals which correlated with increased plasma concentrations of EZE-GLUC in both i.v. and p.o. dosed MCD animals. Hepatic expression of the biliary Abcc2 and Abcb1 transporters were also increased in MCD; and, the biliary efflux of EZE-GLUC was slightly diminished in MCD animals despite no alterations in the biliary concentration of total bile acids. The cellular localization of Abcc2 and Abcb1 appeared to be internalized away from the canalicular membrane in MCD livers, providing a mechanism for the shift to plasma drug efflux. Expression of the EZE metabolizing enzyme Ugtlal was not altered in MCD liver or intestine. Importantly, induction of ABCC2, 3 and ABCB1 protein was also found in human NASH livers. Similarly, ABCC2 is internalized in human NASH as evidenced by immunohistochemical staining and reduced glycosylation status of the protein. The combination of induced expression and altered localization of key efflux drug transporters in rodent and human NASH samples shifts the disposition profile of EZE toward plasma retention and greater exposure. This increased plasma retention of other drugs in NASH may have implications in pharmacologic effect as well as potential toxicity.

\section{P86. Ocular and systemic distribution of INO-8875, a selective adenosine A1 receptor agonist, in rabbits by quantitative whole body autoradiography (QWBA) and tissue analysis}

\author{
Elizabeth Prescott ${ }^{1}$, Craig Struble ${ }^{1}$, Jon Denissen ${ }^{1}$ and Norman Kim²
}

\section{${ }^{1}$ Drug Metabolism Department, Covance, Madison, WI, ${ }^{2}$ Inotek Pharmaceuticals Corporation, Lexington, MA}

INO-8875, a highly selective adenosine $A_{1}$ receptor agonist, is under development for the treatment of ocular hypertension and primary open-angle glaucoma. INO-8875 lowers intraocular pressure (IOP) by increasing conventional outflow facility via the trabecular meshwork (TM). The ocular and systemic tissue distribution of INO-8875 was evaluated in New Zealand White and Dutch-Belted rabbits after topical ocular administration. Ocular and systemic tissue distribution 
and mass balance were studied using $\left[{ }^{14} \mathrm{C}\right]-\mathrm{INO}-8875$. Following topical ocular administration, the radioactivity was distributed in the anterior globe of the eye (cornea, aqueous humor, trabecular meshwork, and ciliary body) and systemic tissues at 0.25 hours post-dose, with greatest ocular exposure in the cornea, without melanin binding. Nasolacrimal drainage was significant and resulted in levels of $\left[{ }^{14} \mathrm{C}\right]$-INO-8875-related material being distributed through the whole body. Significant levels of radioactivity were observed in the kidney and liver, suggesting both hepatic and renal clearance. The percentage of dose in the ocular and systemic tissues was calculated based upon tissue weights for the New Zealand White rabbit. The route of distribution of $\left[{ }^{14} \mathrm{C}\right] \mathrm{INO}-8875$ is thought to be typical of an ocular drug following topical ocular administration. Nasolacrimal drainage can be an important factor in the systemic distribution of a topical ocular compound. The percentage of dose administered found in the ocular tissues and systemic tissues also demonstrates the percentage of drug that is absorbed into the eye and drained into the whole body. The long-lasting drug concentrations in ocular target tissues with low systemic exposure and rapid clearance suggest a favorable safety profile and therapeutic index for INO8875.

\title{
P87. Tissue distribution of ${ }^{51} \mathrm{CR}$-labeled stem cells after intravenous or intrathecal administration in rat
}

\author{
Dragomir I. Draganov, Justin Godsey, Sicco Popma and Terry L. Johnson
}

Metabolism, WIL Research Laboratories, LLC, Ashland, $\mathrm{OH}$

Following in vitro labeling with $\mathrm{Na}_{2}{ }^{51} \mathrm{CrO}_{4}$, rat stem cells were administered by slow bolus injection in a tail vein or by intrathecal infusion to male and female rats. At $0.16,1,6,24,72$ and 168 hours post-dosing, up to 3 animals/sex/time point were euthanized by carbon dioxide inhalation. After in situ perfusion with phosphate buffered saline, selected tissues were collected, processed and analyzed for ${ }^{51} \mathrm{Cr}$ radioactivity by $\gamma$ counting. An additional animal/sex/time point was processed for quantitative whole body autoradiography (QWBA). Very good correlation in tissue distribution and kinetics of elimination was observed between the results obtained by direct $\gamma$-counting and QWBA analysis. The sensitivity of the direct $\gamma$ counting method is hindered by the low counting efficiency of $\gamma$-counting for ${ }^{51} \mathrm{Cr}(<4 \%)$, the short half-live of this isotope ( $<28$ days) and the limitation in sample size that can be analyzed $(<200 \mathrm{mg})$. WBA analysis of the ${ }^{51} \mathrm{Cr}$-derived radioactivity appeared to be more sensitive than the direct $\gamma$ counting, especially at the later collection time points, and had the further advantage of precise localization of the radioactivity in sub-regions of the tissues and organs of interest.

\section{P88. UGT-catalyzed glucuronidation: An important elimination mechanism of GS-1101 (CAL-101), a potent and selective inhibitor of the P110 delta isoform of phosphoinositide 3-kinase (PI3K delta) for treatment of patients with hematologic malignancies}

\author{
$\underline{\text { Hao Chen }}^{1}$, Heather Webb ${ }^{2}$, Jerry Evarts ${ }^{3}$ and Roger Ulrich ${ }^{2}$
}

${ }^{1}$ Oncology Research, Gilead Sciences, Inc, Seattle, WA, ${ }^{2}$ Oncology, Gilead Sciences, Inc, Seattle, WA, ${ }^{3}$ Chemistry, Gilead Sciences, Inc, Seattle, WA

GS-1101 (CAL-101; 5-Fluoro-3-phenyl-2-[(S)-1-(9H-purin-6-ylamino)-propyl]-3H-quinazolin-4-one) is a potent inhibitor of PI3K $\delta$ that is currently in clinical development for treatment of patients with hematologic malignancies. One major and 2 minor glucuronides of GS-1101 $\left(G_{1}, G_{2}\right.$ and $\left.G_{3}\right)$ were readily identified in human urine, indicating that glucuronidation may represent an important disposition mechanism of GS-1101 in human subjects. Structures of $G_{1}$ and $G_{3}$ were proposed and confirmed with synthesized standards as GS-563129 and GS-563119 (both N-glucuronides of GS-1101). In vitro studies on glucuronidation were then conducted using both unlabeled and ${ }^{14} \mathrm{C}$-labeled GS-1101. Activities of 12 human recombinant UGT iso-enzymes in catalyzing glucuronidation of GS-1101 were assessed and categorized as high (UGT1A4), moderate (UGT2B7, 2B17, and 1A1), minimal (UGT1A3, 1A9, and 2B15), and not detectable (UGT1A6, 1A7, 1A8, 1A10, and 2B4). GS-1101 was incubated with human liver, small intestinal, renal, and lung microsomes in the presence of UDPGA at $37^{\circ} \mathrm{C}$. Microsomal generation of GS-563129 and GS-563119 was both tissue- and UDPGA-dependent, demonstrating a UGT-catalyzed N-glucuronidation. UGT catalytic activity observed in human liver microsomes was approximately 4- and 6-fold higher than of that observed in small intestinal and renal microsomes. Catalytic activity in human lung microsomes was negligible. Co-incubation of GS-1101 and tigogenin (a specific substrate of UGT1A4) with human liver microsomes resulted in 50-70\% inhibition of the reaction. Profound species differences were observed when rates of rat, dog, and rabbit liver microsome-catalyzed N-glucuronidation of GS-1101 were compared to rates 
observed in human liver microsomes. These studies demonstrate that $\mathrm{N}$-glucuronidation is a quantitatively important metabolic clearance mechanism of GS-1101.

\title{
P89. A significant bioactivation pathway common to 2-(alkylthio)-1, 3, 4-thiadiazoles
}

\author{
Yanou Yang ${ }^{1}$, Jonathan Josephs ${ }^{2}$, W. Griff Humphreys ${ }^{3}$ and Yue-Zhong Shu ${ }^{4}$ \\ ${ }^{1}$ Biotransformation, Bristol-Myers Squibb Co, Pennington, NJ, ${ }^{2}$ Biotransformation, Bristol-Myers Squibb Co, Princeton, \\ NJ, ${ }^{3}$ Biotransformation, Bristol-Myers Squibb Company, Princeton, $N J,{ }^{4}$ Bristol-Myers Squibb, Princeton, NJ
}

Certain functional groups/structural motifs are known to generate chemically reactive metabolites that can covalently modify essential cellular macromolecules, and therefore have the potential to disrupt biological function and elicit idosyncratic adverse drug reactions. We recently found that 5-substituted 2-(alkylthio)-1, 3, 4 thiadiazole structural motif can be added to the growing list of structural alerts for bioactivation. This bioactivation pathway was first observed for a commercially available 4, 5 substituted 2-(methylthio)-1, 3, 4 thiadiazole compound. When this compound was incubated with human liver microsomes in the presence of NADPH and glutathione (GSH), an unusual GSH adduct was formed. Structure identification of the GSH adduct by high resolution accurate mass instruments indicated the replacement of alkylthio group by GSH. This specific bioactivation pathway has not been reported in the literature based on our knowledge. The possible mechanism for this GSH adduct formation could be attributed to the enzymatic oxidation of alkylthio group to form sulfoxide and sulfone, which are very good leaving groups and are readily displaced due to nucleophilic attack by GSH. We also investigated the presence of this bioactivation pathway in more than a dozen commercially available compounds containing 2-(alkylthio)-1, 3, 4 thiadiazole motifs and having different substitutions at 4 and/or 5 positions of thiadiazoles. The structural identification of the unusual GSH adducts and effect of substitutions of this bioactivation pathway will be presented in detail in this report. These results can help medicinal chemists make necessary chemical modifications and thoughtful drug design to avoid potential liability related to 2-(alkylthio)-1, 3, 4 thiadiazoles.

\section{P90. Bioavailability and in-vivo efficacy of praziquantel-polyvinylpyrrolidone solid dispersion in S. mansoni-infected mice}

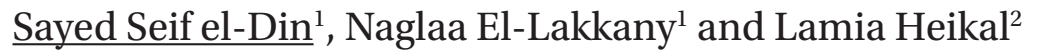 \\ ${ }^{1}$ Pharmacology, Theodor Bilharz Research Institute, Giza, Egypt, ${ }^{2}$ Pharmaceutics, Faculty of Pharmacy, Alexandria \\ University., Alexandria, Egypt
}

Praziquantel (PZQ) is the drug of choice in the control and treatment of schistosomiasis. One of the problems of PZQ is its very low aqueous solubility and its dissolution rate is considered the limiting factor for its bioavailability. The objective of this work was to correlate between physical properties and dissolution behavior of PZQ-polyvinylpyrrolidone (PVP; K25) solid dispersion (SD) at the ratios of $1: 1$ and 3:7 and its oral bioavailability and in-vivo efficacy against S. mansoni. The PZQ and PZQ-PVP SD were characterized by infrared spectroscopy, differential scanning calorimetry (DSC), scanning electron microscopy (SEM) and solubility test. These analyses showed a decrease of crystallinity, possible interaction between PZQ and PVP, and greater increase in the dissolution rate and appreciable reduction in particle size. PZQ-PVP SD in ratio of 3:7 showed better dissolution rates than that in 1:1 ratio. With these results, the solid dispersion of PZQ:PVP (3:7) was chosen for the biological assays. Schistosoma mansoni (S. mansoni)-infected mice treated orally with either pure PZQ or PZQ-PVP at a single dose of $500 \mathrm{mg} / \mathrm{kg}$ showed a higher increase in both AUC ${ }_{(0-8 h r)}$ and $\mathrm{C}_{\max }$ concomitant with significant decrease in $\mathrm{k}_{e l}$ and significant increase in both $\mathrm{K}_{a}$ and $\mathrm{t}_{1 / 2 e}$ versus corresponding uninfected mice. Moreover, normal and S. mansoni-infected mice treated with PZQ-PVP SD showed increase in both AUC $(0-8 \mathrm{hr})$ and $\mathrm{C}_{\max }$ by $(2.3,1.6)$ and $(1.3,1.25)$ fold respectively, with a decrease in $\mathrm{k}_{e l}$ and increase in $\mathrm{t}_{1 / 2 e}$ by about two fold versus the corresponding groups treated with pure PZQ. The percentage worm reduction at all the administered doses $(62.5,125$, 250,500 and $1000 \mathrm{mg} / \mathrm{kg}$ ) was significantly higher by about 1-1.5 fold in mice treated with PZQ-PVP SD $\left(\mathrm{ED}_{50}=40.92\right)$ versus those treated with pure $\mathrm{PZQ}\left(\mathrm{ED}_{50}=99.29\right)$. In addition, total tissue egg load reached the significant level at doses 62.5, 125 and $250 \mathrm{mg} / \mathrm{kg}$ in PZQ-PVP SD-treated group versus pure PZQ. Concomitantly, a significant decrease in total immature eggs with an increase in dead eggs in PZQ-PVP SD treated mice at doses $125,250 \mathrm{mg} / \mathrm{kg}$, and a significant decrease in mature eggs at doses 250 and 500 compared with their corresponding pure PZQ-treated groups. Solid dispersion preparation of PZQ with the carrier PVP could lead to a further improvement in the effectiveness of praziquantel therapy especially with the appearance of some PZQ tolerant S. mansoni isolates. 


\title{
P91. Brain microdialysis of pomalidomide in rats
}

\author{
Mehran Moghaddam, Jason Katz and Yang Tang
}

Department of Drug Metabolism and Pharmacokinetics, Celgene Corporation, San Diego, CA

Pomalidomide is a distinct $\mathrm{IMiD}^{\circledR}$ immunomodulatory drug with potent anti-proliferative and pro-apoptotic activities in vitro, and is effective in patients with relapsed/refractory multiple myeloma (RRMM). Pomalidomide has desirable pharmacokinetic attributes in rodents. Of particular interest is the in vivo brain microdialysis data generated after oral administration of Pomalidomide to rats. In this experiment, a microdialysis probe was implanted in the jugular vein and another directed towards the striatum to continuously monitor unbound concentrations of Pomalidomide from awake, freely moving rats. Samples were collected in 25-minute intervals for approximately 10 hours following oral dosing at $50 \mathrm{mg} / \mathrm{kg}$. In vivo recovery was determined by the retrodialysis method using a positive control compound as the probe calibrator and LC-MS/MS was used to assay for all microdialysate samples. Following p.o. administration, unbound Pomalidomide concentrations in brain were consistently lower than unbound concentrations in blood. Unbound concentrations in brain reached $\mathrm{C}_{\max }$ and $\mathrm{AUC}_{(0-10)}$ values of around $430 \mathrm{ng} / \mathrm{mL}$ and $2700 \mathrm{ng} \cdot \mathrm{hr} / \mathrm{mL}$, respectively. Unbound concentrations in blood reached $\mathrm{C}_{\max }$ and $\mathrm{AUC}_{(0-10)}$ values of around $1100 \mathrm{ng} / \mathrm{mL}$ and $6800 \mathrm{ng} \cdot \mathrm{hr} / \mathrm{mL}$, respectively. The results obtained in this study were comparable to those seen in a concurrent study looking at whole brain Pomalidomide content following its oral administration of to mice. This data suggests that Pomalidomide crosses the blood brain barrier and may be suitable for treatment of brain cancer and primary CNS lymphoma.

\section{P92. Cardiomyocytes from stem cells: Towards "Disease in a dish"}

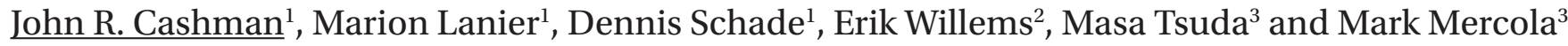 \\ ${ }^{1}$ Human BioMolecular Research Institute, San Diego, CA, ${ }^{2}$ Cell Biology, Sanford Burnham Research Institute, La Jolla, \\ CA, ${ }^{3}$ Sanford Burnham Research Institute, La Jolla, CA
}

In the United States, the need for cardiac cells for transplantation or research applications is immense. While over 300,000 individuals per year seek heart transplants, less than $2 \%$ will receive hearts. Human cardiomyocytes are also needed to investigate the safety of new chemical entities being developed as drug candidates because cardiac toxicity is a major cause of drug development failure. Despite the need for human cardiac cells, progress to provide human cardiac cells has been challenging. One approach is to utilize human pluripotent stem cells to generate large numbers of cardiomyocytes for transplantation purposes or for use in drug discovery and development work. Currently, however, there are few methods to produce large numbers of human cardiomyocytes on a biotechnology scale to address the need. The hypothesis of our work is that small molecules could be identified that direct human pluripotent stem cell differentiation to cardiomyocytes. The long term goal of this work is to create a toolbox of small molecule reagents useful to direct human stem cell differentiation into cardiomyocytes and thus address the need for human cardiac cells. Therefore, we screened a collection of about 550 pathway modulators in a human embryonic stem cell-based assay that probes cardiomyocyte differentiation. One compound from the screen, (i.e., IWR-1), a very potent "hit" was selected for further medicinal chemical refinement as a Wnt/ $\beta$-catenin antagonist and as an agent that directs human stem cell differentiation into cardiomyocytes. In an iterative process of chemical synthesis and parallel evaluation for Wnt/ $\beta$-catenin inhibition and cardiomyogenesis, several potent agents with improved pharmacodynamic and kinetic properties were elaborated. Cardiomyogenesis was verified by quantifying the effects of transcription factors expressed in human stem cells upon differentiation (i.e., Nkx2.5, $\alpha$ MHC, alpha activin) by RT-qPCR, image analysis and for selected compounds, flow cytometry. The results showed that a distinct Wnt/ $\beta$-catenin inhibition structure-activity relationship emerged from the "Dynamic Medicinal Chemistry" approach. Potent Wnt/ $\beta$-catenin antagonists that emerged from this optimization campaign were useful toolbox reagents to produce large numbers of human cardiomyocytes from human stem cells. The conclusion is that small molecules can be used to afford human cardiomyocyte differentiation from pluripotent stem cells. Application of this approach to human induced pluripotent stem (iPS) cells could afford cardiomyocytes that could be used in drug discovery and development applications (i.e., drug toxicity testing) as well as provide a new way to address "Disease in a Dish" issues and create cells for therapeutic utility. 


\title{
P93. Comparison of various nonclinical in vivo/in vitro models to assess CNS penetration of TAK-285, a novel HER2/EGFR inhibitor
}

\author{
ذing-Tao Wu ${ }^{1}$, Justin Gordon ${ }^{1}$, Mingxiang Liao ${ }^{1}$, Qing Zhu ${ }^{1}$, Alex Bulychev ${ }^{1}$, Susan Chen $^{1}$, Karthik \\ Venkatakrishnan ${ }^{2}$ and Cindy $\mathrm{Xia}^{1}$
}

${ }^{1}$ Drug Metabolism and Pharmacokinetics, Millennium Pharmaceuticals, Inc., Cambridge, MA, ${ }^{2}$ Clinical Pharmacology,
Millennium Pharmaceuticals, Inc., Cambridge, MA

TAK-285 is an orally available novel HER2/EGFR inhibitor that is currently in clinical development for HER2 over-expressing (HER2+) breast cancer. Since approximately one third of patients with HER2+ metastatic breast cancer develop brain metastasis, treatment options for patients whose brain metastases have progressed after radiotherapy are urgently needed. The currently approved HER2-targeted agents, trastuzumab and lapatinib, have generally shown limited activity against brain metastases, likely due to the limited brain penetration of these drugs. In this work, we used and compared various nonclinical tools to assess the CNS penetration potential of TAK-285. In a Caco-2 cell model, TAK-285 showed high permeability and was not a substrate for P-gp and BCRP. In comparison, and consistent with literature, lapatinib showed low permeability and were found to be substrates for P-gp/BCRP. The brain penetration of TAK-285 and lapatinib was assessed in vivo in Sprague-Dawley rats. The brain-to-plasma AUC ratio $(\mathrm{B} / \mathrm{P})$ for total (free plus bound) TAK-285 and lapatinib was 0.202 and 0.0243 , respectively, indicating distribution of TAK-285 into brain tissue in contrast to negligible CNS penetration of lapatinib across an intact $\mathrm{BBB}(\mathrm{B} / \mathrm{P}$ being close to the blood volume fraction of brain). Additional nonclinical studies were performed to assess the unbound TAK-285 concentration in rat brain. A tissue binding study was conducted using equilibrium dialysis with rat brain homogenates and the results, taken together with the total B/P ratio, suggested that there was virtually no unbound TAK-285 available in the brain tissue. To assess the potential relevance of this finding, a definitive in vivo rat microdialysis study was performed with dual probes to measure unbound TAK-285 in the brain interstitial fluid (ISF) and in blood simultaneously. The results showed that the exposure of unbound TAK-285 in brain ISF was about $24 \%$ of that in plasma indicating that the brain tissue binding method significantly underestimated unbound TAK-285 brain concentrations. To enable subsequent evaluations of CNS distribution of TAK-285 in humans, recognizing that brain microdialysis is not feasible for use in the clinical setting, a rat CSF pharmacokinetic study was conducted to collect and measure the unbound CSF concentration of TAK-285. Consistent with the microdialysis findings and contrary to the tissue binding results, a substantial amount of unbound TAK-285 (approximately 13\% of that in plasma) was found in the CSF. The generally similar CSF and brain ISF distributional characteristics of TAK-285 support use of unbound CSF exposure in the clinical setting as a surrogate of brain ISF exposure. A clinical study was recently conducted with CSF collection from patients at steady-state conditions after multiple dose oral administration of TAK-285. The human CSF results were in good accordance with the expectations from the rat CSF study and indicated the lack of substantial barriers to CNS penetration of TAK-285 in humans.

\section{P94. Derisking idiosyncratic toxicity concerns with a covalent modifying drug: Retrospective analysis with the pan-ErbB receptor tyrosine kinase inhibitor and clinical candidate (Cl-1033, Canertinib)}

\author{
Jonathan N. Bauman, Xin Yang, Raman Sharma and Amit S. Kalgutkar
}

\section{Pharmacokinetics, Dynamics, and Metabolism, Pfizer Global Reseach and Development, Groton, CT}

The design of controlled target-specific covalent enzyme inhibitors is conceptually very attractive given the increased biochemical efficiency associated with an irreversible mechanism, which can lead to high therapeutic margins. From a practical standpoint, however, this is hard to achieve because of the difficulty in striking the "right" balance between target covalent binding and indiscriminant reactivity with proteins, DNA, and glutathione that can cause acute or delayed immune-mediated toxicity (e.g., drug allergy and/or hypersensitivity). Against this backdrop, 74 enzymes that are inhibited by marketed drugs, 19 are inactivated via selective covalent modification with little to no toxicity. A likely reason for this phenomenon may be the poor intrinsic chemical reactivity of the "warhead" towards nucleophiles under physiological conditions but yet upon appropriate positioning within the active site of the target protein, the electrophilic functionality selectively reacts with an active site amino acid nucleophile. As such, there is little, if any, information available on such attributes on the approved or investigational drugs. Consequently, from a drug discovery perspective, there is currently no experimental paradigm to systematically derisk the toxicity potential with covalent inhibitors in programs that adopt such strategies. CI-1033 (Canertinib) is a pan-ErbB receptor tyrosine kinase inhibitor, 
which irreversibly binds to the kinase through its electrophilic acrylamide motif. CI-1033 also was an advanced clinical candidate for the treatment of advanced non-hematological cancers. In this retrospective analysis we examine the disposition of CI-1033 in animals and humans with a particular emphasis on its potential for indiscriminant alkylation (relative to its pharmacology and overall elimination pathways). The approaches described herein could be applied in drug discovery programs attempting to pursue covalent modifiers.

\title{
P95. Effect of pharmaceutical vehicles on the brain penetration
}

\author{
Nageswararao Muddana, Vishwottam Kandikere, Gopinadh Bhyrapuneni, Ramoji Kosuru, Ranjit \\ kumar Ponnamaneni, Shantaveer Irappanavar, Arun Kumar Manoharan and Ramakrishna Nirogi
}

\author{
Discovery Research, Suven Life Sciences Ltd, Hyderabad, India
}

Accurate assessment of brain penetration for new chemical entities using suitable vehicle at preclinical state is very critical for selection of candidate molecules. The objective of the present investigation is to identify the suitable vehicle in the assessment of brain penetration in preclinical drug discovery. In vivo steady state brain penetration model in male Sprague Dawley rats was chosen to assess the brain penetration. Olanzapine was used as a tool compound and $\%$ captisol equivalent to drug strength, 5\% pharmasolve, 5\% PEG-400, 1\% Tween-80, 5\% Tween-80, $10 \%$ Tween-80 was chosen as vehicles. Rats were cannulated femoral vein for infusion and jugular vein for blood sampling. Olanzapine was dissolved in different vehicles and infused via femoral vein catheter at a dose of $1 \mathrm{mg} / \mathrm{kg} / \mathrm{hr}$ up to 6 hours. Blood samples were collected through the jugular vein at pre-determined time points during the infusion, animals were sacrificed at $6 \mathrm{~h}$ and brains were isolated and homogenized in water, brain and plasma olanzapine concentrations were assessed by LC-MS/MS. Olanzapine was reached steady after $6 \mathrm{~h}$ infusion and plasma concentrations of olanzapine were similar $(279-303 \mathrm{ng} / \mathrm{mL})$ in all groups. However brain concentrations of olanzapine were significantly increased $(3882 \mathrm{ng} / \mathrm{g})$ in $10 \%$ Tween-80 as compared to other vehicles $(1943-2365 \mathrm{ng} / \mathrm{g})$. The brain to plasma ratio $(\mathrm{Cb} / \mathrm{Cp})$ was increased in $10 \%$ Tween-80 treated group of animal compare to other groups (6.95 to 14.19). Olanzapine was reported as a moderate substrate for the P-gp. The result indicates that $10 \%$ Tween- 80 is affecting the olanzapine brain concentrations without altering the plasma concentrations via inhibition of P-gp at the blood brain barrier. In summary, caution to be taken when using 10\% Tween-80 as a vehicle for assessing the brain penetration in CNS drug discovery programs.

\section{P96. Evaluation of cellular uptake of three paclitaxel formulations in cancer cells}

\author{
$\underline{\text { Siu Kwan Wo }}{ }^{1}$, Chen Rui $\mathrm{Li}^{2}$ and Zhong Zuo ${ }^{2}$ \\ ${ }^{1}$ School of Pharmacy, Faulty of Medicine, The Chinese University of Hong Kong, Hong Kong, Hong Kong, ${ }^{2}$ School of \\ Pharmacy, Faculty of Medicine, The Chinese University of Hong Kong, Hong Kong, Hong Kong
}

Aim: Since conventional paclitaxel formulation using Cremophor-ethanol (CrEL) associated with adverse effects, sol-
vent-free formulations in the form of paclitaxel nanoparticles are recently developed so as to improve the therapeutic
effectiveness and reduce the toxicity arisen from CrEL. The present study is to compare the cancer cell uptake kinetics
of three paclitaxel formulations, including paclitaxel with CrEL, and solvent-free albumin-bound and polymer-based
paclitaxel nanoparticles. Methodology: Three cell lines, MCF-7, A549 and OVCAR-3, representing human breast, lung
and ovarian carcinoma respectively, were used in this study. Testing concentrations range and pH environment of the
three formulations were evaluated by MTT test. Cellular uptake of the three paclitaxel formulations at three loading con-
centrations (high, medium and low of their determined concentration range) by the three target cells were assessed at
various time intervals. The collected cell samples were extracted by methanol followed by analysis of paclitaxel content
using a developed and validated LC/MS/MS method. The calculated percentage of cell uptake was compared between
different loading concentrations/incubation times of the same formulation to investigate the effect of the incubation
time/concentration on the cell uptake. In addition, cell uptake of the three formulations at same concentrations was
compared to evaluate the impact of nanoparticles formulations on the cancer cell uptake of paclitaxel. Results: MTT
tests indicated that $>80 \%$ of the cells survive at pH 7.4 with 1-10 $\mu$ M paclitaxel, while only less than $40 \%$ of the cells
remained viable when pH reduced to $5-6$. Cellular uptake for paclitaxel with CrEL showed that the percentage uptake of
paclitaxel was found to less than $5 \%$ for both A549 and MCF-7. Increase of the loading concentrations from $5 \mu \mathrm{M}$ to 10
$\mu \mathrm{M}$ did not significantly increase the uptake, however increase of the incubation time from 8 to $24 \mathrm{~h}$ could enhance the
cellular uptake of paclitaxel when the pH remain the same. Consequently, loading concentration of $1-10 \mu \mathrm{M}$ paclitaxel,
pH 7.4 and incubation period up to $24 \mathrm{~h}$ were selected for subsequent cell uptake kinetic analysis of the three tested

(c) 2011 Informa Healthcare USA, Inc. 
paclitaxel formulations. Our results showed that cellular uptake was generally enhanced in the two solvent-free paclitaxel nanoparticle formulations when compared to paclitaxel with CrEL, which accounts for $\sim 2-3 \%$ uptake after $24 \mathrm{~h}$ incubation at $5 \mu \mathrm{M}$ loading concentration. In addition, roles of cellular binding and drug release from the three formulations have also been evaluated to provide mechanistic explanations on the enhanced cellular uptake. Conclusion: Our results demonstrated that paclitaxel nanoparticles exhibit higher cellular uptake than CrEL containing formulation, suggesting that paclitaxel nanoparticles may achieve better therapeutic effects than its conventional formulation at similar dose. Further in vivo comparison would warrant its clinical impact.

\title{
P97. Abstract withdrawn
}

\section{P98. Improved bioanalysis and pharmacokinetics by integrating radiolabeled metabolism data from a human clinical ADME study of [14C]2-PAP prodrug}

\author{
Gregory K. Poch ${ }^{1}$, Steve Unger ${ }^{1}$, Nadia Martinez ${ }^{1}$, Selynda Garza ${ }^{1}$, Edward Wells ${ }^{1}$, Suma Krishnan ${ }^{2}$ \\ and Schuyler Vinzant ${ }^{1}$
}

${ }^{1}$ Worldwide Clinical Trials Drug Development Solutions, Austin, TX, ${ }^{2}$ Pinnacle Pharmaceuticals Inc, Charlottesville, VA

Water-soluble prodrugs are used to provide better exposure to insoluble, but highly effective drugs. Studies were conducted in our laboratory on a prodrug of phenazopyridine (PAP), a urinary tract analgestic in human subjects. Six male humans each received a single oral administration of a dual labeled $\left[{ }^{14} \mathrm{C}\right]_{2}$-PAP prodrug at a target dose level of $100 \mu \mathrm{Ci}$. Levels of radioactivity in plasma, urine, excreta and whole blood were determined by oxidation and liquid scintillation analysis. The total recovery of the radioactive dose in urine and feces ranged from 82.04 to $93.66 \%$ of the administered dose among the six subjects. Urine was the major route for elimination, accounting for an average $(n=6)$ of $72.59 \pm 1.98 \%$ of the administered radioactive dose. Fecal route of excretion contributed to a minor route of elimination (average $16.75 \pm 3.61 \%$ ) for elimination of the radioactive dose. Prodrug-PAP derived metabolites present in urine, feces and plasma were separated and quantified using HPLC coupled with fractionation followed by radioactivity detection or mass spectrometry. Metabolite profiles of urine samples from 0 -6h revealed that ${ }^{14} \mathrm{C}$-prodrug-derived urinary radioactivity was primarily associated with PAP, three monohydroxylated PAP isomers, three glucuronide conjugates of the respective monohydroxylated PAP isomers, $\mathrm{p}$ - $\mathrm{N}$-acetyl phenol, $\mathrm{N}$-acetyl phenol glucuronide conjugate, and $\mathrm{N}$-acetyl phenol glutathione conjugate. The urinary profiles at $6-12 \mathrm{~h}$ was primarily associated with PAP, a minor dihydroxylated PAP metabolite, two glucuronide conjugates of the respective monohydroxylated PAP, p-N-acetyl phenol, $\mathrm{N}$-acetyl phenol glucuronide conjugate, and $\mathrm{N}$-acetyl phenol glutathione conjugate. Profiles of feces revealed more extensive metabolism. During the assessment of its pharmacokinetics, PAP levels determined using LC-MS/MS were higher than those determined from radiochemical profiling. Repeat analysis showed increased concentration of incurred samples while aged quality control samples showed no change. Acidification of extracts resulted in even greater apparent drug levels. Extracts of clinical samples were monitored for PAP and metabolites over the course of several days. A decrease of monohydroxylated PAP was observed with a subsequent increase in a peak that interfered with PAP. Upon acidification or over time, the metabolite degraded into an interfering isomer of PAP. Reduction of the diazo bond is facilitated at low $\mathrm{pH}$, generating a species that gave abundant MRM response due to its facile $\mathrm{N}-\mathrm{N}$ cleavage (vs. $\mathrm{N}=\mathrm{N}$ bond cleavage in PAP). A similar phenomenon was seen for the prodrug. This degradation was found to be responsible for the failed incurred sample reproducibility (ISR) runs. Modification from SPE to liquid-liquid extraction reduced the amount of unstable monohydroxylated PAP metabolite resulting in reproducible runs. In both analyses, having a selective extraction which optimized the recovery of analyte and reduced the interfering degradant of the hydroxy metabolite afforded accurate pharmacokinetics. Integrating ADME and pharmacokinetic data provided the guidance needed to develop an accurate and reproducible assay for PAP and its prodrug.

\section{P99. Linear ion trap vs. high resolution accurate mass QqTOF MS: Which provides more useful data in a drug discovery program?}

James A. Ferguson ${ }^{1}$, Richard P. Schneider², Veronica A. Zelesky², Brendon Kapinos ${ }^{2}$ and Alfin Vaz ${ }^{2}$

${ }^{1}$ Application Laboratory, AB SCIEX, Framingham, MA, ${ }^{2}$ Global R\&D, Pfizer Global R\&D, Groton, CT

Novel Aspect: Comparison of results acquired with new technologies provides valuable information to DMPK scientists in early drug discovery Introduction: In recent years, several new platforms have been introduced by the leading MS manufacturers which make it easier for biotransformation scientists to acquire both molecular ion and product ion data with a minimal 
number of injections to an HPLC system. Faster scanning and high mass resolution and mass accuracy provide scientists with an unprecedented ability to acquire large volumes of data (both qualitative and quantitative) from single injections, but these benefits may be offset by associated limitations. This investigation will seek to delineate the major advantages to be had from each of two MS platforms as well as the associated limitations that would be important to consider in a drug discovery environment. Methods: Microsomal and hepatocyte incubations ( 1 \&10 ?M) were conducted with well-characterized literature reference compounds, and compounds from existing drug discovery programs. Following incubation, samples were denatured with acetonitrile, mixed well, centrifuged, and injected onto an HPLC system. The HPLC systems used was a standard narrow-bore system flowing at 400-500 ?L/min. Data acquisition modes included enhanced MS and product ion modes on the QTRAP 5500 system, and high mass resolution data from the TripleTOF 5600 system with "on-the-fly" multiple mass defect triggered IDA for collection of MS/MS data. Preliminary Data: Literature reference and discovery compounds were incubated and analyzed with the systems noted above. For nefazodone, numerous metabolites were observed (12 to 21) using the AB SCIEX QTRAP 5500, and those metabolites of lowest intensity yielded excellent EPI spectra. A comparative analysis using the TripleTOF 5600 showed that many of the same metabolites were detected, demonstrating the selectivity of the mass defect-triggered IDA experiments which this platform affords the biotransformation scientist. Low accumulation times allow acquisitions of molecular and product ion of many metabolites, but comparative sensitivity and spectral quality of the minor metabolites was not equivalent to that demonstrated with the QTRAP platform. A significant advantage of the TripleTOF 5600 is the ability for the analyst to acquire high resolution and high mass accuracy molecular ion data over a large $\mathrm{m} / \mathrm{z}$ range and obtain MS/MS information based on mass defect-triggered IDA. Post acquisition extraction of specific ion intensities (drug and metabolites) using the PeakView application offers a qualitative/quantita-tive workflow of notable value to discovery teams that may be trying to provide SAR around absorption/permeability issues, target selectivity, or alterations to intrinsic clearance. Both platforms present distinct advantages; rapid HPLC analysis with high sensitivity and high quality EPI spectra, high mass resolution with the selectivity of multiple mass defect filtering for the drug and metabolites of interest and semi-automated peak finding with metabolite ID software packages appropriate to the data type (Trap of TOF). Experimental objectives will define the criteria and needs to utilize either platform, thereby providing clearance and metabolite identification for drug discovery teams in a rapid time period.

\title{
P100. Meropenem prodrugs for the treatment of tuberculosis
}

\author{
$\underline{\text { Aaron M. Teitelbaum }^{1}}$, Courtney C. Aldrich ${ }^{2}$ and Rory P. Remmel ${ }^{1}$
}

\author{
${ }^{1}$ Medicinal Chemistry, University of Minnesota, Minneapolis, $M N,{ }^{2}$ Center for Drug Design, University of Minnesota, \\ Minneapolis, $M N$
}

Purpose.Mycobacterium tuberculosis (Mtb), the causative agent of tuberculosis (TB), is the leasing cause of bacterial infectious disease mortality worldwide. The WHO estimates that $1 / 3$ of the world's population is infected latently, with $\sim 440,000$ new cases in 2008. TB is also the major cause of death in HIV/AIDS patients in Africa and India. Consequently, new oral agents that can be given once daily are desperately needed to treat multiple and extensively drug resistant TB. Although $\beta$-lactams are the most widely used group of antibiotics, they have never been systemically utilized in TB therapy due to their poor penetration of the mycobacterial cell wall or granulomas. In 2009, Blanchard, Barry et al. shattered this long held dogma demonstrating that meropenem and clavulanate were highly effective against XDR-TB strains in vitro. Due to meropenem's short half life $(1 \mathrm{hr}$ ) and lack of oral bioavailability, we have synthesized and are currently evaluating prodrugs of meropenem as potential therapeutics for the treatment of TB. Methods. An initial goal of the project was to synthesize the proxetil ester of meropenem. This has been accomplished in small scale by a direct carboxylate alkylation with freshly prepared 1-iodoethyl isopropyl carbonate in dimethylacetamide. Several other meropenem prodrugs are currently being synthesized by following the same procedure with the respective iodo alkylating agent. Results. Two prodrugs of meropenem were successfully synthesized and are currently being evaluated in animal plasma and tissue homogenates. Conclusion.Meropenem prodrugs are more lipophillic than the parent meropenem and are expected to have longer halflives and higher bioavailabilities. We also expect these novel prodrugs to be effective in a guinea pig tuberculosis model.

\section{P101. Metabolism and excretion of a potent p38 mitogen-activated protein kinase inhibitor N-cyclopropyl-3-(1-(2,6-difluorophenyl)-7-methyl-6-oxo-6,7-dihydro-1H-pyrazolo[3,4-b] pyridin-5-yl)-4-methylbenzamide}

\author{
ذinyan Lin ${ }^{1}$, Dean Hickman², Gary Skiles ${ }^{2}$ and Faye Hsieh ${ }^{2}$
}

${ }^{1}$ Dept of PKDM/MS 30E-2-B, Amgen Inc., Thousand Oaks, CA, ${ }^{2} P k d m$, Amgen Inc., Thousand Oaks, CA 
Compound I (N-cyclopropyl-3-(1-(2,6-difluorophenyl)-7-methyl-6-oxo-6,7-dihydro-1H-pyrazolo[3,4-b]pyridin-5yl)-4-methylbenzamide) is a potent mitogen activated protein kinase P38 $\alpha$ inhibitor. The metabolic disposition of I was investigated to identify appropriate species for toxicity testing, to assess the clinical drug-drug interaction (DDI) potential, and to inform a circulating metabolite(s) strategy for the first-in-human (FIH) study. The in vitro metabolite profile in hepatocytes of rat and cynomolgus monkey included the products of oxidation and amide hydrolysis and was qualitatively similar to human hepatocyte metabolism. The major metabolite in hepatocytes of all species was the product of amide hydrolysis (M7). In human hepatocytes the turnover of $\mathbf{I}$ was inhibited approximately $50 \%$ by the CYP3A inhibitor ketoconazole $(1 \mu \mathrm{M})$. Following a single oral administration of $1 \mathrm{mg} / \mathrm{kg}\left[{ }^{14} \mathrm{C}\right]$ I to male bile-duct cannulated Sprague-Dawley rats, drug related radioactivity was predominantly recovered as metabolites consistent with the in vitro data. Based on plasma collected at $1 \mathrm{hr}$ post dose, compound I was the major circulating drug related radiolabeled component (62\%) and metabolite M7 accounted for $21 \%$ of the total drug related radioactivity. Analysis of plasma from multiple dose rat and cynomolgus monkey non-GLP toxicology studies indicated the exposure ratio of M7 to I was approximately 0.5 . The in vitro metabolite profiles supported the use of rat and cynomolgus monkey as preclinical tox species. Metabolite M7 was also a circulating metabolite of potential toxicological importance in rat and monkey ( $>10 \%$ of total drug related material in plasma) and is therefore a candidate for further exposure assessment in FIH and GLP toxicology studies.

\title{
P102. Novel synthetic Omega-3 monounsaturated fatty acids inhibit the proliferative activity of breast cancer cells that over-express COX-2
}

\author{
Pei H. Cui ${ }^{1}$, Tristan Rawling ${ }^{1}$, Kirsi Bourget ${ }^{1}$, Nenad Petrovic $^{2}$ and $\underline{\text { Michael Murray }}^{1}$ \\ ${ }^{1}$ Pharmacy, The University of Sydney, Sydney, Australia, ${ }^{2}$ Pharmacy and Medical Sciences, University of South Australia, \\ Adelaide, Australia
}

Tumor metastasis is the primary cause of death due to breast and a range of other cancers but at present there are no effective treatments. Cyclooxygenase-2 (COX-2) is over-expressed in many cancers and converts the $\omega$ - 6 polyunsaturated fatty acid (PUFA) arachidonic acid to prostaglandin (PG)- $\mathrm{E}_{2}$ that enhances the viability and invasion potential of tumor cells. In contrast, epidemiological and experimental studies have indicated that $\omega$-3 PUFA such as eicosapentaenoic acid (EPA) decrease tumor growth and metastasis. We tested the hypothesis that the $\omega-3$ olefinic bond in EPA may selectively mediate the anti-tumor actions of $\omega$-3 PUFA. A series of novel monounsaturated fatty acids (MUFAs) of carbon chain length C16-C22 that contained $\omega-3$ olefinic bonds was designed and synthesized and evaluated in MDA-MB-468 breast cancer cells that were engineered to stably over-express COX-2 (COX-2/MDA-MB-468 cells). The C19-C22 $\omega-3$ MUFA analogues effectively inhibited COX-2/MDA-MB-468 cell proliferation, reflected by the mitochondrial reduction of 3-(4,5-dimethylthiazol-2-yl)-2,5-diphenyltetrazolium bromide, and activated apoptosis, reflected by caspase-3 activity in cell lysates. In contrast, the shorter chain C16-C18 $\omega-3$ MUFA analogues were less effective and exhibited lower anti-proliferative and pro-apoptotic potencies that were comparable to the naturally occurring $\omega$-3 PUFA EPA. In the COX-2/MDA-MB-468 stable transfectants the C19-C22 $\omega$-3 MUFA derivatives also decreased the extent of endogenous and AA-stimulated formation of PG- $\mathrm{E}_{2}$, which is an important COX-2-derived metabolite of AA that maintains cell viability and drives the metastatic phenotype. The C20-C22 $\omega$-3 MUFAs were also found to inhibit the number of COX-2/MDAMB-468 cells that migrated out of matrigel droplets, an assay that reflects tumor cell invasion potential. Considered together these findings indicate that longer-chain $\omega$-3 MUFAs may be prototypes of a novel class of anti-cancer agents, based on the naturally occurring $\omega$-3 PUFA structure, that decrease the formation of pro-migratory PG- $\mathrm{E}_{2}$ by tumor cells that contain high levels of COX-2.

\section{P103. Preclinical assessment of the absorption, distribution, metabolism and excretion of HMPL-013, A novel potent and specific VEGFR inhibitor}

\author{
Yi Gu, Jian Wang, Hongcan Ren, Li Zhang, Lixia Guo, Ke Li, Weihan Zhang, Weiguo Su and Yang Sai
}

Hutchison MediPharma Limited, Shanghai, China

HMPL-013 is a small-molecule VEGFR inhibitor. This study is to evaluate its preclinical ADME/PK profiles. HMPL-013 has high permeability (Papp $(\mathrm{A} \rightarrow \mathrm{B}) \sim 30 \times 10-6 \mathrm{~cm} / \mathrm{s}$ ) across Caco-2 cell monolayer and its efflux ratio is around 1 with or without $10 \mu \mathrm{M}$ varapamil. With low to moderate hepatic extractions predicted from its metabolic stability in liver 
microsomes of mouse, rat, dog and monkey, pharmacokinetic studies confirm its good oral absorption with bioavailability of $42 \% \sim 53 \%$ and Tmax $<4 \mathrm{~h}$ in these species. Oral exposure (AUC) is $1134 \mathrm{~h} . \mathrm{ng} / \mathrm{mL}$ with Cmax of $326 \mathrm{ng} / \mathrm{mL}$ at $1 \mathrm{mg} / \mathrm{kg}$ in rats and $2047 \mathrm{~h} . \mathrm{ng} / \mathrm{mL}$ with $129 \mathrm{ng} / \mathrm{mL}$ at $0.3 \mathrm{mg} / \mathrm{kg}$ in dogs. The exposure are linear from 0.5 to $2 \mathrm{mg} / \mathrm{kg}$ in rats and 0.1 to $1 \mathrm{mg} / \mathrm{kg}$ in dogs. Food has no significant effect on HMPL-013 pharmacokinetic profile in dogs. Vd is around $1 \mathrm{~L} / \mathrm{kg}$ in mouse, rat and dog and $3 \mathrm{~L} / \mathrm{kg}$ in monkey. The plasma protein binding fraction is $88 \sim 95 \%$ in mouse, rat, dog and human, invariable from 1 to $10 \mu \mathrm{M}$. In rat, HMPL-013 is majorly distributed in gastrointestinal tract, liver, kidney, adrenal and fat. Three major Phase I metabolites, generated by demethylation (M1), hydroxylation (M2), and mono-oxygenation (M3) respectively, are found in liver microsomes of different species. Dog is mostly similar to human in terms of metabolism rate, metabolite type and the relative abundance. Reaction phenotyping shows multiple hCYP450s are involved in HMPL-013 metabolism. Pharmacokinetic studies show the systemic clearance is 3.3, 8.0, 1.4 and $8.0 \mathrm{~mL} / \mathrm{min} / \mathrm{kg}$ in mouse, rat, dog and monkey. Metabolite identification in rat excreta confirms the existence of the three major in vitro metabolites and reveals the major metabolic pathway of HMPL-013 in vivo is demethylation, hydroxylation and further glucuronidation. The maximum of excretion of parent HMPL-013 in urine and feces is reached after $24 \mathrm{~h}$ post oral dosing at $1 \mathrm{mg} / \mathrm{kg}$ in rats. $10.4 \%$ of administrated HMPL-013 is recovered as unchanged in feces, while $<1 \%$ in urine. Considering M1, M2 and the possible glucuronide conjugates, the mass balance recovery up to $72 \mathrm{~h}$ is $33.2 \%$ in rats combining the urinary and fecal excretion. A separated biliary excretion study reveals $<0.1 \%$ of the dose is recovered as intact after $24 \mathrm{~h}$, but this excretion accounts for $47.9 \%$ of the dose if further considering M1, M2 and the possible glucuronide conjugates. HMPL-013 has no obviously reversible or mechanism-based CYP inhibition in human liver microsomes, no effect on hPXR-mediated CYP3A4 transcription, and no induction of CYP1A2 and CYP3A4 in human hepatocytes. HMPL-013 has low accumulation risk in rats, as it can be eliminated completely from almost all organs after $14 \mathrm{~h}$ post-dose and its pharmacokinetic parameters do not change significantly after repeated oral dosing at $1 \mathrm{mg} / \mathrm{kg}$ for 7 days. Allometric scaling suggests the clearance and Vd of HMPL-013 in human is $2.4 \mathrm{~mL} / \mathrm{min} / \mathrm{kg}$ and $2.4 \mathrm{~L} / \mathrm{kg}$. Liver microsomal metabolic stability also suggested low hepatic extraction in human. Summarily, HMPL-013 exhibits good preclinical pharmacokinetic profile and is valuable to be further developed.

\title{
P104. Reactive metabolite screening in early ADME using rapid high resolution accurate mass technology
}

\author{
Łames A. Ferguson ${ }^{1}$, Richard P. Schneider ${ }^{2}$, Veronica A. Zelesky² and Hui Zhang ${ }^{3}$ \\ ${ }^{1}$ Application Laboratory, AB SCIEX, Framingham, MA, ${ }^{2}$ Global R\&D, Pfizer Global R\&D, Groton, CT, ${ }^{3}$ Pfizer Global R\&D, \\ Groton, $C T$
}

Introduction - One goal of early ADME is determing whether a candidate drug forms reactive metabolites as early as possible. Screening methods using MRM, neutral loss or other techniques are currently used. MRM methods have excellent sensitivity but are targeted while neutral loss methods, though, a bit more generic, have less sensitivity. We propose a semi-targeted workflow using full scan accurate mass with targeting for expected reactive metabolites based on historical data by either use of inclusion lists or by NL-triggered IDA which can still find metabolites not on the inclusion list. Data can be processed quantitatively or semi-quantitatively and data will be compared to an existing MRM-based screen. Methods - The QqTOF system was operated in either mass defect-triggered IDA mode with an inclusion list for "expected" glutathione adducts or NL-triggered IDA mode with a NL of 129 (for GSH adducts). UHPLC was used to speed the analysis with maximum cycle times of ca. $0.5 \mathrm{~s}$ to do surveys and dependent scans. Data was processed by XIC of the expected adducts or by filtering the dependent product ion scans for accurate mass loss of the 129 fragment from glutathione. Semi-quantitative output can be shown using software which can show the integrated peaks from all wells in a 96-well plate simultaneously. Preliminary Data - Sample plates with several compounds were analyzed using the screening methods described. Quantitative software was used to integrate the expected metabolites and any found by the filtered data. The software can then simultaneously display the data for all of the potential adducts for a quick visual inspection for high levels of metabolites. The accurate mass data was compared with data collected on a high-end triple quadrupole system doing the screening by an MRM-based assay and found to be comparable. The MRM-based method has the advantage of better sensitivity, but is limited to finding only the predicted metabolites. The high end triple quad is also used to do a NL screen. However, since the NL is done at unit resolution, interferences can (and do) crop up. The accurate mass system has the potential to find unpredicted metabolites and can also do accurate mass NL to avoid the interferences found in the unit-resolution NL data. Novel Aspect - Rapid, accurate mass reactive metabolite screening used to find as many potential reactive metabolites as possible while minimizing false positives. 


\title{
P105. Screening newly synthesized curcumin analogues with improved pharmacokinetic properties by in vitro Caco-2 model and in vivo bioavailability studies
}

\author{
Deng Ying 1 , Hu Jingqing², Bi Huichang², Jin Jing², Cai Yike², Bu Xianzhang² and Huang Min² \\ ${ }^{1}$ The Shenzhen Baoan Affilated Hospital, Southern Medical Univerity, Shenzhen, China, ${ }^{2}$ School of Pharmaceutical \\ Sciences, Sun Yat-sen University, Guangzhou, China
}

Objective: T6- and T3- series of compounds are newly synthesized 4-hydroxymethyliene curcumin analogues with great anti-cancer potency, and it was found that most of 4-arylidene curcumin analogues possessed potent NF-kB inhibitory activity ${ }^{[1]}$. In the present work, the in vitro permeability and in vivo pharmacokinetic features of 10 curcumin analogues were investigated. Methods:In vitro Caco-2 cell monolayers and an in vivo animal model were used to investigate their permeability, transport mechanism, and bioavailability. Results: The Caco-2 bi-directional transport experiment tested at $30 \mu \mathrm{M}$ showed the new analogues had significant improved permeability than that of curcumin on both of the directions (the value of $P_{\text {app }}$ in the range of $(1.29-6.35) \times 10^{-6} \mathrm{~cm} / \mathrm{s}$ versus $0.67 \times 10^{-6} \mathrm{~cm} / \mathrm{s}$ of curcumin from the apical to basolateral side, while the value of $P_{\text {app }}$ in the range of $(0.64-4.37) \times 10^{-6} \mathrm{~cm} / \mathrm{s}$ versus $0.42 \times 10^{-6} \mathrm{~cm} / \mathrm{s}$ of curcumin from the basolateral to apical side). The transport of curcumin $(15,30$ and $60 \mu \mathrm{M})$ and $\mathrm{T} 63(10,20$ and $40 \mu \mathrm{M})$ from the apical (AP) to basolateral (BL) side increased significantly with concentrations and the transport of curcumin $(30 \mu \mathrm{M})$ and T63 $(20 \mu \mathrm{M})$ was not affected in the presence of P-glycoprotein inhibitor Verapamil. Based on the results from in vitro permeability screening and in vitro anti-cancer activity screening, T63 was selected into in vivo pharmacokinetic study in rats. Following the oral and intravenous administration of curcumin and T63 $\left(28 \mathrm{mg} \cdot \mathrm{kg}^{-1}\right.$ for oral dose and $5.6 \mathrm{mg} \cdot \mathrm{kg}^{-1}$ for intravenous dose), the results showed that $\mathrm{T} 63$ had greater $A U C_{0-\infty}, A U C_{0-\mathrm{t}}$ and $C_{\max }$ than that of curcumin after oral administration and greater $A U C_{0-\infty}, A U C_{0-t}$ and longer $t_{1 / 2}$ after intravenous administration. The bioavailability of T63 was improved in some extent than that of curcumin (8.7\% versus $5.8 \%$ ). Conclusion: The newly synthesized curcumin analogues had an improved permeability and pharmacokinetic behavior than that of curcumin and some of them are worthy of further development to promising anti-cancer agents.

\section{Reference}

[1] Qiu X, Du Y, Lou B, et al. Synthesis and Identification of New 4-Arylidene Curcumin Analogues as Potential Anticancer Agents Targeting Nuclear

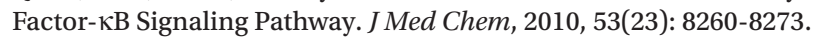

\section{P106. Species differences in the oral bioavailability of a novel MET kinase inhibitor}

\author{
Bianca M. Liederer ${ }^{1}$, Leonid M. Berezhkovskiy ${ }^{1}$, Vikki Dinkel ${ }^{2}$, Emile G. Plise ${ }^{1}$, Susan Wong ${ }^{1}$ and \\ Dan Sutherlin ${ }^{1}$
}

${ }^{1}$ Genentech Inc., South San Francisco, CA, ${ }^{2}$ Array BioPharma Inc., Boulder, CO

GNE-A ( $N$-(4-(3-((1s,4s)-4-(dimethylamino)cyclohexylamino)-1 $H$-pyrazolo[3,4- $b$ ]pyridin-4-yloxy)-3-fluorophenyl)-2 -(4-fluorophenyl)-3-oxo-2,3-dihydropyridazine-4-carboxamide) is a potent and selective small molecule MET kinase inhibitor developed as a potential orally available drug for the treatment of human cancers. The objective of this study was to assess the preclinical ADME characteristics of GNE-A and its potential as a human drug candidate. The pharmacokinetic (PK) properties of GNE-A were evaluated following single IV and PO dose administration to mouse, rat, dog and monkey. GNE-A showed significant species differences in F with very low \%F values (5-6\%) in rodents to moderate/high \%F values in monkey and dog (48-74\%). In contrast, the plasma clearance in preclinical species was similar with moderate CL values of $28,37,15$, and $14 \mathrm{~mL} / \mathrm{min} / \mathrm{kg}$ in mouse, rat, monkey and dog, respectively. The $\mathrm{V}_{\mathrm{ss}}$ ranged from 7.4 to $21.2 \mathrm{~L} / \mathrm{kg}$. Urinary excretion of the parent after IV dose administration in rat, monkey and dog was minor ( $<3 \%$ of administered dose). Results suggest that the observed $\mathrm{F}$ in higher species is likely primarily due to hepatic first pass metabolism while other factors contribute in rodents. GNE-A was stable in blood $\left(\mathrm{t}_{1 / 2}=11-49 \mathrm{~h}\right)$ and in intestinal microsomes $\left(\mathrm{t}_{1 / 2}=3-17 \mathrm{~h}\right)$ in evaluated preclinical species and human. The major metabolites in mouse, rat, dog, monkey and human liver microsomes were an $\mathrm{N}$-oxide and an $\mathrm{N}$-desmethyl metabolite. All preclinical species, and human, exhibited high plasma protein binding (93.2-96.8\% bound). MDR1-transfected MDCK cells showed a marked efflux ratio of 162. P-gp inhibitor GF120198 inhibited this polarized efflux to 1.4, suggesting that GNE-A is a substrate for MDR1. In vitro blood-plasma ratios ranged from 0.5 to 1.6 in all evaluated species, indicating that GNE-A did not preferentially distribute into red blood cells. GNE-A showed no inhibition of CYP isoforms $\left(\mathrm{IC}_{50}>25 \mu \mathrm{M}\right)$ indicating a low potential of this compound for DDIs. Human PK parameters were predicted using allometric scaling with MLP correction with four 
preclinical species and in vitro scaling using human microsomes and hepatocytes. Results projected a low drug clearance and a high $\mathrm{V}_{\mathrm{ss}}$ of approximately $21.7 \mathrm{~L} / \mathrm{kg}$ in human. Overall, GNE-A exhibited desirable preclinical properties and projected human PK estimates. Further investigations are ongoing to better understand the species differences in $\mathrm{F}$ in order to provide valuable information for the prediction of human oral PK and for further guidance in the progression of GNE-A.

\title{
P107. Stereoselectivity: Assessment of in vitro and in vivo preclinical properties including toxicity of carboxamide enantiomers
}

\author{
Bianca M. Liederer, Eric Harstad, Leonid M. Berezhkovskiy, Emile G. Plise, Dan Sutherlin and \\ Xingrong Liu
}

\author{
Genentech Inc., South San Francisco, CA
}

The R- and S-enantiomers of $N$-(4-(3-(1-ethyl-3,3-difluoropiperidin-4-ylamino)-1H-pyrazolo[3,4-b]pyridin-4-yloxy)-3 -fluorophenyl)-2-(4-fluorophenyl)-3-oxo-2,3-dihydropyridazine-4-carboxamide are novel MET kinase inhibitors that were developed as potential orally available anticancer agents. The objective of this study was to assess whether the chirality of these compounds affected their preclinical ADME, toxicity and developability. PK properties were evaluated following single IV and PO dose administration to mice, rats and monkeys. Safety profiles, plasma exposures and tissue distributions (liver, lung, heart, spleen, and bone marrow) were determined in a 14-Day daily oral dose toxicity study in mice. Plasma protein binding, blood-plasma partitioning, permeability/transporter characteristics and CYP inhibition were determined in vitro. After single IV/PO dose administration of each enantiomer, plasma exposure of the S-enantiomer was higher in mice and monkeys but not in rats. The plasma clearance for the S-enantiomer was low in mice and monkeys $(23.7$ and $7.8 \mathrm{~mL} / \mathrm{min} / \mathrm{kg}$, respectively) and high in rats $(79.2 \mathrm{~mL} / \mathrm{min} / \mathrm{kg})$. The R-/S-enantiomer clearance ratio was approximately 1.6 except in rats (0.5). $7.1 \%$ and $5.7 \%$ of the IV dose of the R- and S-enantiomer, respectively, were excreted as the parent into the rat urine. After oral administration the R-/S-enantiomer ratio of $\mathrm{AUC}_{\text {inf }}$ was $0.95,1.9$ and 0.41 in mice, rats, and monkeys, respectively. Following multiple PO dose administration to mice, plasma exposure was approximately 2-fold higher for the R-enantiomer compared to the S-enantiomer and marked stereoselective tissue distribution was observed with approximately 2.5- to 29-fold higher tissue concentrations of the S-enantiomer. Both enantiomers showed low to moderate permeability in MDCK cells with no significant efflux ratios. Both enantiomers had high plasma protein binding ( $>97 \%$ bound) in preclinical species and human, and weak CYP inhibition $\left(\mathrm{IC}_{50}\right.$ values $>10 \mu \mathrm{M}$ ). In vitro blood-plasma ratios ranged from 0.67 to 0.91 for the R-enantiomer and from 0.72 to 0.95 for the S-enantiomer in all species evaluated, indicating that the compounds did not preferentially distribute into red blood cells. Overall, the differences between the enantiomers with respect to in vivo single dose PK and in vitro studies were relatively modest. However, a high degree of stereoselectivity in tissue distribution was observed after multiple dosing which appeared to result in greater toxicity of the S-enantiomer. Further preclinical evaluation is warranted to assess if the stereoselective tissue distribution leads to differences in efficacy.

\section{P108. Abstract withdrawn}

\section{P109. The pharmacokinetics and metabolism of HMPL012, a novel potent inhibitor targeting multiple kinases}

\author{
Jian Wang, Yi Gu, Weifang Xue, Li Zhang, Li Ke, Zheyi Hu, Weihan Zhang, Weiguo Su and Yang Sai
}

\section{Hutchison MediPharma Limited, Shanghai, China}

HMPL012 (Sulfatinib) is a novel small molecular compound that inhibits multiple kinases critical for tumor growth, apoptosis and invasion/metastasis. The aim of this study is to evaluate the pharmacokinetics and metabolism characteristics of HMPL012 using in vivo and in vitro approaches. HMPL012 showed oral bioavailability of $>50 \%$ and $>30 \%$ in rat ( 40 and $160 \mathrm{mg} / \mathrm{kg})$ and $\operatorname{dog}(5,10$ and $20 \mathrm{mg} / \mathrm{kg})$, respectively. But an interesting phenomenon was that the bioavailability of HMPL012 in rats at $10 \mathrm{mg} / \mathrm{kg}$ was only about $10 \%$, which could associate with its efflux based on the Caco-2 result $(\operatorname{Papp}(\mathrm{B} \rightarrow \mathrm{A}) / \operatorname{Papp}(\mathrm{A} \rightarrow \mathrm{B})$ was about 6$)$. The total clearance of HMPL012 in rats was about $58.8 \mathrm{~mL} / \mathrm{min} / \mathrm{kg}$, which was close to the hepatic blood flow $(55.2 \mathrm{~mL} / \mathrm{min} / \mathrm{kg}$ in rats), while $65.7 \mathrm{~mL} / \mathrm{min} / \mathrm{kg}$ in dogs was even much higher than the hepatic blood flow ( $31 \mathrm{~mL} / \mathrm{min}$ in dogs), which was probably caused by an extra-hepatic elimination of HMPL012. 
HMPL012 presented extensive tissue distribution after oral dose in rats. The major distributed organs were lung, spleen, kidney, adrenal and pancreas. HMPL012 had low exposure in brain, indicating that it could not penetrate through blood-brain barrier. HMPL012 was eliminated completely from almost all organs in 24 hrs post-dose and no exposure accumulation in rats after repeated oral doses for 7 days. The low accumulation risk was also exhibited in the 14C radiolabeled experiment, which showed that more than 90\% of the administered HMPL012 was eliminated out of body after $24 \mathrm{hrs}$ post-dose. The major elimination pathways of HMPL012 were bile and feces, and only $10 \%$ of the administered HMPL012 was eliminated via urine. HMPL012 can be metabolized extensively in rats after oral administration, and only $<20 \%$ of prototype can be eliminated out of body in $72 \mathrm{~h}$ post-dose. Total 7 phase I metabolites and 10 phase II metabolites were observed in urine, bile and feces after oral dose, and the major metabolic types were mono-oxidation, carboxylation, N-demethylation, and glucuronide conjugation. Among of them, the carboxyl metabolite was the most important one, which was major excreted via bile with about $10 \%$ recovery of the oral dose. The results of CYP inhibition and induction studies in human liver microsomes and cryo-preserved hepatocytes showed that HMPL012 had weak inhibition and induction potentials on CYP450 isozymes, indicating it has low drug-drug interaction risk. In a summary, HMPL012 exhibits good preclinical pharmacokinetics and is valuable to be for further clinical studies.

\title{
P110. The role of the liver and kidney in the pharmacokinetics of subcutaneously administered teriparatide acetate in rats
}

\author{
$\underline{\text { Aki Sakurai }^{1}}{ }^{\text {, Masashi Serada }}{ }^{1}$, Masayo Igarashi $^{1}$, Koichi Mitsugi ${ }^{2}$, Toshie Takano ${ }^{2}$, Koichi \\ Shibusawa $^{2}$ and Terutomo Kohira ${ }^{1}$
}

\begin{abstract}
${ }^{1}$ Laboratory for Safety Assessment and ADME, Pharmaceuticals Research Center, Asahi Kasei Pharma Corporation, Izunokuni, Shizuoka 410-2321, Japan, ${ }^{2}$ ADME and Tox. Research Institute, Sekisui Medical Co., Ltd., Tokai, Ibaraki 3191182, Japan
\end{abstract}

Teriparatide acetate is a therapeutic agent for the treatment of patients with osteoporosis at a high risk of fracture. Teriparatide is the amino-terminal 1-34 fragment of human parathyroid hormone, namely hPTH(1-34), which increases bone mineral density and reduces fracture risk. While thousands of studies regarding the parathyroid hormone and parathyroid hormone-related proteins have been reported, it is however, still unclear whether the liver or kidneys are more critical for the pharmacokinetics of teriparatide. In the present study, we conducted absorption, distribution, metabolism and elimination studies of teriparatide acetate in normal, and hepatic- or renal-failure rats after subcutaneous administration to investigate the pharmacokinetics of teriparatide acetate. The plasma and urine concentrations of teriparatide acetate were determined by an ELISA method, and the radioactivity derived from the

${ }^{125}$ I-labeled analog was measured by a gamma-well counter and HPLC. After single and repeated administration of teriparatide acetate to normal rats, teriparatide was rapidly absorbed into the circulation and eliminated from plasma with a half life of approximately $0.5 \mathrm{~h}$. The $\mathrm{C}_{\max }$ and AUC increased in proportion to the dose of teriparatide acetate. The accumulation of teriparatide acetate in plasma was not observed during the repeated dosing period. Subsequently, in vivo and in vitro metabolism studies using the ${ }^{125}$ I-labeled analog were performed. After the administration of the ${ }^{125}$ I-labeled analog, the plasma concentration of its metabolites increased time-dependently. Although the total radioactivity concentration in the kidney was more than 3 -fold the level in the liver, the in vitro metabolism study suggested that the radioactivity was derived from the metabolites of the ${ }^{125}$ I-labeled analog. In the excretion study, no intact teriparatide was detected in the urine. Therefore, it was speculated that most of the administered teriparatide acetate was metabolized in the distributed tissues. According to the studies in renal or hepatic failure model rats, renal failure not hepatic failure affected the pharmacokinetics of teriparatide acetate, which accounted for the decrease of clearance of teriparatide. In conclusion, these results suggest that after subcutaneous administration of teriparatide acetate, teriparatide is rapidly absorbed and distributed to the liver and kidneys, where teriparatide was immediately metabolized. In particular, the kidneys were shown to play an important role in the distribution and metabolism but not the excretion of teriparatide.

\section{P111. The use of UV-MS correlation for the quantification of plasma metabolites on a dual-cell linear ion trap}

\author{
$\underline{\text { Tim Stratton }}^{1}$, August Specht ${ }^{2}$, Julie Horner ${ }^{2}$ and Yingying Huang ${ }^{3}$
}

${ }^{1}$ Thermo Fisher Scientific, San Jose, CA, ${ }^{2}$ Ion Trap Marketing, Thermo Fisher Scientific, San Jose, CA, ${ }^{3}$ Metabolism Marketing, Thermo Fisher Scientific, San Jose, CA 
The quantitative assessment of metabolites in samples from development in vivo studies has become an area of increasing study with the release of guidances from regulatory bodies (MIST and M3 R2) and their subsequent interpretation and implementation. The hurdles faced in metabolite identification are joined by obtaining quantitative levels on relevant metabolites for which absolute structure may not be known and for which reference standards are not available. The challenges of matrix suppression, analyte stability, and MS detector response factor make quantitation of metabolites based on a parent drug standard almost impossible. We have applied a UV correlation to assign concentrations to metabolites identified from in vitro generated samples and used the results to quantify plasma samples on the same instrument. Initially, metabolites of dextromethorphan were generated in vitro through incubations with human liver S9 fraction $(2 \mathrm{mg} / \mathrm{mL})$. Dextromethorphan was incubated for 2 hours at $100 \mathrm{uM}$ to assure the creation of significant concentration of metabolites. After an organic quench, samples were analyzed by UHPLC-MS on an LTQ Velos Pro using a combination of collision-induced dissociation fragmentation (CID) and higher-energy collisional dissociation (HCD) to identify the metabolites present in the sample. Analysis of the in vitro sample by UHPLC-UV along with a standard curve of dextromethorphan prepared in microsomal matrix ( $50 \mathrm{uM}$ to $1 \mathrm{uM}$ ) was performed to assign absolute metabolite concentrations based on quantification against dextromethorphan UV response. For this work, as with all UV-MS correlation quantitation, the molar absorptivity of dextromethorphan and its metabolites was assumed to be the same. When quantified against a known UV standard curve of dextromethorphan, the high concentration incubation samples provided concentrations of metabolites ranging from approximately $30 \mathrm{uM}$ (dextrorphan) down to the limit of UV detection. The in vitro sample was diluted using a plasma extraction matrix to give a final standard curve concentration of between 1000 and $2 \mathrm{ng} / \mathrm{mL}$ for both dextrorphan and methoxymorphinan. These two metabolites were selected as they were available commercially. Plasma samples for UHPLC-MS analysis were prepared by spiking known amounts of dextrorphan and methoxymorphinan (between 750 and $5 \mathrm{ng} / \mathrm{mL}$ ) into plasma extraction matrix followed by mixing with in vitro microsomal matrix. A total of 12 spiked concentration values were prepared for each analyte. Quantification of the spiked plasma samples against the UV-generated metabolite standard curve was compared to the known spike values and also confirmed for two metabolites by standard curves created with synthetic standards. For all the samples analyzed, the calculated concentration was within $2 \mathrm{X}$ the known value when quantifying based on the in vitro UV-MS correlation standard curve. The same spiked samples quantified against a dextromethorphan standard curve only (assuming a one to one MS signal correlation between dextromethorphan and metabolites) provided results that were on average greater than $5 \mathrm{X}$ off from the known values. Quantitation was performed by targeted selective reaction monitoring (SRM) as well as full scan $\mathrm{MS}^{2}$ on dextromethorphan, dextrorphan, and methoxymorphinan without the use of timed segments.

\title{
P112. An in vitro test system to evaluate drug-drug interactions with biologics
}

\author{
Maciej Czerwinski ${ }^{1}$, Christina Renneke ${ }^{2}$, Joanne Parker ${ }^{2}$, Chad Pope ${ }^{1}$, Kevin Lyon ${ }^{1}$, Catherine \\ Wiegand ${ }^{1}$, Jason Neat ${ }^{1}$, Faraz Kazmi ${ }^{1}$, David Buckley ${ }^{1}$ and Andrew Parkinson ${ }^{1}$
}

${ }^{1}$ XenoTech, LLC, Lenexa, KS, ${ }^{2}$ Viracor IBT Laboratories, Lee's Summit, MO

Inflammation, infection, vaccination, and some marketed therapeutic proteins (biologics) are associated with cytokinemediated suppression (down-regulation) of drug-metabolizing enzymes (DME). Biologics, such as monoclonal antibodies, can trigger the release of pro-inflammatory cytokines (like IL-1b, IL-6, INFg and TNFa); extreme cases of which are known as a cytokine storm. By affecting DME expression biologics may change the clearance of small molecule drugs and thereby precipitate drug-drug interactions (DDI). In the present study, we devised an in vitro method to evaluate the potential for biologics to elicit DDIs. The method involves treating whole human blood to stimulate the release of pro-inflammatory cytokines from peripheral blood mononuclear cells (PBMCs), after which plasma is prepared and added to primary human hepatocytes co-cultured with Kupffer cells to evaluate simultaneously the direct and indirect effects of biologics on cytochrome P450 (CYP) enzyme expression. We selected LPS (lipopolysaccharide or endotoxin) as an indirect-acting biologic. Treatment of whole blood with LPS $(50 \mathrm{ng} / \mathrm{mL})$ for 24 hours at $37^{\circ} \mathrm{C}$ stimulated the release IL-1b, IL-2, IL-6, IL-8, IL-10, IL-12p70, GM-CSF, INF- $\gamma$, TNF- $\alpha$ as determined with a sector imager and pro-inflammatory 9-plex kit (Meso Scale Discovery). Co-cultures of human hepatocytes and Kupffer cells were treated once daily for three consecutive days with various concentrations of plasma from saline-treated blood (negative control) or LPS-treated blood (test system) or with IL-6 (a direct-acting cytokine; positive control). After 72-hours of treatment, the cells were incubated in situ with marker substrates for CYP1A2 (phenacetin O-dealkylation), CYP2B6 (bupropion hydroxylation) and CYP3A4 (testosterone $6 \beta$ hydroxylation). Interlukin- $6(10 \mathrm{ng} / \mathrm{mL}$ ) reduced the enzymatic activity of CYP1A2, CYP2B6 and CYP3A4 by 65, 66 and 83\%, respectively. Plasma from the LPS-stimulated blood reduced the enzymatic activity of CYP1A2, CYP2B6 and CYP3A4 by 69, 87 and 67\% relative to saline-treated plasma, respectively. Analysis by qRT-PCR revealed corresponding changes in the levels of CYP mRNA. The results suggest that this two-step, in vitro method may 
be suitable to detect the direct and indirect effects of biologics on CYP expression in order to evaluate the DDI potential of therapeutic proteins and other biologics.

\title{
P113. Can lysosomal trapping cause drug-drug interaction?
}

\author{
Mingxiang Liao, Mi-Sook Kim, Qing Zhu, Lili Yao, Bingli Ma, Bei-Ching Chuang, Jing-Tao Wu, \\ Suresh K. Balani, Frank W. Lee and Cindy Xia
}

Drug Metabolism and Pharmacokinetics, Millennium Pharmaceuticals, Inc., Cambridge, MA

Basic lipophilic compounds are characterized by a high volume of distribution as a result of extensive tissue uptake. The main mechanisms of the high tissue distribution have been proposed through the nonspecific binding to membrane phospholipids and the trapping by acidic subcellular compartments, mainly lysosomes. The present studies evaluated the effect of lysosomal trapping on drug tissue distribution and drug-drug interactions of weak bases in hepatocytes from various species and in Sprague-Dawley (SD) rats. The well-known lysosomal inhibitors (monensin and chloroquine) decreased the accumulation of imipramine in hepatocytes from mouse, rat, dog, monkey, and human. In contrast, the inhibition of thioridazine accumulation by lysosomal inhibitors was only observed in mouse hepatocytes, but not in other tested species. In SD rats, lysosomal trapping contributed to the high tissue distribution of imipramine and thioridazine, suggested by the correlation between the rank orders of the tissue distributions with the rank orders of lysosome amount in tissues. Pre-treatment of chloroquine decreased the exposure of imipramine in various tissues, except liver. The heart had the most significant effect. However, co-administration of chloroquine did not change AUC of thioridazine in rat tissues except heart and liver. Thus, cultured hepatocytes can be used as an in vitro model to estimate the contribution of lysosomal trapping on tissue accumulation. The rat tissue distribution study also indicates that ysosomal trapping mediated drug-drug interaction (DDI) in tissues is possible. The inhibition of chloroquine on the clearance of imipramine and thioridazine might also contribute to these DDIs. The combined effects of CYP-mediated metabolism and lysosome trapping need to be further investigated.

\section{P114. Development of a rapid cell-based assay using flow cytometry for assessing drug interactions with human sodium-dependent taurocholate cotransporting polypeptide (NTCP)}

\author{
$\underline{\text { Steve Wright }}^{1}$, J. Li' ${ }^{2}$ Y. Wang ${ }^{2}$, A. Owen ${ }^{2}$ and I.J. Hidalgo ${ }^{2}$ \\ ${ }^{1}$ Department of Biology, Carson-Newman College, Jefferson City, TN, ${ }^{2}$ Absorption Systems LP, Exton, PA
}

Purpose.Sodium-taurocholate cotransporting polypeptide (NTCP) is the major bile acid uptake system in human hepatocytes. Previous studies showed NTCP transported rosuvastatin and its variants demonstrated significant different transport capacities. In addition, about $35 \%$ of rosuvastatin hepatic uptake is sodium dependent, thus implying that NTCP may play a critical role in rosuvastatin disposition in vivo. As a result, inhibition of NTCP-mediated drug transport may reduce liver uptake and/or efficacy of rosuvastatin or other drugs. The objective of this study was to establish a rapid in vitro inhibition assay using flow cytometry for screening NTCP-mediated drug interactions in stably transfected HEK293. Methods.HEK293 cells, stably transfected with NTCP or the control vector, were cultured as monolayers, trypsinized, and then assayed in suspension. Chenodeoxychilyl-(Ne-NBD)-lysine (CDCA-NBD) was used as a probe substrate. Cellular uptake of CDCa-NBD in transfected and control cells was determined by flow cytometry. Results. The suitability of the assays was determined by Z' factor, a simple statistical parameter for evaluating high throughput screening assays. The calculated Z' factors for NTCP was 0.93 , indicating the assay is suitable for measuring inhibition of NTCP-mediated transport. The application of this assay for screening drug interactions with NTCP was illustrated by investigating some well-known NTCP substrates and/or inhibitors including bosentan, BSP, CsA, CDCA, glibenclamide, rifampicin, rifamycin SV, taurocholic acid, and three statins, atorvastatin, rosuvastatin and pravastatin. CDCANBD uptake assays conducted in the presence of well-known compounds accurately reflected their inhibitory potency against NTCP. Compounds known to interact with NTCP inhibited NTCP-mediated CDCA-NBD cellular uptake relative to control cells. Among the three statins, atovastatin at $400 \mu \mathrm{M}$ showed $75 \%$, inhibition followed by rosuvastatin with $20 \%$ inhibition while pravastatin had no effect on CDCA-NBD uptake. Based on the initial screen, concentration-dependent inhibition was investigated to determine the $\mathrm{IC}_{50}$ values for selected compounds. The $\mathrm{IC}_{50}$ values were $49.4 \mu \mathrm{M}$ for CDCA, $131.5 \mu \mathrm{M}$ for rifampicin, $258.0 \mu \mathrm{M}$ for taurocholic acid, $639.3 \mu \mathrm{M}$ for E3S and $763.7 \mu \mathrm{M}$ for bosentan. Conclusion. The in vitro assays using flow cytometry demonstrated utility for identifying potential drug-drug interactions associated with human NTCP. 


\title{
P115. Impact of marker detection on in vitro in vivo extrapolation of CYPs induction. Enzymatic activity versus mRNA
}

\author{
Patrice Dehanne, Estelle Rapine and Olivier Barberan
}

\author{
Aureus Sciences, Paris, France
}

Induction is not generally considered to be a concern for safety; however, activity in the clinic can result in therapeutic failure such as breakthrough bleeding[i] or unwanted pregnancy[ii] due to lower systemic plasma concentration resulting from an increase in metabolic clearance. Despite the potential therapeutic consequences of induction, few progresses have been made in quantitative predictions of induction-mediated drug-drug interactions (DDIs) from in vitro data[iii],[iv]. However, it is of great importance to retrieve the right parameters from in vitro inductions assays $\left(\mathrm{EC}_{50}, \mathrm{Emax}, \mathrm{nh}\right)$. Moreover, detection markers like mRNA or enzyme activity performed to quantify inductions levels of compounds recently appear critical to identify clinically meaning-full inducers[v]. Until now detection markers were compared for classification of compounds only based on in vitro results. The aim of this study is to determine which detection markers should be used to performed in vitroin vivo extrapolation of CYPs induction based on prediction of drug-drug interactions involving clinically relevant inducers of CYPs.

[i] Donley TG, Smith RF, Roy B. Reduced oral contraceptive effectiveness with concurrent antibiotic use: a protocol for prescribing antibiotics to women of childbearing age. Compendium. 1990;11(6):392-6.

[ii] Sabers A. Pharmacokinetic interactions between contraceptives and antiepileptic drugs. Seizure. 2008;17(2):141-4.

[iii] Shou M, Hayashi M, Pan Y, Xu Y, Morrissey K, Xu L, Skiles GL. Modeling, prediction, and in vitro in vivo correlation of CYP3A4 induction. Drug Metab Dispos. 2008;36(11):2355-70.

[iv] Fahmi OA, Hurst S, Plowchalk D, Cook J, Guo F, Youdim K, Dickins M, Phipps A, Darekar A, Hyland R, Obach RS. Comparison of different algorithms for predicting clinical drug-drug interactions, based on the use of CYP3A4 in vitro data: predictions of compounds as precipitants of interaction. Drug Metab Dispos. 2009;37(8):1658-66.

[v] FahmiOA, Kish M, BoldtS, Obach RS. CytochromeP4503A4mRNA is a more reliable marker than CYP3A4 activity for detecting pregnane X receptor-activated induction of drug-metabolizing enzymes. Drug Metab Dispos.;38(9):1605-11.

\section{P116. Inactivation of CYP2C8 by gemfibrozil 1-O-B-glucuronide accumulation in human hepatocytes: Implications for clinical DDI predictions}

\author{
Andrea Whitcher-Johnstone and Tom S. Chan
}

Drug Metabolism and Pharmacokinetics, Boehringer Ingelheim Pharmaceuticals Inc., Ridgefield, CT

CYP2C8 inactivation by gemfibrozil glucuronide has been associated with clinical drug-drug interactions (DDIs) observed between gemfibrozil and cerivastatin. Since hepatocytes can be used to evaluate oxidative and conjugative metabolism, we investigated the possibility that hepatocytes, but not microsomes, could detect inactivation of CYP2C8 when incubated with gemfibrozil using amodiaquine as a specific CYP2C8 substrate. Using hepatocytes, the $\mathrm{K}_{\mathrm{I}}$ and the $\mathrm{k}_{\text {inact }}$ values determined for inactivation of CYP3A4 by gemfibrozil were $4.12 \mu \mathrm{M}$ and $0.04 \mathrm{~min}^{-1}$, respectively. Unexpectedly, the $\mathrm{K}_{\mathrm{I}}$ value was 5-fold lower than what has previously been reported for the inactivation of CYP2C8 by gemfibrozil glucuronide in human liver microsomes. We hypothesized that in hepatocytes, the rate of gemfibrozil formation would exceed its rate of efflux leading to the intracellular accumulation of gemfibrozil glucuronide and that the lower $\mathrm{K}_{\mathrm{I}}$ value would reflect the higher concentration of gemfibrozil glucuronide available to the enzyme. To investigate this hypothesis, the intracellular concentration of gemfibrozil glucuronide in suspended human hepatocytes was estimated using LC/MS/MS and microscopically determining the average hepatocyte volume. Surprisingly, the intracellular concentration of gemfibrozil glucuronide was 191-fold higher than the extracellular concentration. However, the fraction of CYP2C8 metabolizing cerivastatin (0.61) was not high enough to allow the resulting inactivation parameters and accumulation to improve the

DDI prediction observed between gemfibrozil and cerivastatin Consequently,these results suggest the involvement of another mechanism contributing to the observed DDIs for gemfibrozil and cerivastatin.

\section{P117. Abstract withdrawn}

\section{P118. Modulation of hepatic UGT function by IL-6 and TNFá and its relationship with the PXR, CAR, and AhR}

\author{
ఏin Zhou, Shawn Li and Feng Li
}

Alliance Pharma, Inc., Malvern, PA

(c) 2011 Informa Healthcare USA, Inc. 
Pro-inflammatory cytokines, IL-6 and TNF $\alpha$, are known to suppress multiple cytochrome P450s (CYPs). Their impact on the function of UDP-glucuronosyltransferases (UGTs) has not been fully investigated. Furthermore, the mechanism of UGTs modulation by IL- 6 and TNF $\alpha$ has not been explored although previous studies suggested that suppression of CYPs by pro-inflammatory cytokines may be via down-regulation of the nuclear receptors. In this study, we examined the effects of IL-6 and TNF $\alpha$ on the function of UGT1A1, UGT1A4, UGT1A9, and UGT2B7, using estradiol3-glucuronidation (UGT1A1), lamotrigine-N2-glucuronidation (UGT1A4), propofol-glucuronidation (UGT1A9), and zidovudine-glucuronidation (UGT2B7) as probe reactions. Hepatocytes in sandwich culture were treated with different concentrations of IL-6 or TNF $\alpha$ for $72 \mathrm{~h}$. At the end of the treatment, UGT activities were assessed with individual probe substrates. IL-6 and TNF $\alpha$ showed significant and concentration-dependent suppression on UGT2B7-dependent zidovudine glucuronidation. In a concentration range of $0.2-20 \mathrm{ng} / \mathrm{mL}$, IL- 6 decreased zidovudine glucuronidation by up to $60 \%$, and TNF $\alpha$ decreased zidovudine glucuronidation by up to $48 \%$. IL- 6 at $20 \mathrm{ng} / \mathrm{mL}$ also showed significant suppression on UGT1A9-dependent propofol glucuronidation (decreased by 40\%). For estradiol-3-glucuronidation and lamotrigine-N2-glucuronidation, no obvious modulation was observed. The considerable suppression of IL-6 and TNF $\alpha$ on zidovidine glucuronidation suggests the possibility that therapeutic proteins, which modulate IL-6 or TNF $\alpha$ function, may affect UGT2B7-mediated small molecule drug metabolism. We also evaluated the expression of PXR, CAR, and AhR in the hepatocytes after 72-h direct exposure to different concentrations of IL-6 and TNF $\alpha$. The results showed that IL-6 and TNF $\alpha$ modulated the expression of PXR, CAR, and AhR differently. TNF $\alpha$ exhibited small but significant suppression on AhR but had no effects on the expression of PXR and CAR. IL-6 (at higher concentrations), on the other hand, significantly decreased the expression of PXR and CAR, but had no obvious remarkable impact on the expression of AhR. These results suggest that the mechanisms of UGT-suppression by IL-6 and TNF $\alpha$ are different. Down-regulation of PXR and CAR is likely one of the mechanisms for UGT- suppression by high concentrations of IL-6, whereas AhR may be involved in TNF $\alpha$-mediated UGT-suppression.

\title{
P119. N-Glucuronidation of midazolam increases in the presence of a CYP3A inhibitor in both human hepatocytes and human volunteers
}

\author{
$\underline{\text { Kosea S. Frederick }}^{1}$, Jennie Schmenk ${ }^{2}$, Mary F. Paine ${ }^{3}$, Cathrine L. Denton ${ }^{4}$ and Michael Fisher ${ }^{2}$
}

${ }^{1}$ Drug Discovery Support, Boehringer-Ingelheim, Ridgefield, CT, ${ }^{2}$ Drug Discovery Support, Boehringer-Ingelheim Pharmaceuticals, Ridgefield, CT, ${ }^{3}$ Division of Pharmacotherapy and Experimental Therapeutics, UNC Eshelman School of Pharmacy, The University of North Carolina at Chapel Hill, Chapel Hill, NC, ${ }^{4}$ UNC Eshelman School of Pharmacy, The University of North Carolina at Chapel Hill, Chapel Hill, NC

Purpose. The short-acting sedative midazolam is one FDA-recommended "probe" victim drug substrate for CYP3A. In vitro evidence has shown that inhibition of CYP3A shifts the primary route of midazolam metabolism, 1'-hydroxylation, to an alternate pathway, $\mathrm{N}$-glucuronidation. Incomplete characterization of the latter route, mediated by UGT1A4, may result in mis-estimation of the magnitude of a potential drug-drug interaction (DDI). Based on these observations, the interaction between midazolam and the CYP3A inhibitor indinavir was evaluated in both human hepatocytes and human volunteers. Methods. Cryopreserved human hepatocyte lots were selected based on CYP3A and UGT1A4 activities, as measured by the formation kinetics of 1 '-hydroxymidazolam ( $1^{\prime}$-OHMDZ) and midazolam $N$-glucuronide (MDZNG), respectively. Indinavir was selected based on the $>100$-fold greater inhibitory potency against recombinant CYP3A4 compared to recombinant UGT1A4. Incubations consisted of hepatocytes $\left(0.5 \times 10^{6}\right)$ in serum-free William's E medium; midazolam ( $4 \mu \mathrm{M})$; and indinavir (1-200 $\mu \mathrm{M})$, the CYP3A inhibitor ketoconazole $(10 \mu \mathrm{M})$, or the UGT1A4 inhibitor hecogenin $(0.25-30 \mu \mathrm{M})$. After $2 \mathrm{~h}$, culture medium was collected and analyzed for midazolam, 1-OHMDZ,

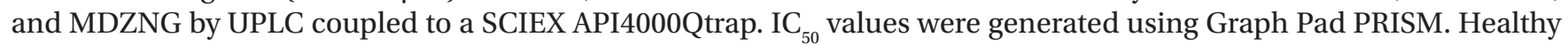
volunteers $(n=8)$ participated in a randomized, open-label, two-way crossover study. Each volunteer was administered water alone or with indinavir $(800 \mathrm{mg})$ the evening of day 1 and the morning of day 2 . Midazolam syrup $(2.5 \mathrm{mg})$ was administered $1 \mathrm{~h}$ after the second dose of water/water+indinavir. Water/water+indinavir was administered again 5 and $11 \mathrm{~h}$ post-midazolam administration. Urine was collected from $0-24 \mathrm{~h}$ post-midazolam administration and analyzed for midazolam, 1'-OHMDZ, 1'-O-MDZ-glucuronide (MDZOG), and MDZNG by UPLC-LC/MS/MS. Results. The IC ${ }_{50}$ of indinavir for CYP3A and UGT1A4 in human hepatocytes were 3 and $>200 \mu \mathrm{M}$, respectively; the IC $_{50}$ for hecogenin was $>30$ and $0.03 \mu \mathrm{M}$ for, respectively. Relative to vehicle ( $<0.5 \%$ methanol), $1^{\prime}$-OHMDZ formation in hepatocytes decreased (by $84 \%$ ), while MDZNG formation increased (by 200\%), in the presence of indinavir $(10 \mu \mathrm{M})$. Ketoconazole $(10 \mu \mathrm{M})$ decreased 1'-OHMDZ formation by $98 \%$; hecogenin $(10 \mu \mathrm{M})$ decreased MDZNG formation by $80 \%$. Relative to water, the cumulative amounts of 1'-OHMDZ and MDZOG excreted into the urine of human subjects decreased (by 98 and $35 \%$, respectively), while those of midazolam and MDZNG increased (by $100 \%$ and $200 \%$, respectively), in the presence of indinavir. Conclusions. Inhibition of CYP3A by indinavir shifted the metabolism of midazolam to the alternate 
UGT1A4-mediated pathway in humans both in vitro and in vivo. $N$-Glucuronidation may represent a significant fraction of midazolam clearance in the presence of a CYP3A inhibitor, which could lead to underestimation of the magnitude of a DDI if using midazolam clearance or oral clearance as an index of CYP3A activity.

\title{
P120. Predicting transporters mediated drug interactions based on the mechanistic static approach. application to statins
}

\author{
Olivier Barberan $^{1}$, Estelle Rapine ${ }^{1}$, Amélie Moreau ${ }^{2}$ and Georges Daviolante ${ }^{2}$ \\ ${ }^{1}$ Aureus Sciences, Paris, France, ${ }^{2}$ Technologie Servier, Orléans, France
}

Until now, and in spite of the advances reached on the identification and functional characterization of transporters[1], transporter mediated DDIs and in particular those involving transporters and cytochromes within the same organ, remain very difficult to predict. Existing models could be categorized in mechanistic static (MSM) and the mechanistic dynamic model $(\mathrm{PB} / \mathrm{PK})$ and were used for the prediction of transporter mediated DDIs with advantages and limitations. Even if the mechanistic dynamic models $(\mathrm{PB} / \mathrm{PK})$ remain the gold standard for predictions of DDIs for complex situations, mechanistic static models appear the most adequate to predict DDIs very early in the development phase and with a large amount of compounds on the market[2]. MSM approach remains poorly used for transporters in the literature (Matsushima et al.[3]) and doesn't take into account interactions between transporters and enzymes mainly cytochromes P450 at target organs where both are expressed. Consequently, the aim of this study is to predict transporters mediated drug drug interactions involving Statins drugs as victims. Statins mainly eliminated through transport process (i.e. Rosuvastatin) are included but also those partially metabolized and actively excreted unchanged (i.e. Pravastatin). Concerning Statins cleared mainly through active excretion the model described by Endres et al.[4]was applied whereas for those cleared through active excretion and metabolism the "Well stirred model" modified by Sirianni et al.[5] and more recently by Endres et al.[6]was investigated.

[1] Shugarts S, Benet LZ. The role of transporters in the pharmacokinetics of orally administered drugs. Pharmaceutical Research (2009); 26(9): 2039-2054

[2] Boulenc X. Schmider W. Barberan O. In Vitro/In Vivo Correlation for Drug-Drug Interactions H. G. Vogel, J. Maas, A. Gebauer (eds.), Drug Discovery and Evaluation: Methods in Clinical Pharmacology Springer-Verlag Berlin Heidelberg (2010) Chapter 10

[3] Kusuhara H, Sugiyama Y. Drug-Drug Interactions involving the membrane transport process. Drugs Pharmaceutical Sciences (2001); 116: 123-188.

[4] Endres J. Christopher, Peng Hsiao, Francisco S. Chung, Jashvant D. Unadkat. (2006) The role of transporters in drug interactions. Eur. J. Pharmaceutical Sciences. 27, 501-517.

[5] Sirianni, G. L.; Pang, K. S. (1997) Organ clearance concepts: new perspectives on old principles. J. Pharmacokinet. Biopharm.,25 (4), 449-70.

[6]Endres C.J., Endres M.G., Unadkat J.D., (2009) Interplay of Drug Metabolism and Transport: A Real Phenomenon or an Artifact of the Site of Measurement? Mol. Pharm. 6(8), 1756-1765

\section{P121. Prediction of clearance changes of drugs that are substrates of major cytochrome P450 enzymes caused by drug-drug interaction or single nucleotide polymorphism}

\author{
$\underline{\text { Akihiro Hisaka }^{1}}$, Yoshiyuki Ohno ${ }^{2}$, Ryo Yamaguchi ${ }^{2}$, Masaki Ueno ${ }^{2}$, Kousei Ito ${ }^{2}$ and Hiroshi Suzuki² \\ ${ }^{1}$ Pharmacology and Pharmacokinetics, The University of Tokyo Hospital, Tokyo, Japan, ${ }^{2}$ Department of Pharmacy, The \\ University of Tokyo Hospital, Tokyo, Japan
}

[Background] Drug-drug interactions (DDIs) caused by inhibition of drug metabolizing enzymes are very important in clinical situations. Regarding the DDIs caused by inhibition of cytochrome P450 (CYP) 3A4, we previously established the CR-IR method to quantitatively and comprehensively predict magnitude of DDIs after oral administration based on the ratio of the contribution of CYP3A4 to clearance after oral absorption $\left(\mathrm{CR}_{\mathrm{CYP3A}}\right)$ and the time-averaged apparent inhibition ratio of CYP3A4 $\left(\mathrm{IR}_{\text {СХР3A4 }}{ }^{1,2}\right.$. The CR-IR method has been extended to predict DDIs which are associated with induction of CYP3A4 $4^{3}$. However, prediction of the magnitude of DDIs caused by inhibition of other important CYP enzymes, such as CYP1A2, CYP2C9, CYP2C19 and CYP2D6, remained to be established. [Objective] The objective of the present study is to examine whether the CR-IR method is applicable to these four CYP enzymes. In addition, we also 
tried to clarify whether it is applicable to single nucleotide polymorphisms (SNPs) in CYP enzymes in the same manner. [Data sources] The analysis was based on 382 DDI studies reported in 246 publishedarticles over a period 1975 2009. In addition, 12 articles published over a period 1995 - 2008, in which increase in the AUC of substrate drugs in SNPs carriers of CYP2D6*10 is reported, were analyzed. [Methods] The increase in the AUC of substrate drugs by co-administration of an inhibitor drug was calculated by 1 / (1-CR-IR). By using the reduction ratio (RR), which represents the ratio for the reduction in metabolizing activity of SNPs type enzyme to that of the wild type enzyme, the increase in the AUC of substrate drugs in SNPs carriers compared to that in the wild type subjects is given by 1 / (1-CR-RR). Regarding the DDIs, we determined CR values of 22, 11, 4 and 6 substrate drugs and IR values of 18, 11, 7 and 3 inhibitor drugs for CYP2D6, CYP2C9, CYP1A2 and CYP2C19, respectively. Besides, CR and IR values of 24 substrate and 5 inhibitor drugs for CYP3A4 were added to those reported previously ${ }^{1}$ ). [Results] The increases in the AUC of substrate drugs of various CYP enzymes associated with inhibition or SNPs were successfully predicted within 50 200\% of the observed values for 375 studies (98.2\%). In addition, from RR obtained in the analysis, it was found that the enzyme activity of CYP2D6*10 homozygotes, which is observed very frequently in Asians, accounts for $23 \%$ compared with that of the wild type CYP2D6. [Conclusion] The CR-IR method to predict increase in the AUC of substrate drugs caused by DDIs and those observed in SNPs carriers has wide applicability. It may be useful to raise alerts appropriately to enable adjusting dose or replacing regimes in DDI situations of various CYP enzymes as has been demonstrated for CYP3A44). Reference: ${ }^{1}$ ) Ohno et al., Clin Pharmacokinet. 2007; 46: 681-96. ${ }^{2}$ ) Hisaka et al., Pharmacol. Ther.2010; 125: 230-48. ${ }^{3}$ ) Ohno et al., Clin Pharmacokinet. 2008; 47: 669-80. $\left.{ }^{4}\right)$ Hisaka et al., Clin Pharmacokinet, 2009: 48: 653-66.

\title{
P122. The usefulness of OATP inhibitor as a tool of in vitro drug-drug interaction study using human hepatocyte
}

\author{
Shinsuke Aoyama, Miki Fujishima, Takami Sarashina, Teruhiko Yanagisawa, Kiyoe Morita, Yasuhisa \\ Adachi and Shin-ichi Ninomiya
}

Sekisui Medical Co., Ltd., Ibaraki, Japan

To date, drug-drug interactions related to hepatic clearance have mainly been reported as drug metabolising enzyme mediated interactions, primarily interactions involving CYP isoforms. Recent progress in drug transporter studies has shown a number of drug-drug interactions mediated by various drug transporters. Organic Anion Transporting Polypeptide (OATP) is predominantly expressed in the liver and shows a broad substrate specificity against various anionic drugs, e.g. statins. Inhibition of OATP mediated uptake causes clinically significant drug-drug interaction (DDI) with OATP substrates. A concept paper on transporter studies in new drug development, given from International Transporter Consortium, indicates that not only metabolic enzymes but also transporters are very important factors to evaluate the hepatic clearance. ${ }^{1}$ Human hepatocyte is a useful tool for evaluation of DDI mediated by both metabolic enzyme and transporter isoforms. In order to separately evaluate DDI mediated by transporters using hepatocyte, we have to use a specific inhibitor against transporters, which does not inhibit CYP enzymes. However, it is not well known whether OATP inhibitors interact with CYP isoforms. We evaluated the effect of an OATP inhibitor, rifampicin, on the CYP mediated metabolism (CYP1A2, CYP2C9, CYP2D6 and CYP3A4). The inhibitory potentials of rifampicin on CYP1A2, CYP2C9, CYP2D6 and CYP3A4 mediated metabolic activities were evaluated by following indices; phenacetin O-deethylation, tolbutamide hydroxylation, dextromethorphan O-demethylation and midazolam l'-hydroxylation, respectively. Rifampicin at $10 \mu \mathrm{M}$ showed no inhibition on each CYP enzyme activities, although rifampicin at $10 \mu \mathrm{M}$ inhibited OATP1B1 and OATP1B3 mediated uptakes significantly. The IC50 values on OATP1B1 and OATP1B3 mediated $\left[{ }^{3} \mathrm{H}\right]-\mathrm{E}_{2} 17 \beta \mathrm{G}$ uptakes using each transporter expressing cells were 0.243 and $0.388 \mu \mathrm{M}$, respectively, suggesting that rifampicin at $10 \mu \mathrm{M}$ inhibits OATP mediated uptakes specifically. The results of DDI study mediated by OATP isoforms using human hepatocyte will be discussed in this presentation.

1) Kathleen M. Giacomini et al., Membrane transporters in drug development, Nature Reviews, 2010 (9), 215-236.

\section{P123. Target delivery in traditional Chinese medicine}

\section{Ruizhi Zhao}

Second Affiliated Clinical college, Guangzhou university of triditional Chinese medicine, Guangzhou, China

Most drugs have a widespread distribution in vivo, and effects beyond the disease site are needs to extrude. To herbs, it contains many components with different structure, always have a lot of effects, how to get the desired effect at the 
desired site is a difficult problem. In ancient China, people usually get this goal by co-administration of other drugs to get the synergy and attenuation effect, and in thousands of years, the experience was summarized as meridian guide theory. About 24 drugs were regarded as the meridian guide drug. Among them, vinegar baked Bupleuri Radix, borneol, platycodi, cassia bark, meridian guide drug of liver, heart, lung, kidney respectively were studied using pharmacological and pharmacokinetic method. Pharmacokinetics study showed that vinegar baked bupleuri radix increased the distribution of resveratrol, rhein and its metabolite, oxymatrine and its metabolite, gentiopicroside in liver, and meanwhile decreased their distribution in other tissues. Platycodi increased the distribution of levofloxacin, florfenicol, difloxacin and roxithromycin in lung, and decreased their distribution in other tissues. Borneol increased the distribution of clindamycin, sodium ferulate, carbamazepine, tetramethylpyrazine, methotrexat, cidomycin, rifampici, puerarin, gastrodin, ginsenoside Rg1, Re, notoginseng R1 in brain. Pharmacological studied showed that platycodi enhanced the curative effect of flos lonicerae and forsythia suspense on treating chronic obstruction pulmonary. Borneol enhanced the curative effect of sodium ferulate, sodium ferulate, scutellarin in brain diseases. Cassia bark enhanced the curative effect of anemarrhenae rhizome and cortex phellodendri in treating hyperplasia of prostate gland. Mechanism study showed that almost all the meridian guide drug inhibited P-gp activity, vinegar baked bupleuri radix increased the permeability and changed the membrane constituents of BRL cell line, borneol loosen the tight junction of blood- brain barrier. Therefore co-administered with meridian guide drug is a simple and effective way for target delivery. This research was financially supported by the found of National Science Foundation of China (Grant No. 30672668, 81073063)

\title{
P124. The rate of hydrolysis of AZD3043 is dependent on the butyrylcholinesterase genotype and independent of other butyrylcholinesterase substrates
}

\author{
Łenny Aasa ${ }^{1}$, Antti Kautiainen ${ }^{1}$, Stéphane Barassin ${ }^{1}$, Anne-Marie Sims ${ }^{2}$ and Clive Brealey ${ }^{3}$ \\ ${ }^{1}$ iMed DMPK, CNSP AstraZeneca R\&D Södertälje, Södertälje, Sweden, ${ }^{2}$ PHC-Biomarker, AstraZeneca R\&D, Alderly Park, \\ UK, Alderly Park, United Kingdom, ${ }^{3}$ iMed DMPK, R\&I AstraZeneca R\&D, Mölndal, Sweden, Mölndal, Sweden
}

AZD3043 is a positive allosteric GABA receptor modulator exhibiting anesthetic and sedative effects and is intended for intravenous administration. AZD3043, an ester, is rapidly hydrolyzed by butyrylcholinesterase (BChE, E.C 3.1.1.8), present e.g. in human plasma and liver microsomes, to its pharmacologically inactive carboxylic acid. The hydrolysis is crucial for the rapid time of onset and/or offset of sedation/anesthesia in patients treated with AZD3043. In addition, polymorphism in the gene encoding $\mathrm{BChE}$ is known to affect the enzyme function. In order to investigate the potential for drug-drug interactions (DDI) of AZD3043 with compounds that are substrates for BChE, $\left[{ }^{14} \mathrm{C}\right]$ AZD3043 was incubated at different time points with remifentanil or succinylcholine, at clinically relevant concentrations, in human liver microsomes and human plasma. The half life $\left(\mathrm{t}_{1 / 2}\right)$ and clearance (CL) for AZD3043 were shown to be unaffected by the co-administration of BChE drugs. It was concluded that there is a low risk for PK interactions in vivo, when administering AZD3043 with other drugs metabolized by BChE, such as remifentanil and succinylcholine. To study the influence of plasma level of BChE on $\mathrm{t}_{1 / 2}$ and $\mathrm{CL},\left[{ }^{14} \mathrm{C}\right] \mathrm{AZD} 3043$ was incubated at clinically relevant concentrations at different time points with human plasma from 18 individuals with different BChE phenotypes/BCHE genotypes. The results showed that there was a strong correlation $\left(\mathrm{r}^{2}=0.83\right)$ between plasma CL, as well as the $\mathrm{t}_{1 / 2}$, and different levels of BChE in human plasma. These in vitro studies demonstrate an appropriate way of supporting the design of in vivo studies. The way forward could be to investigate if there are in vitro-in vivo correlations, with respect to both DDI and activity measurements in subjects with different BChE phenotypes/BCHE genotypes.

\section{P125. Time-dependent inactivation and estimation of CYP3A4 clinical pharmacokinetic drug-drug interactions using plated hepatocytes}

\author{
Daniel R. Albaugh, Cody L. Fullenwider, David P. Joseph, J. Matthew Hutzler and Michael B. Fisher
}

\section{Drug Discovery DMPK, Boehringer-Ingelheim Pharmaceuticals, Inc., Ridgefield, CT}

Time-dependent inactivation (TDI) of cytochrome P450 enzymes is a major concern due to the potential for clinically significant drug-drug interactions (DDI). Therefore, from a drug discovery perspective it is critical to not just identify, but also estimate the magnitude of any potential interaction for new chemical entities prior to preclinical development. Traditionally, kinetic parameters of enzyme inactivation $\left(\mathrm{k}_{\text {inact }}\right.$ and $\left.\mathrm{K}_{\mathrm{I}}\right)$ are measured in a two-staged assay with multiple concentrations and time points using human liver microsomes (HLMs). However, precipitants of TDI are not always products of oxidative metabolism, and non-inactivating enzymatic pathways may also be operable. Therefore, it may be more relevant to assess 
TDI in a system containing the full complement of drug-metabolizing enzymes. The current studies investigate fresh plated primary hepatocytes, cryopreserved plated hepatocytes, and HepaRG plated whole cell systems for their ability to generate in vitro inactivation parameters comparable to those generated using HLMs. This initial assessment was achieved by using a subset of known CYP3A4 inactivators with various potencies: clarithromycin, mibefradil, troleandomycin (TAO), verapamil, and fluoxetine. The $\mathrm{k}_{\text {inact }}$ and $\mathrm{K}_{\mathrm{I}}$ values obtained in various plated whole cell systems were both compared to parameters generated in HLMs, and used to estimate subsequent clinical pharmacokinetic DDIs.

\begin{tabular}{|c|c|c|c|c|c|c|c|c|c|c|c|c|}
\hline \multirow{2}{*}{ Compound } & \multicolumn{3}{|c|}{ Fresh Plated Hepatocytes } & \multicolumn{3}{|c|}{$\begin{array}{c}\text { Cryopreserved Plated } \\
\text { Hepatocytes }\end{array}$} & \multicolumn{3}{|c|}{ Hepa RG Plated } & \multicolumn{3}{|c|}{ HLM $(0.1 \mathrm{mg} / \mathrm{mL})$} \\
\hline & $\mathrm{K}_{\mathrm{I}}$ & $\mathrm{k}_{\text {inact }}$ & DDI $_{\text {pred }}$ & $\mathrm{K}_{\mathrm{I}}$ & $\mathrm{k}_{\text {inact }}$ & DDI $_{\text {pred }}$ & $\mathrm{K}_{\mathrm{I}}$ & $\mathrm{k}_{\text {inact }}$ & DDI $_{\text {pred }}$ & $\mathrm{K}_{\mathrm{I}}$ & $\mathrm{k}_{\text {inact }}$ & DDI $_{\text {pred }}$ \\
\hline Clarithromycin & 12.6 & 0.06 & 11 & 8.1 & 0.09 & 16 & 5.4 & 0.08 & 17 & 17.7 & 0.05 & 9 \\
\hline Mibefradil & 0.6 & 0.10 & 5 & 0.2 & 0.28 & 16 & 1.1 & 0.91 & 13 & 1.1 & 0.70 & 11 \\
\hline TAO & 0.9 & 0.10 & 18 & 0.6 & 0.08 & 18 & 2.2 & 0.17 & 16 & 1.8 & 0.31 & 20 \\
\hline Verapamil & 12.6 & 0.14 & 3 & 1.9 & 0.14 & 6 & 5.5 & 0.12 & 3 & 8.9 & 0.10 & 3 \\
\hline Fluoxetine & \multicolumn{3}{|c|}{ Not Tested } & 17.1 & 0.03 & $<2$ & 35.7 & 0.01 & $<2$ & \multicolumn{3}{|c|}{ Not Detected } \\
\hline
\end{tabular}

$\mathrm{K}_{\mathrm{I}}(\mu \mathrm{M}) ; \mathrm{k}_{\text {inact }}\left(\mathrm{min}^{-1}\right) ; \mathrm{DDI}$ pred $\left(\right.$ predicted interaction $=\mathrm{AUC}_{\text {inhibited }} / \mathrm{AUC}$ of victim drug midazolam) using methods described in

Venkatakrishnan and Obach (2007) Curr Drug Metab 8(5), 449-62; NI = no interaction

Results from these studies suggest that a plated whole cell approach allows for a practical cell-based system for comparable estimation of in vitro inactivation kinetic parameters when compared to the gold standard human liver microsomal system for CYP3A4. In particular, the ability to conduct inactivation experiments for longer periods ( $>30 \mathrm{~min}$ ), allows for more thorough assessment of weak inactivators such as fluoxetine. Next steps will include assessment of plated systems for the ability to capture and model complex interactions such as gemfibrozil-CYP2C8.

\title{
P126. Time-dependent inactivation of cytochrome P450 3A by imatinib: implications for its clinical pharmacokinetics and drug interactions
}

\author{
Yang Xu, Yuping Chen, Yihong Zhou, Shuguang Ma, Mike Hayashi, Yvonne Pan, Gary L. Skiles and \\ Magang Shou
}

Pharmacokinetics and Drug Metabolism, Amgen Inc., Thousand Oaks, CA

It has been reported that oral clearance (CL/F) of imatinib decreased by $\sim 26 \%$ at steady-state compared to that at day 1 in the patients with gastrointestinal stromal tumor or chronic myeloid leukemia. The mechanism underlying this time-dependent change on oral clearance remains unclear. In the present study, we report that imatinib inhibited CYP3A, the enzyme that is partially responsible for the drug's own metabolism, in an NADPH, preincubation time, and concentration-dependent manner. $k_{\text {inact }}$ (maximal rate of enzyme inactivation)and apparent $K_{\mathrm{I}}$ (inhibitor concentration achieving half themaximal rate of inactivation) weredetermined using both human liver microsomes (HLM) and pooled, cryopreserved human hepatocytes. Incubation of fresh human hepatocytes with imatinib (48 hours) resulted in a significant loss of CYP3A activity, but had no impact on CYP3A4 mRNA. The apparent $k_{\text {inact }}$ and $K_{\mathrm{I}}$ values derived from human hepatocytes were used to predict clinical DDIs associated with CYP3A inhibition by imatinib using a populationbased simulator. The prediction correlated well with the reported in vivo DDI data. Moreover, two cyanide conjugates of imatinib formed in HLM supplemented with $\mathrm{NaCN}$ were structurally characterized by LC-MS/MS to elucidate the mechanism of time-dependent CYP3A inhibition. These findings provide a possible explanation for the time-dependent decrease in oral clearance of imatinib and suggest that the reported lack of effect on the steady-state pharmacokinetics of imatinib by acute ritonavir treatment is also due to inactivation of CYP3A by imatinib. In addition, our predictions indicate that imatinib may contribute to the clinical DDIs with CYP3A substrates (e.g., simvastatin) mainly through the mechanism of time-dependent CYP3A inhibition rather than the reversible inhibition as previously reported.

\section{P127. Using the Cynomolgus monkey as a model to assess cytochrome P450 3A (CYP3A) induction potential prior to first-in man: A case study}

\author{
Aberra Fura, Sean Kim, Mary Obermeier, Zheng Yang and David Rodrigues
}

Department of Metabolism and Pharmacokinetics, Bristol-Myers Squibb, Princeton, NJ

BMS-A is a proprietary molecule that induces CYP3A in human hepatocytes ( $>20$-fold increase in CYP3A mRNA; and $\sim 3$-fold increase in 1-hydroxymidazolam activity compared to vehicle control at $5 \mu \mathrm{M})$ and is highly bound $(>99 \%)$ 
to human and monkey serum proteins. To further gauge the drug interaction risk in humans, BMS-A was added to cynomolgus monkey primary hepatocytes. Similar to humans, BMS-A induced CYP3A mRNA expression in a concentration-dependent manner ( $>10$-fold of the control at $10 \mathrm{mM} ;>35 \%$ of rifampicin response at $10 \mathrm{mM}$ ). However, the CYP3A activity, measured as the formation of 1-hydroxymidazolam was decreased $(\sim 40 \%$ of the control at 10 $\mu M)$. Such a reduction was also concentration-dependent. Since no significant reversible or metabolism-dependent inhibition of BMS-A was observed in monkey liver microsomes $\left(\mathrm{IC}_{50}>40 \mathrm{mM}\right)$, the apparent decrease in the formation of 1-hydroxymidazolam was postulated to be due to either the inhibition of the heme synthesis or the induction of the enzymes (such as UGTs) metabolizing 1-OH midazolam. In order to place these in vitro data in context, the oral pharmacokinetics of midazolam was examined in monkeys following daily oral administration of BMS-A at therapeutically relevant doses ( 1 and $5 \mathrm{mg} / \mathrm{kg}$ ) over a six-day period. At these doses, the corresponding steady-state Cmax of BMS-A was 4 and $19 \mathrm{mM}$, respectively. In this study, the oral clearance of midazolam was increased by 29 to $45 \%$ and 57 to $77 \%$ after 2 - and 6-day dosing of BMS-A, consistent with the induction of CYP3A mRNA in monkey hepatocytes. However, a decrease in the plasma concentration of 1-OH midazolam was also observed in vivo (60 to $70 \%$ ) despite an increase in the clearance of the parent compound. This result suggests that the apparent decrease in 1-OH midazolam concentration in hepatocytes may not be due to the inhibition of heme synthesis but rather due to the induction of 1-OH midazolam clearance. Therefore, induction assessment in primary hepatocytes, employing 1-hydroxymidazolam, requires careful interpretation. Furthermore, the in vivo data showed that high plasma protein binding does not necessarily mitigate the risk of induction as predicted from the unbound fraction. Collectively, these results demonstrate the importance of leveraging both in vitro and in vivo preclinical data when assessing CYP3A induction risk prior to first in man.

\title{
P128. What kind of assumptions should we use to improve the predictability of drug-drug interactions involving organic anion transporting polypeptide (OATP) substrates?
}

\author{
Kazuya Maeda, Kenta Yoshida and Yuichi Sugiyama
}

\begin{abstract}
Department of Molecular Pharmacokinetics, Graduate School of Pharmaceutical Sciences, The university of Tokyo, Tokyo, Japan
\end{abstract}

The importance of transporters in the pharmacokinetics of drugs has been well recognized by several clinical observations demonstrating the effects of genetic polymorphisms of certain transporters or drug-drug interactions (DDIs). One of the main topics in a recently-published "FDA whitepaper on drug transporters" (1) is how to quantitatively assess the risk of transporter-mediated DDIs and judge whether clinical DDI studies will be needed for the new drug application. In the early stage of drug development with the limited in vitro information, simple static model using $1+\mathrm{I} / \mathrm{Ki}$ (I: inhibitor concentration, Ki: inhibition constant) value is useful as a first step to estimate the extent of DDIs. Though several case studies demonstrating the prediction of DDIs from in vitro experiments have been reported, few studies have shown the effects of several assumptions on the predictability of multiple DDI cases involving several OATP substrates by using a simple static model. Thus, the purpose of this study is to understand which assumptions we should use for the accurate prediction of the AUC ratios (AUCRs) of OATP substrates in previously reported DDI cases by in vitro inhibition potencies with minimal false-negative predictions. We first performed systematic review of previous literature to dig out the reported DDI cases involving 12 OATP substrates (atorvastatin, cerivastatin, fluvastatin, pitavastatin, pravastatin, rosuvastatin, nateglinide, repaglinide, glibenclamide, glimepiride, irbesartan, and telmisartan) and picked up 54 DDI cases for further analyses. Pharmacokinetic parameters and information about transporters/metabolic enzymes for both substrate drugs and coadministered inhibitors were collected from the previous reports. In some cases, we performed in vitro inhibition experiments for certain transporters and metabolic enzymes if the inhibition potencies of some drugs were not published. Based on these information, we calculated the changes in intestinal availabilities (FaFg), hepatic or renal clearances, and thus AUCRs of OATP substrates following the clearance concept. In 49 out of the 54 cases, the AUCRs of OATP substrates were well predicted within 3-fold difference under original assumptions. Comparing our prediction results to those with other sets of assumptions, we indicated that false-negative predictions are best avoided when assuming that (i) unbound inhibitor concentrations at the inlet to the liver (uptake transporters/metabolic enzymes) are estimated by the method of Ito et al. (2), (ii) FaFg values become 1 when drug interaction number (DIN) (3) is above the thereshold value, and (iii) inhibition of both hepatic uptake and efflux/metabolic processes directly affects the hepatic clearances of OATP substrates. Therefore, observed AUCRs in clinical TP-DDIs can be predicted even with simple static model and in vitro inhibition potencies based on the clearance concept. [reference] (1) International Transporter Consortium, Nat Rev Drug Discov, 9, 215-36 (2010), (2) Ito K et al., Pharmacol Rev, 50(3), 387-412 (1998), (3) Tachibana T et al., Xenobiotica, 39(6), 430-43 (2009) 


\title{
P129. assessing modeling and simulation tools and methods for predicting induction-based drug-drug interactions: A collaborative effort between academic, government regulatory, and pharmaceutical scientists from the IQ consortium (IQC)
}

\author{
$\underline{\text { Heidi Einolf }}^{1}$, Liangfu Chen ${ }^{2}$, Odette A. Fahmi ${ }^{3}$, Christopher R. Gibson ${ }^{4}$, R. Scott Obach ${ }^{5}$, Mohamad \\ Shebley $^{6}$, Michael Sinz ${ }^{7}$, Jose Silva ${ }^{8}$, Jashvant D. Unadkat ${ }^{9}$, Lei Zhang ${ }^{10}$ and Ping Zhao ${ }^{11}$
}

\begin{abstract}
${ }^{1}$ Drug Metabolism and Pharmacokinetics, Novartis Institutes for BioMedical Research Inc., East Hanover, NJ, ${ }^{2}$ Drug Metabolism and Pharmacokinetics, GlaxoSmithKline, King of Prussia, PA, ${ }^{3}$ Pharmacokinetics and Drug Metabolism, Pfizer, Groton, CT, ${ }^{4}$ Drug Metabolism, Merck Research Laboratories, West Point, PA, ${ }^{5}$ Pdm, Pfizer Global Research \& Development, Groton, CT, ${ }^{6}$ Abbott Laboratories, Abbott Park, IL, ${ }^{7}$ Metabolism and Pharmacokinetics, Bristol-Myers Squibb Co, Wallingford, CT, ${ }^{8}$ Drug Metabolism Pharmacokinetics, Johnson \& Johnson Pharmaceutical Research and Development, LLC., Raritan, NJ, ${ }^{9}$ Pharmaceutics, University of Washington, Seattle, WA, ${ }^{10}$ Office of Clinical Pharmacology, U.S. Food and Drug Administration, Silver Spring, MD, ${ }^{11}$ Office of Clinical Pharmacology, CDER, FDA, Silver Spring, $M D$
\end{abstract}

Drug-drug interactions (DDI) that arise by one drug causing an increase in activity of drug-metabolizing enzymes and/ or transporters can result in decreased efficacy and even toxicity of a second drug. The most common underlying biochemical mechanism for this phenomenon is the activation of the pregnane $\mathrm{X}$ receptor (PXR) resulting in increased transcription of several drug metabolizing enzymes and transporters, in particular cytochrome P450 3A4 (CYP3A4). An ability to prospectively predict clinical DDI caused by induction of CYP3A4 is highly sought to aid in the research and development of new drugs. In this collaborative study, the objective was to explore the performance of various DDI prediction models across a set of drugs for which clinical DDI data were available in the scientific literature of their interaction with selected CYP3A4 cleared drugs (e.g. midazolam). Input used in the predictions were derived from in vitro induction studies in cultured human hepatocytes $\left(\mathrm{E}_{\max }\right.$ and $\left.\mathrm{EC}_{50}\right)$, pharmacokinetic parameters of the inducer and probe substrate, and specific aspects of the clinical study design. The methods were judged based on two criteria: (a) overall accuracy in predicting the average magnitude of the interaction, and (b) the ability to categorize drugs into those which cause a $20 \%$ or greater decline in exposure to a CYP3A4 cleared drug vs. those that do not. The following methods and approaches were tested: the correlation between DDI magnitude and (1) $\mathrm{C}_{\max } / \mathrm{EC}_{50}$ or (2) the relative induction score (RIS), (3) the net-effect model ("static" model), and (4) physiologically-based pharmacokinetic modeling ("dynamic" model) using the SimCYP" program. The findings suggest that several of these methods offer reliable predictions of DDI. Average fold-error for the methods were in the range of 2 -fold and was influenced by the type of inducer plasma concentration values used in the methods (e.g. free vs. total; systemic vs. estimated portal). The fidelity of the methods was high with only a couple of mispredictions based on the cutoff of $20 \%$ reduction in exposure. The practical pros and cons of the various approaches will be discussed.

\section{P130. Acrylamide induction of CYP2E1 in the rabbit liver}

\author{
Mine Nuyan Akkeçi, Sevki Arslan, Çigdem Kalin and Emel Arinç
}

Biological Sciences, Middle East Technical University, Ankara, Turkey

Acrylamide is a neurotoxin, reproductive toxin and carcinogen in animals and a potential food carcinogen in humans. Presence of high concentration of acrylamide in some food products including processed potato products, bread, cereals, cookies, and snacks has triggered worldwide concern on acrylamide toxicity. Acrylamide is formed during starch food processing at high temperatures by the Mailard reaction utilizing reducing sugars and amino acid, asparagine. The epoxidation of acrylamide to its epoxide glycidamide by CYP2E1 in the acrylamide metabolism is mostly attributed to be responsible for carcinogenicity of acrylamide. However, the effect of acrylamide on cytochrome P450 isozymes have not been investigated yet. The present study aimed to investigate in vivo effects of acrylamide on CYP2E1 protein levels and associated enzyme activities in rabbit liver. Administration of acrylamide ( $100 \mathrm{mg} / \mathrm{kg}$ s.c., on day 1,5 and 8$)$ to New Zealand male rabbits was found to elevate hepatic microsomal CYP2E1 protein levels, significantly, as determined by Western Blot analysis, by 2 -fold. In addition, CYP2E1-dependent p-nitrophenol hydroxylase, NDMA-N-demethylase and aniline 4-hydroxylase activities were significantly increased approximately 1.80 to 3.0 fold in acrylamide-treated rabbit liver compared to controls. Among the liver damage marker enzymes, no change was observed in alanine amino transferase (ALT) and aspartate amino transferase (AST) enzyme activities, whereas acrylamide treatment led to a significant increase in sorbitol dehydrogenase (SDH) activities in both blood serum and liver (1.68- and 1.27-fold, respectively) with respect to controls. The results of the present 
study demonstrated for the first time that acrylamide treatments induced CYP2E1 protein level and associated enzyme activities in rabbit liver. In conclusion, it has been suggested that the induction of CYP2E1 enzyme due to acrylamide exposure may stimulate not only the formation of epoxide glycidamide, but the activation of other xenobiotics metabolized by CYP2E1 such as nitrosamines, benzene, and pyridine, leading to further increase in mutagenicity and carcinogenicity of these chemicals. Acknowledgment: Partly supported by Turkish Academy of Sciences

\author{
References \\ 1 Arinç, E., Adali, O., and Gençler-Özkan, A.M. (2000) Archives of Toxicology, 74 (6), 329-334. \\ 2 Arinç, E., Arslan, S., Bozcaarmutlu, A. and Adali, O. (2007),Food and Chemical Toxicology,45(1),105-118 \\ 3 Arinç, E., Adali, O., Iscan, M. and Güray, T. (1991) Archives of Toxicology, 65, 186-190 \\ 4 Arinç, E. (2010), Journal of Molecular Catalysis B: Enzymatic, 64 (3-4), 120-122, 2010
}

\title{
P131. Abstract witdrawn
}

\section{P132. CYP1A and CYP3A induction in cryopreserved Cynomolgus monkey hepatocytes pooled from multiple donors}

\author{
$\underline{\text { Utkarsh Doshi }}{ }^{1}$, Qian Yang ${ }^{1}$, Nicole $\mathrm{Li}^{1}$, Albert $\mathrm{Li}^{1}$ and JeanClare Seagrave ${ }^{2}$ \\ ${ }^{1}$ In Vitro ADMET Laboratories LLC, Columbia, MD, ${ }^{2}$ Lovelace Respiratory Research Institute, Albuquerque, NM
}

While primary human hepatocytes are considered the "gold standard" for the evaluation of human P450 induction, animal hepatocytes are useful for the evaluation of species-differences and for the selection of the animal species most relevant to human for in vivo studies. To emphasize the property of the animal species and to minimize individual differences, we developed a procedure for the preparation of cryopreserved monkey hepatocytes pooled from multiple donors. The monkey hepatocytes were isolated from multiple donors $(\mathrm{N}=40 ; 20$ males and 20 females) on the same day. The hepatocytes were pooled followed by cryopreservation. The resulting "multiple donor pooled" monkey hepatocytes were found to have high viability. Most importantly, the hepatocytes retained their ability to be cultured as confluent, monolayer cultures ("Plateable"). We report here the application of the multidonor pooled cryopreserved Cynomolgus monkey hepatocytes in the evaluation of $\mathrm{P} 450$ induction. This study was conducted to compare monkey and human primary hepatocytes to response to prototypical drug inducers such as rifampicin (Rif), omeprazole (Ome), Phenobarbital (PB) and 3-Methylcholanthrene (3-MC) on the CYP1A and CYP3A gene expression. Pooled primary monkey hepatocytes (20 male and 20 female) or pooled primary human hepatocytes ( 5 male and 5 female) were treated with Rif $(0.16$, 1.25 and $10 \mathrm{uM})$, Ome $(0.7,6.25,50 \mathrm{uM}), \mathrm{PB}(31.5,125,250 \mathrm{uM})$, or 3-MC $(0.025,0.25,2 \mathrm{uM})$ for two days following by q-PCR quantification of mRNA of CYP1A1 and CYP3A8 for monkey, and CYP1A2 and CYP3A4 for human. The cryopreserved monkey hepatocytes or cryopreserved human hepatocytes were plated into a 24 well plate at a density 350,000 cells/well and allowed to attach for $4 \mathrm{hrs}$. Following attachment plating medium was removed and the cells were overlaid with $0.25 \mathrm{mg} / \mathrm{ml}$ Matrige $^{\mathrm{m}}{ }^{\mathrm{T}}$ in plating medium and incubated for an addition $20 \mathrm{hrs}$. The plate was cultured in an incubator maintained with a humidified atmosphere of $95 \%$ air and 5\% carbon dioxide. The various doses of Rif, PB, Ome or 3-MC were dissolved in $0.1 \%$ DMSO. The cells without administration of drugs and only with $0.1 \%$ DMSO was used as control. Total RNA was isolated individually from each well followed by cDNA synthesis. Real-time reactions were carried out using Taqman primers. Each PCR cycle threshold (Ct) was normalized to the average Ct of the endogenous housekeeping control gene GAPDH. The comparative ? Ct method was used to calculate relative quantification of gene expression. Treatment of Ome and 3-MC caused dose dependent induction of CYP1A1 in monkey and CYP1A2 in human hepatocytes. Induction fold of CYP1A2 in human hepatocytes was found to be higher than that for CYP1A1 in monkey hepatocytes. Treatment with Rif and PB caused dose dependent induction of CYP3A8 in monkey and CYP3A4 in monkey hepatocytes. The results suggest that monkey hepatocytes responded similarly to human hepatocytes to P450 inducers. The multiple donor pooled monkey hepatocytes should be useful for a case-by-case evaluation of the validity of the Cynomolgus monkey as an in vivo model for the prediction of human clinical findings.

\section{P133. DPX2 cells as a model system for detecting PXR-mediated induction of CYP3A enzymes by therapeutic agents; utility for DDI predictions}

\author{
Odette A. Fahmi ${ }^{1}$, Judy Raucy ${ }^{2}$, Elsa Ponce ${ }^{2}$ and Jerome M. Lasker ${ }^{2}$ \\ ${ }^{1}$ Pharmacokinetics and Drug Metabolism, Pfizer, Groton, CT, ${ }^{2}$ Puracyp, Inc, Carlsbad, CA \\ (c) 2011 Informa Healthcare USA, Inc.
}


The increase in P450 enzyme activity noted after exposure to certain therapeutics can elicit marked drug-drug interactions (DDI) that, in turn, may ultimately result in poor clinical outcome or adverse drug effects. The enhanced oxidative metabolism observed in response to drug treatment is mediated primarily by CYP3A4, the most prevalent hepatic P450 enzyme whose expression is regulated by the orphan nuclear receptor PXR. In vitro systems capable of rapidly and accurately determining whether a given agent activates PXR, and is thus capable of enhancing CYP3A-mediated metabolism, are highly sought-after tools for drug discovery screening. To that end, we assessed the utility of DPX2 cells, a HepG2-derived cell line stably integrated with a PXR expression vector plus a luciferase reporter, to detect therapeutics that not only cause PXR activation/CYP3A induction but also elicit clinical DDI. DPX2 cells were found to express mRNAs encoding CYP1A2, CYP2B6, CYP3A4, CYP3A5 and CYP3A7. Expression of CYP3A mRNA was inducible by treatment with rifampicin (RIF) and carbamazepine, although only CYP3A7 protein was detected after RIF treatment. CYP3A7 was catalytically active in DPX2 cells, as indicated by the RIF-inducible (29-fold) metabolism of luciferin isopropyl acetal, a bioluminescent CYP3A substrate. DPX2 cells expressed markedly higher levels of PXR than non-transfected HepG2 cells, and also contained appreciable levels of the key influx and efflux ABC transporters MDR1, MRP2, MRP3, MRP4, NTCP, OCT1, OAT2 and OATP2B1. The capacity of DPX2 cells to accurately identify clinical agents capable of eliciting CYP3A enzyme induction in vivo was then investigated with 22 clinical inducers and 12 clinical non-inducers. All 22 therapeutic agents with known in vivo inducing properties proved capable in DPX2 cells of activating PXR, with $\mathrm{E}_{\max }$ values ranging from 3.5- to 13-fold. Of the 12 therapeutics without in vivo inducing effects, 6 or 50\% acted as PXR activators while the other 6 failed to activate this receptor. The relative induction score (RIS), which was calculated by combining DPX2 cell activation parameters $\left(\mathrm{EC}_{50}\right.$ and $\mathrm{E}_{\max }$ ) for eleven drugs ( 7 clinical inducers and 4 clinical non-inducers) with their efficacious total plasma concentrations demonstrated a strong correlation $\left(\mathrm{r}^{2}=0.90\right)$ between RIS and the magnitude of induction of midazolam clearance. However, application of the RIS derived from the midazolam curve with other object drugs; such as nifedipine, simvastatin, triazolam and ethinyl estradiol resulted in predictions (mostly over-predictions) with varying degrees of accuracy. Additionally, PXR activation data obtained with the 12 clinical non-inducing agents did not translate into DDI predictions that were significant. In conclusion, the DPX2 cell-based assay exhibits the sensitivity and specificity needed for detecting potential clinical CYP3A inducers, and offers a more rapid and economical tool compared to human hepatocytes for use in drug discovery screening and early development.

\title{
P134. Development of ex-vivo drug metabolizing enzyme assays in the domestic shorthair cat (Felis catus)
}

\author{
Shangara S. Dehal, Thuy Ho, Andrew P. Blanchard, George Zhang, Nathalie Boily and David M. \\ Stresser
}

BD Biosciences Discovery Labware, Woburn, MA

Objective: Pre-clinical safety/toxicity studies are routinely conducted in rodent and non-rodent species. Often, drug metabolizing enzymes activities are determined to assess drug candidate induction potential and gain understanding of pharmacokinetic properties following repeat dosing. Drugs intended for animal use may undergo similar testing but advantageously can be conducted in target species. In vitro enzyme activity assays are well-established for mouse, rat, dog and monkey, but only limited information on the cytochrome P450 (CYP) and uridine 5'-diphosphoglucuronosyl transferase (UGT) activities is available for the domestic shorthair cat. The purpose of this investigation was to develop CYP and UGT enzyme activity assays in cat liver microsomes to assess drug-induced changes ex-vivo. Methods: Male $(n=3)$ and female $(n=3)$ cat livers were homogenized and microsomes prepared by differential centrifugation. Male and female pools were then characterized for various CYP enzyme activities generally considered selective for the major xenobiotic metabolizing CYP families (e.g. CYP1, 2, 3 and 4) in mammals. Activities and target enzyme examined were 7-ethoxyresorufin o-deethylase/CYP1A; 7-benzyloxyresorufin o-dearylase/CYP2B; lauric acid 11- and 12-hydroxylase/ CYP2E and CYP4, respectively and testosterone 6 $\beta$-hydroxylase/CYP3A. In addition, 4-methylumbelliferone (4-MU) and $p$-nitrophenol glucuronosyl transferase activities were assessed. Results: In general, kinetic parameters were not remarkably different compared to other preclinical species examined in this laboratory. It is generally believed that the cat has little or no capacity to glucuronidate drugs. Thus it was somewhat surprising to find that 4-MU was glucuronidated albeit at generally lower activity compared to other preclinical species. $p$-Nitrophenol kinetic parameters were not determined because catalytic activity was not detected. Conclusions: Liver microsomal CYP and UGT enzyme activity assays were developed in domestic cat and are suitable for assessing changes in enzyme levels both male and female animals. 


\title{
P135. Effects of pregnancy-specific somatotropins on cytochrome P450 (CYP) expression in human hepatocytes
}

\author{
Hye Jin Chung, Liam Fischer and Hyunyoung Jeong
}

Department of Pharmacy Practice, College of Pharmacy, University of Illinois at Chicago, Chicago, IL

Pregnancy alters hepatic drug metabolism, but the responsible factors and underlying mechanism remain to be identified. Physiological changes accompanying pregnancy are potentially responsible for the changes, such as rising concentrations of pregnancy-specific somatotropins: prolactin (PRL), placental lactogen (PL), and growth hormone variant $(\mathrm{GH}-\mathrm{V})$. Whether these hormones influence hepatic drug metabolism in humans is unknown. In this study, we evaluated the effects of PRL, PL, and GH-V on expression of major hepatic CYP enzymes. To this end, freshly isolated human hepatocytes (from 3 different donors) were treated with vehicle (phosphate buffered saline), PRL ( $150 \mathrm{ng} / \mathrm{mL}$ ), PL ( $6 \mu \mathrm{g} / \mathrm{mL}$ ), or GH-V $(20 \mathrm{ng} / \mathrm{mL})$ for $72 \mathrm{hr}$, and mRNA expression levels of CYP 1A2, 2A6, 2B6, 2C9, 2C19, 2D6, 2E1, 3A4, 3A5, and Na+taurocholate cotransporting polypeptide (NTCP; used as positive control based on a previous report in rat hepatocytes) were determined by quantitative real-time PCR. PL increased mRNA expression level of CYP2E1 (2-fold) in the hepatocytes while PL did not affect expression of other CYP isoforms. PRL and GH-V had negligible effects on expression of all CYP isoforms tested. Interestingly, NTCP expression was not influenced by any of the hormones, suggesting potential interspecies differences in somatotropin pharmacology between rats and humans. Taken together, these results indicate that except PL, pregnancy-specific somatotropins are unlikely responsible for altered drug metabolism during pregnancy. Further study is needed to elucidate regulatory mechanism underlying upregulation of CYP2E1 expression by PL.

\section{P136. In Vitro evaluation of novel PPAR-sparing insulin sensitizers and their pharmacologically active hydroxy metabolites as inducers of CYP450 expression in cultured human hepatocytes}

\author{
$\underline{\text { Wade J. Adams }}{ }^{1}$, Immaculate Amunon ${ }^{2}$, Jerry R. Colca ${ }^{1}$ and Jason N. Neat ${ }^{3}$ \\ ${ }^{1}$ Metabolic Solutions Development Company, Kalamazoo, MI, ${ }^{2}$ Enzyme Induction Department, XenoTech, LLC, Lenexa, \\ KS, ${ }^{3}$ Enzyme Induction Department, Metabolic Solutions Development Company, Kalamazoo, MI
}

MSDC0602 (5-\{4-[2-(3-methoxyphenyl)-2-oxoethoxy]benzyl\}-1,3-thiazolidine-2,4-dione]) and MSDC-0160 ([5-[4-[2-(5ethyl-2-pyridyl)-1-oxoethoxy]phenylmethyl]-1,3-thiazolidine-2,4-dione]) are being developed for the treatment of Type 2 diabetes. They are insulin sensitizers that are related to the thiazolidinedione pioglitazone (ACTOS ${ }^{\mathrm{m}}$ ) except both compounds have significantly reduced ability to activate the nuclear transcription factor PPAR. These compounds exert their pharmacology by selectively modulating mitochondrial metabolism resulting, among other things, in improved insulin sensitivity and an increase in brown adipose tissue. The PPAR-sparing nature of these compounds and their pharmacologically active hydroxy metabolites (MSDC-0597 and MSDC-0037, respectively) may provide increased therapeutic ratio in clinical practice. Therapeutic Cmax of the parent compounds and hydroxy metabolites in humans are expected to be ? 1.2 and ? $12 \mu \mathrm{M}$, respectively. The potential of MSDC-0602 and MSDC-0160 to induce CYP450 expression was assessed in primary cultures of fresh human hepatocytes in the current study. To evaluate induction, freshly isolated human hepatocytes $(n=3)$ were cultured and treated daily for three consecutive days with vehicle control, one of three concentrations of MSDC-0602 (1.2, 12 or $120 \mu \mathrm{M})$ or MSDC-0160 (1.2, 12 or $120 \mu \mathrm{M})$, or one of three known prototypical CYP inducers, namely omeprazole (AhR, CYP1A2, $100 \mu \mathrm{M})$, phenobarbital (CAR, CYP2B6, $750 \mu \mathrm{M})$ and rifampin (PXR, CYP3A4/5, $10 \mu \mathrm{M}$ ) and then evaluated for CYP activities (namely, CYP1A2, 2B6 and 3A4/5). In addition, the ability of either test compound to cause toxicity was assessed both by daily microscopic evaluation, and by the release of lactate dehydrogenase (LDH) in to the culture medium, to which, neither MSDC-0602, nor MSDC-0160 caused any detectible toxicity, by either method. Under conditions where the prototypical inducers caused anticipated increases in CYP activity, neither MSDC-0602 nor MSDC-0160 induced (less than or equal two fold) CYP1A2 activity (at concentrations up to $120 \mu \mathrm{M}$ ), CYP2B6 activity (at concentrations up to $12 \mu \mathrm{M}$ ) or CYP3A4 (at concentrations up to $12 \mu \mathrm{M}$ ). However, at higher concentrations (120 $\mu \mathrm{M})$ MSDC-0602 and MSDC-0160 induced both CYP2B6 (2.71- and 3.84-fold, respectively) and CYP3A4/5 (9.76- and 10.6-fold, respectively) activity and in some cases, the induction was more than $20 \%$ or $40 \%$ of the positive control effect in one or more of the human preparations. These data suggest that although MSDC-0602 and MSDC-0160 induced CYP2B6 and 3A4/5 activity, they do so only at concentrations which are greater than or equal 10x the expected therapeutic steady state Cmax. 


\title{
P137. Profiling of NCEs in a PXR reporter cell line and human hepatocytes to adopt a best- practice approach for identifying CYP3A inducers in drug discovery
}

\author{
$\underline{\text { Kevin J. Coe }}^{1}$, Judith Skaptason ${ }^{1}$, Robyn Buerger ${ }^{2}$, Chrissa Ferguson², Zhengyin Yan², Gary Caldwell ${ }^{2}$ \\ and Tatiana Koudriakova ${ }^{1}$
}

${ }^{1}$ Dmpk, Janssen Pharmaceutical Companies of Johnson \& Johnson, San Diego, CA, ${ }^{2}$ Dmpk, Janssen Pharmaceutical
Companies of Johnson \& Johnson, Springhouse, PA

Induction of CYP3A by drugs can result in undesirable ADME-properties, such as insufficient drug exposure due to autoinduction, increased risk of drug-drug interactions due to enhanced clearance, and potential for toxicity due to CYP3A-mediated bioactivation of drugs into reactive metabolites. Thus, identification of new chemical entities (NCEs) that induce CYP3A expression is required to guide early chemistry efforts in order to avoid nomination of drugs that are inducers. Drugs that induce CYP3A often activate the nuclear receptor pregnane X receptor (PXR) to increase gene expression of a battery of ADME-related genes, including CYP3A4. The ligand binding domain of PXR is highly divergent across species, leading to potency differences that impede the use of pre-clinical species to extrapolate the risk of CYP3A induction in humans. The study of CYP3A induction is therefore best explored in vitro in human models, such as engineered PXR reporter cell lines and human hepatocytes. Prototypical CYP3A inducers and a chemically diverse test set of NCEs were examined in the Puracyp ${ }^{\mathrm{m}}$ PXR reporter cell line and human hepatocytes to measure transcriptional and activity response of CYP3A, respectively. Although the PXR reporter cell line offers the throughput necessary for SAR building, follow-up work in human hepatocytes is paramount to confirm the success of the current chemistry strategy to address CYP3A induction. In our traditional assay, hepatocytes are seeded in 48-well plates and incubated with a test compound for two days. After two days, the hepatocyte media is replaced with fresh media containing a "CYP probe substrate cocktail" which includes midazolam $(3 \mu \mathrm{M})$. The reaction is carried out for $30 \mathrm{~min}$ followed by LC-MS/MS analysis for the formation of the CYP-selective probe metabolites. In addition to a relatively modest throughput ( 12 compounds) this detection method is sometimes susceptive to ion suppression and other bioanalytical problems (e.g. lack of sensitivity). To overcome these issues, we examined the utility of a luminescent-based assay, using the CYP3A4-selective probe Luciferin-IPA (Promega). Hepatocytes were seeded in 96-well plates, incubated with CYP inducers for two days and the CYP activity was measured using Luciferin-IPA substrate. Comparison of the two methodologies for CYP3A activity reveals a good concordance and applicability of the luminescent-based assay to meet our throughput demands. Findings from these bridging experiments reveal that PXR- hepatocyte relationships are often series-dependent and are especially timely if a series induces CYP3A in hepatocytes but not in the PXR reporter assay. These observations, in turn, allow for a translation of the PXR reporter data to outcomes in hepatocytes, refine strategy for candidate selection, and reveal other possible ADMET liabilities such as CYP3A time dependent inhibition and hepatocyte toxicity. In conclusion, the tandem profiling of compounds in PXR reporter and hepatocytes assays mitigate the risk in drug discovery of extensive characterizing and nominating drug candidates that harbor significant CYP3A induction.

\section{P138. Abstract withdrawn}

\section{P139. Automated 384-well cell-based cytochrome P450 inhibition assays using cryopreserved hepatocytes in suspension}

\author{
Bradley R. Larson ${ }^{1}$, Peter Banks ${ }^{1}$, Timothy A. Moeller ${ }^{2}$, Tracy Worzella ${ }^{3}$, Mary Sobol ${ }^{4}$ and Dongping \\ $\mathrm{Ma}^{4}$
}

${ }^{1}$ BioTek Instruments, Winooski, VT, ${ }^{2}$ Celsis In Vitro Technologies, Baltimore, MD, ${ }^{3}$ Scientific Applications and Technology Integration, Promega Corporation, Madison, WI, ${ }^{4}$ Research, Promega Corporation, Madison, WI

DDI are of serious concern to the pharmaceutical industry and associated regulatory agencies. Cytochrome P450 (CYP) enzymes are key players in the metabolism of drugs within the body, and modulations in their activity have been implicated in many known DDI. Therefore, it is essential to understand how these enzymes can be affected by xenobiotics with regards to inhibition to avoid potential drug-drug interactions. Numerous formats currently exist to monitor the potential effects that a lead compound may have on a CYP. These include the use of recombinant CYP isoforms, microsomes, and immortalized cell lines with hepatocyte-like function. While each of these formats has its advantages, there is an increasing realization that data generated using these methods do not provide a complete picture of the 
effects a compound would have in an in vivo setting. Therefore, assays using primary hepatocytes are becoming increasingly important to accurately determine potential DDI. This is due to the fact that hepatocytes, the primary cell of the liver, possess the full complement of enzymes, nuclear factors and co-factors at physiological levels. Because of the increased desire to perform hepatocyte-based assays, coupled with the need to perform ADME-Tox assays earlier in the drug discovery process, it is essential to be able to perform these assays in a higher-throughput setting, using appropriate laboratory automation. Here we present an application that demonstrates the ability to monitor CYP inhibition using cryopreserved human hepatocytes in an automated format. Using this setup, the inhibitory effects of lead compounds on CYP isoforms 1A2, 2C9, and 3A4 can be tested in a single 384-well plate, in a profiling format. Multiple luminescent assays, each using a luminogenic substrate specific for the isoform being analyzed, were employed for this application. The assay procedure was automated using a microplate dispenser as well as an 8-channel liquid handler. Four known inhibitors were tested with each of the three isoforms included in the profile, CYP1A2, -2C9, and -3A4. IC50 values were derived from a similar 11-point titration curve. Inhibition data was compared to that generated using human liver microsomes in a similar assay format, as well as to IC50 and Km values from the literature, and the current FDA Draft Guidance.

\title{
P140. Can $K_{i}$ values for direct inhibition of CYP enzymes can be reliably estimated from $\mathrm{IC}_{50}$ values?
}

\author{
Lois J. Haupt, Faraz Kazmi, Brian Smith, Sarah Leatherman and Andrew Parkinson
}

XenoTech, LLC, Lenexa, KS

Regulatory agencies recommend that the potential for a drug candidate to cause clinically relevant, direct inhibition of cytochrome $\mathrm{P} 450$ (CYP) enzymes be estimated based on the ratio of $[\mathrm{I}] / K_{\mathrm{i}}\left(\right.$ or $\left.1+[\mathrm{I}] / K_{\mathrm{i}}\right)$ where $[\mathrm{I}]$ is the in vivo concentration of drug candidate and $K_{\mathrm{i}}$ is the inhibition constant for direct inhibition, which requires an in vitro evaluation of the effects of multiple concentrations of the inhibitory drug versus multiple concentrations of CYP probe substrate (the former spanning $K_{\mathrm{i}}$ and the latter spanning $K_{\mathrm{m}}$ ). In the present study we conducted a retrospective analysis of 251 in vit$r o K_{\mathrm{i}}$ determinations to ascertain whether $K_{\mathrm{i}}$ values could be reliably estimated (within a factor of two) simply by dividing the corresponding $\mathrm{IC}_{50}$ values (determined prior to measuring $K_{\mathrm{i}}$ ) by two, based on the relationship that, for competitive inhibition, $K_{\mathrm{i}}=\mathrm{IC}_{50} / 2$ (when $[\mathrm{S}]=K_{\mathrm{m}}$ ). The $K_{\mathrm{i}}$ and $\mathrm{IC}_{50}$ values were determined under the following conditions: (1) the concentration of CYP marker substrate was equal to $K_{\mathrm{m}}$ (for $\mathrm{IC}_{50}$ determinations) and spanned $K_{\mathrm{m}}$ (for $K_{\mathrm{i}}$ determinations); (2) the substrate incubation time was short ( $5 \mathrm{~min}$ or less) to minimize metabolism-dependent inhibition and inhibitor depletion, and (3) the concentration of human liver microsomes was low $(0.1 \mathrm{mg} / \mathrm{mL}$ or less) to minimize nonspecific binding and depletion of the inhibitor. Under these conditions, estimates of $K_{\mathrm{i}}$ based on $\mathrm{IC}_{50} / 2$ correlated strongly with actual $K_{\mathrm{i}}$ determinations $(\mathrm{r}=0.944) .249$ of the 251 values agreed within a factor of two. In the case of noncompetitive inhibitors (where $K_{\mathrm{i}}=\mathrm{IC}_{50}$ ), $K_{\mathrm{i}}$ values estimated from $\mathrm{IC}_{50} / 2$ were overestimated by a factor of nearly two (i.e.,the estimated values were roughly half the measured values), which is to be expected because, for non-competitive inhibitor, $K_{\mathrm{i}}=\mathrm{IC}_{50}$ (not $\mathrm{IC}_{50} / 2$ ). The results of our analysis suggest that, under appropriate experimental conditions, $K_{\mathrm{i}}$ values for direct inhibition can be reliably, albeit somewhat conservatively, estimated from values of $\mathrm{IC}_{50} / 2$.

\section{P141. Characterization and evaluation of in vivo/ex vivo inhibition of monoamine oxidase enzymes activities in rat to support proof-of-concept (POC) studies of the new generation of monoamine oxidase inhibitors}

\author{
Mohammed Taimi, Robert R. Annand, Jon Gilbert and Katya Tsaioun
}

\section{Apredica, Watertwon, MA}

Aim and Background: Monoamines oxidases (MAO) A and B are enzymes responsible for the deamination of the amine
neurotransmitters in the central nervous system and peripheral tissues. MAO inhibitors are an important class of drugs
used in the treatment of many CNS disorders such as depression and Parkinson's diseases. We have developed and
optimized an MAO assay to evaluate the in vivo inhibition of MAO activities in rat plasma and several tissues following a
single oral dose of tranylcypromine (TCP), a non-selective MAO inhibitor.Methods: MAO enzyme activity was measured
in platelet-rich plasma (PRP) and in mitochondria fraction of several rat tissues using an LC/MS/MS-based method. To
this end, the formation 4-hydroxyquinoline, the metabolite produced by oxidation of kynuramine, a substrate for MAO
enzymes, was quantified by LC/MS/MS in ex-vivo PRP and tissue mitochondria fractions. In addition, levels of the brain 
endogenous MAO-A substrate, serotonin, and its respective metabolite, 5-hydroxyindole-3-acetic acid (5-HIAA) and the MAO-B substrate, 2-phenethylamine (PEA), and its respective metabolite, phenylacetic acid (PAA), were monitored as biomarkers in rat brain extract. Results: Dramatic inhibition of MAO activity was observed after oral administration of $3 \mathrm{mg} / \mathrm{kg}$ of the TCP. Twenty-four hours following PO dosing of TCP, the MAO activity was reduced in rat tissues, by more than $90 \%$ in blood, brain and liver and by more than $50 \%$ in intestinal mucosa. In addition, the brain level of 5-HIAA, a metabolite of serotonin generated by MAO-A, was reduced by more than $50 \%$ following dosing with TCP. Conclusion: The present study outlines a methodology for evaluating the in vivo impact of the MAO inhibitors in rat in efforts to evaluate in vivo efficacy of new generation of MAO inhibitors in animal model for in vivo POC studies.

\title{
P142. Choice of solvent for fluorimetric $\mathrm{IC}_{50}$ assays with bactosomes can influence the end result
}

\author{
Caroline F. Peet ${ }^{1}$, Anna W Kaaz ${ }^{1}$, Michael W Voice ${ }^{2}$ and Michael P Pritchard ${ }^{2}$
}

${ }^{1}$ Screening Services, Cypex Ltd, Dundee, Scotland, ${ }^{2}$ Cypex Ltd, Dundee, Scotland

Here we describe a modified 96-well format fluorimetric assay for the determination of $\mathrm{IC}_{50}$ values for 27 compounds across 8 human CYP isoforms (1A2, 2B6, 2C8, 2C9, 2C19, 2D6, 3A4 and 3A5) after incubation with Bactosomes. Formation of fluorescent metabolites was followed for a 30 minute period with reaction slopes plotted in 10 minute segments along the timecourse. $\mathrm{IC}_{50}$ values were plotted as a function of time, to highlight not only direct, but also time and/ or metabolism dependent inhibition. Each compound/isoform pairing was conducted on at least two occasions with separate batches of EasyCYP® Bactosomes where protein and CYP content were matched between batches. The effect of using both dimethylsulfoxide (DMSO) and methanol as the solvent for substrates and inhibitors was investigated at final incubation volumes of $1.5 \%(\mathrm{v} / \mathrm{v})$ and $2.5 \%(\mathrm{v} / \mathrm{v})$ in the assay. Potent and selective inhibition was noted in all isoforms with this compound set particularly with 'gold standard' compounds for specific isoforms recommended for use in interaction studies in vitro and in vivo by the FDA. Consistent $\mathrm{IC}_{50}$ values were determined for all compounds with Pearson Rank Coefficients $>0.94$ being calculated for DMSO results irrespective of solvent levels across the 8 human CYP isoforms investigated. Rank Coefficients were lower for assays containing methanol, especially at $1.5 \%$ solvent levels, probably due to solubility issues observed with some compounds eg Montelukast, Troglitazone and Troleandomycin (TAO). Pearson Rank Coefficients were $>0.85$ when $1.5 \%$ DMSO results were compared to $2.5 \%$ methanol results, and $>0.71$ when $1.5 \%$ DMSO data were compared to $1.5 \%$ methanol data. Although the rank order of different solvent results generally agreed with each other the absolute values calculated were affected by solvent and composition choice: Lower $\mathrm{IC}_{50}$ values were determined for 3A4, 2C9 and 2C19 when methanol was used in the assay than when compared to corresponding DMSO data, but the converse was true for 2C8, 2B6 and 2D6, where DMSO results tended to be lower. Using DMSO at $2.5 \%$ of the assay volume was unsuitable for assaying $3 \mathrm{~A} 4$ activity, due to the inhibitory nature of this solvent at the higher levels in control wells. As such, we recommend using $1.5 \%(\mathrm{v} / \mathrm{v})$ DMSO as solvent in this assay design, especially when comparing data generated over a long experimental period for ranking purposes. $\mathrm{IC}_{50}$ values have been determined across each isoform for every compound to give selective concentrations for prototypical inhibitors of each isoform in Bactosomes. Some inhibitory effects noted here have not been documented previously in the literature.

\section{P143. Development of a fluorimetric assay for determining $\mathrm{IC}_{50}$ values with bactosomes for 8 major human CYP isoforms; incorporation of $\mathrm{IC}_{50}$ value changes as a function of time to predict time/metabolism-dependent inhibition}

\author{
Caroline F. Peet ${ }^{1}$, Anna W Kaaz ${ }^{1}$, Michael W Voice ${ }^{2}$ and Michael P Pritchard ${ }^{2}$ \\ ${ }^{1}$ Screening Services, Cypex Ltd, Dundee, Scotland, ${ }^{2}$ Cypex Ltd, Dundee, Scotland
}

Here we describe for the first time a modified fluorimetric assay to determine $\mathrm{IC}_{50}$ values for prototypical inhibitors of 8 major human CYP isoforms (1A2, 2B6, 2C8, 2C9, 2C19, 2D6, 3A4 and 3A5) with recombinant Bactosomes. This assay follows changes in $\mathrm{IC}_{50}$ values over time, which can give a very early lead optimisation phase indication if there is a time/ metabolism dependent component over and above a direct inhibitory effect of the compound across these isoforms. Inhibitors tested which were known to have a direct inhibition step only were Clopidogrel (2B6), Troglitazone (2C9) N-Benzylphenobarbital (2C19), Quinidine (2D6), Cinacalcet (2D6) and Ketoconazole (3A4/5) giving IC $_{50}$ values based on slopes for the reactions over a 30 minute incubation period against selective isoforms of $\leq 0.27 \mu \mathrm{M}$. In addition, the following compounds are known to bring about an additional inhibition liability as a result of time and/or metabolism: 
Furafylline (1A2), Fluvoxamine (1A2), Montelukast (2C8), Sulfaphenazole (2C9), Tienilic acid (2C9), Ticlopidine (2C19), Paroxetine (2D6) and Troleandomycin (3A4/5). $\mathrm{IC}_{50}$ values for each of these reactions were $\leq 0.28 \mu \mathrm{M}$ (except for Furafylline against 1A2 [55 $\mu \mathrm{M}]$ and TAO against 3A5 $[1.1 \mu \mathrm{M}]$ ) but most paired reactions also showed a consistent decrease in determined value over the 30 minute incubation period, which was not observed with the direct acting compounds. The exceptions to the expected results showing a decrease in $\mathrm{IC}_{50}$ values over time were Paroxetine against 2D6 and Ticlopidine against 2C19 which have been reported in the literature in HLM experiments. Reasons for this disparity will be explained more fully in the poster. The selectivity of all inhibitors across each isoform will be documented for the first time allowing the end user to incubate at selective concentrations in future experiments with Bactosomes. Results showing the time/metabolism dependent component of Isoniazid, Amiodarone and 8-methoxypsoralen (8-MOPS) inhibition on 8 isoforms will also show that despite higher starting $\mathrm{IC}_{50}$ values $(\geq 0.48 \mu \mathrm{M})$, these compounds make suitable positive controls for all isoforms except 2C8. Of the compounds investigated, many brought about inhibition of 2C8 activity but only Montelukast showed a decrease in $\mathrm{IC}_{50}$ value over time.

\title{
P144. Dichloroacetate-dependent inactivation of GSTZ1 in human liver cytosol is modulated by physiologically important anions in a GSTZ1 haplotype-dependent manner
}

\author{
Margaret O. James ${ }^{1}$, Wenjun $\mathrm{Li}^{1}$, Yuan $\mathrm{Gu}^{1}$, Taimour Langaee ${ }^{2}$ and Peter W. Stacpoole ${ }^{3}$ \\ ${ }^{1}$ Medicinal Chemistry, University of Florida, Gainesville, FL, ${ }^{2}$ Pharmacotherapy and Translational Research, University \\ of Florida, Gainesville, FL, ${ }^{3}$ Medicine, Biochemistry and Molecular Biology, University of Florida, Gainesville, FL
}

Dichloroacetate (DCA), an investigational drug used to treat mitochondrial diseases, is metabolized by glutathione transferase Z1 (GSTZ1) to glyoxylate as the first step in its elimination. The clearance of DCA in people is considerably slowed upon chronic administration of therapeutic doses, although the extent of reduction in clearance varies between individuals. The slower clearance is thought to be due to inactivation of GSTZ1 by DCA. Several non-synonymous SNPs are present in the coding regions of GSTZ1. Three of these SNPs, E32K, G42R, T82M, give rise to five known haplotypes in humans, EGT, KGT, EGM, KGM and KRT. Recent studies showed that chloride protected hepatic cytosolic GSTZ1 from inactivation by DCA, although individuals carrying the KRT variant required a greater concentration of chloride than other individuals. The objectives of the present study were to test the hypotheses that chloride would affect the half-life of inactivation of GSTZ1 by DCA, that this would vary with haplotype, and that other anions would have similar effects. Human liver cytosol fractions from EGT/EGT and EGT/KRT individuals were used. In cytosol dialyzed against $0.1 \mathrm{M}$ potassium phosphate buffer $\mathrm{pH} 7.4$ but containing no chloride, the half-life of inactivation of GSTZ1 by $0.5 \mathrm{mM}$ DCA in the presence of $5 \mathrm{mM}$ glutathione was $30 \mathrm{~min}$ in EGT/EGT homozygotes and $23 \mathrm{~min}$ in EGT/KRT heterozygotes. Addition of a physiological concentration of $\mathrm{KCl}, 38 \mathrm{mM}$, to dialyzed cytosol fractions prolonged the half-life of inactivation to $5.4 \mathrm{~h}$ in EGT/EGT homozygotes, and to $2.5 \mathrm{~h}$ in EGT/KRT heterozygotes. To examine the effects of other anions, incubations with dialyzed cytosol were carried out for $2 \mathrm{hr}$ in the presence of $0.5 \mathrm{mM}$ DCA, $5 \mathrm{mM}$ glutathione and the potassium salt of the anion, $38 \mathrm{mM}$. The controls had no DCA and the references had no added potassium salt. Under these conditions, $90 \%$ of GSTZ1 activity was lost in the absence of additional anions and $20 \%$ of activity was lost in the presence of KCl. Addition of sulfate or carbonate did not affect the inactivation of GSTZ1 by DCA, however bromide, iodide and sulfite prevented the inactivation. Intracellular concentrations of bromide, iodide and sulfite are approximately 1000-fold lower than intracellular concentrations of chloride, and levels of all these anions can change with age and disease. While the mechanism of this effect is not yet known, this study has shown that as well as haplotype, anions in the liver can influence the rate of inactivation of GSTZ1 by DCA. Supported in part by US PHS grants ES 07355 and ES 012617

\section{P145. Esomeprazole and omeprazole sulfone are in vitro metabolism-dependent inactivators of CYP2C19: Determination of $K_{1}$ and $k_{\text {inact }}$ values}

\author{
Brian W. Ogilvie, Paul Toren, Faraz Kazmi and Andrew Parkinson
}

\author{
XenoTech, LLC, Lenexa, KS
}

The administration of omeprazole has been implicated in decreasing the efficacy of clopidogrel, which depends (at least in part) on conversion to its active metabolite by CYP2C19. We previously identified omeprazole and esomeprazole, but not Romeprazole, as irreversible (or quasi-irreversible) metabolism-dependent inhibitors of CYP2C19 [1,2]. The inhibitory potential of omeprazole toward CYP2C19 increased 4.2-fold (and that of esomeprazole by 10-fold) after 
a 30-min preincubation with NADPH (based on $\mathrm{IC}_{50}$ shift) and was found to have $k_{\text {inact }} / K_{\mathrm{I}}$ values ranging from 5.1 to $24 \mathrm{~min}^{-1} \cdot \mathrm{mM}^{-1}$ depending on the experimental conditions used. The inhibitory potential of omeprazole sulfone (one of the major metabolites of esomeprazole, but a minor metabolite of $R$-omeprazole) toward CYP2C19 also increased 3.2-fold after a 30-min preincubation with $\mathrm{NADPH}$ (based on $\mathrm{IC}_{50}$ shift) $[1,2]$. We hypothesized that the overall irreversible inhibitory effect of omeprazole (and esomeprazole in particular) on CYP2C19 may be due to a combination of effects of the parent drug and its sulfoxide metabolite. In an effort to characterize the potency with which esomeprazole and omeprazole sulfone inactivate CYP2C19, experiments were undertaken in NADPH-fortified pooled human liver microsomes (HLM). The time-course (zero to $15 \mathrm{~min}$ ) of CYP2C19 inactivation by esomeprazole ( 0.3 to $30 \mu \mathrm{M}$ preincubation concentration) and omeprazole sulfone (1.0 to $100 \mu \mathrm{M}$ preincubation concentration) were examined with $1.0 \mathrm{mg} /$ mL NADPH-fortified HLM followed by a 10-fold dilution and subsequent incubation with $400 \mu \mathrm{M} S$-mephenytoin (10x $\mathrm{Km})$ at a final HLM concentration of $0.1 \mathrm{mg} / \mathrm{mL}$. Under these conditions, we estimated $K_{\mathrm{I}}$ and $k_{\text {inact }}$ to be $17 \mu \mathrm{M}$ and $0.072 \mathrm{~min}^{-1}$, respectively, for esomeprazole $\left(k_{\text {inact }} / K_{\mathrm{I}}\right.$ value $\left.=4.3 \mathrm{~min}^{-1} \cdot \mathrm{mM}^{-1}\right)$, and $4.4 \mu \mathrm{M}$ and $0.025 \mathrm{~min}^{-1}$ for omeprazole sulfone $\left(k_{\text {inact }} / K_{\mathrm{I}}\right.$ value $\left.=5.7 \mathrm{~min}^{-1} \cdot \mathrm{mM}^{-1}\right)$. Because we previously showed that omeprazole is metabolically labile in HLM at $1.0 \mathrm{mg} / \mathrm{mL}\left(\mathrm{t}_{1 / 2}=14 \mathrm{~min}\right)$, we also performed similar experiments with $\mathrm{HLM}$ at a concentration of $0.1 \mathrm{mg} / \mathrm{mL}$ in the preincubation and $0.01 \mathrm{mg} / \mathrm{mL}$ final. Under these conditions, we estimated $K_{\mathrm{I}}$ and $k_{\text {inact }}$ to be $5.7 \mu \mathrm{M}$ and $0.058 \mathrm{~min}^{-1}$, respectively, for esomeprazole $\left(k_{\text {inact }} / K_{\mathrm{I}}\right.$ value $\left.=10 \mathrm{~min}^{-1} \cdot \mathrm{mM}^{-1}\right)$, and $0.80 \mu \mathrm{M}$ and $0.017 \mathrm{~min}^{-1}$ for omeprazole sulfone $\left(k_{\text {inact }} / K_{\mathrm{I}}\right.$ value $\left.=21 \mathrm{~min}^{-1} \cdot \mathrm{mM}^{-1}\right)$. The reversibility of inhibition of CYP2C19 by omeprazole sulfone was also examined. The results of this study suggest that esomeprazole more rapidly inactivates CYP2C19 than does racemic omeprazole, and that omeprazole sulfone (formed by CYP3A4, mainly from esomeprazole) may contribute to the overall in vivo inactivation of CYP2C19 by omeprazole and esomeprazole in particular.

1. Paris BL, Yerino P, Ogilvie BW and Parkinson A (2008) The proton pump inhibitors (PPIs) omeprazole and rabeprazole, but not lansoprazole, are in vitro time-dependent inhibitors of CYP2C19. Drug Metab Rev40(S1):89-90.

2. Ogilvie BW, Yerino P, Kazmi F, Buckley DB, Rostami-Hodjegan A, Paris BL, Toren P, and Parkinson A (2011). The proton pump inhibitor, omeprazole, but not lansoprazole or pantoprazole, is a metabolism-dependent inhibitor of CYP2C19: implications for coadministration with clopidogrel. Submitted to Drug Metab Dispos 6/22/2011.

\title{
P146. Evaluation of mechanism based CYP 450 3A Drug Interaction in Rats
}

\author{
Vishwottam Kandikere, Gopinadh Bhyrapuneni, Nageswararao Muddana, Veera Raghava Chowdary \\ Palacharla, Nilkanth Naik, Ramoji Kosuru, Shantaveer Irappanavar and Ramakrishna Nirogi
}

Discovery Research, Suven Life Sciences Ltd, Hyderabad, India

Mechanism based inactivation of CY3A become a major clinical problem due to loss of enzyme function leads to decrease the clearance of the inactivator due to auto-inhibition or prolongs the clearance of the drugs gets metabolized by the same mechanism. Although in vitro data are routinely used for the prediction of drug interactions, in vivo drug-drug interaction potential may provide the supportive evidence. Therefore, we sought to use the rat as an in vivo screening model to predict pharmacokinetic interactions with known CYP3A mechanism based inactivators. Chronic portal vein cannulated rats were pretreated with mibefradil ( $24 \mathrm{~h}$ before), erythromycin ( $12 \mathrm{~h}$ before) and diltiazem ( $12 \mathrm{~h}$ before) followed by indinavir infusion for $2 \mathrm{~h}$ through the portal vein, blood samples were collected at various time points during the infusion and post infusion. Plasma concentration of indinavir was measured using LC-MS/MS. Area under plasma concentration (AUC) profile found to be increased in the inhibitors pretreatment groups. Mibefradil was found to increase AUC of indinavir significantly by 2.8 fold as compared to vehicle treated. Erythromycin and diltiazem also found to increase AUC of indinavir by 1.9 and 1.2 fold respectively. The clearance and volume of distribution were found to decrease with an increase in intensity of inhibition. In summary, decrease in metabolism of known CYP3A substrate indinavir in the liver after inhibition of metabolic enzyme by known mechanism based inhibitors. This model can be used to screen the compounds at the preclinical stage to co-relate the fold change in AUC in in-vivo system in addition to the in vitro predictions.

\section{P147. Evaluation of dilution, dialysis and ultracentrifugation methods to assess the reversibility of metabolism-dependent inhibitors (MDIs) of cytochrome P450 (CYP) enzymes}

\author{
David B. Buckley, Faraz Kazmi, Phyllis Yerino, Brian Ogilivie and Andrew Parkinson
}


Metabolism-dependent inhibition (MDI) of cytochrome P450 (CYP) enzymes can involve the formation of metabolites that are (1) more potent reversible inhibitors than the parent compound, (2) irreversible inhibitors that covalently modify the CYP enzyme, or (3) quasi-irreversible inhibitors that coordinately bind the heme iron. To assess whether an MDI causes reversible or irreversible inhibition, many researchers employ a dilution, dialysis or ultracentrifugation procedure. The dilution method and, in many cases, the dialysis method require the use of relatively high concentrations of human liver microsomes (HLM) $(\geq 1 \mathrm{mg} / \mathrm{mL})$ in a pre-incubation step. We recently demonstrated that a high concentration of HLM $(\geq$ $1 \mathrm{mg} / \mathrm{mL}$ ) in the pre-incubation step can markedly decrease the free concentration of inhibitors by extensive metabolism and/or non-specific binding (Parkinson et al., 2011 DMD, 39 (8)). In the present study, we evaluated the dilution method for its ability to determine the mechanism of CYP3A4 inactivation by mibefradil (Posicor ${ }^{\circledR}$ ), a metabolically liable drug withdrawn due to its irreversible MDI of CYP3A4 in vivo. The mechanism of CYP3A4 inhibition by mibefradil was assessed with a dilution method and was incorrectly identified as a reversible inhibitor. Similarly, previous publications indicate that the dialysis method can reverse some quasi-irreversible MDIs of CYP3A4, namely troleandomycin and verapamil (Ma et al., $2000 D M D 28(2)$, p125). In the present study, we confirmed that MDI of CYP3A4 by troleandomycin and verapamil was reversed by dialysis alone, which differs from the clinical outcome (irreversible). We next evaluated the reversibility of 19 MDIs that inhibit one or more of eight CYP enzymes (namely CYP1A2, 2A6, 2B6, 2C8, 2C9, 2C19, 2D6 and 3A4) with a centrifugation method that does not require the use of high concentrations of HLM (Buckley et al., 2008). In each case, the mechanism of CYP inactivation determined the centrifugation method correctly identified the mechanism of MDI. The ultracentrifugation procedure described here allows for a drug candidate to be pre-incubated with a low concentration of $\operatorname{HLM}(0.1 \mathrm{mg} / \mathrm{mL}$ or less $)$ at, or near, the concentration of HLM used in non-dilution IC $_{50}$ shift experiments, avoiding problems associated with dilution or dialysis methods. We further demonstrate the utility of potassium ferricyanide treatment coupled with the ultracentrifugation method for distinguishing irreversible inhibitors (due to covalent MDI) from quasiirreversible inhibitors (due to metabolite-inhibitory complex [MIC] formation). Overall, the results of this study support the use of the ultracentrifugation method to evaluate the mechanism of CYP enzyme inactivation.

Buckley et al., (2008) Metabolism-dependent cytochrome P450 inhibitors: In vitro assessment of reversibility by ultracentrifugation methods. Drug Metab Rev40 (S3):172-173.

\title{
P148. Inhibitory effect of Chinese herbal extracts on the activities of human cytochrome P450 enzymes in vitro
}

\author{
Yan $\mathrm{Li}^{1}$, Jiangong Shi ${ }^{1}$, Shen Lin ${ }^{1}$, Qinghao Zhang1, Baolian Wang ${ }^{1}$, Caroline A. Lee ${ }^{2}$, R. Scott \\ Obach $^{3}$, Teresa A. Smolarek ${ }^{3}$ and Jenny Y. Zheng ${ }^{4}$
}

\author{
${ }^{1}$ Institute of Materia Medica, Chinese Academy of Medical Sciences, Beijing, China, ${ }^{2}$ Pfizer Inc., San Diego, CA, ${ }^{3}$ Pfizer \\ Inc., Groton, CT, ${ }^{4}$ Pfizer (China), Shanghai, China
}

Traditional Chinese Medicines (TCMs) are commonly used in Asian countries in combination with many therapeutic drugs. They may produce both pharmacokinetic and pharmacodynamic herb-drug interactions. Limited data on the drug-drug interaction (DDI) liability of TCMs restricts the ability to provide useful advice to patients on potential interactions between prescribed drugs and TCMs. Cytochrome P450s are known to be the major enzymes involved in the metabolism of most clinical drugs and metabolic-based DDI. The aim of the present study is to identify the potential inhibitory constituents in TCMs toward human CYP450s in vitro, with an ultimate intent to predict those herbs most likely to cause DDI with other drugs. Briefly, 50 herbal medicines, many of the most frequently used in China, were selected for testing. The raw materials of each herb were extracted by both water and ethanol, which is consistent with clinical preparation technique in China. Each extract was further fractionated by a reversed-phase (C18) CombiFlash system ( $2 \mathrm{~min}$ fractions, 45 fractions/herb) and tested in a cocktail CYP inhibition assay. The results demonstrated that 20 of 50 tested TCMs showed significant inhibition $(>90 \%)$ on single or multiple CYP activities (1A2, 2C9, 2C8, 2D6, 3A4) mostly in solvent extracts, and less so in water extracts. Furthermore, profound inhibition of more than 70 fractions from 20 TCMs remained even after 10- or 100-fold dilution. Interestingly, CYP2C9 and 2C8 were inhibited the most by tested fractions. In addition, most constituents with inhibitory activity on CYP450s appeared between fractions 20-40, which had similar polarity in HPLC analysis. The subfractions of those fractions showing inhibitory activity will be further isolated by HPLC and LC-MS for structure elucidation of active constituents and determination of mechanism of inhibition through kinetic studies in human liver microsomes.

\section{P149. Studies to further investigate the inhibition of human liver microsomal cytochrome P450 2 C8 (CYP2C8) by the acyl-ß-glucuronide of gemfibrozil}

$\underline{\text { Susan M. Jenkins }}^{1}$, Tatyana Zvyaga ${ }^{1}$, Stephen Johnson ${ }^{2}$, Jeremy Hurley ${ }^{1}$, Andrew Wagner ${ }^{1}$, Richard Burrell $^{1}$, Wesley Turley ${ }^{1}$, John E. Leet ${ }^{1}$, Thomas Philip ${ }^{1}$ and A. David Rodrigues ${ }^{2}$ 
In previous studies, gemfibrozil acyl- $\beta$-glucuronide, but not gemfibrozil, was found to be a mechanism-based inhibitor of cytochrome P450 2C8 (CYP2C8). In order to better understand whether this inhibition is specific for gemfibrozil acyl- $\beta$ glucuronide, or if other glucuronide conjugates are potential substrates for inhibition of this enzyme, we evaluated several pharmaceutical compounds (as their acyl-glucuronides) as direct-acting and metabolism-dependent inhibitors of CYP2C8 in human liver microsomes. Out of eleven compounds that were evaluated as their acyl-glucuronide conjugates, only gemfibrozil acyl-ß-glucuronide exhibited mechanism-based inhibition, indicating that CYP2C8 mechanism-based inhibition is very specific to certain glucuronide conjugates. Structural analogs of gemfibrozil were synthesized and their glucuronide conjugates were prepared in order to further examine the mechanism of inhibition. When the aromatic methyl groups on the gemfibrozil moiety were substituted with trifluoromethyls, the resulting glucuronide conjugate was a weaker inhibitor of CYP2C8 and mechanism-based inhibition was abolished. However, the glucuronide conjugates of mono-methyl gemfibrozil analogs were mechanism-based inhibitors of CYP2C8, although not as potent as gemfibrozil acyl-ß-glucuronide itself. The ortho-monomethyl analog was a more potent inhibitor than the meta-monomethyl analog, indicating that CYP2C8 favors the ortho position for oxidation and potential inhibition. Molecular modeling of gemfibrozil and its analogs in the CYP2C8 active site indicates that the ortho-monomethyl analog has a superior docking pose due to better hydrogen bonding of the glucuronide conjugate, as well as significantly improved hydrophobic contacts with the protein.

\title{
P150. The length of preincubation times in abbreviated cytochrome P450 time-dependent inhibition studies: One size fits all?
}

\author{
David M. Stresser ${ }^{1}$, Andrew K Mason ${ }^{1}$, Charles L. Crespi ${ }^{1}$ and Elke S. Perloff ${ }^{2}$ \\ ${ }^{1} B D$ Biosciences Discovery Labware, Woburn, MA, ${ }^{2} B D$ Gentest SM Contract Research Services, Woburn, MA
}

FDA and EMEA draft guidance recommend pre-incubation of small molecule drug candidates with liver microsomes and NADPH before the addition of substrate to assess the potential for time-dependent inhibition (TDI) of cytochrome P450. A 30 minute preincubation is commonly used as it is believed this would be long enough to permit detection of TDI. But is this adequate in all cases? For example, AMG487 displayed a dose- and time-dependent reduction in oral clearance in a Phase I multiple dose clinical study, however there was no indication of TDI in vitro. Follow on investigation indicated a secondary metabolite with TDI properties was the likely cause of this finding [Tonn, et al. (2009) Drug Metab Dispos 37:502-513]. Results from our laboratory [Perloff, et al (2009) Xenobiotica 39:99] demonstrate that comparing preincubation times of 10 and 30 minutes can distinguish rapid from slower acting time-dependent inhibitors. These findings suggest that extending preincubation times even further may help identify very slow acting time-dependent inhibitors, such as AMG487, that would otherwise go undetected. Compounds requiring conversion to secondary or tertiary metabolites that are precursors to metabolites that elicit a TDI response may benefit, in particular. Similarly, conditions that promote conversion to secondary and tertiary metabolites, such as higher protein concentrations in the preincubation, may also enhance detection of TDI. To examine this possibility, we used diltiazem as a model compound. This is because diltiazem requires sequential metabolism to yield a metabolic intermediate complex and TDI. In a dilution method IC $_{50}$ shift midazolam 1'-hydroxylation and testosterone 6ß-hydroxylation assay assay, where compound was preincubated at 10X protein, we found that the magnitude of the $\mathrm{IC}_{50}$ shift increased approximately 100 -fold when comparing a 30 minute to a 90 minute preincubations. The enhanced shift was the result of a further shift to the left of the "plus NADPH" $\mathrm{IC}_{50}$ value from a mean of $\sim 10 \mu \mathrm{M}$ to $\sim 0.1 \mu \mathrm{M}$ while the "minus NADPH" curve was essentially unchanged, ranging from 100 to $216 \mu \mathrm{M}$. We also found that in a substrate addition method (with $1 \mathrm{X}$ protein in the preincubation), the $\mathrm{IC}_{50}$ shift increased approximately 8-fold. Again, the enhanced shift was due to a further shift to the left of the "plus NADPH" IC ${ }_{50}$ value from a mean of $\sim 49 \mu \mathrm{M}$ to $\sim 14$ $\mu \mathrm{M}$ while the mean "minus NADPH" curve was relatively unchanged, ranging from 93 to $185 \mu \mathrm{M}$. In cases where either borderline $\mathrm{IC}_{50}$ shift values are observed (e.g. 1.5-fold), or where sequential metabolism is suspected of causing significant inhibition, it may be appropriate to conduct TDI assays using conditions that enhance test article metabolism. Such conditions include higher protein concentrations or preincubation times longer than the standard 30 minutes.

\section{P151. Use of dialysis to differentiate between reversible and mechanism-based CYP inhibition}

\author{
$\underline{\text { Stephen Madden }}{ }^{1}$ Jon Welch ${ }^{2}$ and Alison Meechan ${ }^{2}$
}

\author{
${ }^{1}$ Metabolism and Pharmacokinetics, Charles River Laboratories, Edinburgh, United Kingdom, ${ }^{2}$ In vitro Sciences, Charles \\ River Laboratories, Edinburgh, United Kingdom
}


Distinguishing true mechanism-based inhibition from reversible inhibition can be problematic when the rate of dissociation of the inhibitor from the target enzyme is relatively slow. Using dialysis to remove unbound drug (inhibitor) has been widely promoted as the method of choice to make this distinction. In this study, we have investigated the use of dialysis to discriminate between a known reversible and a known mechanism-based inhibitor of human CYP3A4 activity in pooled hepatic microsomes. Midozalam 1'-hyroxylase was utilized as the marker of CYP3A4 activity. Microsomes $(2 \mathrm{mg} / \mathrm{mL}$; obtained from BD Gentest) were preincubated in the presence and absence of the CYP inhibitors ketoconazole (competitive, reversible) and mifepristone (mechanism-based, irreversible) and NADPH. Following this pre-incubation an aliquot of the reaction mixture was removed for the determination of enzyme activity. The remainder of the reaction mixture was either transferred to a Slide-a-lyzer dialysis cassette (10 KDa molecular weight cut-off) prehydrated in phosphate buffered saline (PBS) containing $0.1 \mathrm{mM}$ EDTA (pH 8.) or to a fresh incubation tube. All samples were then incubated at $4^{\circ} \mathrm{C}$ for $24 \mathrm{~h}$. The dialysis buffer was continually stirred and changed periodically throughout the $24 \mathrm{~h}$ incubation period. After $24 \mathrm{~h}$, an aliquot from each incubation mixture was removed and assayed for CYP3A4 activity. Following the pre-incubation with the reversible inhibitor ketoconazole a proportion of CYP3A4 activity was recovered by $24 \mathrm{~h}$ in the absence of dialysis, with virtually complete recovery of activity achieved following dialysis. In contrast, following pre-incubation wth mifepristone there was no recovery in CYP3A4 following dialysis, in fact enzyme activity was slightly reduced in both dialysed and non-dialysed samples. In conclusion, extensive dialysis at reduced temperature is a simple and relatively inexpensive method to differentiate between reversible and non-reversible enzyme inhibitors.

\title{
P152. Adaptive de novo cytochrome $\mathrm{P} 450$ expression by normal human bladder epithelium in vitro in response to xenobiotic exposure
}

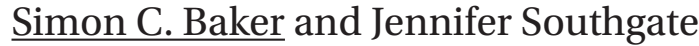

\section{Department of Biology, University of York, York, United Kingdom}

Incomplete detoxification of xenobiotics by the gut and liver may result in toxins being filtered out of the blood by the kidneys and concentrated in the urine. As urine is stored for long periods in the bladder, this raises the possibility that the bladder epithelial lining may be exposed to significant concentrations of xenobiotic and that as a consequence, the urothelium has evolved adaptive mechanisms. Whereas cytochrome P450 expression is well documented in bladder cancer no investigation has been performed on the metabolic capacity of normal human urothelium. Normal human urothelial (NHU) cells expressed transcript for the transcription factors which drive the majority of cytochrome P450 expression in the liver. Aryl hydrocarbon receptor and glucocorticoid receptor transcripts were expressed at comparable levels to those found in human liver; however, constitutive androstane receptor and pregnane $\mathrm{X}$ receptor transcripts were expressed at much lower levels. To examine the metabolic potential of the urothelium, NHU cells were treated with an induction cocktail targeting these receptors which has previously been reported to maintain metabolic activity in cultured primary human hepatocytes. A range of de novo cytochrome P450 enzyme expression was observed in NHU cells following treatment with the induction cocktail $(10 \mu \mathrm{M}$ ITE, $10 \mu \mathrm{M}$ rifampicin and $500 \mu \mathrm{M}$ phenobarbital) for 7 days. Whilst some features of the metabolic profile were comparable to liver, many of the enzymes induced were not those considered to play an important role in hepatic xenobiotic metabolism. This, potentially urothelium specific, extrahepatic metabolic profile requires further study to determine its function with respect to xenobiotic processing. The implication of this study is that urothelium may function as an important site of extra-hepatic metabolism which could contribute to bladder pathogenesis.

\section{P153. Comparison of xenobiotic metabolizing enzyme activities in normal human skin and reconstructed human skin models from skinethic laboratories}

\author{
Joan Eilstein ${ }^{1}$, Guillaume Lereaux ${ }^{1}$, Jean-Roch Meunier ${ }^{1}$, Jacques Leclaire ${ }^{2}$ and Daniel Duche ${ }^{1}$ \\ ${ }^{1}$ Research and Innovation / Advanced Research, L'Oréal, 93600 Aulnay-Sous-Bois, France, ${ }^{2}$ Research and Innovation, \\ L'Oréal, 92585 Clichy, Cedex, France
}

Skin is mainly considered as a physical barrier. However, skin is also recognized to be an important site of extrahepatic metabolism and requires to be characterized in terms of metabolic abilities. Moreover, the $7^{\text {th }}$ European amendment to the cosmetic directive will totally ban, in 2013, the use of animal testing to develop and trade new cosmetic ingredients in the European Community. For more than twenty years, L'Oreal has been producing and improving reconstructed human skin models (skin models) as in vitro alternative methods to animal experimentation. It uses them to predict 
different toxicological endpoints such as skin corrosion and skin irritation on finish products and cosmetic ingredients in development. For these reasons it is essential to assess and compare these skin models with a normal human skin (NHS) in terms of metabolic abilities. In this work, the apparent metabolizing enzyme activities of three SkinEthic" skin models were determined and compared with NHS for the main cytochrome P450-dependent monooxygenases involved in drug metabolism, esterases, alcohol and aldehyde deshydrogenase, glutathione $S$-, $N$-acetyl-, glucuronyl- and sulfotransferases. Results show that the skin is much better equipped in conjugating enzymes and esterases than in P450dependent monoxygenases and define it as a much more detoxifying organ than a bioactivating one. They demonstrate as well that the skin models are equivalent to a NHS in terms of metabolic capabilities. Consequently they are useful as substitutes of human skin samples to assess the local efficacy and safety of new cosmetic ingredients.

\title{
P154. In vitro and in vivo studies implicating CYP4B1 in 4-ipomeanol-induced pneumotoxicity in mice and cattle
}

\author{
Oliver T. Parkinson ${ }^{1}$, Edward J. Kelly² and Allan E. Rettie ${ }^{3}$
}

${ }^{1}$ Medicinal Chemistry, University of Washington, Seattle, WA, ${ }^{2}$ Dept of Pharmaceut, University of Washington, Seattle, WA, ${ }^{3}$ Dept of Med Chem, University of Washington, Seattle, WA

4-Ipomeanol is a pulmonary toxin produced by sweet potatoes that has been linked to severe pulmonary edema and death in livestock, particularly cattle, throughout the world. In vitro studies were conducted to explore the mechanism of cattle's high susceptibility to pneumotoxicity after ingestion of 4-ipomeanol. 4-Ipomeanol bioactivation to a reactive ene-dial was assessed in bovine liver and lung microsomes by LC/MS-MS after trapping with $\mathrm{N}$-acetylcysteine/Nacetyllysine. Bioactivation in cow lung was inhibited potently by the CYP4B1 chemical inhibitor, HET0016 and by a polyclonal antibody to rabbit CYP4B1. Western blotting of bovine pulmonary microsomes confirmed high levels of expression of CYP4B1 protein in the target species. To further test the contribution of CYP4B1 towards the activation of 4-ipomeanol, we conducted in vivo studies in wild-type and CYP4B1 knockout mice to determine if the absence of CYP4B1 was protective for mice treated with the toxin. Wild-type mice injected intraperitoneally with $20 \mathrm{mg} / \mathrm{kg} 4$-ipomeanol (the reported LD50) exhibited signs of distress including lethargy and increased respiration within several hours of dosing, whereas CYP4B1 knockout mice showed no outward signs of toxicity. Additional histopathology data will be presented. We conclude that CYP4B1 is the major determinant of 4-ipomeanol-induced pneumotoxicity in mice and cattle.

\section{P155. Skin metabolism of cinnamic alcohol and cinnamaldehyde: Its role in contact dermatitis}

\author{
Daniel Duche $^{1}$, Joan Eilstein ${ }^{1}$, Guillaume Lereaux ${ }^{1}$, Jacques Leclaire ${ }^{2}$ and Jean-Roch Meunier ${ }^{1}$ \\ ${ }^{1}$ Predictive Methods and Models Development, L'Oreal Research and Innovation - Advanced Research, 93601 Aulnay \\ sous Bois Cedex, France, ${ }^{2}$ Life Sciences, L'Oreal Research and Innovation - Advanced Research, 92585 Clichy Cedex, \\ France
}

Skin is mainly considered as a physical barrier to its environment. However, it is also an organ involved in the metabolism of endogenics and xenobiotics which could have penetrated it. Contact dermatitis results from the interaction between haptens (electrophilic compounds) and the side chain of nucleophilic amino acids of endogenous proteins to form antigenic complexes. Cinnamic alcohol $(\mathrm{CIN}-\mathrm{OH})$ and Cinnamaldehyde (CIN-CHO) are very well known pro-hapten and hapten, respectively. In this work, we studied the cutaneous metabolic fate of these two compounds. We used normal human skin (NHS) and three reconstructed human skin models (skin models) from SkinEthic" to assure the relevance of their use. Results showed that CIN-OH is oxidized into CIN-CHO by alcohol deshydrogenases (ADH) and CIN-CHO is quickly oxidized into cinnamic acid by aldehyde deshydrogenases (ALDH). Epoxide, trans-diol, hydroxylated aromatic ring, glutathione adduct and no other metabolite was detected. Apparent enzymatic parameters such as affinity $(\mathrm{Km})$, maximal velocity (Vmax) and metabolic clearance, estimated by the $\mathrm{Vmax} / \mathrm{Km}$ ratio, were calculated from metabolites assay in dose-effect studies. We observed that Vmax/Km ratio for ALDH $\left(\sim 0.8 \pm 0.1\right.$ and $1.7 \pm 0.6 \mu \mathrm{L} \cdot \mathrm{min}^{-1} \cdot \mathrm{mg} \mathrm{prot}^{-1}$ for models and NHS, respectively) was more important than ADH activity $\left(\sim 0.2 \pm 0.1 \mu \mathrm{L} \cdot \mathrm{min}^{-1} \cdot \mathrm{mg} \mathrm{prot}^{-1}\right.$ for NHS and models). ALDH activity was higher in NHS than in skin models. That could be due to the biggest variability of the measures with NHS samples (several single donors) compared with skin models (keratinocytes pool of several donors which supplies less variable data). Contact dermatitis occurs according to the aldehyde ability to react with proteins. Regarding these results, skin sensitization process will be triggered more likely when the aldehyde local concentration exceeds the 
capacity of ALDH isozymes to metabolize it. Moreover, people who would be deficient in the gene expression of a crucial ALDH isoform could represent a sensitive target to CIN-CHO sensitization. Except for ALDH activity, data obtained with skin models are similar to NHS for these substrates. In conclusion, findings show that, for these metabolic pathways, skin reveals a more detoxifying behavior than a bioactivating one. On the other hand, skin models can replace NHS to study the cutaneous metabolism of primary alcohols and aldehydes and determine the saturating concentration of substrates which could become toxic.

\title{
P156. Unusual oxidative instability of a quinoxaline derivative in rodent plasma: Role of xanthine oxidase
}

\author{
Heather Eng ${ }^{1}$, Raman Sharma², Gregory S. Walker², Angela Wolford², Yue Chen² and Amit S. \\ Kalgutkar $^{2}$
}

${ }^{1}$ Pharmacokinetics, Dynamics, and Metabolism, Pfizer Global Research and Development, Groton, CT, ${ }^{2}$ Pharmacokinetics, Dynamics and Metabolism, Pfizer Global Research and Development, Groton, CT

Compound 1 (1), a quinoxaline derivative, is a potent agonist of the G protein-coupled receptor 119 (GPR 119) with possible application in the treatment of type 2 diabetes. During routine profiling of disposition characteristics, we found that 1 was unstable in plasma $\left(37^{\circ} \mathrm{C}, \mathrm{pH} 7.4\right)$ from rat $\left(T_{1 / 2}=16 \mathrm{~min}\right)$, mouse $\left(T_{1 / 2}=61 \mathrm{~min}\right)$, and guinea pig $\left(T_{1 / 2}=4 \mathrm{~min}\right)$. Compound $\mathbf{1}$, however, proved to be stable in rat plasma when incubations were conducted at $4{ }^{\circ} \mathrm{C}$, which suggested that the plasma lability was mediated through an enzyme(s)-mediated process. The instability noted in rodent plasma was not observed in plasma from dog, monkey, and human plasma $\left(T_{1 / 2}>370 \mathrm{~min}\right.$ at $\left.37^{\circ} \mathrm{C}, \mathrm{pH} 7.4\right)$. Metabolite identification studies in incubations of $\mathbf{1}$ in rat plasma did not reveal the presence of the putative hydrolytic product, which implied that carboxylesterase-mediated hydrolysis of the carbamate moiety was not the reason for the plasma lability. To our surprise, a single metabolite (M1) peak corresponding to a molecular weight $16 \mathrm{Da}$ higher than that of 1 was observed (compound 1: $\mathrm{MH}^{+}=403, R_{\mathrm{t}}=4.03 \mathrm{~min} ; \mathrm{M1}: \mathrm{MH}^{+}=419, R_{\mathrm{t}}=3.51 \mathrm{~min}$ ). The incorporation of ${ }^{18} \mathrm{O}$ label in $\mathbf{M 1}$ in rat plasma incubations of $\mathbf{l}$ conducted in the presence of $\mathrm{H}_{2}^{18} \mathrm{O}$ indicated that the source of oxygen in $\mathbf{M l}$ was derived from water (and not molecular oxygen). The structure of Ml was determined by NMR following isolation from rat plasma, which indicated formation of a dihydroquinoxaline derivative upon hydrolysis of the iminium bond on a quinoxaline ring. In order to investigate whether plasma aldehyde oxidase and/or xanthine oxidase contributed towards M1 formation, stability studies on 1 were conducted in rat plasma pretreated with raloxifene (an aldehyde oxidase inhibitor) or allopurinol (a xanthine oxidase inhibitor). Raloxifene $(1 \mu \mathrm{M})$ did not prevent oxidative turnover of $\mathbf{1}$ to M1, suggesting that plasma aldehyde oxidase does not play a role in the oxidation of $\mathbf{1}$. In contrast, the degradation of $\mathbf{1}$ of $\mathbf{M 1}$ in rat plasma was completely prevented in the presence of allopurinol $(250 \mu \mathrm{M})$, which strongly suggests a role of xanthine oxidase in the metabolism of 1 to M1. Consistent with these findings, the formation of M1 was also demonstrated in incubations of 1 in purified XO. To the best of our knowledge, our studies constitute the first report on the involvement of rodent XO in oxidative drug metabolism in plasma.

\section{P157. A standardized decoction of nigella sativa seeds, hemidesmus indicus roots and smilax glabra rhizomes induces apoptosis in human hepatocellular carcinoma (HePg2) cells}

\author{
$\underline{\text { Sameera R. Samarakoon }}{ }^{1}$, Ira M. Thabrew ${ }^{2}$, Prasanna B. Galhena ${ }^{3}$ and Kamani Tennekoon ${ }^{4}$ \\ ${ }^{1}$ Institute of Biochemistry, Molecular Biology, and Biotechnology, University of Colombo, Colombo 3, Sri Lanka, \\ ${ }^{2}$ Biochemistry, Institute of Biochemistry, Molecular Biology, and Biotechnology, University of Colombo, Colombo 3, Sri \\ Lanka, ${ }^{3}$ Department of Biochemistry and Clinical Chemistry, Faculty of Medicine, University of Kelaniya, Ragama, Sri \\ Lanka, ${ }^{4}$ Institute of Biochemistry, Molecular Biology, and Biotechnology, University of Colombo, Colombo 3, Sri Lanka
}

A decoction comprised of Nigella sativa seeds, Hemidesmus indicus roots and Smilax glabra rhizomes used as a traditional medicine for cancer has been reported to protect against chemically induced hepatocarcinogenesis in rat livers with no significant toxic side effects. Recent studies have shown that the anti-hepatocarcinogenic activity of the decoction is mediated by selective cytotoxicity, antioxidant activity, anti-inflammatory activity and modulation of genes involved in tumour suppression (p53) and cell cycle arrest (p21). Apoptosis, the process of programmed cell death, is now recognized as a vital process in the regulation of tissue development and homeostasis, which is highly conserved throughout evolution. Apoptosis inducing agents are gaining increasing importance globally as weapons for the management of various types of cancer. The present study was carried out to investigate whether induction of apoptosis can 
also be a mechanism through which the decoction mediated its anti-hepatocarcinogenic action. In this study, the apoptotic effects of the decoction were evaluated by (a) expression of mRNA and protein of pro apoptotic gene Bax and anti apoptotic gene Bcl-2 by Reverse Transcription PCR, immunohistochemistry and western blot analysis (b) assessment of the effects on Caspase (Caspase 3 and Caspase 9) activity (c) fluorescent microscopic observation of the morphology of HepG2 cells stained with acridine orange/ethidium bromide staining and Hoechst 33258 staining and (d) DNA fragmentation analysis by agarose gel electrophoresis. The results demonstrated that the decoction can significantly $(\mathrm{P}<0.001)$ enhance the expression of pro-apoptotic gene Bax while reducing expression of anti apoptotic gene Bcl-2 in a time and dose dependent manner in HepG2 cells. Caspase 3 and Caspase 9 proteins were also significantly $(\mathrm{P}<0.001)$ activated by the decoction in a time and dose dependent manner. DNA fragmentation and morphological changes observed under fluorescent microscope further confirmed the apoptosis induced by the standardized decoction in HepG2 cells. Overall findings demonstrate that modulation of genes involved in apoptosis is also a mechanism utilized by the decoction comprised of Nigella sativa seeds, Hemidesmus indicus roots and Smilax glabra rhizomes to mediate anti-hepatocarcinogenic effects.

\title{
P158. Analysis of the endoplasmic reticulum stress response in progressive human non- alcoholic fatty liver disease
}

\author{
April D. Lake ${ }^{1}$, Petr Novak ${ }^{2}$, Rhiannon N. Hardwick ${ }^{1}$ and Nathan J. Cherrington ${ }^{3}$
}

${ }^{1}$ Pharmacology and Toxicology, University of Arizona, Tucson, AZ, ${ }^{2}$ Southwest Environmental Health Sciences Center, University of Arizona and Institute of Plant Molecular Biology in the Czech Republic, Ceske Budejovice, Czech Republic, ${ }^{3}$ University of Arizona, Tucson, AZ

The progression of human non-alcoholic fatty liver disease (NAFLD) from steatosis to non-alcoholic steatohepatitis (NASH) is associated with many alterations in global gene expression. The purpose of the current study was to investigate changes in the expression of gene components of the endoplasmic reticulum (ER) stress pathway in progressive stages of human NAFLD. Differential gene expression between three clinically defined pathological groups including normal, steatosis and NASH was analyzed. The expression levels of ER stress gene components were assayed using gene data from a human NAFLD liver tissue Affymetrix GeneChip Human 1.0ST microarray study. Signaling of the ER stress pathway originates through the activation of three transmembrane sensor proteins that include: inositol-requiring enzyme 1 (IRE1), PKR-like kinase (PERK), and activating transcription factor-6 (ATF6). These three sensors are responsible for activating the unfolded protein response (UPR), which coordinates gene expression changes and cellular processes that reduce the occurrence of misfolded proteins in the ER. A comprehensive list of ER stress genes including those with roles in autophagy and de novo lipogenesis were tested in an enrichment analysis in the human NAFLD gene expression data. It was found that de novo lipogenesis genes were significantly enriched for downregulation. Additionally, activation of the IRE1 ER stress pathway was activated as evidenced by nuclear accumulation by immunohistochemical staining of X-box binding protein-1 (XBP-1). XBP-1 is a critical regulator of the UPR and it is translocated to the nucleus of cells during ER stress. XBP-1 nuclear staining was more prominent in steatosis and NASH human livers (normal-10.05\%, steatosis-48.4\%, NASH fatty-54.4\%, NASH not fatty-58.5\% positive nuclei). Increased nuclear XBP-1 is indicative of ER stress-mediated regulation of JNK, autophagy, and de novo lipogenesis pathways. The results of this study indicate a role for ER stress in the progression of human NAFLD.

\section{P159. Collagen antibody induced arthritis (CAIA) in mice and primary mouse hepatocyte culture as models to study P450 suppression: In vivo/in vitro similarities and differences}

\author{
$\underline{\text { Leslie J. Dickmann }^{1}}{ }^{\text {, Sonal K. Patel }}{ }^{1}$, Helen McBride ${ }^{2}$, Kent Miner ${ }^{3}$, Larry C. Wienkers ${ }^{1}$ and J. Greg \\ Slatter ${ }^{1}$
}

${ }^{1}$ Pharmacokinetics and Drug Metabolism, Amgen Inc., Seattle, WA, ${ }^{2}$ Inflammation Research, Amgen Inc., Thousand Oaks, CA, Inflammation Reserach, Amgen Inc., Thousand Oaks, CA

The murine collagen antibody-induced arthritis (CAIA) model and primary mouse hepatocytes treated with IL-1 $\beta$, IL-6, or TNFa alone and in combination were used to investigate changes in cytochrome P450 expression incurred by inflammatory disease. CAIA in female mice increased serum IL-1 $\beta$, IL-6 and hepatic mRNA of the acute phase response (APR) marker, serum amyloid A (SAA). The CAIA model significantly altered cytochrome P450s mRNA and activity levels and UGTs mRNA levels. Most P450s and UGTs were down-regulated, although some isoforms, such as Cyp3a13, were 
up-regulated. Hepatic P450-related effects of CAIA and the effects of IL-6, IL-1 $\beta$, and TNFa on mouse hepatocytes were compared at in vitro cytokine concentrations similar to those measured in CAIA mouse serum in vivo. In vivo/in vitro suppression by cytokines was congruent for some P450 isoforms (Cyp1a2, Cyp2c29, and Cyp3a11) but not for others (cytochrome P450 oxidoreductase (POR) and Cyp2e1). In mouse hepatocyte culture, IL-6 and IL-1 $\beta$ cytokines in combination in vitro caused a large synergistic increase in SAA expression but not in cytochrome P450 suppression. IL-1 $\beta$ and IL-6 were both equipotent at suppressing cytochrome P450 gene expression while TNFa caused only mild suppression at the highest concentrations used. TNFa in combination with IL-1 $\beta$, IL-6, or both had a protective effect against IL-1 $\beta$ and IL-6 mediated P450 suppression. When IL-1 $\beta$ or IL- 6 was combined with low concentrations of TNFa, several P450 isoforms were induced rather than suppressed. In a pilot study, commercial murine IL-6 and IL-6R antibodies did not lower liver mRNA levels of APR proteins in the CAIA mouse and did not de-suppress cytochrome P450 levels in the CAIA mouse liver. The factors underlying the lack of effect of the commercial anti-cytokine monoclonal antibodies, including dose and role of other cytokines, in mice warrant more detailed experimentation. These data highlight the in vitro/in vivo similarities and differences between the CAIA and cultured hepatocyte models and the pros and cons of each model.

\title{
P160. Constitutive androstane receptor transactivates human ABCG2 in hepatocytes through a novel cis-element in the distal promoter
}

\author{
Kouichi Yoshinari, Satoshi Benoki, Tsubasa Chikada, Jun Imai and Yasushi Yamazoe \\ Graduate School of Pharmaceutical Sciences, Tohoku University, Sendai, Japan
}

ABCG2, also known as breast cancer resistance protein or BCRP, is an efflux transporter that is involved in the excretion of various compounds in multiple tissues and in the multidrug resistance in tumor cells. Its substrates include endobiotics such as estrone sulfate and urate, anticancer agents such as mitoxantrone, topotecan and methotrexate, and metabolites of environmental chemicals such as benzo[a]pyrene-3-sulfate and benzo[ $[a]$ pyrene-3-glucuronide. Recent studies have reported that $A B C G 2$ mRNA levels in cultured human hepatocytes were increased after treatment with phenobarbital and that treatment of epileptic patients with carbamazepine caused the increase in hepatic $A B C G 2 \mathrm{mRNA}$ levels. These results suggest a role of constitutive androstane receptor (CAR) in the transcriptional activation of human $A B C G 2$ (hABCG2). The precise mechanism, however, remains unknown. In the present study, hABCG2 mRNA levels in human hepatocytes were found to increase after infection with adenovirus expressing human CAR (hCAR) and treatment with its activator. Reporter assays using reporter plasmid containing various lengths of $\mathrm{h} A B C G 25^{\prime}$-flanking regions and hCAR expression plasmid suggested that an hCAR-responsive element is located between -8000 and -7485 . With electrophoretic mobility shift assays and chromatin immunoprecipitation assays, a DR5 motif (direct repeat separated by 5 nucleotides), located at -7988 to -7972 in a 3' to 5' orientation, was identified as a binding motif of hCAR/human retinoid X receptor a heterodimer. The motif was termed G2-DR5. The introduction of mutations into G2-DR5 of the hABCG2 reporter genes resulted in the complete loss of the hCAR-mediated increase in reporter activities. In addition, hCAR transactivated the reporter construct containing three copies of wild-type G2-DR5, but not mutated G2-DR5, under thymidine kinase promoter. These results suggest a critical role of G2-DR5 in the hCAR-mediated transactivation of hABCG2. Interestingly, the extent of hCAR-mediated transactivation was higher with the construct containing three copies of G2-DR5 in a natural orientation (3' to 5') was higher than that containing complement G2-DR5 motifs. Finally, we found that human pregnane X receptor, which belongs to the same NR1I subfamily of the nuclear receptor superfamily as CAR, did not activate any reporter gene containing G2-DR5 in reporter assays. Taken together, our results suggest that in human hepatocytes hCAR transactivates $\mathrm{h} A B C G 2$ through the DR5 motif located in the distal promoter of $\mathrm{h} A B C G 2$ and that the motif is specific to CAR but not pregnane $\mathrm{X}$ receptor.

\section{P161. Abstract withdrawn}

\section{P162. Global gene expression changes induced in primary human hepatocytes by thiazolidinediones upon repeat dosing of hepatopac ${ }^{\mathrm{Tm}}$ cultures}

\author{
Michael McVay and Salman Khetani
}

Hepregen Corporation, Medford, $M A$

Global gene expression changes as assessed using whole genome microarrays can provide insights into the mechanism of action of drug candidates. Furthermore, signatures of gene expression may distinguish compounds with potential to cause tissue injury with diverse mechanisms from the non-toxic compounds. In the case of the liver, primary 
human hepatocytes are widely considered to be the most suitable to assess drug-induced gene expression changes in vitro since they contain the full repertoire of regulatory pathways; however, hepatocytes display a precipitous decline in phenotypic functions when left in suspension or cultured in a sandwich of extracellular matrix proteins. Therefore, hepatocytes in these conventional systems are generally unsuitable for assessment of gene expression changes upon chronic exposure in order to mimic clinically relevant dosing regimens. We have previously developed a microscale model of the human liver in which primary human hepatocytes are organized in colonies of empirically optimized dimensions and subsequently surrounded by supportive stromal cells (HepatoPac ${ }^{\mathrm{mI}}$ ). Here, we sought to discern the effects of acute (24 hours) and chronic ( 7 to 14 days) drug exposure on the transcriptome of primary human hepatocytes using the hepatotoxic and non-toxic drug pair Troglitazone and Rosiglitazone, respectively. HepatoPac cultures were dosed from 24 hours to 14 days at a sub-lethal and clinically relevant C[max] dose. Messenger RNA was collected at 24 hours, 7 days, and 14 days after dosing, and hybridized to whole genome human Affymetrix GeneChips ${ }^{\mathrm{TM}}$. Subsequent analysis of gene expression data with GeneSpring software revealed a time-dependent increase in statistically significant gene expression changes induced by both Troglitazone and Rosiglitazone as compared to vehicle controls. However, Rosiglitazone significantly changed the expression of 2, 16, and 221 genes, while Troglitazone changed the expression of 17, 409 and 928 genes over 24 hours, 7 days, and 14 days, respectively. In particular Troglitazone caused up-regulation of several genes involved in cellular pathways such as oxidation-reduction, lipid metabolism and biosynthesis, and stress/wounding response. Studies are currently underway to evaluate drug-induced gene expression changes of multiple toxic/non-toxic pairs in order to evaluate common pathways. In the future, HepatoPac cultures may be utilized with both gene expression and biochemical assays to select optimal drug candidates in both drug discovery and development.

\title{
P163. Identification and characterization of ABCG2 enhancer regions
}

\author{
$\underline{\text { Rachel J. Eclov, Nadav Ahituv and Deanna L. Kroetz }}$
}

\section{Bioengineering and Therapeutic Sciences, University of California of San Francisco, San Francisco, CA}

The mitoxantrone resistance protein (MXR; BCRP; ABCG2), referred to here as MXR, is an efflux membrane transporter expressed apically in several tissues with a broad range of both exogenous and endogenous substrates. The transport activity, tissue distribution and cellular localization of MXR suggest that it plays a pivotal role in endogenous substrate disposition as well as the protection and detoxification of the body from xenobiotics. Overexpression of MXR is associated with drug resistance to a variety of anticancer drugs, and it has been linked with decreased diseasefree survival in several cancers. An individual's susceptibility to certain drug-induced side effects has also been linked to MXR expression or nonsynonymous single nucleotide polymorphisms (SNPs) in the MXR gene ABCG2. Therefore, it is important to not only characterize the functional effects of $A B C G 2$ SNPs, but to also look at other mechanisms which alter MXR expression and function. We hypothesize that there are regulatory regions surrounding $A B C G 2$, and our aim for this study was to identify and characterize regions surrounding $A B C G 2$ that increase gene expression. We computationally analyzed a $\sim 300 \mathrm{~kb}$ stretch of DNA (chr4:89130400-89439035, hg18), which includes the $A B C G 2$ gene, in order to identify putative $A B C G 2$ enhancers and then tested these regions for in vitro and in vivo enhancer activity. Genomic regions with potential enhancer capabilities were identified as having high evolutionary conservation determined by comparative genomics (ECR and Vista browsers) and/or the presence of specialized transcription factor binding sites (TFBS) identified with computational methods (rVista, Transfac, Cister). These regions were cloned from human DNA and inserted into the enhancer assay vector pGL4.23. The constructs were transiently transfected into liver (HepG2), kidney (HEK293T), intestine (HCT116) and breast (MCF-7) cell lines, and the luciferase gene activity was measured as a surrogate for the enhancer capabilities of the genomic region. We tested 29 regions and identified seven separate regions (250-1000 bp in length) surrounding ABCG2 exhibiting significant enhancer activity in vitro. Global and cell-specific enhancers were identified, with at least three enhancer regions in each of the cell lines. HEK293T, MCF-7 and HepG2 cell lines each had two cell-specific enhancer regions. An additional region showed enhancer activity in both the HepG2 and HCT116 cell lines; two global enhancer regions showed activity in all four cell lines. Studies are ongoing utilizing the mouse tail vein injection assay to test these regions for in vivo enhancer activity in the liver. Our study provides evidence that there are genomic regions surrounding $A B C G 2$ capable of increasing gene expression, possibly in a tissue-specific manner. The results from this research will inform future clinical investigations examining how interindividual variation in MXR expression and function contributes to differences in drug response. This work was supported by NIH grant GM61390 and a predoctoral fellowship from the American Foundation for Pharmaceutical Education. 


\title{
P164. In Vitro effects of cytokines on cytochrome P450 expression in human hepatocyte culture
}

\author{
Leslie J. Dickmann, Sonal K. Patel, Larry C. Wienkers and J. Greg Slatter
}

Pharmacokinetics and Drug Metabolism, Amgen Inc., Seattle, WA

The in vitro effects of interleukin $1 \beta$ (IL-1 $\beta$ ), interleukin 6 (IL-6), and tumor necrosis factor alpha (TNF $\alpha$ ), alone and in combination, on cytochrome P450 expression in human hepatocytes were investigated. Human hepatocytes were treated for 72 hours with individual cytokines and cytokine combinations followed by measurement of cytochrome P450 mRNA, CYP1A2 and CYP3A4 marker activities, and the acute phase response marker, c-reactive protein (CRP). Cytokine concentration ranges were chosen to encompass physiologic serum concentrations observed in health and disease. Previous studies had shown that treatment of human hepatocytes ( $\mathrm{N}=8$ donors) with IL-6 caused a pan-suppression of mRNA of 10 major cytochrome $\mathrm{P} 450$ isoenzymes, with $\mathrm{EC}_{50}$ values that differed by isoenzyme. Marker activities for CYP1A2 (average donor $\mathrm{EC}_{50}=1116 \pm 1260 \mathrm{pg} / \mathrm{mL}$ ) and CYP3A4 (average donor $\mathrm{EC}_{50}=67.8 \pm 52.8 \mathrm{pg} / \mathrm{mL}$,) illustrated isoform-specific differences in IL-6 potency. ${ }^{1} \mathrm{In} N=5$ donors, IL- $1 \beta$ suppressed mRNA of the same 10 cytochrome P450 isoenzymes. Suppression was donor dependent and $\mathrm{EC}_{50}$ values of suppression were greater than those obtained with IL-6. Suppression of CYP1A2 marker activity by IL-1 $\beta$ could not be measured in 3 of 5 donors due to lack of response, and in the two remaining donors the average $\mathrm{EC}_{50}$ was $450 \mathrm{pg} / \mathrm{mL}$. For CYP3A4 activity, the $\mathrm{EC}_{50}$ of suppression was $416 \pm 454 \mathrm{pg} / \mathrm{mL}$ indicating that IL- $1 \beta$ was less potent than IL- 6 . When human hepatocytes $(\mathrm{N}=1$ donor) were treated with IL- $1 \beta$ and IL-6 in combination at concentrations ranging from 1-100 pg/mL, IL-6 was the main determinant of mRNA increases in CRP and serum amyloid A (SAA) and mRNA decreases in CYP3A4. IL-1 $\beta$ and IL-6 in combination did not show synergy in either CRP or SAA up-regulation or P450 down-regulation, although the effects of the two cytokines in combination were additive in certain instances. Results obtained with TNF $\alpha$ used in combination with IL-1 $\beta$ and IL- 6 will also be presented. These data indicate that IL- $1 \beta$ and IL- 6 both suppress cytochrome P450 mRNA and enzymes levels in vitro and that, at similar physiologically-relevant concentrations in vitro, IL-6 is more potent than IL- $1 \beta$.

${ }^{1}$ Dickmann LJ et al. Drug Metab Dispos May 9 2011, epub ahead of print.

\section{P165. Influence of inflammatory processes on ADME gene expression in human liver and hepatocytes}

\author{
$\underline{\text { Marcus Klein }}^{1}$, Benjamin A. Kandel ${ }^{1}$, Kathrin Klein ${ }^{1}$, Maria Thomas ${ }^{1}$, Wolfgang E. Thasler ${ }^{2}$, Matthias \\ Schwab $^{1}$ and Ulrich M. Zanger ${ }^{1}$
}

${ }^{1}$ Dr. Margarete Fischer-Bosch Institute of Clinical Pharmacology, Stuttgart, Germany, ${ }^{2}$ Surgery, LM University Munich, Hospital Grosshardern, Munich, Germany

During inflammation local inflammatory cells of the liver (Kupffer cells and liver-infiltrating macrophages) produce pro-inflammatory cytokines such as TNF $\alpha, \mathrm{IL}-1 \alpha, \mathrm{IL}-1 \beta$ and IL-6. These key-mediators play important roles in hepatocellular signaling pathways and in controlling cellular homeostasis. They are responsible for the induction of the acute-phase response that results in dramatic changes in gene expression, involving numerous genes of importance for absorption, distribution, metabolism and excretion (ADME) of drugs. Here we present three different approaches to explore the role of inflammation pathways in ADME gene expression. First, we analyzed genome-wide mRNA expression data from 150 liver samples surgically removed from Caucasian donors with respect to the human acute-phase protein C-reactive protein (CRP), a common marker of inflammation. In seven liver donors presenting with clinically increased CRP numerous genes were identified with significantly decreased (e.g. CYP3A4, CYP1A2, GSTA1) or increased expression (e.g. SULT1C2, SLC2A10). Second, we investigated the role of genetic polymorphisms in inflammatory pathways for expression of ADME genes. For this purpose selected SNPs of the IL-6 and NFKB signaling pathways were genotyped and their association with the mRNA expression levels of several ADME genes was analyzed. We identified polymorphisms in IL6ST (gp130), IL-6 and NFKB genes to be significantly associated with expression of several ADME-genes including CYP3A4. Third, we investigated the influence of IL-6 on ADME gene expression in primary human hepatocytes. For this purpose, human hepatocytes were treated with IL-6 and mRNA-profiles were obtained at different time points by performing high-throughput qPCR. IL-6 significantly down-regulated gene-expression of many CYPs including CYP3A4, whereas few CYPs were up-regulated. The data also indicated IL-6-mediated effects on expression of nuclear receptors, transcription factors and transporters. These combined approaches shed new light on inflammatory influences 
on ADME gene expression and should be useful to develop biomarkers for disease-related changes in drug metabolism capacity. Supported by the German BMBF (Virtual Liver grant 0315755) and the Robert Bosch Foundation, Stuttgart, Germany.

\title{
P166. Investigating receptor crosstalk and ADME gene expression using a high-throughput RT-qPCR method in human hepatocytes and HepaRG cells
}

\author{
Joshua G. DeKeyser, Michael Hayashi and Magang Shou
}

Pharmacokinetics and Drug Metabolism, Amgen Inc., Thousand Oaks, CA

Changes in the expression of absorption, distribution, metabolism and excretion (ADME) genes can result in altered pharmacokinetics of administered drugs and can cause clinical drug-drug interactions. Primary human hepatocytes are the "gold standard" for investigations into changes in ADME gene expression but suffer from an erratic supply and large inter-donor variability. A recently developed cellular model, HepaRG, displays many of the properties of human hepatocytes and has shown promise as a model of ADME gene expression due to expression of the xenobiotic-sensing receptors constitutive androstane receptor (CAR), pregnane X receptor (PXR) and aryl hydrocarbon receptor (AHR) and increased expression of their target genes in response to prototypical inducers. The xenobiotic-sensing receptors demonstrate considerable crosstalk both in the genes that they induce and the chemicals that activate them. The aim of this work was to investigate the crosstalk issue by using a holistic approach. We utilized both primary human hepatocytes and highly differentiated HepaRG cells along with a high-throughput RT-qPCR methodology to simultaneously monitor changes in the expression of 22 or 90 different ADME genes after treatment with different inducers across a broad range of concentrations. By looking at the expression of many different target genes at once we are able to make predictions about the involvement of different receptors at different concentrations of inducers. The results demonstrate a good correlation in the induction response of 90 different ADME genes between primary human hepatocytes and the HepaRG cells after treatment with rifampicin, phenobarbital and omeprazole. Concentration-response analysis of 22 ADME genes in HepaRG cells showed that CITCO, a CAR ligand, induced CYP2B6 at much lower concentrations than CYP3A4 $\left(\mathrm{EC}_{50}=2.5 \mathrm{nM}\right.$ and $2.2 \mu \mathrm{M}$, respectively). In contrast, phenobarbital a non-ligand activator of CAR, did not demonstrate the large difference in potency between CYP2B6 and CYP3A4 induction $\left(\mathrm{EC}_{50}=792 \mu \mathrm{M}\right.$ and $481 \mu \mathrm{M}$, respectively). This result may suggest a difference in the affinity of CAR for its promoters or cofactors depending on whether CAR is being activated through direct or indirect mechanisms. The PXR agonist TO901317 demonstrated saturated induction of CYP2B6 and CYP3A4 at $1.5 \mathrm{nM}$, the lowest concentration tested and also induced the bile-salt export pump (BSEP) at higher concentrations $\left(\mathrm{EC}_{50}=3.1 \mu \mathrm{M}\right)$ suggesting a possible role of the farnesoid $\mathrm{X}$ receptor at this concentration.

\section{P167. Lentiviral knock down of the nuclear receptors CAR and PXR in human hepatocytes}

\author{
$\underline{\text { Benjamin A. Kandel }}{ }^{1}$, Jessica K. Rieger ${ }^{1}$, Maria Thomas ${ }^{1}$, Nina Hofmann ${ }^{1}$, Daniel Knobeloch ${ }^{2}$ and \\ Ulrich M. Zanger ${ }^{1}$
}

${ }^{1}$ Dr. Margarete Fischer-Bosch Institute of Clinical Pharmacology, Stuttgart, Germany, ${ }^{2}$ Div. of Experimental Surgery, BCRT, Charité Universitätsmedizin Berlin, Berlin, Germany

The liver enriched orphan nuclear receptors (NRs) CAR and PXR are the major sensors of drugs and other xenobiotic compounds and are involved in the regulation of many ADME (absorption, distribution, metabolism and excretion) genes including CYP3A4, members of the CYP2C family as well as phase II enzymes and transporters. The commonly used techniques to elucidate NR-mediated regulation of gene expression was mostly done in cell-based reporter assays or in mouse or rat models by using prototypical agonists or antagonists in wild-type or gene knock-out background. Because cell-lines do not express ADME genes well (in particular CYPs) and because of the high species differences with respect to PXR and CAR ligand and target gene specificity, the data collected from these model systems may not be truly reflecting the situation in human liver. The best known in vitro system available to investigate human liver metabolism and its underlying regulatory mechanisms are surgery-derived primary cultures of human hepatocytes. To achieve highly efficient gene knock-down we designed lentiviral vectors to introduce shRNA-expression cassettes into primary human hepatocytes in order to simulate a loss of function phenotype. For comparison, hepatocytes were treated with the prototypical agonists for PXR (rifampicin) and CAR (CITCO). To investigate the occurring alterations in the mRNA-expression profile we performed high-throughput quantitative real-time PCR on a Fluidigm platform. Changes on protein level were analysed by Western blotting. To examine changes in the activity of the CYPs 1A2, 3A4, 
2B6, 2C8, 2C9, 2C19 and 2D6 we used a previously established LC-MSMS-based cocktail-assay.We show that lentiviral particles containing shRNA against CAR or PXR not only reduce the mRNA-levels of CAR and PXR, respectively, but also decrease the mRNA levels of genes regulated by these two NRs, including CYP3A4 and CYP2B6. Furthermore, treatment with the agonists CITCO or rifampicin for CAR or PXR resulted in an increase in the expression of the respective target genes which could be effectively attenuated by lentiviral infection. Whole genome expression-profiling via Affymetrix microarrays will now be used to elucidate the "regulome" of these NRs. Combination of the knock-down technique with other treatments proposed to influence the transcriptional activity of CAR and PXR are ongoing. Additionally other NRs will be included in this type of experiments. This study was supported by the German Federal Ministry of Education and Research (Virtual Liver grant 0315755) and the Robert Bosch Foundation, Stuttgart, Germany.

\title{
P168. MicroRNAs in posttranscriptional regulation of drug metabolism and disposition
}

\section{$\underline{\text { Ai-Ming Yu }}$}

Department of Pharmaceutical Sciences, University at Buffalo, SUNY, Buffalo, NY

Considerable variations in drug metabolism and disposition are well recognized, which may lead to a reduced drug efficacy or an adverse drug reaction in pharmacotherapy. Some of the mechanistic causes are know, e.g., genetic polymorphism, inhibition and transcriptional regulation of drug-metabolizing enzymes or transporters. However, it remains obscure how xenobiotic metabolism and disposition is regulated at the posttranscriptional level. MicroRNAs (miRNAs) represent a large group of short, noncoding RNAs that control posttranscriptional expression of target genes. Currently, more than 1,000 miRNAs have been identified in human genome, which may regulate thousands of proteincoding genes. Some miRNAs have been shown to directly or indirectly control the expression of P450 drug-metabolizing enzymes, $\mathrm{ABC}$ transporters and/or nuclear receptors. Herein we present our findings on noncoding miRNAs in regulation of drug-metabolizing enzyme CYP3A4, ABC transporter BCRP/ABCG2 and MRP1/ABCC1, and nuclear receptor VDR/NR1I1. Consequently, intervention of miRNA signaling may modulate target gene expression, alter the capacity to dispose a drug, and influence the sensitivity of cells to xenobiotic drugs. In addition, the expression of some miRNAs is significantly changed in cells following the exposure to specific xenobiotic drugs. Therefore, miRNA-mediated posttranscriptional gene regulation may represent a new mechanism underlying interindividual variations in drug metabolism and disposition. Acknowledgment: The work is supported by the Interdisciplinary Research Development Fund (IRDF), University at Buffalo, The State University of New York, and in part by the award number R01DA 021172 from National Institute on Drug Abuse, National Institutes of Health.

\section{P169. Molecular regulation of aldehyde dehydrogenase 1a1 by electrophiles: Role of AP-1 transcription factors}

\author{
Ngome L. Makia ${ }^{1}$, Keith Cameron Falkner ${ }^{2}$, Daniel J. Conklin ${ }^{3}$ and Russell A. Prough ${ }^{4}$
}

${ }^{1}$ Biochemistry \& Molecular Biology, U. Louisville School of Medicine, Louisville, KY, ${ }^{2}$ Dept of Medicine/Gastroenterology, University of Louisville, Louisville, KY, ${ }^{3}$ Medicine/Cardiology, U. Louisville School of Medicine, Louisville, KY, ${ }^{4}$ Dept of Biochem \& Molec Biol, University of Louisville School of Medicine, Louisville, KY

The lipid aldehydes, 4-hydroxy-2-nonenal (4-HNE) and propene-2-al (acrolein) are reactive alpha,beta-unsaturated aldehydes generated during the peroxidation of lipids and are implicated in the pathogenesis of several oxidative-stress mediated diseases, including steatohepatitis and cancer. We have established that recombinant mouse liver Aldhlal efficiently metabolizes lipid aldehydes and protects a liver-derived cell line from the toxic effects of these aldehydes. Thus, mechanisms to induce the expression of Aldhlal might be a useful rational for preventing oxidative stress-induced pathologies. The purpose of the study was to investigate whether electrophiles modulate the expression of Aldhlal and to elucidate the signaling pathway involved. We performed microarray analyses to establish the effect of acrolein or BHA on the expression of hepatic Aldhlal. Mice were administered AIN76A (control) diet, diet containing 0.45\% BHA or $5 \mathrm{mg} / \mathrm{kg}$ acrolein by gavage. The mRNA levels of Aldhlal and other electrophiles detoxification enzymes was induced by acrolein and BHA. We confirmed the microarray result by quantitative real-time PCR analysis, which showed 2.5-fold and 3-fold induction of Aldhlal gene expression by BHA and acrolein respectively. BHA- and acrolein-treated mice liver also showed increased cytosolic Aldh activity compared to control. Acrolein and tert-butylhydroquinone (the metabolized products of BHA) are electrophiles that induce oxidative stress in cells and are activators of NF-E2-related factor-2 (Nrf2) and AP-1 transcription factors. To decipher the signaling pathways involved in Aldh1al induction, we 
analyzed the mRNA levels of Aldhlal in the liver of WT and Nrf2-/- mice exposed to BHA. Mice exposed to BHA showed 2-fold increase in mRNA levels of Aldhlal in WT and Nrf2-/- mice compared to control, indicating that electrophileinduced expression is not dependent on Nrf2. However, the mRNA and protein levels of AP-1 and the activity of c-Jun were significantly increased by BHA. We hypothesized that BHA- and acrolein-induced expression of hepatic Aldhlal is mediated by AP-1 transcription factor. Transient transfection experiments were conducted in HepG2 cells with Aldhlal 5'-flanking luciferase reporter vectors. While co-transfection with Nrf2 expression plasmid alone or in the presence of tBHQ had no effect, over-expression of c-Jun/AP-1 resulted in 4-fold induction in Aldhlal transcriptional activity. We further showed that c-Jun mediates Aldhlal promoter activity as a homodimer and not as c-Jun/c-Fos heterodimer. We also established by promoter deletion and mutagenesis analysis that two AP- 1 sites at position -758 and -1069 relative to Aldhlal transcription start site are responsible for induction by c-Jun. EMSA analysis using biotin-labeled probe and super shift with antibodies against c-Jun, c-fos and Nrf2 showed that c-Jun binds to the proximal AP-1 site at -758 but not at -1069. The recruitment of c-Jun to this AP-1 site by BHA was confirmed by ChIP experiment, which showed 10-fold enrichment to the proximal AP-1 site with c-Jun. These results indicate that electrophiles promote the recruitment of c-Jun/AP-1 to the Aldhlal gene promoter, resulting in increased transcription of Aldhlal. Supported in part by USPHS ES11860.

\title{
P170. Temporal changes in CYP3A4 mRNA and activity following treatment of cultured human hepatocytes with interleukin-6 (IL-6): Implications for study design and endpoint selection
}

\author{
Michelle McBride, Jason Neat, David Buckley, Jennifer Simpson, Bradley Klaus, Janet Sawi, Paul \\ Bolliger and Andrew Parkinson
}

XenoTech, LLC, Lenexa, KS

Therapeutic proteins, such as cytokines, monoclonal antibodies and soluble receptors, can increase systemic exposure to small drug molecules by suppressing hepatic cytochrome P450 levels by activating transcription factors like NFKB either directly (as occurs with IL-1 $\beta$, IL-6, IFN $\gamma$ and TNF $\alpha$ ) or indirectly (by stimulating the release of these pro-inflammatory cytokines from Kupffer or circulating blood cells). In this study, we examined the time course of changes in CYP3A4 activity (measured as microsomal testosterone $6 \beta$-hydroxylation) and mRNA levels (measured by qRT-PCR) in three primary cultures of human hepatocytes treated once or once daily for three consecutive days with interleukin-6 (IL-6; $10 \mathrm{ng} / \mathrm{mL}$ ). Under conditions where the control CYP3A4 activity was relatively stable over the three-day treatment period and inducible (more than 10 fold) by the PXR agonist rifampin, the following cytokine-mediated changes were observed:

1. Treatment of human hepatocytes with a single or multiple applications of IL-6 suppressed CYP3A4 mRNA levels and, to a lesser extent, CYP3A4 activity in all three hepatocyte preparations.

2. Following a single application of IL-6, suppression of CYP3A4 mRNA (71-89\%) was maximal after $24 \mathrm{~h}$ whereas suppression of CYP3A4 activity (51-77\%) was maximal after $48 \mathrm{~h}$. Likewise, three days after a single application of IL-6, recovery of CYP3A4 mRNA levels was greater than the recovery of CYP3A4 activity.

3. Compared with a single application, multiple applications of IL-6 (once a day for 3 days) caused greater suppression of both CYP3A4 mRNA $(\sim 80 \% \rightarrow \sim 90 \%)$ and CYP3A4 activity $(\sim 37 \% \rightarrow \sim 70 \%)$.

These data suggest that, for a single application of pro-inflammatory cytokine, mRNA is a suitable endpoint when examined after 24 hours whereas CYP activity is best evaluated 48 hours post-treatment. Consequently, the selection of a treatment regimen (single vs. multiple daily treatments) is an important factor in the selection of both time-points and endpoints (mRNA or activity) when evaluating therapeutic proteins for the potential to modulate CYP3A4 expression.

\section{P171. Comparison of the effects of all-trans-retinoic acid in HepG2 and MCF7 cells using semi- quantitative shotgun proteomics}

\author{
Samuel L.M. Arnold ${ }^{1}$, John D. Chapman², David R. Goodlett ${ }^{2}$ and Nina Isoherranen ${ }^{1}$
}

${ }^{1}$ Pharmaceutics, University of Washington, Seattle, WA, ${ }^{2}$ Medicinal Chemistry, University of Washington, Seattle, WA

Decreased proliferation in a number of cell lines treated with all-trans Retinoic Acid (atRA) has been well documented[1], but the inhibition in proliferation is not seen in all cell lines, and the mechanism is not fully understood. It has been proposed that different expression profiles of nuclear receptors (RAR, PPAR) and soluble binding proteins (CRABP-II, 
FABP5) are responsible for the different effects[2] via specific channeling of atRA between binding proteins and nuclear receptors. Therefore it was hypothesized atRA treatment would be associated with different proteomic profiles in different cell lines due to different nuclear receptor activation. To test this hypothesis, the proteomic signatures of MCF7 and HepG2 cells were characterized after atRA treatment. MCF7 cells were chosen based on the well documented effects of atRA on their proliferation. HepG2 cells were used as based on the observation the proliferation of the cell line is resistant to atRA treatment. The effect of atRA in cell proliferation was determined by MTT assays, and metabolism of atRA was monitored by the formation of known atRA metabolites, which also inhibit proliferation[1]. For proteomic analysis, the cells were treated with $10 \mu \mathrm{M}$ atRA or vehicle DMSO control for 72 hours. All samples were analyzed on a LTQ Velos (Thermo) using the data-independent-acquisition method (PAcIFIC) described by Panchaud et al. [3]. Protein identifications were made using SEQUEST requiring a false discovery rate $<1 \%$ and identification of 2 unique peptides per protein. 73 proteins whose expression was either increased $>20 \%$ or decreased $>20 \%$, were analyzed using DAVID $[4,5]$ to identify atRA associated signaling pathways. A distinct difference in the enriched pathways up-regulated and downregulated between the cell lines was detected and included anti-apoptosis, glycolysis/gluconeogenesis, and pentosephosphate clusters. While down-regulated in atRA treated MCF7 cells, the pathways were up-regulated in HepG2 cells. These responses were associated with a higher rate of atRA metabolism in HepG2 cells compared to MCF7 cells suggesting a potential role of the metabolites in regulation of cellular effect. This data demonstrates that multiplexed shotgun proteomic methods can be used to characterize cell line specific effects of xenobiotics, and will be useful for quantitative comparisons of tissue specific pharmacological of toxicological effects of therapeutic entities.

1. Van heusden, J., et al., The antiproliferative activity of all-trans-retinoic acid catabolites and isomers is differentially modulated by liarozole-fumarate in MCF-7 human breast cancer cells.Br J Cancer, 1998.77(8): p. 1229-35.

2. Schug, T.T., et al., Opposing effects of retinoic acid on cell growth result from alternate activation of two different nuclear receptors.Cell, 2007.129(4): p. 723-33.

3. Panchaud, A., et al., Precursor acquisition independent from ion count: how to dive deeper into the proteomics ocean.Anal Chem, 2009.81(15): p. 6481-8.

4. Huang da, W., B.T. Sherman, and R.A. Lempicki, Systematic and integrative analysis of large gene lists using DAVID bioinformatics resources. Nat Protoc, 2009. 4(1): p. 44-57.

5. Huang da, W., B.T. Sherman, and R.A. Lempicki, Bioinformatics enrichment tools: paths toward the comprehensive functional analysis of large gene lists.Nucleic Acids Res, 2009.37(1): p. 1-13.

\title{
P172. global transcriptomic profiling of LPS, TNFa, and IL-4 in primary human hepatocytes
}

\author{
Michael J. Liguori, Rita Ciurlionis, Amy C. Ditewig, Eric A.G. Blomme and Yi Yang
}

Cell, Molecular, \& Exploratory Toxicology, Abbott Laboratories, Abbott Park, IL

An inflammatory state has been associated with the decreased clearance of multiple drugs via mRNA repression and activity inhibition of multiple cytochrome P450 enzymes (CYPs). This phenomenon disallows accurate assessment of the pharmacokinetics in humans and can lead to changes in drug efficacy and toxicity thresholds. This repression effect has been linked to the increased release of several cytokines, including TNF $\alpha$, IL-2, and interferons. To understand the molecular pathways in the hepatocyte transcriptome upon exposure to inflammatory mediators, we investigated the effects of lipopolysaccharide (LPS) and two well-characterized cytokines, TNF $\alpha$ (proinflammatory) and IL-4 (both pro- and anti-inflammatory), on the global mRNA expression in human hepatocytes. First, naïve human hepatocytes from a panel of 8 donors were shown to express moderate levels of the IL-4, TNF, toll-like, and several other receptors. Subsequently, human hepatocytes plated in collagen coated plates were treated with IL-4, TNF $\alpha$, or LPS for $48 \mathrm{~h}$. The RNA from these cells was then analyzed using whole human genome Affymetrix microarrays. The global gene expression profiles, revealing $>3000$ transcriptomic changes, were similar for LPS and TNF $\alpha$ treated hepatocytes, but remarkably distinct from the IL-4 treatments. In general, several major liver CYPs were repressed in all treatment groups, including CYP3A4 which was repressed to a lesser degree in IL-4 treated hepatocytes. CYP2E1 was induced only upon IL-4 exposure. Few transcripts $(\sim 40)$ were upregulated in common between all treatments with several of these being involved in cell adhesion, migration, and chemotaxis. Considerably more genes $(\sim 150)$ were downregulated similarly in all of the treatments, including GSTs, FMO3, UGTs, and transporters. The PXR xenosensor, a major regulator of CYP expression, was significantly repressed by all treatments. $H N F 4 \alpha$, which also participates in the regulation of phase I enzymes, was significantly downregulated in the presence of TNF $\alpha$ and LPS, but not IL-4. Both LPS and TNF $\alpha$ treatments substantially enhanced the expression of secondary cytokines, such as MCP-1, IL-1, IL-6, and IL-8, but IL-4 treatment had little effect on the level of proinflammatory factors. Both LPS and TNF $\alpha$, but not IL-4, significantly amplified the expression of multiple factors involved with toll-like receptor and NF- $\mathrm{KB}$ signaling. IL-4 had a significant repression on TGF $\beta$ and PDGF 
signaling with only minor changes observed with TNF $\alpha$ and LPS. IL-4 upregulated the expression of several cyclins, whereas TNF $\alpha$ and LPS repressed these factors. These data support the notion that TNF $\alpha$ and LPS are more proinflammatory compared to IL-4 in primary hepatocytes, and may contribute toward further understanding of the molecular interactions involved in phase I enzyme repression.

\title{
P173. Metabolic effects of naphthalene on mouse urine using NMR-based metabolomics
}

\author{
Ching-yu Lin and Hao-Jan Liang
}

Institute of Environmental Health, College of Public Health, National Taiwan University, Taipei, Taiwan

Metabolic Effects of Naphthalene on Mouse Urine Using NMR-based Metabolomics Naphthalene, the most common polyaromatic hydrocarbons, exists widely in the environment with significant human exposure. Naphthalene has been demonstrated possible carcinogenic from animal investigations. Previous studies have also demonstrated that naphthalene causes bronchiolar epithelial necrosis in mouse distal airway, after parenteral administration. The toxic effects are species and site selective. Formation of reactive metabolites and depletion of glutathione have been correlated with naphthaleneinduced cell injury in mice. Our hypothesis is that naphthalene disrupts intracellular homeostasis and causes metabolic turbulences which can be monitored and associated with cell injury in urine. Metabolomic measurements from noninvasive samples can be applied in exposed human populations to assess the health effects of exposure to environmental toxicants in the future. High-resolution 1H nuclear magnetic resonance (NMR) followed by principal component analysis (PCA) was applied to characterize metabolic effects of naphthalene in leading to cell injury in a susceptible species, mouse. Dose-response $(0,100,200 \mathrm{mg} / \mathrm{kg})$ and time course $(12,24,36,48 \mathrm{hrs})$ experiments were conducted in mice, after parenteral administration. Urine samples were collected for NMR-based metabolomic analysis. Both naphthalene metabolites and endogenous metabolites were examined and correlated to the progress of cell injury. Most of naphthalene metabolites including $\mathrm{N}$-acetyl cysteine naphthalene diol epoxide conjugate and $\mathrm{N}$-acetyl cysteinenaphthalene conjugate were appeared in the NMR spectra of urine at the first 12 hours. Results of PCA from $1 \mathrm{H}$ NMR spectra of urine samples demonstrated doseresponse relationship between naphthalene concentration and urine metabolome at early time points. Partially recovery of urine metabolome was observed at the late time point. Several selected metabolites were semi-quantified by comparing to the control group. Significantly decrease of endogenous metabolites including citrate and 2-oxoglutarate were found from urine of mice treated with high concentration of naphthalene for 12 hours. In addition, the amount of taurine from urine of mice was significantly decreased despite naphthalene doses and time periods after doses. In conclusion, metabolic effects of naphthalene to mice are dose-and time-dependent. We suggest that the decrease of taurine may be due to consumption of glutathione to conjugate to naphthalene electrophilic metabolites. Therefore, little amount of cysteine was available to synthesize taurine. The research was supported by National Health Research Institutes

(NHRI-EX100-9915EC) in Taiwan.

\section{P174. Metabolomic study of the organophosphates and the antidotes by using LC-MS/MS}

\section{$\underline{\text { Shan Jin }}$}

\section{Department of Medicine, Harvard Medical School, Charlestown, MA}

Organophosphates are highly toxic chemicals that can be used as pesticides and chemical weapons. However, there are not enough antidotes or post exposure therapies for organophosphate toxicity. An in vivo HTS method has been developed to discover new antidotes by using a zebrafish model and a number of hit compounds have been identified. A metabolomic study has been used for dissecting toxicity pathways and countermeasure mechanisms. The LC-MS/MS approach has been used to monitor a large number of chemicals such as drugs, neurotransmitters, amino acids, lipids, carbohydrates, and their metabolites in zebrafish with or without treating with organophosphates and the antidotes. Several novel mechanisms and new pathways have been found for potential organophosphate antidotes by the metabolomic study.

\section{P175. The human primary hepatocyte transcriptome reveals novel insights into atorvastatin and rosuvastatin action}

\author{
Mateja Hafner ${ }^{1}$, Peter Juvan ${ }^{1}$, Tadeja Rezen ${ }^{1}$, Katalin Monostory ${ }^{2}$, Jean-Marc Pascussi ${ }^{3}$ and Damjana \\ $\underline{\text { Rozman }}^{1}$
}


${ }^{1}$ Institute of Biochemistry, University of Ljubljana, Center for Functional Genomics and Bio-Chips, Ljubljana, Slovenia, ${ }^{2}$ Dept of Biochem Pharmacol, Chemical Research Center, Hungarian Academy of Sciences, Budapest, Hungary, ${ }^{3}$ Cancer signaling, INSERM U661, Nimes, France

Objectives: With particular emphasis on interactions between cholesterol homeostasis and drug metabolism we investigate the global transcriptome of human primary hepatocytes treated by two commonly prescribed cholesterol lowering drugs atorvastatin and rosuvastatin and by rifampicin that serves as an outgroup as well as a model substance for induction of nuclear receptor PXR. Methods: Hepatocytes from human donors have been treated with rosuvastatin, atorvastatin and rifampicin for 12, 24 and 48h. Expression profiling with cholesterol and drug metabolism enriched low density Steroltalk cDNA and whole genome Affymetrix HG-U133 Plus 2.0 arrays has been applied. Differential expression of genes and gene set enrichment analysis of KEGG pathways were performed. Lists of differentially expressed genes and gene sets were cross-compared. Selected genes were confirmed by qPCR. Results: Statins lead to: (a) upregulation of cholesterol-related genes indicating increased LDL uptake and storage of esterified cholesterol, elevated bile acid/drug export and lower capacity to form HDL; (b) perturbation of genes in glucose and fatty acid homeostasis, influencing acetyl-CoA pools, promoting gluconeogenesis and glucose export; (c) elevated expression of ADIPOR2 suggesting increased sensitivity to adiponectin; (d) perturbations in genes of lipoprotein particle formation, differently for each statin; (e) perturbed expression of many metabolic genes that are directly controlled by nuclear receptors CAR and/or PXR. Conclusions: These data provide a novel global insight into hepatic effects of statins, offering biochemical explanations for higher blood glucose in statin-treated patients, and for drug-induced secondary fatty liver disease. Acknowledgements: This work was supported by the EU FP6 project STEROLTALK and by Slovenian Research Agency projects P1-0104 and J2-2197. Mateja Hafner was supported by the fellowship from the Slovenian Research Agency.

\title{
P176. The identification of novel in vivo preclinical biomarkers for metabolism mediated toxicity using two distinct, eletrophilic near-infrared fluorophores
}

\author{
Brooke M. VandenBrink ${ }^{1}$, Helen J. McBride ${ }^{2}$, Marcus D. Soto ${ }^{3}$, Alexandra Ioanoviciu ${ }^{1}$ and Dan A. \\ Rock $^{1}$ \\ ${ }^{1}$ Pharmacokinetics and Drug Metabolism, Amgen Inc, Seattle, WA, ${ }^{2}$ Inflammation, Amgen Inc, Thousand Oaks, CA, \\ ${ }^{3}$ Pharmacokinetics and Drug Metabolism, Amgen Inc, Thousand Oaks, CA
}

Predicting the impact of drug bioactivation and formation of reactive metabolites remains largely ambiguous. Classic experimental approaches that employ covalent binding have failed to provide a clear link between in vitro bioactivation and in vivo toxicity. The identification of novel biomarkers for metabolism-mediated toxicity (bioactivation) could aid in the development of new chemical entities. As a proof of concept experiment to identify novel in vivo biomarkers for drug bioactivation two polar, electrophilic near-infrared fluorophores, IRDye ${ }^{\circledR}$ 800CW Maleimide and IRDye ${ }^{\circledR}$ 800CW NHS were dosed IV to SCID mice. The main objective of the study was to focus on kidney/renal markers that could be used as potential preclinical biomarkers of metabolism-mediated toxicity. In addition, the biodistribution and disposition of both dyes were characterized by organ imaging, whole body imaging and plasma and urine pharmacokinetic analysis. The results demonstrated that after IV administration both dyes rapidly distributed to all tissues. However by 24 hours, the maleimide-based dye demonstrated higher exposure in liver, kidney and carcass relative to the NHS ester-based dye. Analysis of plasma and urine samples by gel electrophoresis demonstrated clear overlap in the major labeled proteins between the two electrophiles. Proteomic analysis of the in vivo samples is described. Ultimately, the use of surrogate electrophiles may aid in the identification of novel in vivo biomarkers that could be developed and utilized for screening the potential toxicity and safety of new chemical entities.

\section{P177. A novel human hepatocyte culture medium for the maintenance of P450, uptake transporters, and efflux transporters}

\author{
Albert P. Li, Qian Yang and Utkarsh Doshi
}

In Vitro ADMET Laboratories LLC, Columbia, MD

Recent research findings have provided strong evidence that the metabolic fate of a xenobiotic is a result of the integrated activities of uptake transporters, drug metabolizing enzymes, and efflux transporters. An ideal in vitro hepatic model will be cultured hepatocytes that retain all of these three major functions. Freshly isolated or cryopreserved human 
hepatocytes in general retain in vivo levels of drug metabolizing enzymes and uptake transporters, but would lack efflux transporter functions. Prolonged (e.g. 5 day) culturing of the hepatocytes as sandwich cultures would allow expression of the efflux transporters, but drug metabolizing enzymes, especially critical P450 isoforms such as CYP3A4 would be decreased to approximately 5 to $10 \%$ of the level of freshly isolated hepatocytes. We report here the development of a novel medium (Li's Differentiation Maintenance Medium (LDMM)) that has the potential to maintain all three critical hepatic functions: uptake transport, efflux transport, and drug metabolism. RT-PCR results showed that human hepatocytes cultured in LDMM for 5 days on collagen without matrigel overlay exhibited significantly higher expression of genes that are known to decrease with culture duration including the following: liver specific genes transthyretin and transferrin; P450 isoforms 1A2, 2B6, 2C8, 2C9, 2C19, 2D6, 3A4, and 3A5; uptake transporters NTCP, OCT1, OAT2; and efflux transporter BSEP. The genes that did not decline with culture duration such as OATP1B1, OATP1B3, OATP2B1, MDR1, MRP2, MRP3, MRP4 and BCRP remained unchanged or slightly increased in LDMM. CYP3A4 activity measured in human hepatocytes cultured in LDMM was found to be similar to that in thawed cryopreserved hepatocytes before culturing. Results with multiple laboratories on drug metabolizing enzyme activities, uptake transporter functions, and efflux transporter functions will be shown. The discovery of LDMM represents a major advance in hepatocyte culturing and should enhance of the application of this in vitro model to predict the metabolic fate and toxicity of a xenobiotic in vivo.

\title{
P178. A long term culture model for primary hepatocytes from Cynomolgus monkeys
}

\section{Simon Aoyama, Sara Lambirth and Salman Khetani}

\author{
Hepregen Corporation, Medford, MA
}

In vitro models of animal liver tissues are of great interest to drug developers and regulatory agencies because of their potential to reduce costs and live animal use during drug development. Furthermore, drug metabolism and toxicity studies in vitro across human and animal species are utilized for selection of the appropriate animal species for in vivo testing in rodent and non-rodent species. Primary monkey hepatocytes are widely considered to be the most suitable for drug metabolism and toxicity studies; however, these cells display a precipitous decline in phenotypic function when kept in suspension or plated in a sandwich of extracellular matrix. We have developed a microscale model of the Cynomolgus monkey liver in which microfabrication tools adapted from the semiconductor industry were utilized to organize primary hepatocytes into colonies of empirically optimized dimensions and these colonies were subsequently surrounded by supportive murine embryonic 3T3-J2 fibroblasts (monkey micropatterned co-cultures or m-MPCC). We have determined the effects of hepatocyte island diameter and spacing, cell seeding densities, donor lots, and fresh versus cryopreserved sources on the magnitude and lifetime of m-MPCCs. Several liver-specific functions were measured over four weeks including urea synthesis, cytochrome P450 and Phase II activities, and formation of functional bile canaliculi. Our results indicate that m-MPCCs display higher levels of measured functions for at least 4 weeks as compared to traditional cell culture models. For instance, bile canalicular transport was observed with 5-(and-6)-carboxy$2^{\prime}, 7^{\prime}$-dichlorofluorescein diacetate for at least 24 days in culture, while urea synthesis was nearly 100 -fold higher in $\mathrm{m}$-MPCCs than in sandwich cultures on Day 10 of culture (653.13 ug/day/million cells vs. $6.84 \mathrm{ug} / \mathrm{day} / \mathrm{million}$ cells). In addition, cultures showed higher functionality when proliferating fibroblasts were used as compared to growth-arrested ones. The utility of m-MPCCs for drug metabolism and toxicity studies is now currently under investigation. In the future, $\mathrm{m}$-MPCCs may be used as an in vitro predictive tool for refining and reducing testing in live monkeys during drug development.

\section{P179. Assessment of uptake contributions of hepatic uptake transporters}

\author{
Yi-an Bi, Charles Rotter, Emi Kimoto, Kevin Whalen, Bo Feng, Manthena Varma, Ayman El-Kattan \\ and Yurong Lai
}

Department of Pharmacokinetics, Dynamics \& Metabolism, Pfizer Inc., Groton, CT

Purpose: The design of drugs with selective distribution in liver is an effective strategy in the development of hepaticallytargeted drugs which liver plays key roles such as metabolic regulation and is the principal tissue affected by disease. Liver specific transporters such as the organic anion transporting polypeptide (OATP) transporters and organic cation transporters (OCT) are responsible in transportation anionic drugs into hepatocytes, while NTCP plays roles not only for bile acid flow, but also transportation of anionic drugs such as statins. In drug discovery, there is interest in development 
of reliable in vitro assays using transfected cell lines and primary hepatocyte systems to assess the active and passive components and the contributions of each specific transporter. This work is to validate assays to assess different transporter contributions using transfected cell lines, hepatocyte suspension and SCHH. Methods: Uptake assays were run using transfected MDCK cell lines and hepatocyte suspension and SCHH with regular and sodium free buffers. Statins such as rosuvastatin, atovastatin and internal compounds were used as substrates. Rifmycin and sodium free buffer were used to assess active and passive and contributions of OATPs and NTCP. BSEP substrates were also evaluated using NTCP/BSEP double transfected cell line and SCHH in the presence and absence of $\mathrm{Ca}^{2+}$ using medium loss and accumulation methods. Results: The results showed that OATPS were responsible for rosuvastatin uptake and no significant NTCP contribution was observed, while for atovastatin uptake, contributions of OATPs, NTCP and passive diffusion were about 50\%, $20 \%$ and $30 \%$ respectively. The results of transfected cell lines were consistent to the results of hepatocyte assay using sodium free buffer. Conclusion: With uptake assays using transfected cells, hepatocyte suspension and SCHH, contributions of OATPs, NTCP and passive diffusion were assessed. NTCP/BSEP substrates were also indentified. These assays will increase the value, confidence and safety awareness for those liver-targeted drugs in discovery stage.

\title{
P180. Comparison of uptake clearance of rat and human hepatocytes
}

\author{
$\underline{\text { Yi-an Bi }}{ }^{1}$, Emi Kimoto ${ }^{2}$, Kevin Whalen², Ayman EI-Kattan² and Yurong Lai ${ }^{2}$ \\ ${ }^{1}$ Pfizer Inc., Groton, CT, ${ }^{2}$ Pharmacokinetics, Dynamics \& Metabolism, Pfizer Inc., Groton, CT
}

Purpose: Hepatic uptake transport is often the rate-limiting step in hepatic clearance. The ability to predict uptake clearance and species difference is therefore critical in drug development. Currently, hepatocyte suspensions and Sandwichcultured Hepatocytes (SCH) are used to measure initial uptake clearance, but more studies are needed to better define these models. The purpose of this study is to compare the uptake clearance of several OATP and NTCP substrates in suspended hepatocytes and SCH of two species. The results indicate that OATP and NTCP uptake clearance is generally well-maintained in SCHH, but significantly down regulated in SCRH. Activities of uptake transporters are well maintained in hepatocyte suspension of both species and rat suspension show 10 folds higher than human suspension. Methods: Uptake assays were run using rat and human hepatocyte suspensions, and SCRH and SCHH with or without inhibitor. Time points were $0.25,0.5,1$ 1.5, 2, 5 and 10 minutes depended on studies. Statins such as rosuvastatin, atovastatin, pitavastatin, cerivastatin fluvastatin, pravastatin and taurocholate were used as substrates. Results: The results showed that activities of uptake transporters were well maintained in SCHH, $14 \mu \mathrm{l} / \mathrm{min} / \mathrm{mg}$ protein in suspension and 11 on day 5 for rosuvastatin, while significant down-regulations were observed in SRCH, $200 \mu \mathrm{l} / \mathrm{min} / \mathrm{mg}$ protein in suspension to 8 on day 5 . In suspensions, uptake activities in rat are about 10 folds higher than in human. Early time points need to be taken to measure initial uptake in rat suspension, uptake rate from $300 \mu \mathrm{l} / \mathrm{min} / \mathrm{mg}$ protein in $0.25-0.5 \mathrm{~min}$ to 200 in 0.5-1.0 min for rosuvastatin. Conclusion: Transporter-mediated uptake clearance is generally well-maintained in sandwich-cultured human hepatocytes SCHH and is similar to that observed in hepatocyte suspensions, but down regulated in SCRH. Early time points are needed to measure initial uptake in rat.

\section{P181. Comparison of different in vitro hepatic systems for evaluation of intrinsic clearance}

\author{
Rongjun Zuo, Sweta Parikh, Charles L. Crespi and Chris Patten
}

\section{BD Biosciences, Bedford, MA}

Purpose: Primary human hepatocytes are considered the "GOLD STANDARD" for drug metabolism studies since they contain full complement drug metabolism enzymes, drug transporters, and enzyme regulation systems. Primary human hepatocytes in plated culture maintain liver specific function longer than suspension hepatocytes (usually less than 4 hours) or human liver microsomes (usually less than 2 hours), allowing for longer incubation time required for metabolic stability study of low turn over drug candidates. The purpose of this study is to compare different hepatic in vitro metabolism systems for evaluation of intrinsic clearance and prediction of in vivo human clearance. Methods: In vitro hepatic metabolism systems in this study include fresh and cryopreserved human hepatocytes cultured on collagen I plate, cryohepatocytes in suspension, and human liver microsomes. Several commercially available compounds (spanning a range of in vivo human clearance data) were incubated with different in vitro systems, incubation samples were taken at different time points and submitted for LC-MS/MS analysis. Kinetic parameters (Vmax, Km) were estimated and the in vitro intrinsic clearance was calculated through parent compound loss or metabolite formation. Rank order of compounds in the different in vitro systems was compared with in vivo human clearance data. Results: Different in 
vitro hepatic systems showed similar rank order of test compounds, however plated primary human hepatocytes culture showed better prediction of low turn over compounds. Conclusions: Platable human cryopreserved hepatocytes, which can maintain hepatic functions for longer periods compared to suspension hepatocytes or human liver microsomes, are a useful tool for evaluating the metabolic stability of slowly metabolized test compounds.

\title{
P182. Cryo-preserved HepaRG ${ }^{\mathrm{Tm}}$ cells: An alternative to primary human hepatocytes for drug- drug interaction and safety applications
}

\author{
Jonathan P. Jackson, Manda Edwards, Erica Deibert and Stephen S. Ferguson
}

ADME/Tox, Life Technologies, Durham, NC

Currently, primary human hepatocytes (PHHs) are used as the 'gold standard' in vitro hepatic model system due to their ability to support mature hepatic phenotypes (e.g. metabolism, transport, and induction) important in drug clearance, drug-drug interaction (DDI), and safety assessments. However, the use of PHHs for earlier screening applications to identify potential DDI or safety liabilities earlier has been limited by availability, lot-to-lot variability, finite lot sizes and cost. Alternative model systems including HepG2 and Fa2N-4 have shown promise for certain screening applications (e.g. AhR-, PXR-mediated induction); however, these cell lines have failed to support the broader complement of PHH functionality (e.g. basal metabolism, biliary polarization, or lack of regulatory factors). Fresh HepaRG ${ }^{\mathrm{mi}}$ cells have been shown to support mature hepatic phenotypes, but the perishable nature of fresh cells has severely limited their availability to the global community. Herein we report the characterization of cryo-preserved HepaRG ${ }^{\mathrm{m}}$ cells for drug metabolism (e.g. CYP1A2, CYP2B6, CYP2C, CYP2D6, CYP3A, UGT, SULT, and FMO) over multiple time points, induction (AhR, PXR, CAR), transport (uptake of taurocholate and estrone sulfate) and cytotoxicity (e.g. aflatoxin B1) with comparisons to multiple PHH preparations. Through these assessments, we demonstrate that cryo-preserved HepaRG ${ }^{\mathrm{m}}$ cells have comparable functionality to PHH. We also show that cryo-preserved HepaRG ${ }^{\mathrm{m}}$ cells maintain CAR responsiveness with specific activators, a critical pathway that is absent in Fa2N-4 cells and is generally lacking in lower quality lots of PHH. Furthermore, we provide induction data of CYP1A2, CYP2B6, and CYP3A4 enzyme activity in response to prototypical AhR, CAR, and PXR activators in multiple lots of both HepaRG ${ }^{\mathrm{m}}$ cells and PHH. These data show that induction responses in three different lots of $\mathrm{HepaRG}^{\mathrm{m}}$ cells were highly reproducible as compared to induction responses observed in 52 different lots of PHH. In conclusion, we present for the first time metabolism, transport, and induction comparisons between cryo-preserved $\mathrm{HepaRG}^{\mathrm{mm}}$ cells and $\mathrm{PHH}$ demonstrating the superior reproducibility of this model system with comparable functionality. These results coupled with demonstrating the longevity ( $\geq 22$ days) of HepaRG ${ }^{\mathrm{m}}$ cells in culture highlight this hepatic model system's potential in the study and prediction of xenobiotic clearance, DDI, and safety assessment applications.

\section{P183. Evaluation of a 96-well plate induction assay: The impact of different extracellular matrix based growth substrates on induction potential of Cytochrome P450, UDP-dependent glucuronyl transferase, transporter proteins with multiple endpoints}

\author{
Rongjun Zuo, Haiyan Xia and Chris Patten
}

\section{BD Biosciences, Bedford, MA}

Purpose: Primary human hepatocyte is the "Gold Standard" for studying drug and other foreign compound (xenobiotic) metabolism since it contains full complement of drug metabolism enzymes and cofactors, transporter proteins, and nuclear receptor pathways for regulating enzymes involved in drug-drug interactions. It has been shown that extracellular matrix (ECM) based growth substrates provide a physiological environment that supports and promotes key hepatocyte functions; specifically for hepatocytes, collagen I has been the conventional substratum for attachment, plated metabolism for low turn-over compounds, induction assay, toxicity assay, and uptake/efflux assay when overlaid with matrigel. The purpose of this study is to evaluate the effect of different extracellular matrix based growth substrates (e.g., collagen I coating at different concentrations, matrigel thin coat, and a collagen mimetic surface) on induction response of CYP450, UGT, and uptake/efflux transporters in human primary hepatocytes cultured in 96-well plates with multiple endpoints including mRNA Western blot analysis, and enzyme activity or transporter activities. Methods: 96-well tissue culture plates are treated with different extracellular matrix based growth substrates including different concentrations of Collagen I proteins, matrigel thin coat, and a mimetic surface. Freshly isolated or cryopreserved human hepatocytes from several donor livers are cultured on these plates and are assessed for induction response of CYP3A4, CYP1A2, 
UGT1A1, BCRP, NTCP using positive control inducers measured with multiple endpoints (mRNA, enzyme activity and Western blot analysis). Results: matrigel thin coat increased both basal and induced CYP450 and UGT activities at the highest extent, but no significant effect on transporter activities; collagen I coating showed a concentration dependent improvement in CYP and transporter induction response, and less effect on UGT response. Conclusion: different growth substratum showed differential effect on induction response of different enzyme/transporter proteins; 96-well matrigel thin coat plates provides a relatively high through-put assay platform for evaluating CYP and UGT induction response in primary hepatocytes.

\title{
P184. Evidence for CYP2C9:CYP3A4 enzyme activity interactions in human hepatocytes through modulation of enzyme levels
}

\author{
Diane B. Ramsden ${ }^{1}$, Tom S. Chan ${ }^{1}$ and Timothy S. Tracy² \\ ${ }^{1}$ Drug Metabolism and Pharmacokinetics, Boehringer Ingelheim, Ridgefield, CT, ${ }^{2}$ College of Pharmacy, University of \\ Kentucky, Lexington, $K Y$
}

Protein-protein interactions have been well documented for recombinant CYP450s. This interaction has not been as clearly demonstrated in a more physiological in vitro system such as human liver microsomes. In this study we sought to determine whether this type of CYP450 protein interaction could be occurring in human hepatocytes. Firstly, an analysis of vendor-supplied CYP2C9 and CYP3A4 activity levels from cryopreserved human hepatocytes was performed. This analysis indicated a positive correlation between CYP2C9 and CYP3A4 activity. This observation is inconsistent with the finding in recombinant systems where increased CYP3A4 levels led to decreased CYP2C9 activity. Therefore, an attempt to dissociate the co-regulation of CYP2C9 and CYP3A4 constitutive enzyme levels, using siRNA, was undertaken. By utilizing a long-term, micropatterned, human hepatocyte and mouse fibroblast co-culture (HepatoPac $\left.{ }^{\circledR}\right)$, we were able to follow CYP2C9 activity in response to selective knockdown and recovery of CYP3A4. CYP3A4 specific gene silencing resulted in a 70\% decrease in CYP3A4 activity (midazolam hydroxylation) commensurate with a $67 \%$ increase in CYP2C9 activity (diclofenac hydroxylation). Conversely, once the siRNA was removed, both CYP3A4 and CYP2C9 activities returned to pre-knockdown levels. Induction of these enzymes with a model PXR activator (rifampicin) with and without concomitant knock down of CYP3A4 with siRNA on CYP2C9 and CYP3A4 activity was evaluated. Knockdown of CYP3A4 levels in the presence of rifampicin, resulted in a 70\% increase in the CYP2C9 inductive response [4.0-fold (-siRNA) to 6.9-fold (+siRNA)]. No measurable increase in CYP3A4 induction response was noted [5.6-fold (-siRNA) to 5.1-fold (+siRNA)]. Taken together, this work demonstrates that modulation of CYP3A4 levels can lead to appreciable increases in CYP2C9 activity in human hepatocytes, although the clinical consequence of these findings is not yet clear. Funded in part by a grant from the National Institutes of Health (GM 086891) to T.S.T.

\section{P185. Expression and activity levels of cytochrome P450 in 1-week spheroid culture of rat hepatocyte on nanopillar sheets}

\author{
Toshito Nakagawa ${ }^{1}$, Atsuko Higashida ${ }^{2}$, Kunihiro Ohta ${ }^{1}$ and Masaki Ishigai ${ }^{1}$ \\ ${ }^{1}$ Preclinical Research Dept, Chugai Pharmaceut Co Ltd, Gotemba, Japan, ${ }^{2}$ Discovery Platform Technology Dept, Chugai \\ Pharmaceut Co Ltd, Gotemba, Japan
}

Primary hepatocyte culture is a useful tool for studying drug metabolism and drug-drug interactions but, since some metabolic enzyme and transport activity is decreased during culture, hepatocytes are mainly used only for short incubation periods. In such short term assays, it is hard to estimate the stability of compounds with low metabolic clearances or to determine their metabolites. Improved cell assays are needed which allow long incubation periods, because recent advances in metabolic stability screening at the early stage of new drug development are producing a lot of metabolically stable candidates. Three-dimensional culture is believed to maintain liver functions in cells longer than conventional monolayer culture and recently a number of devices have been developed by which spheroids are easily formed. Of these, nanopillar sheets have been reported to maintain hepatocyte spheroids with higher mRNA expression levels of Cyp3a3 and Mrp2, so we investigated the changes of cytochrome P450 mRNA expression and activity in rat hepatocyte spheroids during culture compared to those in a conventional monolayer. Cyp3al expression showed a marked difference, being highly expressed in the spheroids over a 4-day culture (at a level comparable to that in freshly isolated cells), but decreased to about $20 \%$ of the initial expression in the monolayer. Cyp3a9 mRNA levels were relatively well maintained, Cypla2, 2e1 and 4a1 mRNA levels were dramatically decreased, and Cypla1 levels were elevated on Day 1 
of the culture in both the spheroids and the monolayer. We also found that all formation levels of testosterone hydroxlyated metabolites were higher in the spheroids. The formation of $2 \beta$ - and $6 \beta$-hydroxyl testosterone in the spheroids was more than $25 \%$ of the initial activity in a 4 -day culture, while that in the monolayer was less than $10 \%$. Activity of 7-ethoxyresorufin O-deethylase was elevated in both the spheroids and monolayer. These observations agreed closely with the results of mRNA expression and also suggested that Cyp3a activity was well maintained in the spheroids but not in the monolayer. We demonstrated that rat hepatocyte spheroids cultured on nanopillar sheets, an easy-to-handle cell system, have higher levels of Cyp3a1 mRNA and Cyp3a activity over longer periods than those cultured in a conventional monolayer. Since Cyp3a isoforms are recognized to be one of the most important drug metabolizing enzymes in liver, this system could be useful for studies of drug metabolism and drug-drug interactions which need to be incubated over a long period.

\title{
P186. GigaMatrix differentiates stem/progenitor cells into mature functional hepatocytes
}

\author{
Marsha Roach, Lola Reid, Richard Malavarca, Shawn Hallowell, Linyan He \\ GigaCyte, LLC, University of North Carolina, GigaCyte, LLC, GigaCyte, LLC, GigaCyte, LLC
}

Human fresh and cryopreserved hepatocytes plated on collagen I coated plates sometimes overlaid with Matrigel are used routinely throughout Drug Discovery for drug metabolism studies, transporter studies, pharmacokinetics and pharmacodynamics studies, gene expression and induction/inhibition studies and toxicity screens. While being the gold standard, these cellular models are met with limitations. Here we provide data to demonstrate that human stem/progenitor cell-derived hepatocytes may offer a better model system with fewer limitations than the current gold standard models. We have developed a hepatic model system that includes human stem/progenitor cells, novel formulations of lineage stage-specific media for expansion and differentiation of stem/progenitor cells and maintenance of mature hepatocytes as well as our GigaMatrix Liver Biomatrix isolated from decellularized liver. Our decellularization methods preserve the matrix biochemistry and structure, retaining $>95 \%$ of its collagens and most of the liver's collagen-associated matrix components, growth factors and cytokines. Consequently, when human stem/progenitor cells were plated onto the biomatrix, they differentiated into mature hepatocytes within a few days without adding exogenous growth factors. Data will be presented showing that CYP3A4 activity was equivalent to primary adult hepatocytes, sustained over a period of several weeks and didn't rapidly decline as with primary adult hepatocytes. Data from other metabolic activity studies will also be presented.

\section{P187. Hepatobiliary disposition of bilirubin and its mono- and di-glucuronide metabolites in sandwich-cultured rat hepatocytes}

\author{
Jin Kyung Lee ${ }^{1}$, Robert Hart ${ }^{2}$ and Brouwer Kenneth $^{3}$ \\ ${ }^{1}$ Biology, Qualyst Inc, Durham, NC, ${ }^{2}$ Chemistry, Qualyst Inc, Durham, $N C,{ }^{3}$ Qualyst Inc, Durham, NC
}

The serum bilirubin level is often used as a biochemical marker to indicate liver injury. In the liver, bilirubin is extensively conjugated by uridine diphosphate-glucuronosyltransferase $1 \mathrm{Al}$ to bilirubin monoglucuronide (bil-mono) and diglucuronide (bil-di), which are then excreted into bile by multidrug resistance-associated protein 2 . Due to the shared metabolic and excretory pathways, the hepatobiliary disposition of bilirubin and its conjugates can be modulated by therapeutic drugs, thus resulting in drug-induced hyperbilirubinemia. The present study was designed to examine the hepatobiliary disposition and conjugation of bilirubin, and to identify the potential drug-bilirubin interaction in sandwich-cultured rat hepatocytes (SCRH) using B-CLEAR ${ }^{\circledR}$ technology. Bilirubin accumulation $(10 \mathrm{uM}, 20$ minute incubation) was negligible after the correction of nonspecific binding. Interestingly, the addition of bovine serum albumin $(0.4 \%)$ in the dosing incubation solution markedly enhanced bilirubin accumulation, and its conjugation to bil-mono and bil-di; the accumulation in cells+bile for bilirubin, bil-mono, and bil-di was $119 \pm 33.9,138 \pm 33.0$, and $260 \pm 67.7$ $\mathrm{pmol} / \mathrm{mg}$ protein, respectively. Similar to the in vivo observations, the biliary excretion index (BEI) for bilirubin, bilmono, and bil-di was extensive $(90.1 \pm 21.4 \%, 83.8 \pm 4.76 \%$, and $83.9 \pm 6.79 \%$, respectively). The potential drug-bilirubin interaction was evaluated using rifamycin (RIF), erythromycin estolate (EE), bromosulfophthalein (BSP), cyclosporine A (CYA), glyburide (GLY), and troglitazone (TGZ). The accumulation of bilirubin in cells+bile was decreased by all tested drugs (13.8-60.7 \% of control); bilirubin BEI was decreased by RIF, BSP, CYA, and TGZ (0-78.2\% of control). The accumulation of bil-mono in cells+bile was reduced by all tested drugs, except BSP (18.8-64.8\% of control); RIF, EE, CYA, and TGZ decreased the BEI of bil-mono (44.9-76.9\% of control). Bil-di accumulation in cells+bile was marginally affected by 
tested drugs, but its BEI was decreased by RIF, CYA and TGZ to 49.1 72.9\% of control. In conclusion, the use of SCRH is a useful in vitro approach for investigating the influence of drugs in the hepatobiliary disposition of bilirubin and its metabolites.

\title{
P188. Long term storage effects on the metabolic activity of cryopreserved primary human and rat hepatocytes
}

\author{
Todd W. Stewart ${ }^{1}$, Christina K. Nolan ${ }^{1}$, Rafal P. Witek ${ }^{1}$, Brian J. Paszkiet ${ }^{2}$, Stephen S. Ferguson ${ }^{1}$ and \\ Cornelia M. Smith ${ }^{1}$
}

\author{
${ }^{1}$ Life Technologies, Durham, NC, ${ }^{2}$ Life Technologies, Frederick, $M D$
}

Cryopreserved hepatocytes represent the current "state of the art" model system for studying xenobiotic metabolism and drug-drug interaction potential. Advances in cryopreservation technology have allowed for the long-term storage of hepatocytes and provided a consistent supply for research applications. Although several laboratories have demonstrated that cell attachment in culture and metabolic activity are not significantly impacted by cryopreservation, little is known in regards to the functionality of hepatocytes which have been stored cryopreserved for extended periods. To test the hypothesis that cell morphology, viability, viability stability, metabolic activity and attachment are sustained after 'long-term' storage, human and rat hepatocytes stored in cryogenic conditions for 4 - 5 years (long-term) were assessed and compared to those stored for 1 - 2 years (short-term). Cell viability and yield were determined by trypan blue exclusion. Metabolic activities CYP1A2, CYP2C9, CYP2D6 and CYP3A were determined using probe substrates phenacetin, diclofenac, dextromethorphan and testosterone, respectively and analyzed by LC-MS-MS. Phase II activities glucuronidation (UGT) and sulfation (SULT) were assessed by 7-hydroycoumarin incubations and HPLC. Human cryopreserved hepatocytes were seeded on collagen-coated plates, overlaid and monitored over several days for attachment. Data from each individual lot was compared to its own historical data obtained post cryopreservation. Viability, yield and viability stability were maintained in both groups with less than 3 percent difference noted between historical and current data. The human lots previously characterized as plateable, maintained this quality, even after long-term storage. CYP activities were sustained also. For example average ( \pm S.E.) CYP3A activities were $631 \pm 224 \mathrm{pmol} / \mathrm{min} / 10^{\wedge} 6$ cells (historical) and $516 \pm 198 \mathrm{pmol} / \mathrm{min} / 10^{\wedge} 6$ cells (current) for stored $4-5$ year lots and $631 \pm 142 \mathrm{pmol} / \mathrm{min} / 10^{\wedge} 6$ cells (historical) and $552 \pm 119 \mathrm{pmol} / \mathrm{min} / 10^{\wedge} 6$ cells (current) for stored 1 - 2 year lots. Likewise, SULT activities were not significantly different between historical and current values in either group. Interestingly, a significant decrease $(\mathrm{p}<0.05)$ in UGT activity was observed in the $4-5$ year old human lots, with average values of $574 \pm 54 \mathrm{pmol} / \mathrm{min} / 10^{\wedge} 6$ cells versus $256 \pm 7 \mathrm{pmol} /$ $\mathrm{min} / 10^{\wedge} 6$ cells. A non-significant decrease in UGT activity was observed for the $4-5$ year old rat lots. UGT activities did not significantly change in the $1-2$ year old human lots $\left[432 \pm 8 \mathrm{pmol} / \mathrm{min} / 10^{\wedge} 6\right.$ cells (historical) versus $498 \pm 40 \mathrm{pmol} /$ $\mathrm{min} / 10^{\wedge} 6$ cells (current)]. In conclusion, no significant changes in viability, viability stability, attachment and analyzed CYP activities were observed in long-term cryogenically stored human and rat hepatocytes. The observed decreases in UGT activity in lots stored for 4- 5 years warrant additional investigation to further characterize the impact of long-term storage on phase II activity. (Legal notice: For Research Use Only. Not intended for any animal or human therapeutic or diagnostic use.)

\section{P189. Metabolite stability screening and hot spot metabolite identification by combining non- selective and selective fragmentation and high resolution accurate mass}

\author{
$\underline{\text { Tim Stratton }}{ }^{1}$, Karen Salomon ${ }^{2}$ and Yingying Huang ${ }^{2}$
}

${ }^{1}$ Thermo Fisher Scientific, San Jose, CA, ${ }^{2}$ Metabolism and Metabolomics, Thermo Fisher Scientific, San Jose, CA

Metabolic stability screening assays are a common in vitro test used to screen drug discovery candidates for favorable properties. In the past these screening assays were commonly analyzed by LCMS using triple quandrupole mass spectrometers which need to be optimized for each compound. While the quantitative quality was sufficient, qualitative data was limited without significant additional method development. Here we show how data obtained on a novel benchtop high resolution accurate mass instrument operating in both all ion fragmentation and selective MS2 modes, combined with screening software, provides both quantitative stability as well as early qualitative metabolite information. The same data, without reinjection, can be further scrutinized for more definitive metabolite structure using selective MS2 scan data. The metabolic stability of multiple compounds (nortriptyline, diclofenac, and propranolol) $(0.5 \mu \mathrm{M})$ in liver microsomes $(0.5 \mathrm{mg} / \mathrm{mL})$ was determined by timecourse incubation with sample aliquots taken at five time points. 
Samples were analyzed by UPLCMS on a novel benchtop quadrupole Orbitrap MS with a higher energy collision dissociation (HCD) cell. Data was acquired as a full scan followed by an all-ion-fragmentation (AIF) scan. Precursor isolated HCD MS2 scans were obtained near the peak maxima on components observed above threshold. The data was acquired using a beta version of MetQuest 1.1 metabolic screening software which performed automatic relative quan/qual data analysis. Further metabolite structure determination was possible using the same data files without reinjection by using a metabolite finding and identification software. Initial processing of the timecourse samples was performed automatically using a list of 50 commonly found biotransformations as well as screening for unexpected metabolites. To be considered an unexpected metabolite, the extracted ion chromatogram of an observed $\mathrm{m} / \mathrm{z}$ value had to follow a peak shape, and not appear in the control. Expected metabolites were included only if the area, after comparison to the control, differed by more than $5 \mathrm{X}$ and their exact mass value was within $5 \mathrm{ppm}$ of the expected value. For unexpected modification, an initial assessment of the formation profiles and isolated MS2 fragmentation scans was performed to determine if they were truly metabolites. Formation profiles for all metabolites were automatically generated and reported. Theoretical fragments for nortriptyline were generated and combined with the list of modifications provided from the Quan/Qual study. The fragments with and without modifications were then used to perform a fragment ion search (FISh) on the acquired data. The same metabolites as identified in the Quan/Qual study were found by FISh and the relative site of modification was identified based on the precursor ion selected fragmentation.

\title{
P190. RNA-Seq reveals the transcriptome changes at gene and transcript levels after treatment of HepaRG cells and primary human hepatocytes with phenobarbital
}

\author{
Lai Peng ${ }^{1}$, Dan $\mathrm{Li}^{1}$, In-Hee Lee ${ }^{2}$, Mahesh Visvanathan² and Xiao-bo Zhong
}

${ }^{1}$ Pharmacology, Toxicology, and Therapeutics, University of Kansas Medical Center, Kansas City, KS, ${ }^{2}$ Molecular and Integrative Physiology, Division of Cancer and Drug Development, Bioinformatics Core Facility, University of Kansas, Lawrence, $K S$

Phenobarbital (PB) is a widely used anticonvulsant, which induces or suppresses the expression of a large spectrum of genes in hepatocytes. About $90 \%$ human genes transcribe multiple transcripts. However, it is unclear how PB changes the expression of multiple transcripts of genes in human hepatocytes. Therefore, we used RNA sequencing (RNA-Seq) to examine the transcriptome changes on the expression of genes and transcripts in HepaRG cells and primary human hepatocytes after PB treatment. RNA-Seq was done on an Illumina HiSeq 2000 with 100-base pair-end reads. About 100 to 200 million reads were generated for each sample. The RNA-seq reads were aligned to human reference genome by TopHat with more than $60 \%$ reads successfully mapped. The abundance of genes and transcripts was estimated by Cufflinks using Ensembl reference annotation. We found that around 14,000 genes were expressed above a threshold of 1 FPKM (fragments per kilobase of exon per million fragments mapped), and identified the predominantly expressed transcripts for each gene. Biological duplicates demonstrated strong correlation in expression levels of genes and transcripts. By using DESeq for analysis of differential expression, we identified 173 genes and 534 transcripts with significant changes (FDR $<5 \%$ ) in expression after PB treatment in HepaRG cells. GO annotation indicated these PB responsive genes mainly belonged to pathways of oxidation reduction, response to wounding and extracellular stimuli, and drug, xenobiotic or steroid metabolic processes. Multiple transcripts of most PB responsive genes displayed differential expression, with some isoforms of one gene showing larger induction or suppression than other isoforms in the PB treated group. Individual variations of expression at transcript levels in response to PB treatment were also found among different primary human hepatocytes. In conclusion, RNA-Seq has revealed the transcriptome changes at both gene and transcript levels in response to PB treatment, and provided new information on the effects of PB in human hepatocytes.

\section{P191. Screening and characterization of chemically reactive metabolites using polarity switching of hybrid linear trap quadrupole orbitrap fourier transform mass spectrometry}

\author{
$\underline{\text { Bo Wen }}^{1}$, Jiansheng Huang ${ }^{2}$ and David Moore ${ }^{3}$
}

\author{
${ }^{1}$ Drug Metabolism and Pharmacokinetics, Hoffmann-La Roche, Nutley, NJ, ${ }^{2}$ Hoffmann-La Roche, Nutley, NJ, ${ }^{3}$ Dept of \\ Non-Clinical Safety, Hoffmann-La Roche Inc., Nutley, NJ
}

A highly sensitive and efficient method has been developed for detection and structural characterization of glutathione ( $\gamma$-glutamyl-cysteinylglycine, GSH)-trapped reactive metabolites using high speed polarity switching on a hybrid linear trap quadrupole Orbitrap fourier transform mass spectrometer. The primary MS/MS method for screening GSH-trapped 
reactive metabolites is a neutral loss (NL) scan of $129 \mathrm{Da}$ (pyroglutamic acid) in the positive ion mode, while a precursor ion (PI) scan of $272 \mathrm{Da}$ (deprotonated $\gamma$-glutamyl-dehydroalanyl-glycine) in the negative ion mode was recently developed to provide broader coverage of different classes of GSH conjugates. However, in most cases, negative MS/ MS spectra provide very limited structurally informative ions. In this study, positive and negative MS/MS spectra were simultaneously acquired using a novel polarity switching approach to unanimously identify and structurally characterize GSH conjugates in a single liquid chromatography/tandem mass spectrometry (LC/MS/MS) analysis. High mass accuracy and high resolution full scan MS data obtained in the positive ion mode provided highly desirable confidence in the molecular composition of detected GSH conjugates. Furthermore, due to its full scan MS acquisition in both positive and negative ion modes, this novel approach is highly suitable for unbiased detection and structural characterization of reactive metabolites trapped by different types of nucleophiles such as cyanide and $N$-acetyl cysteine, as well as stable 'downstream' metabolites in the same LC/MS/MS run. This provides a holistic view of metabolism and/or bioactivation of the compound tested. The effectiveness and reliability of this approach was evaluated using a number of model compounds in human liver microsomal incubations, including acetaminophen, clozapine, diclofenac, imipramine, 4-ethylphenol, and ticlopidine. As a result, the polarity switching approach revealed the presence of known adducts and, in many instances, identified additional conjugates that had not been reported previously. In comparison to the widely used neutral loss (NL) or precursor ion (PI) scanning analysis, this approach provided superior sensitivity and coverage for different types of GSH conjugates with highly efficient structural characterization. More importantly, the novel polarity switching approach is suitable for high-throughput screening of reactive metabolites in the drug discovery process.

\title{
P192. Ultrafast SPE integrated with TOF-MS increases the throughput of metabolic stability assays and enables analysis of metabolites
}

\author{
Michelle V. Romm ${ }^{1}$, Nikunj Parikh¹, Yuqin Dai ${ }^{2}$, Vaughn P. Miller ${ }^{1}$, William A. LaMarr ${ }^{1}$, Keith \\ Waddell $^{2}$ and Can Ozbal ${ }^{1}$
}

${ }^{1}$ Agilent Technologies, Wakefield, MA, ${ }^{2}$ Agilent Technologies, Santa Clara, CA

The metabolic half-life or stability of a drug candidate has important pharmacokinetic and clinical significance, because it influences both oral bioavailability and plasma concentration of a compound, ultimately affecting efficacy. Large compound libraries and advancements in liquid handling have placed demands on the throughput of in vitro metabolic stability assays. Analysis of assay samples, typically accomplished by LC/MS/MS, is a bottleneck in the process because of the required development of MRM methods. We evaluated the ability of the Agilent RapidFire High-throughput Mass Spectrometry system interfaced to a TOF (RapidFire 360) to provide equivalent assay results to LC/MS/MS, but with a more efficient workflow and the additional benefit of metabolite analysis through mining of the TOF's data without a priori knowledge of metabolite ID. A diverse set of compounds were incubated with human liver microsomes over a time course of 0-60 min and analyzed using LC-MS/MS and SPE-TOF systems. SPE-TOF samples were analyzed utilizing generic source parameters and exact mass extraction with cycle times of less than 10 seconds per sample. The metabolic half-life values as determined by parent depletion for a diverse set of compounds were equivalent by the two platforms ( $\mathrm{R}^{\wedge} 2$ greater than 0.9 ). In addition to the greater than 10-fold increase in cycle time of the ultrafast SPE system, these results indicate that the MRM method development can be eliminated for the metabolic stability assay thus providing additional workflow efficiency. Post data acquisition, new Agilent RapidFire Integrator data analysis software was used for the automated extraction of the exact masses for the parent and six common metabolic transformations including putative oxidation, dealkylation and reduction from the TOF MS data. The disappearance of parent was compared with the formation of metabolites for the set of compounds. For most compounds, these common metabolic pathways accounted for a significant percentage of the overall metabolism of the parent compound. For example, oxidation accounted for the majority of the metabolism for midazolam and sequential N-dealkylation was seen for chlorpromazine. Compounds for which the six common metabolic pathways did not account for a significant percentage of the overall metabolism were readily identified and could be selected for further investigation. The results presented here illustrate that the use of SPE-TOF for metabolic stability assays not only results in a greater than 10x increase in throughput with equivalent data to LC-QqQ but also provides initial metabolite identification through the fully automated extraction and quantitation of commonly observed metabolites.

\section{P193. Abstract withdrawn}

\section{P194. A novel approach to generate robust predictive developmental toxicity classification models from highly unbalanced dataset and benchmarking with the reported models}




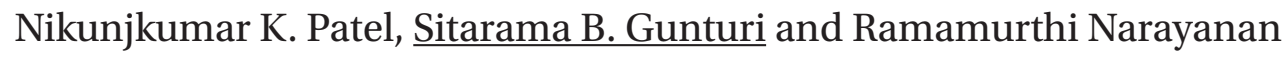

Innovation Labs Hyd, Life Siences R\&D Division, Tata Consultancy Services Limited, Hyderabad, India

Developmental toxicity (DT) has been defined as the adverse effects induced during prenatal, postnatal or as a result of parental exposure that can be manifested at any point in the life span of the organism [1]. It is one of most important toxicological end point under European Union's REACH regulations [2] and US EPA requirements [3]. Cost, time and ethical concerns in using whole animal developmental toxicity testing are driving the need to develop alternatives to animal testing such as in vitro and computational methods. Currently available in vitro tests are not sufficient for replacing the whole animal tests for DT. Moreover, the battery of in vitro tests is required as it is impossible to model the whole mammalian reproductive and the developmental cycle in a single in vitro system. Whereas computational models have the potential to offer significant advantages in terms of time- and cost-effectiveness and animal welfare, there has been little work performed in this area, except for the few reports [5-8]. The reported models are trained based on highly unbalanced data and subsequently lead to biased predictive performance on the external test sets. In our analysis of the reported models, we found that the models are not validated thoroughly for the bias despite of the biased nature of the training data. Various data balancing techniques such as SMOTE (Synthetic Minority Oversampling Technique) have been successfully applied to reduce the learning bias of unbalanced datasets in other fields such as fraud detection, linguistics and electronics. Our continued interest to understand the applications and limitations of computational models in toxicity assessment, prompted us to study the generation of global classification models for DT based on a dataset of 286 (200 toxic, 86 nontoxic) diverse drug and drug like compounds and 450 calculated descriptors. For this study, we employed for the first time, a) SMOTE data balancing technique to reduce the learning bias of unbalanced dataset b) D - Optimal design to select the optimal training and test sets c) Genetic algorithm for parameter selection and d) Machine Learning Techniques (SVM, kNN, CART) for model generation. The models generated were validated extensively and an in-depth analysis was conducted to understand the limitations of the models reported for practical applications. We would like to share our results, in particular 1) the application of SVM along with SMOTE and DOD for the generation and validation of classification models for DT 2) the comparative performance of our models with the reported models 3) the physico chemical meaning of the parameters that contribute to the DT profile of the drug-like compounds and 4) the limitations of the reported DT models. References: 1. UNECE GHS Part 3 Chp. 3.7 (2004), 2. Off. J. Eur. Union, L136, 103 (2006), 3.EPA Guidelines for Developmental Toxicity Risk Assessment, 4. Chem Cent. J., 4:S4 (2010), 5. Reprod. Toxicol., 30, 147 (2010), 6. SAR QSAR Env Res., 15, 1 (2004), 7. SAR QSAR Env Res., 14, 83 (2002)

\section{P195. Automatic generation and validation of QSAR toxicity models}

\section{Matthew D. Segall}

\section{Optibrium Ltd., Cambridge, United Kingdom}

Whether compounds are intended as drugs, cosmetics, agrochemicals or for other industrial application, it is essential to understand their potential to cause toxic effects. This can guide the prioritisation compounds for further research or consideration of the most appropriate downstream experiments to confirm their safety. The ability to predict toxicities based on chemical structure alone would allow these factors to be considered prior to synthesis, allowing the safest options to be pursued and saving time and resources wasted on synthesis and testing of unsuitable compounds. We will describe the generation and validation of Quantitative Structure Activity Relationship (QSAR) models of key toxicity endpoints, based on data made available by the US Environmental Protection Agency (EPA) as part of its Toxicity Estimation Software Tool (T.E.S.T.) [1]. The models were generated using the StarDrop ${ }^{\mathrm{mt}}$ Auto-Modeller ${ }^{\mathrm{mt}}$ [2][3]. Multiple models were automatically generated using different statistical methods including Gaussian Processes, Radial Basis Functions and Partial Least Squares. The predictive power of the models generated were compared with each other and with the models provided in T.E.S.T. using independent validation sets. The resulting toxicity models conform to the Organization for Economic Cooperation and Development (OECD) principles for validation of QSAR models for regulatory purposes [4] and the best models for each endpoint were found to perform equivalently or better than the EPA T.E.S.T. models on the same validation sets. This demonstrates that the automatic methods employed are capable of building models that compare favorably with those created with more 'manual' methods. Furthermore, the models produce a Glowing Molecule ${ }^{\mathrm{m}}$ visualization [5] that highlights regions of a compound with a significant influence on a predicted property. This provides a link between the predicted toxicity and the chemical mechanism and guides users in the redesign of compounds with improved safety. 
[1] US Environmental Protection Agency. Quantitative Structure Activity Relationship.[Internet]. 2011 [cited 2011 May 20]. Available from: http://www.epa.gov/nrmrl/std/cppb/qsar/\#TEST.

[2] Obrezanova O, Gola JMR, Champness E, Segall MD. Automatic QSAR modeling of ADME properties: blood-brain barrier penetration and aqueous solubility. J Comp Aid Mol Design. 2008 431-440.

[3] Optibrium. [Internet]. [cited 2011 March 3]. Available from: http://www.optibrium.com/stardrop.

[4] OECD. OECD PRINCIPLES FOR THE VALIDATION, FOR REGULATORY PURPOSES, OF (QUANTITATIVE) STRUCTURE-ACTIVITY RELATIONSHIP MODELS.[Internet]. 2004 [cited 2011 May 20]. Available from: http://www. oecd.org/dataoecd/33/37/37849783.pdf.

[5] Segall MD, Champness E, Obrezanova O, Leeding C. Beyond Profiling: Using ADMET models to guide decisions. Chemistry and Biodiversity. 2009 2144-2151.

\title{
P196. Development of two-dimensional template system for the prediction of |CYP2b6-mediated reaction sites
}

\author{
Noriyuki Koyama $^{1}$ and Yasushi Yamazoe ${ }^{2}$
}

${ }^{1}$ Department of Drug Metabolism, Tokushima Research Institute, Otsuka Pharmaceutical Co., Ltd., Tokushima, Japan, ${ }^{2}$ Graduate School of Pharmaceutical Sciences, Tohoku University, Sendai, Japan

We have developed a template-based system for predicting the regioselectivity of CYP2B6-mediated oxidation of chemicals. Chemical structures of substrates drawn by Chem Draw or Chem 3D software were directly overlaid on a grid of hexagons corresponding to each six-membered aromatic ring on a personal computer. An atom of chemicals, which was the presumed reaction site, was first fitted to a corner of the template corresponding to the site of oxidation, and the neighbor atom was rotated by 60 degrees to fit to the next corner of the template and to keep the rest of the structure within the border. If all the structure was kept within the templates, the placement was judged as a possible candidate to contribute to the metabolite formation. Two types of planar templates (Templates A and B) were extracted from overlapping regions of the polyaromatic hydrocarbon-type substrates. These templates were found to link to each other at specific positions (pinching points) through the application of various substrates having non-planar structures to Templates $\mathrm{A}$ and $\mathrm{B}$. The rates of their occupancies on the corner of the templates were evaluated using more than 40 substrates, and the heavily utilized area (trigger region) was envisioned on Template A. This CYP2B6 template system is suggested to interact in at least three positions (trigger, pinching/bending and oxidation sites) with substrates. Both CYP2B6 substrates and non-substrates were applied to assess the validity of this template system. A substrate was initially placed to interact at the trigger region, and extended to the distal part of this template system. Bulky structures that were not placed on the template system were accommodated in the specific wing-shaped area. When the whole of the structure was put within the template system, this placement was judged to be acceptable and the atom locating at the oxidation site of the template was predicted to be oxidized. In the case where the placement was judged to be unacceptable in the system, this chemical was judged to be a non-substrate of CYP2B6. Results were consistent between the predicted and the in vitro experimental data with high accuracy. The simulated results for the 109 reactions of 82 chemicals mediated by CYP2B6 have been consistent with the in vitro experimental data. This system is a virtual model determined with size and shape of various substrates, which does not necessarily reflect either the structure of the enzyme or the mechanisms of reaction between substrates and the enzyme. We think that this system may be useful for studies on drug metabolism and new drug development.

\section{P197. Evaluation of ADMET properties predictions using the advanced in silico modeling tool ADMET predictor}

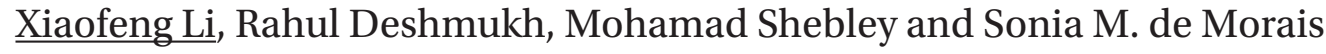

\section{Dmpk-BA, Abbott, Abbott Park, IL}

Xiaofeng Li, Rahul Deshmukh, Mohamad Shebley, Sonia M de Morais Drug Metabolism, Development Sciences, GPRD, Abbott Laboratories, Abbott Park, IL 60064, USA Abstract The demand for fast read out of physiochemical properties at an early discovery stage is great. In silico tools that can provide an early assessment of these properties and reduce cycle time are very important. ADMET predictor is a computer software for advanced predictive modeling of absorption, distribution, metabolism, elimination and toxicological properties based on chemical structures. ADMET predictor was used to predict the biopharmaceutical properties of Abbott proprietary compounds. Three properties were evaluated to 
test the predictive capability of the software, including solubility ( $\mathrm{pH}$ 7.4), $\log \mathrm{D}(\mathrm{pH} 7.4)$, and CYP inhibition properties (CYP1A2, 2C9, 2D6 and 3A4). All ADMET predicted values were compared to the experimentally measured results. For comparison, compound solubility was binned as: poor $(<10 \mu \mathrm{M})$, low (between 10 and $50 \mu \mathrm{M}$ ), moderate (between 50 and $200 \mu \mathrm{M})$, and high $(>200 \mu \mathrm{M})$. For $\log \mathrm{D}$, compounds were classified as follows: $\log \mathrm{D}<3,3<=\operatorname{LogD}<=5$, and $\log$ $\mathrm{D}>5$. CYP inhibition was classified using a binary classification for a compound- an inhibitor (IC50 $<20 \mu \mathrm{M})$ or not an inhibitor (IC50<20 $\mu \mathrm{M})$. For log D comparison, about $91 \%$ of the compounds $(\mathrm{n}=1431)$ compared well with the experimental $\log \mathrm{D}$, when compounds had a $\log \mathrm{D}<3$. For solubility comparison $(\mathrm{n}=1249)$, when compounds were determined to have experimental solubility $>200 \mu \mathrm{M}$, ADMET predictor was about $70 \%$ accurate. For CYP inhibition, ADMET predictor had about $50 \%$ accuracy in predicting the potential of the compound as an inhibitor or not an inhibitor. Amongst compounds determined as inhibitors, ADMET predictor has accuracy of about 50 - 85\% across all the CYPs tested. The greatest accuracy was for CYP3A4 with about $85 \%$. The false positive prediction was high, about $25-40 \%$. ADMET predictor provides a fast and easy read out of compound physiochemical properties and can be a useful tool in virtual space to design compounds with more favorable drug like properties. Compound synthesis and high throughput experimental screening can also be prioritized based on the in silico readout.

\title{
P198. Factoring depth of metabolic sequence reasoning into the in silico prediction of phase II metabolism
}

\author{
Catherine O'Leary-Steele ${ }^{1}$ and Anthony Long ${ }^{2}$ \\ ${ }^{1}$ Knowledge base, Lhasa Limited, Leeds, United Kingdom, ${ }^{2}$ Knowledge Base, Lhasa Limited, Leeds, United Kingdom
}

The metabolic fate of xenobiotics is predicted in our knowledge-based software program Meteor, through a comprehensive system of biotransformations and reasoning rules developed by expert scientists. The reasoning framework assigns a specific likelihood to the potential metabolites and has been carefully designed to allow users to prioritise biotransformation searches and extract the most likely metabolites from all the possible outcomes. The absolute reasoning rules are developed based on factors such as occurrence rates in the literature, the species involved and physiochemical properties such as lipophilicity and molecular weight. Also taken into consideration is the depth within the metabolic sequence for which the query substrate occurs, i.e. whether the substrate is the administered compound or whether it is a generated metabolite from a previous reaction sequence. Depth of sequence considerations in absolute reasoning are particularly significant in modelling drug detoxification. Phase I oxidation reactions often generate metabolites that are good substrates for phase II conjugation reactions. These often occur in preference to further phase I processes. Although there are some exceptions to this general observation, there are several points of consideration that support this biphasic metabolism hypothesis over sequential phase I oxidations. Firstly, the products of phase I oxidations are typically less lipophilic than their respective parent compounds and are potentially poorer substrates for further CYP450-mediated biotransformations. Phase II enzymes are generally polymorphic with a broad substrate specificity which, depending on cofactor availability, makes them efficient catalytic systems with low affinities and high capacities. This high turnover rate is particularly relevant, considering that the concentration of phase I metabolites within the cell is typically low with respect to the parent compound. Furthermore, membrane-bound phase II enzymes may be predisposed to supramolecular recognition effects, placing phase I and phase II enzymes in close proximity so that substrates can be transferred efficiently between them, for example cytochromes and glucuronyl transferases on the endoplasmic reticulum. Many phase II enzymes are also cytosolic and may therefore sequester any phase I products that are released in the cytosol. Despite their broad substrate specificities and turnover efficiencies, phase II metabolites generated directly from suitably-functionalised test compounds are often not seen experimentally. Consequently, these are expressed as optimistic unconfirmed positive predictions. This paper will discuss how enhanced reasoning models can result in the prediction of phase II biotransformations at lower levels of likelihood than phase I biotransformations at the first generation of metabolite formation, and at higher levels for subsequent generations. This work is complementary to existing reasoning models that lower the absolute reasoning of phase I biotransformations for substrates which are generated metabolites rather than administered compounds. The increased selectivity in prediction (the reduction in the number of optimistic unconfirmed positive predictions) will be exemplified with reference to the test compound (S)-1,1,1-trichloro-2-(4-hydroxyphenyl)-2-(4-methoxyphenyl)ethane.

\section{P199. Generation of CYP2D6 model structures for docking studies to explore interactions with substrates at molecular level}

Prashant V. Desai ${ }^{1}$ and Michael A. Mohutsky ${ }^{2}$ 
${ }^{1}$ Computational ADME-Tox, Drug Disposition, Eli Lilly and Company, Indianapolis, IN, ${ }^{2}$ Drug Disposition, Eli Lilly and Company, Indianapolis, IN

Cytochrome P450s (CYPs) are a large family of heme containing enzymes that are responsible for the oxidative metabolism of a wide variety of endogenous compounds and xenobiotics. Amongst the several isozymes of CYPs, Cytochrome P450 2D6 (CYP2D6) is an important isozyme involved in metabolism of $\sim 25 \%$ of currently marketed drugs, including several antidepressants and antipsychotics. In this study, molecular docking studies for CYP2D6 substrates were conducted. Developing such models for CYP2D6 is rather challenging due to low resolution of the published crystal structure (PDB ID: 2F9Q), several missing residues and lack of bound ligand. Since the binding site of this structure appeared to be 'collapsed', it was necessary to develop additional models of CYP2D6 that could accommodate structurally diverse substrates with a range of molecular volume. The Induced-fit strategy including loop refinement was utilized to generate two additional model structures of CYP2D6. Docking experiments were carried out using these models and compared with those using the original crystal structure. Significant improvement were observed using these model structures in terms of generating acceptable binding poses that would correlate with corresponding sites of metabolism for a collection of diverse CYP2D6 substrates. These newly identified CYP2D6 models, along with the docking methodology developed using the current study, could be employed for understanding substrate-enzyme interactions for CYP2D6 at molecular level which, in turn, could be utilized to generate hypotheses toward addressing CYP2D6 metabolism-related issues.

\title{
P200. Preference prediction of site and order of human CYP2E1 metabolism
}

\author{
Yasushi Yamazoe $^{1}$, Kouichi Yoshinari² and Kazumi Ito ${ }^{2}$ \\ ${ }^{1}$ Graduate School of Pharmaceutical Sciences, Tohoku University, Sendai, Japan, ${ }^{2}$ Dm\&mt, Tohoku University, Sendai, \\ Japan
}

An in-silico system for the prediction of CYP2E1-mediated reaction has been devised using a two dimensional template of a hexagonal-block shape. Using published data for the metabolism on more than 80 substrates of CYP2E1, the draft template was further refined to verify the adjacent area and to know the contribution of each position from the relative usages. The positions on the template were classified into four-different point (0-3) groups depending on the relative usage. In addition, we set independent points (-5 to 3 ) for specific positions to incorporate three-dimensional or functional information. For the verification of a chemical as CYP2E1 substrate, a part of chemical atoms is first fitted to a corner of the template (for example; to the site of oxidation position) and rotated other parts at every 60 degrees to fit to corners of the template and to stay within the border. Inverted structures are also estimated in this way. A conformer yielded on the template is judged as a possible candidate to contribute to metabolite formation, if all the parts of the structure are kept within the border of the template. Chemicals overlaid on the template are verified by the score for both the site of the oxidation and order of occurrence. Using chemicals which are not used for the construction of CYP2E1 template, CYP2E1 template developed was tested. Among 109 reactions of 79 chemicals, judgments of 105 reactions were consistent with experimental results. These consisted of 68 CYP2E1-mediated and 37 CYP2E1-nonmediated reactions, showing more than $96 \%$ accuracies for the present prediction system.

\section{P201. Quantitative prediction of aqueous toxicity using spline based regression methods}

\author{
Sitarama B. Gunturi, Jyotsna Bahl, Nikunjkumar K. Patel and Ramamurthi Narayanan
}

\section{Tata Consultancy Services Limited, Hyderabad, India}

Aqueous Toxicity is one of the most important and one of the most extensively studied eco-toxicological end point under the REACH regulation [1]. In-silico prediction of aqueous toxicity continues to be a viable approach to reduce both the number of efforts and the cost of experimental toxicity assessment. There have been models in the literature with good predictive ability. But most of these models suffer from the lack of interpretability. To address this issue, we applied spline based regression methods, namely, Random Function Approximation (RFA) [2] and Multivariate Adaptive Regression Splines (MARS) [3] to develop easily interpretable models. In this study, we have taken carefully compiled data from [4-5]. We built the models on the training set of 644 compounds, validated them on test sets I and II consisting of 339 and 110 compounds respectively and compared the results with those of the published ones. From our analyses we observe that the models i) have excellent predictive ability ii) are robust and iii) are easily interpretable. References: 1 . REGULATION 
(EC) No. 1907/2006 of the european parliament and of the council of 18 December 2006 concerning the Registration, Evaluation, Authorisation and Restriction of Chemicals (REACH), Off. J. Eur. Union, L136 (2006), pp. 103-106, 2. Jyotsna Bahl, Narayanan Ramamurthi and Sitarama B Gunturi, Novel Algorithm to select basis functions in Spline Regression: Applications in QSAR studies, Communicated to J. Chem. Intel. Lab. Sys; 3.J. H. Friedman, Multivariate Adaptive Regression Splines, Ann. Stat. 19 (1991) 1-141; 4. Hao Zhu, Alexander Tropsha, Denis Fourches, Alexandre Varnek, Ester Papa, Paola Gramatica, Tomas Oberg, Phuong Dao, Artem Cherkasov and Igor V. Tetko, Combinatorial QSAR Modeling of Chemical Toxicants Tested against Tetrahymena pyriformis, J. Chem. Inf. Model. 2008, 48, 766-784; 5. Igor V. Tetko, Iurii Sushko, Anil Kumar Pandey, Hao Zhu, Alexander Tropsha, Ester Papa, Tomas Oberg, Roberto Todeschini, Denis Fourches and Alexandre Varnek, Critical Assessment of QSAR Models of Environmental Toxicity against Tetrahymena pyriformis: Focusing on Applicability Domain and Overfitting by Variable Selection, J. Chem. Inf. Model. 2008, 48, 1733-1746

\title{
P202. A novel cellular model for drug-induced adipogenesis
}

\author{
Aarti Uzgare, Utkarsh Doshi and Albert Li
}

\author{
In Vitro ADMET Laboratories LLC, Columbia, MD
}

Obesity is a serious public health problem and a major risk factor for cardiovascular disease, certain types of cancer, and type 2 diabetes. Hence there is a need for relevant cellular models that can be used to test for drugs that may inhibit adipogenesis. Currently, most cellular models utilize cultures of fibroblastic 3T3-L1 preadipocytes that can be induced to undergo adipogenesis. However the effect of other cell types on adipocyte function has not yet been elucidated. Since hepatocytes are the major xenobiotic metabolizing cells of the body, drug testing in the presence of hepatocytes is relevant. In this study we have used a proprietary technology, developed in our laboratory, termed Integrated Discrete Multi Organ Co-culture (IdMOCTM) for the co-culture of 3T3-L1 preadipocytes and hepatocyes. Using quantitative PCR for the amplification of an early marker for adipogenic differentiation namely AP2 we show that hepatocyte presence causes an increase in 3T3-LI adipogenesis. Using Rosiglitazone as a positive control for adipogenesis and insulin sensitization, we demonstrate further increases in 3T3-LI adipogenesis in the presence of hepatocytes. To determine the specificity of Rosiglitazne action, which occurs presumably via its metabolites, N-desmethyl rosiglitazone (N- and para-hydroxy rosiglitazone, an inhibitor of the major metabolizing enzyme-CYP2C8, namely trimethoprim was used. A decrease in adipogenesis in the presence of trimethoprim indicated the role of metabolism in driving drug-induced differentiation. Such a model is physiologically relevant in testing drugs that may induce adipogenesis and as such are likely to cause weight gain and other complications.

\section{P203. A robust method to identify compounds that undergo intracellular lysosomal sequestration}

\author{
$\underline{\text { Faraz Kazmi }}^{1}$, Ryan Funk ${ }^{2}$, Chad Pope ${ }^{1}$, Maciej Czerwinski ${ }^{1}$, David Buckley ${ }^{1}$ and Andrew Parkinson ${ }^{1}$ \\ ${ }^{1}$ XenoTech, LLC, Lenexa, KS, ${ }^{2}$ Department of Pharmaceutical Chemistry, University of Kansas, Lawrence, KS
}

Lysosomes are acidic organelles ( $\mathrm{pH} 4-5$ ) that play a key role in various metabolic processes such as the turnover of phospholipids, the breakdown of waste products (including bacteria and viruses) and apoptosis. Lipophilic and amphiphilic drugs (a.k.a. cationic amphiphilic amines or CADs) with ionizable amines ( $\mathrm{pKa}>6$ ) can accumulate in lysosomes (a process known as lysosomal trapping), which contributes to presystemic clearance in lysosome-rich organs (such as liver and lung) and, together with the binding of lipophilic amines to phospholipids, is associated with a large volume of distribution of numerous cardiovascular and CNS drugs. The prolonged accumulation of lipophilic amines in lysosomes and their binding to phospholipids, both of which disrupt lysosomal function, have been implicated as the major cause of phospholipidosis, where an excessive accumulation of phospholipids occurs in various tissues. In the present study, we describe an in vitro fluorescence-based method to identify lysosomotropic agents. Lysotracker Red or Neutral Red was used as a lysosome-specific probe (excitation/emission wavelengths of 530/590 nm and 530/645 nm respectively) with immortalized human hepatocytes (Fa2N-4 cells), which were incubated with test drugs (at seven concentrations) in a 96-well microtiter plate at $37^{\circ} \mathrm{C}$. Lysotracker Red and Neutral Red were shown by fluorescence microscopy to concentrate in the lysosomes of Fa2N-4 cells. Following a 10-30min co-incubation of drug and fluorescent probe, the Fa2N-4 cells were washed and subsequently solubilized with an organic solvent. Fluorescence was measured and $\mathrm{IC}_{50}$ values for the drug-dependent inhibition of lysosomal trapping of the fluorescent dye were determined. Cell viability was assessed based on the release of LDH into the medium or cytoplasmic staining with the fluorescence probe CMFDA. 
The compounds tested consisted of diverse physiochemical properties, such as acids, lipophilic amines and zwitterions. All of the lipophilic amines tested (which included propranolol, imipramine, astemizole and dextromethorphan) caused a concentration-dependent decrease in the cellular concentrations of Lysotracker Red or Neutral Red, confirming that these compounds are lysosomotropic (lysosomally trapped) drugs. Negative controls such as acetaminophen and diclofenac showed no evidence of Lysotracker Red or Neutral Red inhibition. These findings indicate that the Fa2N-4 immortalized hepatocyte cell line can be used as a test system in conjunction with fluorescent lysosomotropic probes to predict the potential of drug candidates to undergo lysosomal trapping.

\title{
P204 . An effective screening approach to assess the impact of $a-1$ acid glycoprotein binding on the unbound fraction of a drug
}

\author{
Karin D. Brown, Gary P. Hingorani and Ronald B. Franklin
}

Dmpkcp, Array BioPharma Inc., Boulder, CO

Binding to plasma proteins can be an important determinant of drug action and pharmacokinetics. Human Serum Albumin (HSA) and $\alpha-1$ Acid Glycoprotein (AAG) are the most abundant plasma proteins with average physiological concentrations of approximately $40 \mathrm{~g} / \mathrm{L}$ and $0.6-1.2 \mathrm{~g} / \mathrm{L}$, respectively. AAG plasma concentrations can vary due to physiological, pathological and genetic factors and the fluctuations in AAG plasma levels can have a direct effect on concentrations of unbound drug, and consequently alter its pharmacokinetics and pharmacodynamics. Here we describe a screening paradigm to quickly ascertain which compounds have a greater affinity for AAG and the subsequent evaluation of those compounds by a more thorough determination of the effect of increasing AAG concentrations on protein binding. Two techniques were utilized to determine the binding affinity $\left(\mathrm{K}_{\mathrm{d}}\right)$ and free fraction for test compounds: (i) the TRANSIL ${ }^{\circledR}$ binding methodology was used to establish the $K_{d}$ values for AAG and HSA and (ii) Rapid Equilibrium Dialysis (RED) using physiological concentrations of HSA $(40 \mathrm{~g} / \mathrm{L})$ in the presence of increasing concentrations of AAG $(0-3 \mathrm{~g} / \mathrm{L})$ was used to assess the effect of AAG binding on the unbound fraction $\left(\mathrm{F}_{\mathrm{u}}\right)$ of a drug. Drugs with a greater than 10 -fold preference for AAG demonstrated a greater than three-fold decrease in the free fraction with increasing AAG levels, e.g., ritonavir, imatinib, nelfinavir, docetaxel, chlorpromazine, imipramine, erythromycin, and propranolol. Test compounds that had a moderate preference (5-10 fold) for AAG showed two-fold or lower decreases in free fraction with increasing AAG concentrations, e.g., paclitaxel, saquinavir and ketoconazole, while those compounds that displayed similar affinity for AAG and HSA did not exhibit a decrease in the free fraction with increasing AAG concentrations, e.g., sulfasalazine, phenytoin, warfarin, and carbamazepine. These techniques were used to assess the involvement of AAG binding on the free fraction for several compounds under development at Array BioPharma Inc.

\section{P205. An improved approach to characterize unbound drug concentrations}

\author{
David A. Erickson, Kari Landsverk, Nicole Engh, Kristen Cardinal, Michele Hamrick, Kristine \\ Horabik, Jody Wanta and Richard Ridgewell
}

\section{Covance Labs Inc, Madison, WI}

The traditional approach for determination of differences in unbound drug concentrations between normal and special human populations, e.g., those with renal or hepatic impairment, follows an ex vivo study design. This approach entails the collection of blood samples from human subjects following administration of a candidate drug and subsequent analysis of isolated plasma for unbound drug concentration. However, there are several shortcomings with this approach. While much attention is given to sample analysis using a validated analytical method, clinical samples are often prepared using unsuitable procedures. In addition, the inability to predict or control the concentration of drug in post-dose plasma samples often results in unbound fraction below the limit of quantitation. Furthermore, the traditional ex vivo approach does not adequately factor drug concentration dependence on plasma protein binding. Therefore, the use of typical ex vivo data in pharmacokinetic analyses may be questionable when unbound fractions vary with total plasma concentrations. Here, we present a simple in vitro approach to determine unbound drug concentrations involving special populations based on the accepted practice used in normal human populations. This accepted approach begins with fortifying plasma with a known range of drug concentrations to establish relationship between drug concentration and unbound drug in plasma. This concentration-dependence relationship is applicable to subsequent clinical studies and leads to more informative data for pharmacokinetic analyses. This same strategy applies to special human populations, where pre-dose plasma from each subject is fortified with known 
concentrations of the drug, thereby offering several advantages. For example, precise control of in vitro plasma drug concentrations ensures that the measured unbound drug concentration will fit the quantifiable range of the analytical method. In addition, this approach can use a radiolabeled form of the drug, even when a nonradiolabeled form is used in the clinical study. The use of a radiolabeled form of the drug improves analytical accuracy and provides a more definitive measure of non-specific binding. We present in vitro data demonstrating good correlation with ex vivo data for accurate characterization of unbound drug concentrations, thereby verifying the in vitro approach for use with normal and special populations.

\title{
P206. Approaches to understanding the impact of blood partitioning in drug discovery
}

\author{
Tim J. Potter ${ }^{1}$, Clive Dilworth ${ }^{1}$, Sally Lee ${ }^{1}$, Hollie Adey ${ }^{1}$ and Bernard P. Murray ${ }^{2}$
}

${ }^{1}$ Cyprotex Discovery, Macclesfield, United Kingdom, ${ }^{2}$ Drug Metabolism, Gilead Sciences, Inc., Foster City, CA

Assessment of the binding of pharmaceutical agents to red blood cells is important in understanding the pharmacokinetics of a compound. Pharmacokinetic parameters are usually determined by analysis of drug concentrations in plasma rather than whole blood, which may be misleading if the compound of interest partitions into red blood cells or is excluded from them. Additionally, fraction unbound values determined in plasma may not be correlated with fraction unbound in whole blood for compounds which bind to erythrocytes. This could lead to over-prediction of whole blood potency and misinterpretation of in-vivo pharmacokinetic data if not taken into account. Since some compounds show specific binding to blood cells selection of the most appropriate concentration to measure blood partitioning is crucial. If too high a concentration is selected then binding sites in the blood become saturated and partitioning is not effectively observed; too low and detection issues start to become apparent and the physiologically relevant range is missed. We have developed robust methods for assessment of both blood to plasma ratio and fraction unbound in blood, presented herein. It was found that measurement of blood to plasma ratio was more reliable using methodology where both blood and plasma samples were analysed. This is preferable to indirect methods involving analysis only of the plasma fraction, which were found to perform poorly for compounds exhibiting low blood to plasma ratios. Concentration dependency studies revealed that a concentration of $500 \mathrm{nM}$ was most appropriate for the measurement of blood to plasma ratio. Above 2000nM, it was found that the ratio was diminished for certain compounds to an extent that it could be missed in screening. A method for measuring fraction unbound in blood was developed using equilibrium dialysis and it was found that this was well correlated with fraction unbound in plasma, except in the case of known red blood cell binders where the measured blood plasma ratio could be used to improve the correlation. A strategy for utilising these methods in drug discovery is suggested and the use of such data in a drug discovery project exemplified. It is concluded that measurement of blood-plasma ratio at the appropriate concentration at an early stage of drug discovery leads to an early understanding of potential issues, and enables effective decisions to be made on follow up screening strategy.

\section{P207. Characterization of a human hepatocelluar carcinoma cell line on micro-space cell culture plate}

\author{
Yoko Ejiri ${ }^{1}$, Akane Yoshida ${ }^{2}$, Masaya Hosoda ${ }^{1}$, Kaoru Kobayashi $^{2}$ and Kan Chiba ${ }^{2}$ \\ ${ }^{1}$ Tsukuba Research Center, Kuraray Co., Ltd., Tsukuba, Japan, ${ }^{2}$ Laboratory of Pharmacology and Toxicology, Graduate \\ School of Pharmaceutical Sciences, Chiba University, Chiba, Japan
}

Human hepatocellular carcinoma cell lines cultured in monolayer are good tools for drug metabolism study, but have limitations due to their low activities of drug-metabolizing enzymes such as cytochrome P450s (CYPs). To overcome the problem, 3-dimensional (3D) culture systems have been proposed to maintain the drug-metabolizing activities. However, special equipments and complex techniques are often required for those 3D culture systems. Simple and easyto-use 3D culture system would enable human hepatocellular cell lines to be a good tool as an alternative to human primary hepatocytes. To this end, we studied 3D culture method with micro-space cell culture plate, which has regularly arrayed micro-compartments on its surface. In this study, we evaluated the drug metabolizing enzymes of a human hepatocellular carcinoma cell line, FLC-4, cultured on the micro-space cell culture plate. To optimize cell culture conditions, FLC-4 cells were cultured on two micro-patterns ( $200 \mu \mathrm{m}$ width X $100 \mu \mathrm{m}$ depth and $200 \mu \mathrm{m}$ width X $50 \mu \mathrm{m}$ depth). Also, surface coating and seeding density were optimized. As a result, FLC-4 formed spheroid at 7-day culture on the $200 \mu \mathrm{m} \mathrm{X} \mathrm{100 \mu m} \mathrm{pattern} \mathrm{and} \mathrm{the} \mathrm{3D} \mathrm{structure} \mathrm{was} \mathrm{maintained} \mathrm{for} \mathrm{a} \mathrm{week.} \mathrm{mRNA} \mathrm{expression} \mathrm{of} \mathrm{CYP1A1,} \mathrm{1A2,} \mathrm{2C9,} \mathrm{3A4}$ and UGT1A1 on $200 \mu \mathrm{m} \mathrm{X} 100 \mu \mathrm{m}$ micro-space plate were higher than those in monolayer culture. mRNA expression of 
nuclear receptors, CAR and PXR, were also higher on micro-space culture plate than those in monolayer culture. On the other hand, spheroid formation was not observed on $200 \mu \mathrm{m}$ X $50 \mu \mathrm{m}$ micro-space plate and mRNA expression of the enzymes were much lower than those on $200 \mu \mathrm{m}$ X $100 \mu \mathrm{m}$ plate. Enzyme induction studies showed that phenobarbital induced mRNA expression of CYP3A4 and UGT1A1 significantly on micro-space culture plate. The metabolites of triazolam and SN-38, substrates of CYP3A4 and UGT1A1, were significantly formed compared to those in monolayer culture. These results suggest that the micro-space cell culture plates are useful tools for drug metabolism study using the human hepatocellular carcinoma cell line.

\title{
P208. Development of an in vitro system with human liver microsomes for phenotyping of CYP2C9 genetic polymorphisms using a mechanism-based inactivator
}

\author{
Darcy R. Flora ${ }^{1}$ and Timothy S. Tracy ${ }^{2}$ \\ ${ }^{1}$ Experimental and Clinical Pharmacology, University of Minnesota, Minneapolis, $M N,{ }^{2}$ College of Pharmacy, University \\ of Kentucky, Lexington, $K Y$
}

Polymorphisms in cytochrome P450 enzymes can significantly alter the rate of drug metabolism as well as the extent of drug-drug interactions. Individuals homozygotically expressing the *3 allele (I359L) of CYP2C9 have an $~ 80 \%$ reduction in oral clearance, and in heterozygotic individuals the reduction in clearance is $\sim 40-50 \%$. Although these polymorphisms result in a decrease in the activity of an individual enzyme molecule, we hypothesized that decreasing the total number of active enzyme molecules in an in vitro system (CYP2C9*1/*1 human liver microsomes) by an equivalent percentage could be used to mimic the same net change in overall metabolic capacity observed with CYP2C9*1/*3 and CYP2C9*3/*3. Secondarily, we hypothesized that a selective mechanism-based inactivator could be used to change this overall metabolic capacity. Thus, the CYP2C9 selective mechanism-based inactivator tienilic acid was used to irreversibly reduce total CYP2C9 activity in human liver microsomes and, therefore, mimic the CYP2C $9 * 1 / * 3$ and CYP2C9*3/*3 genotypes. Probe substrates representing the other major P450 enzymes (CYP1A2; ethoxyresorufin, CYP2B6; bupropion, CYP2C8; amodiaquine, CYP2C19; omeprazole, CYP2D6; bufuralol and dextromethorphan, CYP2E1; chlorzoxazone, and CYP3A4; testosterone) were not affected by the addition of tienilic acid, suggesting that this model is selective for CYP2C9. In lieu of using rare human liver microsomes from CYP2C $9 * 1 / * 3$ and CYP2C9*3/*3 individuals, a tienilic acidcreated knockdown in human liver microsomes may be an appropriate in vitro model to determine metabolism of a given substrate, to determine whether other enzymes may compensate for reduced CYP2C9 activity, and to predict the extent of genotype-dependent drug-drug interactions.

\section{P209. Evaluating the potential for lysosomal trapping in immortalized human hepatocytes (Fa2N-4 cells)}

\author{
Tiffini Hensley, Greg J. Loewen, Faraz Kazmi, Brian Ogilvie, David Buckley and Andrew Parkinson
}

XenoTech, LLC, Lenexa, KS

Sequestration of high concentrations of lipophilic amines (a.k.a. cationic amphiphilc drugs or CADs) in the acidic (pH 4-5) environment of lysosomes contributes to their presystemic clearance (by lysosomal trapping in liver and lung), their large volume of distribution (Vd) and their propensity to cause phospholipidosis. In a related study with Lysotracker Red, a fluorescent probe that, by virtue of being a lipophilic amine, accumulates in lysosomes, we demonstrated that Fa2N-4 cells (immortalized human hepatocytes) contain functional lysosomes, similar to those observed in non-transformed human hepatocytes. However, Fa2N-4 cell do not retain uptake transporter activity (Harisparsad et al. Drug Metab. Dispos. 36: 1046-1055, 2008). Therefore, we hypothesized that Fa2N-4 cells could be used to investigate the uptake (by passive diffusion) and lysosomal trapping of lipophilic amines (or CADs). To test this hypothesis, we measured the accumulation of two lysosomotropic probe drugs, namely propranolol and imipramine (lipophilic amines) and an acidic drug, atorvastatin (OATP substrate), in cultured Fa2N-4 cells in the presence and absence of lysosomotropic and nonlysosomotropic drugs. Plated Fa2N-4 cells were incubated with substrates at $37^{\circ} \mathrm{C}$, after which the cells were washed twice with ice-cold PBS and lysed with acetonitrile containing an internal standard. The resulting lysate was centrifuged and the supernatant fraction was analyzed by LC/MS/MS to determine the amount of probe drug that had accumulated in the Fa2N-4 cells. Propranolol (lipophilic amine) was incubated with Fa2N-4 cells in the presence of various concentrations of chloroquine (to inhibit sequestration of the probe drugs) for up to $20 \mathrm{~min}$. Accumulation of propranolol was time dependent and inhibited by chloroquine in a concentration-dependent manner. The uptake of propranolol and 
imipramine was inhibited by ammonium chloride and lysosomotropic drugs. Conversely, the uptake and accumulation of these lipophilic amines was affected to a lesser extent by atorvastatin. The accumulation of atorvastatin in Fa2N-4 cells was much lower than that observed for the lipophilic amines and its accumulation was not notably affected by lysosomotropic compounds, consistent with atorvastatin being an OATP substrate that does not undergo lysosomal trapping. Taken together, these data suggest that lipophilic amines accumulate in the lysosomes of Fa2N-4 cells and the uptake is inhibited by lysosomotropic drugs. Fa2N-4 cells may be a useful and practical alternative to cultured human hepatocytes to determine whether drug candidates are subject to lysosomal trapping.

\title{
P210. Functional evaluation of 3D-culture of human hepatocytes on cell-able under newly optimized condition
}

\author{
$\underline{\text { Shin Enosawa }}{ }^{1}$, Yuriko Takahashi ${ }^{1}$, Tomoko Jomura ${ }^{2}$, Emiko Ozeki ${ }^{2}$ and Takeshi Ikeya ${ }^{2}$ \\ ${ }^{1}$ Clinical Research Center, National Center for Child Health and Development, Tokyo, Japan, ${ }^{2}$ Transparent Inc., Chiba
}

A multi-well plate microfabricated with highly hydrophilic polymer, Cell-able facilitates 3-dimensional (3D) culture of primary hepatocytes and induces long-term well-functioning culture, comparing to conventional monolayer. We report here the improved outcome using novel culture medium specialized for human hepatocytes and mouse fibroblasts as feeder cells. [Methods] Primary human hepatocytes were isolated from surgically resected liver tissue (Etical permission No.385 and 396) or purchased as cryopreserved hepatocytes (Xenotech). Hepatocytes $\left(2 \times 10^{\wedge} 4 /\right.$ well) were seeded on 96-weell type Cell-able that had been precultured with mouse fibroblasts (JCRB9019 or ATCC CCL-163). Cells were cultured with RM101 (Transparent) based on Williams medium E containing 1\% fetal bovine serum. Hepatocyte functions were evaluated by testosterone metabolism and transportation of tritiated-taurocholic acid and carboxy-dichlorofluorescein diacetate (CDF-DA). [Results and Discussion] Hepatocytes cultured on Cell-able with RM101 showed 1.55 to 3.43 fold higher activity of testosterone 6-beta hydroxylation of other 5 media ( 3 commercially available and 2 previously reported media) examined on day 7 and the superiority increased thereafter until day 21 . Activity of testosterone 6-beta hydroxylation was maintained well for 54 days. Noticeably, the cells on day 54 increased the activity from 0.15 to $0.80 \mathrm{fmol} / \mathrm{cell} / \mathrm{min}$ in response to rifampicin. Time course changes in taurocholate uptake were $24.1 \mathrm{fmol} /$ cell (day of isolation), 2.84 (day 3), and 6.83 (day 7), while those of monolayer hepatocytes were 0.49 (day 3) and not detectable on day 7. The increase of the activity from day 3 to 7 on Cell-able was thought to be due to maturation of spheroid structure. The evidence of bilially efflux was examined by cofocal microscope using CDF-DA. The clear fluorescent spots were detectable intercellular area of the spheroids. When calcium ion was withdrawn from the culture medium, the intensity and sharpness of fluorescent spots became weaker and vaguer, suggesting the formation of small bile duct-like structure between hepatocytes. The present results indicate that Cell-able culture system suits for the examination of a sequence of reactions of DMPK research.

\section{P211 - In vitro reactivity assessment of covalent drugs targeting Bruton's tyrosine kinase}

\author{
Matthew Labenski, Prasoon Chaturvedi, Erica Evans, Hormoz Mazdiyazni, Michael Sheets, Sharon \\ Aslanian, Mariana Nacht, Russell Petter, Juswinder Singh and William Westlin
}

\section{Avila Therapeutics, Waltham, MA}

Covalent inhibitors have proven to be very successful therapies for a wide range of indications. To ensure that the potential for non-specific reactivity is minimized, all new covalent inhibitors should be screened against both the intended target as well as potential off-target proteins. Bruton's tyrosine kinase (Btk) is a member of the Tec family of kinases that is involved in B-lymphocyte development, differentiation, and B cell receptor signaling. CNX-774 is a potent, selective, and orally available small molecule inhibitor of Btk that forms a ligand-directed covalent bond with Cys-481, a non-conserved amino acid within the active site of the enzyme. In this study, we have assessed the off-target reactivity and specificity of CNX-774, employing a panel of HPLC and LC-MS based assays. In biochemical assays, CNX-774 has demonstrated potent inhibition of Btk with an IC50 of $<1 \mathrm{nM}$ in a continuous-read assay (Omnia, Invitrogen). The covalent bonding of CNX-774 to Btk was confirmed by incubating recombinant Btk protein with a 10-fold molar excess of CNX-774 for 1 hour at room temperature and analysis by MALDI-TOF MS. A shift of protein mass corresponding to the molecular weight of CNX-774 confirmed the covalent bonding of CNX-774 to Btk. Digestion of the covalently bonded Btk with pepsin followed by MSMS analysis established the bonding of CNX-774 to Cys-481. Cellular potency as well as prolonged duration of action of CNX-774 was demonstrated in Ramos cells by using a biotinylated covalent probe 
that targets the same Cysteine residue as CNX-774. In order to assess the reactivity and potential off-target non-specific binding of CNX-774, it was tested in a panel of assays that included glutathione (GSH) reactivity, plasma protein bonding in an albumin-depleted human plasma preparation, and general extractability in whole blood. Conjugation of CNX-774 with GSH did not occur after incubation with $10 \mathrm{mM} \mathrm{GSH}$ at pH 7.4 for 4 hours at $37^{\circ} \mathrm{c}$. Off-target protein reactivity was assessed by incubating CNX-774 with albumin-depleted human plasma at a final concentration of $0.1 \mathrm{mM}$ for $1 \mathrm{hr}$ at $37^{\circ} \mathrm{C}$, followed by analysis by MALDI-TOF MS. No change was observed in the molecular mass of any protein in the compound-treated plasma protein sample demonstrating that CNX-774 does not bond covalently to any of the highto mid-level abundance human plasma proteins. The potential covalent bonding of CNX-774 to off-target proteins in blood was determined by incubating CNX-774 in fresh rat or human whole blood at $37^{\circ} \mathrm{C}$ and monitoring the compound concentration over time by mass spectrometry. CNX-774 was found to be $>90 \%$ extractable after $2 \mathrm{hrs}$ of incubation in both rat and human whole blood. These results demonstrate that CNX-774 has potent inhibitory activity towards the intended target, Btk, while achieving remarkable specificity in a variety of assays designed to assess off-target reactivity towards abundant cellular thiols and blood proteins. These studies demonstrate the utility of intelligently designed in vitro assays to determine specificity and off-target reactivity of targeted covalent inhibitors at the discovery stage of the drug development process minimizing the potential for unintended non-specific reactivity.

\title{
P212. Increase of glucuronidation in human liver microsomes by PLA2 inhibition: Implications for improved PK predictions
}

\author{
Erin F. Mulrooney ${ }^{1}$, Sebastien Gagné2 ${ }^{2}$ Cody van Dijk², Julien Breault-Turcot' ${ }^{2}$, Jean-François \\ Lévesque $^{2}$ and Deborah A. Nicoll-Griffith ${ }^{3}$ \\ ${ }^{1}$ Drug Metabolism and Pharmacokinetics, Merck, Boston, $M A,{ }^{2}$ Preclinical Drug Metabolism and Pharmacokinetics,
Merck Frosst Canada \& Co., Kirkland, QC, Canada, ${ }^{3}$ Global DMPK, Merck \& Co, Rahway, NJ
}

In recent years, compounds eliminated wholly or in part by glucuronidation have represented a significant proportion of Merck's pipeline. However, these compounds generally have uncertainty around their human PK predictions making it difficult to assess the probability of success early in development. Commonly employed in vitro tools to assess metabolism, such as liver microsomes, generally under-predict clearance for compounds eliminated by glucuronidation, more so than for compounds eliminated by oxidative metabolism. In recent studies it was suggested that longchain unsaturated fatty acids, in particular arachidonic acid, present in human liver microsomes (HLM) can be potent inhibitors of glucuronidation through UGT1A9 and UGT2B7 (Tsoutsikos et al, 2004, Rowland et al., 2007, 2008). In this study we have explored this theory further by quantifying the inhibitory potential of arachidonic acid and other free fatty acids on recombinant human UGT1A9 and UGT2B7. It was found that arachidonic acid is an inhibitor of UGT1A9 and UGT2B7 with $\mathrm{IC}_{50, \text { app }}$ values of 0.7-0.8 $\mu \mathrm{M}$ and 6-24 $\mu \mathrm{M}$ respectively. Additionally, we have investigated the use of a potent, irreversible Phospholipase $\mathrm{A}_{2}\left(\mathrm{PLA}_{2}\right)$ inhibitor (methyl arachidonyl fluorophosphonate, MAFP) and bovine serum albumin (BSA) during microsomal preparation to minimize the production of arachidonic acid. HLM prepared from a pool of 3 donors with BSA and MAFP contained almost 7 -fold less arachidonic acid compared to control preparations and almost 9-fold less arachidonic acid following incubation at $37^{\circ} \mathrm{C}$ for $3 \mathrm{~h}$. This translated to an increase in the rate of zidovudine (a UGT2B7 specific substrate) and propofol (a UGT1A9 specific substrate) glucuronidation by 10 and 8-fold respectively in these HLM preparations with no significant difference in microsomal binding between control preparations and preparations containing both BSA and MAFP for either substrate. In vitro-in vivo extrapolations using the well stirred model and data from control microsomes under-predicted human in vivo $\mathrm{CL}_{\text {int }}$ by more than 100-fold. This under-prediction was improved to 9-fold when microsomes prepared with BSA and MAFP were used. This work provides an alternative microsomal preparation technique that does not rely on altering the commonly employed incubation conditions. As the under-prediction of glucuronidation observed when extrapolating from HLM systems is most likely a multi-faceted issue it is unlikely to be rectified by one explanation alone. However, this work might be one piece of the puzzle towards developing more predictive in vitro tools for predicting human PK of certain compounds eliminated by glucuronidation.

\section{P213. Integrated discrete multiple organ co-culture (IdMOC) system for the evaluation of multiple organ drug distribution, drug metabolism, and organ specific drug toxicity}

\author{
Albert P. Li, Utkarsh Doshi, Aarti Uzgare, Hariharan Saminathan and Qian Yang
}


The metabolic fate of a xenobiotic in vivo often involves multiple organs. However, investigation on drug metabolism is routinely performed using experimental systems derived from a single organ, especially liver-derived systems such as liver microsomes and hepatocytes. The Integrated Discrete Multiple Organ Co-culture (IdMOC $\left.{ }^{\mathrm{m}}\right)$ system has been developed in our laboratory as an in vitro experimental system to overcome this major deficiency of single-organ systems. The IdMOC employs a wells-in-a well concept, with multiple inner wells located within a larger, containing well. Multiple cell types are firstly cultured individually in each inner well, followed by flooding and interconnection of the inner wells with an overlying medium. The IdMOC thereby models an organism with multiple organs that are connected by the systemic circulation. We report here the application of IdMOC in the co-culturing of major cell types of the major human organs: hepatocytes (liver), renal proximal tubule cells (kidney), neurons (nervous system), adipocytes (fat) and skeletal myocytes (skeletal muscle). Using IdMOC, in vitro pharmacokinetic/toxicokinetic studies can be performed via quantification of parent disappearance and metabolite formation in the medium, quantification of drug distribution in each cell type, as well as the evaluation of toxicity in each cell type. Results with model drug metabolizing enzyme substrates, transporter substrates, and organ-specific toxicant including midazolam (CYP3A substrate), L-glutamic acid gamma (4- methoxy-beta naphthylamide) (GGT substrate), metformin (OCT1 substrate), simvastatin (SLCO1B1 substrate and skeletal muscle toxicant), and aflatoxin B1 (hepatotoxicant and hepatocarcinogen) are shown, using LC/MS/MS for the quantification of parent and metabolites and multiple endpoints (MTT metabolism, cellular ATP content, cellular GSH content) for cytotoxicity. The results demonstrate that IdMOC is as an effective in vitro experimental tool for the evaluation of multiple organ drug distribution, metabolism, and toxicity.

\title{
P214. Plasma protein binding: A comparison of techniques to help overcome common issues encountered during in vitro testing
}

\section{Guy Webber}

\author{
Department of In Vitro Sciences, Quotient Bioresearch Ltd, Rushden, United Kingdom
}

The extent to which a drug binds to plasma proteins is a commonly derived parameter during drug discovery and development. It is assumed that only free, unbound, systemic drug is available to cause an efficacious effect in vivo, although this view has recently been challenged ${ }^{[1]}$. Nevertheless, plasma protein binding remains an integral parameter to assess to aid in the evaluation of pharmacological, pharmacokinetic and toxicological data. In common with other areas of in vitro science, recent product development in this area has been extensive and there are now different apparatus available for use to assess plasma protein binding in the modern DMPK laboratory. To assess the utility of some of these new apparatus in the laboratory, we have compared the human plasma protein binding of atenolol (low plasma protein binding), testosterone (medium plasma protein binding) and warfarin (high plasma protein binding) using recently launched apparatus. These include: Fast-Micro-equilibrium DIALYZER ${ }^{\mathrm{m}}$ devices, DispoEquilibrium DIALYZER ${ }^{\mathrm{m}}$ devices, RED $^{\mathrm{mm}}$ devices and the HTDialysis $96^{\mathrm{m}}$ well plate and we have compared the binding values obtained with more traditionally encountered techniques such as equilibrium dialysis using Dianorm ${ }^{\mathrm{mt}}$ dialysis cells, ultrafiltration (Centrifree ${ }^{\mathrm{Tt}}$ devices) and ultracentrifugation. As anticipated, for our highly bound drug, warfarin, generally, there was good agreement between most of the techniques used (range 98.4098.47\% bound). Binding was slightly higher with RED $^{\mathrm{mm}}$ devices $(98.68 \%)$ and highest using DispoEquilibrium DIALYZER ${ }^{\mathrm{m}}$ devices (99.25\%). With testosterone, which is less bound than warfarin, greater variation was observed across the apparatus used. There was excellent agreement between ultrafiltration and ultracentrifugation (93.05 and 93.14\%, respectively). Slightly higher binding values were obtained using the other devices (range 94.16-95.02\%). Similar to warfarin, the highest binding value was observed using DispoEquilibrium DIALYZER ${ }^{\mathrm{m}}$ devices (96.51\%). With atenolol, where plasma protein binding is low, there was greatest variation with binding ranging from $0 \%$ bound using the traditional Dianorm ${ }^{\mathrm{m}}$ dialysis cells, up to a maximum of $35.46 \%$ bound using the DispoEquilibrium DIALYZER ${ }^{\mathrm{mm}}$ devices. This work has been conducted as part of an on-going validation plan within Quotient Bioresearch and it is hoped the outcomes will aid in overcoming some of the commonly encountered issues that are associated with assessing plasma protein binding in vitro, namely, low throughput, problematic compounds (typically highly lipophilic compounds) and low sample volumes (typically from neonatal plasma).

[1] The effect of plasma protein binding on in vivo efficacy:misconceptions in drug discovery, Dennis A. Smith, Li Di and Edward H. Kerns, Nature Reviews: Drug Discovery, 9 December 2010, 929 - 939.

\section{P215. TRANSIL high sensitivity binding assay: A novel tool for assessing plasma binding of hardly soluble and sticky drugs}

\author{
Hinnerk Boriss and Kathleen Boehme
}


Partitioning approaches, like erythrocyte partitioning, have been used over several decades to assess plasma binding of drugs that prove difficult to handle in dialysis or ultrafiltration devices. However, these methods have never gained great popularity since they are cumbersome as well as time and resource demanding. Here we present validation data for an assay that is easy to use, scalable to various needs of automation, and generates results in less time than dialysis. The assay is based on a binding equilibrium shift method to determine the affinity of drugs to plasma. By providing pre-determined ratios of membrane surface areas and plasma concentrations, the partitioning between membrane and plasma results in a series of different equilibria, which in turn define the affinity to plasma. In a standard configuration the assay allows accurate measurement of unbound fractions of at least $0.0001 \%$. At the same time recovery typically remains above $70 \%$, because all assay compartments contain plasma which stabilizes highly lipophilic and barely soluble drugs in solution. For a set of ten less lipophilic and well soluble drugs we demonstrate that the assay yields identical unbound fractions of drug in plasma as dialysis. While for drugs like cyclosporine, the assay yields results comparable to the ultracentrifugation method. We therefore conclude that the assay yields more reliable results for tightly bound and hardly soluble drugs than dialysis or ultrafiltration.

\title{
P216. A comparative kinetic study of [14C]-eugenol and [14C]-methyleugenol activation and detoxification in human, mouse, and rat liver fractions
}

\author{
Emmanuel Minet ${ }^{1}$, Daniela Gentile ${ }^{2}$, Clive Meredith ${ }^{1}$, Nik Newland $^{1}$ and Eian Massey ${ }^{1}$ \\ ${ }^{1}$ Risk Characterization, British American Tobacco, Southampton, United Kingdom, ${ }^{2}$ In Vitro Sciences, Charles River \\ Laboratories, Edinburgh, United Kingdom
}

Introduction: Eugenol (CAS № 97-53-0) is one of a series of alkenylbenzene compounds (including safrole, methyleugenol and isoeugenol) that occur naturally in plants. It is used in a variety of consumer products as a flavoring agent, antiseptic or anesthetic. There is limited evidence for the carcinogenicity of eugenol to experimental animals. However, in vitro tests for the genotoxic potential of eugenol, whilst usually negative, have on occasion reported a positive result. In contrast, the structurally related compound methyleugenol is consistently reported as genotoxic and carcinogenic in a variety of in vitro and in vivo mammalian models. Objective: The absence of unequivocal translation of toxicity data obtained from animal models to human is a limiting factor for eugenol toxicity assessment. Thus, we decided to compare the bioactivation and detoxification of eugenol and methyleugenol at a mechanistic level in human, rat and mouse to further assess their potential toxicity. Method: The formation of a 1'-hydroxy proximate carcinogen, and a cytotoxic quinone methide, two key toxic metabolites for alkenylbenzenes, were quantified in hepatic microsomes and S9 fractions in the presence of phase I and phase II cofactors. Kinetic constants ${ }_{\text {app }} \mathrm{Km}$ and ${ }_{\text {app }}$ Vmax were measured for these reactions. Results: We report that methyleugenol generates a significant amount of the 1 '-hydroxy proximate carcinogen while eugenol glucuronidation prevents the formation of both 1'-hydroxyeugenol and the quinone methide. Species comparison and kinetics highlighted key metabolic differences between human, mouse, and rat, providing a mechanistic insight into the bioactivation and detoxification of eugenol and methyleugenol in these species.

\section{P217. A simple, robust automated multiplexed cell-based assay process for the assessment of mitochondrial dysfunction and cytotoxicity}

\author{
Bradley R. Larson ${ }^{1}$, Peter Banks ${ }^{1}$, Tracy Worzella ${ }^{2}$, Timothy A. Moeller ${ }^{3}$ and Andrew Niles ${ }^{4}$ \\ ${ }^{1}$ BioTek Instruments, Winooski, VT, ${ }^{2}$ Scientific Applications and Technology Integration, Promega Corporation, Madison, \\ WI, ${ }^{3}$ Celsis In Vitro Technologies, Baltimore, MD, ${ }^{4}$ Research and Development, Promega Corporation, Madison, WI
}

Mitochondrial perturbation is a common mechanism of drug-induced toxicity. Recent advances in mitochondrial study have revealed that numerous drugs that were withdrawn from the market demonstrate strong mitochondrial impairment in the liver or the heart. These include troglitazone (Rezulin), cerivastatin (Baycol), and nefazodone (Serzone). Therefore it is becoming increasingly important to focus on earlier identification of lead compounds that impact mitochondrial function during the discovery phase of drug development. Here we demonstrate the utility of a multiplexed assay to assess cell membrane integrity changes (cytotoxicity), as well as mitochondrial function. Cytotoxicity is first assessed by measuring the presence or absence of a distinct protease activity associated with necrosis using a fluorogenic peptide substrate (bis-AAF-R110) to measure "dead cell protease activity". The substrate cannot cross the intact membrane of live cells and therefore gives no signal with viable cells. Mitochondrial function is then measured by adding an ATP detection reagent, resulting in cell lysis and generation of a luminescent signal proportional to the amount of ATP present. 
The combination of the two readouts allows for distinguishing between compounds that exhibit mitochondrial toxicity and those that inhibit ATP production due to cytotoxic effects, such as primary necrosis. The entire process was automated, including cell dispense, compound titration and transfer, and reagent addition. Primary hepatocytes, as well as the HepG2 cell line were used as model systems. Automated assay validation, effect of glucose-containing and nonglucose media (Crabtree effect), and pharmacology experiments were performed. Results demonstrate the utility of this automated, multiplexed assay to rapidly profile compounds for effects on mitochondrial function.

\title{
P218. A fluorescence based screening approach for determination of drug-phospholipid complex formation and prediction of phospholipidosis
}

\author{
Liping Zhou' ${ }^{1}$ Gina Geraci², Linhong Yang' ${ }^{2}$ Jianling Wang ${ }^{2}$ and $\underline{\text { Upendra A. Argikar }}{ }^{2}$
}

${ }^{1}$ Chemical and Pharmaceutical Profiling, Novartis Institutes for Biomedical Research Inc., Cambridge, MA, ${ }^{2}$ Metabolism and Pharmacokinetics, Novartis Institutes for Biomedical Research Inc., Cambridge, MA

Drug-induced phospholipidosis (PLD) is a lipid storage disorder characterized by reversible accumulation of phospholipids in tissues. It is generally considered to be an adaptive response upon administration of certain drug-typically cationic amphiphilic drugs (CADs)-rather than a toxic manifestation. However, tissue and organ damage have been reported in association with PLD for some cases. There is only a limited pool of available screening methods to predict a drug's PLD-inducing potential. We have developed a high-throughput fluorescence non-cell based assay to screen for the drug-phospholipid interaction, which correlates to phospholipidosis. Anionic amphiphilic phospholipids can form complexes in aqueous solution and its critical micelle concentration (CMC) is determined using the fluorescence probe N,N-dimethyl-6-propionyl-2-naphthylamine (Prodan). Upon interaction with drug candidates, this CMC may shift to a lower value due to the association between lipids and drug candidates. The relative change, CMCDL/CMCL, provides a direct measurement of the drug-lipid association. Metabolism of a drug can change the degree of phospholipidosis depending on the rate of metabolism and the nature of the metabolite(s). Our data from forty-five drugs, along with metabolites of ten drugs, in this fluorescence assay demonstrate a good correlation with phospholipidosis as reported in cellular assays, in vivo tests, and human studies in the literature. We therefore report the first fluorescence based non-cell in vitro assay to screen for PLD-inducing potential in drug candidates, which is additionally fast, reliable and cost-effective.

\section{P219. A novel in vitro approach for risk assessment of reactive metabolites in drug discovery}

\author{
$\underline{\text { Richard A. Thompson }}{ }^{1}$, Emre M. Isin ${ }^{1}$, Yan $\mathrm{Li}^{2}$, Lars Weidolf ${ }^{1}$, Ian Wilson ${ }^{3}$, Ken Page ${ }^{3}$, Steve Swallow \\ and Gerry Kenna ${ }^{4}$
}

${ }^{1}$ Dmpk IM, AstraZeneca R\&D Molndal, Mölndal, Sweden, ${ }^{2}$ Dmpk IM, AstraZeneca, Waltham, MA, ${ }^{3}$ Dmpk IM, AstraZeneca, Macclesfield, United Kingdom, ${ }^{4}$ Safety Assessment, AstraZeneca, Macclesfield, United Kingdom

The Biotransformation of drug molecules may result in the formation of stable as well as electrophilic reactive metabolites (RM). These chemically reactive species have the propensity to react with cellular macromolecules and have been implicated in an array of adverse drug reactions (ADRs), with the liver being the most frequently affected organ. Drug induced liver injury (DILI) accounts for over $50 \%$ of the cases of acute liver failure in the United States. Historically, the approach within the Pharmaceutical Industry to the threat from RMs has been one of minimizing RM formation. While this approach has been useful in numerous cases, it has had major drawbacks for use as a decision making tool for the progression of compounds. We have previously proposed that addressing potential RM risks needs to be done alongside other potential mechanisms of DILI. (1) We present now an approach for risk assessing compounds based on combining scores from two independent in vitro tools:

1. Estimated RM Body Burden based on the fraction of metabolism leading to covalent binding in human hepatocytes and the estimated therapeutic dose.

2. An in vitro hepatic screening panel composed of assays designed specifically to assess processes that have the potential to initiate liver injury. The panel consists of assays to assess i) cell toxicity - immortalized human hepatocyte derived cell lines (THLE) with no CYP activity or with 3A4 activity, ii) mitochondrial impairment- HepG2 cytotoxicity in galactose $v$ s. glucose medium, iii) inhibition of hepatic biliary transporter activity-Bile Salt Export Pump (BSEP) and Multi-drug resistance protein Type 2 (Mrp2) 
Using a set of marketed drugs we have shown that this combination approach gives a unique new way of assessing risk related to RMs. We have found that the use of a quantitative measure of the propensity to form RMs in combination with the in vitro hepatic screening panel assessing cellular processes with the potential to initiate liver injury, gives a much more holistic appreciation of potential hazards behind DILI. The results from this assessment and potential for use in the Pharmaceutical industry will be discussed.

(1) R.A. Thompson, E.M. Isin, Y. Li, R. Weaver, L. Weidolf, I. Wilson, A. Claesson, K. Page, H. Dolgos, J.G. Kenna, Risk assessment and mitigation strategies for reactive metabolites in drug discovery and development, Chem. Biol. Interact. 192 (2011) 65-71. doi:10.1016/j.cbi.2010.11.002.

\title{
P220. Bioactivation and dehydrogenation of naltrexone and oxycodone in human liver microsomes: Identification of the reactive quinone methide species
}

\author{
$\underline{\text { Bo Wen }}^{1}$, Jiansheng Huang ${ }^{2}$ and David Moore ${ }^{3}$ \\ ${ }^{1}$ Drug Metabolism and Pharmacokinetics, Hoffmann-La Roche, Nutley, NJ, ${ }^{2}$ Hoffmann-La Roche, Nutley, NJ, ${ }^{3}$ Dept of \\ Non-Clinical Safety, Hoffmann-La Roche Inc., Nutley, NJ
}

Naltrexone, a potent narcotic antagonist used in maintenance treatment for opiate dependence, has been associated with very rare but severe incidences of hepatotoxicity in patients. Because of its hepatotoxicity, FDA has issued a black box warning concerning its potential to cause hepatocellular injury when given in excessive doses. While the mechanism of idiosyncratic hepatotoxicity remains unknown, it is possible that metabolic activation and subsequently covalent binding of reactive metabolites to cellular proteins play a causative role. Studies were initiated to determine whether naltrexone undergoes cytochrome P450 (P450)-mediated bioactivation in human liver microsomes to electrophilic intermediates. LC/MS/MS analysis of incubations containing naltrexone in the presence of NADPH and glutathione (GSH) revealed formation of a GSH conjugate derived from the addition of the sulfydryl nucleophile to the unchanged parent molecule of naltrexone. Formation of reactive intermediates of naltrexone was primarily mediated by heterologously expressed recombinant CYP3A4, and to a less extent CYP2D6, CYP1A1, and CYP1A2. Oxycodone, a close analog of naltrexone which also causes hepatotoxicity in some patients, formed a similar GSH conjugate after initial $O$-demethylation of 3-methoxyl group. A tentative pathway states that a bioactivation sequence involves P450-catalyzed two-electron oxidation and dehydrogenation of the hydroxymorphinan core to an electrophilic quinone methide intermediate, which is subsequently attacked by glutathione yielding the benzylic sulfydryl conjugate. The results from the current investigation constitute the first report on the cytochrome P450-catalyzed bioactivation of the morphinan drugs naltrexone and oxycodone. In summary, these findings are of significance in the understanding of the bioactivation pathways of the hydroxymorphinan core and their potential link to mechanisms of toxicity of naltrexone and oxycodone.

\section{P221. Abstract withdrawn}

\section{P222 . Abstract withdrawn}

\section{P223. Effect on GSH and GSSG levels in rats treated with acetaminophen and tamoxifen using LC-MS/MS}

\author{
Helen L. Shen, Lata Venkatarangan, Bruce Aungst and Zamas Lam
}

Dmpk, QPS, LLC, Newark, DE

Many drug candidates form glutathione conjugates in treated animals. Glutathione levels and glutathione depletion may be associated with hepatotoxicity for some compounds. During lead optimization a common approach is to minimize reactive metabolite formation through appropriate structural modification. In this study, we investigated compounds that are known to form glutathione conjugate and measured their effects on levels of glutathione (GSH) and glutathione disulfide (GSSG) in liver, bile, and plasma in rats. Bile duct-cannulated rats were treated by oral administration with vehicle, tamoxifen, or acetaminophen for 4 days. Plasma and bile samples were collected daily up to $4 \mathrm{~h}$ post-dose, and liver tissues were harvested on Day 4 after the collection of plasma and bile samples. Plasma, bile, and liver samples were stabilized by the addition of acid and/or Ellman's reagent to prevent the oxidation of GSH to GSSG. LC-MS/MS methods were developed for the analysis of 
GSH and GSSG in these matrices. Stability test was carried out for each matrix with stabilizing agent to ensure proper storage condition for the samples. Because of the high endogenous levels of GSH and GSSG in the plasma, bile, and liver, surrogate matrices were used for the preparation of calibration standards. GSH assays were carried out with and without derivatization with Ellman's reagent. Extent of derivatization in the surrogate matrix and matrix effect were also investigated. LC-MS/ MS responses for the standards at high concentration were shown to be similar between the sample matrices and surrogate matrices. GSH and GSSG concentrations in the plasma, bile, and liver tissue from the treated animals were compared to those from the vehicle control animals. The effect was also compared among the three matrices from the treated animals. The advantage of this plasma/bile bioanalytical method over liver analysis is the ability of timed sample without needing terminal time-point, and to correlate GSH/GSSG ratio with the plasma concentration of the drug candidate.

\title{
P224. Abstract withdrawn
}

\section{P225. Intrauterine origin of caffeine-induced high susceptibility to adult metabolic syndrome: Alleviated glucocorticoid inactivation alters the neuroendocrine metabolic programming of fetal rats}

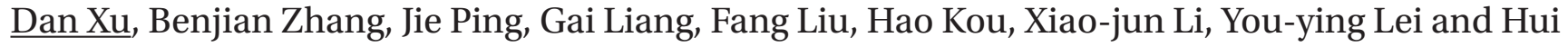 \\ Wang
}

Pharmacology, Basic Medical School of Wuhan University, Wuhan 430071, China, Wuhan, China

Epidemiological investigations suggest that there is a fetal origin of metabolic syndrome - intrauterine growth retardation (IUGR). However, no complete and systematic theoretical system has yet reached on the mechanism of IUGR and increased susceptibility to adult metabolic syndrome. Xenobiotics are known as the most precise and dangerous incentives of IUGR and it has been demonstrated that caffeine as a xanthine alkaloid widely present in coffee, tea, cola beverage and some analgesic drugs, could lead to reproductive and embryo toxicity. Recent epidemiological investigation also shows that while caffeine were ingested by child and adolescent, individual obesity incidence and adult metabolic syndrome susceptibility are significantly increased. In the present study, we systematically investigated caffeine induced IUGR and related adult metabolic syndrome. The underlying mechanism "fetal neuroendocrine metabolism programming" was also demonstrated. We initially confirmed the phenomenon through animal test that caffeine exposure during pregnancy could cause IUGR, and subsequent increased hypothalamic-pituitary-adrenal axis (HPA) sensitivity, disordered glucose and lipid metabolism after maturity. Based on the established IUGR rat model, we demonstrated the development of fetal HPA axis was suppressed, manifested as the significantly decreased mRNA and protein expressions of corticotropin release hormone (CRH) in fetal hypothalamic and sharply decreased mRNA expressions of steroidogenic acute regulatory protein (StAR) and cytochrome $\mathrm{P} 450$ cholesterol side chain cleavage (P450scc) in fetal adrenal, in caffeine-treated groups. And we also found that the expression of hippocampus glucocorticoid receptor (GR), a regulation center of HPA axis, was enhanced, after prenatal caffeine exposure. Meanwhile, glucose and lipid metabolism pathways in liver were changed as well, manifested as that prenatal caffeine exposure could inhibit insulin-like growth factor-1 (IGF-1)/insulin signaling pathways in fetal liver and enhance the adiponectin/leptin signaling pathways with typical characteristics of functional changes in pre-receptor and/or receptor level. Further, on cultured primary fetal hippocampal neurons in vitro, we found that caffeine could increase the methylation frequency in the promoter region of 11b-hydroxysteroid dehydrogenase-2 (11b-HSD-2) which is recognized as the metabolic enzyme of glucocorticoid. These results suggest that caffeine exposure during pregnancy can change fetal HPA axis neuroendocrine-related metabolic programming and then increase the susceptibility to adult metabolic syndrome; caffeine induced-glucocorticoid (GC) metabolic activation in fetal tissue may mediate and be responsible for these alternations previously mentioned. All of these works will help, at least partly, to resolve fetal development origin of insulin resistance and metabolic syndrome, and it is also significant for understanding some new risk factors of adult metabolic syndrome comprehensively. *This work is supported by the National Nature Science Foundations of China (No. 30830112, 81072709), Innovative Reaearching Group for the Nature Science Foundation of Hubei Province (No. 2009CDA079) and Fundamental Research Funds for the Central Universities (No. 3081015).Correspondence to: wanghui19@whu.edu.cn

\section{P226. Pyrrolizidine alkaloids induce fetal developmental toxicities in mice}

\author{
Hao Kou ${ }^{1}$, Jie Xiong ${ }^{1}$, Zhen-guo $\mathrm{Ma}^{1}$, Jiang Zheng ${ }^{2}, \underline{\mathrm{Yu} \mathrm{Guo}}^{1}$ and Hui Wang ${ }^{1}$ \\ ${ }^{1}$ Pharmacology, Basic Medical School of Wuhan University, Wuhan, China, Wuhan, China, ${ }^{2}$ Pediatrics/Seattle \\ Children's Hospital Research Institute, University of Washington, Seattle, WA
}


Background: Pyrrolizidine alkaloids (PAs) are toxic alkaloids widely presented in traditional herbs, food supplements and tea drinks. Previous studies show that, in addition to hepatotoxicity2, PAs also result in reproductive and developmental toxicities. However, such toxic effects of retrorsine (RTS) and monocrotaline (MCT) in mice, two typical PAs, have not been totally identified yet. Methods: We investigated the effects of PAs on fetal mice by exposing the pregnant mice to RTS $(20 \sim 10 \mathrm{mg} / \mathrm{kg} \bullet \mathrm{d})$ and MCT $(80 \mathrm{mg} / \mathrm{kg} \bullet \mathrm{d})$ respectively from gestational day (GD) 7 to GD17. The control mice were treated with equal volume of vehicle. The indices, namely body weight, body length and tail length, were examined on GD 17 to indicate the fetal physical development. The level of adrenocorticotrophic hormone (ACTH) and corticosterone (CORT) in fetal plasma were measured by ELISA kit. HE staining was used to observe morphological change of fetal liver and lung. Enlrich reagent was applied to determine the bound pyrrole metabolite of PAs in fetal liver and lung, and the content of glutathione (GSH) in these tissues was detected simultaneously. In fetal liver, the activity of alanine transaminase/ aspartate aminotransferase (ALT/AST) and of glutathione redox system including glutathione-Stransferase (GST), glutathione peroxidase (GPx), glutathione reductase (GR) as well as other oxidative parameters such as superoxide dismutase (SOD) activity and malonaldehyde (MDA) content were measured as well. Results: When compared with control, $10 \mathrm{mg} / \mathrm{kg}$ RTS decreased the fetal body weight and length $(\mathrm{P}<0.01, \mathrm{P}<0.05)$ while both $20 \mathrm{mg} / \mathrm{kg}$ of RTS and $80 \mathrm{mg} / \mathrm{kg}$ of MCT restricted the fetal physical development of mice to some extent. The level of fetal plasma CORT in RTS groups were higher than that of control, notably, $20 \mathrm{mg} / \mathrm{kg}$ RTS significantly elevated the level of fetal plasma ACTH. Local congestion was found in fetal liver and cellular necrosis was observed in fetal lung. A certain amount of bound pyrrole metabolites could be detected in fetal liver and lung from any PA-treated group. RTS reduced the GSH content in fetal liver and lung compared to the control but without obvious dose-effect relationship, while MCT had no significant effect on GSH content. The activities of ALT/AST, GST, GPx and GR were all decreased to a certain degree. The content of MDA was augmented especially in $20 \mathrm{mg} / \mathrm{kg}$ of RTS group $(\mathrm{P}<0.05)$, while the activity of SOD in all PA groups were lower than that of control $(\mathrm{P}<0.01)$. Conclusions: Both RTS and MCT could induce developmental toxicities in mice, notably for RTS which severely retarded fetal physical development. These toxicities may be closely associated with metabolic injury of PA that conjugation of PA metabolites with GSH caused a depletion of GSH then lead to oxidative injury in local tissues. Besides, the elevated level of fetal plasma glucocorticoids (GC) may result from the fetal overexposure to maternal GC.*This work is supported by the National Nature Science Foundations of China (No. 30830112,30901835) and the Research Fund for the Doctoral Program of Higher Education of China (No:20090141110009).Correspondence to:Yu Guo, lily_0514@163.com

\title{
P227. Risk assessment for idiosyncratic hepatotoxicity of a BACE1 inhibitor by covalent binding in human $\mathrm{S9}$ fractions
}

\author{
Louis Leung ${ }^{1}$, Zhuang Miao ${ }^{1}$, Michael Brodney ${ }^{2}$, Kevin Ogilvie $^{2}$, Klass Schildknegt ${ }^{3}$ JianHua Liu ${ }^{1}$ \\ and R. Scott Obach ${ }^{1}$
}

${ }^{1}$ Pharmacokinetics Dynamics and Metabolism, Pfizer Inc., Groton, CT, ${ }^{2}$ Neuroscience Med Chem, Pfizer Inc., Groton, CT, ${ }^{3}$ Research API-Radiochemistry, Pfizer Inc., Groton, CT

Metabolic activation of a drug molecule to reactive intermediates, which subsequently form covalent adducts with tissue macromolecules, is believed to be one of the mechanisms that can lead to liver toxicity or idiosyncratic adverse reactions. A BACE1 (Beta-site Amyloid precursor protein Cleavage Enzyme 1) inhibitor (Compound B) was found to form glutathione adducts in incubations with human liver microsomes in a NADPH-dependent manner. The mechanism of bioactivation was postulated to be due to hydroxylation on the phenol group of the molecule to form catechols followed by subsequent oxidation to a reactive O-quinone. The covalent binding of a tritium-labeled analog of Compound B was determined in human S9 fractions in the absence or presence of various cofactors (UDPGA, SAM, PAPS or GSH) to assess the role of non-P450-mediated metabolism as detoxicating pathways by potentially reducing the extent of covalent binding. The fraction of metabolism comprised by covalent binding was deduced by determining the intrinsic clearance of covalent binding and the total intrinsic clearance in S9 fractions. Finally, the hypothetical total body burden of covalent binding was estimated by taking into consideration of the possible human dose range, and compared with literature data of 18 marketed drugs(1). Compound B underwent NADPH-dependent covalent binding in human S9 fractions, the extent of which was reduced to varying degrees in the presence of cofactors required for non-P450-mediated metabolism. Taking into consideration of the estimated hypothetical total body burden of covalent binding and the possible human dose range, the risk of idiosyncratic hepatotoxicity for Compound B may be considered to be moderate to low. The current methodology for risk assessment of idiosyncratic hepatotoxicity may be useful in candidate optimization and selection during early drug discovery. (1)Bauman JN et al., Can in vitro metabolism-dependent covalent binding data distinguish hepatotoxic from nonhepatotoxic drugs ?An analysis using human hepatocytes and liver S9 fraction. Chem Res Toxicol 2009;22(2):332-340. 


\title{
P228. Abstract 1ithdrawn
}

\section{P229. Thyroxine $\left(T_{4}\right)$ catabolism in human and rat hepatocytes increases following exposure to prototypical hepatic enzyme inducers}

\author{
$\underline{\text { Vicki M. Richardson }^{1} \text {, Yusupha M. Sey }}{ }^{2}$ and Michael J. DeVito ${ }^{3}$ \\ ${ }^{1}$ ORD/Nheerl/Istd/PB, USEPA and UNC at Chapel Hill, Research Triangle Park, NC, ${ }^{2} \mathrm{ORD} / \mathrm{Nheerl} / \mathrm{Istd} / \mathrm{PB}, \mathrm{USEPA}$, \\ Research Triangle Park, NC, ${ }^{3}$ Toxicology Branch, NIEHS/NTP, Research Triangle Park, NC
}

Nuclear receptor agonists such as phenobarbital (PB), 3-methylcholantrene (3MC), 2,2',4,4,5,5'-hexachlorobiphenyl (PCB 153), and, pregnenolone-16 $\alpha$-carbonitrile (PCN) decrease serum thyroxine $\left(\mathrm{T}_{4}\right)$ concentrations in rats. This decrease is thought to occur through the induction of hepatic metabolizing enzymes resulting in the enhanced catabolism and elimination of $\mathrm{T}_{4}$. There is some evidence that this decrease in serum $\mathrm{T}_{4}$ occurs in humans, but the mechanism by which this transpires is uncertain. Using primary rat and human hepatocytes, we compared the effects of aryl hydrocarbon receptor (AhR), constitutive androstane receptor (CAR), and pregnane $\mathrm{X}$ receptor (PXR) agonists on $\mathrm{T}_{4}$ catabolism. 48 hours after plating, fresh sandwich-cultured hepatocytes from male human or Sprague-Dawley rats were treated with the following: CAR agonists, PB (1000uM); PCB 153 (30uM) or AhR agonist, 3-MC (5uM). 5uM rifampicin (Rif) and 10uM PCN were used as PXR agonists for human and rat hepatocytes, respectively. Control and treated cells were exposed to a final concentration of $0.1 \%$ DMSO. After 72 hours, media were removed and replaced with $0.05 \mathrm{uM}$ (rat median serum concentration) or $0.1 \mathrm{uM}\left[{ }^{125}\right]-\mathrm{T}_{4}$ (human median serum concentration). After 24 hours, media were collected for analysis. $\mathrm{T}_{4}$ and its metabolites were separated on an UPLC from which fractions were collected and quantified on a gamma counter. Of the conjugated metabolites, $\mathrm{T}_{4} \mathrm{G}$ is the most abundant as compared to $\mathrm{T}_{4} \mathrm{~S}$. Studies show a 50 -fold higher activity in $\mathrm{T}_{4} \mathrm{G}$ formation in unexposed rat hepatocytes as compared to unexposed human hepatocytes (5.2 vs. $0.1 \mathrm{fmol} \mathrm{T}_{4} \mathrm{G} / \mathrm{min} / \mathrm{mg}$ protein). In rat hepatocytes, there was no significant increase in $\mathrm{T}_{4} \mathrm{G}$ appearance with $\mathrm{PB}$ treatment compared to control; PCN, 3-MC and PCB 153 treatment increase $\mathrm{T}_{4} \mathrm{G}$ formation by 1.4-, 5.0-, and 3.8-fold, respectively. In contrast, experiments with human hepatocytes showed significant increases in $\mathrm{T}_{4} \mathrm{G}$ appearance following PB, Rif, 3-MC, and PCB 153 treatment by as much as 8-, 10-, 12-, and 12-fold, respectively. Increases in $\mathrm{T}_{4} \mathrm{G}$ showed to be variable between human donors suggesting possible polymorphisms in human UGT genes. As a possible in vitro screening tool for environmental toxicants, the results highlight the utility of primary hepatocytes in evaluating potential species differences and human health risk.(This abstract of a proposed presentation, does not necessarily reflect USEPA or NIH policy.)

\section{P230. Zomepirac acyl glucuronide migration}

\section{Rongfang Fran $\mathrm{Xu}^{1}$, Heang Keang $\operatorname{Lim}^{1}$ and Michael McMillian ${ }^{2}$}

${ }^{1}$ Drug Metabolism, Johnson \& Johnson Pharmaceutical Research and Development, Raritan, $N J,{ }^{2}$ Mechanistic Toxicology, Johnson \& Johnson Pharmaceutical Research and Development, LLC., Raritan, NJ

Zomepirac (Zomax) is an NSAID which produced effective pain relief, but was withdrawn from the market due to a high incidence of anaphylaxis reactions. The 1-O-B-acyl glucuronide of zomepirac migrates to other glucuronide positions with a half life of 27 minutes, and the aldehyde form of the migrating glucuronide is very reactivel. However, it is unclear how this reactive acyl glucuronide of zomepirac contributes to the idiosyncratic anaphylaxis reactions observed in patients. In the present study, a multi-step hypothesis was tested: 1 . Zomepirac is glucuronidated in the hepatocyte and the least reactive 1-O-B-acyl glucuronide is immediately pumped out; 2 . More reactive acyl glucuronides form only outside the cells at pharmacological concentrations, so tissue damage is minimized (consistent with clean preclinical toxicity studies); and 3. Migrating reactive acyl glucuronides react with extracellular molecules (such as human serum albumin), producing antigens that ultimately give rise to pronounced immune reactions in patients. Co-culturing HepG2 cells with old human hepatocyte cultures (stromal cells, fibroblasts) improved their responses and some differentiated functions over HepG2 cells cultured alone on collagen. LC-MS was used to detect zomepirac and its acyl glucuronide metabolite from HepG2 co-cultures. Zomepirac 1-O-B-acyl glucuronide synthesized with human liver macrodomes was observed to chemically degrade (migrate to other glucuronide positions) in $0.1 \mathrm{M}$ phosphate buffer at $\mathrm{pH} 7.4$; these experiments served to characterize the migrated forms by LC-MS. Only the 1-O-B-acyl glucuronide metabolite is observed in cells, and only at high concentrations. The 1-O-B-acyl glucuronide and its migrated forms are observed in media. Protein binding is easily detected when the migrated acyl glucuronide metabolites are present. In summary, 
1-O-B-acyl glucuronide of zomepirac is generated in in HepG2 cells grown on old human hepatocytes (and confirmed with cryopreserved human hepatocytes). The generated 1-O-B-acyl glucuronide is pumped out of cell into the media rapidly. 1-O-B-Acyl Glucuronide migrates to other reactive acyl glucuronides species outside the cell. If the same occurs in human patients and preclinical species, intracellular toxicity would not occur, but extracellular (ie, immune mediated by antigen generation from reactive acyl glucuronides) toxicity would predominate, as observed in the clinics. We are in the process of examining other NSAIDs and reactive acyl glucuronides in this model. References 1.Disposition and Chemical Stability of Telmisartan 1-O-Acylglucuronide. T. Ebner, G. Heinzel, A. Prox, K. Beschke, and H. Wachsmuth. Drug Metabolism and Deposition. 27, (10) 1143-1149, 1999

\title{
P231. Automated metabolite cross-correlation as a tool for faster decision making
}

\author{
$\underline{\text { Hesham Ghobarah }}^{1}$, Russell J. Mortishire-Smith ${ }^{2}$, Pascale Proost ${ }^{2}$, Alina Dindyal-Popescu ${ }^{1}$ and Hai \\ Ying ${ }^{1}$
}

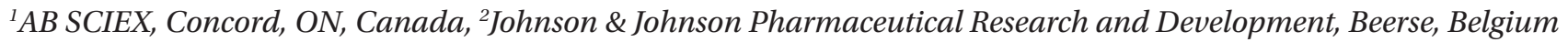

Recent advances in both hardware and software have greatly facilitated the identification of metabolites in complex matrices. However, conclusions are rarely drawn on the basis of one sample alone. For example, identification of metabolites that are disproportionate in one species relative to others is important if the metabolite is pharmacologically active, or potentially toxic. Selection of appropriate species for toxicology studies, and in vitro-in vivo correlation also require comparison of the metabolic profile from multiple samples. Where a compound has low metabolic clearance, isoform phenotyping is typically performed based on appearance of metabolites across a sample population. Finally, PK/PD relationships for metabolites require understanding of kinetics. Currently, most existing metabolite identification workflows typically process and report each sample independently (relative to one or more controls). Correlation of results across multiple samples is subsequently performed manually. Here we describe automated cross-correlation of metabolite identification samples. Sets of samples were taken from standard metabolic clearance, isoform phenotyping, and cross-species comparison experiments prepared using standard methodologies. Data acquisition was performed on a hybrid QqTOF accurate mass analyzer (TripleTOF ${ }^{\mathrm{m}} 5600$ system, AB SCIEX, Concord, Canada) coupled with a Shimadzu Prominence UFLC-XR HPLC system using a generic acetonitrile / water / $0.1 \%$ formic acid gradient. System was operated in positive electrospray mode using a DuoSpray ${ }^{\mathrm{mm}}$ source. The Information DependentAcquisition (IDA) method consisted of a TOF MS survey scan (m/z 100- 1000) followed by 2 TOF MS/MS dependent scans (m/z 50- 1000) operated in high resolution mode $(\mathrm{R}=30,000)$. Accumulation times were $70 \mathrm{~ms}$ for TOF MS, and $50 \mathrm{~ms}$ for each MS/MS scan. Dynamic Background Subtraction was applied in the IDA criteria for dynamic exclusion, and a collision energy of $35 \mathrm{eV}$ with a spread of $+/-10 \mathrm{eV}$ was used for the MS/MS scans. External mass calibration was performed automatically using a calibrant delivery system connected to the DuoSpray source. Real time multiple mass defect filtering was also used for IDA criteria in non-exclusive mode to give higher MS/MS trigger priority to ions falling within the selected mass defect windows. Data analysis was performed using MetabolitePilot ${ }^{\mathrm{m}} \mathrm{s}$ software. Briefly, data from each sample was processed using standard peak finding and assignment strategies. Results from each sample were then loaded into the correlation tool along with independent variables defining the nature of each sample (e.g. species, time point, isoform). The cross-correlation algorithm matches each metabolite across multiple samples on the basis of observed accurate mass and retention time, then reports MS and UV responses in tabular format. The software then provides options for visualizing the trends using line or bar charts for metabolites selected by the user. Chromatographic, full scan, and MS/ MS data is automatically overlaid for the selected samples. A summary table is also generated and can be exported for further analysis if needed.

\section{P232. Deuterium-substituted lapatinib isotopologs exhibit reduction in 0-debenzylation and metabolic shunting}

Vinita Uttamsingh, Craig Masse, Sophia Nguyen, Adam Morgan, Changfu Cheng, Bhaumik Pandya, Richard Gallegos, Gary Bridson, Lijun Wu, Roger Tung and Scott Harbeson

CoNCERT Pharmaceuticals, Inc., Lexington, MA

Lapatinib (Tykerb $\left.{ }^{\circledR}\right)$ is a potent, orally available small-molecule reversible dual EGFR and HER-2 tyrosine kinase inhibitor. It is indicated, in combination with capecitabine, for the treatment of advanced or metastatic breast cancer. In humans, lapatinib undergoes extensive metabolism and is eliminated primarily in the feces. The predominant metabolite 
observed in human feces is the potentially reactive and hence undesired phenol M1 resulting from CYP3A4/5 mediated $O$-debenzylation of lapatinib. CoNCERT prepared several precision deuterium-modified isotopologs of lapatinib to examine the effects of deuteration on the metabolic fate of the analogs. While deuterium isotope effects on CYP450mediated metabolism are well documented, the consequences of deuteration on the overall metabolite profile of a drug are unpredictable. Hence, in vitro and in vivo testing is required to determine the effects of deuterium substitution on the metabolism and pharmacokinetics of a drug. We investigated the formation of the $O$-debenzylated metabolite M1 from nine deuterated lapatinib isotopologs in human liver microsomes (HLM). Only a select subset of the isotopologs showed a substantial reduction in the formation of the phenolic metabolite compared to lapatinib. Interestingly, several of the more heavily deuterated lapatinib analogs showed a less marked effect on the formation of the phenolic metabolite. Kinetic isotope effects, $\mathrm{D}_{\mathrm{V}}$ and $\mathrm{D}_{\mathrm{V} / \mathrm{K}}$, for a d2-isotoplog were also observed. In addition, metabolite profiling in hepatocytes was performed. The results of these experiments supported the observations from the HLM studies and are indicative of metabolic shunting.

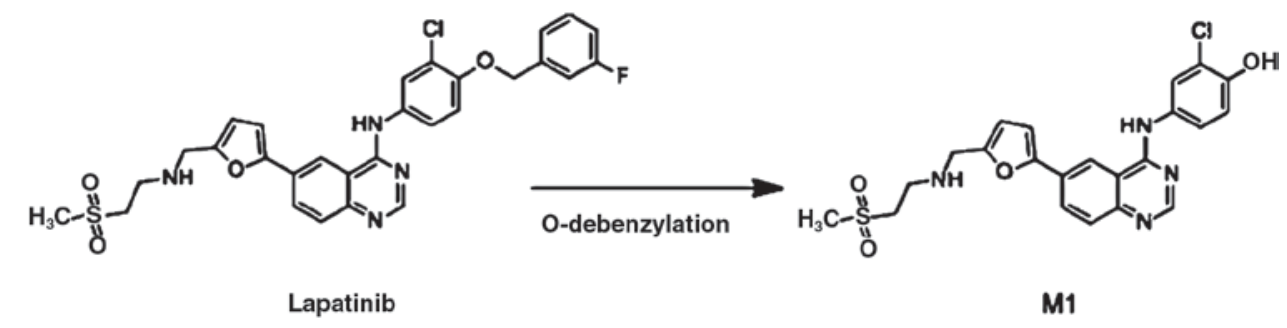

P233. High-resolution accurate mass-measurements and metabolite identification: An
automated approach using fragment prediction in combination with fragment ion search
(FISh)

Janine Wank ${ }^{1}$, Winfried Wagner-Redeker ${ }^{1}$, Anthony Taylor ${ }^{2}$, Rose L. Herbold $^{3}$ and Paul-Gerhard Lassahn $^{1}$

${ }^{1}$ Swiss Bioanalytics, Basel, Switzerland, ${ }^{2}$ Thermo Fisher Scientific, Reinach, Switzerland, ${ }^{3}$ Thermo Fisher Scientific, San Jose, $C A$

Structural elucidation of putative metabolites via LC/MS is a time-consuming process of manual interpretation of MS data and requires a strong background in metabolism pathways as well as gas-phase fragmentation pathways. In order to evaluate an automated software workflow for metabolite identification and structural elucidation, we processed high resolution accurate mass data using the Fragment Ion Search (FISh) processing tool in Thermo Scientific Mass Frontier 7.0 software. The investigated metabolites were derived from Ticlopidine, a potent thienopyridine antiplatelet drug which is known to be extensively metabolized. Ticlopidine was incubated with human liver S9 enzymes. Final experimental concentrations in the $1 \mathrm{~mL}$ incubation mixture were $100 \mathrm{mM} \mathrm{KPO}_{4}(\mathrm{pH} 7.4), 3 \mathrm{mM} \mathrm{NADPH}$, and $3.8 \mu \mathrm{M}$ Ticlopidine. The incubation mixtures were homogenized gently and placed into a water bath $\left(37^{\circ} \mathrm{C}\right)$. Aliquots of the reaction solution were withdrawn after 0,30 , and $60 \mathrm{~min}$ and the reaction was quenched by the addition of acetonitrile. The individual samples were centrifuged and analyzed by LC/MS on a Thermo Scientific LTQ Orbitrap XL mass spectrometer equipped with an electrospray ionization source (ESI) interfaced to a Thermo Scientific Accela 600 pump and Open Accela Autosampler. The high resolution accurate mass data was directly exported to the Mass Frontier 7.0 software program. After the determination of the theoretical fragments of Ticlopidine and specification of common Phase I and Phase II modifications, component detection and FISh processing were used to screen for putative metabolites. By defining the possible modifications of Ticlopidine, the list of predicted fragments was automatically extended by the mass shifts of the modifications. All known and published metabolites were detected and identified by the automated FISh processing approach. FISh further provided the explanation of the MS/MS data including the structures of the fragments and the localization of the biotransformations for each detected component. For the metabolites M1 and M5, the putative structures of the metabolites were confirmed by a comparison between the measured and theoretical fragments. All MS/MS fragment ions were explained. In addition to these five common metabolites, an unusual $S$-oxide dimer metabolite M6 was detected by FISh based on an in-source fragment. The measured and simulated Full MS data of the co-eluting metabolites M3 and M4 demonstrated the need of high resolution accurate mass data. The exact mass and isotopic pattern clearly helped to verify the putative identification of both metabolites and supported the effectiveness of both in the metabolite identification workflow. 


\title{
P234. Identification and determination of the percent AUC of five metabolites of a tetrahydropyran-containing drug, co-eluting as a broad peak due to the presence of metabolites undergoing conformational change during HPLC radiochromatography
}

\author{
Heasook Kim-Kang ${ }^{1}$, Zheming Gu${ }^{1}$, LiQuan Wang ${ }^{1}$, Shelby R. Anderson ${ }^{2}$ and Elizabeth Joshi ${ }^{2}$
}

\author{
${ }^{1}$ Drug Metabolism and Biotransformation, XenoBiotic Laboratories, Inc., Plainsboro, NJ, ${ }^{2}$ Drug Disposition, Eli Lilly and \\ Company, Indianapolis, IN
}

Currently the use of a radiolabeled compound provides the most accurate assessment of the exposure of animals or humans to metabolites. In general, reverse phase HPLC coupled with radioactive detection is used to obtain metabolite profiles in different matrices in order to achieve the separation of all the major metabolites. However, one HPLC method may not be applicable for the separation of all the metabolites if the metabolite profile is complex. Sometimes, the major radioactive peak can contain multiple components, and the co-eluting metabolites can be isolated and subjected to secondary chromatography. The secondary chromatography may include the modification of HPLC conditions or the use of TLC instead of HPLC. However, TLC is not desirable to be used for quantitative analyses because many metabolites can undergo degradation on the TLC plate due to the direct exposure to air. Most co-eluting metabolites can be successfully separated by modification of HPLC conditions. However, when one or two major components in the region undergo conformational change, the change of HPLC condition may not be sufficient to separate the co-eluting compounds. Metabolites which contain the 1-hydroxy-tetrahydropyran moiety can exist in an open-chain (acyclic) form or a cyclic hemiacetal form in equilibrium, resulting in a broad peak (ca. $10 \mathrm{~min}$ ) containing two or three different forms for each molecule. For the drug in this study, the broad peak region was isolated, and the isolate was subjected to derivatization using butyl PABA/sodium cyanoborohydride to prepare reduced Schiff base products of the two 1-hydroxy-tetrahydropyran-containing metabolites. Radioprofiles and LC/MS data before and after the derivatization were obtained. The derivatization was complete and RP-HPLC analysis of the derivatization mixture could successfully separate the five metabolites, which enabled the accurate assessment of their AUC values. The ID of each peak was then obtained using LC/MS. Since the reaction condition was mild, three other metabolites co-eluting in the same region remained intact. A TLC radiochromatogram of the isolate was also obtained, and as expected it showed degradation of some of the metabolites on the TLC plate.

\section{P235. Identification and Semi-quantification of circulating metabolites of BIIB021 in first-in- human (FIH) studies}

\author{
Lin Xu, Caroline Woodward, Min Chang, Samina Khan and Chandra Prakash
}

Dmpk, BiogenIdec, Cambridge, MA

To improve patient safety and to avoid late-stage clinical trial delays, FDA and International Conference on Harmonization (ICH) recommends that the drug metabolites present in humans should also be present in at least one of the animal species used for long term safety assessment. Therefore, comparison of metabolic profiles between preclinical species and humans should be assessed as early as possible during the drug-development process. In vitro systems (microsomes and/or hepatocytes are used in early discovery to predict human metabolites. However, these systems predict the primary and secondary metabolites with only 12-70\% success. BIIB021, a synthetic HSP90 inhibitor, is being developed for the oral treatment of breast cancer. The objectives of this study were to obtain both quantitative and qualitative circulating metabolites of BIIB021 in humans. Six individual plasma samples at the drug steady state were pooled via "Hamilton" approach and samples were analyzed by LC/UV/MS for assessment of systemic exposure of BIIB021 and its metabolites. The identified metabolites were semi-quantified by radioactivity-UV response factors, which were obtained from preclinical radiolabelled ADME studies. The results showed that BIIB021 was extensively metabolized and presented from $9 \%$ to $54 \%$ circulating drug components in patients. Monohydroxylated M7 and M10 were major primary circulating metabolites, which were catalyzed by CYP450 and aldehyde oxidase, respectively. Each presented from 11\% to $37 \%$ circulating components. Other major circulating metabolites were formed by further oxidation and glucuronidation of M7, respectively. They presented from $5 \%$ to $34 \%$ circulating components. The estimated concentrations of M3 and M7 via this internal response factor were consistent with those obtained by traditional bio-analysis with a calibration curve of metabolites. The exposure of M4 and M10 were subsequently estimated by this validated approach for comparisons between humans and preclinical species for metabolite safety assessments. Two major metabolic pathways of BIIB021 were identified in human. M3 and M10 were identified as minor metabolites in human liver microsomes and hepatocytes, which did not predict both as major systemic metabolites in human. 


\title{
P236. In vivo disposition and pharmacokinetics and in vitro inhibition and induction profiles of saxagliptin, a potent inhibitor of dipeptidyl peptidase 4 , in human
}

\author{
$\underline{\text { Hong Su}}{ }^{1}$, Lisa J. Christopher ${ }^{1}$, Ramaswamy A. Iyer ${ }^{1}, \mathrm{Kai} \mathrm{Cao}^{2}$, Samuel Bonacorsi², David Boulton ${ }^{3}$ \\ and William G. Humphreys ${ }^{1}$ \\ ${ }^{1}$ Department of Biotransformation-PCO, Bristol-Myers Squibb, Princeton, NJ, ${ }^{2}$ Radiochemistry, Bristol-Myers Squibb, \\ Princeton, NJ, ${ }^{3}$ Discovery Medicine and Clinical Pharmacology, Bristol-Myers Squibb, Princeton, NJ
}

ONGLYZA $^{\mathrm{mw}}$, (saxagliptin, BMS-477118) is a potent dipeptidyl peptidase (DPP4) inhibitor indicated for the treatment of type 2 diabetes mellitus. In this study, the pharmacokinetics and disposition of $\left[{ }^{14} \mathrm{C}\right]$ saxagliptin were determined following administration of a single $50 \mathrm{mg}, 91.5 \mu \mathrm{Ci}$ oral dose to 6 healthy male subjects. Saxagliptin was rapidly absorbed with a Tmax of approximately $0.5 \mathrm{~h}$. Thereafter, both saxagliptin and total radioactivity (TRA) concentrations declined rapidly with elimination half-life values of $<3 \mathrm{~h}$. Saxagliptin and 5-hydroxysaxagliptin (M2), an active metabolite, were the most prominent drug-related components in the plasma; together they accounted for the majority of the AUC of circulating radioactivity. Parent drug and M2 were also the prominent drug-derived components in urine, while M2 was a prominent fecal metabolite. Approximately $97 \%$ of the administered radioactivity was recovered within 7 days post-dose, of which $74.9 \%$, and $22.1 \%$ were excreted in the urine and feces, respectively. The parent compound and M2 comprised approximately $24 \%$ and $44 \%$ of the excreted dose, respectively, indicating that saxagliptin was highly metabolized and the primary pathway for metabolism of saxagliptin was hydroxylation of the adamantyl group to form M2. Formation of other hydroxylated metabolites, along with glucuronidation, and sulfation were minor pathways. Neither saxagliptin nor M2 inhibited or induced CYP enzymes, in vitro, suggesting that saxaliptin has low potential to affect the clearance of co-administered drugs that are substrates for CYP enzymes.

\section{P237. In vitro metabolism of BIIB021, an oral heat shock protein HSP90 inhibitor, in liver microsomes and hepatocytes of rats, dogs, and humans}

\author{
$\underline{\text { Lin Xu}}$, Caroline Woodward and Chandra Prakash
}

Dmpk, BiogenIdec, Cambridge, $M A$

Inhibition of heat shock protein 90 (HSP90) results in the degradation of oncoproteins that drive malignant progression, making HSP90 a target of cancer therapy. BIIB021, a synthetic HSP90 inhibitor, was being developed for the oral treatment of breast cancer. The objectives of this study were to obtain both quantitative and qualitative metabolite profiles of [14C] BIIB021 after incubations in rat, dog and human liver microsomes and hepatocytes. BIIB021 is extensively metabolized in both liver microsomes and hepatocytes. In liver microsomes for all species, the major metabolic pathway involves the monooxygenation on the methoxy-dimethylpyridine moiety to form M7, accounting for 35-53\% of total radioactivity. M7 formation was also observed as a major metabolic pathway in human and rat hepatocytes, accounting for 37-49\% of total radioactivity. However, in dog hepatocytes, the major metabolic pathways were due to the glucuronidation of M7 to form M4 accounting for $45 \%$ of total radioactivity and O-demethylation of methoxy-dimethylpyridine moiety to form M2 accounting for $26 \%$ of total radioactivity. Oxidative N-dealkylation (M11 and M12) is found solely in the rat. They represented $7 \%$ and $11 \%$ of the radioactivity in rat LM and hepatocytes, respectively. Non-enzymatic oxidative dechlorination of BIIB021 (M6) and its metabolites (M13 and M15) observed during the incubation was indicative of instability of BIIB021 chlorine moiety in aqueous solution. M6 and its derivative M15 were found dominant in human hepatocytes, representing $4 \%$ and $3.7 \%$ of the radioactivity, respectively. Direct displacement of chlorine on the amino-chloropurine moiety by glutathione to form M18 accounted for 9-13\% of the radioactivity in rat and dog hepatocytes. Subsequent minor secondary metabolites M14 and M17 were observed in human, dog, and rat hepatocytes accounted for less than $10 \%$ radioactivity in each species. Mono-hydroxylation on the purine moiety (M10) was catalyzed probably via cytosolic enzyme aldehyde oxidase.

\section{P238. In-vitro and in-vivo identification of new domperidone metabolites by LC-ESI-MS/MS}

\author{
$\underline{\text { Amir S. B. Youssef }}{ }^{1}$, Murali Pathikonda ${ }^{2}$, Upendra Argikar ${ }^{3}$, Henry P. Parkman $^{4}$ and Swati V. Nagar ${ }^{5}$ \\ ${ }^{1}$ Department of Pharmaceutical Sciences, Temple University School of Pharmacy, Philadelphia, PA, ${ }^{2}$ Gastroenterology \\ Section, Temple University School of Medicine, Philadelphia, PA, ${ }^{3}$ Metabolism and Pharmacokinetics, Novartis
}


Institutes for Biomedical Research Inc., Cambridge, MA, ${ }^{4}$ Gastroenterology Section, Department of Medicine, Temple University School of Medicine, Philadelphia, PA, ${ }^{5}$ Dept of Pharmaceut Sciences, Temple University School of Pharmacy, Philadelphia, PA

Purpose: Domperidone is a dopamine type 2 receptor antagonist that is used as a prokinetic agent to treat gastroparesis. After oral absorption, it is extensively metabolized in the liver. Published studies point toward oxidative metabolites of domperidone, detected by radiometric-HPLC or single quadrupole mass spectrometeric(LC-MS) techniques. Our aim was to identify domperidone 'phase I' (oxidation/reduction) and 'phase II' (conjugation) metabolites using liquid chromatography (LC) combined with electrospray ionization (ESI) enabled tandem mass spectrometry (MS/MS). Methods: Oxidative metabolites of domperidone were identified in human liver microsomes (HLM) under conditions favorable for cytochrome P450 (CYP)- catalyzed reactions. Domperidone was also incubated with recombinant purified CYP3A4. Glucuronides were generated by further incubation of CYP incubations with uridine diphosphate glucuronic acid (UDPGA) to evaluate UDP-glucuronosyltransferases (UGT)- catalyzed reactions. Sulfated metabolites were similarly evaluated by addition of human liver cytosol and the co-factor 3'-Phosphoadenosine-5'-phosphosulfate (PAPS). Metabolic oxidative and conjugative reactions were screened for metabolites using LC-ESI-MS/MS. For the in-vivo study, gastroparesis patients $(\mathrm{n}=11,18-65$ years old $)$ currently being treated with domperidone at a dose of 10-20 mg 3-4 times/day were recruited. Subjects were nonsmokers and were not on other medications known to be substrates, inducers or inhibitors of CYP3A4. At the start of the study, a saliva sample for DNA isolation was collected. Patients then were administered their usual domperidone dose. A blood sample was collected one hour after the dose. All blood samples were stored immediately upon collection at $-20^{\circ} \mathrm{C}$ until further analysis. Results:In-vitro, domperidone was metabolized to a mono-hydroxylated metabolite (M1), a de-alkylated metabolite (M2), an alcohol metabolite (M3) possibly formed from an aldehyde intermediate, and a di-hydroxylated metabolite (M4). M4 has not been reported previously. M1 and M4 were further glucuronidated (M5 and M6 respectively) and M1 was observed to subsequently form a sulfate conjugate (M7). To the best of our knowledge, M5, M6 and M7 have not been reported previously. Preliminary analysis of patient plasma samplesshowed metabolite (M1). Interestingly, up to 5-fold interpatient variability in circulating levels of M1 was detected among patients. The M1 was observed to subsequently form a sulfate conjugate (M7). Other metabolites observed in-vitro were not detected in patient plasma. Conclusion: In total, 7 domperidone metabolites including 3 new metabolites have been identified in-vitro in human subcellular fractions. The formation of M1 to M4 is CYP-mediated. Furthermore, M1-M4 were identified in CYP3A4 incubations indicating that it contributes to domperidone metabolism. In blood samples, only M1 and M7 were detected indicating that the sulfated form is a circulating conjugate for domperidone. Moreover, metabolite M1 levels in blood exhibit high inter-patient variability. These results will ultimately allow a better understanding of domperidone disposition in-vivo.

\title{
P239. Pharmacokinetics, disposition and metabolism of AZD5455, a selective enkephalinergic agonist, in rats and dogs
}

\author{
$\underline{\text { Yan Li }}^{1}$, Down Perry ${ }^{2}$ and Scott Grimm ${ }^{1}$
}

${ }^{1}$ Dmpk, AstraZeneca, Waltham, MA, ${ }^{2}$ Clinical Bioanalysis, AstraZeneca, Wilmington, DE

AZD5455 is a selective enkephalinergic agonist being developed for the treatment of anxiety disorders. In this study, the pharmacokinetics, disposition and metabolism of AZD5455 in rats and dogs were reported. After intravenous administration, high systemic clearance was observed in rats $(6.7 \mathrm{~L} / \mathrm{h} / \mathrm{kg})$ and dogs $(4.1 \mathrm{~L} / \mathrm{h} / \mathrm{kg})$ with large volume distribution (18-26 and 11-14 L/kg for rats and dogs, respectively). The terminal half-life was $1.7-4.2 \mathrm{~h}$ and $2.9-3.5 \mathrm{~h}$ for rats and dogs, respectively. Moderate bioavailability was observed in rats (65-115\%) and dogs (35-69\%). After a single oral and intravenous administration of $\left[{ }^{14} \mathrm{C}\right]$ AZD5455 to male and female rats and dogs, the majority of the radioactivity (57-81\%) was recovered in the feces, whereas $21-35 \%$ was eliminated in urine. In bile duct-cannulated rats dosed intravenously, 35\% of the administrated radioactivity was recovered in the bile, with $25 \%$ of the radioactivity was found in the feces, suggesting direct intestinal secretion. AZD5455 underwent extensive metabolism in both species with $<5 \%$ of the administrated radioactivity recovered as AZD5455 in most of the excreta samples. Unchanged AZD5455 was a prominent drug-related component in the plasma from both species, but multiple metabolites contributed significantly to the circulating radioactivity, particularly for dogs, in which metabolites comprised more than $90 \%$ of the area under the time curve (0-72h). Circulating metabolites included M7, a primary amide metabolite, M12, a carboxylic acid metabolite, and M13, a desethyl metabolite. Overall, the results from these studies suggested that AZD5455 was well absorbed and extensively metabolized through multiple pathways in these preclinical species. 


\title{
P240 - Unexpected mass changes as a result of hydrogen/deuterium exchange
}

Austin Li, Steven Ring, Erya Yu and \ames P. Chovan

Drug Safety \& Disposition, Cephalon, Inc., West Chester, PA

While online hydrogen-deuterium exchange (HDX) has evolved as a routine approach to confirm proposed structures of drug metabolites, it has been mostly used to distinguish between hydroxylation and other oxidative reactions in that the former biotransformation increases the number of exchangeable protons, while the latter does not. In this communication, we report on unexpected additional exchangeable $\mathrm{H}$ atoms in 2 drug metabolites that had imine or enamine partial structures. The "additional" $\mathrm{H}$ atoms were beyond the obvious exchangeable protons such as those on hydroxyl, amino or thiol functionalities and were ultimately determined to be the methylene protons adjacent to an imine or enamine. They were exchanged due to imine-amine or enamine-amine equilibrium in the presence of $\mathrm{D}_{2} \mathrm{O}$. Metabolite MC-1B (Figure A) showed 2 more exchangeable $\mathrm{H}$ atoms during online HDX analysis than the 5 expected exchangeable protons on hydroxyl and amine functionalities. Metabolite MC-2 (Figure B) showed 4 more exchangeable protons during the HDX experiment in addition to the 1 expected exchangeable amine proton. Based on the evidence of accurate mass measurements in both full-scan and product ion-scan modes, these "additional" exchangeable $\mathrm{H}$ atoms further confirmed the structural assignments of these metabolites. The equilibrium schemes for HDX of metabolites MC-1B and MC-2 are shown in Figures A and B, respectively. The keto-enol tautomerization for MC-1B is postulated but not possible to confirm at this time.

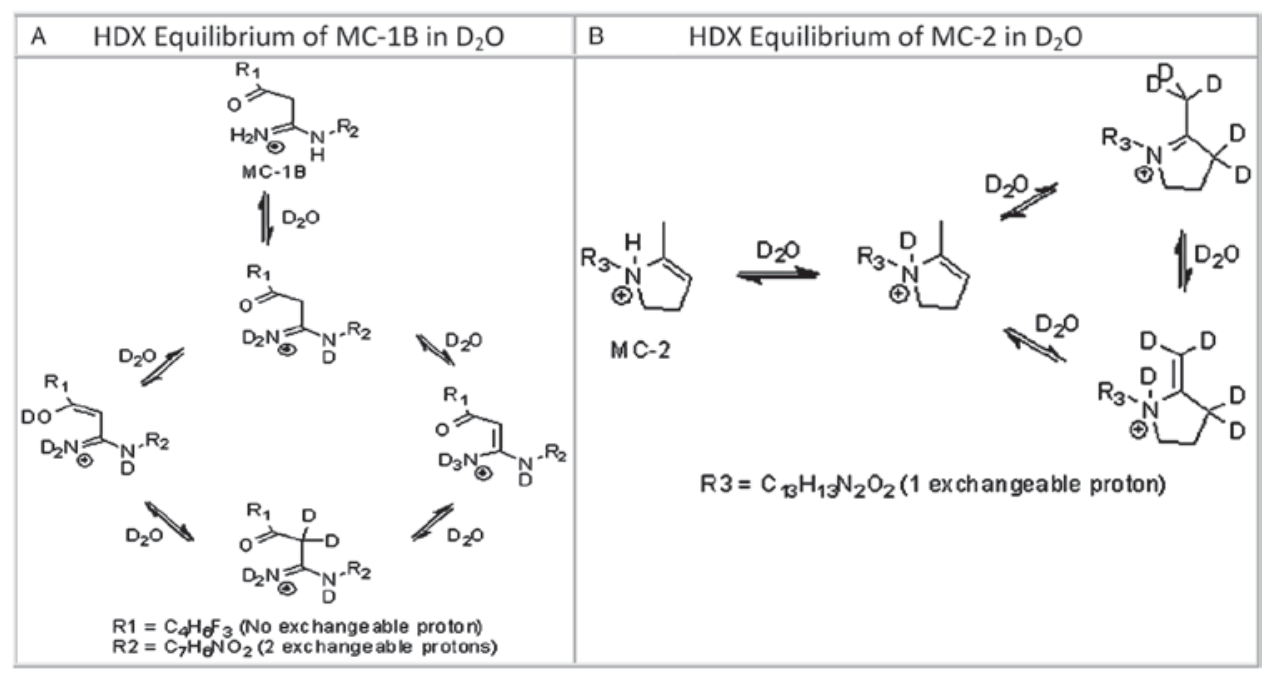

\section{P241. gADME: Characterization of CYP2D6 activity of mutant proteins using control substrates and CNS-active drugs}

\author{
$\underline{\text { Robert R. Annand }}^{1}$, Mohammed Taimi ${ }^{1}$, Takayuki Nakano ${ }^{1}$, Helen Gill ${ }^{2}$, Clive Dilworth ${ }^{3}$ and Katya \\ Tsaioun $^{4}$ \\ ${ }^{1}$ Apredica, Watertwon, MA, ${ }^{2}$ Cyprotex, Macclesfield, United Kingdom, ${ }^{3}$ Cyprotex Discovery, Macclesfield, United \\ Kingdom, ${ }^{4}$ Apredica, Watertown, MA
}

Genetic variability of drug metabolism is a recurrent problem in drug development. Cytochrome P450 (CYP) is a family of enzymes that are primarily responsible for the oxidative metabolism of most pharmaceuticals. Of the over fifty isoforms of CYPs encoded by the human genome, ten are responsible for the majority of drug metabolism. CYP2D6 isoforms, though they account for only $2 \%$ of the total CYP enzymes in the liver, are responsible for the oxidative metabolism of $30 \%$ of the approved drugs including a number of psychiatric drugs. More than 100 different genetic variants of CYP2D6 have been described, including a number that involve alterations in the coding sequence for the CYP2D6 protein. We have characterized a number of purified CYP2D6 mutant proteins and characterized their activity against the standard substrates bufuralol, dextromethorphan, and debrisoquine. We have measured the metabolic activity of this panel against a set of drugs composed of antidepressants (TCAs and SSRIs), antipsychotics, and other psychiatric drugs. Through this analysis, we have gained insight into the underlying mechanism of phenotypic variation in psychiatric drug metabolism. This information can be utilized in stratifying populations of potential responders and non-responders in 
clinical studies and in the market, thus increasing the chances of the drug candidate to be successful in clinical studies and in the marketplace. This is particularly important for CNS drugs, where failure in the clinic is particularly high, compared to other therapeutic areas, and any tool that increases the probability of success has a potential to have a large impact on the outcome.

\title{
P242. A novel ring oxidation of 4- or 5-substituted 2(H)-oxazole to corresponding 2-oxazolone catalyzed by cytosolic aldehyde oxidase
}

\author{
Vinod Arora ${ }^{1}$, Thomas Philip ${ }^{1}$, Xiaohua (Stella) Huang ${ }^{2}$ and Yue-Zhong Shu ${ }^{3}$ \\ ${ }^{1}$ Biotransformation, Bristol-Myers Squibb, Wallingford, CT, ${ }^{2}$ Discovery Analytical Sciences, Bristol-Myers Squibb, \\ Wallingford, CT, ${ }^{3}$ Biotransformation, Bristol-Myers Squibb, Princeton, NJ
}

5-Membered heterocycles like oxazoles are frequently used in medicinal chemistry as ester and amide bioisosteres for improved metabolic stability and other drug-like properties. Literature indicates that oxazoles are primarily metabolized by cytochrome $\mathrm{P} 450$ s to ring opened products and that 2-amino substituted oxazoles undergo oxidative deamination to 2-oxazolone (1). In the present paper, we report a novel conversion of 4- or 5-substituted 2(H)-oxazole to corresponding 2-oxazolone. Using 5-(3-bromophenyl) oxazole as a model compound, its 2-oxazolone metabolite was fully characterized by LC/MS/MS and NMR. The reaction occurred in the liver cytosolic fraction without the requirement of P450 enzymes and co-factor NADPH. Investigations into the mechanism of formation of 2-oxazolone suggested that the reaction was primarily catalyzed by aldehyde oxidase and significantly inhibited by an aldehyde oxidase inhibitor, raloxifene. The rank order of oxazolone formation was mouse>monkey>>rat and human liver cytosols, whereas the metabolite was not formed in dog liver cytosols. The species difference was similar to some published aldehyde oxidase-catalyzed reactions $(2 \& 3)$.

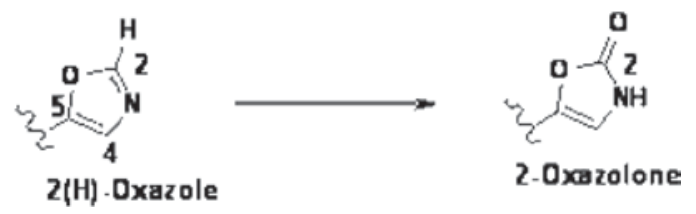

1: Dalvie, D. K., Kalgutkar, A. S., Khojasteh-Bakht, S. C., Obach, R. S., and O'Donnell, J. P., Biotransformation Reactions of Five Membered Aromatic Heterocyclic Rings, Chemical Research in Toxicology, 2002,15, 269-299.

2: Klecker,R. W., Cysyk, R. L., and Collins, J. M., Zebularine Metabolism by Aldehyde Oxidase in Hepatic Cytosol from Humans, Monkeys, Dogs, Rats, and Mice: Influence of Sex and Inhibitors, Bioorganic \& Medicinal Chemistry, 2006, 14, 62-66

3: Khan, K. K., Cameron, M. D., Wright, J. K., and Black, C. B., Investigation of Aldehyde Oxidase Activity in Human, Monkey, Dog, Rat, and Mouse Cytosols and Cryopreserved Hepatocytes, from http://www.cellzdirect.com

\section{P243. A unique metabolic pathway of sipoglitazar, a novel anti-diabetic agent: Cytochrome P450-catalyzed oxidation of sipoglitazar acyl glucuronide and the mutation effect of the UGT2B15 enzyme responsible for glucuronidation of sipoglitazar}

\author{
Mitsuhiro Nishihara, Yuto Hiura, Miyako Sudo, Naohiro Kawaguchi, Junzo Takahashi, Yutaka \\ Kiyota, Takahiro Kondo and Satoru Asahi \\ Drug Metabolism and Pharmacokinetics Research Laboratories, Pharmaceutical Research Division, Takeda \\ Pharmaceutical Company Limited, Osaka, Japan
}

Sipoglitazar, 3-[3-ethoxy-1-[[4-[(2-phenyl-1,3-thiazol-4-yl)methoxy]phenyl]methyl]pyrazol-4-yl]propanoic acid was tried to develop as a novel anti-diabetic agent. The deethylated metabolite (M-I) and the glucuronide conjugate of sipoglitazar (sipoglitazar-G) appeared to be the key metabolites in the elimination process for sipoglitazar in rats and monkeys. M-I was also detected as the major metabolite of the plasma in the human clinical studies. In vitro metabolic studies with human hepatocytes and hepatic microsomes indicated that M-I was not formed directly from sipoglitazar and that sipoglitazar-G was involved in the metabolism from sipoglitazar to M-I. Further studies for the metabolism of sipoglitazar-G revealed that (1) HPLC, LC-MS/MS and NMR analyses for sipoglitazar-G showed that sipoglitazar-G 
was composed of two glucuronides; an $\alpha$-2-O-acyl glucuronide (sipoglitazar-G1) and a $\beta$-1-O-acyl glucuronide (sipoglitazar-G2). (2) UGT2B15 is principally responsible for the formation of sipoglitazar-G2 from the UGT identification studies (their activities, enzyme kinetics and correlation studies between the glucuronidation of sipoglitazar and that of the probe substrate for each UGT). (3) The stability studies of these glucuronides suggested that sipoglitazar-G2 could be converted to sipoglitazar-G1 and sipoglitazar, but sipoglitazar-G1 could not be converted to sipoglitazar-G2. (4) M-I was formed only from sipoglitazar-G2, but not from sipoglitazar or sipoglitazar-G1 and CYP2C8 was mainly involved in this process. From these results, sipoglitazar is considered to be initially metabolized to sipoglitazar-G2 by UGT2B15 and then sipoglitazar-G2 is metabolized to M-I by $O$-dealkylation by CYP2C8 and deconjugation. Sipoglitazar-G1 is sequentially formed by the migration of the $\beta$-site of sipoglitazar-G2. Furthermore, the effects of the D85Y mutation of UGT2B15 on sipoglitazar glucuronidation were investigated by kinetic studies using His-tagged UGT2B15*1 (wild) and UGT2B15*2 (D85Y) membrane fractions. The affinity of sipoglitazar to UGT2B15*1 (Km: $101.3 \mu \mathrm{mol} / \mathrm{L})$ was considerably higher than that to UGT2B15*2 (Km: $763.6 \mu \mathrm{mol} / \mathrm{L})$, which could be coincident with the pharmacokinetics of sipoglitazar showing two different clearances and the existence of extensive and poor metabolizers in the clinical study (Stringer F, et al (2009) Genotyped versus phnotyped dosing to account for UGT polymorphism of the novel PPAR agonist sipoglitazar. PAGE 18:Abstract 1606). In conclusion, the metabolic pathway of sipoglitazar to M-I includes a very unique pathway, in which glucuronidation was essential for hydroxylation as the intermediate and the genomic polymorphism of the glucuronidation enzyme could affect the human pharmacokinetics of sipoglitazar.

\title{
P244. Assessment of pharmacokinetics using chimeric mice with humanized livers
}

\author{
Kristin Samuelsson ${ }^{1}$, Kathryn Pickup ${ }^{1}$, John Swales ${ }^{2}$, James Yates ${ }^{3}$, Christopher Smith ${ }^{1}$, Richard \\ Gallagher $^{1}$, Yoshio Morikawa ${ }^{4}$ and Ian Wilson ${ }^{1}$ \\ ${ }^{1}$ Drug Metabolism and Pharmacokinetics IM, AstraZeneca, Macclesfield, United Kingdom, ${ }^{2}$ Discovery Drug Metabolism \\ and Pharmacokinetics, AstraZeneca, Macclesfield, United Kingdom, ${ }^{3}$ AstraZeneca, Macclesfield, United Kingdom, \\ ${ }^{4}$ Marketing, New York Branch, PhoenixBio Co., Ltd., New York, NY
}

An in vivo animal model with the ability to accurately predict the pharmacokinetics (PK) and metabolism of a drug candidate in man would provide significant advantages in drug development. The chimeric mouse from PhoenixBio (PXB) has $>70 \%$ of the hepatocytes replaced with human hepatocytes and is of significant interest as a model to investigate human-specific metabolism, CYP-inhibition, CYP-induction, CYP-polymorphism and toxicity. The aims of this work were to evaluate the PXB mouse to predict human in vivo pharmacokinetics and metabolism, assess the suitability of the model for use in drug-drug interaction (DDI) studies and provide mass spectrometry response factors for semiquantification of human metabolites relevant to safety testing of metabolites (MIST). Two compounds were selected for investigation in the PXB mouse model, an AstraZeneca compound known to be a time dependent inhibitor of CYP3A4, and midazolam, a CYP3A4 substrate. As a control to the "humanized" mice the SCID mouse was used. In the first study the pharmacokinetics of midazolam were investigated following oral administration at 3 dose levels $(0.1,1 \mathrm{and} 10 \mathrm{mg} /$ $\mathrm{kg}$ ) to determine which dose level to use in a DDI study. The second study looked at the PK of midazolam alone, the PK of the AZ candidate drug following dosing at steady state, and finally the PK of the two compounds dosed together. Blood samples were collected at selected timepoints following oral dosing with PK profiles generated using $5 \mu \mathrm{l}$ aliquots of blood for LC-MS/MS analysis. Following midazolam dosing, a clear difference in exposure was seen between the two strains of mice with the $\mathrm{C}_{\max }$ and the area under the curve (AUC) seen to be significantly higher in the chimeric compared to the SCID mice. From these studies a dose level of $1 \mathrm{mg} / \mathrm{kg}$ in the chimeric mouse was found to correspond to the $\mathrm{C}_{\max }$ reached in humans at a therapeutic dose. The use of these data to design the subsequent DDI study, together with the results of this investigation will be described, together with comments on the usefulness of the model for predictions in human subjects.

\section{P245. Co-culture of cryopreserved primary rat and human Kupffer cells and hepatocytes to study APAP cytotoxicity}

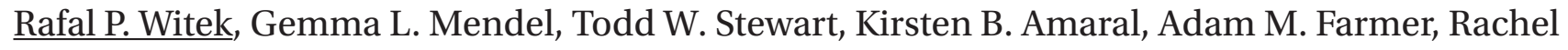
M. Witek, Manda Edwards, Kimberly Freeman, Erica Deibert, Brian J. Paszkiet, Jonathan P. Jackson and Stephen S. Ferguson

Life Technologies, Durham, NC 
Introduction: Kupffer cells (KCs) directly interact with hepatocytes during liver inflammation by modifying immunity and xenobiotic metabolism. KCs are known to suppress the expression of multiple cytochrome P450 enzymes following activation with lipopolysaccharide (LPS). Since KCs are involved in liver diseases that are characterized with inflammation including viral and autoimmune hepatitis and non-alcoholic steatohepatitis (NASH), there is a need for a KCs and hepatocyte co-culture model for drug discovery and ADME/Tox applications. This study hypothesizes that the use of cryopreserved rat and human KCs in co-culture with hepatocytes will recreate liver inflammation and model acetaminophen (APAP) cytotoxicity observed during liver inflammation. Methods: Fresh and cryopreserved Kupffer cells were characterized by morphology, plateability, phagocytosis, immunohistochemistry staining for CD68 and CD163, and cytokine release for IL-6 and TNF $\alpha$ by ELISA in response to $24 \mathrm{hrs}$ treatment with $1 \mu \mathrm{g} / \mathrm{mL}$ LPS. KCs and hepatic co-cultures were cultured in DMEM medium supplemented with non-essential amino acids (NEAA), Insulin-Transferring-Selenium (ITS+) and Penicillin/Streptomycin. For plating and maintenance media, additional $10 \%$ or $2 \%$ fetal bovine serum (FBS) was used, respectively. Since corticosteroids inhibit inflammation, dexamethasone or hydrocortisone were not used with KCs. For experiments with APAP cytotoxicity, inflammatory ratios of approximately 1:2-1:4 KCs to hepatocytes were used. Co-cultures were cultured for 24 hours and then pre-treated with $1 \mu \mathrm{g} / \mathrm{mL}$ LPS for 24,4 and 0 hours prior to addition of $10 \mathrm{mM}$ APAP for additional $24 \mathrm{hr}$ in the same media containing LPS. Cells were evaluated for viability using PrestoBlue" reagent, morphology and cytokine release (e.g. IL-6, $\mathrm{TNF} \alpha$ ) by ELISA. Real time qRT-PCR was performed to establish gene expression changes in metabolism, inflammation and viability. Results: Fresh and cryopreserved rat KCs were found to be similar after comparison of morphology, plateability, culture purity via immunofluorescent staining. The purity of KCs was found to be at $85.6 \%( \pm 1.9 \mathrm{SE})$ for CD68 and at 93.5\% ( $\pm 0.4 \mathrm{SE})$ for CD163, commonly recognized cell surface markers of KCs. Using cytokine panel, activated fresh and cryopreserved co-cultures revealed comparable upregulated expression of IL-1 $\alpha$, IL-1 $\beta$ and IL-2 by at least two fold, and IL-6 IL-10 and TNF $\alpha$ by at least four fold; conversely, they revealed down regulated expression in IL-4 and IL-12 by at least two fold. For the cytotoxicity experiments, viability and morphology data analysis revealed time-dependent hepato-protection in co-cultures at 4 hours pre-treatment with LPS, but not at 0 and 24 hours. A strong correlation was observed with cytokine release of IL-6 and TNF $\alpha$, and expression of CYP1A2 and CYP2E1. Conclusions: Cryopreserved KCs can maintain known characteristics and functions as compared to freshly isolated cells. APAP cytotoxicity data show that these cells can be effectively used for modeling liver inflammation and reveal hepato-protective role of KCs as shown at 4 hour pre-treatment with LPS. Cryopreserved KCs when co-cultured with hepatocytes, provide a powerful in vitro ADME/Tox tool to evaluate the effects of xenobiotic metabolism during inflammation.

\title{
P246. Comparison of metabolism of donepezil in human, following oral and dermal administration, and in an in vitro model of human epidermis
}

\author{
$\underline{\text { Sandra Dennis }^{1}}$, Renata Murgasova $^{1}$ and Susan Meier-Davis ${ }^{2}$
}

${ }^{1}$ Charles River Laboratories, Senneville, QC, Canada, ${ }^{2}$ Teikoku Pharma USA, Inc, San Jose, CA

Donepezil hydrochloride, (RS)-2-[(1-benzyl-4-piperidyl) methyl]- 5,6-dimethoxy-2,3-dihydroinden-1-one monohydrochloride], is a centrally acting reversible acetyl cholinesterase inhibitor, with therapeutic use in the treatment of Alzheimer's disease. A transdermal delivery is being investigated for donepezil administration. Plasma metabolites of donepezil hydrochloride were compared following two routes of drug delivery, dermal and oral, in human and in a reconstituted human epidermis (RHE) in vitro model. Metabolite profiling of the test article in the extracted samples and structural elucidation of metabolites were carried out in a semi-quantitative manner by LC/quadrupole-Ion trap MS/MS. In plasma from human subjects $(\mathrm{n}=12)$, five metabolic pathways were observed, i.e: a) Odemethylation to metabolites M1 and M2; b) aromatic hydroxylation to metabolite M3; c) N-dealkylation to metabolite M4; d) N-oxidation to metabolite M6; and e) glucuronidation of O-demethylated metabolites (M1 and M2) to metabolites M11 and M12. Chemically intact donepezil was present in all of the plasma samples. The mean concentration levels of the unchanged drug and its metabolites in both dosing groups decreased in a similar order, there were no substantial differences in the plasma profiles of the individual subjects and the metabolite yields in the general circulation were not affected by the dosing route. In samples from the RHE model, unchanged drug and metabolites M1/M2, M3, M4, and M6 were identified, with unchanged drug being the most abundant component, followed in descending order by M4, M6, and the isomeric M1/ M2 metabolites. Percutaneous transport across RHE was accompanied by metabolism of the compounds and there were quantitative and qualitative similarities between the metabolites produced by the RHE and those obtained in the circulation after topical dosing. Data from the present study demonstrate that the RHE model is a potentially valuable model for skin pharmacological studies. 


\title{
P247. Comparison of novel luciferin-1A2 substrate and phenacetin for measuring CYP1A2 induction in human hepatocytes
}

\author{
Timothy A. Moeller ${ }^{1}$, Dongping $\mathrm{Ma}^{2}$, Stephen Dennel ${ }^{1}$ and James J. Cali ${ }^{3}$
}

${ }^{1}$ Celsis In Vitro Technologies, Baltimore, MD, ${ }^{2}$ Research, Promega Corporation, Madison, WI, ${ }^{3}$ Research Department, Promega Corporation, Madison, WI

Induction of drug metabolizing enzymes is one critical component in assessing drug-drug interactions. The process is controlled through drugs binding to receptors which regulate gene transcription. One mechanism entails the aryl hydrocarbon receptor (AhR), a transcription factor responsible for the up-regulation of phase I and phase II enzymes such as CYP1A1/2 and UGT1A1. The most common method to determine a drug's induction potential through AhRdependent pathway is to measure the activity of CYP1A2 in hepatocytes using phenacetin as a substrate after exposure to the drug and compare it to the basal rate. The quantification of the metabolism of phenacetin requires bioanalytical methods like UPLC/MS/MS which demands special equipment and expertise. In contrast, the P450-Glo ${ }^{\mathrm{m}}$ assay with the luminescent CYP1A2 substrate Luciferin-1A2 (6-methoxybenzo[d]thiazole-2-carbonitrile) is a simple and effective method to measure CYP1A2 activity requiring a plate reader and minimal expertise which will expedite data acquisition and simplify workflow. Herein, we describe the comparison of phenacetin and Luciferin-1A2 substrates for CYP specific and to determine induction of CYP1A2 in human hepatocytes using omeprazole as the inducer. The induction ratios were similar for most of the 16 donors tested for both lytic and non-lytic P450-Glo ${ }^{\mathrm{m}}$ Luciferin-1A2 substrate methods as compared to phenacetin with significant correlation found ( $\mathrm{P}$ value $<0.0001, \mathrm{R}^{2}$ of 0.7$)$. This new CYP1A2 substrate is an alternative for measuring CYP1A2 activity to traditional probe substrates that require HPLC or MS methods.

\section{P248. Differences in biotransformation of raloxifen, tamoxifen and tamoxifen metabolites in Ishikawa human endometrial and MCF7 human breast cancer cell lines}

\author{
Teresa Wilborn ${ }^{1}$, Erika Cretton-Scott ${ }^{1}$, Lori Coward ${ }^{2}$, Gregory Gorman ${ }^{2}$ and Charles N. Falany ${ }^{3}$ \\ ${ }^{1}$ Department of Pharmaceutical, Social and Administrative Sciences, McWhorter School of Pharmacy, Samford \\ University, Birmingham, AL, ${ }^{2}$ Pharmaceutical Sciences Research Institute, McWhorter School of Pharmacy, Samford \\ University, Birmingham, AL, ${ }^{3}$ Pharmacology and Toxicology, University of Alabama at Birmingham, Birmingham, AL
}

Tamoxifen and raloxifene are selective estrogen receptor modulators (SERM) with similar effects on breast and bone tissue. Tamoxifen however is associated with endometrial hyperplasia while raloxifene is not. Although the molecular mechanism underlying the differential tissue effects is unknown, the metabolic disposition of raloxifene and tamoxifen or the tamoxifen metabolites, 4-hydroxytamoxifen, N-desmethyl-4-hydroxytamoxifen (endoxifen) and alpha-hydroxytamoxifen in endometrial and breast tissue may be involved. The purpose of this investigation is to determine if there are differences in the metabolic disposition of raloxifene, tamoxifen and tamoxifen metabolites in MCF-7 breast cancer cells and Ishikawa human endometrial adenocarcinoma cells that may contribute to their differential tissue effects in vivo. MCF-7 cells and Ishikawa cells were incubated with 10 $\mu \mathrm{M}$ raloxifene, tamoxifen, endoxifen, 4-hydroxy- and alphahydroxytamoxifen up to 48 hours. Unchanged drug and metabolite profiling in both media and cellular samples was carried out by LCMS. Expression ofSULT1A1 and SULT1E1 in the two cell lines was determined by real-time quantitative PCR. Formation of endoxifen, 4-hydroxytamoxifen and alpha-hydroxytamoxifen was noted in MCF-7 and Ishikawa cells following exposure to tamoxifen; no measurable levels of sulfate or glucuronide conjugates were observed in either cell type. When cells were exposed to 4-hydroxytamoxifen, sulfation of 4-hydroxytamoxifen only occurred in Ishikawa cells while only glucuronidation was observed in MCF-7 cells. Although formation of endoxifen after 4-hydroxytamoxifen treatment occurred in both cell types, neither sulfation nor glucuronidation of endoxifen was observed. When cells were exposed directly to endoxifen or alpha-hydroxytamoxifen, no measurable levels of sulfate or glucuronide conjugates were observed in either cell type. In contrast to these observations with tamoxifen and its metabolites, both sulfation and glucuronidation of raloxifene was observed in MCF-7 and Ishikawa cells, with more sulfation occurring in Ishikawa cells. PCR analysis indicated SULT1A1 message was present in both Ishikawa and MCF-7 cells, whereas SULT1E1 was only detected in Ishikawa cells. The most notable observation of these findings is the sulfation of 4-hydroxytamoxifen in Ishikawa cells but not MCF-7 cells. SULTs 1A1 and 1E1 are known to be capable of sulfating 4-hydroxytamoxifen. Since sulfation of 4-hydroxytamoxifen was observed only in Ishikawa cells, our study suggests that SULT1E1 catalyzes 4-hydroxytamoxifen sulfation in these cells. The consequences of 4-hydroxytamoxifen sulfation in endometrial cells and implications related to tamoxifen associated endometrial hyperplasia warrants further investigation. 


\title{
P249. Evaluation of non-CYP mediated metabolism: Comparison of recombinant enzymes and
} sub-cellular fractions in identifying non-CYP mediated pathways

\author{
Philip J. Butler, Katie Fox, Michael Griffin and Clive Dilworth
}

Cyprotex, Macclesfield, United Kingdom

Traditional screening strategies for assessing metabolic stability of new chemical entities measure intrinsic clearance $\left(\mathrm{CL}_{\text {int }}\right)$ in human liver microsomes. Microsomal stability assays are convenient test systems, with the appropriate cofactors, for assessing phase I and II metabolism, providing important information on the metabolic liabilities of new drug candidates. Recent improvements in medicinal chemistry however, have developed the design of new drug candidates to address metabolic liability to cytochrome P450 enzymes. As a result this has uncovered weaknesses to other metabolic pathways and differences are being observed between the in vivo clearance and in vitro microsomal data. These can be attributable to non-CYP mediated interactions or transporter effects. Rather than moving straight into hepatocytes which are not amenable to high-throughput screening, a common answer to this is to screen using liver S9 fraction which comprises of both microsomal and cytosolic fractions of the cell. However, due to lower enzyme activity resulting in a lack of sensitivity this may not always pick up on possible problems. An early screening panel of the most active non-CYP450 enzymes helps to highlight any problems earlier in the screening cascade, before undergoing the cost of an in vivo study, or can help later down the screening cascade to account for any differences observed for in-vitroin-vivo scaling. We have developed a screening panel of recombinant enzymes covering the main non-CYP450 mediated pathways. The enzymes we have focused on include carboxylesterases, aldehyde oxidases, flavin monooxygenase, monoamine oxidases, sulfotransferases, $\mathrm{N}$-acetyltransferases, glutathione S-transferases and epoxide hydrolases. Here we compare standard stability screens using microsomes/S9/cytosol (supplemented with cofactors NADPH, UDPGA and PAPs) with our recombinant enzyme stability panel for a diverse list of test compounds to demonstrate the benefit in early drug screening strategies. The in vitro $\mathrm{CL}_{\text {int }}$ of test compounds was determined based on disappearance of compound analysed by LC-MS/MS. Utilising this recombinant stability screen in conjunction with a traditional microsomal stability screen improves the prediction of human drug clearance especially for compounds metabolised by multiple metabolic pathways which otherwise would have been under-predicted from an in vitro microsomal stability screen.

\section{P250. Human metabolism of JWH-018 and JWH-073 and in vitro characterization of major omega and omega-1 metabolites}

\author{
Krishna C. Chimalakonda ${ }^{1}$, Lisa K. Brents ${ }^{2}$, Cindy L. Moran ${ }^{3}$, Paul L. Prather ${ }^{4}$, Laura P. James ${ }^{5}$, Anna \\ Radominska-Pandya ${ }^{6}$ and Jeffery H. Moran ${ }^{7}$
}

${ }^{1}$ Pharmacology \& Toxicology, University of Arkansas for Medical Science, Little Rock, AR, ${ }^{2}$ Pharmacology and Toxicology, University of Arkansas for Medical Sciences, Little Rock, AR, ${ }^{3}$ Arkansas State Crime Lab, Little Rock, AR, ${ }^{4}$ Pharmacology and Toxicology, University of Arkansas for Medical Science, Little Rock, AR, ${ }^{5}$ Univ of Arkansas for Medical Sciences and Arkansas Children's Hospital, Department of Pediatrics, Little Rock, AR, ${ }^{6}$ Biochemistry and Molecular Biology, University of Arkansas for Medical Science, Little Rock, AR, ${ }^{7}$ Department of Health, AR Public Health Laboratory, Little Rock, AR

Herbal mixtures being marketed as "K2" or "SPICE” are sold as 'legal marijuana' to those trying to skirt existing regulations or avoid detection in standard drug screens. These products are often laced with synthetic cannabinoids characterized as aminoalkylindoles (AAIs; e.g. JWH-018 and JWH-073). Use of these products is on the rise in the US and limited case reports suggest that use is associated with severe adverse reactions. Previous data has shown that these products are $\mathrm{CB}_{1}$ cannabinoid receptor agonists, and that they undergo phase I oxidation at the C-terminus ( $\left.\omega\right)$ of the small alkyl side. Our recent data has shown that the $(\omega)$-hydroxyl and $(\omega)$-carboxyl metabolites of these two compounds are excreted in urine primarily as glucuronic acid conjugates. In this study, we tested the hypothesis that humans also metabolize JWH-018 and JWH-073 to form glucuronic acid conjugates of ( $\omega$-1)-hydroxyl derivatives prior to excretion and that ( $\omega-1)$-hydroxyl metabolites retain affinity and activity towards the $\mathrm{CB}_{1}$ cannabinoid receptor. Solid-phase extraction and LC-MS/MS procedures, are presented that simultaneously identify and quantify ( $\omega)$-hydroxyl, $(\omega)$-carboxyl, and novel ( $\omega$-1)-hydroxyl metabolites of JWH-018 (JWH-018 N-(4-hydroxypentyl)) and JWH-073 (JWH-073 N-(3-hydroxybutyl)) and their glucuronic acid conjugates in urine collected from individuals that self-administered either JWH-018 or a mixture of JWH-018 and JWH-073. Each of these metabolites were excreted primarily as glucuronides at fairly high concentrations (>17 ng/mL), with the ( $\omega$-1)-hydroxyl glucuronides being present in the highest concentrations (40-230 ng/ $\mathrm{mL}$; relative ranking of JWH-018 metabolites being $(\omega-1)$-hydroxyl $>(\omega)$-hydroxyl > $(\omega)$-carboxyl ). Results from $\mathrm{CB}_{1}$ 
competition receptor binding assays employing the cannabinoid receptor radioligand $\left[{ }^{3} \mathrm{H}\right] \mathrm{CP}-55,940$, followed by determination of intrinsic efficacy at $\mathrm{CB}_{1}$ receptors using $\left.{ }^{35} \mathrm{~S}\right] \mathrm{GTP} \gamma \mathrm{S}$ binding, showed that $(\omega$-1)-hydroxyl derivatives of JWH-018 and JWH-073 have significant $\mathrm{CB}_{1}$ receptor affinity and activity. Both the $(\omega)$ - and $(\omega$-1)-hydroxyl metabolites of JWH-018 and JWH-073 bound to $\mathrm{CB}_{1}$ receptors with high, nanomolar affinity. The ( $\omega$ )-hydroxyl metabolites also retained $\mathrm{CB}_{1}$ receptor activity equivalent to that of the respective parent compound, acting as either partial (JWH-073) or full (JWH-018) agonists to stimulate G-proteins. The ( $\omega$-1)-hydroxyl metabolites acted as partial agonists. Finally, studies examining the $(\omega)$-carboxyl metabolites demonstrated that these derivatives have extremely low affinities for $\mathrm{CB}_{1}$ receptors and appear to be inactive. This is the first report to identify and simultaneously quantify oxidized and glucuronidated urinary metabolites of JWH-018 and JWH-073 in humans. These data indicate that P450 oxidation does not necessarily lead to the loss of $\mathrm{CB}_{1}$ receptor affinity or intrinsic activity. Further studies to examine the role of conjugation by UGTs in facilitating the inactivation or excretion of JWH-018 and JWH-073 is the next crucial step to elucidate the clinical pharmacology of these emerging drugs of abuse. (NIH-GM075893 to AR-P; APHL Innovation Award to JHM; UAMS Arkansas CCTR grant to LPJ,AR-P,JHM,CLM, \& PLP)

\title{
P251. Identification of the human enzymes involved in the oxidative metabolism of crizotinib
}

\author{
Theodore R. Johnson ${ }^{1}$, Sue Zhou ${ }^{1}$, Caroline A. Lee ${ }^{1}$, Andrea Clouser-Roche ${ }^{1}$, Sascha Freiwald ${ }^{1}$, \\ Kuresh A. Youdim², Karen Aherne ${ }^{2}$, Minerva R. Batugo ${ }^{1}$, Judith Skaptason ${ }^{3}$, Shinji Yamazaki ${ }^{1}$, \\ Michael R. Wester ${ }^{1}$ and Bill J. Smith ${ }^{1}$
}

${ }^{1}$ Pharmacokinetics, Dynamics \& Metabolism, Pfizer Inc., San Diego, CA, ${ }^{2}$ Pharmacokinetics, Dynamics \& Metabolism, Pfizer Inc., Sandwich, Kent, United Kingdom, ${ }^{3}$ Dmpk, Janssen Pharmaceutical Company of Johnson \& Johnson, San Diego, CA

Crizotinib [PF-02341066: $\quad(R)$-3-[1-(2,6-dichloro-3-fluoro-phenyl)-ethoxy]-5-(1-piperidin-4-yl-1H-pyrazol-4-yl)pyridin-2-ylamine] is a small-molecule inhibitor of the anaplastic lymphoma kinase (ALK) receptor tyrosine kinase and its oncogenic variants and is currently in development for the treatment of ALK-positive advanced non-small cell lung cancer. In vivo studies have shown that the primary metabolic pathways of crizotinib are $O$-dealkylation (M4, PF-03255243) and oxidation of the piperidine ring to form crizotinib lactam (M10, PF-06260182). The objective of this study was to identify the human enzymes involved in the oxidative metabolism of crizotinib and in the formation of key metabolites. Crizotinib was incubated with human liver microsomes (HLM) in the presence of cytochrome P450 (CYP) isozyme-specific chemical inhibitors and with human recombinant CYP (rCYP) enzymes. Liquid chromatography/mass spectrometry (LC/MS) methods were used to monitor either the loss of parent drug or metabolite formation (M4 or M10) in HLM or recombinant enzyme incubations. Studies in HLM with selective chemical inhibitors demonstrated that CYP3A enzymes were the major enzymes contributing to the metabolism of crizotinib in vitro ( $84 \%$ inhibition by troleandomycin). Incubations in rCYP enzymes confirmed that CYP3A4 was the predominant CYP isozyme involved in the oxidative metabolism of crizotinib, and a subsequent study demonstrated that crizotinib was also a CYP3A5 substrate. Formation of M4 appeared to be mediated solely by CYP3A4 based on studies with rCYP enzymes. Consistent with these findings, ketoconazole (CYP3A inhibitor) reduced formation of M4 in HLM by 99\%. Formation of crizotinib lactam (M10) appeared to be mediated by both CYP enzymes and aldehyde oxidase (AO) in a probable two-step reaction. The proposed mechanism for the role of AO in formation of M10 involves an initial CYP-catalyzed oxidation of the piperidine ring of crizotinib to an imine intermediate, followed by an AO-catalyzed oxidation of the intermediate to the corresponding lactam. Supporting this hypothesis was the observation that formation of M10 decreased markedly in rCYP enzymes in the absence of human liver cytosol (source of AO). Studies in HLM fortified with human liver cytosol in the presence of selective chemical inhibitors demonstrated that CYP3A was the major enzyme (65\% relative contribution) involved in formation of M10, with minor contributions from CYP2C8, CYP2C19, and CYP2D6. Results from clinical drug interaction studies with ketoconazole (CYP3A inhibitor) and rifampin (CYP3A inducer) confirmed the major role of CYP3A4/5 in the metabolism of crizotinib and M10 in vivo. In conclusion, in vitro studies demonstrated that CYP3A4/5 were major enzymes involved in the metabolic clearance of crizotinib and in the formation of key metabolites.

\section{P252. Identification of novel phase I and phase IImetabolites of maraviroc using human liver microsomes}

$\underline{\text { Yanhui Lu}}^{1}$, Craig W. Hendrix² and Namandje N. Bumpus ${ }^{2}$

${ }^{1}$ Department of Pharmacology and Molecular Sciences, The Johns Hopkins University, School of Medicine, Baltimore, $M D,{ }^{2}$ Department of Medicine, The Johns Hopkins University, School of Medicine, Baltimore, MD 
Maraviroc (4,4-difluoro-N-\{(1S)-3-[3-(3-isopropyl-5-methyl-4H-1,2,4-triazol-4-yl)-8-azabicyclo[3.2.1]oct-8-yl]-1 -phenylpropyl\}cyclohexanecarboxamide) is a human immunodeficiency virus (HIV) co-receptor CCR5 antagonist that is approved for the treatment of HIV infection. In addition, there is increased interest in maraviroc for oral and topical microbicide prevention of HIV. The present study sought to perform a comprehensive analysis of the biotransformation of maraviroc. Using human liver microsomes the formation of both phase I and phase II metabolites was examined. The resulting metabolites were separated and detected by ultra performance liquid chromatrography-mass spectrometry (UPLC-MS). Under these conditions, three previously unreported metabolites were identified. These metabolites were determined to be a novel dihydroxylated product of maraviroc and two glucuronide metabolites. The precursor ion of the dihydroxylated metabolite of maraviroc had a mass-to-charge ratio (m/z) of 546.4, with two primary product ions of $\mathrm{m} / \mathrm{z} 421.3$ and 312.0. Further fragmentation of the product ions using MS/MS/MS indicated that both of the oxygen insertions were on the difluorocyclohexane ring. A glucuronidated metabolite of maraviroc was found at $\mathrm{m} / \mathrm{z}$ of 706.2. By performing MS/MS/MS analysis of a predicted product ion with a m/z of 581.3, fragment ions were generated indicating that the glucuronide conjugation occurs on the difluorohexane ring subsequent to the insertion of one oxygen. MS/MS analysis of the second $706.2 \mathrm{~m} / \mathrm{z}$ ion resulted in the detection of a primary product ion of $389.0 \mathrm{~m} / \mathrm{z}$ indicating that the conjugation takes place on the triazole ring subsequent to monooxygenation. In conclusion, three previously unreported metabolites of maraviroc were identified using human liver microsomes. Increased knowledge regarding the biotransformation of maraviroc may inform the development of maraviroc for use in HIV prevention.

\section{P253. Abstract withdrawn}

\section{P254 - Mechanistic studies on Nefazodone side chain cleavage using 1802: A Baeyer-Villiger oxidation specifically catalyzed by CYP3A4 through a ferric peroxide intermediate}

\section{$\underline{\text { Amin Kamel }}$}

Metabolism and Pharmacokinetics, Novartis Institutes for Biomedical Research Inc., Cambridge, MA

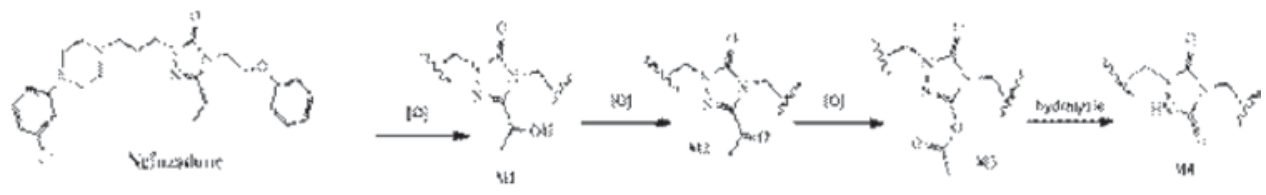

Nefazodone undergoes. an unusual metabolic pathway which involves cleavage of carbon-carbon bonds to form the triazoledione metabolite (M4). A possible mechanism for this unusual metabolic pathway has been reported for progesterone and lanosterol which involves Baeyer-Villiger rearrangement through a ferric peroxide intermediate. Mayol et al also proposed a similar mechanism for the formation of triazoledione nefazodone from the acetyl intermediate. However, and to the best of our knowledge, such an intermediate was never detected/isoltaed and other supportive data to confirm the proposed mechanism for nefazodone was not reported. Attempts to detect and isolate the 5-O-acetyl nefazodone intermediate (M3) were successful and its structure was confirmed using accurate mass measurement, CID studies and incorporation of ${ }^{18} \mathrm{O}_{2}$. Furthermore, the formation of this peroxy intermediate could proceed via P450-, horseradish peroxidase (HRP)- and/or flavine monoxygenase (FMO)-mediated Baeyer-Villiger oxidation. Results indicated that the formation of the peroxy intermediate is specifically mediated by CYP3A4 and HRP and FMO are not involved in its formation. A mechanism which involves Baeyer-Villiger rearrangement through a ferric peroxide intermediate is invoked for nefazodne via a P-450-mediated attack (the ferric-dioxo species) on the carbonyl carbon of the acetyl chain of M2 to form an enzyme-bound peracid intermediate. Rearrangement of the $\mathrm{C}-5$ ferric peroxide intermediate of nefazodone via Baeyer-Villiger rearrangement would result in the formation of 5-O-acetyl nefazodone (M3) which undergoes subsequent ester hydrolysis to form M4. Accurate mass measurement and CID studies under normal conditions and in an atmosphere of ${ }^{18} \mathrm{O}_{2}$ were crucial to confirm the structural assignments for all metabolites and intermediate. Additionally, the formation, detection and characterization of 5-OacetylNefazodone (M3) intermediate directly implicated the insertion of two atoms of molecular oxygen in a carboncarbon bond side chain which is most readily accomplished via rearrangement of a peroxy intermediate and added confidence to the proposed mechanism. 


\title{
P255. Abstract withdrawn
}

\section{P256. Oxidative metabolism of 20(S)-protopanaxadiol in human intestinal and hepatic microsomes}

\author{
Nga Ting Colette Chiu ${ }^{1}$, Subrata Deb ${ }^{2}$, Hans Adomat ${ }^{2}$, William Jia ${ }^{1}$ and Emma Guns ${ }^{2}$ \\ ${ }^{1}$ Department of Surgery, University of British Columbia, Vancouver, BC, Canada, ${ }^{2}$ Vancouver Prostate Centre, \\ Vancouver, BC, Canada
}

20(S)-protopanaxadiol (aPPD), a gut microflora metabolite of ginseng saponin, is currently undergoing early clinical trials as an antidepressant agent. As a gastrointestinal metabolite, aPPD must first undergo absorption and metabolism in the intestinal epithelium before further metabolism in the liver. The present study identifies and compares the metabolism profile and enzyme kinetics of aPPD in human intestinal microsomes (HIM) and human liver microsomes (HLM). Pooled HIM and HLM of mixed gender were obtained from XenoTech, LLC (Lenexa, KS). aPPD $(22 \mu \mathrm{M})$ was incubated with HIM $(1 \mathrm{mg} / \mathrm{ml})$ and HLM $(0.25 \mathrm{mg} / \mathrm{ml})$ for $60 \mathrm{~min}$ and $10 \mathrm{~min}$, respectively, to profile the metabolite formation. In vitro reaction mixtures for enzyme kinetic studies contained $67 \mathrm{mM}$ potassium phosphate buffer ( $\mathrm{pH} 7.4), \mathrm{HIM}(0.25 \mathrm{mg} /$ $\mathrm{ml})$ or HLM $(0.15 \mathrm{mg} / \mathrm{ml})$, NADPH-regenerating system and varying concentrations of aPPD $(0-33 \mu \mathrm{M})$ in a final volume of $0.1 \mathrm{ml}$ incubated for $10 \mathrm{~min}$. A liquid chromatography-matrix-assisted laser desorption/ionization time-of-flight (LC-MALDI-TOF) mass spectrometry in positive ionization mode was used for metabolite identification study, whereas for enzyme kinetic studies of metabolite formation and substrate depletion a Waters Acquity Ultraperformance liquid chromatography (UPLC) system coupled to a Quattro Premier XE triple quadrupole mass spectrometer (MS) was used to resolve the hydroxy metabolites of aPPD and the parent molecule on a BEH MS $\mathrm{C}_{18} \operatorname{column}(1.7 \mu \mathrm{m}, 2.1 \times 100 \mathrm{~mm})$. Three major monohydroxylated metabolites (M1, M2 and M3) and five minor dihydroxylated metabolites were identified in both HIM and HLM incubation mixtures. Monohydroxylated metabolites of aPPD were identified by multiple reaction monitoring of the $\mathrm{m} / z 477>459$ and $459>441$ transitions, whereas $\mathrm{m} / z 443>425$ and $425>123$ transitions were used to detect aPPD in the LC/MS assay. MASSLYNX version 4.1 software was used for data acquisition and Quantlynx analyses. The incubation conditions, e.g. amount of protein and incubation time, were optimized for linearity of aPPD substrate depletion and monohydroxy metabolite formation. Enzyme kinetic parameters (Km and Vmax) were determined using nonlinear regression analysis in SigmaPlot Enzyme Kinetics Module (version 1.3) and were evaluated by substrate inhibition or Hill equations. The Km values for the monohydroxy metabolite formation were in the range of $15 \mu \mathrm{M}$ to $39 \mu \mathrm{M}$. M1 and M2 have higher Km values in HLM than in HIM while the opposite holds for M3. Results from the present study allow us to compare the metabolite formation profile and enzyme kinetics of aPPD in two major xenobiotic metabolizing sites and provides an insight into the metabolic fate of aPPD in humans.

\section{P257. Oxidative metabolism of a methylsulfonyl moiety}

\author{
Nick Neitzel $^{1}$, Brian Baer ${ }^{1}$, Brad Fell $^{2}$, Lance Williams ${ }^{3}$ and Ron Franklin ${ }^{1}$
}

${ }^{1}$ Drug Metabolism and Pharmacokinetics, Array BioPharma Inc., Boulder, CO, ${ }^{2}$ Medicinal Chemistry, Array BioPharma Inc., Boulder, CO, ${ }^{3}$ Pharmacology, Array BioPharma Inc., Boulder, CO

AR-0001 contains a phenyl ring to which is attached 1) a carbon-containing R group at the 1position, 2) an electron withdrawing group (EWG) at the 2-position, and 3) a methyl sulfone functional group at the 4position. It was hypothesized that attachment of the methylsulfonyl moiety at the 4position would not pose a metabolic liability. The metabolism of AR-0001 was evaluated in NADPH-fortified liver microsomes of mouse, rat, dog, monkey, and human, as well as in the plasma of mouse, rat, dog, and monkey following oral administration of AR-0001. The extraction of AR-0001 and its metabolites from all samples was accomplished using protein precipitation, and detection was achieved using high performance liquid chromatography with in-line ultraviolet detection (HPLCUV) and electrospray tandem mass spectrometry (ESI-MS/MS) in the positive ionization mode. Daughter ion fragments observed in the product ion spectra of parent AR-0001 ( $\mathrm{m} / \mathrm{z} 468)$ were verified using an isotope pattern overlay provided by an EWG atom contained within the R group. Metabolites of AR0001 were detected in both microsomal and plasma extracts. Two metabolites that were +2 amu $(m / z$ $470)$ over parent and $14 \mathrm{amu}(\mathrm{m} / z 454)$ different than parent were of particular interest because their product ion spectra relative to parent suggested that biotransformation likely was occurring on the methyl sulfone functionality of AR-0001, and it was hypothesized that these were the sulfonic and sulfinic acid metabolites, respectively. Subsequently, a deuterated standard of AR-0001 ( $d_{3}$-AR-0001), with three deuteriums incorporated into the methyl group of the methylsulfonyl 
moiety, was synthesized. This deuterated material was incubated with NADPH-fortified liver microsomes in the same manner as non-deuterated AR-0001. Analysis of $d_{3}$ AR-0001 microsomal extracts resulted in two metabolites with identical mass and product ion spectra as those previously observed in non-deuterated AR-0001 microsomal and plasma extracts, signifying loss of the deuterated methyl group. This result strongly suggests that metabolism proceeds via oxidative demethylation of the methyl sulfone, a phenomenon recently documented in the literature ${ }^{1}$. While needing to consider the methylsulfonyl moiety as a metabolic liability, it also suggests great caution against placing a radiolabel in this position unless it has been shown to be metabolically stable.

1. Kassahun K., Black W.C., Nicoll-Griffith D., McIntosh I., Chauret N., Day S., Rosenberg E., and Koeplinger K. 2011. Pharmacokinetics and Metabolism in Rats, Dogs, and Monkeys of the Cathepsin K Inhibitor Odanacatib: Demethylation of a Methylsulfonyl Moiety as a Major Metabolic Pathway. Drug Metab Dispos 39(6):1079-1087.

\title{
P258. Pharmacokinetics, metabolism and excretion of ${ }^{14} \mathrm{C}$-monoethyl phthalate (MEP) and ${ }^{14} \mathrm{C}$ - diethyl phthalate (DEP) after single oral and IV administration in the juvenile dog
}

\author{
Clair I. Smart ${ }^{1}$, L. M. Kao ${ }^{2}$, Brian John ${ }^{1}$, Bruce Ruoff ${ }^{3}$, G. Mannens ${ }^{4}$, N. Bower ${ }^{5}$ and T. Aoki ${ }^{6}$ \\ ${ }^{1}$ Drug Metabolism, Huntingdon Life Sciences, Huntingdon, United Kingdom, ${ }^{2} J o h n s o n$ and Johnson Pharmaceutical \\ Research and Development, L.L.C., Raritan, NJ, ${ }^{3}$ Global Preclinical Compound Development, Johson \& Johnson \\ Pharmaceutical Research and Development, L.L.C., Titusville, NJ, ${ }^{4}$ Johnson \& Johnson Pharmaceutical Research and \\ Development, a division of Janssen Pharmaceutica N.V., Beerse, Belgium, ${ }^{5}$ Eisai Inc., NJ, ${ }^{6}$ Eisai Co., Ltd., Japan
}

Objective: The pharmacokinetics, metabolism and excretion of MEP and DEP were studied in the juvenile dog to compare the MEP exposure after intravenous or oral administration of ${ }^{14} \mathrm{C}-\mathrm{MEP}$ or ${ }^{14} \mathrm{C}-\mathrm{DEP}$ in juvenile beagle dogs. Methods: Four male juvenile beagle dogs (12-weeks old at the beginning of treatment) were treated with a single oral or bolus intravenous dose of either ${ }^{14} \mathrm{C}$-MEP or ${ }^{14} \mathrm{C}$-DEP (ca.1200 $\mu \mathrm{g}$ or $164 \mu \mathrm{g} / \mathrm{kg} ; 89 \mu \mathrm{Ci} / \mathrm{dog}$ ). Following dose administration, blood samples were collected up to $24 \mathrm{~h}$ post-dose, and urine and faeces were quantitatively collected for up to $72 \mathrm{~h}$ postdose. Plasma, urine, and faeces were analysed for total radioactivity (TR). Urinary and plasma metabolites were profiled by HPLC equipped with a radioactivity detector and the attempt to identify major urine metabolites was performed by LC-MS. Pharmacokinetic parameters of MEP and DEP were determined. During the initial phases of the study, dogs were (for companionship) housed in pairs and a crossover dosing regime was used to eliminate bias caused by ageing. Results: The absorption, metabolism and excretion of ${ }^{14} \mathrm{C}$-MEP were near identical to that of ${ }^{14} \mathrm{C}$-DEP. ${ }^{14} \mathrm{C}$-DEP was rapidly and near completely metabolized to ${ }^{14} \mathrm{C}-\mathrm{MEP}$ following either intravenous or oral administration. The peak plasma levels of TR were reached within 30 minutes after oral administration with either ${ }^{14} \mathrm{C}-\mathrm{MEP}$ or ${ }^{14} \mathrm{C}$-DEP, the elimination half-life of MEP was estimated to be $1 \mathrm{~h}$. TR was mainly excreted in urine (90-96\% of the dose) with faecal excretion accounting for only a minor fraction of the total excreted radioactivity ( $2-3 \%$ of the dose). In plasma, MEP accounted for the majority of TR, in addition, three minor metabolites (M1, M2 and M3) were detected. In urine, MEP was the major component, and the three minor plasma metabolites were also observed with M3 accounting for approximately $12 \%$ of the dose. The metabolites are neither phthalic acid, nor glucuronide/sulfate conjugates. Conclusions: The present data demonstrated that DEP was rapidly and near completely metabolized to MEP after intravenous or oral administration. The absorption, metabolism and excretion of ${ }^{14} \mathrm{C}$-MEP and ${ }^{14} \mathrm{C}$-DEP in juvenile beagle dogs were indistinguishable, consequently the pharmacokinetics of MEP and DEP was near identical. The near identical metabolism and pharmacokinetics of MEP and DEP in juvenile dogs provided the scientific rationale of possible use of DEP data for the risk assessment of MEP exposure in humans, especially in pediatric population.

\section{P259. Substrate specificity for FMO- and CYP-Mediated reactions and enzyme inactivation in HLM: Methyl p-tolyl sulfide versus benzydamine}

\author{
Zhiming Wen, Robert Strab, Elizabeth Henry, Kenneth Roberts, Ramesh Bambal, Sid Bhoopathy, \\ Albert Owens and Ismael Hidalgo
}

Absorption Systems LP, Exton, PA

Methyl p-tolyl sulfide (MTS) and benzydamine (BZD) are two commonly used probe substrates for flavin-containing monooxygenase (FMO) in vitro. However, the substrate specificity of MTS and BZD, and the enzyme inactivation characterization of FMO in human liver microsomes (HLM) have not been systematically investigated. In addition, the 
involvement of individual FMO isoforms and the contributions of cytochrome P450 (CYP) enzymes in the metabolism of MTS (S-oxygenation) and BZD (N-oxygenation) are not completely understood. In this study, the enzyme inactivation for the S-oxygenation of MTS and the N-oxygenation of BZD in HLM was investigated by (1) heat treatment $\left(50^{\circ} \mathrm{C}, 5 \mathrm{~min}\right)$ in the presence and absence of NADPH; (2) incubation with and without methimazole (a non-specific FMO inhibitor); (3) incubation with and without 1-aminobenzotriazole (ABT, a non-specific CYP inhibitor); and (4) combination of heat treatment and incubation with ABT. The contributions of FMO and CYP to the metabolism of MTS and BZD were evaluated using human recombinant FMOs (FMO-1, FMO-3, and FMO-5) and CYPs (CYP1A2, 2A6, 2B6, 2C8, 2C9, 2C19, 2D6, 2E1, and 3A4). The results show that the S-oxygenation of MTS is catalyzed by FMO (mainly FMO-1 and FMO-3) and CYP (mainly CYP2E1, 1A2, and 2B6), whereas the N-oxygenation of BZD is catalyzed predominantly by FMO (mainly FMO- 1 and FMO-3) and the contribution of CYP is minor. In HLM, the S-oxygenation of MTS was inhibited by $51 \%$ with heat treatment, $33 \%$ with methimazole, $87 \%$ with heat treatment and ABT, and $59 \%$ with ABT alone. The N-oxygenation of BZD was inhibited by $97 \%$ with heat treatment, $87 \%$ with methimazole, $98 \%$ with heat treatment and ABT, and no inhibition with ABT alone. For FMO reaction phenotyping, BZD is a good probe substrate for either HLM or recombinant FMO, whereas MTS can only be used with recombinant FMO. The combination of heat treatment and co-incubation with an inhibitor of CYP (e.g., ABT) or FMO (e.g., methimazole) is a more definitive approach to distinguish the involvement of FMO or CYP in metabolism by HLM.

\title{
P260. Time-dependent cytochrome P450-mediated metabolism of etravirine in primary human hepatocytes
}

\author{
Lindsay Yanakakis $^{1}$ and Namandje N. Bumpus ${ }^{2}$
}

${ }^{1}$ Department of Biology, The Johns Hopkins University, Baltimore, $M D,{ }^{2}$ Department of Medicine, The Johns Hopkins University, School of Medicine, Baltimore, MD

Etravirine (4-[[6-amino-5-bromo-2-[(4-cyanophenyl)amino]-4-pyrimidinyl]oxy]-3,5-dimethylbenzonitrile) is a secondgeneration non-nucleoside reverse transcriptase inhibitor used to treat HIV-1. Although first-generation members of this drug class are well established cytochrome P450 (CYP) substrates and autoinducers of metabolism comprehensive information regarding the metabolism of etravirine has yet to be reported. Using ultra performance liquid chromatography-mass spectrometry (UPLC-MS) the formation of etravirine metabolites was examined. Twelve hours following treatment of primary human hepatocytes with etravirine the concentrations of metabolites resulting from monomethylhydroxylation (M1) and dimethylhydroxylation (M2) of the etravirine benzonitrile moiety methyl substituents as well as monooxygenation of the benzonitrile ring (M3) were increased 4.6-, 4.0-fold, 7.1-fold, respectively. Twentyfour hours following treatment with etravirine, the metabolite concentrations of M1, M2 and M3 further increased to 6.1-, 6.0- and 9.0-fold, respectively, as compared to the initial metabolite concentrations measured. In samples from chronically-treated (treated every $24 \mathrm{~h}$ for $72 \mathrm{~h}$ ) hepatocytes the concentrations of M1, M2 and M3 were increased 8.7-, 7.5- and 17-fold, respectively. Interestingly, two additional metabolites, one monohydroxylated and one dihydroxylated, were detected in the chronic-treated samples that were not observed at any of the other time points. The fragmentation pattern of the dihydroxylated metabolite suggests monomethylhydroxylation of the dimethyl benzonitrile moiety and monooxygenation of the benzonitrile ring itself. To determine which cytochrome P450 (CYP) mRNAs may have been upregulated by etravirine real-time PCR arrays were used. Of the 42 CYPs that were analyzed only the upregulation of CYP3A4 mRNA was statistically significant (2.8-fold 6 hours following treatment; 5.8 -fold following chronic treatment) $(p<0.05)$. CYP2B6 and CYP2C9 mRNAs were elevated at all time points, however, due to interindividual variability of the hepatocytes these increases did not reach statistical significance. These data indicate that etravirine is an autoinducer of metabolism and that differential metabolite formation may be observed following chronic- versus acute-treatment.

\section{P261. Using 'Humanized' TK-NOG mice to predict human drug metabolism and drug-drug interactions}

\author{
Yajing $\mathrm{Hu}^{1}$, Toshi Nishimura ${ }^{1}$, Manhong $\mathrm{Wu}^{1}$, Hiroshi Suemizu ${ }^{2}$, Menashe Elazar ${ }^{3}$, Jeffrey Glenn ${ }^{3}$, \\ Masami Hasegawa ${ }^{4}$, Masato Nakamura ${ }^{4}$, Tatsuji Nomura ${ }^{4}$, Yuan Chen ${ }^{5}$, Ming Zheng ${ }^{1}$, William L. \\ Fitch $^{1}$ and Gary Peltz ${ }^{1}$
}

${ }^{1}$ Anesthesia, Stanford University, Stanford, CA, ${ }^{2}$ Biomedical Research, Central Institute for Experimental Animals, Kawasaki, Japan, ${ }^{3}$ Medicine, Stanford University, Stanford, CA, ${ }^{4}$ Central Institute for Experimental Animals, Kawasaki, Japan, ${ }^{5}$ Drug Metabolism and Pharmacokinetics, Genentech Inc., South San Francisco, CA 
Inter-species differences in drug metabolism can make it difficult to use pre-clinical animal testing data to predict drug metabolites, drug-drug interactions and potential toxicities that will occur in humans. To address this problem, we investigated whether chimeric TK-NOG mice with 'humanized livers' could provide a more predictive assessment of human drug metabolism for clemizole (1), which is a new treatment for hepatitis $C$ virus infection (HCV) that is in clinical development (2). We first examined clemizole's pharmacokinetic profile in a commonly used, conventional mouse strain. Clemizole had an un-expectedly short plasma half-life (measured at $0.15 \mathrm{hr}$ ); it was very rapidly biotransformed into a glucuronide (M14) and a dealkylated metabolite (M12), and into a variety of lesser metabolites in C57BL/6J mice. Then, the pattern of clemizole metabolism in TK-NOG mice with 'humanized livers' was compared with that in control (NOG) mice with the same genetic background. The relative amount of clemizole and 6 metabolites in plasma $30 \mathrm{~min}$ after administration of a single $(25 \mathrm{mg} / \mathrm{kg}$ PO) dose of clemizole was measured. Based upon the human albumin concentration (range 1.3 to $4.2 \mathrm{mg} / \mathrm{ml}$ ) in their sera, the extent of liver humanization ranged from $12-40 \%$. The relative amounts of clemizole and 5 metabolites did not significantly differ between control and humanized mice $(p>0.10)$. However, all 'humanized' TK-NOG mice had a substantially larger amount of metabolite M1 in their plasma (2.3 fold, $\mathrm{p}<0.05)$, and the relative abundance of M1 was highest in the mouse with the most extensive liver humanization. Comparison of the drug metabolite profile in humanized TK-NOG mice with control mice correctly indicated that the human and rodent patterns of clemizole metabolism would differ, which enabled the human-predominant drug metabolite to be identified before human subjects were exposed to the drug. After the murine studies were completed, clemizole metabolism was analyzed in plasma samples obtained from ten human subjects after administration of a single (100 mg P.O.) dose of clemizole. Clemizole had a much longer plasma half-life (measured at $3.4 \mathrm{hrs)} \mathrm{in} \mathrm{humans,} \mathrm{and} \mathrm{a} \mathrm{very} \mathrm{different} \mathrm{pattern} \mathrm{of}$ drug metabolites relative to mice. Clemizole was rapidly converted to a single major metabolite (M1), which accounted for $55 \%$ of all drug and metabolites present in human plasma. A detailed in vitro analysis of clemizole metabolism was then performed to characterize the metabolic pathways and the basis for the inter-species differences. Analyses performed in the humanized mice also correctly indicated that a drug-drug interaction would occur when clemizole was co-administered with a CYP3A4 inhibitor. This analysis demonstrates that TK-NOG mice with 'humanized' livers can more reliably predict human drug metabolism and potential drug-drug interactions, and that their use could improve the quality of pre-clinical drug assessment.

\title{
Reference
}

1. M. Hasegawa et al.,Biochem Biophys Res Commun. 2011 Feb 18;405(3):405-10. Epub 2011 Jan 14.

2. S. Einav et al., Nat Biotechnol. 2008 Sep; 26(9):1019-27.

\section{P262. An investigation into the prediction of in vivo clearance for a range of aldehyde oxidase substrates using a mechanistic population-based pharmacokinetic model}

\author{
Helen Cubitt ${ }^{1}$, Karen Rowland-Yeo ${ }^{1}$, Amin Rostami-Hodjegan ${ }^{2}$ and Zoe Barter ${ }^{1}$
}

\section{${ }^{1}$ Simcyp Ltd, Sheffield, United Kingdom, ${ }^{2}$ Simcyp Limited and The University of Manchester, Sheffield, United Kingdom}

There is a growing interest in the importance of metabolism by aldehyde oxidase (AO) within the drug industry [1]. Experimental and in vitro-in vivo extrapolation (IVIVE) strategies for AO related metabolism are less robust when compared to those available for P450-mediated metabolism [1,2]. Indeed, Zientek et al (2010) recently reported that their attempt to predict intravenous clearance $\left(\mathrm{CL}_{\mathrm{iv}}\right)$ values for four compounds partially metabolised by AO led to 3to 52- fold lower values than those observed. We have developed this analysis, incorporating additional in vitro and clinical elimination data in order to rationalise the poor prediction accuracy. Values of AO-mediated intrinsic clearance $\left(\mathrm{CL}_{\text {int,A0 }}\right)$ for six compounds (O6-benzylguanine, carbazeran, DACA, XK-469, zaleplon and zoniporide) were obtained from published studies using both human liver cytosolic (HLC) and S9 (HLS9) fractions, with a preference for metabolite formation $\left(\mathrm{V}_{\max } / \mathrm{K}_{\mathrm{m}}\right)$ data. $\mathrm{CL}_{\mathrm{int}, \mathrm{AO}}$ values were combined with any known microsomal $\mathrm{P} 450 \mathrm{CL}_{\mathrm{int}}$ (O6-benzylguanine and zaleplon). In vitro data were scaled using the Simcyp ${ }^{\circledR}$ Population-based Simulator (Version 10 SP1) in order to incorporate system and population-specific variability for in vitro scaling factors (mg cytosolic, S9 or microsomal protein per gram liver), liver weight, cardiac output and haematocrit. Any known in vivo renal or biliary excretory clearance data were also included. Taking into account additional elimination mechanisms, the contribution of AO metabolism ranged between $20 \%$ (O6-benzylguanine) and $100 \%$ (carbazeran and DACA) of total in vivo clearance. Observed in vivo $\mathrm{CL}_{\text {iv }}$ ranged between 0.5 and $154 \mathrm{~L} / \mathrm{h}$ (for XK-469 and carbazeran, respectively). On average, in vitro $\mathrm{CL}_{\text {int }}$ under-predicted observed $\mathrm{CL}_{\text {iv }}$ by 2.0- to 5.2- fold using $\mathrm{CL}_{\text {int,AO }}$ from HLC (zoniporide and XK-469, respectively) and 2.0- to 7.7- fold using $\mathrm{CL}_{\mathrm{int,AO}}$ from HLS9 (zoniporide and DACA, respectively). Estimation of intestinal availability by comparing intravenous and oral data for carbazeran and zaleplon $\left(\mathrm{F}_{\mathrm{G}}\right.$ of 0.20 and 0.64 , respectively) and correction for intestinal firstpass metabolism improved the prediction accuracy of oral clearance (4-fold under-prediction) for both compounds. 
Previously, where no extrahepatic metabolism was assumed, the error was 21- and 9- fold, respectively [2]. A significant improvement of intravenous and oral in vivo clearance prediction accuracy has been achieved by carefully selecting and combining additional sources of elimination data for these compounds. However, there is also a need for further evaluation of extrahepatic metabolism by AO and of absolute abundance, in order to accurately assess population variability and drug-drug interaction potential. References: 1. Garattini, E. and M. Terao, Increasing recognition of the importance of aldehyde oxidase in drug development and discovery. Drug Metab Rev, 2011. 2. Zientek, M., et al., In vitro-in vivo correlation for intrinsic clearance for drugs metabolized by human aldehyde oxidase. Drug Metab Dispos, 2010. 38(8): p. 1322-7.

\title{
P263. Characterization of hydralazine as a specific aldehyde oxidase inhibitor to assess fraction metabolized in human hepatocytes
}

\author{
Christine C. Orozco, Timothy J. Strelevitz and Ronald S. Obach
}

Pharmacokinetics, Pharmacodynamics and Metabolism, Pfizer, Groton, CT

In vitro systems are routinely used in pharmaceutical research to understand clearance mechanisms and make clinical predictions for drug candidates. Methods for identifying metabolism by cytochrome P450 (CYP) enzymes are well established and frequently used in the search for new drug candidates. Often, structural modifications to compounds are introduced in order to improve metabolic stability in liver microsomes, which has resulted in a greater prevalence of compounds metabolized by enzymes absent in liver microsomes. In pharmaceutical research, there has been an increased number of compounds metabolized by aldehyde oxidase (AO), a cytosolic enzyme involved in the metabolism of aza-aromatic compounds. This work presents a method for determining the fraction metabolized by human AO using the specific AO inhibitor, hydralazine in human hepatocytes. Specific assay conditions for substrate and inhibitor concentration, as well as incubation time were optimized and applied to zaleplon, a substrate with clearance mediated by both AO and CYP. Hydralazine completely inhibited the AO catalyzed metabolism of zaleplon to its oxo metabolite, without disrupting the CYP catalyzed deethylation pathway. The conditions determined from this first study were applied to several substrates with multiple metabolic pathways and varying degrees of AO clearance. For each substrate, the contribution of AO mediated clearance to total hepatic clearance was estimated. The data were corroborated by examination of metabolite profile in human hepatocytes and making an estimate of the relative amounts of metabolites generated by $\mathrm{AO}$ vs. other pathways. In addition, inter-lot variability for $\mathrm{AO}$ activity of five hepatocyte donors was assessed. This characterization of hydralazine as a specific $\mathrm{AO}$ inhibitor provides a method to identify the fraction metabolized by human $\mathrm{AO}$ which can be applied to unknown substrates and potential future drug candidates.

\section{P264. Characterization of aldehyde oxidase (AO) activity in cryopreserved human hepatocytes}

\author{
James Matthew Hutzler, Young-Sun Yang, Daniel Albaugh, Cody Fullenwider, Diane Wong, Jennie \\ Schmenk and Michael Fisher
}

Drug Discovery Support, Boehringer-Ingelheim Pharmaceuticals, Ridgefield, CT

Extensive metabolism by the cytosolic enzyme aldehyde oxidase (AO) has recently been highlighted as having significant impact to drug development programs, with numerous clinical candidates halted due to low oral exposure and/ or high systemic clearance by this mechanism. Cryopreserved hepatocytes are commonly used for generating in vitro intrinsic clearance data to enable scaling to predict clearance in humans, yet little characterization of AO activity in hepatocytes has been reported. Substrates of AO for which human clinical pharmacokinetics is known were selected and evaluated in pooled mixed-gender cryopreserved human hepatocytes from two vendors in effort to characterize $\mathrm{AO}$ activity in this in vitro system. Estimated hepatic clearance $\left(\mathrm{Cl}_{\mathrm{h}}\right)$ for BIBX1382, carbazeran, $\mathrm{O}^{6}$-benzylguanine, zaleplon, and XK-469 using cryopreserved hepatocytes from Life Technologies was 18.2, 17.4, 12.8, $<4.3$, and $<4.3 \mathrm{~mL} / \mathrm{min} /$ $\mathrm{kg}$, respectively, while in hepatocytes from Celsis/IVT, estimated hepatic clearance values were 17.6, 17.0, 11.2, $<4.3$, and $<4.3 \mathrm{~mL} / \mathrm{min} / \mathrm{kg}$, respectively, indicating marginal differences between hepatocyte lots. The low exposure following oral doses of BIBX1382 and carbazeran $(<5 \% \mathrm{~F})$ would have been fairly well predicted using hepatic extraction $\left(\mathrm{f}_{\mathrm{h}}\right)$ values derived from cryopreserved hepatocytes (assuming $f_{a}=1$ and $f_{G}=1$ ). In addition, the estimated hepatic clearance value for $\mathrm{O}^{6}$-benzylguanine was within $73-84 \%$ of the observed total clearance in humans following IV administration $(\mathrm{CL}=15 \mathrm{~mL} / \mathrm{min} / \mathrm{kg})$, indicating a reasonable level of predictivity from this in vitro system. However, at least a 3.5-fold under-prediction of total clearance was observed for zaleplon, which currently remains unexplained. The observed 
metabolic clearance in cryopreserved hepatocytes was confirmed to be a result of AO metabolism via two approaches. Metabolite identification following incubations in the presence of $50 \% \mathrm{H}_{2} \mathrm{O}^{18}$ confirmed that the predominant oxidative metabolite was generated by $\mathrm{AO}$, as expected isotope patterns in the mass spectra $(\sim 1: 1$ ratio of +16 and +18 amu mass shifts) were observed following analysis by high resolution (LTQ-OrbiTrap) mass spectrometry. Secondly, observed clearance values were efficiently attenuated upon co-incubation with $50 \mu \mathrm{M}$ hydralazine, a well-known inhibitor of AO. Overall, these data suggest that the use of cryopreserved hepatocytes may be a practical approach for assessing AO-mediated metabolism in discovery, as well as predicting hepatic clearance of substrates of AO.

\title{
P265. Novel human hydrolase carboxymethylenebutenolidase (CMBL): A bioactivating enzyme of prodrug-type ARB olmesartan medoxomil
}

\author{
Tomoko Ishizuka ${ }^{1}$, Izumi Fujimori ${ }^{1}$, Mitsunori Kato ${ }^{2}$, Motoko Saito ${ }^{1}$, Yasushi Yoshigae ${ }^{1}$, Kazuishi \\ Kubota $^{2}$, Atsushi Kurihara ${ }^{1}$ and Takashi Izumi ${ }^{1}$ \\ ${ }^{1}$ Drug Metabolism \& Pharmacokinetics Research Laboratories, Daiichi Sankyo Co., Ltd., Tokyo, Japan, ${ }^{2}$ Translational \\ Medicine \& Clinical Pharmacology Department, Daiichi Sankyo Co., Ltd., Tokyo, Japan
}

Olmesartan medoxomil (OM) is a prodrug type angiotensin receptor antagonist (ARB) and is prescribed worldwide as monotherapy or in combination with a thiazide diuretic and/or a calcium channel blocker as an antihypertensive agent. We, for the first time, purified the OM-hydrolyzing enzyme from human liver cytosol and identified it to be Carboxymethylenebutenolidase (CMBL), a homolog of Pseudomonas dienelactone hydrolase, as the OM bioactivating enzyme that hydrolyzes the prodrug and converts it to the pharmacologically active metabolite olmesartan, in human liver and intestine. Interestingly, the biological and enzymatic functions of CMBL remain unrevealed in humans as well as in other higher eukaryotes. Quantitative reverse transcription PCR shows the ubiquitous expression of human CMBL gene transcript in various tissues. The comparable chemical inhibition properties and enzyme kinetic parameters for OM bioactivation between the recombinant CMBL and human liver and intestinal cytosols, were observed. Positive correlation between the CMBL protein expression and OM-hydrolyzing activity in a variety of individual human liver cytosols demonstrated that CMBL is the primary OM bioactivating enzyme in the liver. The recombinant CMBL converted several prodrugs having the same medoxomil moiety as OM to their active metabolites, but did not hydrolyze typical esterase substrates for carboxylesterases and choline esterases. CMBL exhibited a unique sensitivity to chemical inhibitors, thus, being distinguishable from other known esterases. Site-directed mutagenesis on the putative active residue $\mathrm{Cys}^{132}$ of the recombinant CMBL abolished the OM-hydrolyzing activity, suggesting that human CMBL is a member of cysteine hydrolases with an active cysteine residue in the active center. In conclusion, we have for the first time brought light on the uninvestigated human hydrolase CMBL as the OM-bioactivating enzyme, demonstrating its involvement in the metabolism of xenobiotics in humans. Further research is needed to clarify the physiological functions of CMBL proteins which are evolutionarily conserved from bacteria to mammals, including humans.

\section{P266. Quantitative targeted absolute proteomics (QTAP) for the analysis of UGT1As and UGT2Bs in human liver and intestinal microsomes using nanoUPLC-MS/MS with selected reaction monitoring}

\author{
Philip C. Smith ${ }^{1}$, John K. Fallon ${ }^{2}$, Hendrik Neubert ${ }^{3}$ and Ruth Hyland ${ }^{4}$
}

\author{
${ }^{1}$ Division of Molecular Pharmaceutics, Eshelman School of Pharmacy, University of North Carolina at Chapel Hill, \\ Chapel Hill, NC, ${ }^{2}$ Eshelman School of Pharmacy, University of North Carolina at Chapel Hill, Chapel Hill, NC, ${ }^{3}$ Pfizer, \\ Cambridge, MA, ${ }^{4}$ Pfizer Inc., Sandwich, United Kingdom
}

Introduction. Quantification of enzymes of metabolism and transporters allows estimation of drug clearance and may be utilized to evaluate mechanism of drug-drug interactions or the effects of disease states on drug disposition. Development of specific antibodies to proteins is often difficult and Western blots only provide relative quantification, being semi-quantitative at best. Here we provide method validation for novel QTAP analysis of 14 active UGTs in human liver and intestinal microsomes (M).Methods. M (10 mg) were denatured with heat, reduced with dithiothreitol, then alkylated with iodoacetamide. Digestion was optimized with trypsin $(1: 20 \mathrm{w} / \mathrm{w})$ at $37^{\circ} \mathrm{C}$ for $4 \mathrm{~h}$. The reaction was stopped with cold acetonitrile, heavy labeled peptide ( $1 \mathrm{pmol}$, Thermo) was added, the supernatant dried and reconstituted in $50 \mathrm{~mL}$ mobile phase A. Precision was evaluated by replicated assay of pooled M. Accuracy of the assay could not be definitively determined, relying upon assumptions of complete digestion and stability of the proteotypic (signature) 
peptides. LC-MS/MS with Selected Reaction Monitoring was done with a Waters NanoAcquity coupled to an AB SCIEX QTRAP ${ }^{\circledR} 5500$ using a $0.15 \times 100 \mathrm{~mm}$ BEH C18, $1.7 \mathrm{~mm}$ particle (Waters) preceded by a Symmetry C18 trap column $(0.18 \times 2 \mathrm{~mm})$. The mobile phase of A: $1 \%$ acetonitrile, B:acetonitrile, was at $2 \mathrm{~mL} / \mathrm{min}$, from $0-35 \%$ B over $20 \mathrm{~min}$. Two MRMs were collected per peptide and heavy peptide standards, then averaged. Results. The QTAP assay was able to detect UGT1A1, 1A3, 1A4, 1A6-10 and 2B4, 2B7, 2B10, 2B15 and 2B17 in all samples with LOD below 0.5 pmole/mg M protein for all isoforms except 1A9. UGT1A5 was not detectable in any M sample. The assay had excellent linearity up to $100 \mathrm{pmol} / \mathrm{mg}$. Intraday CVs were below $20 \%$ at the LOD for most isoforms and often below $10 \%$ at expressed levels of the UGTs $(3.0,3.5,2.2,1.5$ and $10.8 \%$ for UGT1A1, 1A6, 1A9, $2 \mathrm{~B} 7$ and $2 \mathrm{~B} 10$ respectively $[\mathrm{n}=3])$. Matrix effects were minimized by injecting less than $0.5 \mathrm{mg}$ digested total protein on-column. Data confirmed that UGT1A5, 7, 8 and 10 were not measurable in pooled liver M, but UGT1A8 and 10 measurable in pooled intestinal M. All five UGT2Bs measured were found in liver M, but only UGT2B7 and 2B17 were detected in intestinal M. Conclusions. This is the first report of a quantitative proteomic analysis of all 14 functional human UGTs. Using capillary LC-MS/MS, the QTAP assay is sensitive enough to be applied to mg amounts of total protein, commonly available from cell culture and small tissue samples. Validation of QTAP showed that the approach can provide robust and reproducible data that is satisfactory for the demands of drug development. Assay of UGTs using QTAP provides quantitative data not previously available and provides valuable information for drug development, as demonstrated here by the assay of human UGTs in hepatic and intestinal M. QTAP methods are generally applicable and can be applied across species and a wide range of proteins with detection reaching to low abundance proteins.

\title{
P267. Abstract withdrawn
}

\section{P268. Strategic use of EDTA-free subcellular fractions for better IVIVC in early drug discovery}

\author{
Liling Liu ${ }^{1}$, Karl B. Frank ${ }^{1}$, Adrian J. Fretland ${ }^{1}$, Barry Goggin², David Moore ${ }^{1}$ and Rob T. Taylor ${ }^{1}$ \\ ${ }^{1}$ Non-Clinical Safety, Hoffmann-La Roche Inc., Nutley, NJ, ${ }^{2}$ Onclology, Hoffmann-La Roche Inc., Nutley, NJ
}

In vitro metabolic stability and intrinsic clearance determined from sub-cellular fractions are key contributors to the early drug discovery process. Unique challenges arise when predictions from early in vitro screening data do not correlate with early pharmacokinetic data. A lack of IVIVC was noted for an amide-containing chemical class where in vitro liver microsomal intrinsic clearances predicted moderate to high clearance, yet in vivo clearances greater than liver blood flow were observed. Follow up in vitro studies indicated non-P450-mediated metabolism and indicated that esterases may play a role in the metabolism of these compounds. As some esterase families of drug metabolizing enzymes have been shown to be inhibited by metal chelators such as EDTA, we hypothesized that the addition of EDTA in the sub-cellular fractions was distorting the in vitro clearance results. In this study we investigate the degree to which EDTA-treated sub-cellular fractions (rat liver tissue homogenate, rat liver S9, human liver S9, and rat whole blood) affect metabolic stability and intrinsic clearance of various discovery compounds. We demonstrate that the in vitro metabolic stability of these various compounds are affected by the presence of EDTA in the sub-cellular preparations. In addition, we identify whole rat blood as a better in vitro system to establish IVIVC with rat PK.

\section{P269. Underestimation of the bioavailability of aldehyde oxidase substrates, example of FK3453. Is there a good strategy to predict AO clearance?}

\author{
Nicolas Gerst, Takafumi Akabane, Bindu Upadhyay, Jeffrey Masters and Kouichi Tamura
}

Astellas Rsch Inst of America, Skokie, IL

FK3453 was a lead compound that failed in clinical trials due to poor bioavailability. Preclinical data showed that FK3453 is metabolized by CYPs and aldehyde oxidase (AO) in human and rat subcellular liver fractions. The low systemic exposure of FK3453 observed in humans was the result of unpredictably high AO metabolism despite the favorable pharmacokineticprofiles in rats and dogs. A single metabolite was found in vivo in human studies, and subsequently found to be due to AO metabolism. The appearance of this AO metabolite in the incubation mixture with human cytosol, hepatocytes and liver slices, mirrored the disappearance of FK3453. The CLints observed from human liver Cytosol, S9 fraction, hepatocytes and liver slices (CLint in ml/min/kg, Cytosol: 21.6; S9 fraction: 17.9; 
hepatocytes: 31.2; Liver slices: 17.3 ) were very comparable, however there was a significant under estimation to the observed human in vivo clearance (CLint in vivo; $486 \mathrm{ml} / \mathrm{min} / \mathrm{kg}$ in dispersion model). Conclusions: All in vitro assays under predict in vivo clearance of FK3453 in human. The cytosol and S9 fractions gave similar data to more comprehensive systems like hepatocytes and liver slices indicating that the fractionation is not the cause of underestimation We suggest a 20 -fold scaling factor might be appropriate to predict in vivo clearance from these in vitro screening methods.

\title{
P270. Deficiency of PXR decreases atherosclerosis in apolipoprotein E-deficient mice
}

\author{
Changcheng Zhou, Yipeng Sui, Jinxian Xu and Jennifer Rios-Pilier
}

Graduate Center for Nutritional Sciences, University of Kentucky, Lexington, KY

The pregnane X receptor (PXR, also known as SXR) is a nuclear hormone receptor activated by xenobiotics as well as diverse sterols and their metabolites. PXR functions as a xenobiotic sensor to coordinately regulate xenobiotic metabolism via transcriptional regulation of xenobiotic-detoxifying enzymes and transporters. Recent evidence indicates that PXR may also play an important role in lipid homeostasis and atherosclerosis. To define the role of PXR in atherosclerosis, we generated PXR and ApoE double knockout $\left(\mathrm{PXR}^{-/} \mathrm{ApoE}^{-/}\right)$mice. Here we show that deficiency of PXR did not alter plasma triglyceride and cholesterol levels in $\mathrm{ApoE}^{-/-}$mice. However, $\mathrm{PXR}^{-/} \mathrm{ApoE}^{-/-}$mice had significantly decreased atherosclerotic cross sectional lesion area at the both aortic root and brachiocephalic artery by $40 \%(\mathrm{p}<0.01)$ and $60 \%$ $(\mathrm{p}<0.001)$, respectively. Interestingly, deficiency of PXR reduced the expression levels of CD36, lipid accumulation and CD36-mediated oxidized LDL uptake in peritoneal macrophages of $\mathrm{PXR}^{-/-} \mathrm{ApoE}^{-/-}$mice. Furthermore, immunofluorescence staining showed that PXR and CD36 were expressed in the atherosclerotic lesions of $\mathrm{ApoE}^{-/-}$mice and the expression levels of PXR and CD36 were diminished in the lesions of $\mathrm{PXR}^{-/} \mathrm{ApoE}^{-/-}$mice. Our findings indicate that deficiency of PXR attenuates atherosclerosis development, which may result from decreased CD36 expression and reduced lipid uptake in macrophages.

\section{P271. A functional SNP in aldo-keto reductase 1D1 (AKR1D1) affects the expression and activity of multiple cytochrome's P450 (CYPs)}

\author{
Amarjit S. Chaudhry ${ }^{1}$, Ranjit K. Thirumaran², Xia Yang ${ }^{3}$, Frederick Peter Guengerich ${ }^{4}$, Stephen C. \\ Strom $^{5}$ and Erin G. Schuetz ${ }^{1}$ \\ ${ }^{1}$ Department of Pharmaceutical Sciences, St. Jude Children's Research Hospital, Memphis, TN, ${ }^{2}$ Pharmaceutical Sciences, \\ St. Jude Children's Research Hospital, Memphis, TN, ${ }^{3} L L C$, a wholly owned subsidiary of Merck \& Co., Inc., Rosetta \\ Inpharmatics, Seattle, WA, ${ }^{4}$ Department of Biochemistry and Center in Molecular Toxicology, Vanderbilt University \\ School of Medicine, Nashville, TN, ${ }^{5}$ Pathology, University of Pittsburgh, Pittsburgh, PA
}

A recent study (1) exploited network and pathway analysis tools to illuminate the primary hepatic CYP network and to identify the upstream regulators AKR1D1 and SLC10A1, whose variation perturbs expression of the downstream regulated CYPs. These regulators seemed biologically plausible because SLC10Al (the major hepatic bile acid uptake carrier) and AKR1D1 (a central enzyme in cholic acid and chenodeoxycholic acid synthesis) each regulate hepatocellular concentrations of bile acids, endogenous ligand activators of PXR and CAR, known transcriptional regulators of CYP3A, CYP2C9 and CYP2C19. The hypothesis was tested that genetic variation in AKR1D1 contributes to observed variation in hepatic CYP expression and activity. Adenoviral mediated AKR1D1 overexpression in primary human hepatocytes increased expression of major CYPs including CYP3A4, CYP2C8, CYP2C9, CYP2C19, and CYP2B6. Conversely, Lentiviral mediated AKR1D1 knockdown in primary human hepatocytes significantly decreased expression of these same CYPs. Having established that AKR1D1 expression influences expression of human hepatic CYPs, we went on to identify a functional SNP (rs1872930) in the 3' untranslated region of AKR1D1. Transfection of wild type and variant AKR1D1 3' UTR reporter constructs in HepG2 cells demonstrated that the variant allele is associated with increased AKR1D1 transcript stability and/or translation efficiency. These results were further corroborated by the finding that AKR1D1 rs1872930 genotypes correlated with AKR1D1 mRNA expression in human livers. Human livers with the rs187930 AKR1D1 variant allele had higher AKR1D1 mRNA, and higher mRNA expression of multiple CYP enzymes including CYP3A4, CYP2C8, CYP2C9, CYP2C19, and CYP2B6, compared to livers with the AKR1D1 wt allele. These results demonstrate that genetic variation in AKR1D1 is associated with CYP mRNA expression in human livers. 


\title{
Reference
}

1. Yang X, Zhang B, Molony C, Chudin E, Hao K, Zhu J, Gaedigk A, Suver C, Zhong H, Leeder JS, Guengerich FP, Strom SC, Schuetz E, Rushmore TH, Ulrich RG, Slatter JG, Schadt EE, Kasarskis A and Lum PY. Systematic genetic and genomic analysis of cytochrome P450 enzyme activities in human liver. Genome Res 2010; 20(8): 1020-36

\section{P272. Extensive pathway oriented candidate gene approach allows identification of factors influencing CYP3A4 expression and function}

\author{
$\underline{\text { Kathrin Klein }}{ }^{1}$, Maria Thomas ${ }^{1}$, Stefan Winter ${ }^{1}$, Thomas Weiss ${ }^{2}$, Matthias Schwab ${ }^{1}$ and Ulrich M. \\ Zanger $^{1}$
}

${ }^{1}$ Dr. Margarete Fischer-Bosch Institute of Clinical Pharmacology, Stuttgart, Germany, ${ }^{2}$ Surgery, University of Regensburg, Regensburg, Germany

\begin{abstract}
Objectives: Members of the CYP3A enzyme family participate in the metabolism of up to 50\% of clinically used drugs and many endogenous substances. Large variability in CYP3A expression and function contributes to adverse events. Although high heritability of CYP3A phenotype has been proposed, genetic polymorphisms have so far only been recognized as important predictive factors for the minor CYP3A5 but not for the major CYP3A4. In contrast, known non-genetic factors contributing to CYP3A4 variation include sex and inflammation. We used a pathway-oriented pharmacogenomics approach to assess the role of various regulatory pathways for expression and function of hepatic CYP3A4 expression. Methods: A set of 150 human liver samples of Caucasian origin was phenotyped for CYP3A4 mRNA and protein expression and for two prototypical biotransformation reactions, verapamil $\mathrm{N}$-demethylation and atorvastatin ortho-hydroxylation. Candidate gene polymorphisms compiled from literature and databases were analyzed by maldi-tof mass spectrometry and by microarray analysis. Validation experiments were performed in human primary hepatocytes by lentiviral gene knock-down. Results: CYP3A4 expression was not normally distributed and varied widely at mRNA, protein and activity levels. A total of 334 SNPs in 40 trans-genes including nuclear receptors and other transcription factors, inflammation-related genes, drug transporters and activity-related genes were analyzed. Statistical analysis identified SNPs in the Ah receptor pathway, inflammationrelated genes and in PPAR $\alpha$ as most consistent markers of CYP3A4 phenotype. Western immunoblotting revealed reduced expression of PPAR $\alpha$ in homozygous carriers of rs4253728. Gene knock-down experiments by shRNA-encoding lentivirus confirmed a pronounced effect of PPAR $\alpha$ on CYP3A4 expression in human hepatocytes. Conclusions: Our pathway-oriented pharmacogenomics approach identified several polymorphisms as novel consistent predictors of CYP3A4 expression and function in human liver. We propose that PPAR $\alpha$ plays a hitherto underestimated role in coordinating different control mechanisms for hepatic CYP3A4 expression. This study was supported by the German Federal Ministry of Education and Research (Virtual Liver grant 0315755 and grant 03 IS 2061C) and the Robert Bosch Foundation, Stuttgart, Germany.
\end{abstract}

\section{P273. Functional assessment of CNV's in human liver}

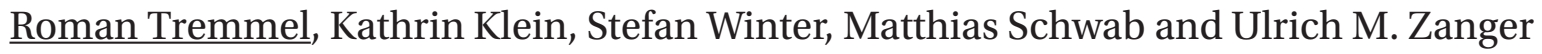

\section{Dr. Margarete Fischer-Bosch Institute of Clinical Pharmacology, Stuttgart, Germany}

Objective: Common variations in the human genome occur as single nucleotide polymorphisms (SNPs) and as structural variations, e.g. cytogenetically visible chromosome aberrations, or more frequent variations at the DNA-level which include deletions, insertions or duplications some of which lead to gene copy number variations (CNVs). Microarray-based analyses and new sequencing technologies have increased the total number of known CNVs to approximately $12 \%$ of the human genome. However their effects on gene expression remained largely unexplored. In our study we used a large human liver bank and the database of genomic variants (http://projects.tcag.ca/variation) to investigate the extent of CNVs in genes involved in absorption, distribution, metabolism and excretion (ADME) and to assess their potential impact on gene expression. Methods: The database was screened for structural variants in ADME genes including drug metabolizing cytochromes P450 (CYP), drug transporters, nuclear receptors and other liver expressed genes. For verification we used genomic DNA of 150 human liver samples for analysis with TaqMan 7500 and Applied Biosystems predeveloped copy number variation assays. RnaseP was supposed to have a constant copy number of two and was used as reference gene. Relative expression levels were calculated and the copy numbers of the target gene were estimated using the CopyCaller software (Applied Biosystems). Expression data (mRNA, protein, activity) for some of the selected ADME genes were available for the sample set from previous studies and were correlated to the genotype. Results: The well known CNVs in the CYP2D6 gene were previously genotyped using the TaqMan method and were shown to be correlated to expression and function. For CYP2A6, which 
is involved in the metabolism of nicotine and several pharmaceuticals and carcinogens, structural variants (duplication, deletion $C Y P 2 A 6 * 4)$ samples with heterozygous deletion $(n=3)$ or duplication $(n=5)$ were identified. The CYP2A6 genotype was significantly associated with phenotype (protein expression and coumarin 7-hydroxylation; $\mathrm{p}<0.05$ ). Further genes are currently under investigation and results will be presented on the poster. Conclusions: Copy number variations contribute to variable ADME gene expression. The combined analysis of available knowledge of the location of structural variants accessible by databases and their investigation in a large human liver bank should lead to novel insight on the impact of CNVs on ADME gene expression and drug response. Supported by German Federal Ministry of Education and Research (Virtual Liver grant 0315755) and the Robert-Bosch Foundation, Stuttgart, Germany

\title{
P274. Influence of MDR1 and NFKBIA polymorphisms on cyclosporin A concentrations in Chinese renal transplant recipients
}

\author{
$\underline{\text { Huang Min }}{ }^{1}$, Zhang $\mathrm{Yu}^{2}$, Li Jiali ${ }^{2}$, Wang Xueding ${ }^{2}$ and Wang Changxi ${ }^{3}$ \\ ${ }^{1}$ School of Pharmaceutical Sciences, Sun Yat-sen University, Guangzhou, China, ${ }^{2}$ School of Pharmaceutical Sciences, Sun \\ Yat-sen University, GuangZhou, China, ${ }^{3}$ Transplant Center of the First Affiliated Hospital of Sun Yat-sen University, Sun \\ Yat-sen University, Guangzhou, China
}

Cyclosporine (CsA) is the first-line immunosuppressants widely used to prevent allograft rejection after renal transplantation. But it has a narrow therapeutic index and shows considerable inter-individual differences in its pharmacokinetics. At the same time, Cyclosporine is a substrate of CYP3A4 and P-glycoprotein (P-gp, encoded by MDR1 gene). Nuclear factor

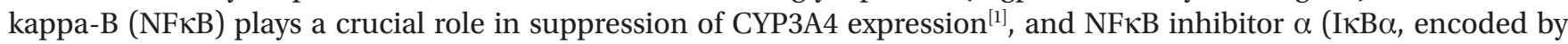
NFKBIA gene) has been reported to downregulate the activity of NFKB. Thus, inter-individual differences in the activity and/ or expression of CYP3A4, P-gp ${ }^{[2]}$ and NFאB may contribute considerably to cyclosporine A pharmacokinetics. Objective: To investigate the association of MDR1 and NFKBIA polymorphisms with cyclosporine concentrations in Chinese renal transplant recipients. Method: A total of 88 renal transplant recipients receiving cyclosporine were genotyped by polymerase chain reaction-restriction fragment length polymorphism for MDR1 G2677T/A, C3435T, and NFKBIAG2758A. Cyclosporine whole blood levels were measured by fluorescence polarization immunoassay.Trough concentration $\left(\mathrm{C}_{0}\right)$ of cyclosporine during days 6-18 after transplantation was determined. Results: Dose-adjusted $\mathrm{C}_{0}$ of cyclosporine in MDR1 $2677 T T$ carriers was significant higher than those in GG plus GT carriers $(90.38 \pm 24.46 \mathrm{vs} .66 .89 \pm 25.66 \mathrm{ng} / \mathrm{mL}$ per mg/ $\mathrm{kg}, P<0.001$ ). MDR1 $3435 T T$ carriers had significant higher dose-adjusted $\mathrm{C}_{0}$ of cyclosporine than those in CC plus CT carriers $(91.96 \pm 24.01$ vs. $68.95 \pm 26.82 \mathrm{ng} / \mathrm{mL}$ per $\mathrm{mg} / \mathrm{kg}, P=0.002)$. When combined the effects of MDR1 G2677T/A and C3435T, the carriers of haplotype T-T had an obviously higher dose-adjusted $\mathrm{C}_{0}$ of cyclosporine than carriers of other haplotypes $(97.23 \pm 21.78$ vs. $68.38 \pm 26.36 \mathrm{ng} / \mathrm{mL}$ per $\mathrm{mg} / \mathrm{kg}, P<0.0001)$. In addition, dose-adjusted $\mathrm{C}_{0}$ of cyclosporine in NFKBIA 2758AA carriers were significant higher than those in GA plus GG carriers $(81.93 \pm 27.37 \mathrm{vs.} 59.77 \pm 22.97 \mathrm{ng} / \mathrm{mL}$ per $\mathrm{mg} / \mathrm{kg}, P<0.0001)$. In this analysis, MDR1 G2677T/A and NFKBIA G2758A contributed most to the inter-individual variability in cyclosporine trough concentration, accounting for $24.6 \%$ and $14.6 \%$, respectively. Conclusion: These results indicated that MDR1 2677TT, 3435TT and NFKBIA 2758AA genotype were closely correlated with cyclosporine trough concentrations. It suggested that these SNPs may play a key role in optimal dosing regimen of cyclosporine.

\section{References}

[1] Gu, X. "Role of NF- $\mathrm{KB}$ in Regulation of PXR-mediated Gene Expression: a mechanism for the suppression of cytochrome P-450 3A4 by proinflammatory agents. Journal of Biological Chemistry. 2006. 281(26): 17882-17889.

[2] Wang, Y., C. Wang, et al. Effect of genetic polymorphisms of CYP3A5 and MDR1 on cyclosporine concentration during the early stage after renal transplantation in Chinese patients co-treated with diltiazem. European Journal of Clinical Pharmacology. 2008.65(3): 239-247.

\section{P275. Pharmacogenomics of thiopurine response: Use of a lymphoblastoid cell line (LCL) model system to identify SNPs associated with clinical response and functional validation of candidate genes}

\author{
Alice Matimba ${ }^{1}$, Fang Li ${ }^{1}$, Alina Livshits ${ }^{1}$, Cher S. Cartwright ${ }^{2}$, Brooke Fridley ${ }^{3}$, Gregory Jenkins ${ }^{3}$, \\ Anthony Batzler ${ }^{3}$, Richard Weinshilboum ${ }^{1}$ and Lynne Lennard ${ }^{2}$
}
${ }^{1}$ Department of Pharmacology and Experimental Therapeutics, Mayo Clinic, Rochester, MN, ${ }^{2}$ Academic Unit of Clinical Pharmacology, University of Sheffield, Sheffield, United Kingdom, ${ }^{3}$ Division of Biomedical Statistics and Informatics, Mayo Clinic, Rochester, MN


Genetic factors may influence response to the thiopurine drugs, 6-thioguanine (6-TG) and 6-mercaptopurine (6-MP) that are used to treat childhood acute lymphoblastic leukemia (ALL). It is known that single nucleotide polymorphisms (SNP) in genes encoding proteins in the "thiopurine pathway", primarily thiopurine S-methyltransferase (TPMT), contribute to variation in response. However, SNPs in other genes may also modify thiopurine response. Genome-wide approaches integrating SNP association analysis, followed by functional validation, have been used to discover novel mechanisms influencing drug response ${ }^{1}$. The first step in this study involved the use of the "Human Variation Panel" lymphoblastoid cell line (LCL) model system to perform a GWAS using 1.3 million SNPs, with 6-TG or 6-MP cytotoxicity (IC50) as a phenotype. 289 top associated SNPs $\left(\mathrm{p}<10^{-4}\right)$ not located in or near "thiopurine pathway" genes were selected and genotyped using DNA from 589 Caucasian UK-ALL97/99 patients on maintenance therapy with 6-TG and 6-MP². Association analysis was performed with a range of phenotypes including survival, relapse, TPMT activity and red cell levels of the thiopurine metabolites, 6-thioguanine nucleotides (6-TGN) and 6-methylmercaptopurines (6-MeMPs). Top SNPs associated with at least one clinical phenotype $(\mathrm{p}<0.01)$ and located in or within $200 \mathrm{~kb}$ of a gene were used to select candidate genes for functional validation. siRNAs targeting these genes were then used to perform gene knockdown, followed by cytotoxicity assays in HeLa, OVCAR10, U251 and U87MG cell lines, using the CellTiter 96® Aqueous Non-Radioactive Cell Proliferation Assay, 72 hours after incubation with a series of 6-TG and 6-MP concentrations. IC50 values were calculated using a four parameter logistic model. The clinical association analysis identified top SNPs located in CDH12, FRMD4B and GNG2 that were associated with reduced levels of 6-MeMPs (p<0.01) and SNPs in PAG1, NME1, NME2 and SPAG9 thatwere associated with decreased 6-TGN levels, while SNPs in KCNMA1 were associated with increased 6-TGN levels. SNPs in A2BP1, ATP6AP2, RAD51AP2, VSNL1 and ZP4 were associated with worse outcomes (HR values from 1.8 to 2.7), validating results from the LCL GWAS in which these SNPs were associated with increased thiopurine drug resistance. Functional validation by siRNA knock-down of ATP6AP2, FRMD4B, GNG2, NME1 and KCNMA1 showed significant cytotoxicity curve shifts resulting in increased resistance to both 6-MP and 6-TG in the cancer cell lines. These observations suggest a possible role for these five genes in variation in thiopurine response. This study was supported in part by Leukaemia and Lymphoma Research, United Kingdom and National Institutes of Health Grants RO1 CA132780 and U19 GM61388.

\title{
References
}

1. Li L, Fridley BL, Kalari K, Jenkins G, Batzler A, Weinshilboum RM, Wang L. Gemcitabine and arabinosylcytosin pharmacogenomics: genomewide association and drug response biomarkers. PLoS One. 2009 4(11):e7765.

2. Vora A, Mitchell CD, Lennard L, Eden TO, Kinsey SE, Lilleyman J, Richards SM; Medical Research Council; National Cancer Research Network Childhood Leukaemia Working Party. Toxicity and efficacy of 6-thioguanine versus 6-mercaptopurine in childhood lymphoblastic leukaemia: a randomised trial. Lancet. 2006 368(9544):1339-48.

\section{P276. Prevalence and functional characterization of non-coding polymorphisms in the cytochrome $b_{5} /$ NADH cytochrome $b_{5}$ reductase detoxification pathway}

\author{
$\underline{\text { Kristina L. Blanke}}^{1}$, James Saccoㄹ, Robert Millikan ${ }^{3}$ and Lauren Trepanier ${ }^{4}$
}

\author{
${ }^{1}$ Molecular \& Environmental Toxicology Center, University of Wisconsin Madison, Madison, WI, ${ }^{2}$ Department of Medical \\ Sciences, University of Wisconsin Madison, Madison, WI, ${ }^{3}$ Gillings School of Global Public Health, University of North \\ Carolina at Chapel Hill, Chapel Hill, NC, ${ }^{4}$ Department of Medical Sciences, Molecular \& Environmental Toxicology \\ Center, University of Wisconsin Madison, Madison, WI
}

Cytochrome $b_{5}$ (b5) and NADH cytochrome $b_{5}$ reductase (b5R) catalyze the detoxification of reactive hydroxylamine metabolites of arylamine and heterocyclic amine carcinogens, to include 4-aminobiphenyl (4-ABP) and 2-amino1-methyl-6-phenylimidazo [4,5- $b]$ pyridine (PhIP), which are found in cigarette smoke and grilled meats, respectively. Polymorphisms in the genes encoding b5 (CYB5A) and b5R (CYB5R3) may play a role in the detoxification of these compounds, which are linked to bladder, colon, and breast cancer. The aim of this study was to determine the functional significance of single nucleotide polymorphisms (SNPs) in the non-coding regions of CYB5A and CYB5R3, and to determine their prevalence in 2037 patients (766 African American) and 1816 controls (681 African American) from the Carolina Breast Cancer Study population.NoncodingSNPs were identified in the promoter regions of $C Y B 5 A(-382 \mathrm{C}>\mathrm{T},-389 \mathrm{G}>\mathrm{A})$ and $C Y B 5 R 3(-231 \mathrm{C}>\mathrm{A},-251 \mathrm{G}>\mathrm{T})$, and the $3^{\prime} \mathrm{UTR}$ of $C Y B 5 R 3(* 392 \mathrm{G}>\mathrm{C})$ that we had previously observed in association with low protein expression and hydroxylamine reduction activities in human liver and breast. The variants were generated by site directed mutagenesis and expressed in HepG2 cells using a dual-luciferase reporter system. The CYB5R3 SNP $-251 \mathrm{G}>\mathrm{T}$ showed a significant decrease in promoter function compared to the wild-type CYB5R3 construct (58\% decrease; $\mathrm{P}=0.042$ ). This SNP was found with a minor allele frequency (MAF) of 0.071 in African American women, which was significantly higher than that found in 
non-African American women (MAF 0.002; P < 0.0001). Evaluation of the functional significance of the 3'UTR SNP in $C Y B 5 R 3, * 392 \mathrm{G}>\mathrm{C}$, is underway. This SNP was also found in a significantly higher number of African American women (MAF 0.133) compared to non-African Americans (MAF 0.006; $\mathrm{P}<0.0001$ ). These results to date indicate that a functionally defective promoter polymorphism in $C Y B 5 R 3,-251 \mathrm{G}>\mathrm{T}$, is significantly over-represented among African American women.

\title{
P277. Regulatory polymorphism in promoter region of CYP2C9 affects mRNA expression in human livers
}

\section{Danxin Wang}

\author{
Pharmacology, The Ohio State University, Columbus, $\mathrm{OH}$
}

Cytochrome P450 2C9 (CYP2C9) is one of the most important drug metabolizing enzymes, involving in metabolism of nearly $25 \%$ of clinical used drugs. Coding region polymorphisms CYP2C9*2 and *3 were known to play an important role in inter-person variability in drug dosage and clinical outcomes. However, it was not clear whether other regulatory polymorphism(s) also contribute to CYP2C9 inter-person variability in CYP2C9 activity or expression. In this study, by measuring allelic RNA expression in human 87 liver samples combined with genotyping and sequencing in genomic DNA samples, we identified and characterized a promoter tandem repeat polymorphism that is fully accounted for allelic RNA expression of CYP2C9 in human livers. According to different length and distinct structure, this tandem repeat polymorphisms can be grouped into three different variants, designating as short, medium and long alleles. Short allele reduced allelic CYP2C9 mRNA level by 25-60\% compared to medium (reference) allele. Consistently, in promoter reporter gene assays, short allele reduced constitutive CYP2C9 promoter activity by $25 \%$ compare to reference construct regardless of presence or absence of previously identified other promoter SNPs/haplotypes. Short allele is in high linkage disequilibrium with CYP2C $9 * 3$, may contribute to the loss of function effects of *3. Moreover, because short allele has higher allele frequency than *3, may also exert its loss of function effect independent of *3. The clinical significant of short allele requires further investigation.

\section{P278. Bioactivity in vitro and pharmacokinetics in vivo of hypoglycemic protein E2HSA}

\section{$\underline{\text { Guifang Dou }}^{1}$ and Zhiyun Meng ${ }^{2}$}

\begin{abstract}
${ }^{1}$ Laboratory of Drug Metabolism and Pharmacokinetics, Beijing Institute of Transfusion Medicine, Beijing, China, ${ }^{2}$ Beijing institute of transfusion medicine, Beijing Institute of Transfusion Medicine, Beijing, China
\end{abstract}

Objective: This thesis was designed to study the bioactivity in vitro and pharmacokinetics in vivo of hypoglycemic protein E2HSA Methods: The change of cAMP concentration in RINm-5F cells can reflect the vitro bioactivity of Exendin-4 and E2HSA.Meanwhile the cAMP levels be measured by a cAMP immunoassay kit. A chemiluminescent enzyme immunoassay (CLEIA) for analyzing E2HSA and Exendin-4 in blood samples was established and validated. CLEIA was employed to study pharmacokinetics of E2HSA and Exendine-4. Furthermore, ammonium sulfate and CLEIA were applied in studying metabolic conversion and active styles of E2HSA in rhesus monkey. Results and Conclusions:In response to the GLP-1 molecule and its analogs (e.g. Exendin-4 and E2HSA) binding, GLP-1R directly stimulates adenyl cyclase, leading to a rise of intracellular cAMP. The change of cAMP concentration in RINm-5F cells reflected thebioactivity of Exendin-4 and E2HSA. The cAMP levels were measured using a cAMP immunoassay kit. E2HSA and Exendin- 4 exhibited a dose-dependent stimulation of cAMP accumulation in RINm-5F cells. E2HSA proved to retain only $1 \%$ activity of Exendin-4 in vitro. Following a single subcutaneous administration with a dosage $0.3 \mathrm{mg} / \mathrm{kgand} 15$ $\mu \mathrm{g} / \mathrm{kg}$ of E2HSA and Exendin-4 to rhesus monkey, the blood samples were collected at different time points. All collected blood samples were centrifuged to obtain serum and the concentration of E2HSA and Exendin-4 in serum were determined by CLEIA method. Pharmacokinetic parameter calculations and pharmacokinetic modeling were carried out using the WinNonlin 5.2 Statistical software. It indicated that the exposure level of E2HSA in serum was increased significantly. There was obviously prolongation of the $t_{1 / 2}$ for E2HSA comparing with Exendin-4. Proteins with different molecular weight can be separated by ammonium sulfate precipitation according to their solubility. Results showed that $60 \%$ saturated ammonium sulfate can completely precipitate the E2HSA in the serum, while Exendin-4 remained in the supernatant. Ammonium sulfate precipitation with CLEIA can be used to detect the E2HSA metabolism features in vivo, which included the concentration of both E2HSA and its metabolite Exendin-4. Key words: E2HSA Exendin-4 CLEIA bioactivity 


\title{
P279. CYP2B6 genetic polymorphism and its impact on efavirenz plasma levels in HIV/AIDS out-patients of African origin
}

\author{
$\underline{\text { Benjamin U. Ebeshi }}{ }^{1}$, Margaret Oluka ${ }^{2}$, Oluseye O. Bolaji ${ }^{3}$, Anastasia N. Guantai ${ }^{2}$ and Collen M. \\ Masimirembwa ${ }^{4}$
}

${ }^{1}$ Pharmaceutical \& Medicinal Chemistry, Faculty of Pharmacy, Niger Delta University, Wilberforce Island, Bayelsa
State, Nigeria, ${ }^{2}$ Pharmacology and Pharmacognosy, School of Pharmacy, University of Nairobi, Nairobi, Kenya,
${ }^{3}$ Pharmaceutical Chemistry, Faculty of Pharmacy, Obafemi Awolowo University, Ile-Ife, Nigeria, ${ }^{4}$ African Institute of
Biomedical Science \& Technology, Harare, Zimbabwe

Background: The non-nucleoside reverse transcriptase inhibitor, efavirenz is metabolized primarily by the polymorphic cytochrome P450 2B6 (CYP2B6), and high plasma concentrations of the drug are associated with a single nucleotide substitution at position $516(516 \mathrm{G}>\mathrm{T})$ of CYP2B6 giving rise to CYP2B6*6 allelic variant with functional or non-functional activities. The pharmacokinetics of efavirenz is associated with a long steady state half-life of $40-55 \mathrm{~h}$, which is suitable for once daily dose regimen [1-3]. Objectives: The study was carried out to investigate the steady state plasma levels of efavirenz in HIV/AIDS out-patients with a view to recommending a genotype-based dose adjustment on the administration of efavirenz. Methods: Following written informed consent, one hundred and six (106) HIV/AIDS sero-positive black African patients of Kenya origin (made up of 40 males and 66 females) undergoing treatment at the Comprehensive Care Center of the Kenyatta National Hospital, Nairobi were enrolled in the study. Plasma concentrations of efavirenz were determined using a high performance liquid chromatographic (HPLC-UV) technique. Results: Out of the 106 HIV/AIDS patients investigated, $4 \%$ had efavirenz plasma levels below $1 \mathrm{mg} / \mathrm{L}$, predicted to be the minimum effective concentration and $70 \%$ had plasma concentrations above the predicted maximum safe concentrations of $4 \mathrm{mg} / \mathrm{L}$, whereas, $25 \%$ had plasma levels within the predicted lower and upper limit of the safety margin (i.e. $>1 \mathrm{mg} / \mathrm{L}<4 \mathrm{mg} / \mathrm{L}$ ). Plasma concentration based on gender differences was identified as covariates of efavirenz disposition. The findings suggest that a possible daily dose reduction in patients with high plasma levels could be effected without any negative impact on the therapeutic efficacy. Furthermore, previous study on CYP2B6*6 $(\mathrm{Q} 172 \mathrm{H})$ polymorphism revealed a poor metaboliser (PM) allele frequency of $35 \%$ in healthy Kenya populations [4]. However, genotyping for the CYP2B6*6 functional and non-functional genotypes on the HIV/AIDS patients will be necessary to corroborate these findings, so that a definite statement on the genotype-base dose adjustment can be inferred. Conclusion: The occurrence of high plasma levels of efavirenz in population of African origin has grave implication on the dosage of efavirenz. Preliminary data in this study suggests that a genotype inspired dose optimization of efavirenz may be necessary in African populations. References 1. Ward BA, Gorski JC, Jones DR, Hall SD, Flockhart DA, Desta Z. The cytochrome P450 2B6 (CYP2B6) is the main catalyst of efavirenz primary and secondary metabolism: implication for HIV/AIDS therapy and utility of efavirenz as a substrate marker of CYP2B6 catalytic activity. J Pharmacol Exp Ther 306:287-300; 2003. 2. WHO. Scaling up antiretroviral therapy in resource-limited settings: treatment guidelines for a public health approach, 2003 revision. http://www.who.int/pub/ prev-care/arvrevision; 2003.3. De Clercq E. Anti-HIV drugs, Verh K Acad Geneesked Belg 69:81-104; 2007 4. Matimba A, Oluka MN, Ebeshi BU, Sayi J, Bolaji OO, Guantai AN, Masimirembwa CM. Establishment of a biobank and pharmacogenetics database of African populations. Eur J Hum Genetics 49:1-4; 2008.

\section{P280. Comparison of dried blood spot vs. plasma sampling utilizing the Culex-I automated blood collection system for canine pharmacokinetic studies}

\author{
$\underline{\text { Brad Gien }}^{1}$, L. David Hopper ${ }^{1}$, Vance Cooper ${ }^{2}$, Tyler DeGraw ${ }^{3}$, Lori Payne ${ }^{2}$, Candace Rohde \\ Johnson $^{3}$, S. Calugaru' ${ }^{2}$, A.J. Hoehn' ${ }^{1}$, P. Downing ${ }^{1}$ and R. Sun ${ }^{3}$ \\ ${ }^{1}$ BASi-Evansville, Mount Vernon, IN, ${ }^{2} B A S i$, McMinnville, OR, ${ }^{3} B A S i$-WL, West Lafayette, IN
}

The aim of the current research was to demonstrate that pharmacokinetic data in plasma and Dried Blood Spots (DBS) can yield equivalent results. Four male beagle dogs were implanted with a subcutaneous access port (VAP) with a catheter inserted into the femoral vein. After surgery the dogs were allowed to recover 7 days. During the recovery period the dogs were acclimatized to metabolism cages and dog jackets for 2 hours a day. On the study start day the dogs were placed in jackets and individual metabolism cages. The dogs were connected to the Culex-L automated blood collector (Culex-L ABC), a computer-controlled robotic blood collection system, and dosed once orally with $50 \mathrm{mg} / \mathrm{kg}$ of acetaminophen. Samples of $600 \mu \mathrm{L}$ whole blood were collected into chilled vials prior to dosing and at 0.5, 1, 2, 4, 6 and 8 hours post dosing. Within two hours after collection, four $20 \mu \mathrm{L}$ spots of whole blood from each time point were pipetted 
onto Culex DBS collection cards. The DBS cards were allowed to air dry overnight at room temperature and stored at room temperature in a sealed bag containing a desiccant. The remaining blood was processed to plasma and frozen. Twenty eight whole blood samples were collected, seven from each dog. Twelve samples were several minutes later than scheduled (from 5 to $14 \mathrm{~min}$ ) due to either a software problem, or dislodgement of the needle accessing the VAP. Samples were analyzed by liquid-liquid extraction with separation and detection by LC-MS/MS. The pharmacokinetic profiles from each sampling method indicate that there is no difference between the sampling techniques. The mean concentrations from both sampling methods across the time points were indistinguishable. DBS methods offer many advantages for the collection, storage and shipping of blood samples for drug analysis. Smaller volume of sample is required for analysis allowing more frequent and multiple samples compared to a traditional PK method. Processing, handling, and shipping of DBS samples is less time consuming, less laborious, and incurs lower cost than frozen plasma. Automation of the blood sampling and delivery process ensures consistent volume, quality and location of DBS samples on the collection cards. It can also reduce labeling and handling of the cards.

\title{
P281. Different response of cilostazol and its metabolites, 3,4-dehydrocilostazol and 4-transhydroxy cilostazol, in relation to platelet accumulation
}

\author{
Boram Kang ${ }^{1}$, Su Jeong Lim ${ }^{1}$, Yeong-Lim Choi ${ }^{1}$, Min Hye Kim , Ho-Jung Shin ${ }^{1}$, Ji Eun Seo ${ }^{2}$, Yun Mi \\ Lee $^{2}$, Jae-Gook Shin ${ }^{1}$ and Im-Sook Song ${ }^{1}$
}

${ }^{1}$ Pharmacogenomics Research Center, Inje University College of Medicine, Busan, South Korea, ${ }^{2}$ Clinical Pharmacology, Inje University College of Medicine, Busan, South Korea

Cilostazol and two major metabolites, 3,4-dehydrocilostazol and 4-transhydroxy cilostazol, are inhibitors of phosphodiesterase (PDE) III, with relative activity ratios of 1: 4-7: 0.2. Moreover, ratios of plasma concentration, protein binding and urine excretion are different, suggesting that cilostazol, 3,4-dehydrocilostazol, and 4-transhydroxy cilostazol may have different transport characteristics. Therefore, the purpose of this study was to investigate the correlation between the platelet accumulation and VASP phosphorylation, the response of cilostazol and its metabolites and to investigate transport characteristics of cilostazol and two metabolites. The platelet to medium ratio was constant meaning that accumulation of cilostazol and its metabolites in platelet is not related with transport system within the concentration range $(5-1000 \mathrm{ng} / \mathrm{mL})$ tested. Phosphorylation of VASP was increased by the treatment of cilostazol and its two metabolites in a concentration dependent manner. $\mathrm{EC}_{50}$ values calculated from the drug-response curve were $0.57 \mu \mathrm{M}$ for cilostazol, $0.22 \mu \mathrm{M}$ for 3, 4-dehydrocilostazol, and 5.46 $\mu \mathrm{M}$ for 4-transhydroxy cilostazol. The ratios of platelet accumulation of cilostazol and its two metabolites were comparable to the $\mathrm{EC}_{50}$ values for phospho-VASP (1: 1.28: 0.19 for platelet to medium ratio, 1: 2.57: 0.10 for $\mathrm{EC}_{50}$ values). Platelet expressed MRP4 with the highest level and MDR1, MRP1, MRP2, MRP3, and OCT1 when measured their mRNA level by realtime PCR. In conclusion, platelet accumulation of cilostazol and its metabolites was correlated with drug response, VASP phosphorylation with the limited contribution of drug transporters although they are expressed in platelet.

\section{P282. Effect of aripiprazole in an animal model of alcohol addiction and its pharmacokinetics and dopamine $\mathrm{D} 2$ receptor occupancy in rats}

\author{
Gopinadh Bhyrapuneni, Ramanatha Saralaya, Pradeep Jayarajan, Vishwottam Kandikere, \\ Nageswararao Muddana, Devender Reddy Ajjala, Ramakrishna Suraneni and Ramakrishna Nirogi
}

\section{Discovery Research, Suven Life Sciences Ltd, Hyderabad, India}

Drug abuse is a serious health problem in many areas of the world, yet there are no widely effective medications available to treat its underlying pathology or its clinical manifestations. Recent calculations have estimated that drug addiction accounts for more than $40 \%$ of the financial cost to society of all major neuropsychiatric disorders. Globally alcohol dependence ranks $3^{\text {rd }}$ in the list of preventable causes of morbidity and mortality. The aim of present research was to study the effect of aripiprazole in an animal model of alcohol addiction and evaluate its pharmacokinetic, dopamine $\mathrm{D}_{2}$ receptor occupancy in normal as well as alcohol addicted rats and also substantiate the potential use of aripriprazole in alcohol dependent patients with psychotic disorders. Methods: Male Sprague Dawley (SD) rats were trained to drink 4 $\%$ alcohol in a $45 \mathrm{~min}$ schedule induced ethanol polydipsia paradigm. Effects of acute administration of aripiprazole ( 0 , 1,3 and $10 \mathrm{mg} / \mathrm{kg}$, p.o.) on alcohol addicted rats were studied. The pharmacokinetic and dopamine $\mathrm{D}_{2}$ receptor occupancy of aripiprazole were studied in alcohol addicted as well as normal rats. Results: Aripiprazole dose dependently 
decreased the alcohol consumption in alcohol addicted rats. The oral bioavailability of aripiprazole at $10 \mathrm{mg} / \mathrm{kg}$, p.o. in alcohol addicted rats and normal rats were 28 and $8 \%$ respectively. The oral exposure of aripiprazole was found to be 3 fold higher in alcohol addicted rats compared to normal rats. Acute treatment with aripiprazole at $1 \mathrm{and} 3 \mathrm{mg} / \mathrm{kg}$, p.o. had no effect on alcohol drinking in alcohol addicted rats and at a dose of $10 \mathrm{mg} / \mathrm{kg}$, p.o. significantly decreased the alcohol consumption. In normal rats aripiprazole showed 5, $19 \& 40 \%$, in alcohol addicted rats aripiprazole showed 22, 49 \& $81 \%$ dopamine $\mathrm{D}_{2}$ receptor occupancy at dose levels of $1,3 \& 10 \mathrm{mg} / \mathrm{kg}$ p.o. respectively. Conclusion: As reported earlier aripiprazole dose dependently decreased the alcohol consumption. A significant difference in exposure and dopamine D2 receptor occupancy profile was observed between alcohol addicted rats and normal rats. So care should be taken to monitor the blood levels of aripiprazole in addicted individuals. Since there is no medication for the specific treatment of dually diagnosed patients with alcohol abuse and mental illnesses such as schizophrenia, schizoaffective disorder and bipolar disorder this study provide further support for the potential therapeutic utility of aripiprazole in alcohol dependent patients with psychotic disorders.

\title{
P283. Effect of generic warfarin sodium formulation on its clinical steady state concentrations
}

\author{
$\underline{\text { Zhou Limin }}^{1}$, Wo Siu Kwan' ${ }^{1}$ You Hoi Sze ${ }^{1}$, Bolger MB² and Zuo Zhong ${ }^{1}$ \\ ${ }^{1}$ School of Pharmacy, Faculty of Medicine, The Chinese University of Hong Kong, Hong Kong, Hong Kong, ${ }^{2}$ Simulations \\ Plus Inc., Lancaster, CA
}

Aim: As a BCS class I drug, it is expected that the dissolution profile of warfarin sodium tablet would be able to predict its in vivo performance. The current study aims to evaluate the impact of different generic warfarin sodium tablets in China on its clinical steady state concentrations. Method: We measured dissolution profiles and collected from the literature, physicochemical properties of three generic warfarin sodium tablets in Hong Kong and mainland China market. These included: APO-Warfarin (APO) manufactured by APOTEX INC; IVAX Warfarin Tablet (IVAX) manufactured by IVAX Pharmaceuticals Ireland; Warfarin Sodium Tablet (SINE) manufactured by Shanghai Sine Pharmaceutical Co. Ltd. The dissolution test was conducted using an ERWEKA DT80 dissolution apparatus according to USP pharmacopeia. Samples were analyzed by ESI/MS/MS in the negtive ion mode with MRM monitoring transitions of 307/161 for warfarin. Differential scanning calorimetry (DSC) profiles of the three warfarin sodium tablets generated from Perkin Elmer Pyris 1 differential scanning calorimeter were obtained to provide mechanistic explanations on their dissolution profiles. The in vivo steady state concentrations of the three warfarin sodium tablets were obtained either from clinical patients (for IVAX) or GastroPlus" (Simulation Plus Inc.) and compared. Results: All the tablets fully disintegrated within 30 min.It was shown that the dissolution rate of IVAX-warfarin and APO-warfarin at 30 min were significantly higher than that of SINE-warfarin $(p<0.05)$. There was $77.2 \%$ of IVAX-warfarin and $67.2 \%$ of APO-warfarin dissolved at $30 \mathrm{~min}$ of dissolution test, whereas, only $31.5 \%$ of SINE-warfarin was dissolved at $30 \mathrm{~min}$ of the dissolution test. The physicochemical properties of three warfarin sodium products were investigated by DSC. IVAX-warfarin and APO-warfarin showed an endothermic peak due to their melting at $143.5^{\circ} \mathrm{C}$ and $141.3^{\circ} \mathrm{C}$, respectively. However, the SINE-warfarin showed no endothermic peak. Comparison of the steady state concentrations of the three tablets indicated that the time to achieve steady state did not differ too much although they demonstrated different dissolution profiles. However, differences between the peak and trough concentrations at the steady state significantly increased for the tablet with the poor dissolution performance, indicating its potential clinical risk. Conclusion: There existed significant different dissolution profiles among the tested generic warfarin sodium tablets, which could be caused by different physicochemical properties of the formulations. Such differences will further increase the variations on the steady state concentrations of warfarin in the patient.

\section{P284. Gender differences in pharmacokinetics of fluoxetine in newborn lambs up to 1 month of age}

\author{
$\underline{\text { Timothy Chow }}^{1}$, Tuan-Anh Nguyen ${ }^{2}$, Dan Rurak ${ }^{2}$ and Wayne Riggs ${ }^{1}$ \\ ${ }^{1}$ Faculty of Pharmaceutical Sciences, University of British Columbia, Vancouver, BC, Canada, ${ }^{2}$ Faculty of Medicine, \\ University of British Columbia, Vancouver, BC, Canada
}

The disposition of many drugs in infant life is not well studied. Drug therapy is commonly used in newborns and neonates, but drug administered to the mother during the perinatal period and passively transferred to the fetus in utero constitutes a major route of drug exposure in newborns. In utero, the fetus has maternal routes for drug disposition, however after birth, newborns must rely on their own immature mechanisms. Therefore, a thorough understanding of 
the ontogeny of drug metabolizing pathways is essential to determine the development of these clearance mechanisms from birth to adult life with the goal of providing clinicians with guidelines for optimal drug dosing. In this study, sheep are used to determine the pharmacokinetics of the commonly used antidepressant fluoxetine at $\sim 3$ and 10 days, and 1 month of age. Under isoflurane anesthesia and aseptic surgical techniques, heparin bonded polyurethane catheters were implanted in a carotid artery and jugular vein in lambs at 2 days of age. Racemic fluoxetine $(1 \mathrm{mg} / \mathrm{kg}) \mathrm{was}$ administered as an IV bolus injection and arterial blood samples were (2ml) collected at 5, 15, 30, 45, 60 min., and 2, 4, 6, 9, 12, 24, $36,48,60$, and 72 hours. Plasma was separated and stored at $-80^{\circ} \mathrm{C}$ until analysis with a validated stereoselective LC/MS/ MS assay. ${ }^{1}$ Pharmacokinetic parameters calculated include AUC, elimination half-life, clearance, volume of distribution, and mean residence time. Fluoxetine is primarily metabolized by the enzyme CYP2D6, which is minimally expressed in human and sheep fetuses and progressively develops after birth. The drug is also a chiral compound and exhibits stereoselective disposition with the levels of the $\mathrm{S}$ isomer being higher than the $\mathrm{R}$ isomer. Our results indicate that in terms of age, there is no statistical difference in the pharmacokinetic parameters at $\sim 3$ and 10 days, and 1 month of age; however, when adult sheep data from our previous study (Kim et al, 2004) ${ }^{2}$ is considered, the pharmacokinetic parameters of the adult sheep are significantly different from those of the lambs. At each age group and for both genders, stereoselective disposition of fluoxetine was observed in AUC, elimination half-life, volume of distribution, clearance, and mean residence time. There appears to be statistically significant gender differences in the pharmacokinetic parameters at $\sim 3$ and 10 days, and 1 month of age. In general, the data suggests that female lambs have a greater metabolic capacity for fluoxetine than male lambs. For example, the gender differences in AUC for S-fluoxetine is shown in the following graph:

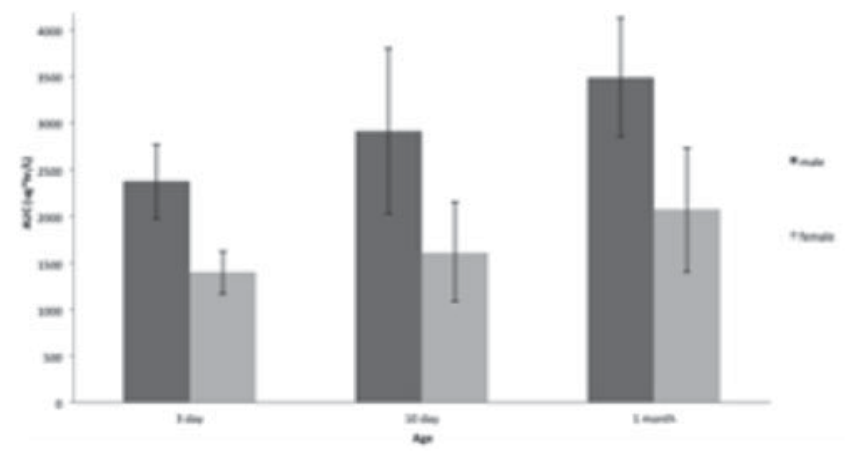

1. Chow. TW et al. A Validated Enantioselective Assay for the Simultaneous Quantitation of (R)-, (S)-fluoxetine and (R)-, (S)-norfluoxetine in Ovine Plasma Using Liquid Chromatography with Tandem Mass Spectrometry (LC/MS/MS). J. Chromatogr. B 879 (2011) 349.

2. Kim, J. Phd Thesis, The University of British Columbia (2000).

\title{
P285. Grouping plasma pharmacokinetic profiles using kohonen self-organizing maps
}

\author{
Jason L. Boyd ${ }^{1}$, Robert Fraczkiewicz², Grazyna Fraczkiewicz¹, Robert D. Clark² and Walter S. \\ Woltosz $^{2}$
}

${ }^{1}$ Simulations Plus Inc., Lancaster, CA, ${ }^{2}$ Life Sciences, Simulations Plus Inc., Lancaster, CA

Pharmacokinetic data from large clinical trials is often highly variable, even in well-controlled trials involving homogeneous cohorts. Common sources of such variability are genetic polymorphism in the CYP enzymes, developmental and demographic differences, and dietary or lifestyle choices. Pharmacokinetic scientists are tasked with quantitating variability in these data sets and identifying mechanistic sources of variability. Rapid processing of data to identify and graphically depict trends is standard practice in pharmacokinetic modeling and when implemented appropriately can effectively guide modeling efforts. Kohonen self-organizing mapping is an unsupervised clustering tool used in many scientific fields to organize and group data. Using the software ADMET PredictorTM from Simulations Plus, Kohonen mapping was applied to highly variable plasma concentration versus time ( $\mathrm{Cp}$-time) data from 93 subjects selected for a particular trial using criteria intended to minimize variability. These criteria are typical for the pharmaceutical industry: narrow range of age, body weight, and body mass index. Conditions of the clinical trial were also controlled to limit variability, including, for example, restricting and controlling dietary intake, concomitant medications, and physical activity. Prior to grouping, Cp-time profiles were characterized by peak concentrations which varied by three orders of magnitude, with the time to reach peak concentration ranging from one hour to over 20 hours. Applying Kohonen mapping to the data yielded Cp-time profile groups organized by shared features such as sharp early peaks, sharp late peaks, rounded late peaks, and profiles with multiple peaks. In this example, the 93 profiles were grouped into six easily 
identified clusters. The smallest groupings contained two profiles and the largest grouping contained 27 profiles. This grouping positively impacted the pharmacokinetic analysis by rapidly identifying relevant subgroups among subjects. In this case, through subsequent simulation it was determined that tablet disintegration effects were responsible for pharmacokinetic profiles of subjects in some subgroups, but that intersubject differences in physiology were responsible for Cp-time profile in other subgroups. This paper demonstrates that Kohonen mapping can be a useful tool for clinical pharmacologists, pharmacokinetic scientists, and toxicologists who are faced with increasingly complicated clinical trials, highly technical formulations, and ever-shrinking deadlines. Application of this technique to pharmacokinetic data analysis can improve efficiency and productivity in the already burdened pharmaceutical industry.

\title{
P286. Impact of CYP2D6 and MAO-A in indolealkylamine metabolism, pharmacokinetics and dynamics
}

\author{
$\underline{\text { Ai-Ming Yu }}$
}

Department of Pharmaceutical Sciences, University at Buffalo, SUNY, Buffalo, NY

Indolealkylaminesareamajorclassofnaturalpsychoactivedrugsthatactmainlyonserotonergicsystem. Indolealkylamines have seen widespread abuse while some show potential use for psychopharmacotherapy. Overdosing or concomitant use of indolealkylamines causes severe or even fatal "serotonin toxicity". 5-Methoxy- $N, N$-dimethyltryptamine (5-MeODMT) and 3,4-dihydro-7-methoxy-1-methyl-beta-carboline (harmaline) represent two indolealkylamine drugs that are commonly used together. 5-MeO-DMT is known as a nonselective 5-HT receptor agonist, and harmaline is a selective MAO-A inhibitor (MAOI). CYP2D6-mediated $O$-demethylation is revealed as an important pathway for harmaline metabolism, and MAO-A-catalyzed deamination is the predominant route for 5-MeO-DMT metabolism. Further studies using genotyped/phenotyped human hepatocytes indicate that CYP2D6 status significantly affects harmaline depletion, and co-incubation with harmaline blocks 5-MeO-DMT deamination metabolism. In vivo studies in CYP2D6-humanized versus wild-type mice show that CYP2D6 genotype/phenotype has significant impact on harmaline pharmacokinetics and pharmacodynamics. In addition, coadministration of harmaline leads to an increased and prolonged exposure to 5-MeO-DMT, as well as a potentiation of 5-MeO-DMT-induced hyperthermia and toxicity. Our findings suggest that CYP2D6 genetic polymorphism may cause considerable variability in indolealkylamine metabolism, pharmacokinetics and dynamics. Concurrent use of MAOI will increase the risk of 5-MeO-DMT intoxication. Acknowledgment: This project is supported by the award number R01DA 021172 from National Institute on Drug Abuse, National Institutes of Health.

\section{P287. Pharmacokinetic and pharmacodynamic implications of disease-associated changes in plasma protein binding}

\author{
Rahul Deshmukh ${ }^{1}$, Neil Wishart ${ }^{2}$, Jeffrey Voss ${ }^{2}, \underline{\text { Robert Carr }}^{1}$, Michael Firedman ${ }^{2}$, Deborah Hyland ${ }^{2}$, \\ Anwar Murtuza ${ }^{2}$ and Heather Blanchette ${ }^{3}$
}

${ }^{1}$ Dmpk-BA, Abbott, Abbott Park, IL, ${ }^{2}$ Abbott Immunology, Abbott, Abbott Park, IL, ${ }^{3}$ Cubsit Pharmaceuticals, Lexington

The extent of plasma protein binding (PPB) is considered one of the important physiological factors affecting the disposition profiles of the drug. Concentration of acute phase plasma proteins such as $\alpha_{1}$-acid glycoprotein $\left(\alpha_{1}\right.$-AGP) can be altered under various disease conditions, including inflammation. We present a case of a preclinical drug candidate showing highly dynamic plasma protein binding between disease states and its implication on the pharmacokinetic properties of the compound. Pharmacokinetic studies for Compound 1 exhibited about 5 times higher exposure in preclinical diseased rats compared to healthy animals at the same dose. The clearance and volume of distribution of Compound 1 both consistently decreased, though the half-life did not change. This led us to evaluate the protein binding properties of the compound to explain the discrepancy. PPB studies showed that the protein binding in diseased rat plasma was much higher compared to that for healthy animals. Furthermore, additional experiments with plasma proteins, suggested that Compound 1 binds to $\alpha_{1}$-AGP in a concentration dependent manner and the binding affinity of Compound 1 was greater for $\alpha_{1}$-AGP than for albumin. In the diseased animals, the level of $\alpha_{1}$-AGP can be significantly increased relative to healthy animals, resulting in a higher degree of overall plasma protein binding. This can explain the altered pharmacokinetic properties observed with Compound 1. We also looked at the free-drug concentrations of the compound by correcting for the free fraction of the drug. The free-drug exposures were essentially unchanged. Based on the assumption that only free drug available for uptake into tissue, subject to elimination and exerts the pharmacological 
response, changes in protein binding due to disease state will impact the pharmacokinetic properties of a drug. The halflife and the unbound exposure should remain unchanged and the impact on changing the pharmacological response will be minimal. This case study highlights the fact that plasma protein binding effects can be highly dynamic and an understanding of the degree of free fraction across disease models is critical to understanding the pharmacokinetic and pharmacodynamic relationship.

\title{
P288. Pharmacokinetic effect of voriconazole on cyclosporine in the treatment of aspergillosis after renal transplantation
}

\section{$\underline{\text { Choi Da-Hyeon }}$}

Pharmacogenomics research center, Inje University College of Medicine, Busan, South Korea

Azole antifungal agents are essential drugs in the treatment of fungal infections in renal transplant patients. As azoles, these antifungal agents are inhibitors of CYP3A4 and Pglycoprotein (P-gp); and thus therapeutic drug monitoring is important. We evaluated a patient with cutaneous and pulmonary aspergillosis who was successfully treated with voriconazole and a low cyclosporine trough level $(3.2-27.9 \mathrm{ng} / \mathrm{mL})$ for 3 months. During that period, the patient showed good allograft function with the co-administration of voriconazole and cyclosporine. We measured the patient genotype of MDR1, CYP3A4, CYP3A5, and CYP2C19 enzymes in addition to the intracellular concentration of cyclosporine in peripheral blood mononuclear cells (PBMCs). The intracellular concentration of cyclosporine in PBMC is 3.2 times higher with no functionally defected alleles in MDR1, CYP3A4, CYP3A5, or CYP2C19 enzymes when cyclosporine is coadministered with voriconazole ex vivo. Although other confounding factors causing immunological modulation may exist, it is plausible that low serum and highintracellular cyclosporine concentrations, due to the inhibition of P-gp activity by voriconazole, also contribute to an immunosuppressive state. Key Words: pharmacokinetic effect, voriconazole, cyclosporine, aspergillosis, renal transplantation

\section{P289. Abstract withdrawn}

\section{P290. Pharmacokinetics in rats: Small blood volume sampling and Ic analysis}

\author{
M.A.M Wenker, M. Eman, T. Noij and H.H. Emmen
}

\section{NOTOX B.V., 's Hertogenbosch, Netherlands}

Rats are often used to evaluate the safety of new chemical entities and are therefore also the rodent species of choice to generate pharmacokinetic data. One important drawback in the use of these rodents is their relative small size and therefore the inability to draw large amounts of blood from these animals. Often composite plasma concentrationversus-time curves are prepared, whereby different animals are used at different time points. However, this means a higher use of animals and a more ambiguous interpretation on pharmacokinetic data. New analytical techniques like UPLC allow the analysis of very small sample volumes while still preserving the sensitivity of conventional HPLC-MSMS analysis. When a smaller volume of blood is needed, a complete PK profile from one rat can be drawn. In this study, we have compared the pharmacokinetics of two known compounds, Nimesulide and Moxifloxacine, obtained by composite (retro-orbital puncture) and single-profile curves (tail and saphenous vein). Rats were orally dosed with either $50 \mathrm{mg} / \mathrm{kg}$ Moxifloxacine or $3 \mathrm{mg} / \mathrm{kg}$ Nimesulide and sampled at 7 time points after dosing by the retro-orbital punction, tail vein puncture or saphenous vein puncture. Blood samples of 100 microliter were drawn, and plasma samples of 10 microliter were analyzed by UPLC/MSMS. For Moxifloxacine, the calculated PK parameters (Cmax, AUC and tl/2) were quite similar between all blood sampling sampling methods. The variability observed in the PK parameters was highest for the tail vein sampling, but variability of all blood sampling methods was within $20 \%$. For Nimesulide, the calculated PK parameters (Cmax, AUC and tl/2) were quite similar between the tail vein and saphenous vein sampling methods. The obtained plasma concentration time curve showed a double peak pattern for data obtained by the retro-orbital punction, as a result of the composite sampling. The variability observed in Cmax concentration was roughly $30 \%$ for the orbita sampling while this was only $10 \%$ for both other blood sampling techniques. In summary, using a combination of small volume blood sampling (preferably saphenous vein) and UPLC analysis, a single PK profile of one rat can be obtained, which leads to the use of less animals, less stress and discomfort to the animals and a better interpretation of pharmacokinetic data. 


\title{
P291. Pharmacokinetics and brain permeability of 3,5,4'-trimethoxy-trans-stilbene (TMS)
}

\author{
$\underline{\text { Satish Sharan }}^{1}$ and Swati Nagar ${ }^{2}$
}

${ }^{1}$ Pharmaceutical Sciences, Temple University, Philadelphia, PA, ${ }^{2}$ Pharmaceutical Sciences, Temple University School of Pharmacy, Philadelphia, PA

Purpose: 3,5,4' -trimethoxy-trans-stilbene (TMS) is a naturally occurring analog of resveratrol. Its anti-neoplastic, anti-allergic and anti-angiogenic activities have been reported recently. TMS has been reported to be more potent than resveratrol in various in vitro studies. The objective of this study was to understand the TMS pharmacokinetics and permeability in brain in an in vivo mouse model. Methods: Based on in silico predictions with Chemdraw software we hypothesized that TMS had favorable physicochemical properties to cross the blood-brain barrier. We further evaluated our hypothesis by conducting a pharmacokinetic study in which TMS $(5 \mathrm{mg} / \mathrm{kg}$, IA) was administered to C57BL/6J male mice $(\mathrm{n}=2)$. Carotid artery was cannulated and blood samples were serially collected at 2.5, 5, 10, 15, 45, 90, 180, 300, 420 and 600 min. Samples were analyzed with a validated LC-ESI/MS/MS method. Noncompartmental pharmacokinetic parameters of TMS were estimated with WinNonlin. Based on the pharmacokinetic parameters and simulations, an infusion study to achieve steady state was designed to measure TMS permeability in brain at steady state. Thus, a single IV bolus loading dose of $20 \mathrm{mg} / \mathrm{kg}$ of TMS and a constant-rate infusion of $10 \mathrm{mg} / \mathrm{kg}$ was administered to 3 healthy C57BL $/ 6$ male mice. Brain and plasma samples were collected by euthanizing the animals at $30 \mathrm{~min}$ and the TMS concentrations in brain and plasma were measured using a validated LC-ESI/MS/MS method. Results: The table below lists the physicochemical properties of TMS with Chemdraw:

\begin{tabular}{lcccc}
\hline & Mol Wt. & tPSA & $\log \mathrm{P}$ & ClogP \\
\hline TMS & 270.32 & 27.69 & 3.85 & 4.761 \\
\hline
\end{tabular}

Average TMS plasma exposure $\left(\mathrm{AUC}_{0-10 \mathrm{hrs}}\right)$ was found to be $162.96 \mathrm{~min} * \mathrm{ug} / \mathrm{ml}$. The predicted steady state plasma concentration of TMS at $30 \mathrm{~min}$ was $889 \mathrm{ng} / \mathrm{ml}$ and the experimental plasma concentration at $30 \mathrm{~min}$ was found to be $774.67 \pm 176.6 \mathrm{ng} /$ $\mathrm{ml}$. This was well within the range of the predicted plasma concentration. The brain to plasma ratio of TMS at steady state was found to be $10.4 \pm 1$ 15. Conclusion: TMS concentration in mouse brain was found to be 10 fold higher than plasma at steady state plasma concentrations. Due to significantly higher brain concentrations of TMS than those reported for resveratrol, and its previously reported anti-cancer and anti-angiogenic activities, TMS can be considered a promising candidate for further evaluation for treatment of brain tumors. (Supported by NIH grant 1RO3CA133943-01 to SN)

\section{P292. Pharmacokinetics of $40 \mathrm{kDa}$ PEG in rodents using high-field NMR spectroscopy}

\author{
Purnima Khandelwal $^{1}$, Lisa Zhang ${ }^{2}$, Anjaneya Chimalakonda ${ }^{2}$, Janet Caceres-Cortes ${ }^{3}$, Christine \\ Huang ${ }^{2}$, Punit Marathe ${ }^{2}$ and Michael D. Reily ${ }^{3}$ \\ ${ }^{1}$ Discovery Analytical Sciences, Bristol-Myers Squibb, Pennington, NJ, ${ }^{2}$ MAP Discovery Support, Bristol-Myers Squibb, \\ Pennington, NJ, ${ }^{3}$ Discovery Analytical Sciences, Bristol-Myers Squibb, Princeton, NJ
}

A quantitative NMR-based method was developed and successfully applied to measuring double branched polyethylene glycol 40kDa (PEG40) in in-vivo serum samples, enabling determination of pharmacokinetic (PK) parameters of PEG40 in preclinical species. Conjugation of macromolecular drugs to PEG improves their therapeutic potential by altering their PK profile, but literature on complete PK profiling for PEG40 itself is lacking. Other analytical techniques have difficulty measuring PEG40 due to its large, sticky nature which frequently causes contamination of mass spectrometer (MS) sources. This is further confounded by the fact that PEG is a polymer which contains many individual analytes, complicating chromatographic or MS-based measurement. NMR is ideal for measuring such polymers because, among other advantages, a single, sharp peak is obtained for all the equivalent methylene protons which is insensitive to polymeric heterogeneity. High field NMR $(600 \mathrm{MHz})$ with cryoprobe technology allowed for analysis of samples in 250nM range. Results from IV PK profiling of PEG40 in CD-1 mice are presented here. Mice received $50 \mathrm{mg} / \mathrm{kg}$ of PEG40 as a bolus IV injection in $100 \%$ saline and serum samples were collected at 3m, 15m, 30m, 1h, 3h, 6h, 8h, 24h, 48h, and 72h after IV dosing ( $\mathrm{n}=3$ ). The serum samples were analyzed for PEG40 using the NMR method and PK parameters were calculated using non-compartmental analysis. The volume of distribution at $0.17 \mathrm{~L} / \mathrm{kg}$ indicated limited distribution to the plasma and interstitial space. A low clearance and a half-life of $18 \mathrm{~h}$ for PEG40 is consistent with literature (1) where PK properties of 7 different PEG molecules - MW 3kDa to 190kDa (including PEG50, but not PEG40), have been reported after IV administration of radioactive ${ }^{125}$ I-labeled PEG to BALB/cCrSIc mice. Compared to the radioactive assay in (1), the current NMR technique is quite user-friendly and does not involve preparation of any special PEG. Additionally, this is the first report, to our knowledge, 
of NMR spectroscopy application to PK profiling in serum. Reference: (1) Distribution and Tissue Uptake of Poly(ethylene glycol) with Different Molecular Weights after Intravenous Administration to Mice, Tetsuji Yamaoka, Yasuhiko Tabata and Yoshito Ikada; Journal of Pharmaceutical Sciences, Vol. 83, No. 4, April 1994, 601-606.

\title{
P293. Preclinical disposition and pharmacokinetics-pharmacodynamic modeling of tumor growth inhibition in xenograft mouse models with a MEK inhibitor, GDC-0973
}

\author{
Edna F. Choo ${ }^{1}$, Marcia Belvin², Jason Boggs ${ }^{1}$, Alan Deng ${ }^{1}$, Klaus Hoeflich ${ }^{2}$, Justin Ly' ${ }^{1}$, Mark \\ Merchant $^{2}$, Christine Orr ${ }^{2}$, Kirk Robarge ${ }^{3}$ and Harvey Wong ${ }^{1}$ \\ ${ }^{1}$ Drug Metabolism and Pharmacokinetics, Genentech Inc., South San Francisco, CA, ${ }^{2}$ Cell Signaling Pathways and \\ Translational Oncology, Genentech Inc., South San Francisco, CA, ${ }^{3}$ Chemistry, Genentech Inc., South San Francisco, CA
}

The mitogen-activated protein kinase (MAPK)/extracellular signal-regulated kinase kinase (MEK) pathway regulates cell proliferation, cell cycle regulation, cell survival, angiogenesis, and cell migration. GDC-0973 is potent and selective orally administered small molecule allosteric MEK inhibitor. The objectives of these studies were to characterize the disposition of GDC-0973 in preclinical species and to determine the relationship of GDC-0973 plasma concentrations to efficacy in Colo205 (colon), HCT116 (colon) and H2122 (lung; NSCLC) mouse xenograft models. The clearance (CL) of GDC-0973 was moderate in mouse $(33.5 \mathrm{~mL} / \mathrm{min} / \mathrm{kg})$ rat $(37.9 \pm 7.2 \mathrm{~mL} / \mathrm{min} / \mathrm{kg})$ and monkey $(29.6 \pm 8.5 \mathrm{~mL} / \mathrm{min} / \mathrm{kg})$. CL in dog was low $(5.5 \pm 0.3 \mathrm{~mL} / \mathrm{min} / \mathrm{kg})$. The volume of distribution was large in mouse $(9.4 \mathrm{~L} / \mathrm{kg})$, rat $(34.6 \mathrm{~L} / \mathrm{kg})$ and monkey $(23.3 \mathrm{~L} / \mathrm{kg})$ and moderate in $\operatorname{dog}(4.0 \mathrm{~L} / \mathrm{kg}$ ); half-lives ranged from 4 to $13 \mathrm{~h}$. In intact and bile duct cannulated rats and dogs, [14C]-GDC-0973 was well absorbed in rat $(\sim 80 \%)$ and $\operatorname{dog}(\sim 71 \%)$. The majority of [14C]-GDC-0973 related radioactivity was recovered in the bile of rat (74-81\%) and dog (65\%), suggesting that biliary excretion is a major route of elimination of GDC-0973. Protein binding in mouse, rat, dog, monkey and human ranged between 94-99\%. To characterize the relationship between plasma concentration of GDC-0973 and tumor growth inhibition PK-PD modeling was applied using an indirect response model.

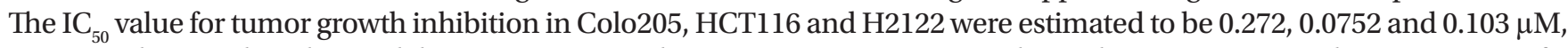
respectively. Based on the model, concentrations where tumor stasis is expected in Colo205, HCT116 and H2122 xenografts were $0.1,0.05$ and $0.07 \mu \mathrm{M}$, respectively. The CL and volume of distribution of GDC-0973 in human predicted by allometry was $3.5 \mathrm{~mL} / \mathrm{min} / \mathrm{kg}$ and $11 \mathrm{~L} / \mathrm{kg}$, respectively. Collectively these data are useful in assessing the disposition of GDC-0973 and estimating the target concentrations/doses required in the clinic and for determining appropriate clinical dosing regimens.

\section{P294. Predictability of glutathione transferase zeta 1 haplotype on kinetics of dichloroacetate in human trials}

\author{
Peter W. Stacpoole ${ }^{1}$, Erin M. Dunbar ${ }^{2}$, Albert L. Shroads ${ }^{1}$, Bonnie S. Coats ${ }^{1}$ and Taimour Langaee ${ }^{3}$ \\ ${ }^{1}$ Department of Medicine, University of Florida College of Medicine, Gainesville, FL, ${ }^{2}$ Department of Neurosurgery, \\ University of Florida College of Medicine, Gainesville, FL, ${ }^{3}$ Department of Pharmacotherapy and Translational Research, \\ University of Florida, Gainesville, FL
}

Dichloroacetate (DCA) holds an almost unique position at the interface between environmental science and allopathic medicine, being viewed as both a population health hazard and as a drug of intriguing clinical potential. DCA is dechlorinated by glutathione transferase zeta 1 (GSTzl) that is identical to the penultimate enzyme of the phenylalanine/ tyrosine catabolic pathway, maleylacetoacetate isomerase (MAAI). DCA is a suicide inhibitor of GSTzl/MAAI, although its biotransformation is also influenced inversely by age (J Pharmacol Exper Ther. 324:1163-1171, 2008) and by haplotype variation in the GSTzl/MAAI gene (J Clin Pharmacol. In press, 2011, PMID:21642471). Interest in DCA's therapeutic potential has recently intensified, based on reports of its selective pro-apoptotic and anti-proliferative actions in human cancers and in pulmonary arterial hypertension that have led to several early-phase clinical trials. However, the clinically limiting side effect of chronic DCA is reversible peripheral neuropathy. The most common human GSTz1/MAAI haplotypes are EGT (Glu32/Gly42/Thr82), KGT (Lys32/Gly42/Thr82), EGM (Glu32/Gly42/Met82) and KRT (Lys32/ Arg42/Thr82). Carriers of the wildtype (EGT) allele have more rapid plasma clearance of DCA than those who are not EGT carriers, as vividly illustrated by the following case comparison. A 68-year-old man with a left frontal lobe glioblastoma multiforme enrolled in a phase I trial of DCA for adults with recurrent brain tumors conducted at the University of Florida, in which retrospective GSTzl haplotype analysis was an exploratory endpoint. At enrollment, he had ataxia but normal liver and renal function. He received sodium DCA $8 \mathrm{mg} / \mathrm{kg}$ body wt $/ 12 \mathrm{hrs}$ by mouth, at which time DCA levels were undetectable in plasma and urine. After 26 days of treatment he presented with worsening gait and DCA 
was stopped. The patient's GSTz1/MAAI genotype was subsequently found to be EGM/EGM, which, based on previous studies, would predict a very slow rate of plasma drug clearance. Three, 8 and 9 days after DCA was discontinued, the patient's plasma (urine) DCA concentrations ( $\mu \mathrm{g} / \mathrm{ml}$ ) were: 280 (1); 150 (58); and 140 (73). In contrast, a 57 -year-old male in the same trial, who was homozygous for the wildtype (EGT) allele and who received the same DCA dose, had trough plasma drug levels of 10.5 and $11.2 \mu \mathrm{g} / \mathrm{ml}$ after 90 and 120 days of continuous drug administration. These data emphasize the importance of prior knowledge of GSTzl/MAAI genotype in assigning dosing regimens to subjects participating in clinical trials of DCA.

\title{
P295. Rapid PK: An efficient in vivo preclinical pharmacokinetic approach to support drug discovery
}

\author{
Chun Li, Bo Liu, Jonathan Chang, Todd Groessl, Liang Wang, YouQun He, Matt Zimmerman, John \\ Isbell and Tove Tuntland
}

Metabolism and Pharmacokinetics, Genomics Institute of the Novartis Research Foundation, San Diego, CA

In modern drug discovery, the implementation of high throughput in vitro absorption, distribution, metabolism and elimination (ADME) assays has greatly facilitated early elimination of poor drug-like compounds and allowed selection of potential candidates for in vivo pharmacokinetic profiling. Despite the advances in the in vitro technologies and in silico approaches for prediction of in vivo PK parameters, in vitro to in vivo extrapolation and predictive power of these approaches are not always reliable and accurate. In vivo rodent PK studies are critical to ensure compounds have appropriate pharmacokinetic properties to be evaluated in preclinical pharmacology and safety studies. However, in vivo animal pharmacokinetic studies are still conducted in a traditional low throughput manner, and therefore, are often the bottlenecks of discovery projects in many pharmaceutical companies. We present a new paradigm which we call "Rapid PK" for preclinical rodent in vivo PK studies to support drug discovery projects. We have applied this approach for both mouse and rat in vivo PK studies, which typically include both IV and PO arms with 3 animals in each dosing arm. Six blood samples are collected for each dosing arm up to $24 \mathrm{~h}$ via serial blood sampling, and blood or plasma samples for the same time point within a dosing arm were pooled, resulting in a total of 12 plasma samples per study. The rapid PK approach has several integrated components, including automated compound preparation; standardized formulation and protocols for dosing and sampling; sample pooling across animals to reduce number of samples; automated plasma sample preparation using a Beckman FXp liquid handler; automated LC/MS/MS tuning and analysis; incorporation of in house developed non-compartmental PK regression tool for batch analysis of multiple compounds, and PK reports generated to internal database using web publishing tools. In this approach, compound is dosed and analyzed discretely, thereby eliminating any drug-drug interaction concerns and analysis complications typically associated with cassette dosing or cassette analysis. We have shown that this approach has significantly improved throughput and turn-around time of preclinical PK studies, yet generating high quality full set of pharmacokinetics parameters sufficient to support discovery drug projects. When compared with standard full PK studies, very good correlation of the PK parameters CL, $\mathrm{V}_{\mathrm{ss}}$ and $\mathrm{T}_{1 / 2}$ was obtained in rapid PK studies after IV dosing of 26 compounds to rodents when the rapid and full PK studies were done months apart. Moreover, excellent correlation of dose-normalized AUC and $\mathrm{C}_{\max }$ values between Rapid and Full PK studies was achieved after oral dosing of 33 compounds.

\section{P296. Stereoselective pharmacokinetics of $( \pm)$-praeruptorin A in rats}

\author{
Yuelin Song, Ruina Zhou, Ru Yan and Yitao Wang
}

State Key Laboratory of Quality Research of Chinese Medicine, Institute of Chinese Medical Sciences, Macao, China

Background ( \pm )-Praeruptorin A (PA, [9-[(Z)-but-2-enyl]-8,8-dimethyl-2-oxo-9, 10-di-hydropyrano[2,3-f]chromen-10 -yl] acetate) is the major active component and a chemical marker of Peucedani Radix (Chinese name Qian-hu), a medicinal herb used as antitussive and mucolytic agent for centuries in China. In the present study, we characterized the chiral discrimination in pharmacokinetics of PA enantiomers in the rat, for the first time. Methods Male SpragueDawley rats $\left(220 \pm 20 \mathrm{~g}\right.$ ) were divided into 6 groups (6 rats/group) and received PA (i.v. $4 \mathrm{mg} \cdot \mathrm{kg}^{-1}$; p.o. $\left.40 \mathrm{mg} \cdot \mathrm{kg}^{-1}\right)$, $(+)$-praeruptorin $(d \mathrm{PA})$ or (-)-praeruptorin( $(\mathrm{PA})$ (i.v. $2 \mathrm{mg} \cdot \mathrm{kg}^{-1}$; p.o. $20 \mathrm{mg} \cdot \mathrm{kg}^{-1}$ ), respectively. Blood samples were collected at appropriate time intervals for $18 \mathrm{~h}$ (i.v.) and $48 \mathrm{~h}$ (p.o.) to obtain plasma. After extraction with ethyl acetate, samples were analyzed by chiral rapid resolution liquid chromatography (RRLC) coupled with MRM or EMS-IDA-EPI mode. LM1 and LM2, the in vivo major metabolites of $l \mathrm{PA}$, were prepared by a scale-up incubation of $l \mathrm{PA}$ with rat 
plasma, isolated using semi-preparative HPLC, and identified by NMR analysis. ( \pm )-cis-Khellactone was obtained from basic hydrolysis of PA and enantiomers separated by chiral semi-preparative HPLC to aid structural identification. Enzymatic kinetics of $l \mathrm{PA}$ in rat plasma was determined from 15 -min reactions at $1 \mathrm{mg} \cdot \mathrm{mL}^{-1}$ of plasma protein and 0.05-30 $\mu \mathrm{mol} \cdot \mathrm{L}^{-1}$ of $l \mathrm{PA}$. Samples were analyzed using an achiral RRLC-MRM method. Results After i.v. administration, $d \mathrm{PA}$, given individually or in the racemate form, was eliminated rapidly at similar rates $\left(t_{1 / 2}, 1.88 \pm 0.77 \mathrm{~h}\right.$ (individual) vs $1.82 \pm 0.86 \mathrm{~h}$ (racemate)) and comparable systemic exposures $\left(\mathrm{AUC}_{0-\infty}: 1221.7 \pm 145.4 \mathrm{ng} \cdot \mathrm{h} \cdot \mathrm{mL}^{-1} v s 1339.1 \pm 145.4\right.$ $\left.\mathrm{ng} \cdot \mathrm{h} \cdot \mathrm{mL}^{-1}\right)$, while its antipode was only detected at the first time point ( $3 \mathrm{~min}$ ) of sampling in both cases. A total of five metabolites were identified in rat plasma after the racemate was injected, with three (DM1-DM3) yielded from $d \mathrm{PA}$ via mono-oxidation and two (LM1: (3’R, 4'R)-4'-angeloyl-khellactone; LM2: ( $\left.3^{\prime} \mathrm{R}, 4^{\prime} \mathrm{R}\right)-3^{\prime}$-angeloyl-khellactone) from $l \mathrm{PA}$ via hydrolysis and/or acyl migration. In the case of oral administration, the parent PA or individual enantiomer were not detectable, yet cis-khellactone, a metabolite formed from hydrolysis of both substituents at C-3' and C- $4^{\prime}$ of the parent compound, was the main drug-related form in rat plasma in all cases. cis-Khellactone exhibited rapid elimination and multiple peaks were observed in plasma concentration-time curves. Additionally, $d$ PA yielded one mono-oxidation (DM2) and two hydrolysis ((-)-cis-khellactone and DM4) products, lPA produced three hydrolysis metabolites ((+)-cis-khellactone, LM1 and LM2) and all these 6 metabolites were found with PA. When incubated with rat plasma, $d \mathrm{PA}$ kept intact over $1 \mathrm{~h}$ incubation, yet $l \mathrm{PA}$ showed rapid elimination and formations of LM1 and LM2 fitted typical Michaelis-Menten model $\left(\mathrm{Ml}: \mathrm{K}_{\mathrm{m}}=4.33 \pm 1.40 \mu \mathrm{mol} \cdot \mathrm{L}^{-1}, \mathrm{~V}_{\max }=0.402 \pm 0.0715 \mathrm{nmol} \cdot \mathrm{min}^{-1} \cdot \mathrm{mg}^{-1} \mathrm{protein}\right.$; M2: $\mathrm{K}_{\mathrm{m}}=9.97 \pm 3.55 \mu \mathrm{mol} \cdot \mathrm{L}^{-1}, \mathrm{~V}_{\max }=0.663 \pm 0.165 \mathrm{nmol} \cdot \mathrm{min}^{-1} \cdot \mathrm{mg}^{-1}$ protein). Conclusions $( \pm$ )-Praeruptorin A exhibited stereoselectivepharmacokinetics and extensive first-pass elimination in the rat. Enantioselective hydrolysisof the levorotatory enantiomer in the plasma should account for the chiral discrimination. (Supported by 973 program (No.2009CB522707) and UL016/09-Y1/CMSWYT01/ICMS)

\title{
P297. Targeting the PI3K pathway in the brain - pharmacokinetics and efficacy of a PI3K inhibitor optimized to cross the blood-brain barrier
}

Laurent Salphati, Jodie Pang, Jonathan Cheong, Emile G. Plise, Bruno Alicke, Xiao Ding, Jennafer Dotson, Dingzhou Feng, Tim Heffron, Allan Joachico, Leslie B. Lee, Chudi Ndubaku, Merry Nishimura, Jim Nonomiya, Alan Olivero, Heidi Phillips, Setve Sideris, Lan Wang and Xiaolin Zhang

\section{Genentech Inc., South San Francisco, CA}

The phosphatidylinositol 3-kinase (PI3K) pathway is a major determinant of cell cycling and proliferation. It is one of the most frequently activated pathways in tumors, with mutations in one of its components detected in up to $30 \%$ of human cancers. In addition, activation or mutation of the p110 $\alpha$ subunit or loss of the phosphatase and tensin homolog have been identified in 70 to $80 \%$ of malignant gliomas. Thus, PI3K represents an attractive therapeutic target for brain cancers. However, the blood-brain barrier (endothelial cells tight junctions and efflux transporters) that limits brain access of anticancer agents represents a major hurdle in the treatment of CNS tumors. GNE-317 is a novel small molecule inhibitor of PI3K that has been optimized for brain penetration. The objectives of these studies were to assess its properties in vitro, to characterize its pharmacokinetics and brain penetration in vivo as well as to evaluate the PI3K pathway suppression achieved following administration to mice. GNE-317 was tested in MDCK cells over-expressing efflux transporters. Its binding to plasma protein and brain components was also measured. Following oral administration to mice, GNE-317 brain concentrations and PI3K pathway suppression were also measured. In vitro studies with MDCK cells transfected with P-glycoprotein or Bcrpl suggested that this compound was not a substrate of either transporter. The apparent permeability $\left(\mathrm{P}_{\text {app }}\right)$ in the apical to basolateral $(\mathrm{AB})$ and basolateral to apical (BA) directions were comparable, ranging from 9 to $18 \cdot 10^{-6} \mathrm{~cm} / \mathrm{sec}$. The efflux ratio $\left(\mathrm{P}_{\text {app, } \mathrm{BA}} / \mathrm{P}_{\text {app, } \mathrm{AB}}\right)$ was lower than 2 . Binding to plasma proteins and brain components was 77 and $94 \%$, respectively. Following IV or oral administrations to CD-1 female mice, GNE-317 brain-to-plasma ratios ranged from 0.7 to 1.1. The PI3K pathway was markedly inhibited in the brain with up to $60 \%$ suppression of the pAkt signal 1 hour post-dose. In addition, GNE-317 was efficacious in mouse xenograft models, such as the orthotopic U87 and GS-2 neurosphere models.

\section{P298. A genetic strategy to identify, validate and use gene signatures of xenobiotic-responsive transcription factors in prediction of pathway activation in the mouse liver}

\author{
Keiyu Oshida ${ }^{1}$, Naresh Vasani ${ }^{1}$, Mitch Rosen ${ }^{1}$, Barbara Abbott ${ }^{1}$, Christopher Lau ${ }^{1}$, Brian Chorley ${ }^{1}$, \\ Susan Hester ${ }^{1}$, Grace Guo ${ }^{2}$, Thomas W. Kensler ${ }^{3}$, Curtis D. Klaassen ${ }^{4}$ and Jon C. Corton ${ }^{1}$
}


${ }^{1}$ EPA, Research Triangle Park, NC, ${ }^{2}$ University of Kansas Medical Center, Kansas City, KS, ${ }^{3}$ Department of Environmental Health Sciences, Johns Hopkins Bloomberg School of Public Health, Baltimore, MD, ${ }^{4}$ Pharmacology, Toxicology, and Therapeutics, The University of Kansas Medical Center, Kansas City, KS

Many drugs and environmentally-relevant chemicals activate xenobiotic-responsive transcription factors (TF). Identification of target genes of these factors would be useful in predicting pathway activation in in vitro chemical screens as well as to determine involvement of the TFs in disease states. Starting with a large compendium of Affymetrix files (>2000), we developed a genetic and genomic strategy to identify sets of signature genes that are dependent on specific transcription factors (TF) including peroxisome proliferator-activated receptor alpha (PPARalpha), constitutive activated receptor (CAR), pregnane X receptor (PXR), aryl hydrocarbon receptor (AhR), the oxidant-induced Nrf2, farnesoid X receptor (FXR), and glucocorticoid receptor (GR). Target genes were identified by comparing the profiles after exposure to activators of a TF in wild-type and TF-null mice. In most cases, three chemical comparisons were used to identify common gene targets. In addition, we used the mouse liver compendium to identify sets of genes associated with phenomena that are linked to liver toxicity/cancer including feminization/masculinization, hypoxia, inflammation and cytotoxicity. The identified genes were validated in silico by characterization of the expression of the signature genes in independent experiments of chemical exposure or perturbation known to alter the TF. By using the signatures to query the compendium, a number of novel observations were made. 1) PPARalpha is activated in a large number of nullizygous mouse models that exhibit liver toxicity and steatosis. 2) PXR and PPARalpha activation appear to be intertwined. Many chemicals or gene mutations activate both TFs. 3) Nrf2 is activated by a large number of conditions including environmentally relevant chemicals, gene mutations, and bacterial infections. 4) GR is activated by caloric restriction and systemic damage outside the liver including the heart and colon. 5) Feminization of male-specific signature genes occurred after castration, in GH-defective dwarf mice, bacterial infections and exposure to some chemicals not previously associated with endocrine disruption. Our approach allows for creation of new signatures diagnostic of other pathways or phenomenon in the mouse liver that can be validated using the compendium. We are presently using the signature sets to predict pathway activation and mode of action after chemical exposure in mouse primary hepatocytes. (This abstract does not represent EPA policy.)

\title{
P299. Influences of testosterone and estrogen on the P2Y12 expression in human megakaryocytes: Testosterone regulates P2Y12 expression
}

\author{
$\underline{\text { Su-Jun Lee }}{ }^{1}$, Jung-A Kwon' ${ }^{1}$ Yazun Bashir Jarrar², In-Sun Jung ${ }^{3}$, Sang Seop Lee ${ }^{3}$ and Jae-Gook Shin ${ }^{3}$
}

\begin{abstract}
${ }^{1}$ Department of Pharmacology and Pharmacogenomics Research Center, Inje University College of Medicine, Busan, South Korea, ${ }^{2}$ Pharmacogenomics Research Center, Department of Pharmacology, Inje University, Busan, TN, South Korea, ${ }^{3}$ Pharmacogenomics Research Center, Inje University College of Medicine, BuSan, South Korea
\end{abstract}

P2Y12 is an important G protein coupled receptor involved in ADP-induced platelet aggregation which is essential to maintain normal hemostasis. Gender differences in the incidence of cardiovascular diseases have been proposed to be linked to the hormonal effects on the cardiovascular related genes. We aimed to determine the influences of testosterone and estrogen on the expression level of P2Y12 gene in the human megakaryocyte which is the cell line of highly specialized precursor for platelets. RNA was isolated from the megakaryocytes after treatment of estrogen and testosterone, respectively. Quantitative real time PCR revealed that P2Y12 mRNA levels were apparently induced by testosterone in dose dependent manner (4-fold, $\mathrm{P}<0.05$ ), whereas estrogen didn't alter the P2Y12 gene expression. The induction of P2Y12 protein by testosterone was also evidenced by Western blot analysis. The increased level of P2Y12 protein by testosterone was repressed by the androgen receptor inhibitor bicalutamide. Treatment of testosterone exhibited a decreased level of vasodilator-stimulated phosphoprotein compared to the control group, suggesting that this would be from the decreased intracellular cyclic AMP level caused by the increased P2Y12 activation. This decreased level of vasodilator-stimulated phosphoprotein was reverted by the bicalutamide. These findings suggest that a novel regulatory pathway by different sex homones on P2Y12 expression may, at least in part, mediate differences in platelet function and pro-thrombotic diseases in humans.

\section{P300. PXR mediated up-regulation of CYP3A expression by Praeruptorin A, C and D}

\author{
Zhong Guoping, Huang Ling, Bi Huichang and Huang Min
}


Objective: Praeruptorin A (PA), Praeruptorin C (PC) and Praeruptorin D (PD) are active compounds isolated from a popular Chinese herbal medicine, Peucedanum praeruptorum Dunn ( Qian Hu). In this study, the effect of PA, PC and PD on the PXR-mediated transactivation of CYP3A4 was investigated. Method: Mice hepatocytes were isolated according to an improving two-step collagenase perfusion technique. Mice primary hepatocytes were treated with PA, PC and PD at a concentration of 2.5, 10, $40 \mu \mathrm{M}$ for $48 \mathrm{~h}$, respectively. The effect of PA, PC and PD on the Cyp3a11 and mPXR mRNA expression in mice primary hepatocytes was measured using a real-time PCR. LS174T cells were transfected with PXR expression plasmids. Appropriate cell samples were exposed to PA, PC and PD at a concentration of 2.5, 10, $40 \mu \mathrm{M}$ for $48 \mathrm{~h}$ or $72 \mathrm{~h}$, The gene expression, protein expression and catalytic activity of CYP3A4 in the transfected LS174T cells were determined by using real-time PCR, Western blot analysis and LC-MS/MS based substrate assay. Results: The level of Cyp3a11 mRNA in mice primary hepatocytes was increased to 3.08 fold when exposed to PA at a concentration of $40 \mu \mathrm{M}$ for $48 \mathrm{~h}$; the level of Cyp3al1 mRNA was increased to 2.99 fold and 3.69 fold at 10 and $40 \mu \mathrm{M}$ of PC treatment; the level of Cyp3a11 mRNA was increased to 4.14 fold at 10 and $40 \mu \mathrm{M}$ of PD treatment. In the PXR-overexpressed LS174T cells, CYP3A4 mRNA expression was induced to 3.97-fold when treated with $40 \mu \mathrm{M}$ PA; CYP3A4 mRNA expression was induced to 3.6, 3.85-flod, respectively when treated with $10 \mu \mathrm{M}$ and $40 \mu \mathrm{M}$ PC; PD could significantly induce CYP3A4 mRNA expression to 4.1, 5.1- fold at 10, $40 \mu \mathrm{M}$ concentrations, respectively. In the PXR-overexpressed LS174T cells, the CYP3A4 protein expression was significantly induced 1.79 fold by PA at $10 \mu \mathrm{M}$; PC could significantly induce CYP3A4 protein expression to $2.19,2.22$ fold at $10,40 \mu \mathrm{M}$, respectively; PD also could significantly induce CYP3A4 protein expression to 2.26, 1.93 fold at 10, $40 \mu \mathrm{M}$, respectively. LS174T cells transfected with PXR-encoded plasmid yielded significant 1.74-fold increase in CYP3A4 catalytic activity after $72 \mathrm{~h}$ exposure to PA at a concentration of $10 \mu \mathrm{M}$ compared with control group; PC could significantly induce CYP3A4 catalytic activity to $1.81,2.1$ fold at 10 and $40 \mu \mathrm{M}$, respectively; after $72 \mathrm{~h}$ exposure to $\mathrm{PD}$ at a concentration of $40 \mu \mathrm{M}$, CYP3A4 catalytic activity was increased to 1.83 fold. Conclusion: These findings suggest that PA, PC and PD from Peucedanum praeruptorum Dunn can significantly up-regulate CYP3A4 gene via the PXR-mediated pathway and this has important implication in herb-drug interaction.

\title{
P301. Phospho-ERK1/2 interacts with the nuclear receptor CAR and represses de-phosphorylation of threonine 38 to inactive CAR in Huh-7 cells
}

\author{
Makoto Osabe and Masahiko Negishi
}

\section{Pharmacogenetics Section, LRDT, NIEHS, NIH, Research Triangle Park, NC}

The nuclear xenobiotic receptor CAR (NR1I3) is the key transcription factor that regulates drug metabolism and excretion in the liver (1). Inactive CAR is sequestered as in the cytoplasm of hepatocytes. Various xenobiotics, typified by the classic CAR activator phenobarbital, indirectly activate CAR and translocate it into the nucleus. While the complete molecular mechanism remains elusive, de-phosphorylation has been thought to regulate this indirect CAR activation. This is evidenced by the protein phosphatase $2 \mathrm{~A}$ inhibitor okadaic acid repressing activation of CAR in mouse primary hepatocytes (2). Recently we have characterized the conserved threonine residue (Thr38 and 48 in human and mouse CARs, respectively) within the DNA binding domain as the target of this de-phosphorylation responsible for CAR activation and nuclear translocation in mouse primary hepatocytes (3). Growth factors have been implicated in the repression of CAR via the activated extracellular signal-regulated kinase ERK1/2 (4). Here, we have determined that growth factors repress de-phosphorylation of threonine 38 and CAR activation. FLAG-tagged CAR T38A and CAR T38D mutants were expressed in Huh-7 cells and coprecipitated by an anti-phospho-ERK1/2 antibody. Phospho-ERK1/2 co-precipitated only the phosphorylation-mimicking CART38D mutant via the XRS peptide localized near the C-terminus of CAR molecules. Correlating with this interaction which occurred in growth factor-dependent manner; the results obtained from our newly developed ELISA revealed that threonine 38 is de-phosphorylated in Huh-7 cells after treatment with U0126. These results indicate that growth factors signal ERK1/2 to directly interact with the phosphorylated form of CAR and inhibit de-phosphorylation, thereby repressing CAR activation. This growth factor regulation was also observed in mouse primary hepatocytes.

\section{References}

1. Honkakoski et al., The nuclear orphan receptor CAR-retinoid X receptor heterodimer activates the phenobarbital-responsive enhancer module of the CYP2B gene. Mol. Cell. Biol.18, 5652-5658, 1998

2. Kawamoto et al., Phenobarbital-responsive nuclear translocation of the receptor CAR in induction of the CYP2B gene. Mol. Cell. Biol. 19,63186322, 1999.

3. Mutoh et al., Dephosphorylation of threonine 38 is required for nuclear translocation and activation of human xenobiotic receptor CAR (NR1I3). J. Biol. Chem. 284, 34785-34792, 2009.

4. Koike et al., Extracellular signal-regulated kinase is an endogenous signal retaining the nuclear constitutive active/androstane receptor (CAR) in the cytoplasm of mouse primary hepatocytes. Mol. Pharmacol. 71,1217-1221, 2007. 


\title{
P302. Phosphorylation of estrogen receptor in reproductive organs of female mice
}

\author{
Sawako Shindo, James Squires, Tsutomu Sakuma, Tomohiko Kanayama, Rick Moore and Masahiko \\ Negishi
}

Pharmacogenetics Section, LRDT, National Institute of Environmental Health Sciences, NIH, Research Triangle Park, NC

Recently our laboratory has characterized threonine 38 as the target of de-phosphorylation responsible for indirect activation of CAR by phenobarbital in mouse primary hepatocytes (1). Threonine 38 is located within the DNA binding domain of CAR and this residue is conserved in 40 out of 45 human nuclear receptors. In the DNA binding domain of Estrogen receptors $\mathrm{ER} \alpha$ and $\mathrm{ER} \beta$, this threonine residue is conserved as serine 212 and 216 in human and mouse $\mathrm{ER} \alpha$, respectively and serine 176 and serine 195 in human and mouse ER $\beta$, respectively. Western blot analysis with an antiphospho-serine 216 peptide antibody ( $\alpha$ P-S216) indicated that these conserved serine residues can be phosphorylated by protein kinase C. Utilizing the phosphorylation-mimicking human ER $\alpha$ S212D mutant, we first investigated the nature of the phosphorylated form of ER $\alpha$. YFP-tagged ER $\alpha$ S212D was localized in the nucleus of Huh7 cells as indicated by both Western blots of nuclear extracts and confocal analysis of intact cells and this mutant retained its trans-activation activity in a Luc-reporter assay. These features of the ER $\alpha$ S212D mutant contrast with those observed with the CART38D mutant which is sequestered in the cytoplasm and loses its trans-activation activity. In addition to the $\alpha \mathrm{P}-\mathrm{S} 216$ antibody, two ER $\alpha$ antibodies ( $\alpha \mathrm{ER} \alpha \mathrm{N}$ and $\alpha \mathrm{ER} \alpha \mathrm{C}$ which recognize the $\mathrm{N}$ - and C-terminal peptides, respectively) were employed to identify ER $\alpha$ and examine whether or not serine 216 of ER $\alpha$ is phosphorylated in mouse reproductive organs. Immunohistochemistry indicated that some, but not all cells that stained positive with ER $\alpha$ antibodies were also stained by the $\alpha \mathrm{P}-\mathrm{S} 216$ antibody. Western blot analysis further reutilized the presence of the phosphorylated ER $\alpha$ in mouse uterus.

\section{References}

Mutoh, et al., Dephosphorylation of threonine 38 is required for nuclear translocation and activation of human xenobiotic receptor CAR (NR1I3). J. Biol. Chem. 284:34785-34792, 2009

\section{P303. Regulation of drug metabolizing enzymes \& transporters by toll-like receptor-2 is mediated by CAR}

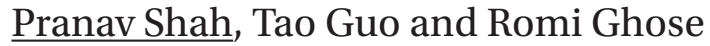

\section{Pharmaceutical and Pharmacological Sciences, University of Houston, Houston, TX}

Purpose and Hypothesis: During inflammation, the expression and activity of key phase I and phase II drug metabolizing enzymes (DMEs) and transporters are altered in the liver, leading to impaired drug metabolism and clearance. Inflammation in the liver is primarily mediated by the Toll-like receptors (TLRs). It is known that the gene expression of DMEs and transporters is regulated by basal transcription factors and the nuclear receptors, constitutive androstane receptor (CAR) and pregnane X receptor (PXR). We have shown that activation of TLR2 by lipotechoic acid (LTA), which is a gram positive bacterial component, leads to a rapid and profound suppression of CAR and its target DME and transporter genes. We hypothesize that down-regulation of hepatic DMEs and transporters is mediated by CAR. Methods: Adult, male (6-8 weeks old) $\mathrm{CAR}^{+/+}$and $\mathrm{CAR}^{-/-}$mice (C57BL/6) were treated with saline or LTA (6 mg $/ \mathrm{kg}$ ) for 4 or 8 hours. Liver tissues were harvested and RNA expression was analyzed by real-time PCR analysis. For in vitro studies, primary hepatocytes were isolated from $\mathrm{CAR}^{+/+}$and $\mathrm{CAR}^{-/-}$mice and treated with saline or LTA $(50 \mathrm{ng} / \mathrm{ml})$ for 8 hours. RNA was analyzed by real-time PCR analysis. Results: As expected, down-regulation of gene expression of the DMEs, Cytochrome P450 (Cyp) 2b10, Cyp2a4 and Amine-N-Sulfotransferase was significant ( 50-60\%) in LTA-treated CAR ${ }^{+/+}$mice. Similarly, the gene expression of the drug transporter, multidrug resistance-associated protein (MRP) 2 was significantly downregulated (30-40\%) by LTA treatment in $\mathrm{CAR}^{+/+}$mice. The down-regulation of these DMEs and transporters was attenuated in the $\mathrm{CAR}^{-/}$mice treated with LTA. LTA-mediated reduction in Cyp3al1 RNA and catalytic activity was attenuated in the $\mathrm{CAR}^{-/}$mice. The basal levels of Cyp2b10 were significantly reduced in the $\mathrm{CAR}^{-/-}$mice as compared to the $\mathrm{CAR}^{+/+}$ mice. A similar down-regulation ( 40-45\%) was observed in primary hepatocytes isolated from $\mathrm{CAR}^{+/+}$mice treated with LTA whereas this down-regulation was attenuated in hepatocytes from $\mathrm{CAR}^{-/}$mice. Conclusion: For the first time, we show that the nuclear receptor CAR is involved in mediating the down-regulation of gene expression of select DMEs and transporters during inflammation induced by gram positive bacterial components. 


\title{
P304. Rifaximin toxicity on hepatocellular fatty degeneration via activation of human pregnane $X$ receptor
}

\section{Łie Cheng}

\section{Laboratory of Metabolism, National Cancer Institute, National Institutes of Health, Bethesda, MD}

Rifaximin is a semi-antibiotics derived from rifamycin, which is minimally absorbed in the circulation and excreted into feces with pro-drug form. It has been approved by FDA on clinical therapy of traveler's diarrhea (2004) and hepatic hepatophelothapy (2010), in addition to being processed in the phase III clinical trial of remedy of irritable bowel disease. Previous report demonstrates rifaximin is gut-specific agonist of human pregnone X receptor (PXR), and short-term administration of rifaximin exerts the protective and therapeutic role on inflammatory bowel disease via cross-inhibition of NF-kB pathway through human PXR activation. PXR is a ligand-activated transcription factor important for its induction of drug transport and metabolism, and mediate endobiotic balance in hepatic steatosis, lipid homeostasis, bile acid homeostasis and steroid homeostasis. Although rifaximin is well known as well-tolerant drug without drug-induced hepatotoxicity addressed hitherto, the effect of long-term administration of rifaximin and subsequent function on PXR-involved body metabolism and physiology is still underdetermined. In the current study, human PXR inserted mice which lack mouse PXR was treated with control and rifaximin for 1 week, 1 month, 3 month till 6 month respectively by food intake, coupling with wild type and Pxr-null mice treated with rifaximin for 6 month as parallel cohorts. Histological analysis revealed time-dependent intense hepatocellular fatty degeneration in $\mathrm{hPXR}$ mice, while not in WT and Pxr-null mice. Hepatic triglyceride level was massively increased when rifaximin treated for 3 and 6 month in hPXR mice concomitant with slight increase of cholesterol and decreased free fatty acid level in liver. hPXR target genes including phase I, II, III enzyme was induced in small intestine and increased in liver, coupling with significant up-regulation of hepatic genes related to triglyceride synthesis and lipid accumulation. However, metabolomic analysis of serum and liver revealed no accumulation of rifaximin in liver even after 6 month treatment, though several nucleotide phosphates (AMP, ADP, ATP etc.) were up-regulated upon occurrence of fatty liver. In contrast, microarray and real-time PCR analysis of small intestine show the massive increase of genes involved fatty acid uptake and triglyceride, cholesterol transportation, including CD36, Dgat2 and ApoA4 etc. Lipid contents measurement of intestinal epithelial cell revealed the increase of triglyceride, fatty acid andcholesterol in hPXR mice while not in WT and Pxr-null mice. Thus, our findings suggested that long-term administration of rifaximin leads to hepatocellular fatty degeneration via hPXR-involved activation of lipid metabolism in small intestine and subsequent effect on lipid accumulation in liver, thus suggesting that human PXR is a key factor on long-term clinical therapy of rifaximin.

\section{P305. The stimulus of piperine on the PXR signaling pathway in drug metabolism}

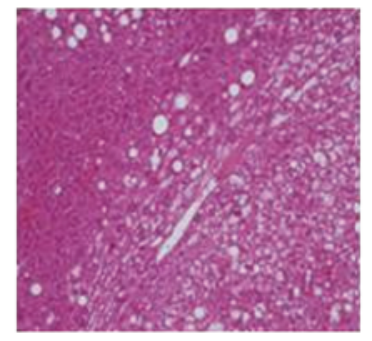

FPXR liver with 6-morth treated rifaximin
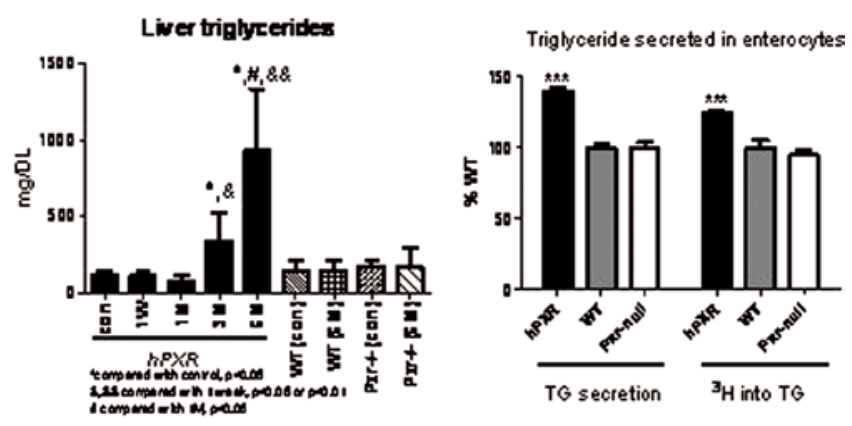

\section{Yue-Ming Wang, Wenwei Lin, Jing Wu, Su Sien Ong and Taosheng Chen*}

\author{
Chemical Biology \& Therapeutics, St. Jude Children's Research Hospital, Memphis, TN \\ * Corresponding author
}

It has been long recognized that dietary substances can cause significant diet-drug and/or drug-drug interactions. An increased awareness has been given to dietary components that could potentially induce changes of drug disposition via the alteration of drug transporters and metabolizing enzymes. Piperine (PIP), a major component extracted from black pepper, is widely used as daily spice and in some forms of traditional medicine. In the present study, we investigated 
whether and how piperine can affect the expression of two important genes, CYP3A4 and MDR1, which encode proteins responsible for drug metabolism and transportation. In reporter gene assays using LS74T and LS180 cells, PIP was able to activate PXR-mediated CYP3A4 promoter activity (with an $\mathrm{EC}_{50}$ of 3.6 and $3.9 \mu \mathrm{M}$, respectively) and PXR-mediated MDR1 promoter activity (with an $\mathrm{EC}_{50}$ of 1.6 and $3.0 \mu \mathrm{M}$, respectively) in a dose-dependent manner. The activity of PIP was blocked by $50 \mu \mathrm{M}$ of ketoconazole, an inhibitor of PXR activity, further confirming the dependency of PIP's activity on PXR. In addition, PIP treatment up-regulated endogenous MDR1 protein level in LS180 cell line and endogenous CYP3A4 protein levels in both LS180 and human primary hepatocytes in a dose dependent manner. However, PIP did not directly bind to PXR as revealed in a ligand binding assay and a coactivator recruitment assay. Further study demonstrated that PIP upregulated PXR protein level in LS174T and LS180 cells, providing the first evidence that the induction of CYP3A4 and MDR1 by PIP was through the increased PXR protein level. In view that PXR is a xenobiotc sensor with broad function on genes responsible for drug transportation and metabolism, our results suggest that cautions should be taken for PIP consumption during drug treatment in patients, particularly, who favor daily pepper spice or rely on certain pepper remedy.

\title{
P306. Metabolic chiral inversion of brivanib
}

ఏiachang Gong ${ }^{1}$, Jinping Gan ${ }^{1}$, Eric Masson², Shariq Syed ${ }^{2}$, Daphne Williams' ${ }^{2}$, Alban Allentoff 3 , Michael Lago ${ }^{3}$, Scott Tran ${ }^{3}$, Janice Pursley ${ }^{1}$, S. Nilgun Commezoglu ${ }^{1}$, W. Griff Humphreys ${ }^{1}$ and Ramaswamy A. Iyer ${ }^{1}$

${ }^{1}$ Pharmaceutical Candidate Optimization, Bristol-Myers Squibb Company, Princeton, NJ, ${ }^{2}$ Dmcp, Bristol-Myers Squibb Company, Princeton, NJ, ${ }^{3}$ Chemistry, Bristol-Myers Squibb Company, Princeton, NJ

Brivanib is a novel dual inhibitor for both VEGF and FGF signaling pathways that regulate angiogenesis and tumor cell proliferation. Brivanib alaninate, the oral alanine prodrug of brivanib, is currently in phase III clinical trials in hepatocellular carcinoma and colorectal cancer. Preclinical and clinical studies have demonstrated that brivanib was highly effective and well tolerated in humans. Brivanib has a single asymmetric center derived from a secondary alcohol with an R-configuration. The potential for chiral inversion was investigated in incubations with liver subcellular fractions, and in humans and animals following oral doses of brivanib alaninate. Incubation of [14C]brivanib alaninate with liver microsomes from rats, monkeys and humans in the presence of NADPH resulted in two radioactive peaks corresponding to brivanib and its enantiomer. The percent of the enantiomeric metabolite formed relative to brivanib ranged from $12-25 \%$ across species. Following oral doses of brivanib alaninate to humans, brivanib underwent minimal metabolic chiral inversion with the plasma concentration of the enantiomer less than $3 \%$ of brivanib concentration in humans. Following oral doses of brivanib alaninate to rats and monkeys, plasma concentration of the enantiomer ranged from 16-186\% of brivanib concnetration. The proposed mechanism involves oxidation of brivanib to a ketone metabolite, followed by reduction to yield brivanib or its enantiomer.

\section{P307. Stereochemistry of mitoglitazone carbonyl reduction in rat, monkey and man}

\author{
$\underline{\text { Wade J. Adams }^{1} \text {, Heather M. Dotzauer }}{ }^{2}$, Ted VanNoord ${ }^{2}$ and Jerry R. Colca ${ }^{1}$ \\ ${ }^{1}$ Metabolic Solutions Development Company, Kalamazoo, MI, ${ }^{2}$ Eurofins AvTech Laboratories, Inc., Portage, MI
}

The stereochemistry of the carbonyl reduction of mitoglitazone, a thiazolidinedione insulin sensitizer drug candidate, was investigated in rat, monkey and man following repeated-dose oral administration of mitoglitazone. A chiral HPLC-UV analytical method that resolved all four diastereomers of hydroxymitoglitazone in plasma was used in this investigation. Sodium heparin plasma samples from subchronic toxicity studies in male and female CD® $[\mathrm{Crl:CD}(\mathrm{SD})]$ rats and cynomolgus monkeys, and from a repeated-dose tolerance study in human volunteers were obtained after the first and last doses of mitoglitazone. Calibration standards and the analytical internal standard (structural analog) were prepared in diluent solution (methanol:deionized water, 1:1, v:v). Aliquots of plasma (500 $\mu \mathrm{L}$ ) were fortified with $50 \mu \mathrm{L}$ of the appropriate calibration standard or diluent (quality control and incurred samples) and $60 \mu \mathrm{L}$ of internal standard. The samples were buffered with $0.5 \mathrm{~mL} 0.1 \mathrm{M}$ potassium phosphate, vortex mixed, and transferred to SPE cartridges that had been activated by successive addition of $1 \mathrm{~mL}$ acetonitrile and $0.1 \mathrm{M}$ potassium phosphate. After gravity elution, the cartridges were successively washed with $2 \mathrm{~mL}$ methanol:potassium phosphate (30:70, v:v) and $1 \mathrm{~mL} 0.1 \mathrm{M}$ potassium hydrogen phosphate before drying. Analytes were eluted by successive addition of $500 \mu \mathrm{L}$ of acetonitrile:deionized water (40:60, v:v) and $500 \mu \mathrm{L}$ acetonitrile:deionized water (50:50, v:v). The eluate was evaporated under nitrogen at 
$40^{\circ} \mathrm{C}$ to dryness and reconstituted in $200 \mu \mathrm{L}$ ethanol:methanol (50:50, v:v) with sonication and vortex mixing. Aliquots $(125 \mu \mathrm{L})$ of reconstituted samples were chromatographed on a CHIRALPAK® IA column (Chiral Technologies, Inc., West Chester, PA) at a flow rate of $1 \mathrm{~mL} / \mathrm{min}$ and gradient elution chromatography using mobile phases containing methanol:ethanol:diethylamine (50:50:0.2) and methanol:ethanol:methane sulfonic acid (50:50:0.2). Analytes were detected by UV at $265 \mathrm{~nm}$ and quantitation was accomplished using peak height ratios (analyte/internal standard). The analytical range of the method was $50-1000 \mathrm{ng} / \mathrm{mL}$. Comparison of hydroxymitoglitazone concentrations determined by a non-chiral LC-MS/MS method and the chiral HPLV-UV method demonstrated good agreement in total hydroxymitoglitazone concentrations for the two analytical methods; mean total hydroxymitoglitazone concentrations determined by the chiral method in rat, monkey and man were $100 \%, 106 \%$ and $108 \%$, respectively, of the LC-MS/MS method. The R-diastereomers were the major hydroxymitoglitazone species observed in all three species, with mean percentages of R-hydroxymitoglitazone in rat, monkey and man being $97 \%, 61 \%$ and $90 \%$, respectively. Except for the rat, the levels of the R,R- and R,S-diastereomers were equivalent and the levels of the S,S- and S,R-diastereomers were equivalent. In the rat, the ratio of the R,R- and R,S-diastereomers differed by approximately 2-fold.

\title{
P308. A time-course study on the effects of interleukin- 6 on gene expression of P450 isoforms, uptake transporters, and efflux transporters in human hepatocytes
}

\author{
Qian Yang, Utkarsh Doshi, Nicole Li and Albert Li
}

In Vitro ADMET Laboratories LLC, Columbia, MD

Pro-inflammatory cytokines such as interleukin-6 (IL-6) are known to alter expression of drug metabolism enzymes and transporter in rodent liver. However, their effects on human hepatocytes remain poorly characterized. The current study was to conduct a comprehensive analysis of the time course of the effects of IL-6 on the expression of human major P450 enzyme and transporters in human hepatocytes. Cryopreserved human hepatocytes were plated into a 24 well plate at a density of 350,000 cells/well. Following attachment plating medium was removed and the cells were overlaid with $0.25 \mathrm{mg} / \mathrm{ml}$ Matrigel $^{\mathrm{m}}$ in plating medium and incubated for an addition $20 \mathrm{hrs}$. The cells were then treated with 0 , $0.1,0.5$ and $5 \mathrm{ng} / \mathrm{mL}$ of IL-6 in serum-free William's E medium supplemented with ITS and dexamethasone for the time periods of 2, 6, 12, 24 and 48 hrs. Total RNA was isolated individually from each well and cDNA was synthesized from each sample. Real-timer PCR was performed using Taqman primers for P450 isoforms (CYPs 1A2, 2B6, 2C8, 2C9, 2D6, 3A4, 3A5), uptake transporters (SLC10A1, SLC22A1m SLC22A7, SLCO1B1, SLCO1B3, SLCO2B1) and efflux transporters (ABCB1, ABCB11, ABCC2, ABCC3, ABCC4, ABCG2) using the ABI 7500 Real-Time System. Each PCR cycle threshold (Ct) was normalized to the average $\mathrm{Ct}$ of the endogenous housekeeping control gene GAPDH. The comparative ? Ct method was used to calculate relative quantification of gene expression. Results showed that in the control (untreated) human hepatocytes, $\mathrm{P} 450$ isoforms and uptake transporters in the untreated cultures decreased rapidly with time and stabilized between 2 and $12 \mathrm{hrs}$ and remained relative stable from 12 to $48 \mathrm{hrs}$ at approximately $20 \%$ of that for the $2 \mathrm{hr}$ time point. In contrast, efflux transporters remained relatively stable or increased during the time period of 2 to 48 hrs. IL- 6 caused down regulations of most of the genes studied. The most significant changes were observed at either 12, 24 or 48 hours, depending on each specific gene. The results showed that a time-course study is a useful approach to evaluate the effects of cytokines such as IL-6 on ADME gene expression.

\section{P309. A novel in vitro MRP2 assay to predict in vivo biliary excretion}

\author{
Federico Colombo, Catherine Armstrong, Jianmin Duan and Nathalie Rioux
}

\section{Biological Sciences, Boehringer Ingelheim Canada Ltd, Laval, QC, Canada}

Purpose: Prediction of biliary excretion in vivo has been one of the challenges for drug discovery scientists due to the lack of an established predictive in vitro assay. The present study explores the possibility of establishing a simple high throughput screen assay to predict in vivo biliary excretion. Method: Canalicular plasma membrane vesicle (cLPM) were prepared from Sprague-Dawley rat livers and the kinetics of ATP dependent uptake of 5(6)-carboxy-2',7'dichlorofluorescein (CDCF) were determined in a 96-well format. Uptake of CDCF $(2 \mu \mathrm{M}) \mathrm{in} \mathrm{cLPM}(100 \mu \mathrm{g} / \mathrm{mL}) \mathrm{was}$ carried out at $37^{\circ} \mathrm{C}$ for 10 minutes in a buffer containing $250 \mathrm{mM}$ sucrose, $10 \mathrm{mM}$ Tris-Hepes, $10 \mathrm{mM}$ creatine phosphate, $100 \mu \mathrm{g} / \mathrm{mL}$ creatine kinase and $10 \mathrm{mM} \mathrm{MgCl}_{2}$ with or without the inclusion of $3 \mathrm{mM}$ ATP (Controls). Vesicle-associated CDCF was separated from the matrix by rapid filtration. The samples were then lysed, filtered and submitted to fluorescent reading. Assay specificity was verified by competition studies with established mrp2, P-gp, and BSEP modulators 
(substrates and inhibitors). The relationship of in vitro $\mathrm{IC}_{50}$ values against ATP-dependent mrp2 uptake and in vivo biliary excretion (expressed as \% of the total dose) of nine literature compounds were examined. Results: The uptake of CDCF in cLPM vesicles was time- and concentration-dependent, with a $\mathrm{K}_{\mathrm{m}}$ value of $2.2 \pm 0.3 \mu \mathrm{M}$ and a $\mathrm{V}_{\max }$ of $115 \pm 26 \mathrm{pmol} / \mathrm{mg} /$ $\min (\mathrm{n}=3)$. CDCF transport was strongly inhibited by the well-documented mrp2 inhibitors, benzbromarone, MK-571 and cyclosporine $\mathrm{A}$, with $\mathrm{IC}_{50}$ values $\leq 1.2 \mu \mathrm{M}$. In contrast, taurocholate (inhibitor of BSEP), and digoxin (inhibitor of P-gp) had very low potency against the mrp2 mediated uptake ( $\mathrm{IC}_{50}$ values of 57 and $101 \mu \mathrm{M}$, respectively). A highly significant linear correlation $(\mathrm{R}=0.939, \mathrm{P}<0.001)$ between the in vitro $\mathrm{IC}_{50}$ values from the described mrp2 assay and in vivo biliary excretion in rats was observed for the 9 tested mrp2 inhibitors/substrates. Potent inhibitors $\left(\mathrm{IC}_{50}\right.$ values $\left.<5 \mu \mathrm{M}\right)$ of CDCF uptake correlated with $>90 \%$ of the dose excreted into the rat bile in vivo, whereas weak inhibitors $\left(\mathrm{IC}_{50}\right.$ values $>100 \mu \mathrm{M})$ had $<5 \%$ of the dose excreted into the bile. Conclusion: The present study describes a novel high throughput assay that selectively identifies potential inhibitors of mrp2 using an in vitro system prepared from normal rat livers. In addition, the highly significant correlation between in vitro mrp2 IC $_{50}$ values from this described assay and in vivo biliary excretion suggest its potential use in drug discovery to minimize mrp2-mediated excretion and mrp2-associated drug-drug interactions.

\title{
P310. Application of receiver operating characteristics to assess digoxin drug interaction potential
}

Caroline A. Lee ${ }^{1}$, Joseph Bentz ${ }^{2}$, Michael O'Connor ${ }^{2}$, Johan Palm ${ }^{3}$, Harma Ellens ${ }^{4}$, Krisztina HerediSzabo $^{5}$, Dallas Bednarczyk ${ }^{6}$, Mitchell Taub ${ }^{7}$, Elke S. Perloff ${ }^{8}$, Christoph Funk ${ }^{9}$, Praveen Balimane ${ }^{10}$, Laurent Salphati ${ }^{11}$, Ailan $\mathrm{Guo}^{12}$, Lalitha Podila ${ }^{13}$, Imad Hanna ${ }^{14}$, Cindy Xia ${ }^{15}$, Libin $\mathrm{Li}^{16}$, Guangqing Xiao $^{17}$, Heleen M. Wortelboer ${ }^{18}$, Dietmar Weitz ${ }^{19}$, Youngeen A. Pak ${ }^{20}$, Eric Reyner ${ }^{21}$, Jesse Taur ${ }^{22}$, Xiaoyan $\mathrm{Chu}^{23}$, Ulrike Gradhand ${ }^{24}$, Mark Warren ${ }^{25}$, Ganesh Rajaraman ${ }^{26}$ and Lei Zhang ${ }^{27}$

\begin{abstract}
${ }^{1}$ Pharmacokinetics, Dynamics \& Metabolism, Pfizer Inc., San Diego, CA, ${ }^{2}$ Drexel University, Philadelphia, PA, ${ }^{3}$ AstraZeneca, Mölndal, Sweden, ${ }^{4}$ GlaxoSmithKline, King of Prussia, PA, ${ }^{5}$ SOLVO Biotechnology, Szeged, Hungary, ${ }^{6}$ Novartis, Cambridge, MA, ${ }^{7}$ Boehringer-Ingelheim Pharmaceuticals, Inc., Ridgefield, CT, ${ }^{8} B D$ Biosciences, Woburn, MA, ${ }^{9}$ Dept of Non-Clin Drug Safety, Hoffmann-La Roche Ltd, Basel, Switzerland, ${ }^{10}$ Bristol-Myers Squibb, ${ }^{11}$ Genentech Inc., South San Francisco, CA, ${ }^{12}$ Non-Clin Drug Safety, Hoffmann-La Roche Inc., Nutley, NJ, ${ }^{13}$ Drug Metabolism \& Pharmacokinetics, Boehringer Ingelheim Pharmaceuticals, Inc., Ridgefield, CT, ${ }^{14}$ Drug Metabolism and Pharmacokinetics, Novartis Institutes for BioMedical Research Inc., East Hanover, NJ, ${ }^{15}$ Drug Metabolism and Pharmacokinetics, Millennium Pharmaceuticals, Inc., Cambridge, MA, ${ }^{16}$ Absorption Systems LP, Exton, PA, ${ }^{17}$ Biogen Inc, Cambridge, MA, ${ }^{18}$ Pharmacokinetics, TNO Quality of Life, Zeist, Netherlands, ${ }^{19}$ Sanofi-Aventis, ${ }^{20}$ Drug Disposition, Eli Lilly and Company, Indianapolis, IN, ${ }^{21}$ Pfizer Global Research \& Development, San Diego, CA, ${ }^{22}$ Eisai, ${ }^{23}$ Drug Metabolism and Pharmacokinetics, Merck \& Co Inc-Merck Rsch Lab, Rahway, NJ, ${ }^{24}$ Merck KGaA, ${ }^{25}$ Optivia, ${ }^{26}$ Life Technologies - CellzDirect, Austin, TX, ${ }^{27}$ Office of Clinical Pharmacology, U.S. Food and Drug Administration, Silver Spring, $M D$
\end{abstract}

Clinically significant drug-drug interactions (DDIs) involving P-glycoprotein (P-gp, MDR1) are typically not as concerning as P450-mediated DDIs. Regulatory agency guidance criteria for in vitro cut-off values establish whether or not a new chemical entity inhibits P-gp in vitro and may subsequently alter the pharmacokinetics of digoxin in vivo. The current FDA draft publication and guidelines provided by the International Transporter Consortium (ITC) whitepaper recommend that a clinical DDI study be considered for a new chemical entity when the maximum concentration at steady state $\left(\mathrm{I}_{1}\right)$ divided by its in vitro P-gp inhibitory potency $\left(\mathrm{IC}_{50}\right)$ is $\geq 0.1$ or when the nominal gut concentration $\left(\mathrm{I}_{2}\right)$ divided by its $\mathrm{IC}_{50}$ is $\geq 10$. Because the in vitro $\mathrm{IC}_{50}$ value is fundamental to the calculation, an appropriate determination of the $\mathrm{IC}_{50}$ is critical to avoid false negative or false positive predictions. The objective was to determine in vitro P-gp IC values for sixteen compounds and to assess the extent of variability. Twenty-two pharmaceutical and contract research laboratories collaborated to generate $\mathrm{IC}_{50}$ data using four in vitro cell systems (Caco-2, MDR1-MDCKII, MDR1- LLCPK1 and MDR1-expressing inside-out membrane vesicles); six equations in the form of efflux ratio, unidirectional flux, or net secretory flux; and multiple commercial nonlinear regression software packages. P-gp inhibition data for each of the compounds were fitted to several logistic and nonlinear regression analyses. Inhibition variability was assessed separately for each in vitro system and equations, and together. In general, MDR1-expressing vesicles exhibited IC ${ }_{50}$ variability ranging from 2- to 38-fold; variability in the cell-based systems was larger, ranging from 2- to 120 -fold. Much of the variability in the cells was due to intrinsic variability between the cells, even within the same cell line. Receiver Operating Characteristic (ROC) analysis was applied to determine the appropriate in vitro cutoff values for $\mathrm{I}_{1} / \mathrm{IC}_{50}$ and $\mathrm{I}_{2} / \mathrm{IC}_{50}$ based on the ROC area under the curve (AUC') to minimize false negative predictions under two conditions 1) all data collated 
together to determine "universal" cutoff values and 2) individual ROC analysis for each laboratory to determine in vitro cutoff values to define digoxin DDI potential. Recommendations will be discussed regarding which cell lines, equations, and calculation methodologies are more reliable for predicting digoxin-related DDIs.

\title{
P311. Automated 96-well assay for the assessment of passive permeability and P-gp efflux during CNS drug discovery
}

\author{
Colin Lorenzten ${ }^{1}$, Shawn Gauby ${ }^{1}$, David Nakamura ${ }^{1}$, Heather Zhang ${ }^{1}$, Erich Goldbach ${ }^{1}$, Shaila \\ Hoque $^{1}$, Bhushan Samant ${ }^{1}$, Pamela Santiago ${ }^{2}$, Patrick Rudewicz ${ }^{1}$, John-Michael Sauer ${ }^{1}$, George \\ Tonn $^{1}$ and Kevin Quinn ${ }^{1}$
}

\author{
${ }^{1}$ Drug Metabolism and Pharmacokinetics, Elan Pharmaceuticals, South San Francisco, CA, ${ }^{2}$ Safety Evaluation, Elan \\ Pharmaceuticals, South San Francisco, CA
}

The role of the ADME scientist in the drug discovery setting is to partner with discovery teams to influence the StructureProperty-Relationship towards compounds with tractable drug-like properties along with potency and selectivity. For CNS drug targets, in addition to suitable solubility, metabolic stability and permeability, avoiding P-gp efflux liabilities are critical. Cell based assays for determining permeability and P-gp efflux using traditional laboratory techniques is time consuming and laborious. With that in mind, we have developed an automated screen to assess passive permeability and P-gp efflux in a 96-transwell format on a Microlab Star (Hamilton) liquid-handler coupled to a Cytomat cell culture incubator (Thermo). We describe our assay workflow, which utilizes the liquid-handler to prepare dosing solutions and manipulate confluent monolayer cultures of MDR1 transfected MDCK-II cells (NKI) from pretreatment through to sample collection and processing. Briefly, the passive permeability of each test-article $(5 \mu \mathrm{M})$ is assessed in the forward (apical-to-basolateral) direction following pre-treatment of cells with a P-gp inhibitor (Tariquidar). P-gp efflux is assessed by the reverse (basolateral-to-apical) over forward (apical-to-basolateral) ratio in the absence of inhibitor. Controls are included to monitor tight-junctions (Atenolol), test-article recovery and assess boundary performance for permeability and P-gp efflux. Test and control article concentrations are determined with a rapid LC-MS/MS assay following automated tuning (e.g. DQ2 software; Applied Biosystems). Compounds are categorized to aid in the selection of lead series and the attrition during lead optimization. Assay performance was verified using compounds of known permeability and P-gp liability. In addition, in vitro data were compared to in vivo P-gp efflux determined from partition $\left(\mathrm{K}_{\mathrm{p}}\right)$ ratios in the Mdrl a/b (-/-) deficient mouse and wild type controls, with the assumption that efflux is approximately similar between human and mouse. The assay was evaluated to test for the occurrence of false negatives and false positives to better understand the limitations of the assay. With the proper use of controls to monitor for monolayer integrity and recovery, false positives were negligible. False negatives were observed for highly permeable substrates (i.e. verapamil and quinidine) where efflux may be saturated at $5 \mu \mathrm{M}$. In some cases this could be circumvented by testing these compounds at lower substrate concentrations. Overall, a strong concordance was shown between our data, literature data and in vivo results. This automated assay has enabled us to routinely and efficiently deliver high quality data to our discovery teams. The rational use of these data by our teams has resulted in the advancement of various compound series, minimizing P-gp efflux liabilities and improving CNS penetration.

\section{P312. Biliary excretion and pharmacokinetics of ABCG2 substrates in Abcg2 knockout rats}

\author{
Liyue Huang ${ }^{1}$, Xuhai Be ${ }^{1}$, Adria Colletti ${ }^{1}$, John Roberts ${ }^{1}$, Meghan Langley ${ }^{1}$, Min-Hwa J. Lin ${ }^{1}$, Zhiyang \\ Zhao $^{1}$, Bradley K. Wong ${ }^{2}$ and Lixia Jin ${ }^{2}$
}
${ }^{1}$ Pharmacokinetics \& Drug Metabolism, Amgen Inc., Cambridge, MA, ${ }^{2}$ Pharmacokinetics \& Drug Metabolism, Amgen Inc., So San Francisco, CA

ATP-binding cassette G2 protein (ABCG2, also known as breast cancer resistance protein) is expressed in various tissues including intestine, liver and kidney. Abcg2 has been shown to play a predominant role in the biliary excretion and pharmacokinetics of nitrofurantoin in mice (Merino et al, Mol Pharmacol 67:1758, 2005). In the present study, we evaluated the contribution of Abcg2 to the biliary excretion and pharmacokinetics of three Abcg2 substrates (nitrofurantoin and proprietary compounds A and B) in Abcg2 knockout (KO) rats. Methods: Biliary and urinary excretion of the three test compounds following IV administration was determined in bile duct catheterized Abcg2 KO and wild-type (WT) SpragueDawley rats (Sigma Sage ${ }^{m}$ lab). In vitro bi-directional transport was performed in MDR1-LLC-PK1 and ABCG2-MDCK 
cells at $5 \mu \mathrm{M}$ in the presence of $0.1 \%$ BSA. Results: Nitrofurantoin had efflux ratios of 1.7 and $\sim 10$ in MDR1-LLC-PK1 and ABCG2-MDCK cells, respectively. Compounds A and B were substrates for both MDR1 and ABCG2 (efflux ratio >10). Biliary excretion of nitrofurantoin accounted for $1.6 \%$ of the dose in the WT rats and was reduced 10-fold in the Abcg2 KO animals. In contrast, urinary excretion accounted for $18.2 \%$ of the dose in the WT rats and was similar in the Abcg2 KO animals. In the WT rats, approximately $40 \%$ and $50 \%$ of the dose was excreted into bile as parent for compounds A and $\mathrm{B}$, respectively. Consistent with the corresponding in vitro efflux status, biliary excretion was decreased 3 to 5 -fold in the Abcg2 $\mathrm{KO}$ rats. In the intact animals, clearance of nitrofurantoin, compound A and compound B was $26 \%, 32 \%$ and $58 \%$ lower in the Abcg2 KO rats as compared to that in the WT animals, respectively. Oral absorption of nitrofurantoin (10 mg/ kg) was high and comparable in both WT and Abcg2 KO rats. Conclusion: Bcrp played an important role in the biliary, but not urinary, excretion of Abcg2 substrates nitrofurantoin compound A and compound B in rats. However, the impact of Bcrp on clearance was minimal to modest for these compounds. Although further studies are needed, initial results indicate that Abcg2 KO rats may be a useful model for understanding the role of Abcg2 in drug disposition.

\title{
P313. Characterization of ABC transporters and SLC transporters in sandwich cultured human cryopreserved hepatocytes
}

\author{
$\underline{\mathrm{Na} \mathrm{Li}}^{1}$, Rongjun Zuo ${ }^{1}$, Kirsten Cooper ${ }^{1}$, Peter Petrov ${ }^{1}$, Joanne Bourgea ${ }^{1}$, Charles Crespi ${ }^{2}$ and \\ Christopher J. Patten ${ }^{1}$
}

${ }^{1} B D$ Biosciences, Bedford, MA, ${ }^{2}$ Dept of Discovery Labware, BD Biosciences, Woburn, WA

Cryopreserved hepatocytes have been widely employed to characterize drug ADME features in pharmaceutical industry. The aim of this study is to characterize the uptake and efflux transporters in human cryopreserved hepatocytes. More than 30 lots of human cryopreserved hepatocytes were tested for the ability to form bile canaliculi network upon culturing in the sandwich configuration. Briefly, cryopreserved hepatocytes were thawed and seeded on a 24-well BD BioCoat" Collagen I plate at density of 400,000 cells/well. After overnight incubation, the cells were overlaid with $0.5 \mathrm{~mL}$ of ice cold $0.25 \mathrm{mg} /$ mL BD Matrigel" prepared in Williams' E media. The culture media was replaced every 24 hrs. On day 5 post culture, cells were rinsed with regular HBSS, and for efflux assays, $5 \mu \mathrm{M}$ CDFDA or $5 \mu \mathrm{M}$ CLF in regular HBSS with or without specific transporter inhibitors was added into cell culture. Bile canaliculi accumulation of fluorescence compounds was assessed by fluorescence microscope. For uptake assay, $1 \mu \mathrm{M}$ of taurocholic acid, $2 \mu \mathrm{M}$ rosuvastatin prepared in regular HBSS buffer was added into cell culture. The accumulation of these probe substrates inside of hepatocytes were evaluated at $2 \mathrm{~min}$, $10 \mathrm{~min}$ and 15min post treatment. In sandwich cultured configuration, platable cryopreserved hepatocytes can form bile canaliculi network which was evidenced by bile accumulation of two fluorescence substrates, CDF and CLF, which was sufficiently inhibited by MRP2 and BSEP inhibitor respectively. The results demonstrate that plated cryopreserved hepatocytes maintain the functionality of major $\mathrm{ABC}$ transporters in sandwich culture. In addition, the dramatic uptake activity of selected substrates demonstrates the functionality of major hepatic uptake transporters, e.g. NTCP and OATP. Functional hepatic uptake and efflux transporters are detected in cryopreserved human hepatocytes in sandwich culture, which provide a useful tool to characterize the drug-drug interaction or drug induced hepatic toxicity during drug discovery.

\section{P314. Characterization of BCRP knock-out rat model using gene expression analysis}

\section{Eskouhie H. Tchaparian, Adria Colletti, John Roberts and Lixia Jin}

\section{Pkdm, Amgen Inc., South San Francisco, CA}

Purpose: Breast cancer resistance protein, BCRP (ABCG2) is an important efflux transporter that plays a role in the absorption, distribution, and excretion of some drugs. Animal gene knock out technology is a valuable research tool for understanding gene function. To understand its potential use as an animal model for drug discovery support, the aim of the present study was to characterize the newly developed Bcrp Knockout (KO) rat model (SAGE Labs, St Louis, $\mathrm{MO}$ ). Gene expression analysis was used to assess the effect of Bcrp gene deletion on drug metabolizing enzymes and transporters. Methods: RNA was extracted from liver, kidney, small intestine (jejunum, ileum, and duodenum), colon, and brain capillary enriched samples. Relative gene expression changes of drug metabolizing enzymes and transporters in the KO Bcrp rat relative to their wild type (WT) were evaluated by real-time RT-qPCR.Results: As expected, the results demonstrated the deletion of the target gene (Bcrp). Additionally, low expression levels of hepatic Mdr1 (2.7 fold mdrlb and 5 fold mdrla), Mrp1 (3 fold) and Mrp5 (more than 10 fold) were detected. No significant change in the kidney gene expression was observed, while about three fold increase in Slc10a2 (Ileum and colon segments) and Mrp6 (duodenum 
and jejunum segments) was seen. The results also indicated significant decrease in the Cyp3a18 mRNA levels ( 10 fold) in the ileum. Conclusion: Overall the result of this study indicates that in addition to Bcrp deletion, the expression level of some transporters is changed, specifically in liver. Further investigation is ongoing to determine whether changes observed at mRNA levels impacts protein expression and /or function of select transporters.

\title{
P315. Comparison of digoxin efflux inhibition in Caco-2 and MDR1-LLC-PK1 cell monolayers
}

\author{
Elke S. Perloff, Andrew K Mason, Sudarshan Kapadnis, Wendy Khun, Lisa G Fox and David M. \\ Stresser
}

BD Gentest ${ }^{S M}$ Contract Research Services, Woburn, MA

\begin{abstract}
Objective: Assessment of P-gp inhibition in vitro has become a routine aspect of drug development, however, literature data as well as the recent results of the P-gp IC I0 $_{50}$ Working Group Industry Initiative indicate large variability in IC $_{50}$ values for the same inhibitor, presumably due to differences in test systems, experimental methodology, and data processing approaches between labs. In this study, we compared the inhibition of digoxin efflux by 16 inhibitors in two different models, Caco-2 and MDR1-LLC-PK 1 cell monolayers, using identical experimental methods in the same lab. Methods: Bidirectional permeability of digoxin as a probe substrate for P-gp was determined in 21- to 25-day Caco-2 and 7-day MDR1-LLC-PK 1 cell monolayers. Cells were cultured under cell line specific conditions, however, all experimental work was performed using identical methodologies for both cell lines (HBSS buffer pH 7.4 with 10 mM HEPES, 90 min incubation, $5 \mu \mathrm{M}$ digoxin). Bidirectional transport of digoxin was assessed in the presence or absence of six concentrations of 16 reported P-gp inhibitors. $\mathrm{IC}_{50}$ values were determined based on the decrease in digoxin efflux ratio and decrease in net secretory flux using non-linear regression to a 2-parameter Hill equation in XLfit v5.2. Results: $\mathrm{IC}_{50}$ values for inhibition of digoxin efflux ratio obtained for the 16 inhibitors ranged from $0.34 \mu \mathrm{M}$ (elacridar in Caco-2) to $36 \mu \mathrm{M}$ (troglitazone in MDR1-LLC-PK ) and varied up to 3.9-fold between Caco-2 and MDR1-LLC-PK 1 cells. Nine out of the 16 inhibitors showed less than 2-fold variation between models. $\mathrm{IC}_{50}$ values for inhibition of net secretory flux showed a similar degree of variability (up to 4.6-fold), but were on average 2.5 -fold higher than corresponding $\mathrm{IC}_{50}$ values based on efflux ratios. Baseline activity for positive (25 $\mu \mathrm{M}$ ketoconazole) and negative (no inhibitor) control digoxin transport was reproducible with inter-day variability in $\mathrm{P}_{\text {app }}$

values and efflux ratios ranging from $8 \%$ to $30 \%$ CV. Conclusions: Variability in IC $_{50}$ values determined using different cell models but identical experimental methodologies in the same lab did not exceed 5-fold for 16 inhibitors tested. These findings suggest that while intrinsic differences between test systems such as different P-gp expressing cell lines do contribute to the variability in $\mathrm{IC}_{50}$ values observed in the literature and in the results from the P-gp $\mathrm{IC}_{50}$ Working Group Industry Initiative, other factors, including differing experimental methodology and technique, as well as alternative data processing approaches likely play a large role in inter-laboratory variability in P-gp inhibition data.
\end{abstract}

\section{P316. Creation and characterization of ATP-binding-cassette transporter knockout rat models}

\author{
$\underline{\text { Iara D. Carbery }}{ }^{1}$ Lara Little ${ }^{2}$, Edward Weinstein ${ }^{2}$ and Xiaoxia Cui ${ }^{2}$ \\ ${ }^{1}$ SAGE Labs, Sigma-Aldrich, St Louis, MO, ${ }^{2}$ Sigma-Aldrich, St Louis, MO
}

Animal models are an essential element of drug development and toxicity assessment. Rats are preferred over mice for their closer resemblance to human anatomy and physiology and their larger size. Here we report the creation and characterization of ATP-binding-cassette (ABC) transporter knockout rats, including Mdrla, Mrp1, Mrp2, and Bcrp using ZFN technology. Homozygous knockout animals were confirmed by the lack of respective proteins in Western Blots. Toxicology analysis showed the accumulation of ABC transporter substrates in knockout animals. In addition, we created and characterized humanized Mdrla rats, where the human Mdrl cDNA was site-specifically inserted under the rat Mdrla promoter and disrupted the rat gene. Species-specific substrates and inhibitors for P-gp can be tested in this model.

Keywords: ATP-binding cassette transporters, Mdrla, zinc finger nuclease

\section{P317. Creation and assessment of adenovirus-mediated drug transporter model expression system for the prediction of pharmacokinetic profiles in humans}

Daisuke Sugiyama, Wendy A. Teft, Mike J. Knauer, Marianne K. DeGorter, Rommel G. Tirona and Richard B. Kim 
Introduction. Predicting the in vivo role and effects of functional genetic polymorphisms to observed PK profile of drugs in humans is important for optimal drug therapy. Currently, IVIVE (in vitro to in vivo extraporation) algorithms are widely utilized for the prediction of drugs which are metabolized by CYP enzymes, but not transporters. However, for many drugs, the rate-limiting step in their elimination is transporters, particularly those that mediate uptake into tissues or target organs. In addition, the extent of drug transporter expression in human tissues or organs, particularly in a quantitative fashion, has not been clearly delineated. Therefore, our goal is to create an in vitro transporter expression system capable of expressing multiple drug transporters simultaneously so that the cell-based system better reflects human organs such as liver. Towards this goal, we have cloned a number of hepatic bile acid and drug transporters into an adenovirus-based expression construct and assess their expression as well as transport activity in a number of cell lines. Methods.Adenovirus constructs containing drug transporters such as NTCP (Na/taurocholate cotransporting polypeptide), members of the human OATPs (organic anion transporting polypeptides) were constructed, and their transport activities as well as cell viabilities were assessed. Results.After incubation with adenoviruses containing NTCP, OATP1B1, 1B3 and 2B1, their substrate transport activities were determined. For OATP1B1 and 1B3 in HeLa cells transport activity at $48 \mathrm{hr}$ incubation with adenovirus was higher than at $72 \mathrm{hr}$, and cell viability after $48 \mathrm{hr}$ was also better that at $72 \mathrm{hr}$ for the same Multiplicity of Infection (MOI). Although transport activity for NTCP and OATP2B1 could be readily detected in HeLa, LLC-PK1, MDCKII and Caco-2 cells, overall activity was greatest in HeLa cells and lowest in Caco-2 cells. In terms of cell viability, Hela, LLC-PK1 and Caco-2 were able to tolerate an MOI of 1000, but for MDCKII cells an MOI of 300. Interestingly, MDCKII cells exhibited higher transport activity compared to LLC-PK1 cells when expressing NTCP, but LLC-PK1 was better when expressing OATP2B1.Conclusions.Our findings show that although a number of cell lines can be transduced to express hepatic transporters using the adenoviral expression system, the effects of adenovirus-mediated transporter expression differed significantly among the tested cell lines. Thus an optimal condition has to be identified based on the cell line as well as the transporter of interest. In our hands, it would appear that HeLa cells may be better for optimal expression of uptake transporters and the LLC-PK1 cells better for expressing efflux transporters. Overall, our newly created in vitro system appears to have the desired properties to serve as a physiologically relevant model for predicting in vivo PK.

\title{
P318. Determination of OATP mediated drug-drug interaction potential in pooled cryopreserved human hepatocytes
}

\author{
Leena Laitinen, Pamela McCarthy, Brian Boucher and Niresh Hariparsad
}

\author{
Dipk, Vertex Pharmaceuticals, Inc., Cambridge, MA
}

Determination of OATP Mediated Drug-Drug Interaction Potential in Pooled Cryopreserved Human Hepatocytes Leena Laitinen, Pamela McCarthy, Brian Boucher and Niresh Hariparsad Early identification and risk assessment for the potential for drug-drug interactions (DDIs) has become an integral component of the drug discovery process. Since the liver is the main drug eliminating organ, it is of great importance to have preclinical tools which accurately predict DDIs. In certain instances, even though drugs have poor permeability into the liver due to their physicochemical properties, they are efficiently taken up into the liver and excreted into bile. Recent evidence suggests that uptake transporters such as organic anion-transporting polypeptides (OATPs) are involved in facilitating this process. Among these transporters, OATP1B1 and OATP1B3 are specifically expressed in the liver and have broad substrate specificities. Both of these transporters are known to be involved in DDIs in the clinic. When rifampicin was co-administered with bosentan, a $500 \%$ increase in the AUC of bosentan was observed. Consequently, it is important in the early drug development process to accurately predict the potential for DDIs associated with these transporters. While cell lines overexpressing these transporters form a key component of assessing the DDI potential, human hepatocytes have a major advantage, since they have the full complement of phase I and phase II drug metabolizing enzymes and transporters. As such, we conducted validation studies with a five-donor pool of cryopreserved human hepatocytes. For this validation, we chose three compounds (cyclosporine, rifampicin and lopinavir) known to cause DDIs in the clinic when coadministered with statins and three compounds (amprenvir, ritonavir and gemfibrozil) that do not cause DDIs in the clinic when coadministered with statins. We used pitavastatin as the probe substrate for all our inhibition studies. Using the R-value approach (Hirano et al., (2006) DMD 34, 1229-1236) we were able to qualitatively predict the DDI potential in the clinic. Having established the utility of the R-value approach, we chose two protease inhibitors used in the treatment of Hepatitis-C. Since the general approach to the treatment of HCV involves administering a cocktail of drugs to patients, there is an increased chance of DDIs. Our studies suggest that both HCV compounds are actively taken up into hepatocytes and therefore could be victims of DDIs if coadministered with compounds such as rifampicin or cyclosporine which are 
known to be potent OATP inhibitors. In our studies, compound A was an inhibitor of OATP1B with an IC50=9.6 $\pm 0.4 \mu \mathrm{M}$, while compound B had an IC50 value of approximately $50 \mu \mathrm{M}$. Additional studies are currently under way in our lab to further assess the DDI potential of these compounds both as perpetrators and victims. Overall, our studies suggest that cryopreserved human hepatocytes can be used as a preclinical tool to identify DDI potential of compounds during the drug development process.

\title{
P319. Development and validation of liquid chromatography-tandem mass spectrometry method for inside-out vesicle uptake assay
}

\author{
$\underline{\text { Na Li }^{1}}$, Sweta N. Parikh ${ }^{1}$, Deqing Xiao ${ }^{2}$, David Stresser ${ }^{2}$, Charles L. Crespi ${ }^{2}$ and Christopher J. Patten ${ }^{1}$
}

${ }^{1}$ BD Biosciences, Bedford, MA, ${ }^{2}$ Discovery Labware, BD Biosciences, Woburn, MA

Due to lack of robust detection method, the application of inside-out vesicle in characterizing drug interaction with $\mathrm{ABC}$ transporter has been largely limited to radiolabeled chemicals or inhibitory screening assay. The aim of this study is to develop a robust detection method using liquid chromatography-Tandem mass spectrometry for characterizing drug interaction with two major ABC transporters using vesicle uptake assay, e.g. human MRP2 and human BCRP. Inside-out vesicles were prepared from insect Sf9 cells infected with baculovirus expressing hMRP2 and hBCRP. The MRP2 uptake of radiolabeled chemicals or non-radiolabeled counterparts: ${ }^{3} \mathrm{H}$ - leukotriene $\mathrm{C}_{4}$ or $\mathrm{LTC}_{4}$ and ${ }^{3} \mathrm{H}$-estradiol-17 $\beta$-glucuronide or E17ßG, BCRP uptake of ${ }^{3} \mathrm{H}$-estrone-3-sulfate or E3S and ${ }^{3} \mathrm{H}$-methotrexate or MTX were characterized using a rapid filtration system as described previously. For non-radiolabeled chemicals, the dried glass fiber filter plate is extracted with $200 \mu \mathrm{L}$ of elution buffer. The extracts were eluted into a 96-well receiver plate by centrifugation at $4000 \mathrm{rpm}$ for $5 \mathrm{~min}$, which were subjected to LC-MS/MS quantification. Kinetic assay $\left(K_{m}\right.$ and $\left.V_{\max }\right)$ and $\mathrm{IC}_{50}$ inhibition assays for benzbromarone on MRP2 uptake activity, Sulfasalazine on BCRP uptake activity were conducted using both radiolabeled and non-radiolabeled substrates. The kinetic parameters of selected MRP2 substrates and BCRP substrates and IC ${ }_{50}$ of selected MRP2 and BCRP inhibitor generated using LC-MS/MS method is comparable to those generated using radiolabeled chemicals. No significant difference was observed for the solvent based elution buffer compared with aqueous based elution buffer for both kinetic assay and inhibitory assay. In this study, the assay method was developed for inside-out vesicle uptake assay for non-radiolabeled chemicals using rapid filtration system and LC-MS/MS detection approach. The method was validated using selected probe substrates for both human MRP2 and BCRP transporters.

\section{P320. Digoxin is not a substrate for organic anion transporting polypeptide transporters OATP1A2, OATP1B1, OATP1B3, and OATP2B1 but is a substrate for a sodium dependent transporter expressed in HEK293 cells}

\author{
$\underline{\text { Mitchell E. Taub }}^{1}$, Kirsten Mease ${ }^{1}$, Rucha S. Sane ${ }^{1}$, Cory A. Watson ${ }^{2}$, Liangfu Chen², Harma Ellens ${ }^{2}$, \\ Brad Hirakawa ${ }^{3}$, Eric L. Reyner ${ }^{4}$, Márton Jani ${ }^{5}$ and Caroline A. Lee ${ }^{6}$ \\ ${ }^{1}$ Drug Metabolism \& Pharmacokinetics, Boehringer Ingelheim Pharmaceuticals, Inc., Ridgefield, CT, ${ }^{2}$ Drug Metabolism \\ and Pharmacokinetics, GlaxoSmithKline, King of Prussia, PA, ${ }^{3}$ Drug Safety Research \& Development, Pfizer \\ Global Research \& Development, San Diego, CA, ${ }^{4}$ Structural and Computational Biology, Pfizer Global Research \& \\ Development, San Diego, CA, ${ }^{5}$ Research \& Development, SOLVO Biotechnology, Budaors, Hungary, ${ }^{6}$ Independent, San \\ Diego, $C A$
}

Digoxin, an orally administered cardiac glycoside cardiovascular drug, has a narrow therapeutic window. Circulating digoxin levels $\left(\mathrm{C}_{\max } \sim 1.5 \mathrm{ng} / \mathrm{mL}\right)$ require careful monitoring, and the potential for drug-drug interactions (DDI) is a concern. Increases in digoxin plasma exposure caused by inhibition of P-glycoprotein (P-gp) have been reported. Digoxin has also been described as a substrate of various organic anion transporting polypeptide (OATP) transporters, posing a risk that inhibition of OATPs may result in a clinically relevant DDI similar to what has been observed for P-gp. While studies in rats have shown that Oatps contribute to the disposition of digoxin, the role of OATPs in the disposition of digoxin in humans has not been clearly defined. In independent studies, Boehringer-Ingelheim, GlaxoSmithKline, Pfizer, and Solvo observed that digoxin is not a substrate of OATPs 1A2, 1B1, 1B3 and 2B1. However, digoxin inhibited the uptake of probe substrates of OATP1B1 ( $\mathrm{IC}_{50}$ of $\left.47 \mu \mathrm{M}\right)$, OATP1B3 $\left(\mathrm{IC}_{50}>8.1 \mu \mathrm{M}\right)$, and OATP2B1 ( $\left.\mathrm{IC}_{50}>300 \mu \mathrm{M}\right)$, but not OATP1A2 in transfected cell lines. Interestingly, digoxin is a substrate of a sodium dependent transporter endogenously expressed in HEK293 cells, as uptake of digoxin was significantly greater in cells incubated with sodium-fortified media compared to incubations conducted in media where sodium was absent. Thus, while digoxin is not a substrate for the 
human OATP transporters evaluated in this study, in addition to P-gp mediated efflux, its uptake and pharmacokinetic disposition may be partially facilitated by a sodium-dependent transporter.

\title{
P321. Establishment of stably transfected cell lines expressing transporters key to drug disposition
}

\author{
Gang Luo, Kristen Cardinal, Manuela Deavila, Michele Hamrick, Kristine Horabik, Jeffrey Zuvon, \\ Liberty Liuperez and Richard Ridgewell
}

Drug Metabolism and Disposition, Covance Laboratories Inc., Madison, WI

Over the last decade, pharmaceutical and academic research has shown an increasing relevance of transporters in the disposition of drugs. With this information, the International Transporter Consortium highlighted several transporters of emerging importance in drug development. These include the uptake transporters organic anion transporting peptide (OATP) $1 \mathrm{~B} 1$ and 1B3, organic anion transporter (OAT) 1 and 3, and organic cation transporter (OCT) 2. Also included are the efflux transporters Pglycoprotein (P-gp/MDR1) and breast cancer resistance protein (BCRP). In addition, OCT1 and multidrug resistance protein (MRP) 2 also play significant roles in the disposition of certain drugs. To assess drug-transporter interactions, we established stably transfected cell lines for these human drug transporters. The cDNA encoding each of these transporters was inserted into a pCMV6-entry vector containing a neomycin resistance gene as the selection marker. Chinese hamster ovary (CHO) and Madin-Darby canine kidney (MDCK) II cells were both transfected with either a sham vector pCMV6 or pCMV6 containing a single uptake or efflux transporter cDNA, respectively. Stably transfected cells were established through selection with geneticin (G418). For each transporter, approximately 40 colonies were screened for functional activity using a radiolabeled substrate. All uptake experiments were conducted by determining radioactivity uptake. OATP1B1 showed 7.2-fold uptake of ${ }^{3}$ Hestradiol-17b-D-glucuronide, OATP1B3 8.5fold uptake of ${ }^{3} \mathrm{H}$-cholecystokinin, OAT1 26.0-fold uptake of ${ }^{14} \mathrm{Cpaminohippurate,} \mathrm{OAT3} \mathrm{5.5-fold} \mathrm{uptake}$ of ${ }^{3}$ Hestrone-3-sulfate, while OCT1 and OCT3 showed 19.7- and 19.6-fold uptake, respectively, of ${ }^{14}$ Ctetraethylammonium compared to the control cells. In preliminary screening of P-gp, the apparent permeability of ${ }^{3}$ Hdigoxin from the basolateral to apical direction using 24-transwell plates wasdetermined. Also in preliminary screening, the diminished accumulation of radioactivity in the BCRP and MRP2 colonies compared to control cells was measured following dosing of ${ }^{3} \mathrm{Hmitoxantrone}$ (for BCRP) and ${ }^{14} \mathrm{C} 1$-chloro-2,4-dinitrobenzene (for MRP2). For secondary screening and further qualification of the efflux transporters, efflux ratios were calculated from the apparent permeability of both the basolateral to apical and apical to basolateral directions using 24-transwell plates. P-gp, BCRP, and MRP2 showed more than two-fold higher efflux ratios with their respective radiolabeled substrates compared to the control cells. Known inhibitors, namely, cyclosporine A for OATP1B1 and OATP1B3, probenecid for OAT1 and OAT3, quinidine for OCT1 and OCT2, zosuquidar for P-gp, Ko-143 for BCRP, and MK-571 for MRP2, showed significant inhibition of the functional activity of each of these cell lines.

\section{P322. Expression of organic anion-transporting polypeptides (OATPs) 1B1 and 1B3 in ovarian cancer cells: Relevance for paclitaxel transport}

\author{
Walter Jaeger ${ }^{1}$, Martin Svoboda ${ }^{2}$, Katrin Wlcek ${ }^{1}$, Barbara Taferner ${ }^{3}$, Steffen Hering ${ }^{3}$, Bruno Stieger ${ }^{4}$ \\ and Theresia Thalhammer ${ }^{5}$
}

\begin{abstract}
${ }^{1}$ Department of Clinical Pharmacy and Diagnostics, University of Vienna, Vienna, Austria, ${ }^{2}$ Center for Physiology and Pathophysiology, Medical University of Vienna, Vienna, Austria, ${ }^{3}$ Department of Pharmacology and Toxicology, University of Vienna, Vienna, Austria, ${ }^{4}$ Department of Medicine, University Hospital, Zürich, Switzerland, ${ }^{5}$ Centre for Physiology and Pathophysiology, Medical University of Vienna, Vienna, Austria
\end{abstract}

Organic anion transporting polypeptides (OATPs) are known to mediate the uptake of many endogenous compounds and xenobiotics and therefore play a critical role in their absorption, distribution and excretion. Although some of the eleven known human OATP family members have been detected in breast, gastric, liver, pancreatic, and colon carcinomas, the knowledge of their expression in ovarian cancer is still limited. Hence we investigated the transcriptional expression of all 11 OATPs in human malignant ovarian tissue samples $(\mathrm{n}=10)$ and in estrogen-responsive OVCAR-3 and estrogen-independent SK-OV-3 ovarian carcinoma cell lines. Furthermore, by using OATP-transfected Xenopus laevis oocytes we also investigated whether OATPs contribute to cellular paclitaxel uptake. Our data showed that OATP1B1 and OATP1B3 are active paclitaxel transporters $\left(\mathrm{V}_{\max }: 12.3 \pm 0.4 \mathrm{fmol} /\right.$ oocyte $/ \mathrm{min}, \mathrm{K}_{\mathrm{m}}: 0.6 \pm 0.04 \mu \mathrm{M}$. vs. $\mathrm{V}_{\max }: 30 \pm 1.6 \mathrm{fmol} /$ 
oocyte/min, $\mathrm{K}_{\mathrm{m}}: 1.6 \pm 0.13 \mu \mathrm{M}$ ) in transfected X. laevis oocytes. Real-time RT-PCR analysis revealed expression of both OATPs in human ovarian cancer tissue specimens and in cancer cell lines but not in normal ovary tissue. The higher mRNA levels for OATP1B1 and OATP1B3 found in SK-OV-3 as compared with OVCAR-3 cells correlated with a higher initial uptake rate for paclitaxel $\left(\mathrm{V}_{\max }: 1660 \pm 126\right.$ and $517 \pm 86 \mathrm{pmol} / \mathrm{mg}$ protein $\left./ \mathrm{min}\right)$. In addition, cytotoxicity studies

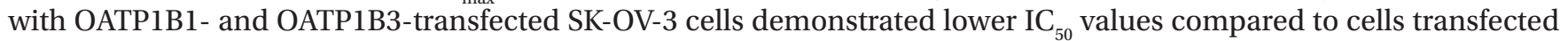
with the empty vector. In conclusion, our results revealed OATP1B1 and OATP1B3 as high-affinity paclitaxel transporters expressed in ovarian cancer cell lines and tumor tissues, suggesting a role for these polypeptides in the disposition of paclitaxel during therapy.

\title{
P323. Effects of antiretroviral protease inhibitors, lopinavir and ritonavir, on the hepatobiliary disposition of bile acids in sandwich-cultured rat hepatocytes
}

\author{
LaToya M. Griffin and Kim L.R. Brouwer
}

\begin{abstract}
Division of Pharmacotherapy and Experimental Therapeutics, University of North Carolina at Chapel Hill Eshelman School of Pharmacy, Chapel Hill, NC
\end{abstract}

Despite the success of protease inhibitor (PI)-based antiretroviral therapy, hepatotoxicity has been associated with the concomitant use of PIs. Inhibition of transport proteins by combined administration of PIs may enhance hepatocyte accumulation of drugs and/or endogenous bile acids (BAs), increasing the risk of drug-induced liverinjury (DILI). This study was designed to examine the effects of two frequently used PIs, lopinavir (LPV) and ritonavir (RTV), alone and in combination, on: (1) hepatic transport of the model BAs taurocholate (TC) and chenodeoxycholate (CDCA) during acute (10 $\mathrm{min}$ ) exposure, and (2) hepatobiliary disposition of endogenous BAs after 24-hr exposure. Studies were conducted in day 4 rat sandwich-cultured hepatocytes (SCH) seeded on 24- or 6-well plates. For transport studies, [ $\left.{ }^{3} \mathrm{H}\right]-\mathrm{TC}$ and $\left[{ }^{14} \mathrm{C}\right]$-CDCA $(1 \mu \mathrm{M}, 10 \mathrm{~min}, \mathrm{n}=3)$ accumulation in cells+bile and cells was determined during co-incubation with LPV (5-50 $\mu \mathrm{M})$, with and without RTV $(5 \mu \mathrm{M})$, using B-CLEAR ${ }^{\circledR}$ technology. The TC biliary excretion index (BEI), which represents the fraction of accumulated substrate in the bile compartment, was determined. Treatment with LPV orRTV $(5 \mu \mathrm{M})$ decreased the TC BEI by $29 \%$ and $70 \%$, respectively, and the CDCA BEI by $86 \%$ and $88 \%$, respectively. LPV concentrations exceeding $10 \mu \mathrm{M}$ ablated TC biliary excretion, irrespective of RTV co-incubation. Cellular TC accumulation was increased by 10 and $25 \mu \mathrm{M} \mathrm{LPV}$, and further increased when combined with RTV. Cellular CDCA accumulation was increased by LPV (5-50 $\mu \mathrm{M})$, and further increased in the presence of RTV. In separate studies, SCH were incubated for $24 \mathrm{hr}$ with LPV ( 5 or $50 \mu \mathrm{M})$ or RTV $(5 \mu \mathrm{M})$, alone and in combination, and cellular concentrations of endogenous BAs (TC, taurochenodeoxycholate, glycocholate and glycochenodeoxycholate) were quantified by LC/MS/MS.Cellular TC concentrations were decreased from control values of 4.79 to 1.65 and $0.86 \mathrm{pmol} / \mathrm{mg}$ protein in the presence of RTV and RTV+LPV $(5 \mu \mathrm{M})$, respectively. Cellular taurochenodeoxycholate concentrations were decreased from control values of 1.65 to 0.72 and $0.31 \mathrm{pmol} / \mathrm{mg}$ protein in the presence of RTV and RTV+LPV $(5 \mu \mathrm{M})$, respectively. Co-incubation of RTV with 5 or $50 \mu \mathrm{M}$ LPV decreased total BA cellular concentrations from 6.98 to 1.17 and $0.95 \mathrm{pmol} / \mathrm{mg}$ protein, respectively; cellular glycocholate and glycochenodeoxycholate concentrations were negligible. In conclusion, acute (10 min) PI exposure increased cellular BA accumulation; conversely, prolonged (24hr) PI exposure decreased total BA cellular concentrations. The SCH model is a useful tool to evaluate the effects of drugs on BA disposition. This system enables direct quantification of cellular concentrations of endogenous BAs, and may aid in predicting risk of DILI. Supported by NIH GM41935.

\section{P324. Effects of protein binding on transporter inhibitions}

\author{
Qing Zhu, Mingxiang Liao, Bei-Ching Chuang, Suresh K. Balani and Cindy Xia
}

Drug Metabolism and Pharmacokinetics, Millennium Pharmaceuticals, Inc., Cambridge, MA

Transporter-mediated DDIs have drawn a lot of attention recently. The International Transporter Consortium and FDA have provided some decision trees for the circumstances under which transporter studies need to be evaluated in the clinic. In the suggested decision trees, the unbound maximum concentration at steady state has been proposed as $[\mathrm{I}]_{1, \mathrm{u}}$ for P-gp inhibition or uptake transporter inhibition. In the present study, we aimed at evaluating the effects of protein binding on transporter inhibitions for both efflux transporters and uptake transporters. Ten P-gp inhibitors and 10 hepatic uptake transporter inhibitors (including OCT1 and OATP) were studied in the presence and absence of human plasma, using Caco-2 cells for P-gp inhibitor evaluations and human hepatocytes for hepatic uptake transporter inhibitor 
evaluations. Compared to the $\mathrm{IC}_{50}$ produced in protein free buffer, the $\mathrm{IC}_{50}$ did not change ( $\mathrm{shift}<1.5$ fold) in the presence of human plasma for the compounds which have protein binding less than 95\%; but increased dramatically (shift $>2$ fold) in the presence of human plasma for most of compounds which have protein binding more than 95\%. The results suggested that one needs to be cautious when using $[\mathrm{I}]_{1, \mathrm{u}} / \mathrm{IC}_{50}>0.1$ as a criteria for the need of a clinical DDI study.

\title{
P325. Evaluation of uptake transporters in human adult and pediatric hepatocytes
}

\author{
$\underline{\text { Michael Hayashi }}{ }^{1}$, Andrew Hui ${ }^{2}$, Josh DeKeyser ${ }^{2}$, Steven Louie ${ }^{2}$, Magang Shou ${ }^{2}$ and Lilly Xu \\ ${ }^{1}$ Pharmacokinetics and Drug Metabolism, Amgen Inc., Thousand Oaks, CA, ${ }^{2}$ Pkdm, Amgen Inc., Thousand Oaks, CA
}

The expression and induction of ADME genes in hepatocytes can alter the pharmacokinetic properties of many xenobiotics. An ontogenic switch in expression of CYP3A isoforms from CYP3A7 in pediatrics to CYP3A4 in adults has been observed in humans. Differential expression of ADME genes in adult and pediatric patients could result in differential dosing paradigms in these two patient populations; therefore, the aim of this study was to evaluate the ADME gene expression profiles in adult $(\mathrm{AH})$ and pediatric hepatocytes $(\mathrm{PH})$ treated with and without known CYP inducers. Human hepatocytes from pediatric and adult donors were evaluated in both suspension and plated format for ADME gene expression and transporter uptake activities (OATP1B1/3, OCT1, and OAT2). For the gene expression study, plated cells were treated with omeprazole (OME), Phenobarbital (PB), rifampicin (RIF) or vehicle control (VC) for 48 hours. The relative expression of 22 ADME genes was determined using RT-qPCR. For transporter activity, cells untreated and treated with the inducers ( 24 and $48 \mathrm{hrs}$ post dosing) were exposed to transporter-specific radiolabelled substrates for $10 \mathrm{~min}$ at either $37^{\circ} \mathrm{C}$ or $4^{\circ} \mathrm{C}$ followed by aspiration and washing $4 \mathrm{X}$ with ice-cold hanks balanced salt solution. Cells were lysed and the uptake of substrates was determined by liquid scintillation and normalized to uptake activity (amount per unit of protein). Our results indicated that the gene expression level of OATP1B1/3, OAT2 and OCT1 in PH has no significant difference compared to AH. The uptake activity of OATP1B1 and OCT1 is significantly lower in $\mathrm{PH}$ relative to AH; however at $24 \mathrm{~h}$ and $96 \mathrm{~h}$ post plating, the uptake in $\mathrm{PH}$ is significantly higher than AH. OATP1B3 uptake in PH is significantly higher than in AH at all time points. OAT2 uptake is similar in both populations except for that at $24 \mathrm{~h}$ post-plating in which uptake in PH is higher. Relative to VC, RIF treatments inhibited uptake for OATP1B3 and OCT1 while OATP1B1 and OAT2 uptake were unchanged. PB inhibited all transporters relative to VC and there was no difference in uptake between adults and pediatrics. After $48 \mathrm{~h}$ of OME treatment, uptake of OAT1B3 and OCT1 in both AH and PH, OATP1B1 in PH, and OAT2 in AH was shown to be inhibited compared to VC. Differences in ADME gene expression and uptake between the two populations was determined using a Student's t-Test. Modest differences in induced and un-induced ADME gene expression profiles were observed between adult and pediatric hepatocytes; however, there is a significant uptake transporter difference in these two populations.

\section{P326. Evaluation of P-glycoprotein expression in pain relevant tissues across species for understanding translation of efflux from preclinical species to human}

\author{
$\underline{\text { Renu S. Dhanikula }}^{1}$, Cyrla Hoffert ${ }^{2}$, Rebecca Grant ${ }^{2}$, Denis Projean ${ }^{1}$, Dajan O’Donnell ${ }^{2}$ and Marie \\ Roumi $^{1}$
}

${ }^{1}$ Dmpk, AstraZeneca R\&D Montreal, Montreal, QC, Canada, ${ }^{2}$ Translational Science, AstraZeneca R\&D Montreal, Montreal, QC, Canada

Translation of drug exposure and efficacy from preclinical species to human is imperative for the success of the drug discovery and development process. However, it is often a challenging endeavour, in particular, when the entry of molecules to the site of action is very limited and when little is known about the species differences in the expression and functionality of proteins or transporters expressed in the target tissue. Various efflux transporters, such as P-glycoprotein (Pgp), are now widely accepted to have profound influence on the disposition of substratesl. Nevertheless, there is paucity of information about their expression and functionality in pain relevant tissues (brain, spinal cord (SC) and dorsal root ganglia (DRG)) across various species. Therefore, our attempts were directed at evaluating the expression of Pgp at these target regions and understanding its effect on CNS disposition. Expression of Pgp protein in the fresh frozen brain, SC and DRG of Sprague Dawley rats, Cynomolgus monkeys and Human was examined using indirect immunohistochemistry with C219 and H241 antibodies (Calbiochem and Santacruz Biotechnologies). Diaminobenzidine was used as a chromogen for the purpose. Subsequently, cellular localization of Pgp was assessed by performing immunofluorescence based co-localization with C219 antibody and endothelial cell marker (Von-Willebrand factor antibody, Abcam). 
Leica DM4000B microscope and Zeiss confocal laser scanning TCS SPE microscope were used for capturing brightfield and fluorescence images, respectively. Evident differences were observed in the expression of Pgp in rat, monkey and human tissues. In the brain cortex, the expression of Pgp was relatively higher in rat than in monkey and human, while it was comparable in later two species (human and monkey). A similar trend was observed in the spinal cord expression of Pgp. Interestingly, there were no species differences in the Pgp expression in the DRG. Regional differences were observed within the spinal cord in all species, with the grey matter exhibiting higher protein levels relative to the white matter. Co-localization studies in all the examined tissues indicated that Pgp was primarily localized in the endothelial cells. These findings suggest that the exposure of Pgp substrates in human brain and SC could be higher than in rats but comparable to that in monkeys. Quantification of Pgp by LC-MS/MS is in progress to substantiate these findings. To the best of our knowledge this is the first report in the literature, wherein expression of Pgp in the DRG of various species has been demonstrated.

\title{
P327. Evaluation of transporter-mediated herb-drug interaction potential of Berberine, Saikosaponin, and Glycyrrhizin
}

\author{
Da-Hyeon Choi ${ }^{1}$, Ho-Jung Shin², Min Hye Kim², Su Jeong Lim², Boram Kang ${ }^{2}$, Jae-Gook Shin ${ }^{2}$ and \\ Im-Sook Song ${ }^{2}$
}

${ }^{1}$ Pharmacology, Inje University College of Medicine, Busan, South Korea, ${ }^{2}$ Pharmacogenomics Research Center, Inje University College of Medicine, Busan, South Korea

Herbal medicines and their active ingredients are widely used worldwide, and they have become an important part of clinical medicine. However, the combined use of herbs and drugs has increased the possibility of pharmacokinetic and pharmacodynamic interactions. To determine the underlying mechanisms of transporter-mediated herb-drug interaction, we investigated the inhibitory effects of berberine, saikosaponin A, and glycyrrhizin on the functions of organic cation transporter ( $\mathrm{OCT1}$ ), organic anion transporterl (OAT1), and organic anion transporting polypeptide1B1 (OATP1B1), and evaluated the interaction potential of berberine, saikosaponin A, and glycyrrhizin with substrate drugs for these transporters. Berberine $(10 \mathrm{mM})$ and saikosaponin $\mathrm{A}(20 \mathrm{mM})$ inhibited the uptake of $\mathrm{MPP}^{+}$by $70-99 \%$ in oocytes expressing OCT1. However, glycyrrhizin did not affect on OCT1-mediated MPP+ uptake. The $\mathrm{K}_{\mathrm{i}}$ values for berberin and saikosaponin A on OCT1-mediated $\mathrm{MPP}^{+}$uptake were $0.047 \pm 0.033 \mathrm{mM}$ and $3.1 \pm 0.3 \mathrm{mM}$, respectively. Glycyrrhizin $(100 \mathrm{mM})$ and saikosaponin A $(20 \mathrm{mM})$, but not berberine, inhibited the OATP1B1-mediated uptake of estron-3-sulfate by $80-90 \%$ and the $\mathrm{K}_{\mathrm{i}}$ values for glycyrrhizin and saikosaponin A on OATP1B1-mediated estrone-3-sulfate uptake were $16.63 \pm 4.2 \mathrm{mM}$ and $7.2 \pm 3.2 \mathrm{mM}$, respectively. In case of OAT1, there were no interactions with berberine, saikosaponin A, and glycyrrhizin. In conclusion, berberine, saikosaponin A, and glycyrrhizin, major constituent in antispasmodic herbal medicine, showed differential interaction potential for OAT1, OCT1, and OATP1B1 transporters. Among them, berberine showed potent inhibitory potential for OCT1 with no effect on OAT1 and OATP1B1 function. Saikosaponin A had an inhibitory effect on both OCT1 and OATP1B1 activity and glycyrrhizin had moderate inhibitory potential for OATP1B1.

\section{P328. Functional analysis of pharmacogenetic variants of human organic cation/carnitine transporter 2 (hOCTN2) identified in Singaporean populations}

\author{
Dorothy Su Lin Toh ${ }^{1}$, Michael Murray ${ }^{1}$, Tan Kuan Pern², Edmund Lee ${ }^{3}$ and Fanfan Zhou ${ }^{1}$ \\ ${ }^{1}$ Pharmacy, University of Sydney, Sydney, Australia, ${ }^{2}$ Bioinformatics Institute, Agency for Science, Technology and \\ Research, Singapore, ${ }^{3}$ Department of Pharmacology, National University of Singapore, Singapore, Singapore
}

The human organic cation/carnitine transporter-2 (hOCTN2; SLC22A5) mediates the cellular influx of organic cations such as carnitine, which is essential for fatty acid oxidation, and a number of cationic drugs. Primary carnitine deficiency has been associated with a wide range of hOCTN2 gene mutations that may encode functionally defective hOCTN2 transporter variants. Previously we identified six novel nonsynonymous single nucleotide polymorphisms in the SLC22A5 gene in individuals from Chinese and Indian populations of Singapore ${ }^{1}$. The present study evaluated the impact of these polymorphisms on hOCTN2 function and expression in transfected HEK-293 cells. Transport function with the substrates L-carnitine and tetraethylammonium was markedly impaired in variants that encoded amino acid substitutions D122Y ( $<20 \%$ of wild-type control) and K302E ( $45 \%$ of wild-type); these amino acid substitutions are located respectively in the large extracellular and large intracellular loops of hOCTN2. In contrast, the function of the 
other four variants was unimpaired (E109K, V175M, K191N and A214V). Subsequent biotinylation and immunofluorescence experiments indicated that the expression of the D122Y and K302E-hOCTN2 variants at the plasma membrane of HEK-293 cells was decreased relative to the wild-type hOCTN2, but that total cellular expression was unchanged. This is also consistent with findings from transporter kinetic studies that the $\mathrm{V}_{\max }$ for L-carnitine uptake was decreased in K302hOCTN2 to $\sim 50 \%$ of wild-type control, while $\mathrm{K}_{\mathrm{m}}$ remained unchanged; kinetic evaluation of D122Y-hOCTN2 was not possible due to variable rates of transport function at low L-carnitine concentrations. The K302E-hOCTN2 variant was also more susceptible than the wild-type transporter to inhibition by the drugs cimetidine, pyrilamine and verapamil. Considered together these findings indicate that impaired plasma membrane maturation of the previously unidentified D122Y and K302E-hOCTN2 variants that occur in Singaporean populations contributes to decreased carnitine influx.

${ }^{1}$ D.S.L. Toh, J.Y. Yee, S.H. Koo, M. Murray and E.J.D. Lee, Genetic Variations of the SLC22A5 Gene in the Chinese and Indian Populations of Singapore. Drug Metab Pharmacokinet (2010) 25: 112-119

\title{
P329. Human organic transporter 1(hOAT1) affected the renal tubular secretion of antofloxacin
}

\author{
Kehua Shao ${ }^{1}$, Minji Wei ${ }^{1}$, Xiuli Zhao ${ }^{2}$ and Chao $\mathrm{Jia}^{2}$ \\ ${ }^{1}$ Institute of Clinical Pharmaclogy, Peking University, Beijing, China, ${ }^{2}$ Base for Clinical Trial, Tongren Hospital, Capital \\ Medical University, Beijing, China
}

Aim: Antofloxacin(ANT) is a newly developed fluoroquinolone antibacterial in China. Pharmacokinetic study in healthy volunteers shows that $40 \%-45 \%$ of ANT administered is eliminated by kidney. The effect of human organic transporter 1 on renal tubular secretion of ANT was investigated in present study. Methods: HK-2 cell monolayer model was established and its integrity of the model was confirmed by transephitherial electric resistance (TEER) and the diffusion rate of fluorescein-labeled dextran (molecular mass $40 \mathrm{kDa}$ ). The activity of hOAT1 was evaluated by p-aminohippurate (PAH). Bi-directional transport of ANT solely or co-used with probenecid (PRO) were studied by using HK-2 cell monolayer model to determine whether hOAT1 affected renal tubular secretion of ANT. The concentrations of ANT collected from the transport assay were determined by high performance liquid chromatography and the transport parameters such as clearance (CLA-B and CLB-A) were calculated and compared when the ANT was used solely and co-used with PRO. Results: When cells was growing in 5-7 d, TEER became stable and the range was from 140 to $150 \mathrm{OM} . \mathrm{cm}^{\wedge} 2$. CLA-B and CLB-A of PAH and ANT were 0.192+/-0.027, 0.325+/-0.184 and 0.475+/-0.200, 0.636+/-0.060 of 60 min transport, respectively. While co-transported with PRO, CLA-B and CLB-A of PAH and ANT became $0.399+/-0.035,0.171+/-0.017(\mathrm{p}<0.05)$ and $0.772+/-0.120,0.422+/-0.032$ respectively $(\mathrm{p}<0.05)$. Conclusion: The above results proved HK-2 cell monolayer model can be used to study the hOAT - drug interaction and hOATl affect the secretion of ANT from renal tubular basal side (B) into apical side (A).

\section{P330. Identification of a novel function of SNBT1 as a sodium-dependent urate transporter}

\author{
Chihiro Nishijima, Katsuhisa Inoue, Kinya Ohta and Hiroaki Yuasa
}

\section{Graduate School of Pharmaceutical Sciences, Nagoya City University, Nagoya, Japan}

We have recently identified sodium-dependent nucleobase transporter 1 (SNBT1, Slc23a4), ${ }^{\text {) }}$ from the rat small intestine as a first nucleobase transporter in mammals. Rat SNBT1 (rSNBT1) was found to mediate sodium-dependent transport of several nucleobases such as uracil, thymine, guanine, hypoxanthine and xanthine, but not adenine and cytosine, and was suggested to be responsible for the intestinal nucleobase absorption. The substrate specificity of rSNBT1 suggests a role of a oxo group at the 4th position (C-4) of pyrimidine ring (or corresponding position of purine ring) in substrate recognition. To further clarify the role of this transporter in the intestinal disposition of nucleobases and relevant compounds, we examined the transport of urate, which is an important metabolite of purine nucleobases and has an oxo group at the required position. The uptake of $\left[{ }^{14} \mathrm{C}\right]$ urate was evaluated at $37^{\circ} \mathrm{C}$ and $\mathrm{pH} 7.4$ for a 2 -min period, using Madin-Darby canine kidney (MDCK) II cells stably expressing rSNBT1. The uptake of $\left[{ }^{14} \mathrm{C}\right]$ urate was also evaluated by using everted intestinal tissue sacs prepared from the small intestine of male Wistar rats, with the uptake condtion of $37^{\circ} \mathrm{C}$ and $\mathrm{pH} 7.4$ for a 2-minperiod. Urate was found to be efficiently transported by rSNBT1 in a sodium-dependent manner. rSNBT1-mediated urate transport was saturable with the Michaelis constant $\left(\mathrm{K}_{\mathrm{m}}\right)$ of $433 \mu \mathrm{M}$ and, at a low concentration of $5 \mu \mathrm{M}$, inhibited by uracil, hypoxanthine, thymine and guanine with the $\mathrm{IC}_{50}$ values of $28.4,10.1,2.7$ and $8.8 \mu \mathrm{M}$, respectively. Particularly, the $\mathrm{IC}_{50}$ value for uracil was comparable with the $\mathrm{K}_{\mathrm{m}}(21.2 \mu \mathrm{M})$ of uracil transport by rSNBT1, suggesting that urate shares the substrate recognition site with uracil, and rSNBT1 could mediate absorption of urate as well as 
uracil in the small intestine. However, the urate uptake was not detectable in the evered sacs of the rat small intestine, although the marked uptake of nucleobases such as hypoxanthine, uracil and thymine could be observed. Thus, rSNBT1 was found to transport urate by a sodium-dependent mechanism shared by nucleobases, although the uptake of urate was not observed physiologically in the rat small intestine. These results suggest that urate absorption is regulated by futile cycling via SNBT1 and other transporters such as Abcg2 and/or Glut9, both of which have been known to mediate the efflux of urate. Further studies will be needed to clarify the role of SNBT1 in urate disposition in the small intestine.

1) Yamamoto S, Inoue K, Murata T, Kamigaso S, Yasujima T, Maeda J, Yoshida Y, Ohta K, and Yuasa H: Identification and functional characterization of the first nucleobase transporter in mammals: implication in the species difference in the intestinal absorption mechanism of nucleobases and their analogs between higher primates and other mammals. J Biol Chem, 285:6522- 6531, 2010.

\title{
P331. Involvement of sodium-coupled monocarboxylate transporter-like activity in nicotinate uptake by brush border membrane vesicles from the rat small intestine
}

\author{
Hiroaki Yuasa, Masahiko Ohkubo, Kinya Ohta and Katsuhisa Inoue
}

Graduate School of Pharmaceutical Sciences, Nagoya City University, Nagoya, Japan

Carrier-mediated transport systems have been suggested to be present in the small intestine for the efficient absorption of nicotinate, a water soluble vitamin (vitamin $\mathrm{B}_{3}$ ) that has various biological activities including lipid-lowering effects, and indeed recent studies have identified $\mathrm{Na}^{+}$-dependent monocarboxylate transporter 1 (SMCT1) and SMCT2 as those that may be involved in that. However, the roles of these transporters in intestinal nicotinate absorption have not been fully verified yet. Although our latest study using the everted tissue sacs successfully demonstrated the prevailing involvement of $\mathrm{Na}^{+}$-dependent SMCT-like transporter activities in nicotinate uptake in both the jejunal and ileal segments of the rat small intestine, there is an earlier study that contradictorily indicated the absence of SMCT-like transporter activity, with demonstrating the involvement of $\mathrm{H}^{+}$-dependent monocarboxylate transporter 1 (MCT1)like transporter activity, in brush border membrane vesicles (BBMVs) from the rat small intestine. To address the issue and gain information for optimizing the absorption of nicotinate and relevant drugs, we examined the uptake of $\left[{ }^{3} \mathrm{H}\right]$ nicotinate in rat intestinal BBMVs. In the present study, BBMVs were prepared from the 20 -cm segments of jejunum and ileum, using 8 male Wistar rats for a preparation, by a calcium precipitation method and uptake experiments were performed by a rapid filtration technique. The uptake of nicotinate $(0.4 \mu \mathrm{M})$ was higher initially in the presence of $\mathrm{Na}^{+}$ than in its absence at pH 7.4 in ileal BBMVs, successfully demonstrating the presence of SMCT-like transporter activity. However, in jejunal BBMVs, the initial uptake of nicotinate at the same concentration was independent of $\mathrm{Na}^{+}$at $\mathrm{pH} 7.4$ and also at pH 5.5, but was enhanced at the lower $\mathrm{pH}$ of 5.5 than at $\mathrm{pH} 7.4$ and saturable only at $\mathrm{pH} 5.5$. These results are indeed in agreement with the earlier indication of the presence of MCT1-like transporter activity alone in BBMVs and conflict with what we recently found in the everted sacs. MCT1-like transporter activity was similarly observed also in ileal BBMVs, but at least we could also observe the presence of $\mathrm{Na}^{+}$-dependent transporter activity at pH 5.5 as well as at $\mathrm{pH}$ 7.4. Particularly at $\mathrm{pH}$ 7.4, where MCT1-like transporter activity was absent, the $\mathrm{Na}^{+}$-dependent SMCT-like transporter activity was suggested to represent a major uptake component. This is the first to demonstrate SMCT-like transporter activity in intestinal BBMVs, although the reason why SMCT-like transporter activity is less evident in BBMVs than in everted sacs remains to be elucidated.

\section{P332. In vitro characterization of transporters mediating hepatic uptake and biliary excretion of AMG009 in rats}

\author{
Eskouhie H. Tchaparian, Guifen Xu, Yun Ling, Tom Huang, Mark P Grillo and Lixia Jin
}

\section{Pkdm, Amgen Inc., South San Francisco, CA}

AMG009 is a CRTH2 (chemoattractant receptor-homologous molecule expressed on Th2 cells) and DP (prostanoid D receptor) dual-antagonist with a low to moderate clearance and good bioavailability. AMG009 is eliminated primarily as unchanged drug (52\%) and acyl glucuronide M1 (39\%) via biliary excretion in rats. Hence, it was hypothesized that hepatic uptake and efflux transporters could play an important role in the disposition and metabolism of AMG009. In the present study, interaction of AMG009 with major rat hepatic transporters were evaluated using 1) rat primary hepatocytes, 2) stably tranfected cell lines overexpressing rat multidrug resistance protein 1 (Mdr1), multidrug resistanceassociated protein 2 (Mrp2),or breast cancer resistant protein (Bcrp), and 3) xenopus laevis oocytes overexpressing rat 
organic anion-transporting polypeptide (Oatps), organic anion-transporter (Oat2), or organic cation-transporter (Oct1). Hepatocytes uptake studies indicated that uptake transport of AMG009 was saturable, and time- and concentrationdependent with an apparent Km value of $187 \mu \mathrm{M}$. Inhibition of AMG009 uptake by chemical inhibitors in primary hepatocytes identified AMG009 as a substrate of Oatps, but not Octl or Oat2. Xenopus oocytes uptake studies revealed that AMG009 uptake was mediated by Oatp1 and Oatp2 with Km values of $39 \mu \mathrm{M}$ and $417 \mu \mathrm{M}$ respectively. Efflux studies identified AMG009 as a substrate of hepatic apical transporters Mdr1 and Mrp2, but not Bcrp. Taken together, these data suggest possible contribution of these transporters in the biliary excretion and hepatic uptake of AMG009. In vivo investigations are underway to determine the significance of transporters in the hepatic metabolism and clearance of this compound in rats.

\title{
P333. Interaction of milk with the intestinal PEPT1-mediated absorption of oseltamivir in rats and humans
}

\author{
$\underline{\text { Kaori Morimoto }}^{1}$, Kozue Kishimura $^{1}$, Megumi Tosaka ${ }^{1}$, Hideaki Asamizu${ }^{1}$, Takaaki Nagami ${ }^{1}$, Nao \\ Kodama $^{1}$, Takashi Kano ${ }^{1}$, Ikumi Tamai ${ }^{2}$ and Takuo Ogihara ${ }^{1}$ \\ ${ }^{1}$ Faculty of Pharmacy, Takasaki University of Health and Welfare, Takasaki, Japan, ${ }^{2}$ Faculty of Pharmacy, Institute of \\ Medical, Pharmaceutical and Health Sciences, Kanazawa University, Kanazawa, Japan
}

Purpose: Our purpose was to study the importance and predictability of food and drug interaction on proton-coupled peptide transporter (PEPT1)-mediated drug absorption by examining oseltamivir - milk interaction in rats and humans. Methods: Oseltamivir uptake profile and its inhibition with excess peptides were examined by using Caco-2 and HeLa cells stably expressing human PEPT1 (HeLa/ PEPT1). To examine the contribution of PEPT1 in gastrointestinal absorption of oseltamivir in vivo, simultaneous administration of casein, glycyl-sarcosine (Gly-Sar) and milk with oseltamivir was conducted in rats. We also evaluated the effect of milk on the pharmacokinetics of oseltamivir in healthy volunteers. The predictability of the extent of milk inhibition of intestinal oseltamivir absorption in humans was examined by using duodenal transit time and inhibitory rate by excess peptides in Caco-2 cells. Results: Oseltamivir uptake by Caco-2 and HeLa/ PEPT1 cells were temperature- and concentration-dependent $\left(\mathrm{K}_{\mathrm{m}}=6.5 \mathrm{mM}\right.$ and $8.6 \mathrm{mM}$, respectively $)$ and one-half to two-third was inhibited by PEPT1 inhibitors such as $10 \mathrm{mM}$ Gly-Sar or Trp-Gly. Plasma concentration of oseltamivir after oral administration in rats was greatly decreased by milk (62\%), casein (82\%) and 125 mM Gly-Sar (82\%) compared with the control group. Plasma concentration of oseltamivir was lower in humans from time 0 to $2 \mathrm{hrs}$, when taken with milk, while $\mathrm{AUC}_{0-\infty}$ was not affected significantly. Urinary excretion of oseltamivir and active metabolite Ro 64-0802 was decreased to $78 \%$ of control by milk. The prediction was roughly consistent with the observed value for the inhibitory ratio of milk for oseltamivir absorption in humans. Conclusions: Our in vitro study suggested that the gastrointestinal absorption of oseltamivir was mediated by PEPT1 at least in part and inhibited by dietary peptides. In vivo pharmacokinetic study confirmed that intestinal absorption of oseltamivir was interacted with milk peptides in both rats and humans, while the extent of the inhibition was different. This may be due to differences in the regional expression and the contribution of PEPT1 on the gastrointestinal absorption of oseltamivir between rats and humans.

\section{P334. Interaction of penciclovir with renal drug transporters}

\section{$\underline{\text { Adrienne Natrillo }^{1}}$, Arpine Vapurcuyan ${ }^{2}$, Yaofeng Cheng ${ }^{2}$ and Ryan Pelis ${ }^{2}$}

\author{
${ }^{1}$ Drug Metabolism and Pharmacokinetics, Novartis Institutes for BioMedical Research Inc., East Hanover, NJ, ${ }^{2}$ Dmpk, \\ Novartis Institutes for BioMedical Research Inc., East Hanover, NJ
}

Penciclovir is the active drug of famciclovir, which is used to treat herpes virus. Penciclovir is primarily cleared from the body via renal excretion, a process that involves robust active proximal tubular secretion. Thus, we expected that renal drug transporters, including organic anion transporters (OATs), urate transporter 1 (URAT 1), organic cation transporter 2 (OCT2), multidrug and toxin extrusion transporters (MATEs) and multidrug resistance-associated proteins (MRPs) could be important in mediating proximal tubular secretion of penciclovir. The interaction of $\left[{ }^{3} \mathrm{H}\right]$ penciclovir as a substrate of renal drug transporters was studied either in human embryonic kidney (HEK) cells stably expressing the individual transporters (OAT1, OAT2, OAT3, OAT4, OCT2, MATE1, MATE2K and URAT1) or in inside-out membrane vesicles (MRP2 and MRP4). Of the renal uptake transporters tested (OAT1, OAT2, OAT3 and OCT2), only OAT2 supported active penciclovir transport. The uptake of penciclovir into HEK cells stably expressing OAT2 was $\sim 20$-fold higher than in cells not expressing the transporter. The $\mathrm{J}_{\max }$ and $\mathrm{K}_{\mathrm{m}}$ value associated with OAT2-mediated penciclovir transport 
were $284 \pm 10 \mu \mathrm{M}$ and $6.7 \pm 0.58 \mathrm{nmol} \cdot \mathrm{mg} \mathrm{protein}^{-1} \cdot \mathrm{min}^{-1}$, respectively. Of the efflux transporters tested (OAT4, MATE1, MATE2K, URAT1, MRP2 and MRP4), only MRP4 appeared to transport penciclovir; penciclovir transport was $\sim 2$-fold higher in the presence versus absence of ATP. These data suggest that renal tubular secretion of penciclovir involves a limited number of drug transporters.

\title{
P335. Interactions of FTY720 and FTY720-P with ATP-binding cassette transporters
}

\author{
Joy Wang ${ }^{1}$, Eric Sands ${ }^{2}$, Cheryl Black ${ }^{2}$, David Williams ${ }^{1}$, Monica Chuong ${ }^{1}$, Eman Atef ${ }^{1}$, Barbara \\ LeDuc $^{1}$, Lawrence $\mathrm{Gan}^{2}$ and Guangqing Xiao ${ }^{2}$
}

${ }^{1}$ Pharmaceutical Sciences, Massachusetts College of Pharmacy and Health Science, Boston, MA, ${ }^{2}$ BiogenIdec, Cambridge, MA

The immunomodulator pro-drug fingolimod (FTY720) is the first oral drug for relapsing multiple sclerosis, an immunemediated disease of the CNS. FTY720 is a structural analogue of sphingosine and is phosphorylated in vivo by sphingosine kinase-2. The phosphorylated FTY720 (FTY720-P) acts as an agonist on four of the five known S1P receptors by triggering a signal to prevent lymphocytes from migrating to sites of inflammation. While the importance of FTY720 in the immune system and CNS has been established, possible interactions of FTY720 and FTY720-P with CNS drug transporters remain unclear. In this study, the in vitro interactions of FTY720 and FTY720-P with Multidrug Resistance Proteins (MRPs), P-glycoprotein (P-gp), and Breast Cancer Resistance Protein (BCRP) were investigated using the human primary brain endothelia/astrocyte co-culture model and the Caco-2 cell model. Our results showed that both FTY720 and FTY720-P induced MRP1 gene expression in human brain endothelial cells. Investigation of similar effects on other transporters expressed in human brain endothelial cells is ongoing. Furthermore, FTY720 inhibited the transport of digoxin, a reference substrate of P-gp, and estrone-3-sulfate, a reference substrate of BCRP, across Caco-2 monolayer in a dose dependent manner, displaying IC50 values of $35 \mathrm{mM}$ and $51 \mathrm{mM}$, respectively. The results show that FTY720 and FTY720-P modulate-MRP1 gene expression, thus suggesting they might affect MRP1-mediated drug transport. Whether FTY720 and FTY720-P are substrates of P-gp, BCRP and MRPs is under investigation.

\section{P336. Interactions of selected antivirals with human organic anion transporter 1 in vitro and potential influence of drug competitors}

\author{
Jana Mandikova, Zbynek Novy, Marie Volkova, Petr Pavek and Frantisek Trejtnar
}

\begin{abstract}
Department of Pharmacology and Toxicology, Charles University in Prague, Faculty of Pharmacy in Hradec Kralove, Hradec Kralove, Czech Republic
\end{abstract}

Transporters for organic anions play an important role in the excretion of a variety compounds by the kidney. Many antivirals used for treatment of HIV infection or chronic hepatitis B may be excreted via the kidney by human organic anion transporter 1 (hOAT1) located characteristically in the proximal renal tubules. Concomitant administration of antivirals with drugs from other therapeutic groups (e. g. antifungals, antibiotics) may lead to potential interactions with this transport system. Such interactions in the kidney may result in significant changes in the renal excretion rate of the interacting compounds. The aim of this study was to compare the affinity of three antiviral drugs (tenofovir, elvitegravir, entecavir) and several potentially concomitantly used drugs (amphotericin B, gentamicin) to hOAT1 using a human cellular in vitro model and evaluate possible transport interactions of the studied drugs at this transport system. Human cervical cancer cell line (HeLa) transiently transfected with hOAT1 was used for the experiments. A possible positive or negative impact of the interactions of the tested drugs at hOAT1 on renal cytotoxicity was tested in human renal cell line HK-2. The used HeLa ${ }^{\text {hOATl }}$ cell model showed probenecid-sensitive uptake of $\left[{ }^{3} \mathrm{H}\right]$ para-aminohippuric acid, a prototypical hOAT1 substrate. In addition, temperature-dependent accumulation of $\left[{ }^{3} \mathrm{H}\right]$ tenofovir in $\mathrm{HeLa}{ }^{\text {hOATl }}$ cells was demonstrated. These findings confirmed proper functioning of hOAT1 in HeLa ${ }^{\text {hOAT1 }}$ cells. In the affinity transport studies, the rate of inhibition of intracellular accumulation of $\left[{ }^{3} \mathrm{H}\right]$ para-aminohippuric acid was induced by gradually increasing concentration of the tested compound. The found values for affinity to hOAT1 can be ranged from the highest to the lowest affinity: amphotericin B > entecavir > tenofovir >> elvitegravir. An interaction of gentamicin at hOAT1 was not proved using available incubation concentrations. Due to the very high affinity to the hOAT1, amphotericin B was selected for interaction experiment with $\left[{ }^{3} \mathrm{H}\right]$ tenofovir. A significant interaction at hOAT1 between these two compounds was demonstrated. Inhibition of $\left[{ }^{3} \mathrm{H}\right]$ tenofovir transport by amphotericin B was stronger than that of $\left[{ }^{3} \mathrm{H}\right]$ para-aminohippuric acid. Although efficiently transported by hOAT1, tenofovir showed lower cytotoxic effect than amphotericin B in HK-2 cells. In conclusion, the study confirms that hOAT1 may contribute to the influx of all three tested antiviral drugs into 
cells. However, the rate of transport by hOAT1 will be various due to the significant differences in the affinity to the transporter. The found competition of amphotericin B and tenofovir at hOAT1 may potentially change renal excretion parameters of the competitors during concomitant administration. The study was supported by Charles University in Prague (Project SVV 263003), grant GAUK No. 360811/FaF/C-LEK and grant IGA MZ No. NT12398-4/2011.

\title{
P337. Intestinal OATP1A2 transport of NOV123 and its inhibition as a mechanism to explain reduced human bioavailability when co-administered with grapefruit juice
}

\author{
Natalya Alexander, Arpine Vapurcuyan, Sam Rebello and Imad Hanna
}

\author{
Drug Metabolism and Pharmacokinetics, Novartis, East Hanover, NJ
}

The potential of the organic anion transporting polypeptides 1A2 (OATP1A2) or 2B1 (OATP2B1) to transport NOV123 (a proprietary compound) was examined using mammalian cells either transiently express OATP1A2 or stably express OATP2B1. These investigations were done to provide a mechanistic explanation for the apparent reduction in the systemic bioavailability of NOV123 upon oral administration with grapefruit juice. In vitro transport activity was evaluated by comparing the uptake of $\left[{ }^{14} \mathrm{C}\right] \mathrm{NOV} 123$ or the positive control substrates, $\left[{ }^{3} \mathrm{H}\right]$ fexofenadine (OATP1A2) or $\left[{ }^{3} \mathrm{H}\right]$ estrone-3-sulfate (OATP2B1) into OATP-expressing cells to that into control cells. Under these assay conditions, NOV123 was found to be a substrate for OATP1A2 but not OATP2B1. In the absence of transport inhibitors, $\left[{ }^{14} \mathrm{C}\right] \mathrm{NOV} 123(10$ $\mu \mathrm{M})$ uptake into OATP1A2-expressing cells was 2.7-fold higher than that in control cells following the 15 min uptake assay. Moreover, the accumulation of $\left[{ }^{14} \mathrm{C}\right] \mathrm{NOV} 123$ or the positive control substrate $\left[{ }^{3} \mathrm{H}\right]$ fexofenadine were reduced in the presence of the positive control OATP1A2 inhibitor verapamil. In contrast, $\left[{ }^{14} \mathrm{C}\right] \mathrm{NOV} 123$ accumulation OATP2B1expressing cells was essentially the same as that into control cells. Also examined in this study was the potential for naringin (a flavonoid component of grapefruit juice) to inhibit the transport activity of OATP1A2. Naringin was found to inhibit OATP1A2-mediated NOV123 or fexofenadine transport by 86-89\%, at the highest concentration tested (1000 $\mu \mathrm{M})$. The estimated $\mathrm{IC}_{50}$ values of OATP1A2-mediated NOV123 or fexofenadine transport inhibition by naringin were $75.5 \pm 11.6 \mu \mathrm{M}$ and $24.2 \pm 2.0 \mu \mathrm{M}$, respectively. These $\mathrm{IC}_{50}$ values are within the concentration range of naringin found in grapefruit juice. In conclusion, NOV123 was shown to be a substrate of human OATP1A2 but not OATP2B1. Naringin, a flavonoid component of grapefruit juice inhibited OATP1A2 activity and thus may provide a mechanistic explanation for the reduced exposure to NOV123 in vivo when co-administered with grapefruit juice.

\section{P338. Investigating the role of UGT1A1, MRP2 and OATP1B1 in drug induced liver toxicity}

\author{
$\underline{\text { Emile G. Plise }}^{1}$, Gillian Smelick², Jonathan Cheong ${ }^{1}$ and Jae H. Chang ${ }^{1}$
}

\section{${ }^{1}$ Drug Metabolism and Pharmacokinetics, Genentech Inc., South San Francisco, CA, ${ }^{2}$ Clinical Phamacology, Genentech Inc., South San Francisco, CA}

Purpose. Severe drug-induced hepatotoxicity has been responsible for drugs being withdrawn from the market. Some adverse drug reactions (ADR) can be predicted early in drug discovery so that the clinical risk-benefit can be assessed. However, unexpected, or idiosyncractic, ADRs may also occur, leading to liver injury that can lead to liver failure and/ or death. Hy's Law predicts that the combination of increased liver transaminase and bilirubin levels following drug administration may foreshadow hepatotoxicity. Here, we aim to examine the mechanism of Hy's Law by investigating the inhibition potency of bromfenac, labetalol, troglitazone and ritonavir against UGT1A1, MRP2 and OATP1B1 that modulate the elimination of bilirubin. Method. Due to limited solubility and ionization of bilirubin, various probe substrates were utilized to examine the activities of UGT1A1, MRP2 and OATP1B1. Specifically, the inhibition of UGT1A1 activity was examined in human liver microsomes and recUGT1Al by monitoring the formation of estradiol 3-glucuronide (E3G) in the absence or presence of various concentrations of inhibitors. The inhibition of MRP2 activity was examined by monitoring the accumulation of carboxy dichlorofluorescein (CDCF) in MRP2 membrane vesicles in the absence or presence of various concentrations of inhibitors. The inhibition of OATP1B1 activity was examined by monitoring the flux of pitavastatin in OATP1B1 expressing HEK cells in the absence or presence of various concentrations of inhibitors. Results. Ritonavir was the most potent inhibitor of $\mathrm{E} 3 \mathrm{G}$ formation with $\mathrm{IC}_{50}$ value of $0.8 \mu \mathrm{M}$. This was followed by troglitazone, bromfenac and labetalol at $\mathrm{IC}_{50}$ value of $7.7,10$ and $55 \mu \mathrm{M}$, respectively. Troglitazone was the more potent inhibitor of MRP2 with IC50 value of $16 \mu \mathrm{M}$. Labetalol and ritonavir did not inhibit MRP2 while bromfenac had only a modest affect on MRP2 activity. Ritonavir and troglitazone were potent inhibitors of OATP1B1 with IC50 value of 0.4 and 0.5 $\mu \mathrm{M}$, respectively. Conclusions. UGT1Al inhibition alone is not sufficient to explain hepatotoxicity characterized by Hy's 
Law because the more potent inhibitor of UGT1Al activity was ritonavir which does not induce elevated bilirubin levels. Troglitazone exhibits significant hepatotoxicity and can be described by Hy's Law. It was shown to block the activity of UGT1A1, MRP2 and OATP1B1. However, although bromfenac and labetalol can also be described by Hy's Law, they were poor inhibitors of OATP1B1 and MRP2. These results suggest that classification under Hy's Law cannot be predicted by UGT1A1, MRP2 nor OATP1B1, and that other factors such as BSEP may be important.

\title{
P339. Involvement of P-glycoprotein in the brain distribution of blonanserin
}

\author{
Tomoko Inoue $^{1}$, Masaaki Tagawa ${ }^{1}$, Kenichi Osada ${ }^{2}$, Yuriko Ogawa ${ }^{2}$, Toshihisa Haga ${ }^{2}$, Yoshihisa \\ Sogame ${ }^{1}$, Takashi Katsumata ${ }^{1}$, Noboru Yamaguchi ${ }^{2}$ and Masashi Yabuki ${ }^{1}$

\begin{abstract}
${ }^{1}$ Pharmacokinetics Research Laboratories, Dainippon Sumitomo Pharma Co., Ltd., Osaka, Japan, ${ }^{2}$ Department of Neuropsychiatry, St. Marianna University School of Medicine, Kawasaki, Japan
\end{abstract}

Blonanserin (BNS), a new atypical antipsychotic drug with dopamine $\mathrm{D}_{2}$ and serotonin $5-\mathrm{HT}_{2 \mathrm{~A}}$ antagonistic properties, is efficacious for use in patients with schizophrenia, and has been launched in Japan and South Korea. Several clinical trials suggest that BNS is noninferior to risperidone (RIS) for the primary endpoint (PANSS total score), and BNS has fewer reports than RIS of hyperprolactinemia (a major adverse effect of antipsychotics). BNS is a liposoluble compound and displays good brain distribution properties in preclinical studies, but its distribution mechanism has not been clarified. By contrast, RIS and its active metabolite, paliperidone (PAL), are known to be substrates of P-glycoprotein (P-gp/ MDR1), an efflux transporter expressed in various tissues. Importantly, P-gp expressed in cerebral vascular endothelial cells limits entry of drugs (e.g. antipsychotics) into the central nervous system (CNS). In this study, using in vitro and in vivo approaches, we evaluated whether BNS, like RIS and PAL, is a P-gp substrate. In the in vitro study, affinity for P-gp at efficacious concentrations of BNS, RIS and PAL (around $\mathrm{C}_{\max }$ in clinical dosing) was investigated by transcellular transport system across LLC-PK1 and LLC-PK1 expressing human MDR1. In the in vivo study, the exposure of these drugs in the brain and plasma (B/P ratio) was measured in $m d r 1 a / 1 b$ knockout (KO) and wild-type (WT) mice (ip, $1 \mathrm{mg} /$ $\mathrm{kg}$ ). The results of our in vitro study indicated that P-gp actively transports RIS and PAL, but not BNS, as substrates (net flux ratio: 3.4, 5.2 and 1.0, respectively). Furthermore, in the in vivo study, BNS gave comparable B/P ratios in KO and WT mice, whereas, B/P ratios for RIS and PAL differed markedly between these animals (8.2 and 17.1-fold for RIS and $\mathrm{PAL}$, respectively). These results suggest that P-gp is likely to influence the brain distribution of RIS and PAL, but not that of BNS.

\section{P340. Loxapine P-glycoprotein interactions in vitro}

\author{
Andrea Reed ${ }^{1}$, Elke Perloff $^{2}, \underline{\text { Keith Huie }}^{1}$, Lori H. Takahashi ${ }^{1}$ and James Cassella ${ }^{3}$ \\ ${ }^{1}$ PK/ADME, Alexza Pharmaceuticals Inc, Mountain View, CA, ${ }^{2}$ Dept of Discovery Labware, BD Sioscis, Woburn, MA, \\ ${ }^{3}$ Alexza Pharmaceuticals Inc, Mountain View, CA
}

Loxapine is a dibenzoxazepine antipsychotic used in the treatment of schizophrenia. Because antipsychotic drugs such as risperidone, paliperidone, olanzapine, have been reported to have various degrees of interaction (substrate or inhibitor) with the multidrug resistance transporter, P-glycoprotein (P-gp), the interaction of loxapine with P-gp was evaluated. The permeability, bidirectional transport and P-gp interaction potential of loxapine was determined in vitro using Caco-2 cells. Caco-2 cell monolayers were used after 21-23 days of culture in 24-well-format diffusion chambers. The apical (A) to basolateral (B) transport study was determined using a loxapine concentration of $1 \mu \mathrm{M}$ in transport media with 1\% DMSO in the donor (A) chamber ( $\mathrm{pH}$ 7.4). The bidirectional transport of loxapine was conducted in the presence or absence of the known P-gp inhibitor ketoconazole. For assessing P-gp inhibition, bidirectional transport of radiolabeled digoxin was determined in the presence and absence of loxapine at concentrations ranging from $0.05-50 \mu \mathrm{M}$. Permeability comparators (propranolol and mannitol) were included in all studies, and ketoconazole, cyclosporine and verapamil were used as comparator controls for P-gp inhibition. Lucifer yellow and TEER values were used in all studies to assess monolayer integrity. Loxapine concentrations were determined by LC/MS/MS, and digoxin, comparator compounds and positive controls were measured by liquid scintillation. $\mathrm{IC}_{50}$ calculations were calculated by non-linear regression based on the Hill equation. The apparent permeability coefficient $\left(\mathrm{P}_{\text {app }}\right.$ for loxapine in the A to B direction was $67 \mathrm{~nm} / \mathrm{s}$, and $73 \mathrm{~nm} / \mathrm{s}$ in the B to A direction, resulting in a polarization ratio close to unity. In addition, ketoconazole had no influence on the polarization ratio. Thus, unlike risperidone, olanzapine and aripiprazole, loxapine is not a substrate for P-gp. Loxapine demonstrated concentration dependent inhibition of digoxin efflux, reducing the digoxin efflux ratio 
to 1.4 at a loxapine concentration of $50 \mu \mathrm{M}$ suggesting that loxapine, like other antipsychotics olanzapine, paliperidone and risperidone, is a moderate inhibitor of P-gp in vitro.

\title{
P341. Molecular identification of a novel prostaglandin $\mathrm{E}_{2}$ transporter
}

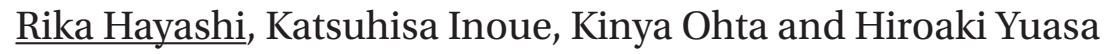 \\ Graduate School of Pharmaceutical Sciences, Nagoya City University, Nagoya, Japan
}

Prostaglandin $\mathrm{E}_{2}\left(\mathrm{PGE}_{2}\right)$ is a primary product of arachidonic metabolism, and plays a key role in the regulation of physiological and pathological homeostasis within the body. $\mathrm{PGE}_{2}$ is synthesized by the sequential actions of cyclooxygenase- 1 and -2 , and specific prostaglandin synthases, and then released outside the cells. Since $\mathrm{PGE}_{2}$ is a charged anion and diffuses poorly across the cellular membranes, carrier-mediated transport systems are needed for its efficient trafficking. However, the export mechanism of prostaglandins has not been fully elucidated yet. Therefore, we attempted to identify one of transporters involved in the export of prostaglandins synthesized intracellularly. In the present study, we selected the candidate genes that may encode a prostaglandin transporter by in silico screening and identified the one that is expressed in LNCaP human prostate adenocarcinoma cells, which are capable of producing prostaglandins. Its cDNA cloned from LNCaP cells was introduced into Madin-Darby canine kidney (MDCK) II cells for stable transfection. Functional characterization of the transporter, which is herein named prostaglandin efflux transporter (PGET), was conducted by evaluation of the uptake of $\left[{ }^{3} \mathrm{H}\right] \mathrm{PGE}_{2}$ into the transfected cells. The specific uptake of $\mathrm{PGE}_{2}$ mediated by PGET was saturable with a Michaelis constant of $24 \mu \mathrm{M}$, which indicates a fairly high affinity. The specific uptake was found not to depend on $\mathrm{Na}^{+}$, but was dependent on the extracellular $\mathrm{pH}$, being optimal at acidic pHs. At pH 7.4, interestingly, $\mathrm{PGE}_{2}$ preloaded in the cells was found to be rapidly effluxed. Therefore, PGET may be involved in the export of $\mathrm{PGE}_{2}$ from the cells physiologically. PGET-mediated $\mathrm{PGE}_{2}$ uptake was inhibited by sulfobromophthalein, an inhibitor of anion transporters, indicating its characteristic as an anion-sensitive transporter for PGE $_{2}$. Furthermore, it was found that many other prostaglandins, except for arachidonate, inhibited PGE $_{2}$ uptake by PGET, suggesting that PGET can specifically recognize prostaglandin derivatives, and mediate their transports. Thus we have successfully identified PGET as a novel prostaglandin transporter that may be involved in the export of prostaglandins from the cells. PGET may play a role in the cell signalings by prostaglandins by modulating their extracellular concentrations.

\section{P342. Mapping transporter interactions of reference P-glycoprotein inhibitors}

\section{Márton Jani}

Research \& Development, SOLVO Biotechnology, Budaors, Hungary

Specific inhibition of P-gp is crucial in drug - transporter interaction studies, especially in assay systems based on primary or immortalized cells, or in in vivo studies. However transporter inhibition profile data are scarcely available on commonly used compounds that are considered P-gp specific. The inhibitory interactions of P-gp inhibitors on a set of human drug transporters was undertaken in cellular uptake and vesicular transport settings. The panel of compounds was taken from the 2006 FDA Drug Interaction Draft Guidance. Generally third generation inhibitors provided the lowest IC50s as well as highest selectivity. Notable cross reactions were observed on OATPs, BSEP as well as OCTs in the case of cationic inhibitors.

\section{P343. Oatp1a/1b knockout mouse model useful for evaluating the importance of Oatp1a/1b transporters for hepatic uptake of SN-38, but not irinotecan}

\author{
Dilek Iusuf $^{1}$, Marion Ludwig ${ }^{1}$, Ahmed Elbatsh ${ }^{1}$, Anita van Esch ${ }^{1}$, Evita van de Steeg ${ }^{1}$, Els Wagenaar ${ }^{1}$, \\ Olaf van Tellingen ${ }^{2}$ and Alfred H. Schinkel ${ }^{1}$
}

\author{
${ }^{1}$ Molecular Biology, Netherlands Cancer Institute, Amsterdam, Netherlands, ${ }^{2}$ Department of Clinical Chemistry, \\ Netherlands Cancer Institute, Amsterdam, Netherlands
}

Organic anion transporting polypeptides (OATPs in humans, Oatps in other species) are sodium-independent transmembrane proteins that mediate cellular uptake of a broad range of endogenous and exogenous compounds. Polymorphisms 
affecting the uptake capacity of OATP1B1 (the most studied of the human OATPs) are associated with life-threatening toxicity upon irinotecan treatment, due to increased plasma concentrations of its cytotoxic metabolite, SN-38 (1). Here, we use the recently generated Oatpla/1b knockout mouse model $\left(\right.$ Slcola/1 $b^{-/}$, (2)) to investigate the role of Oatpla/lb in the disposition of irinotecan and SN-38 in vivo. Upon intravenous administration of $10 \mathrm{mg} / \mathrm{kg}$ irinotecan to male wildtype and Slcola/l $b^{-/}$mice, plasma levels of both irinotecan and SN-38 were significantly increased in the Oatpla/1b knockout mice, leading to 2- and 3-fold increased AUCs respectively. Despite the increased plasma exposure, the liver concentrations were not significantly different between the two strains of mice, although the liver concentrations tended to be slightly lower in the Oatpla/lb knockout mice. However, an impaired liver uptake of irinotecan and SN-38 was evident in the significantly lower liver-to-plasma ratios in the absence of Oatpla/lb. We also investigated if irinotecan and/or SN-38 distribute extensively to erythrocytes in vitro, and what is the role of Oatpla/1b in this process. We added irinotecan or SN-38 to fresh isolated blood from mice, and then determined the amount recovered in plasma and erythrocytes. Unexpectedly, upon addition of irinotecan to blood isolated from Oatpla/lb knockout mice, there was a much higher conversion rate of irinotecan to SN-38 in comparison with wild-type blood. Irinotecan is converted to SN-38 by carboxyesterases (CES) occurring in plasma and liver. We subsequently demonstrated that liver mRNA levels of Ces 3 , the gene encoding one of the enzymes responsible for the conversion of irinotecan to SN-38, were 600 -fold higher in the Slcola $/ 1 b^{-/}$mice. To circumvent this confounder, we administered SN-38 directly intravenously $(1 \mathrm{mg} / \mathrm{kg})$ and evaluated the biliary output of SN-38 in 10 min bile fractions collected in gall bladder cannulated-mice. Absence of Oatpla/1b resulted in a significantly decreased cumulative biliary excretion of SN-38 starting within 20 min after administration. Based on this data, we conclude that the Oatpla/lb knockout model is not a suitable model to investigate the disposition of irinotecan (because of the Ces3 upregulation), but there is some evidence that murine Oatpla/lb uptake transporters have an impact on the disposition of SN-38 after direct administration of this metabolite.

1. Takane H, Kawamoto K, Sasaki T, Moriki K, Moriki K, Kitano H, et al. Life-threatening toxicities in a patient with UGT1A1*6/*28 and SLCO1B1*15/*15 genotypes after irinotecan-based chemotherapy. Cancer Chemother Pharmacol 2009 May;63(6):1165-1169.

2. van de Steeg E, Wagenaar E, van der Kruijssen CM, Burggraaff JE, de Waart DR, Elferink RP, et al. Organic anion transporting polypeptide $1 \mathrm{a} / 1 \mathrm{~b}$-knockout mice provide insights into hepatic handling of bilirubin, bile acids, and drugs. J Clin Invest 2010 Aug 2;120(8):2942-2952.

\title{
P344. PET Imaging of P-glycoprotein inhibition at the human blood-brain barrier by quinidine
}

\author{
$\underline{\text { Li Liu }}^{1}$, Ann C. Collier², Jeanne M. Link ${ }^{3}$, Karen B. Domino4, David A. Mankoff ${ }^{3}$, Janet F. Eary ${ }^{3}$, Peng \\ Hsiao $^{1}$, Anand K. Deo ${ }^{1}$ and Jashvant D. Unadkat ${ }^{1}$
}

${ }^{1}$ Pharmaceutics, University of Washington, Seattle, WA, ${ }^{2}$ Medicine, University of Washington, Seattle, WA, ${ }^{3}$ Nuclear Medicine, University of Washington, Seattle, WA, ${ }^{4}$ Anesthesiology \& Pain Medicine, University of Washington, Seattle, WA

Permeability-glycoprotein (P-glycoprotein, P-gp), an efflux transporter at the blood-brain barrier (BBB), is believed to be one of the primary obstacles in the CNS delivery of many P-gp substrate drugs. The functional importance of P-gp at the human BBB was first demonstrated by our laboratory using a model P-gp substrate $\left({ }^{11} \mathrm{C}\right.$-verapamil), a model P-gp inhibitor (cyclosporine-A, CsA), and Positron Emission Tomography (PET) imaging. CsA, at supertherapeutic blood concentration $(\sim 3 \mathrm{mM})$, modestly increased (by $79 \%$ ) the brain-to-plasma AUC ratio of ${ }^{11} \mathrm{C}$-verapamil radioactivity ${ }^{1}$. To determine if this interaction would be greater with another P-gp inhibitor, we determined the inhibition of P-gp at the human BBB using a FDA-approved potent P-gp inhibitor, quinidine. After obtaining institutional review board approval and each subject's consent, 7 healthy volunteers ( 4 women and 3 men) were administered ${ }^{11} \mathrm{C}$-verapamil ( $\sim 0.1$ $\mathrm{mCi} / \mathrm{kg}, \sim 0.07 \mu \mathrm{g} / \mathrm{kg})$ intravenously before and after 1 hour of quinidine IV infusion $(0.153 \mathrm{mg} / \mathrm{kg} / \mathrm{min})$. Prior to each ${ }^{11} \mathrm{C}$-verapamil administration, ${ }^{15} \mathrm{O}-\mathrm{H}_{2} \mathrm{O}(\sim 0.5 \mathrm{mCi} / \mathrm{kg})$ was administered to measure cerebral blood flow (Q). During each PET-imaging session, brain images and frequent arterial blood samples were obtained for $5\left({ }^{15} \mathrm{O}-\mathrm{H}_{2} \mathrm{O}\right)$ or $20\left({ }^{11} \mathrm{C}-\right.$ verapamil) mins to measure radioactivity content in these tissues. Plasma ${ }^{11} \mathrm{C}$-verapamil and metabolites' radioactivity were quantified by rapid solid-phase extraction. The tissue uptake $\left(\mathrm{AUC}_{\text {tissue }} / \mathrm{AUC}_{\mathrm{plasma}}\right.$ ratio), distribution clearance $\left(\mathrm{CL}_{12}\right)$ and extraction ratio, $\mathrm{ER}\left(\mathrm{CL}_{12} / \mathrm{Q}\right)$, of ${ }^{11} \mathrm{C}$-radioactivity for the whole brain, gray and white matter during the first 10 mins (when metabolism of ${ }^{11} \mathrm{C}$-verapamil is minimal) were determined in the presence and absence of quinidine, and compared using the Student's paired t-test. In the presence of quinidine (at $3.1 \pm 0.4 \mu \mathrm{g} / \mathrm{mL}$ pseudo steady-state plasma concentration), the $\mathrm{AUC}_{\text {tissue }} / \mathrm{AUC}_{\text {plasma }}$ ratios were significantly $(\mathrm{p}<0.005)$ increased for the whole brain $(84 \pm 42 \%)$, gray matter $(84 \pm 42 \%)$, or white matter $(87 \pm 43 \%)$. Quinidine did not affect the extent of ${ }^{11} \mathrm{C}$-verapamil metabolism. A 2 -tissue compartment model using a single input function of total plasma radioactivity was determined to be the best model for estimating $\mathrm{CL}_{12}$. In the presence of quinidine, the average $\mathrm{CL}_{12}$ and $\mathrm{ER}$ estimates were significantly increased $(\mathrm{p}<0.05)$ 
for the brain $(78 \pm 68 \%, 62 \pm 64 \%)$, gray matter $(68 \pm 40 \%, 63 \pm 81 \%)$ and white matter $(94 \pm 76 \%, 78 \pm 83 \%)$. These data demonstrate that the inhibition of P-gp activity at the human BBB observed with CsA can bereproduced using another P-gp inhibitor, quinidine. However, the level of inhibition was comparable to that obtained with CsA, indicating that quinidine at the plasma concentrations achieved is equipotent to CsA in inhibiting P-gp in vivo. Therefore, at therapeutic concentrations, quinidine cannot be used to maximally inhibit P-gp at the human BBB in order to increase CNS delivery of P-gp substrate drugs. More potent FDA-approved P-gp inhibitors, with a wider therapeutic window, are needed to maximally inhibit P-gp at the human BBB to significantly increase CNS delivery of P-gp substrate drugs. Supported by NIHRCNS06804 and TL1RR025016

1. Sasongko L, Link JM, Muzi M, MankoffDA, Yang X, Collier AC, Shoner SC, Unadkat JD. Imaging P-glycoprotein transport activity at the human blood-brain barrier with positron emission tomography. Clin Pharmacol Ther. 77(6):503-14. 2005

\title{
P345. Primary human renal proximal tubule cell model for study of transepithelial transport
}

\author{
Caitlin Brown $^{1}$, Yuko Kosaka², Marc J. Evanchik², Scott Heyward ${ }^{1}$ and Ji Young Lee ${ }^{1}$ \\ ${ }^{1}$ Celsis In Vitro Technologies, Baltimore, $M D,{ }^{2} 3-V$ Biosciences, Menlo Park, CA
}

Renal elimination is a major clearance route for many drugs and their metabolites. This includes active tubular secretion, which takes place primarily in the proximal tubule (PT) cells, and is mediated by uptake and efflux transporters. Currently, most in vitro kidney transporter data are obtained from renal slices, isolated tubule fragments, or animalderived cell lines that can exhibit differing transport characteristics or altered transporter behavior compared to human. Primary cells cultured on transwells have been shown to be an effective in vitro model and may represent a more physiologically relevant system. ${ }^{1,2}$ To that end, freshly isolated primary kidney cells were plated onto semipermeable membrane filters and assessed for monolayer integrity and transporter functionality. A gamma glutamyltransferase (GGT) assay was used to determine percentage of PT cells with respect to distal tubule (DT) cells. An approximate 3:1 ratio of PT to DT cells has been determined to be optimal for appropriate tight junction formation. ${ }^{2}$ In addition, transepithelial electrical resistance (TEER) measurements and mannitol permeability verified monolayer integrity. Prototypical substrates, para-aminohippuric acid ( $\mathrm{PAH}$ ) and 1-methyl-4-phenylpyridinium $\left(\mathrm{MPP}^{+}\right)$, were used to evaluate expression of the key transporter pathways OATs to MRP2/4 and OCT2 to MATE1, respectively. A flux ratio of secretory to absorptive (B $\rightarrow$ A/ $\mathrm{A} \rightarrow \mathrm{B}$ ) greater than 1.5 was defined as significant and corresponded well to TEER measurements starting at about 110 $\mathrm{ohms} / \mathrm{cm}^{2}$. Transporter function diminished or was abolished at much higher TEER values. Shipment of these transwell cultures on agarose media caused a drop in TEER values and a loss of net flux after transfer to liquid media. However, after some days in culture, transepithelial transport was re-established as measured by $\mathrm{PAH}$ and $\mathrm{MPP}^{+}$movement. Thus, a primary kidney cell model has been shown to present appropriate transporter functioning and retention of these transporters upon shipment in an agarose system.

1. Lash LH, Putt DA, Hongliang C. 2006 Aug 24. Membrane transport function in primary cultures of human proximal tubular cells. Toxicology 228: 200-218.

2. Brown CDA, Sayer R, Windass AS, Haslam IS, De Broe ME. 2008 Sept 18.Characterisation of human tubular cell monolayers as a model of proximal tubular xenobiotic handling. Toxicol Appl Pharm 223: 428-438.

\section{P346. Abstract withdrawn}

\section{P347. Rapid screening of human OATP1B1 and OATP1B3 mediated drug interactions in stably transfected human embryonic kidney HEK-293 cell lines using flow cytometry and fluorescence microplate methods}

\author{
Steve Wright ${ }^{1}$, Ying Wang ${ }^{2}$, Jibin $\mathrm{Li}^{2}$, Albert Owen² and $\underline{\text { Ismael Hidalgo }}{ }^{2}$ \\ ${ }^{1}$ Department of Biology, Carson-Newman College, Jefferson City, TN, ${ }^{2}$ Absorption Systems LP, Exton, PA
}

Purpose.Human organic anion transporting polypeptides (OATP) 1B1 and 1B3 are predominantly expressed at the sinusoidal membrane of hepatocytes and play an important role in the hepatic uptake of numerous drugs including statins. Inhibition of OATP1B1/1B3-mediated drug transport may reduce statin liver uptake and/or efficacy, and elevate systemic exposure. Therefore, it is imperative to have reliable assay systems for identifying potential 
OATP1B1- and OATP1B3-mediated adverse drug reactions. The objective of this study was to establish rapid in vitro inhibition assays for screening OATP1B1- and OATP1B3-mediated drug interactions in stably transfected HEK293 cells using either flow cytometry or a fluorescence microplate reader. Methods. For flow cytometry, HEK293 cells, stably transfected with OATP1B1 or OATP1B3 or the control vector, were cultured asmonolayers, trypsinized, and then assayed in suspension; for the fluorescence microplate reader, cells were cultured in 96-well plates. Fluoresceinmethotrexate (FMTX) was used as a probe substrate. Cellular uptake of FMTX in transfected and control cells was determined by flow cytometry or the fluorescence microplate reader. Results. The suitability of the assays was determined by Z' factor, a simple statistical parameter for evaluating high throughput screening assays. The calculated Z' factors for the OATP1B1 and OATP1B3 assays were 0.83 and 0.89 , respectively, by flow cytometry, and 0.76 and 0.75 , respectively, by the fluorescence microplate reader method. Those $\mathrm{Z}$ ' factor values indicate that both methods are suitable for measuring inhibition of OATP1B1- and OATP1B3-mediated transport. The application of these two methods for screening drug interactions with OATP1B1 and OATP1B3 transporters was illustrated by investigating some well-known OATP substrates and/or inhibitors including statins, E3S, and digoxin. FMTX uptake assays using the fluorescence microplate reader conducted in the presence of those compounds accurately reflected their inhibitory potency against OATP1B1 and OATP1B3. Compounds known to interact with OATPs inhibited OATP-mediated FMTX cellular uptake relative to control cells and displayed concentration-dependent inhibition. IC $_{50}$ values were determined for those individual compounds. E3S and pitavastatin had lower IC $_{50}$ values in OATP1B1 cells than OAPT1B3s, indicating higher inhibition potency towards OATP1B1. For example, the $\mathrm{IC}_{50}$ value of E3S was $0.08 \mu \mathrm{M}$ in OATP1B1 but $25.95 \mu \mathrm{M}$ in OATP1B3 cells. Such results are consistent with previous findings that E3S and pitavastatin are more OATP1B1 specific substrates. Conclusion.The in vitro assays using either flow cytometry or fluorescence microplate methods demonstrated utility for identifying potential drug-drug interactions associated with human OATP1B1 and OATP1B3 transporters.

\title{
P348. Regulation of hepatic uptake and efflux transporters in vitro
}

\author{
Gabrielle M. Hawksworth $^{1}$, Muhammad Farooq ${ }^{1}$, Colin J. Henderson ${ }^{2}$ and Richard J. Weaver ${ }^{3}$
}

${ }^{1}$ Division of Applied Medicine, University of Aberdeen, Aberdeen, United Kingdom, ${ }^{2}$ Biomedical Research Institute, Cancer Research UK Molecular Pharmacology Unit, Dundee, United Kingdom, ${ }^{3}$ Drug Safety Assessment Centre, Biologie, Servier, Gidy, France

The nuclear receptors, pregnane $\mathrm{X}$ receptor, PXR (Nrli2), and farnesoid X receptor, FXR (Nrlh4), have been implicated in the regulation of hepatic uptake transporters: the multispecific organic anion transporter, Oatpla4 (Slcola4), the sodium-dependent taurocholate co-transporting polypeptide, Ntcp (Slc10a1), and efflux transporters: multidrug resistance-associated protein 2, Mrp2 (Abcc2), the bile salt export pump, Bsep (Abcb11)1. We have previously shown differential expression of Oatps in response to PXR ligands, with a correlation between Oatpla4 mRNA, protein and enzyme activity ${ }^{2}$. The aim of this study was to establish the relative roles of PXR and FXR in the regulation of these transporters using ligands specific for these nuclear receptors. Hepatocytes isolated from male Sprague Dawley rats

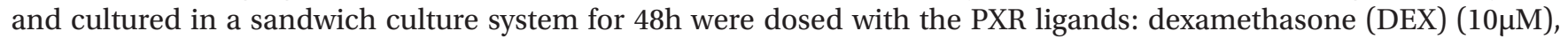
spironolactone (SPR) $(10 \mu \mathrm{M})$ and pregnenolone 16a-carboninitrile $(\mathrm{PCN})(10 \mu \mathrm{M})$ and the FXR ligands: chenodeoxycholic acid (CDCA) $(10 \mu \mathrm{M})$, GW4064 (GW) $(10 \mu \mathrm{M})$ and 6-ethyl chenodeoxycholic acid (6-ECDCA) (10 $\mu \mathrm{M})$. After 96h the cells were harvested, lysed and mRNA levels quantified using quantitative Taqman ${ }^{\circledR}$ real-time PCR and expressed as copy number/ng total RNA. The PXR ligands caused 4-6 fold induction of PXR (Control = 162) without showing any effect on FXR (control = 174) expression, whilst FXR ligands resulted in 5-7 fold induction of FXR along with up regulation of PXR expression, suggesting a potential role of FXR in the regulation of PXR. The PXR ligands induced the expression of Oatpla4 (Control=210), Mrp2 (Control=175) and Bsep (Control=316) 5-8 fold and resulted in 2-3 fold up regulation of Ntcp (Control = 118), whereas the FXR ligands induced Mrp2 and Bsep expression 7-9 fold and down-regulated Oatpla4 and Ntcp expression. We concluded from this study that the expression of PXR and FXR is increased at the transcriptional level in vitro and FXR appears to regulate PXR expression. PXR up regulates both uptake and efflux transporters, whereas FXR up regulates efflux transporters and down regulates the uptake transporters. These results correlate with our previously reported in vivo findings and have implications for both drug-drug interactions (Oatpla4 and Mrp2) and drug-induced cholestasis (Ntcp and Bsep).

\section{References}

1 Cui, Y.J., Aleksunes, L., Tanaka, Y., Goedken, M.J., Klaassen, C.D. 2009 Toxicology 110(1), 47-60

2 Cowie, D.E., Weaver, R.J., Hawksworth, G.M. 2006 Toxicology 226(1), 42-43 


\title{
P349. Abstract withdrawn
}

\section{P350. The impact of grapefruit juice on the active uptake of drugs by hepatic transporters}

\author{
Ron Scialis, Bo Feng, Emi Kimoto, Yurong Lai, Kevin Whalen and Ayman El-Kattan
}

Pharmacokinetics, Dynamics and Metabolism, Pfizer Inc., Groton, CT

It is well known that grapefruit juice (GFJ) possesses components that can modulate drug pharmacokinetics. For instance, furanocoumarins (e.g., bergamottin) found in GFJ could inhibit cytochrome P450 3A4 thereby affecting firstpass metabolism of xenobiotics. In addition, flavonoids in GFJ have been demonstrated to inhibit organic anion-transporting polypeptides (OATPs). The effects, however, were thought to occur locally in the intestinal lumen. Recently, therapeutics targeting the liver via hepatic-specific OATPs have shown promise at increasing efficacy while reducing off-target effects. Therefore, the objective of our current work was to elucidate the potential systemic effect of GFJ on liver OATPs using cellular in vitro models. First, two commercially available ruby red GFJ brands were used at a single dilution and co-incubated with probe compounds in human embryonic kidney (HEK 293) cells stably transfected with major hepatic uptake transporters including OATP1B1, OATP1B3, or OATP2B1. The data indicated that GFJ, even at a dilution of $1 \%(\mathrm{v} / \mathrm{v})$, was able to significantly reduce the active uptake of pravastatin or rosuvastatin in the HEK-OATP cells. The extent of inhibition was compatible between the two GFJ brands. Diluted GFJ did not have any effect on compounds that are passively taken up into cells indicating that OATP inhibition was the primary cause for the observed outcome. Furthermore, active uptake of probes significantly decreased as the concentration of GFJ increased. At 10\% GFJ, pravastatin uptake fell by $80 \%$ compared to controls in HEK-OATP1B1 and HEK-OATP1B3 cells, whereas the same level of GFJ was attributed to a 50\% decline in rosuvastatin uptake in HEK-OATP2B1. Additionally, sandwich-cultured human hepatocytes (SCHH) were utilized to ascertain the extent of GFJ modulation on a more physiologically representative model. As pravastatin does not undergo extensive metabolism, its application in the SCHH model permitted assessment of GFJ inhibition on OATP-mediated uptake. At 5\% GFJ concentration, pravastatin uptake in SCHH was reduced by 67 to $100 \%$ compared to controls for the two GFJ brands. In summary, the results indicate that GFJ, even at low concentrations, has the potential to significantly modulate hepatic OATP function in two separate in vitro models. Moreover, the uptakes of OATP substrates were inhibited by GFJ in a dose-dependent manner. Further work is ongoing to explore the degree of GFJ inhibition of OATP uptake in vivo.

\section{P351. Transporter-based in vitro-in vivo extrapolation (IVIVE)}

\author{
Viera Lukacova $^{1}$, Michael B. Bolger ${ }^{1}$, Walter Woltosz ${ }^{1}$, Neil Parrott ${ }^{2}$, Agnès Poirier ${ }^{3}$ and Thierry Lavé ${ }^{3}$ \\ ${ }^{1}$ Simulations Plus Inc., Lancaster, CA, ${ }^{2}$ Non-clinical development - Drug safety, F.Hoffmann-La Roche Ltd., Basel, \\ Switzerland, ${ }^{3}$ Dept of Non-Clin Drug Safety 69/154, Hoffmann-La Roche Ltd, Basel, Switzerland
}

The use of in vitro data to predict pharmacokinetics of drugs whose disposition is mediated by transporters is complicated due to unknown transporter expression levels in individual tissues in vivo and in different in vitro cell culture systems. The contribution of passive diffusion to drug transfer between extracellular and intracellular space in individual tissues is another important aspect to consider for drugs with low permeability and slow diffusion through cellular membranes. The permeability-surface area product (PStc) is commonly used to describe the rate of passive diffusion through membranes. Estimation of PStc values for different tissues incorporated in PBPK models is not well-established due to some unknown physiological aspects of individual tissues, e.g., cell surface areas. We propose a new method for describing the passive diffusion in different tissues by scaling the PStc values to the tissue cell volumes. The method was further extended to predict the contribution of passive and carrier-mediated transport in vivo from in vitro measurements. Valsartan was used as a model compound with distribution and clearance dependent on the transporter activity. GastroPlus" 7.0 with its PBPKPlus"' Module (Simulations Plus, Inc., Lancaster, CA) was used to simulate plasma concentration-time (Cp-time) profiles utilizing physiologically based pharmacokinetic (PBPK) models based on animal and human physiologies. Drug partitioning into the extracellular space was described by an extracellular water-plasma partition coefficient, taking into account binding of drug to plasma and extracellular tissue proteins. The carrier-mediated transport kinetics in liver was predicted from previously reported in vitro parameters measured in cultured hepatocytes (Poirier 2009). Passive diffusion between the extracellular and intracellular spaces in liver was described by the permeability-surface area product (PStc), which was also predicted from previously reported values measured in vitro (Poirier 2009). The passive diffusion in other tissues was described by PStc values scaled from the liver PStc according to 
the individual tissue volumes. Simulations using a combination of kinetic parameters from the relevant in vitro system (cultured hepatocytes) with physiologically based scaling of the passive diffusion rate across all tissues resulted in very good prediction of total valsartan exposure and provided the correct shape of the predicted $\mathrm{Cp}$-time profile in rat and human based on in silico and in vitro parameters. This method is a promising tool for prediction of the pharmacokinetics of drugs whose disposition cannot be described by well-stirred tissue models and it expands the predictive capabilities of PBPK modeling approaches for prediction of pharmacokinetics based on in vitro data to a wider range of compounds.

Poirier A. et al. (2009) Prediction of pharmacokinetic profile of valsartan in human based on in vitro uptake transport data. J Pharmacokinet Pharmacodyn 36: 585-611

\title{
P352. Using Lentiviral Vector-Based shRNA to silence the expression and function of ABC Transporter(s) in Caco-2 Cells
}

\author{
Chris Bode, Sid Bhoopathy and Ismael J. Hidalgo
}

\section{Absorption Systems LP, Exton, PA}

Due to the fact that almost all pharmacologic reagents (probe substrates and inhibitors) for drug transporters are not sufficiently selective, there is an unmet need for more definitive in vitro test systems for drug transporters. To address this need, lentiviral vectors expressing shRNA targeting individual human efflux transporters were created. Human Caco-2 cells, derived from a colon adenocarcinoma, were exposed to the vectors, and clones were selected with substantial and stable knockdown of the apical efflux transporters P-gp (MDR1, ABCB1), BCRP (ABCG2), or MRP2 (ABCB2). Various applications of the knockdown cell lines (CPT-P1, P-gp knockdown; CPT-B1, BCRP knockdown; CPT-M1, MRP2 knockdown) have been characterized. Substrates of P-gp and BCRP are identified by performing bidirectional monolayer transport assays with test compound in parental and knockdown cells and comparing efflux ratios in one line vs. the other. The transporters involved in the biliary efflux of a test compound is/are identified in a similar manner, but with the entire panel of knockdown cell lines. Inhibition of BCRP is evaluated in the P-gp knockdown cells using cladribine, a relatively specific BCRP probe substrate whose off-target interaction with P-gp (detected with MDR1-MDCK cells) could confound the interpretation of results obtained in parental Caco-2 cells. False positive and false negative results obtained with other test systems are avoided through the use of the knockdown cells. Finally, the use of knockdown cells with a complement of relevant human uptake transporters enables the elucidation of complex interactions among uptake and efflux transporters, which has been studied with the knockdown cells. These applications will be covered in this presentation.

\section{P353. Zinc finger nuclease (ZFN) technology to selectively knockout the expression of MDR1 and BCRP individually and in combination}

\author{
Amanda Brinker, Neetu Venkatraman, Jennifer Pratt, Yongling Xiao, Tim Brayman and Maureen \\ Bourner
}

Sigma-Aldrich, Saint Louis, MO

ATP-binding cassette (ABC) transporters are a family of transmembrane proteins that utilize ATP hydrolysis for translocation of substrates across membranes. Importantly, ABC transporters are known to play a crucial role in the development of multidrug resistance. Evaluation of membrane transporter pharmacology in drug absorption, disposition, and drugdrug interactions (DDI) is critical to the pharmaceutical industry's safety evaluation of new drug entities. With this goal in mind, the ABC transporters MDR1 and BCRP were selected for study. Selection was based on the considerable body of evidence supporting their crucial role in the development of multidrug resistance. BCRP and MDR1 were independently evaluated in C2BBel intestinal cells, a subclone of the extensively studied Caco-2 cell line. Both transporters are expressed in the luminal membrane and are ATP-dependent efflux pumps. The objective is to generate human knockout cell lines in relevant cell types that will clearly address the contribution of membrane transporters to the efficacy and safety of potential new drug candidates. Toward this end, Sigma Life Science is exploiting the zinc finger nuclease (ZFN) technology to selectively knockout the expression of BCRP and MDR1 individually as well as in combination in C2BBe1 cells for the generation of ADME toxicology cell lines. These transporter knockout cell lines are clinically significant, as they will be employed to identify the specific transporters involved in the clearance or exposure of new compounds. The development of these lines will help overcome the challenges of overlapping substrate and inhibitor specificities across transporters. All three of these cell lines were validated in A-B/B-A drug transporter studies. 